\title{
Relationship between room shape and acoustics of rectangular concert halls
}

\author{
Klosak, Andrzej K.; Gade, Anders Christian
}

Published in:

Acoustical Society of America. Journal

Link to article, DOI:

$10.1121 / 1.2933354$

Publication date:

2008

Document Version

Publisher's PDF, also known as Version of record

Link back to DTU Orbit

Citation (APA):

Klosak, A. K., \& Gade, A. C. (2008). Relationship between room shape and acoustics of rectangular concert halls. Acoustical Society of America. Journal, 123(5), 3199-3199. https://doi.org/10.1121/1.2933354

\section{General rights}

Copyright and moral rights for the publications made accessible in the public portal are retained by the authors and/or other copyright owners and it is a condition of accessing publications that users recognise and abide by the legal requirements associated with these rights.

- Users may download and print one copy of any publication from the public portal for the purpose of private study or research.

- You may not further distribute the material or use it for any profit-making activity or commercial gain

- You may freely distribute the URL identifying the publication in the public portal

If you believe that this document breaches copyright please contact us providing details, and we will remove access to the work immediately and investigate your claim. 


\title{
Session 2aAAa
}

\section{Architectural Acoustics: Acoustics of Concert Halls II}

\author{
Takayuki Hidaka, Cochair \\ Takenaka R\&D Institute, 1-5-1, Otsuka, 270-1395 Inzai, Chiba, Japan \\ Daniel E. Commins, Cochair \\ Commins Acoustics Workshop, 15 rue Laurence Savart, Paris, 75020, France
}

\section{Contributed Papers}

\section{8:00}

2aAAa1. Acoustical design consideration for the new IFEZ concert hall. Jin Yong Jeon (Hanyang University, Department of Architectural Engineering, 133-791 Seoul, Republic of Korea, jyjeon@hanyang.ac.kr), Yong Hee Kim (Hanyang University, Department of Architectural Engineering, 133-791 Seoul, Republic of Korea, kimyonghee@gmail.com), Shin-Ichi Sato (Hanyang University, Department of Architectural Engineering, 133791 Seoul, Republic of Korea, s_sato@mac.com)

The design of a new concert hall for the Incheon Free Economic Zone (IFEZ) Arts Center in Korea is in progress. The hall is supposed to be the home of the Asia Philharmonic Orchestra. It has over 1,700 seats, including 150 choirs. A vineyard seating arrangement will be applied, but the seats around the stage area will be minimized in order to form a solid stage enclosure. Lateral walls are designed for each seating block to increase acoustical intimacy. Average width between lateral walls has been designed to be less than $15 \mathrm{~m}$, and every seat is arranged within $7.5 \mathrm{~m}$ to the closest lateral wall. All lateral walls are designed to be inclined to lead first reflections and to improve spatial impression at the audience area. Diffusers are functionally installed to the effective reflecting surfaces from the source on stage. The design considerations are investigated through both computer simulation and scale modelling.

\section{8:20}

2aAAa2. Objective and subjective analysis of acoustical response in newly renovated Palais Moncalm, Quebec City, Canada. Jean-Philippe Migneron (Lab. d'acoustique, École d'architecture, 1, Côte de la Fabrique, Vieux Séminaire, Université Laval, Québec, QC G1K 7P4, Canada, jean-philippe.migneron.1@ulaval.ca), Jean-Gabriel Migneron (Lab. d'acoustique, École d'architecture, 1, Côte de la Fabrique, Vieux Séminaire, Université Laval, Québec, QC G1K 7P4, Canada, jgmigneron@hotmail.com), Jean-François Hardy (Lab. d'acoustique, École d'architecture, 1, Côte de la Fabrique, Vieux Séminaire, Université Laval, Québec, QC G1K 7P4, Canada, jean-francois.hardy.1@ulaval.ca)

To celebrate the 75th anniversary of Palais Montcalm, the building has been almost entirely rebuilt to convert it into the House of Music. The main room, named Salle Raoul-Jobin, has been designed specifically for the resident chamber orchestra: Les Violons du Roy. Collaboration between acoustical consultant, Larry S. King, and architect Jacques Plante of MUSE consortium results in a 979 seats concert hall with variable acoustic to accommodate different kinds of musical events. Since reopening in March 2007, the general acoustic has been enthusiastically acclaimed by professionals, by world known musicians, and by the public. The principal objective of this study is to characterize acoustical response of the room with various adjustments of wall curtains and motorized canopy. This important reflective area is software-controlled in a range of 3 meters depending on musical needs. Acoustical objective parameters, such as reverberation time, early decay time, impulse response or C80 factor will be analyzed and compared to musical subjective perception of a few instruments played at multiple positions on stage and for different listening locations.

\section{8:40}

2aAAa3. New design tendencies in modern concert hall design. Alban A. Bassuet (Arup Acoustics, 155 avenue of the americas, New York, NY 10013, USA, alban.bassuet@arup.com)

New concert hall design trends are emerging as seen from recently completed halls around the world and recent international architectural competitions. Analyzing the current situation, the paper starts by differentiating the acoustical characters of major traditional concert hall forms such as the large shoe-box, the Vineyard and the surround hall. It discusses in particular the balance of acoustical energy that characterize these different basic hall shapes, the sensation of intimacy in relation to the listener's distance to the performance area and the different early reflection patterns inherent from these hall shapes. As an example of modern concert halls design tendencies, the paper then describes a design option chosen by Arup Acoustics for the Paris Concert Hall Architectural design competition with Zaha Hadid architect. The paper describes the development of the form and shape of the hall in response to the brief, the concept chosen for the design of sendin$\mathrm{g} /$ receiving surfaces to improve the early reflection "efficiency" and "stability" and the use of an overhead reflector to improve the balance between soloist and orchestra and the balance between the orchestra and the reverberation in the hall.

\section{9:00}

2aAAa4. Acoustic design and evaluation of a multi-purpose hall of a new conference centre. Attila Balázs Nagy (Kotschy Bt., Álmos vezér u. 4, 2045 Törökbálint, Hungary, nagyab@hit.bme.hu), Ferenc Tamás (Kotschy Bt., Álmos vezér u. 4, 2045 Törökbálint, Hungary, tamas.ferenc@kotschy.hu), András Kotschy (Kotschy Bt., Álmos vezér u. 4, 2045 Törökbálint, Hungary, bandi@kotschy.hu)

At Forum Acusticum 2005 the building and room acoustic design of a new Conference Centre was presented. Since then the construction work has been finished and the Centre is now open. The Conference Centre itself is a multi-functional building, having three wings of different functions. The Main Hall of the Centre is a room for 750 people which can be extended with adjacent section rooms for 1100 persons, giving a total volume of $14000 \mathrm{~m}^{3}$. It was designed to host conferences, lectures, and - most of the time - to be used as a concert hall. The extremely different acoustical demands of being a concert hall and a lecture room had been fulfilled with appropriate room acoustic design and with variable acoustics by employing a DCR (Digital Control of Reverberation) system. In this paper we give a report on the achieved acoustical performance of the Main Hall of the Conference Centre. We have performed extensive room acoustic measurements in the Main Hall, the results of which are compared to the designed values and are presented in this paper. The design of the DCR system is discussed in a different paper. 


\section{9:20}

2aAAa5. Renovation of the concert hall The Doelen: A case study on the impact of a stage canopy on stage and room acoustics. Margriet $\mathrm{R}$. Lautenbach (Peutz BV, PO Box 696, 2700 AR Zoetermeer, Netherlands, m.lautenbach@zoetermeer.peutz.nl), Martijn Vercammen (Peutz, De Grippen 1124, 6605 TA Wijchen, Netherlands, m.vercammen@mook .peutz.nl), Klaus-Hendrik Lorenz-Kierakiewitz (Peutz BV, Kolberger Strasse 19, D-40599 Duesseldorf, Germany, khl@peutz.de)

When opened in 1966, the main concert hall in De Doelen, Rotterdam, the Netherlands, was provided with six canopies above the stage platform. Their function was twofold: to provide a large part of the audience with early reflections; to create good ensemble conditions for the musicians on stage. Despite good reviews after the opening, a few years later the canopies were removed to get rid of unwanted reflections at the recording microphones positions just below the canopy. Since then, a significant percentage of the orchestra is unhappy about the acoustic conditions on stage. During the design process of the renovation, possibilities to re-introduce a stage canopy are investigated. Objective acoustic parameters obtained by carrying out measurements in the hall as well as in a 1:10 scale model and by calculations with a ray-tracing computer model are examined. Parallel, three questionnaire rounds gave an impression about the musicians' subjective judgement about the stage acoustics. In this paper the acoustic differences of three situations (without canopy, with original canopies and with new canopy design) and the search for an optimal balance between improving the stage acoustics without altering room acoustical conditions in the audience are discussed.

\title{
Invited Paper
}

$$
\text { 9:40 }
$$

2aAAa6. Evaluation of virtual acoustic stage support for musical performance. Wieslaw Woszczyk (McGill University, Schulich School of Music, 555 Sherbrooke Street West, Montreal, QC H3A 1E3, Canada, wieslaw@music.mcgill.ca), William L. Martens (McGill University, Schulich School of Music, 555 Sherbrooke Street West, Montreal, QC H3A 1E3, Canada, wlm@music.mcgill.ca)

The effects of performance space acoustics on musical performance can be evaluated most effectively by eliminating the influence of powerful non-auditory factors, such as the visual appearance of the performance space. To allow for such relatively unbiased evaluations, a virtual acoustic stage support system was set up for a live musical performance, and the performer was asked to make blind comparisons between a variety of architectural acoustic simulations. While results support the broad generalization that the preferred reverberation time for acoustical stage support depends upon the piece of music to be performed, it was also clear that preferences strongly depended upon performers' aural familiarity with architectural acoustic spaces in which they had considerable experience in previous performances. After some exposure to descriptive analysis techniques, performers could explain their preferences in terms of the perceptual characteristics that differed between presented aural architectures.

TUESDAY MORNING, 1 JULY 2008

ROOM 253, 8:00 TO 9:00 A.M.

\section{Session 2aAAb}

\section{Architectural Acoustics and Noise: Low Frequency Absorption: Mechanisms, Measurement Methods and Application II}

\author{
Peter D'Antonio, Cochair \\ RPG Diffusor Systems, Inc., 651-C Commerce Drive, Upper Marlboro, MD 20774, USA \\ Christian Nocke, Cochair \\ Akustikbüro Oldenburg, Katharinenstr. 10, Oldenburg, 26121, Germany
}

\section{Contributed Papers}

\section{8:00}

2aAAb1. Effects of Low-frequency Absorption on Perceived Tightness of Bass Imagery in Music Reproduction. William L. Martens (McGill University, Schulich School of Music, 555 Sherbrooke Street West, Montreal, QC H3A 1E3, Canada, wlm@music.mcgill.ca)

In order to enable unbiased observation of the effects of low-frequency absorption on auditory imagery associated with multichannel loudspeaker reproduction, binaural recordings were made of surround sound program material that was reproduced over full-range loudspeakers located in a room that was specially constructed to allow for variation in low-frequency acoustical treatment. These recordings were then presented via headphones to allow for double-blind comparison of the variation in auditory imagery associated with selected changes in room acoustics while holding listener and loudspeaker locations constant. Several perceptual attributes were examined, but the listeners were able to make the most clear distinctions between auditory spatial images in terms of the attribute identified as the "perceived tightness of bass imagery." Analysis of the signals presented to the listeners' ears in these binaurally-reproduced multichannel music samples showed that the tightest bass imagery was associated with high values of interaural coherence, with lower values producing more "muddy" bass imagery. [Work supported by Canada Foundation for Innovation.]

\section{$8: 20$}

2aAAb2. Acoustical tests of custom-made, low-cost bass absorptive treatment for small rooms. Ioana Pieleanu (Acentech Incorporated, 33 Moulton Street, Cambridge, MA 02138, USA, ipieleanu@acentech .com), Jeffrey Fullerton (Acentech Incorporated, 33 Moulton Street, Cambridge, MA 02138, USA, jfullerton@acentech.com), Marc Choiniere (University of Nebraska, 101A Peter Kiewit Institute, Omaha, NE 68182, USA, mchoiniere@gmail.com)

Small music classrooms, practice rooms or small budget recording studios and control rooms are a few of the numerous applications where low frequency absorption is sought and necessary for a well-balanced acoustical spectrum. Often, low frequency absorption is achieved by using preengineered products, such as tuned panel resonators. Unfortunately, the cost of these pre-engineered products can be excessive for small budget projects, 
or projects that involve a large number of such spaces. For these applications, more affordable, custom-made low frequency absorbers are desired. Several types of custom-made treatments were physically tested, to determine if the degree of low frequency absorption they provide would make them an effective replacement for the pre-engineered counterparts. The test samples included glass fiber panels mounted as corner traps, custom-made plywood panel resonators and others. The testing was conducted in a space comparable in size to a small studio room/control room, or a medium practice room. This presentation describes the test method and measurement results.

\section{8:40}

2aAAb3. The importance of bass clarity in pop and rock venues. Niels W. Adelman-Larsen (Flex Acoustics, Diplomvej 377, 2800 Lyngby, Denmark, nwl@flexac.com), Eric R. Thompson (Centre for applied hearing research, Technical University of Denmark, DTU, Bygn. 352, 2800 Lyngby, Denmark, et@oersted.dtu.dk)

High levels of bass sound have been shown to stimulate the part of the brain that controls such basic instincts as sexual desire and hunger [Todd, 2000]. In rock and pop music, the bass frequencies from $40-125 \mathrm{~Hz}$ get amplified to very loud levels. Easily half of the electrical power of the PA and monitor system goes to these 1.5 octaves. A recent survey [Adelman-Larsen et al., 2007] showed that the most important subjective parameter for a rock and pop music hall to score a high rating was 'bass clarity' which correlated with a coefficient of 0.74 to 'overall impression'. Informal discussions with audio engineers and bass players give the perspective that artificial reverberation is rarely, if ever, added to bass-frequencies. In fact the ideal hall should be as dry as possible at low-frequencies. In the mid-treble frequency range, sound absorption, and thereby 'clarity', is easily obtained through the presence of the audience that absorbs 4-6 times more mid/high frequency sound energy than bass sound energy. In the low-frequency range 'clarity' is not so easily obtained. This paper discusses the challenge in depth and proposes design solutions.

TUESDAY MORNING, 1 JULY 2008

ROOM 202/203, 8:20 A.M. TO 12:40 P.M.

\title{
Session 2aAAc
}

\section{Architectural Acoustics and Engineering Acoustics: Acoustics and Electroacoustics of Small Rooms}

\author{
Jiri Tichy, Cochair \\ Pennsylvania State University, 5552 N. Citation Road, Toledo, OH 43615, USA \\ Mendel Kleiner, Cochair \\ Chalmers Rm. Acoust. Group, Dept. of Appl. Acoust., Chalmers Univ. of Technology, Gothenburg, 41296, Sweden
}

\section{Invited Papers}

2aAAc1. Investigation of Bonello criteria as a practical tool in small room acoustics. Todd Welti (Harman International Industries, Inc., R\&D Group, 8500 Balboa Blvd, Northridge, CA 91329, USA, twelti@harman.com)

The Bonello criteria are often used as an objective metric for assessing room dimensions with respect to modal response. In reality, the modal response is not simply a property of the room dimensions, but is quite dependent on source/receiver locations as well. The legitimacy of Bonello's criteria when used in rooms with typical subwoofer and seating configurations has not been well investigated. In this study, a room model is used to calculate room responses for a large number of combinations of room dimensions, subwoofer positions, and seating locations. The Bonello criteria are evaluated for each combination and compared to perceptually relevant criteria such as flatness and seat to seat consistency of the calculated acoustical responses.

\section{8:40}

2aAAc2. Region of control for low frequency modal equalisation in small listening rooms. Aki Mäkivirta (Genelec Oy, Olvitie 5, FIN-74100 Iisalmi, Finland, aki.makivirta@genelec.com)

Small-size listening rooms are characterized by sparse modal density with pronounced spectral colouration when the decay times at low frequencies are large. Various active approaches for reducing the modal decay time have been suggested in the literature. A review of the salient principles of these approaches is given. Active control of the modal decay time at the primary listening position by using the primary radiator for control is demonstrated and evaluated for performance. The locality and size of the region of control, and the amount of control achieved, are studied in light of the experiment. Effect of the modal equalizer filter to the perceived response flatness, audibility of low frequency resonances, and the required amount of modal equalization are discussed in light of the experiment. 
2aAAc3. Improving room acoustics at low frequencies with multiple loudspeakers and time based room correction. Sofus Birkedal Nielsen (Aalborg University, Fredrik Bajers Vej 7 B, 9220 Aalborg Ø, Denmark, sbn@es.aau.dk), Adrian Celestinos (Oticon A/S, Kongebakken 9, 2765 Smørum, Denmark, adc@oticon.dk)

Small and medium size rectangular rooms are often used for sound reproduction. These rooms have substantial acoustical problems at low frequencies primarily caused by the reflections from the room boundaries. The spatial variation in sound pressure level (SPL) can be up to $30 \mathrm{~dB}$ in a room at low frequencies, and appear not only at modal frequencies. The problem is an acoustical issue in time, and should therefore be analyzed in the time-domain, instead of the traditional steady state frequency domain. The construction of a finitedifference time-domain approximation program (FDTD) has lead to a simple and untraditional solution called CABS (Controlled Acoustical Bass System) that makes use of multiple loudspeakers. With the proper placement of low frequency loudspeakers, CABS can create a plane wave from the front wall which will be absorbed by additional low frequency loudspeakers at the back wall. With the back wall reflection removed a homogeneous sound field will be created in the whole room at low frequencies. Simulations and measurements of normal size listening rooms show that 4 loudspeakers are enough to even the sound field in a room. The CABS system is controlled by a developed DSP system.

9:20

2aAAc4. Applications of a plane wave based room correction system for low frequencies using multiple loudspeakers. Adrian Celestinos (Oticon A/S, Kongebakken 9, 2765 Smørum, Denmark, adc@oticon.dk), Sofus Birkedal Nielsen (Aalborg University, Fredrik Bajers Vej 7 B, 9220 Aalborg Ø, Denmark, sbn@es.aau.dk)

When low frequency sound is radiated inside small listening spaces by loudspeakers, large uniformities occur over the sound field. This is due to the multiple reflection and diffraction of sound on the walls and different objects in the room. A developed system named Controlled Acoustically Bass System (CABS) produces uniform sound field at low frequencies. This is performed by utilizing loudspeakers at the front wall and extra loudspeakers at the opposite wall, processed to remove the rear-wall reflection of a rectangular room. Effectiveness of CABS on different room scenarios has been evaluated by using a computer simulation program based on the Finite Difference Time Domain Method (FDTD). CABS has been simulated in a small car cabin and in an irregular room. Non-ideal placement of loudspeakers in CABS have been evaluated. The influence of utilizing CABS with different types of loudspeakers has been evaluated by simulations and measurements.

9:40

2aAAc5. Active Sound Field Equalization. Akira Omoto (Kyushu University, 4-9-1, Shiobaru, Minamiku, 815-8540 Fukuoka, Japan, omoto@design.kyushu-u.ac.jp), Hisaharu Suzuki (Kyushu University, 4-9-1, Shiobaru, Minamiku, 815-8540 Fukuoka, Japan, hisaha@souldsp.jp), Akihiro Kakiuchi (Kyushu University, 4-9-1, Shiobaru, Minamiku, 815-8540 Fukuoka, Japan, kakirikakki @hotmail.com)

Dominant acoustic modes often result in the inevitable non-uniform distribution of the acoustic quantities such as sound pressure, especially in the small sized enclosure. In our study, active control technique is thus introduced to overcome the adverse effects of such modes. Instead of the squared sound pressure which is normally adopted in active noise control, the quantity to be controlled is selected as the weighted values of acoustic intensities or the acoustic impedances in rectangular directions such as $\mathrm{x}, \mathrm{y}$ and $\mathrm{z}$ measured at plural points. The reasonably selected combinations of weights yield the control outputs which can alter the directions of propagating waves and result in reducing the complicated standing waves. Further, results of numerical simulations suggest that the well balanced arrangement of the primary and the secondary sources can 'rectify' the propagating direction and result in the uniform distributions of sound pressure. The strategies used in this study have possibilities of realizing useful controller for active sound field equalization.

\section{0:00}

2aAAc6. Different approaches for efficient finite element modelling of absorbers in small rooms. Marc Aretz (RWTH Aachen University, Institute for Technical Acoustics, Neustraße 50, 52066 Aachen, Germany, Marc.Aretz@akustik.rwth-aachen.de)

The FEM is a powerful tool for the numerical simulation of sound fields in enclosures. It accounts for the modal characteristics of the sound field, which are dominant at frequencies below the Schroeder frequency and it is also possible to model the mutual coupling between airborne and structure borne sound fields, when an appropriate structure model is implemented. When applying the FEM to complex room acoustics applications, like e.g. a control room in a recording studio, it is a challenging task to specify realistic boundary conditions. Different kinds of acoustical absorbers like Helmholtz resonators, plate absorbers and complex layered porous absorbers are found in these environments. While it is possible in principle to use complex and exhaustive models for these acoustical absorbers, it is often computationally much more efficient to use acoustic impedances or two-port network FEM elements to represent the fluid structure interactions. In the course of this study we compare different approaches for the modelling of Helmholtz resonators (without and without flow resistance), since it has proven very costly in terms of computation time to explicitly model every single hole in the resonator boxes. The simulation results are compared with results measured in a model room. 
2aAAc7. Direct and modal frequency response analysis of sound fields in small rooms by finite element method. Reiji Tomiku (Faculty of Engineering, Oita University, Dannoharu 700, 870-1192 Oita, Japan, tomiku-reiji@cc.oita-u.ac.jp), Toru Otsuru (Faculty of Engineering, Oita University, Dannoharu 700, 870-1192 Oita, Japan, otsuru@cc.oita-u.ac.jp), Noriko Okamoto (Faculty of Engineering, Oita University, Dannoharu 700, 870-1192 Oita, Japan, nokamoto@cc.oita-u.ac.jp), Yuka Kurogi (Faculty of Engineering, Oita University, Dannoharu 700, 870-1192 Oita, Japan, kurogi@cc.oita-u.ac.jp)

In this paper, sound pressures are computed by two techniques using finite element method. One is a technique by solving the system of linear equations directly (direct analysis) and the other is a technique by modal superposition (modal analysis). To confirm the accuracy of the direct analysis, sound pressures obtained by the technique are compared with those obtained by the modal analysis in a room with the volume of $10 \mathrm{~m}^{3}$. Then, as in the modal analysis, two methods are employed: one is a simplified method based on a real eigenvalue problem assuming that the damping matrix, [C], has orthogonality; and another is the method based on complex eigenvalue problem. Those obtained by the direct analysis are in good agreement with those obtained by the two kinds of modal analyses regardless of absorption conditions, even if the analysis is carried out at the frequency close to an eigen frequency. Next, diffuseness of sound field below $315 \mathrm{~Hz}$ in a room, which is used in the measurement of ISO140-3, is investigated by the direct analysis from the viewpoint of mean sound pressure level measurements.

\section{1:00}

2aAAc8. The effect of diffusers on low frequency modes. Jamie A. Angus (University of Salford, 12 Lister Way, YO30 6NL York, UK, j.a.s.angus@salford.ac.uk)

Small listening rooms are becoming prevalent, due to the availability of production facilities on personal computers, and the financial pressures faced by the industry. In these rooms, modal behaviour at low frequencies significantly affects the quality of the reverberant decay, due to their non-diffuse nature compared with other frequencies. Diffusion and absorption can help and may be a useful way of improving the low frequency performance of such rooms. This paper discusses the properties of these rooms at low frequencies, in particular the effect of diffusing boundaries on the modal behaviour of such rooms. The paper will first discuss what is meant by a mode and modal decay. It will then go on to examine the effect of diffusing boundaries on the frequency and density of modes. In particular, it will examine the effect of the scale of the diffuser on its efficacy in this task. For ease of visualisation this will be done using a two dimensional model and, for accuracy a finite element, element simulation. The effect of going to three dimensions on the results will also be discussed. Finally, the effect of absorption based diffusing boundaries will be examined.

\section{1:20}

2aAAc9. Power output regularization in the active reproduction of sound fields in rooms. Nick Stefanakis (National Technical University of Athens, School of Electrical and Computer Engineering, Heroon Polytechniou 9, 15773 Athens, Greece, nstefan@mobile.ntua.gr), Sotiris Dalianis (National Technical University of Athens, School of Electrical and Computer Engineering, Heroon Polytechniou 9, 15773 Athens, Greece, dalias@central.ntua.gr), Tilemachos Karatzas (National Technical University of Athens, School of Electrical and Computer Engineering, Heroon Polytechniou 9, 15773 Athens, Greece, tilkar6@hotmail.com), George Cambourakis (National Technical University of Athens, School of Electrical and Computer Engineering, Heroon Polytechniou 9, 157 73 Athens, Greece, gcamb@cs.ntua.gr)

In this paper we address the problem of using a multi-channel active control system in order to reproduce a harmonic sound field in a large part of the volume of a reverberant room. The problems associated with the calculation of the inverse system matrix are confronted by introducing a term that is proportional to the sound power-output of the system in the cost function that is obtained by the multiple point method. Simulation results show that this technique results to a better conditioning of the system matrix at low frequencies, comparing to other traditional regularization techniques. Moreover, it is shown that this method can be employed to increase the spatial robustness of the control sensor array inside the listening room.

\section{1:40}

2aAAc10. Low-frequency response in active acoustic practice rooms. Ronald Freiheit (Wenger Corporation, 555 Park Drive, Owatonna, MN 55060, USA, ron.freiheit@wengercorp.com)

Integrating active (virtual) acoustics into relatively small practice rooms to create a sense of envelopment is critical to the satisfaction of the musicians using these spaces for learning. The number of speakers and their locations play an important role in minimizing the ability to localize to the sources supporting this sense of envelopment. Equally important is the frequency response required for the speakers used in these applications, to more accurately simulate the acoustics of a performance environment. Excessive high frequencies increase the ability to localize and decrease the sensation of a larger space, since the air in larger spaces naturally absorbs many high frequencies. Another challenge is creating the sense of envelopment for instruments in the bass region (125Hz octave band and below) by providing enough low-frequency energy response. Work will be presented on low-frequency response desired to satisfy a sampling 
of solo cello players using active acoustic practice rooms. The optimal sources for these low frequencies - single or multiple - will be discussed. Updated information will also be provided on the sound field coverage in active rooms with extended low-frequency response.

\title{
Contributed Papers
}

\section{2:00}

2aAAc11. Active playback of acoustic quadraphonic sound events. Domenico Stanzial (Italian National Research Council, FSSG-CNR Lab Acustica, c/o Fondazione Cini, Isola di San Giorgio Maggiore, 30124 Venezia, Italy, domenico.stanzial@cini.ve.cnr.it), Giorgio Sacchi (Physics Department - University of Ferrara, V. Saragat, 44100 Ferrara, Italy, giorgio.sacchi@student.unife.it), Giuliano Schiffrer (Physics Department University of Ferrara, V. Saragat, 44100 Ferrara, Italy, schgln@unife.it)

The reproduction in a given confined space - such as a cinema hall or a smaller room - of a sound event previously recorded in a completely different acoustical environment is an interesting and still open acoustical problem. A new method for hi-fi audio playback based on the general solution of the acoustic inverse problem is here pourposed. A feed-forward control based on overdetermination of conditions at active contours - i.e. loudspeakers - in order to obtain an optimal stable solution via least square approach is here proposed. This is easily possible even for complex configurations thanks to acoustic quadraphony, the application of sound intensimetry to audio technology developed in the last years within the IST-2511316-IP European project denominated IP-RACINE. After a short explanation of the model theory, the experimental application to the simplest case of 1-D confined field is presented and some obtained results are shown.

12:20

2aAAc12. Acoustics in a small control room. Sergio Beristain (Mexican Institute of Acoustics, P.O. Box 12-1022, Narvarte, 03001 Mexico, D.F., Mexico, sberista@hotmail.com)

Acoustics in small rooms is allways a problem, particularly at the low frequency range, because of their size and the fact that there is very limited space to install all the acoustic materials needed in order to obtain the desired acoustic response, but when an electroacoustic system has to be employed as it is the case in a small Control Room for a small recording studio, matters become even worse. First of all, the space is further reduced in order to accomodate the required sound system, and with the presence of hard to control low resonant frecuencies, which can be excited at any moment during any recording session, it can be produced highly different sound presure levels at those frequencies, changing the timbre of sounds. Some measurement results are presented.

TUESDAY MORNING, 1 JULY 2008

ROOM 253, 9:20 A.M. TO 1:00 P.M.

\section{Session 2aAAd}

\section{Architectural Acoustics and Noise: Acoustics and Privacy in Healthcare Facilities I: Emerging Policy Around the World}

\author{
David M. Sykes, Cochair \\ ANSI S12 Workgroup 44 and the Joint ASA/INCE/NCAC Subcommittee on Healthcare Acoustics \& Speech Privacy, 23 \\ Buckingham Street, Cambridge, MA 02138, USA \\ Kerstin Persson Waye, Cochair \\ Dept. of Environ. Medicine, The Sahlgrenska Acad. of Gothenburg Univ., Box 414, Gothenburg, 405 30, Sweden
}

\section{Invited Papers}

2aAAd1. Waves of change: global policies \& their impacts on the acoustics profession. David M. Sykes (ANSI S12 Workgroup 44 and the Joint ASA/INCE/NCAC Subcommittee on Healthcare Acoustics \& Speech Privacy, 23 Buckingham Street, Cambridge, MA 02138, USA, david.sykes@ remington-partners.com)

In a decade, five waves of change swept across the globe stimulating new interest in acoustics. This session examines their effects on the EU, North America and Japan. The Internet caused the first wave-the EU's privacy Directive 9546EC drove nations everywhere to develop privacy laws, many covering "Speech Privacy." A second wave, in 2000 when the "tech bubble" burst, produced laws to improve financial accountability and forcing organizations to find ways to shield their leaders. A third wave arose in 2001 with the increase in terrorism, producing a surge in security laws that challenge the right to privacy enshrined in the 1948 Declaration of Human Rights. The fourth wave is demographic: racing to build healthcare facilities for "boomers," this giant industry is now wrestling with a "noise epidemic" resulting from decades of neglecting the health effects of noise. The fifth wave came from the "green" movement-the concept of "indoor environmental quality" has renewed interest in noise as a pollutant. How countries balance such concerns as state security, citizen privacy and human health will be decided by courts but these five waves have catalyzed demand for understanding, insight, expertise, standards, codes, manpower and solutions from the acoustical profession. 
2aAAd2. Developing acoustical policies around in EU countries. Katrin Bergmark (Saint-Gobain Ecophon AB, Box 500, 26061 Hyllinge, Sweden, katrin.bergmark@ecophon.se), Marc R. Janssen (Saint-Gobain Ecophon AB, Box 500, 26061 Hyllinge, Sweden, marc.janssen@ecophon.se)

According to research, noise levels in hospitals worldwide have increased. At the same time, the awareness of the negative effects of noise on patients and healthcare staff has grown. To manage or eliminate noise in hospitals, standards and policies are a great help. An overview of international standards will be presented, showing various parameters and differences in values and angles of approach, e.g based on activity or room type. To further optimize the content, and stimulate the use of the standards, some countries have even defined healthcare specific standards. These can support the planning and the evaluation of the sound environment in those premises. New insights from research and case studies, as well as emerging laws, provide opportunities or even force us to extend the existing content of standards to meet future demands. Suggestions for that include extension of the amount of parameters, such as decrease of sound pressure level $(\triangle \mathrm{SPL})$ and parameters addressing privacy (PI, AC). Finally, revision of standards provides opportunities for end users to increase their understanding and interest in acoustic and to supply possibilities to define the desired quality of the sound environment.

\section{0:00}

2aAAd3. Effects of healthcare acoustics on medical outcomes. Roger Ulrich (Texas A\&M University, Department of Architecture, 3137 TAMU, College Station, TX 77843, USA, rulrich@archmail.tamu.edu)

Although considerable research has examined detrimental effects of noise on patient sleep quality in healthcare buildings, few studies have investigated the extent to which noise may worsen other types of patient clinical outcomes. Studies are also scarce concerning the effects of noise and poor acoustics on healthcare staff. The presentation describes a prospective controlled study conducted with colleagues in Sweden that examined the impact of higher versus lower noise levels, and longer in contrast to shorter reverberation times, on several patient and staff outcomes in a hospital coronary critical care unit (CCU). Acoustics were altered during the study period by changing the ceiling tiles throughout the CCU from sound-reflecting tiles to sound-absorbing tiles of similar appearance. Regarding patients, an improved acoustics environment significantly reduced physiological stress, increased satisfaction with quality of care, improved sleep quality, and lessened incidence of costly re-hospitalizations following discharge. Better acoustics also improved speech intelligibility, and healthcare staff experienced reduced work demands and less pressure and strain. The discussion concludes by outlining research directions, including the need for more outcomes studies to enable development of a strong business case for better healthcare acoustics.

\section{Contributed Papers}

\section{0:20}

2aAAd4. Influence of intenesive coronary care acoustics on the quality of care and phyiological state of patients. Inger Hagerman (Dept of Cardiology, Karolinska University Hospital, M52, Huddinge, 14186 Stockholm, Sweden, inger.hagerman@karolinska.se), Gundars Rasmanis (Dept of Cardiology, Karolinska University Hospital, M52, Huddinge, 14186 Stockholm, Sweden, gundars.rasmanis@karolinska.se), Vanja Blomkvist (Dept of Cardiology, Karolinska University Hospital, M52, Huddinge, 14186 Stockholm, Sweden, vanja.blomkvist@ pubcare.uu.se), Roger Ulrich (Texas A\&M University, Department of Architecture, 3137 TAMU, College Station, TX 77843, USA, rulrich@archmail.tamu.edu), Töres Theorell (Dept of Cardiology, Karolinska University Hospital, M52, Huddinge, 14186 Stockholm, Sweden, tores.theorell@stressforskning.su.se)

Background unstable coronary disease is a stressful situation and environmental influences may increase stress mechanisms important for the cardiovascular status. Aim to evaluate acoustic influence on patients with coronary artery disease and to test if the effects of poor sound absorption on work environment affects quality of care and medical status of patients. Methods 94 patients admitted to the intensive coronary heart unit for chest pain evaluation participated. Patient groups were recruited during bad and good acoustic conditions respectively. Blood pressure, pulse amplitude, heart rate and heart rate variability were monitored. Patients were asked to fill in a questionnaire about the quality of the care and a follow up of rehospitalisation and mortality was made at 1 and 3 months. Results good and bad acoustics differed significantly with respect to pulse amplitude in acute myocardial infarction and unstable angina pectoris groups, with lower values at night during the good acoustics period. Re-hospitalisations were higher for the bad acoustics group. During the good acoustics period patients considered the staff attitude much better than during the bad acoustics period. Conclusion, bad acoustics environment during acute illness may have important physiological effects of importance for rehabilitation.

\section{0:40}

2aAAd5. The future of UK hospital design. Adrian Popplewell (Arup Acoustics, St Giles Hall, Pound Hill, CB3 0AE Cambridge, UK, adrian .popplewell@arup.com)

The importance of acoustic conditions within hospitals is highlighted by recent research indicating that patient recovery times are significantly influenced by the noise levels within wards. Added to this are concerns about speech privacy and confidentiality, patient and staff comfort, communication between patient and doctor, and the effects of vibration on sensitive medical equipment, all of which makes the acoustic design a key parameter in any hospital development. This paper will discuss the practical issues associated with the implementation of the current national guidance on hospital acoustics (Health Technical Memorandum 2045) and the new, currently unpublished replacement, Health Technical Memorandum 08-01. The experiences of the author with several large Private Finance Initiative projects, designed to the current standard, have illustrated the practical difficulties of fully complying with all its requirements and the design solutions which can be adopted. Speech privacy and confidentiality has been a critical issue in developing the new design standard. With Arup Acoustics as key contributors to this document, and acoustic designers of one of the first major projects in the UK to comply with this standard, the author is in a prime position to be able to assess the practical and technical implications of the future guidance.

11:00-11:20 Break 


\section{Invited Papers}

\section{1:20}

2aAAd6. Development of 'sound and vibration design guidelines for health care facilities'. Kurt Rockstroh (Steffian Bradley Architects, 100 Summer Street, Boston, MA 02110, USA, kurtr@ steffian.com)

"The Guidelines for Design \& Construction of Healthcare Facilities" is utilized by the majority of the United States and its federal agencies involved in funding health care projects. As part of the development of the 2010 Edition of "The Guidelines" a joint subcommittee of ASA, INCE, NCAC and the Health Guidelines Revision Committee was formed to develop sound and vibration guidelines. This presentation will explain the collaborative process used to develop, publicize, review and formally vote acceptance of the sound and vibration guidelines. The presentation will also outline the major components of the new guidelines.

\section{1:40}

2aAAd7. Acoustics in green buildings: refining the concept of environmentally quality while improving occupant health and productivity synergistically. Sholem Prasow (Teknion Furniture Systems, 1150 Flint Rd., Thornhill, ON L3T 4 M9, Canada, sholem .prasow@teknion.com)

Environmental Quality has always been a keystone of the green building movement. The concept has evolved from "Indoor Air Quality", for example, in the first version of LEED seven years ago to a much broader and enriched concept - a concept that fully embraces Acoustics - today. This paper explores the evolution of Environmental Quality in both general and health care environments, and demonstrates the emerging role of Acoustics as an equal partner with Air Quality and Lighting as determinants of both health and productivity in the built environment. Specific references will be made to LEED rating systems and to emerging acoustical design requirements within LEED.

\section{2:00}

2aAAd8. Getting noise and speech privacy issues heard in design of healthcare facilities. Anjali Joseph (The Center for Health Design, 1850 Gateway Blvd., Suite 1083, Concord, CA 94520, USA, ajoseph@ @ealthdesign.org)

Hospitals are extremely noisy, and noise levels in most hospitals far exceed recommended guidelines. The high ambient noise levels, as well as peak noise levels in hospitals, have serious impacts on patient and staff outcomes ranging from sleep loss and elevated blood pressure among patients to emotional exhaustion and burnout among staff. Poorly designed acoustical environments can pose a serious threat to patient confidentiality if private conversations between patients and staff or between staff members can be overheard by unintended listeners. At the same time, a poor acoustical environment impedes effective communication between patients and staff and between staff members by rendering speech and auditory signals less intelligible or detectable. This has serious implications for patient safety. A well-designed acoustical environment is critical in addressing these problems related to noise and communication of information. The purpose of this presentation is to examine how different aspects of sound - noise, speech privacy, speech intelligibility, and music - impact patient and staff outcomes in healthcare settings and the specific environmental design strategies that can be used to improve the acoustical environment of healthcare settings.

2aAAd9. Noise confounds in functional MRI research and potential solutions. Marc J. Kaufman (McLean Hospital, 115 Mill St., Brain Imaging Center, Belmont, MA 02478, USA, kaufman@mclean.harvard.edu), Blaise D. Frederick (McLean Hospital, 115 Mill St., Brain Imaging Center, Belmont, MA 02478, USA, BBFrederick@mclean.harvard.edu), Eric E. Ungar (Acentech Inc, 33 Moulton St., Cambridge, MA 02138, USA, eungar@ACENTECH.com), Jonathan D. Kemp (Acentech Inc, 33 Moulton St., Cambridge, MA 02138, USA, jdkemp@acentech.com), David M. Sykes (ANSI S12 Workgroup 44 and the Joint ASA/INCE/NCAC Subcommittee on Healthcare Acoustics \& Speech Privacy, 23 Buckingham Street, Cambridge, MA 02138, USA, david.sykes@ remingtonpartners.com)

High field functional MRI (fMRI) is becoming a neuroscience research technique of choice because it is noninvasive and can reveal brain circuitry regulating sensory, motor, and cognitive functions. Unfortunately, rapid scan fMRI results in high noise levels (100-140 $\mathrm{dB}$ ) that can alter auditory, visual, and pain system function, and also can induce stress, which itself modulates brain responses to various stimuli. These effects can confound fMRI data interpretation. A number of solutions for this problem have been proposed including modifying MRI scanner hardware to reduce noise output, an expensive proposition limited to willing manufacturers, and modifying fMRI pulse sequences to reduce noise output, which is effective within certain limitations. Another approach is to develop acoustic noise isolating equipment that separates subjects from noise. This passive approach confers maximum flexibility because it is both hardware- and fMRI scan sequence-independent. We present initial data documenting efficacy of first generation acoustic noise isolating equipment for animal fMRI studies. As nearly $25 \%$ of patients referred for clinical MRIs refuse scans because they are stressful, acoustic noise isolating equipment scaled for clinical MRI scanner use may decrease stress and increase patient compliance, thereby decreasing morbidity and mortality, and improve MRI center workflow. 
2aAAd10. Experimental study on applicability of sound masking system in medical examination room. Kanako Ueno (Institute of Industrial Science, University of Tokyo, Komaba 4-6-1, Meguro-ku, 153-8505 Tokyo, Japan, ueno@iis.u-tokyo.ac.jp), Hyojin Lee (Institute of Industrial Science, University of Tokyo, Komaba 4-6-1, Meguro-ku, 153-8505 Tokyo, Japan, leehj@iis.u-tokyo.ac.jp), Shinichi Sakamoto (Institute of Industrial Science, University of Tokyo, Komaba 4-6-1, Meguroku, 153-8505 Tokyo, Japan, sakamo@iis.u-tokyo.ac.jp), Atsuko Ito (Center for Advanced Sound Technologies, Yamaha, 203 Matsunokijima, 4380192 Iwata, Shizuoka, Japan, atsuko1_ito@gmx.yamaha.com), Mai Fujiwara (Center for Advanced Sound Technologies, Yamaha, 203 Matsunokijima, 4380192 Iwata, Shizuoka, Japan, mai_fujiwara@gmx.yamaha.com), Yasushi Shimizu (Center for Advanced Sound Technologies, Yamaha, 203 Matsunokijima, 4380192 Iwata, Shizuoka, Japan, yasushi_shimizu@gmx yamaha.com)

Recently, speech privacy to avoid oral information leakage in healthcare facilities has become an important issue. This study investigated effectiveness of sound masking system in regard to masking efficiency, annoyance and its influence on speech conversation for medical examination rooms in an experimental approach. Considering actual application, two adjacent medical examination rooms partitioned by a low sound insulation wall in a typical healthcare facility were selected as an experimental field and sound masking system was temporally installed. In the rooms, acoustic environment was measured and reproduced in an anechoic room with a 3-D sound field simulation system using a 6-ch sound recording/reproduction technique. In the simulated acoustic condition, subjective tests were designed to quantify the masking efficiency and annoyance caused by the masking sound. The annoyance test was conducted in listening condition (with high attention to the sound) and in talking condition (with low attention). As a result, mixed maskers composed by water stream, synthesized speech signals, and steady state noise showed high performance in both aspect of masking efficiency and annoyance.

TUESDAY MORNING, 1 JULY 2008

ROOM 252B, 10:20 A.M. TO 1:00 P.M.

\title{
Session 2aAAe
}

\section{Architectural Acoustics, Musical Acoustics, Physical Acoustics, and Noise: Acoustics of Opera Houses I}

\author{
Robin Glosemeyer Petrone, Cochair \\ 918 16th St., Apt 3, Santa Monica, CA 90403, USA \\ Roberto Pompoli, Cochair \\ Engineering Dept. - Univ. of Ferrara, Via Saragat, 1, Ferrara, 44100, Italy
}

\section{Invited Papers}

10:20

2aAAe1. Generous opera house acoustics for lyric and symphonic performances. Daniel E. Commins (Commins Acoustics Workshop, 15 rue Laurence Savart, 75020 Paris, France, d.commins@comminsacoustics.com)

It is believed that a short acoustic response is needed in an opera house to guarantee good intelligibility of lyrics. Some of the best opera houses have a relatively long reverberation time associated to good clarity. They are more suitable to symphonic concerts than "damped" rooms. The recently opened 1350-seat Grand Théâtre de Provence in Aix-en-Provence, France, is an example of an opera house with a long response and good clarity. An adequate orchestra shell and proper permanent acoustical treatment of the stage tower lead to suitable acoustical parameters in the symphony configuration. The measurements show that in an opera house of this capacity, it is possible to create conditions compatible with opera, symphony, chamber music and recitals. The rich opera configuration sound generates mostly positive reactions. Most concert goers consider that this room is now the reference for the region. One must note however that this approach is risky since the values that are considered ideal for opera must be stretched far. It may lead to excessive reverberation and even to unwanted reflections. This target requires genuine investigations, from early concept to final design, using the most advanced prediction and modeling techniques. The design of larger rooms under the same principle requires even more care.

10:40

2aAAe2. Opera House Reverberation Times - Design Considerations. Eckhard Kahle (Kahle Acoustics, 188 avenue Molière, 1050 Brussels, Belgium, kahle@kahle.be), Thomas Wulfrank (Kahle Acoustics, 188 avenue Molière, 1050 Brussels, Belgium, twulfrank@kahle.be), Yann Jurkiewicz (Kahle Acoustics, 188 avenue Molière, 1050 Brussels, Belgium, yjurkiewicz@kahle.be), Brian F. Katz (LIMSI-CNRS, B.P. 133, 91403 Orsay, France, brian.katz@limsi.fr)

The intimacy of many historic European Opera Houses, especially of the traditional Italian style, is highly cherished and many of these halls are considered to be among the best halls acoustically. From an acoustical point of view the generally small dimensions often combined with a moderate seat count - provide excellent source presence and clarity. On the other hand, the corresponding small volume leads to short reverberation times, and in recent decades higher reverberation times have been preferred and asked for by clients and audiences in many countries. Ideas will be presented on how this apparent dilemma between the preference for small dimensions (for intimacy, source presence and definition) and increased volume (in order to create longer reverberation times) can be addressed. 
2aAAe3. The "Teatro di San Carlo" in Naples and its smaller clone "Teatro Verdi" in Salerno. Raffaele Dragonetti (DETEC, University of Naples Federico II, Piazzale Tecchio 80, 80125 Naples, Italy, dragonet@ unina.it), Carmine Ianniello (DETEC, University of Naples Federico II, Piazzale Tecchio 80, 80125 Naples, Italy, ianniell@ unina.it), Francesco Mercogliano (DETEC, University of Naples Federico II, Piazzale Tecchio 80, 80125 Naples, Italy, francesco.mercogliano@ unina.it), Rosario A. Romano (DETEC, University of Naples Federico II, Piazzale Tecchio 80, 80125 Naples, Italy, rosroman@unina.it)

The Teatro di San Carlo in Naples (Italy) is a well preserved baroque-type theatre. It was opened in 1737 and flourished up to a destructive fire. Nine months after the disastrous event the San Carlo was rebuilt completely as it was and reopened in 1817. It is still praised for its sound quality for opera performance. The Teatro Verdi was conceived by the Municipality of Salerno (Italy) in 1843. However, the "querelles" of local parties delayed much its construction so that it could be opened only in 1872 . The architects who designed the Teatro Verdi were influenced very much by the successful Teatro di San Carlo and tried to copy the older and not-far-one in a smaller scale. The Verdi has undergone few minor changes during its life and is used also for opera shows. The main halls of the two are in a scale about 1:5. This paper reports a comparison of their acoustical features in terms of objective room-acoustics parameters accepted for opera-house sound quality. Acoustic measurements were performed with the same instrumentation set. Similarities and differences are discussed.

\section{1:20}

2aAAe4. Teatro 'La Fenice', Venice --- The secrets of the acoustical reconstruction of the destroyed theater according to historical and modern requirements. Jürgen Reinhold (Müller BBM GmbH, Robert Koch Strasse 11, D 82152 Planegg/München, Germany, Juergen.Reinhold@ MuellerBBM.de)

The reconstruction of this artistically and architecturally very important opera house that was completely destroyed by a fire and which was famous throughout the world not least for its excellent acoustical qualities, was a brilliant challenge also for an acoustician. The historical planning requirements - complete reconstruction "as it was; where it was" - the latest findings in room acoustics as well as a huge number of "modern" requirements are to be brought in line. The theatre hall as heart of the Teatro "La Fenice" was reconstructed in its classical horseshoe shape with five tiers in pure wooden construction. Modern standard specifications had to be met for ventilation and air conditioning, the installation of a modern stage machinery as well as an improved safety and fire prevention concept. Furthermore the space allocation plan had to be extended by the integration of new rehearsal rooms and technical equipment rooms. From the point of view of building and room acoustics this was a very complex task - if in addition the cramped conditions of the Teatro "La Fenice" in Venice are taken into consideration. The lecture provides an insight into the building and room acoustical planning, its realization and the achieved acoustical results.

\section{1:40}

2aAAe5. The acoustics of the Beijing National Grand Theatre of China. Isabelle Schmich (CSTB, 24, Rue Joseph Fourier, 38400 Saint Martin D’Hères, France, isabelle.schmich@cstb.fr), Paul Chervin (CSTB, 24, Rue Joseph Fourier, 38400 Saint Martin D’Hères, France, paul.chervin@cstb.fr), Zhu Xiangdong (School of Architecture, Tsinghua University, Room 104, Centre Main Building, 100084 Beijing, China, zxd@abcd.edu.cn), Yan Xiang (School of Architecture, Tsinghua University, Room 104, Centre Main Building, 100084 Beijing, China, yx@abcd.edu.cn), L Guo-Qi (The National Grand Theatre, 2, Chang an sast street, 100031 Beijing, China, liguoqi@fujisound.com)

The National Grand Theatre of China opened in December 2007 in Beijing. It is a complex of three performance halls: the Opera (2400 seats), the Concert Hall (2000 seats) and the Theatre (1100 seats). These three halls are covered by a super-ellipsoidal shell made out of titanium and glass. The history of the acoustic design evolution from the winning of the competition in 1999 by the Architect Paul Andreu to the final construction will be retraced including the preliminary design, the acoustic simulations and auralisations. The acoustic design of each hall will be presented with its specifications and detailed particularities. Final acoustic measurements have been done in the three empty halls and in the occupied concert hall. The results and acoustic criteria will be discussed. In addition, a subjective evaluation has been done with psychoacoustic questionnaires and the results will be presented and analysed.

\section{Contributed Papers}

\section{2:00}

2aAAe6. Acoustical design and scale model test for the opera house of Zhongshan city. Shuo Xian Wu (State Key Laboratory of Subtropical Building Science, South China University of Technology, 381 Wushan Street, 510640 Guangzhou, China, arshxwu@scut.edu.cn), Yue Zhe Zhao (State Key Laboratory of Subtropical Building Science, South China University of Technology, 381 Wushan Street, 510640 Guangzhou, China, arzhyzh@scut.edu.cn)

The opera house of Zhongshan City in Guangdong province of China is the performing art center of the city. It was completed in Oct. 2005. It has 1400 seats including 833 stall seats and another 484 seats on two levels of balcony. The volume of the auditorium is $12000 \mathrm{~m} 3$ and the $\mathrm{V} / \mathrm{N}$ is $9.1 \mathrm{~m} 3$ per seat. The main purpose of the building is for the performances of opera and ballet. Some music and conference events are also to be held there. Therefore, the occupied reverberation time at medium frequencies is set to be 1.6s and its background noise level has to meet NR-20. During the acoustical design, a 3D computer simulation model established with ODEON software and a 1:20 scale model was made to analyze the sound fields of the auditorium with and without a music shell on the stage. After the completion of the building, an acoustics test was taken. Several performances show that its acoustics reaches a quite high level and has won high praises.

$12: 20$

2aAAe7. Subjective Assessments of Acoustical Environments for Un-assisted Traditional Peking Opera Performances. Wei-Hwa Chiang (National Taiwan University of Science and Technology, 43, sec. 4 Keelung Rd, Taiwan, 106 Taipei, Taiwan, edchiang1224 @ hotmail.com), Wei Lin (National Taiwan University of Science and Technology, 43, sec. 4 Keelung Rd, Taiwan, 106 Taipei, Taiwan, D9313001@mail.ntust.edu.tw), Ya-Jhen Yu (National Taiwan University of Science and Technology, 43, sec. 4 Keelung Rd, Taiwan, 106 Taipei, Taiwan, ianmelody@hotmail.com)

The prevalence of Western performing arts has impacted on how traditional Peking opera is performed. It is, however, necessary to preserve and pass on traditional Peking opera for it integrates the essence of Chinese 
opera. The research has been conducted regarding subjective assessment of the acoustical environment for traditional Peking opera. Recording of dry sound sources was also performed. Sound strength and listening direction were found to be the principle factors that determined evaluation for overall impression while reverberation time is less important. The $6000 \mathrm{~m} 3$ can be used as the upper limit for room volume when a thrust stage is used.

\section{2:40}

2aAAe8. Considerations about the acoustical properties of Teatro Nuovo in Spoleto after the restauration works. Alessandro Cocchi (University, DIENCA Dept. Facoltà di Ingegneria, Viale Risorgimento 2, 40136 Bologna, Italy, alessandro.cocchi@mail.ing .unibo.it), Marco Cesare Consumi (University, DIENCA Dept. Facoltà di Ingegneria, Viale Risorgimento 2, 40136 Bologna, Italy,
marcocesare.consumi@mail.ing.unibo.it),Ryota Shimokura(AIST, 1-8-31 Midorigaoka, Ikeda, 563-8577 Osaka, Japan, ryota.shimokura@aist.go.jp)

In 2003 the municipality of Spoleto decided to stop the performances within the Teatro Nuovo, among the others also of "Two World Annual Festival", as it was necessary to modify some material, repair the floor, refurbish pictures and so on: the first author was charged with all the acoustical aspects and he decided to perform extensive acoustical measurements. During this measurement campaign, a flutter echo was detected in the stalls, so it became necessary to detect the origin of this problem, then to find some modification able to remove this problem: we have already presented our studies on this subject, from which a new design of the orchestra pit was derived. For fire safety reasons it was necessary also to remove some elastic panels from the boxes, and an acoustically equivalent technical solution was adopted. Even the stalls floor was removed and a new solution was adopted incorporating the heating plant and an acoustical solution. In this paper we will present the results of measurements performed after the opening of the theatre (a typical Italia Opera House of the end of the Eighteen Century) and some consideration about the acoustical results so obtained.

\title{
Session 2aAB
}

\section{Animal Bioacoustics and ECUA: Animal Bioacoustic Censusing I}

\author{
Marie A. Roch, Cochair \\ San Diego State University, Dept. of Computer Science, San Diego, CA 92182-7720, USA \\ Cédric Gervaise, Cochair \\ E3I2 - EA3876, 2 rue François Verny, Brest Cedex, 29806, France
}

\section{Invited Papers}

\begin{abstract}
8:00
2aAB1. Passive acoustic detection of grouper sound production. David Mann (University of South Florida, College of Marine Science, 140 7th Ave. S., St. Petersburg, FL 33701, USA, dmann@marine.usf.edu), James Locascio (University of South Florida, College of Marine Science, 140 7th Ave. S., St. Petersburg, FL 33701, USA, locascio@marine.usf.edu), Michelle Schärer (University of Puerto Rico-Mayaguez, Department of Marine Sciences, Isla Magueyes, La Parguera, 00667 Lajas, Puerto Rico, m_scharer@hotmail.com), Chris Koenig (Florida State University, Coastal and Marine Laboratory, St. Teresa Beach, FL 32358, USA, koenig@bio.fsu.edu), Michael Nemeth (University of Puerto Rico-Mayaguez, Department of Marine Sciences, Isla Magueyes, La Parguera, 00667 Lajas, Puerto Rico, michaelnemeth@hotmail.com), Misty Nelson (University of South Florida, College of Marine Science, 140 7th Ave. S., St. Petersburg, FL 33701, USA, mnelson@marine.usf.edu), Felicia Coleman (Florida State University, Coastal and Marine Laboratory, St. Teresa Beach, FL 32358, USA, coleman@bio.fsu.edu), Richard Appeldoorn (University of Puerto Rico-Mayaguez, Department of Marine Sciences, Isla Magueyes, La Parguera, 00667 Lajas, Puerto Rico, rappeldo@uprm.edu)
\end{abstract}

Passive acoustic recordings were used to study the behavior of red hind (Epinephelus guttatus) at spawning aggregation sites off of Puerto Rico and Mona Island, and goliath grouper (Epinephelus itajara) and red grouper (Epinephelus morio) on the West Florida Shelf. The sounds produced by each species were unique, low-frequency pulsed sounds and associated with reproductive-related behavior. Male red hind produced sounds composed of a series of pulses that graded into a tonal-like sound, mostly during territorial patrolling. Long-term acoustic recorder (LARS) data from the west coast of Puerto Rico and Mona Island showed similar diel periodicities of sound production of red hind, but had different monthly peaks in sound production. Goliath grouper sounds consisted of low-frequency individual pulses $(50-100 \mathrm{~Hz})$ that are consistent with sounds produced by a large fish, and showed lunar periodicity in sound production. Peaks in sound production occurred nightly after midnight, but sounds were also produced throughout the day. Sound production and spawning by red grouper was recorded using a remotely operated vehicle (ROV) at depths of 80-100 m. Passive acoustic techniques can provide synoptic, long-term time series of sound production associated with reproductive activities of soniferous species at widely spaced sites.

$8: 20$

2aAB2. The value of acoustic technologies for monitoring bird migration. Andrew Farnsworth (Cornell Laboratory of Ornithology, 420 E 54th St, 38J, New York, NY 10021, USA, af27@cornell.edu)

Many North American species of birds give distinctive flight calls during nocturnal migration. Monitoring these calls can be a powerful method for studying the timing and magnitude of migration, as well as for confirming the presence of individual species and 
potentially for quantifying passage rates. Recent technological advances in acoustic monitoring, such as increased processor speeds of computers, automated detection software, increased data storage capacities, and a comprehensive identification guide, permit recording of the vocalizations of passing migrants over entire nights for entire migration seasons, thus yielding data on species composition, migration timing and routing, and the magnitude of migration traffic. I will discuss several recent studies that have used acoustic technologies to monitor nocturnal migration, presenting data on species composition and relative abundance. Additionally, I will present some recent approaches to addressing quantification of passing migrants by their calls. I will also discuss wider applications of this technology beyond the realm of North American migrant species.

\section{Contributed Papers}

\section{8:40}

2aAB3. Automatic detection of short time periodic bird calls in realistic monitoring scenarios. Daniel Wolff (Department of Computer Science III, University of Bonn, Roemerstr. 164, 53117 Bonn, Germany, wolffd@bonn.edu), Klaus H. Tauchert (Humboldt-University Berlin, Institute of Biology, Invalidenstr. 43, 10115 Berlin, Germany, klaus.tauchert@gmx.de), Karl H. Frommolt (Humboldt-University Berlin, Museum für Naturkunde, Tierstimmenarchiv, Invalidenstr. 43, 10115 Berlin, Germany, karl-heinz.frommolt@rz.hu-berlin.de), Rolf Bardeli (Department of Computer Science III, University of Bonn, Roemerstr. 164, 53117 Bonn, Germany, bardeli@iai.uni-bonn.de), Frank Kurth (FGAN-FKIE, Abteilung KOM, Neuenahrer Str. 20, 53343 Wachtberg-Werthhoven, Germany, kurth@fgan.de), Michael Clausen (Department of Computer Science III, University of Bonn, Roemerstr. 164, 53117 Bonn, Germany, clausen@iai .uni-bonn.de)

In this contribution, we propose a method for the automatic detection and localisation of bird calls featuring simple as well as highly repetitive structures. Reporting from a research project focused at computer aided acoustical monitoring, a detector for Locustella luscinoides (Savi's Warbler) is presented, performing reliably even on highly distorted recordings. In cooperation with the Animal Sound Archive at the Humboldt University, Berlin, this detector was incorporated into a multimodal censusing method combining GPS-data and automatically annotated audio recordings to perform line mapping. An evaluation of the proposed techniques for unsupervised monitoring purposes was also performed on 20 hours of multichannel recordings from Lake Parstein, a cane brake area in Germany. Finally, exem- plifying the discriminative potential of the underlying features, we will point out the application to other members of the Warbler family as well as the detection of cricket and toad sounds.

\section{9:00}

2aAB4. Animal censusing using seismic cues: techniques used for African elephants. Jason D. Wood (Beam Reach Marine Science and Sustainability School, 7044 17th Ave NE, Seattle, WA 98115, USA, jason@beamreach.org), Caitlin E. O'Connell-Rodwell (Stanford University, Department of Otolaryngology, Head and Neck Surgery, Stanford, 94305, USA, ceoconnell@stanford.edu), Simon L. Klemperer (Stanford University, Department of Geophysics, Stanford, 94305, USA, sklemp @ stanford.edu)

Counting populations of animals has proven to be difficult and inexact for species that are difficult to detect visually. A growing number of researchers have successfully turned to detecting animals by their acoustic signals in order to count their numbers. To predict the number of animals present a regression line is generated from the relationship between such measures as calling rate and the number of animals documented visually during that recording by the researcher. Depending on the communication system of the species in question, a significant amount of the variation in calling rate can be attributed to other factors such as group behavior rather than the number of individuals present. For species where these other sources of variation are high it may be more appropriate to use acoustic cues to detect and count the number of animals. This paper will present techniques used to detect and estimate elephant numbers from geophone recordings of their footfalls; cues that are a byproduct of their locomotion and are thus not as dependent on group behavior.

\section{Invited Papers}

2aAB5. Species identification and measurement of activity in odontocete species of Palmyra Atoll by acoustic monitoring. Simone Baumann (Scripps Institution of Oceanography- UCSD, 9500 Gilman Dr. \#0205, La Jolla, CA 92093, USA, sbaumann@ucsd.edu), John A. Hildebrand (Scripps Institution of Oceanography- UCSD, 9500 Gilman Dr. \#0205, La Jolla, CA 92093, USA, jhildebrand@ucsd.edu), Sean M. Wiggins (Scripps Institution of Oceanography- UCSD, 9500 Gilman Dr. \#0205, La Jolla, CA 92093, USA, swiggins@ucsd.edu), Hans-Ulrich Schnitzler (Eberhard-Karls-Universität Tübingen, Zool. Institut, Abt. Tierphysiologie, Auf der Morgenstelle 28, 72076 Tübingen, Germany, hans-ulrich.schnitzler@uni-tuebingen.de)

Acoustic monitoring has been used to study odontocete presence at Palmyra Atoll, a remote island in the Northern Line Islands chain. Long-term recordings of high-frequency, broadband acoustic data have become possible with recent technological advances. A High-frequency Autonomous Recording Package (HARP) has been developed which samples at $200 \mathrm{kHz}$ with a duty cycle of $1 / 4$ for up to seven months. This instrument has recorded since October 2006 at Palmyra Atoll. Visual and acoustic surveys were conducted around Palmyra Atoll using a four-element towed hydrophone array sampling real-time at $200 \mathrm{kHz}$ to obtain species-specific acoustic data. These data are used as reference for automatic detection algorithms applied on the long-term recordings. To date, acoustically and visually detected odontocetes include bottlenose dolphins (Tursiops truncatus), spinner dolphins (Stenella longirostris), melon-headed whales (Peponocephala electra) and beaked whales of the genus Mesoplodon. The long-term HARP data reveal acoustic activity primarily at night time and predominantely odontocete clicks. Both the beaked as well as the melon-headed whales are present year round and show a distinct daily acoustic activity cycle.

2aAB6. Distribution patterns of delphinids in the California Current Ecosystem observed through acoustic monitoring of species-specific echolocation clicks. Erin M. Oleson (Scripps Institution of Oceanography- UCSD, 9500 Gilman Dr. \#0205, La Jolla, CA 92093, USA, eoleson@ucsd.edu), Melissa S. Soldevilla (Scripps Institution of Oceanography- UCSD, 9500 Gilman Dr. \#0205, La Jolla, CA 92093, USA, msoldevilla@ucsd.edu), John Calambokidis (Cascadia Research Collective, 218 1/2 W. 4th Ave., Olympia, WA 98501, USA, calambokidis@cascadiaresearch.org), Curtis Collins (Naval Postgraduate School, 833 Dyer Road, Rm 328, 
Monterey, CA 93943, USA, Collins@nps.edu),Sean M. Wiggins (Scripps Institution of Oceanography- UCSD, 9500 Gilman Dr. \#0205, La Jolla, CA 92093, USA, swiggins@ ucsd.edu), John A. Hildebrand (Scripps Institution of Oceanography- UCSD, 9500 Gilman Dr. \#0205, La Jolla, CA 92093, USA, jhildebrand@ucsd.edu)

Visual surveys along the U.S. west coast conducted in the 1980s-90s suggested that Pacific white-sided and Risso's dolphins likely undergo annual movement between California during winter-spring, and Oregon-Washington in the summer-fall. Using high-frequency autonomous acoustic recordings within the southern California, central California, and Washington regions of the California Current System, we evaluated the seasonal occurrence of these dolphin species through detection of their echolocation clicks. The clicks of Pacific white-sided and Risso's dolphins are characterized by a unique combination of local frequency peaks for each species, providing a statistically robust means of identification from autonomous acoustic records. Based on these unique spectral characters, we extracted periods of clicking by each species from concurrently sampled coastal and shelf/slope locations off southern California and Washington, and a single offshore site off central California. The number of hours that each species was heard per day was quantified and compared among seasons and regions. Both species were heard year-round at shelf/slope sites off southern California and Washington; however their patterns of occurrence at coastal sites differed somewhat. Our results indicate year-round presence of Pacific white-sided and Risso's dolphins in some regions, suggesting that the seasonal distribution of these species may have recently changed.

\section{0:00-10:20 Break}

\section{Contributed Papers}

10:20

2aAB7. Radial distance sampling with passive acoustics: The prospect of estimating absolute densities of cetaceans from static acoustic datalogger data. Jakob Tougaard (National Environmental Research Institute, University of Aarhus, Frederiksborgvej399, DK-4000 Roskilde, Denmark, jat@dmu.dk)

Recording animal vocalisations with a static acoustic datalogger can be viewed as a form of point transect sampling and results analysed within the framework of distance sampling theory. The key element is the radial detection function, which specifies the probability of detecting a vocalisation as a function of distance from the datalogger. This function can be modelled theoretically or preferably determined experimentally and from it the efficient radial detection distance can be determined. Radial detection functions were determined for two different harbour porpoise dataloggers (T-PODs, version 1 and version 3) by means of concurrent visual tracking of porpoises. $52 \%$ and $82 \%$ of the porpoises within $100 \mathrm{~m}$ from the T-PODs were detected by the V1 and V3 T-POD, respectively and $11 \%$ and $30 \%$, respectively were detected between 100 and $200 \mathrm{~m}$ from the T-PODs. Effective detection radius (EDR) was $97 \mathrm{~m}$ and $150 \mathrm{~m}$ for the two T-PODs, respectively. Porpoises echolocate almost continuously. If it is assumed that silent periods rarely exceeds 1 minute in duration an average detection rate on the version 3 T-POD for the study site of 2.7 detection positive minutes per hour can be converted into a density estimate of 0.69 porpoises $/ \mathrm{km}^{2}$.

\section{0:40}

2aAB8. Computer-aided detection of non-stereotyped bowhead whale calls in the presence of seismic airgun signals. Aaron Thode (Marine
Physical Laboratory, Scripps Institution of Oceanography, 9500 Gilman Dr, MC 0238, La Jolla, CA 92093-0238, USA, athode@ucsd.edu), Delphine Mathias (Marine Physical Laboratory, Scripps Institution of Oceanography, 9500 Gilman Dr, MC 0238, La Jolla, CA 92093-0238, USA, delphine.mathias@gmail.com), Miles McLennan (Greeneridge Sciences, Inc., 4512 Via Huerto, Santa Barbara, CA 93110, USA, billm @ greeneridge.com), Charles R. Greene (Greeneridge Sciences, Inc., 4512 Via Huerto, Santa Barbara, CA 93110, USA, cgreene@greeneridge.com)

In 2007 thirty-five autonomous recording packages were deployed over a 150 mile swath in the Beaufort Sea to monitor the annual migration of the bowhead whale (Balaena mysticetus) population during seismic exploration activities. Over 1350 days worth of acoustic data were gathered, generating a need for computer-aided assistance in detecting calls. Bowhead whales produce over ten types of frequency modulated calls, and the frequency range, duration, and fine structure of individual calls vary considerably even within each call type, creating difficulties when using simple matchedfiltering or spectrogram correlation. Numerous airgun signals also display frequency-modulated structure, complicating the challenge. In this presentation a three-stage process is presented for detecting arbitrary bowhead whale calls in the presence of seismic airgun signals, implemented in JAVA and MATLAB, with components extracted from the industry-supported PAMGUARD software package. The first stage runs several "energy-based" detectors simultaneously across multiple frequency bands to capture events, and a second stage analyzes the timing of these detections to remove regular periodic sequences, such as those expected from airguns. The final stage attempts to trace contours on spectrograms. Both genetic algorithms and direct optimization are used to optimize the program's 21 input parameters. [Work supported by Shell Exploration and Production Company].

\section{Invited Papers}

\section{1:00}

2aAB9. Comparison of feature extraction methods for the identification of odontocete species based upon echolocation clicks. Marie A. Roch (San Diego State University, 5500 Campanile Dr, Dept of Computer Science, San Diego, CA 92182-7720, USA, marie.roch@sdsu.edu), Holger Klinck (Alfred Wegener Institute, P.O. Box 120161, 27515 Bremerhaven, Germany, holger.klinck@awi.de), David K. Mellinger (Oregon State Univ. and NOAA, 2030 SE Marine Science Dr., Newport, OR 97365, USA, David.Mellinger@oregonstate.edu), Melissa S. Soldevilla (Scripps Institution of Oceanography- UCSD, 9500 Gilman Dr. \#0205, La Jolla, CA 92093, USA, msoldevilla@ucsd.edu), John A. Hildebrand (Scripps Institution of Oceanography- UCSD, 9500 Gilman Dr. \#0205, La Jolla, CA 92093, USA, jhildebrand@ucsd.edu)

Recent work by several groups has shown that odontocete echolocation clicks contain information that can be used to detect or identify specific species. In this study, we compare the relative performance of cepstral and wavelet features on various Pacific Ocean species of odontocetes. Comparison of features within different systems is often complicated by the large number of variables unrelated to feature extraction that change between systems. By experimenting within a proven state of the art classification framework, it is possible to make meaningful comparisons of feature extraction performance with respect to common machine learning algorithms such as neural networks, support vector machines, and Gaussian mixture models. 
2aAB10. Long-term Passive Acoustic Monitoring of Delphinids in the Southern California Bight. Melissa S. Soldevilla (Scripps Institution of Oceanography- UCSD, 9500 Gilman Dr. \#0205, La Jolla, CA 92093, USA, msoldevilla@ucsd.edu), John A. Hildebrand (Scripps Institution of Oceanography- UCSD, 9500 Gilman Dr. \#0205, La Jolla, CA 92093, USA, jhildebrand@ucsd.edu), Sean M. Wiggins (Scripps Institution of Oceanography- UCSD, 9500 Gilman Dr. \#0205, La Jolla, CA 92093, USA, swiggins@ucsd.edu), Marie A. Roch (San Diego State University, 5500 Campanile Dr, Dept of Computer Science, San Diego, CA 92182-7720, USA, marie.roch@sdsu.edu)

Spectral characteristics of clicks are described for five species of delphinids in the Southern California Bight (long-beaked common dolphins, Delphinus capensis, short-beaked common dolphins, Delphinus delphis, Risso's dolphins, Grampus griseus, Pacific whitesided dolphins, Lagenorhynchus obliquidens, and bottlenose dolphins, Tursiops truncatus). Recent technological advancements allow long-term, broadband (100 kHz bandwidth), passive acoustic monitoring from autonomous sea-floor mounted instruments. The ability to record higher frequencies allows study of a broader range of odontocete vocalizations including echolocation clicks. To determine whether delphinid species could be identified by their clicks, concurrent ship-based visual and acoustic surveys were conducted. We find that clicks from Pacific white-sided and Risso's dolphins contain spectral peaks and notches that are unique and consistent for each species. These spectral patterns are also apparent in long-term autonomous acoustic recordings throughout the Southern California Bight. Utilizing this spectral classification method, we examine of diel, seasonal, and habitat use patterns of acoustically active Risso's and Pacific white-sided dolphins. The ability to monitor animals through the night provides insight into distinct diel patterns of acoustic activity for both species while the high temporal resolution acoustic data can be used to relate oceanographic time series to dolphin activity.

\section{Contributed Papers}

11:40

2aAB11. Sound production and spawning by black brum (Pogonias cromis) in southwest Florida. James Locascio (University of South Florida, College of Marine Science, 140 7th Ave. S., St. Petersburg, FL 33701, USA, locascio@marine.usf.edu), Ernst Peebles (University of South Florida, College of Marine Science, 140 7th Ave. S., St. Petersburg, FL 33701, USA, epeebles@marine.usf.edu), David Mann (University of South Florida, College of Marine Science, 140 7th Ave. S., St. Petersburg, FL 33701, USA, dmann@ marine.usf.edu)

We used the Long Term Acoustic Recording System (LARS) to investigate patterns of sound production in spawning aggregations of black drum (Pogonias cromis) during 2004 - 2006 in southwest Florida. Our purposes were to document trends in black drum sound production on daily and seasonal time scales and to investigate the temporal and quantitative relationships between egg production and sound production. Sound production was strongly diel, beginning near dusk and lasting for several hours. Sound production occurred from October through April and peaked in FebruaryMarch, consistent with prior descriptions of the spawning season for this species based on the gonado-somatic index (GSI). To investigate the relationship between sound production and egg production, surface plankton tows were conducted hourly between 1600 - 0400 on two consecutive nights while continuous underwater acoustic recordings were made. This was done five times between January and April, 2006. Neither the timing nor quantity of sound production was correlated with egg production on a nightly basis. These results indicate that patterns in sound production are not useful for predicting patterns in egg production by black drum on a daily scale but do provide accurate characterization of spawning behavior on a seasonal basis.

\section{2:00}

2aAB12. Nearest-neighbor techniques for automated monitoring of nocturnal flight calls. Harold Figueroa (Cornell University, Bioacoustics Research Program, 159 Sapsucker Woods Road, Ithaca, NY 14850, USA, harold.figueroa@gmail.com), Andrew Farnsworth (Cornell Laboratory of Ornithology, 420 E 54th St, 38J, New York, NY 10021, USA, af27@ cornell .edu)

Flight-calls are short vocalizations used primarily during nocturnal flight . Their observation provides a means for studying the timing, location, and composition of nocturnal migrations. As part of a three-year study the Cornell Lab of Ornithology is using autonomous recorders to sample flight-calls of nocturnal migrants in the Northeastern US. The resulting tens of thousands of hours of recording, make software-assisted detection and classification essential. Automatic processing and human evaluation have yielded a considerable collection of flight-calls, 5-1000 examples for $\sim 100$ species. The many-class classification problem, along with the availability of many examples from most of the classes, and established (condensation and editing) and recent (metric-trees) techniques used in prototype-based classification nearest-neighbor techniques, have led us to develop nearestneighbor based techniques and software to assist in the analysis of this data. We will present classification results on two examples, a set of thrushes (genera Catharus and Hylocichla, family Turdidae) consisting of six species and wood-warblers (family Parulidae) consisting of 48 species. The thrush flight-calls are visually and aurally distinctive, usually $100-400 \mathrm{~ms}$ in duration and occupy and the $2-5 \mathrm{kHz}$ band. Wood-warbler flight-calls are typically between $20-100 \mathrm{~ms}$ in duration and occupy the $5-10 \mathrm{kHz}$, and are difficult for many experienced observers to distinguish.

\section{Invited Paper}

\section{2:20}

2aAB13. Sperm whale monitoring with a deep acoustic platform: Results from NEMO ONDE experiment and way ahead. Gianni Pavan (CIBRA - Università di Pavia, Via Taramelli 24, 27100 Pavia, Italy, gianni.pavan@unipv.it)

Within the INFN NEMO Project on the underwater detection of high energy neutrino, a deep hydrophone station, named ONDE (Ocean Noise Detection Experiment), has been deployed on the seafloor $21 \mathrm{~km}$ offshore Catania (Sicily, Italy), at $2000 \mathrm{~m}$ depth. Acoustic data recorded in 2005 and 2006 provided long term information on the underwater noise and a huge amount of sperm whales' detections that indicate a presence of the species higher than previously believed. Only few sightings are available for the previous years and scarce literature is available for the area. With ONDE, in year 2005 sperm whales were detected in 117 of the 231 recorded days and in 31 of the 83 days in year 2006. Clicks were the most common vocalizations recorded. Chirrups and codas (dominated by the $3+1$ pattern) were present frequently, but creaks, possibly indicating feeding actions, were seldom heard. The whales were often detected, solitary or in groups, for time segments of only a few hours. This, with the low rate of creaks, may mean they were just in transit. Based on these results, a new project named (Listening Into the Deep Ocean) has been set with INGV to create a Mediterranean wide network by upgrading existing underwater seismic detectors with broadband acoustic sensors. 


\section{Contributed Paper}

12:40

2aAB14. Detection and classification of call types in the vocalizations of north-east pacific blue whales. Jack McLaughlin (University of Washington, 1013 NE 40th St, Seattle, WA 98105-6698, USA, jackm@apl.washington.edu), Nicolas Josso (GIPSA-lab, dep. DIS, 961, rue de la Houille Blanche, 38402 St Martin d'Hères, France, nicolas.josso@gispa-lab.inpg.fr), Cornel Ioana (GIPSA-lab, dep. DIS, 961, rue de la Houille Blanche, 38402 St Martin d'Hères, France, cornel.ioana @ gipsa-lab.inpg.fr)

Characterization of marine mammal vocalizations is an essential part of any program of marine mammal monitoring as well as being of great help for furthering understanding of subjects such as underwater communication, sonar, etc. The vocalizations of the North-East Pacific (NEPAC) blue whales are known to be made of at least three different call types: the A call, the B call and the $\mathrm{C}$ call. This study aims at the development of a wholly automatic process of detection and classification for the two most common call types of the NEPAC population which are the A call and the B call. We created one template for the A call and one for the B call in order to extract features with matched filtering operations. We show that a simple Gaussian Mixture Model classifier can be used to accurately track and identify the call types in 24-hour long records. The proposed methodology is applied to real data sets recorded by seismic sensors.

\section{Invited Paper}

\section{1:00}

2aAB15. Experience with VoxNet: a rapidly-deployable acoustic monitoring system for bio-acoustic studies. Lewis Girod (MIT/CSAIL, 32G-918, 32 Vassar St, Cambridge, MA 02139, USA, girod@nms.csail.mit.edu), Michael Allen (Cogent Computing ARC, Coventry University, Coventry, UK, allenm@lecs.cs.ucla.edu), Travis Collier (UCLA Dept. of Biology, 3563 Boelter Hall, Los Angeles, CA 90095, USA, travc@taylor0.biology.ucla.edu), Daniel T. Blumstein (UCLA Dept. of Biology, 3563 Boelter Hall, Los Angeles, CA 90095, USA, marmots@ucla.edu), Deborah Estrin (UCLA Dept. of Biology, 3563 Boelter Hall, Los Angeles, CA 90095, USA, destrin@cs.ucla.edu), Charles Taylor (UCLA Dept. of Biology, 3563 Boelter Hall, Los Angeles, CA 90095, USA, taylor @ biology.ucla.edu)

Terrestrial bioacoustic census is a difficult problem because of propagation characteristics, obstructions, the diversity of bioacoustic sources, and the impact of noise. To address this problem we have developed VoxNet, a complete hardware and software platform for distributed acoustic monitoring applications. Each VoxNet node is a portable, self-contained processor with a small four-channel acoustic array. Using a distributed set of VoxNet nodes, a forested habitat can be monitored and the behavior of animals can be recorded and analyzed acoustically. In this work we present our experiences applying VoxNet to bioacoustic census. This work is based on data collected using the system during a deployment in Chiapas, Mexico at the Chajul Biological Field Station. The Chajul station is located in a region of dense rain forest and is home to Mexico's most diverse ecosystem. Using VoxNet in this harsh environment, we obtained census estimates based on observation of bird calls. 


\title{
Session 2aAOa
}

\section{Acoustical Oceanography and ECUA: Marine Ecosystem Acoustics II}

\author{
Kenneth G. Foote, Cochair \\ Woods Hole Oceanographic Institution, Woods Hole, MA 02543, USA \\ Olav R. Godoe, Cochair \\ Institute of Marine Research, PO Box 1870, Bergen, 5817, Norway
}

Invited Papers

2aAOa1. Spawning Behaviour and Spatial Distribution of Atlantic Herring on Georges Bank Revealed by Ocean Acoustics Waveguide Remote Sensing. Purnima Ratilal (Northeastern University, 302 Stearns Center, Rm 311, 360 Huntington Ave, Boston, MA 02115, USA, purnima@ece.neu.edu), Zheng Gong (Northeastern University, 302 Stearns Center, Rm 311, 360 Huntington Ave, Boston, MA 02115, USA, zgong@ece.neu.edu), Daniel Cocuzzo (Northeastern University, 302 Stearns Center, Rm 311, 360 Huntington Ave, Boston, MA 02115, USA, dcocuzzo@ece.neu.edu), Mark Andrews (Northeastern University, 302 Stearns Center, Rm 311, 360 Huntington Ave, Boston, MA 02115, USA, Andrews.mar@neu.edu), Srinivasan Jagannathan (Massachusetts Institute of Technology, Room 5-435, 77 Massachusetts Avenue, Cambridge, MA 02139, USA, jsrini@mit.edu), Ioannis Bertsatos (Massachusetts Institute of Technology, Room 5-435, 77 Massachusetts Avenue, Cambridge, MA 02139, USA, ibertsat@mit.edu), Tianrun Chen (Massachusetts Institute of Technology, Room 5-212, 77 Massachusetts Avenue, Cambridge, MA 02139, USA, trchen@ mit.edu), Hector Pena (Institute of Marine Research, PO Box 1870, 5817 Bergen, Norway, hector.pena@imr.no), Ruben Patel (Institute of Marine Research, PO Box 1870, 5817 Bergen, Norway, ruben.patel@imr.no), Olav R. Godo (Institute of Marine Research, PO Box 1870, 5817 Bergen, Norway, olav.rune.godoe@imr.no), J. Michael Jech (NOAA/Northeast Fisheries Science Center, 166 Water Street, Woods Hole, MA 02543, USA, michael.jech@noaa.gov), Thomas C. Weber (University of New Hampshire, Ctr. for Coastal and Ocean Mapping, 24 Colovos Road, Durham, NH 03824, USA, weber@ccom.unh.edu), Nicholas Makris (Massachusetts Institute of Technology, Room 5-212, 77 Massachusetts Avenue, Cambridge, MA 02139, USA, makris@mit.edu)

An ocean acoustics waveguide remote sensing (OAWRS) system was deployed in the Gulf of Maine, near Georges Bank to image Atlantic herring and other fish populations from Sep-Oct 2006. OAWRS provides spatially unaliased imaging of herring over wide areas, spannning over $100 \mathrm{~km}$ diameter. Migration and spawning behaviour of Atlantic herring was observed using OAWRS over several diurnal periods, including massive movements on and off the bank to spawn. Measurements made simultaneously with a conventional fish-finding echosounder (CFFS) and a multibeam sonar provide the depth distribution and local 3D morphology respectively of the herring schools in the water column. Concurrent trawl surveys provide identification of the fish species. Measurements mades by OAWRS and CFFS systems are highly correlated. Examples will be provided of the co-registration between the two systems over a one week period. Calibration of the OAWRS system using CFFS estimates of fish population densities along with a full-field scattering model that takes into account both coherent and incoherent scattering from a fish group is discussed. The fish swimbladder is modelled as a spheroidal bubble. Resonance scattering behaviour of herring is observed in the OAWRS system with significant changes in scattering amplitude over the $300 \mathrm{~Hz}$ to $1.5 \mathrm{kHz}$ frequency range of the OAWRS system.

2aAOa2. Passive acoustic mapping of marine biological choruses. Gerald D'Spain (Marine Physical Lab, Scripps Institution of Oceanography, 291 Rosecrans St., San Diego, CA 92106, USA, gld@mpl.ucsd.edu), Heidi Batchelor (Marine Physical Lab, Scripps Institution of Oceanography, 291 Rosecrans St., San Diego, CA 92106, USA, heidi@mpl.ucsd.edu), Catherine Berchok (Marine Physical Lab, Scripps Institution of Oceanography, 291 Rosecrans St., San Diego, CA 92106, USA, cberchok@ucsd.edu)

Since underwater sound is a critical aspect of the ocean environment for marine animals, passive acoustic recordings provide important information on marine habitats. Conversely, because biological sounds can be a dominant component of the ocean sound field, knowledge of their characteristics is important for assessing sonar system performance. This presentation summarizes the properties of three biological choruses observed in experiments off the southern California coast. Measurements were made with large aperture, well-filled hydrophone arrays, yielding highly resolved estimates of the chorus directionality. The first chorus generated energy in the 200-800 Hz band, occurred predominantly at night in spring and summer, and displayed an unusual spatiotemporal pattern where the region of chorusing periodically propagated upcoast over $25 \mathrm{~km}$ of coastline at nearly $1.5 \mathrm{~km} / \mathrm{sec}$. The other two choruses occurred in the 1-10 kHz frequency band and come from slowly-moving, or fixed, sources at, or near, the ocean bottom. One chorus was present only on two consecutive nights during a summer experiment, whereas the second occurred at all times throughout the 10-day duration of a late winter experiment. Numerical models predict some of the characteristics of the choruses. [Work supported by the Office of Naval Research]. 
2aAOa3. Understanding potential effects of using active sonar to study marine ecosystems. Mardi C. Hastings (Penn State University, Applied Research Laboratory, 801 North Quincy Street, Suite 120, Arlington, VA 22203-1708, USA, mch26@psu.edu)

Biological organisms can suffer various kinds of effects when exposed to high intensity sound. Active sonar systems used to investigate marine ecosystems typically have source levels exceeding $220 \mathrm{~dB}$ re: $1 \mu \mathrm{Pa}$ at $1 \mathrm{~m}$, which are high enough to have an effect on organisms in relatively close proximity. Although these systems operate at frequencies from the low kilohertz to megahertz ranges that are not usually associated with potentially harmful effects in the marine environment, fundamental interactions with sound occur at various biological scales that strongly depend on the size of the organism with respect to acoustic wavelengths. These effects range from subtle changes in behavior to various types of trauma that can result in temporary or permanent hearing loss, or in hemorrhage or even mortality. Examples from the literature will be used to illustrate the basic relationships between biological effects of sound and the size and structure of marine organisms, as well as potential concerns associated with using active sonar to study the marine environment.

\section{9:00}

2aAOa4. How toothed whales echolocate to find and capture prey in the deep ocean. Peter Tyack (Woods Hole Oceanographic Institution, Applied Ocean Physics \& Engineering Dept., Woods Hole, MA 02543, USA, ptyack@whoi.edu), Mark Johnson (Woods Hole Oceanographic Institution, Applied Ocean Physics \& Engineering Dept., Woods Hole, MA 02543, USA, majohnson@whoi.edu), Peter T. Madsen (University of Aarhus, Biological Sciences, Zoophysiology, C. F. Møllers Allé, Building 1131, DK-8000 Aarhus, Denmark, peter.madsen@biology.au.dk), Walter M. Zimmer (NATO Undersea Research Centre, Viale San Bartolomeo 400, 19126 La Spezia, Italy, walter@nurc.nato.int), Natacha A. Soto (University of La Laguna, BIOECOMAC Department of Animal Biology, 38207 La Laguna, Spain, naguilar@ull.es)

Sperm and beaked whales dive to feed on squid and deepwater fish. We have attached sound-and-orientation recording tags to study how these whales use echolocation to forage at depth. Tagged whales are usually silent when starting a dive, but start producing echolocation clicks at a few hundred meters depth, shallower than the depth at which they feed, suggesting that descending whales scan the deep layers where they will feed. Once sperm or beaked whales encounter prey, they switch from regular search clicks to a buzz of rapid clicks. Tags on beaked whales not only record outgoing clicks, but also echoes from prey at ranges out to 10-20 m. Beaked whales produce clicks every 0.2-0.4 sec when searching. Echoes from several targets are often detected after each beaked whale click. Beaked whales will pass by many targets before selecting one. Whales may switch from the search clicks to a buzz as they close within a body length of the prey. Sperm and beaked whales have an angular acceleration at the end of the buzz, which probably indicates turning to catch the prey.

9:20

2aAOa5. Hypotheses regarding exploitation of bubble acoustics by cetaceans. Timothy G. Leighton (Institute of Sound and Vibration, Univ. of Southampton, University Road, Highfield, SO17 1BJ Southampton, UK, T.G.Leighton@ soton.ac.uk), Paul R. White (Institute of Sound and Vibration, Univ. of Southampton, University Road, Highfield, SO17 1BJ Southampton, UK, prw@isvr.soton.ac.uk), Daniel C. Finfer (Institute of Sound and Vibration, Univ. of Southampton, University Road, Highfield, SO17 1BJ Southampton, UK, d.c.finfer@soton.ac.uk)

Bubbles are the most acoustically active naturally occurring entities in the ocean, and cetaceans are the most intelligent. Having evolved over tens of millions of years to cope with the underwater acoustic environment, cetaceans may have developed extraordinary techniques from which we could learn. This paper outlines some of the possible interactions, ranging from the exploitation of acoustics by humpback whales (Megaptera novaeangliae) in bubble nets to trap prey, to techniques by which coastal dolphins (e.g. of the genus Cephalorhynchus) could successfully echolocate in bubbly water (a hypothesis which has led to the development of a man-made sonar which can penetrate bubble clouds, and a range of possibilities for homeland security).

\section{9:40}

2aAOa6. Marine ecosystem acoustics: contributions of sonar technology. Kenneth G. Foote (Woods Hole Oceanographic Institution, Woods Hole, MA 02543, USA, kfoote@whoi.edu)

Sonar is considered in the widest sense as an acoustic system consisting of a transmitter and receiver of underwater sound. It may be monostatic, bistatic, or multi-static; narrowband or broadband; with one or more fixed, rotating, or electronically formed beams. Transducers may be mounted on hulls of vessels and autonomous underwater vehicles, fixed structures, or seafloor; towed; or suspended. Spatial scales may be sampled from millimeters to thousands of kilometers; corresponding temporal scales vary from a fraction of a second, as in target-tracking, to seasons, as in observing animal migration. Several sonar types are described. Contributions of sonar technology to ecosystem studies, both actual and potential, are noted. These include, for example, fine-scale surveying of pelagic and semi-demersal fish by scientific echo sounder; mapping of the three-dimensional structure of fish aggregations and bathymetry by multibeam sonar; quantification of benthic egg beds of the squid Loligo opalescens by sidescan sonar; and large-scale surveying of swimbladdered fish by parametric sonar. Each of these sonar systems has potential for detection, quantification, and classification of marine organisms, as well as for characterization of the habitat, hence yielding the kind of information that is required for ecosystem assessment and management. 
2aAOa7. Use of broadband active acoustics to study marine organisms. Timothy K. Stanton (Woods Hole Oceanographic Institution, Applied Ocean Physics \& Engineering Department, 98 Water Street, MS \#11, Woods Hole, MA 02543, USA, tstanton@whoi.edu), Dezhang Chu (NOAA/NMFS/NWFSC/FRAMD, Montlake Blvd., E. Seattle, WA 98112, USA, dchu@whoi .edu)

Broadband signals inherently have more information than narrowband signals. In essence, they have more "channels" of information due to the wider range of frequencies spanned. It is therefore advantageous to use broadband signals in active acoustic studies of marine organisms and to apply techniques that best exploit the broadband nature of the signals. In this presentation, a brief background on the use of narrowband systems for studying marine organisms by these authors and other investigators is given. Limitations to those studies are outlined, with arguments made for the use of broadband acoustics. The past twenty years of studies centered at the Woods Hole Oceanographic Institution are subsequently reviewed in which broadband signals are used both in the laboratory and field to study a variety of organisms-- swimbladder-bearing fish and three major anatomical categories of zooplankton. The analyses are divided broadly into two major categories-- time and frequency domain-- for various types of analyses, including pulse compression processing and spectral analysis. Results are first shown based on studies, one organism at a time, in the laboratory. Those laboratory approaches are then applied to ocean studies of fish and large zooplankton using a new towed instrument spanning the frequency range $1.7 \mathrm{kHz}-100$ $\mathrm{kHz}$.

TUESDAY MORNING, 1 JULY 2008

ROOM 342A, 10:40 A.M. TO 6:40 P.M.

Session 2aAOb

\title{
Acoustical Oceanography, Underwater Acoustics, Signal Processing in Acoustics, and ECUA: Geoacoustic Characterization of the Ocean Bottom and Geoacoustic Inversion I
}

\author{
Peter Gerstoft, Cochair \\ Marine Physical Laboratory, Scripps Institute of Oceanography, 8602 La Jolla Shores Drive, La Jolla, CA 92093-0238, USA \\ Dag Tollefsen, Cochair \\ Norwegian Defence Research Establishment (FFI), Box 115, Horten, 3191, Norway
}

Invited Papers

10:40

2aAOb1. Characterization of seabed geo-acoustic structure for a shallow water environment. David Knobles (Applied Research Laboratories, UT at Austin, P. O. Box 8029, Austin, TX 78713, USA, knobles@arlut.utexas.edu)

Full-field inversion methods over large frequency bands and spatial scales attempt to exploit the sensitivity of the frequency and spatial dependence of the acoustic field of the properties to the seabed. A comprehensive set of broadband impulsive and narrowband tonal acoustic recordings in the 5-3000 Hz band from two L-arrays separated by $20 \mathrm{~km}$ were made in a shallow water area on the New Jersey continental shelf during the Shallow Water 2006 (SW06) experiment. In addition, surface ship of opportunity passages on both arrays were recorded along with noise during the passage of tropical storm Ernesto. Reverberation and transmission loss data in the 25-9200 Hz band from explosive sources were also collected at a nearby site prior to the SW06 experiment. Numerous full-field geoacoustic inversion methods and analyses of all these acoustic data with supporting range and azimuth-dependent geo-physical measurements are employed to characterize the seabed properties. For example, the analyses of these data permit inferences about the frequency-dependence of the seabed sound speed and attenuation to be made. The overlapping data types act to reduce the intrinsic ambiguities associated with inversion, quantify the spatial variability, and serve as independent validation.

\section{1:00}

2aAOb2. Effect of ocean sound speed uncertainty on matched-field geoacoustic inversion. Chen-Fen Huang (Department of Marine Environmental Informatics/National Taiwan Ocean University, 2 Pei-Ning Road, 202 Keelung, Taiwan, chenfen@ mail.ntou.edu .tw)

The effect of ocean sound speed uncertainty on matched field geoacoustic inversion is investigated using data from the SW06 experiment along a nearly range-independent bathymetric track. Significant sound speed differences were observed at the source and receiving array and several environmental parameterizations were investigated for the inversion including representing the ocean sound speed at both source and receivers with empirical orthogonal function (EOF) coefficients. A GA-based global optimization method was applied to the candidate environmental models. Then, a Bayesian inversion technique was used to quantify uncertainty in the environmental parameters for the best environmental model, which included an EOF description of the ocean sound speed. 


\section{Contributed Papers}

11:20

2aAOb3. Quantifying the uncertainty of geoacoustic parameter estimates in a dynamic environment using oceanographic data. Ross Chapman (University of Victoria, 3800 Finnerty Rd, Vcitoria, BC V8W 3P6, Canada, chapman@uvic.ca), Yong-Min Jiang (University of Victoria, 3800 Finnerty Rd, Vcitoria, BC V8W 3P6, Canada, minj@uvic.ca)

This paper presents results of Bayesian matched field geoacoustic inversion in a temporally and spatially varying shallow water environment. The acoustic data were collected by a vertical line array at ranges of 1,3 and 5 $\mathrm{km}$ in the SW06 (Shallow Water 06) experiment carried out in August 2006. The sound speed profiles measured at the array and at the source positions during the experiment showed significant variation in the thermocline in the middle part of the water column. The resulting uncertainty in the sound speed profile in the water impacts the performance of matched field inversion methods in estimation of geoacoustic model parameters. In previous work, an effective sound speed profile was estimated in the inversion, based on empirical orthogonal functions (EOFs) derived from a limited number of sound speed profiles measured at the time of the experiment. This paper explores the effect of using a larger number of profiles measured over several days in the SW06 experiment to include a greater diversity of ocean conditions in generating the EOFs. The inversion results for the two approaches are compared in terms of marginal probability distributions of the estimated geoacoustic parameters. (Work supported by Office of Naval Research).

\section{1:40}

2aAOb4. Short range geoacoustic inversion with a vertical line array Yong-Min Jiang (University of Victoria, 3800 Finnerty Rd, Vcitoria, BC V8W 3P6, Canada, minj@uvic.ca), Ross Chapman (University of Victoria, 3800 Finnerty Rd, Vcitoria, BC V8W 3P6, Canada, chapman@uvic.ca), Peter Gerstoft (Marine Physical Laboratory, Scripps Institute of Oceanography, 8602 La Jolla Shores Drive, La Jolla, CA 92093 0238, USA, gerstoft@ucsd.edu)

During the SW06 (Shallow Water 06) experiments, short range geoacoustic inversion experiments were carried out at a source-receiver range of $230 \mathrm{~m}$ to determine the sea bed properties at fine spatial scales. Acoustic signals were collected at 16 hydrophones on a vertical line array from source depths of $15-65 \mathrm{~m}$ in $10 \mathrm{~m}$ intervals. For this experimental geometry, the angular coverage for reflectivity versus grazing angle is $7-25^{\circ}$. This paper combines the information extracted from low-frequency $(100-900 \mathrm{~Hz})$ and mid-frequency $(1500-4500 \mathrm{~Hz})$ LFM signals transmitted from the same source position to estimate multi-layered geoacoustic models. The model parameterization was based on the number of resolved sub-bottom reflections in the data. An inversion approach using adaptive simplex differential evolution was applied to the data to invert for the sub-bottom sound speeds and layer thicknesses. The temporal variation of the water column sound speed profile was included in the inversion. The estimated sound speeds are consistent with the range-averaged profile that was estimated previously from longer range data for the same radial path from the array. (Work supported by Office of Naval Research).

\section{2:00}

2aAOb5. Does a depth variable sound-speed profile matter for SW06 geoacoustic inversion? Alexandra Tolstoy (ATolstoy Sciences, 1538 Hampton Hill Circle, McLean, VA 22101, USA, atolstoy@ieee.org)

This work will discuss the effects of depth variability for a sound-speed profile with regard to goeacoustic inversion on some simulated SW06 "data". First, broadband $(400-800 \mathrm{~Hz})$ simulated "data" are generated using
RAM_PE (by Collins) in the time domain for some short range (less than 1 $\mathrm{km}$ ) SW06 scenarios. Next, the first 4 boundary reflection arrivals (from surface, bottom, surface-bottom, bottom-surface) are studied as a function of source range and depth, phone depth, and sound-speed variability. Finally, we examine the effects of sound-speed depth variability on geoacoustic inversion via MFP. We note that knowledge of array phone locations has a large impact on inversion capabilities.

\section{$12: 20$}

2aAOb6. Three dimensional geoacoustic inversion on the New Jersey shelf. Megan S. Ballard (Penn. State University, P.O. Box 30, State College, PA 16804, USA, msd200@psu.edu), K.m. Becker (Penn. State University, P.O. Box 30, State College, PA 16804, USA, kmbecker@psu.edu)

Perturbative inversion, based on a linearized relationship between sound speed in the sediment and modal eigenvalues, is applied to data from the Shallow Water Experiment 2006. Data were collected by towing a lowfrequency sound source out and back along radials, spanning a 90 degree angular sector, from a common receiver location. Range-dependent estimates of horizontal wave numbers are obtained along each of the radials using high-resolution signal processing techniques capable of detecting and localizing changes in sub-bottom properties, and that are particularly sensitive to changes in layer structure. Wave number estimates at each range are used in a linearized inversion algorithm to estimate local sediment properties. Locations of the R-reflector and other layering information are used as a priori information in the inversion algorithm. The additional information both constrains the solution of an otherwise ill-posed problem and emphasizes the layered structure of the sediment. These methods have been shown to yield accurate estimates of the sound speed profile deep into the sediment using very few perturbations to the forward model. Combining the local inversion results, a three-dimensional map of sediment sound speed structure is obtained for a $25 \mathrm{~km} 2$ region of the seafloor. [Supported by NDSEG and ONR]

\section{2:40}

2aAOb7. Experimental verification of range-dependent inverse method for geoacoustic parameters from modal dispersion data. Subramaniam Rajan (Scientific Solutions, Inc, 99 Perimeter Road, Nashua, NH 03063 , USA, srajan@ scisol.com), K.m. Becker (Penn. State University, P.O. Box 30, State College, PA 16804, USA, kmbecker@psu.edu)

The procedure for determining the sediment compressional wave speed profile from modal dispersion data in a range-independent case is well documented in the literature. In this paper, the procedure developed for the range-independent case is extended to cover the range-dependent case. In this procedure the region under investigation is divided into discrete range intervals and the sediment characteristics at each range interval estimated assuming that the sediment properties of each range interval are range independent. The approach has been demonstrated to yield range-dependent sediment properties when applied to synthetic data generated for an experiment consisting of multiple source/receiver combinations. During the Shallow Water Experiment 2006, broadband data transmitted by a single source were measured on multiple receivers. These data are used here to estimate range-dependent properties of the sediment. The results obtained in this manner are compared with inversion results obtained for the same region by other investigators who employed different approaches for estimating the geoacoustic properties. [Work supported by ONR, Ocean Acoustics].

1:00-2:00 Lunch Break 


\section{Invited Paper}

2:00

2aAOb8. Modal inversion using the acoustic field emitted by a moving source and measured on a vertical line array of hydrophones. K.m. Becker (Penn. State University, P.O. Box 30, State College, PA 16804, USA, kmbecker@psu.edu), Megan S. Ballard (Penn. State University, P.O. Box 30, State College, PA 16804, USA, msd200@psu.edu)

As part of the Shallow Water Experiment 2006 (SW06), a low-frequency continuous wave acoustic source was towed out and back along radials from a fixed vertical line array of hydrophones. The resulting modal pressure field, measured on synthetic range apertures created by the relative source motion, is used to derive input data to inversion algorithms for estimating bottom properties. The inversion algorithms considered are based on well established relationship between modal eigenvalues and geoacoustic properties of the sediment. In this talk, linear inversion methods based on modal input data will be reviewed for their application to the SW06 data. The first step in these methods is the accurate estimation of modal eigenvalues from the pressure field data. For a moving source, theory predicts a Doppler shift along with frequency spreading proportional to both the transmitted frequency and tow speed. Propagating modes and their corresponding modal eigenvalues are predicted to correspond with the Doppler shifted frequencies. An emphasis of this work is on Doppler shift as an observable in the data and properly accounting for it in inversion. Sediment sound speeds estimated from data are consistent with sandy sediments found in the experimental area. [Work supported by ONR and NDSEG]

\section{Contributed Paper}

\section{2:20}

2aAOb9. Geoacoustic inversion using combustive sound source signals. Gopu R. Potty (University of Rhode Island, Department of Ocean Engineering, Narragansett Bay Campus, Narragansett, RI 02882, USA, gopu@uri.edu), James H. Miller (University of Rhode Island, Department of Ocean Engineering, Narragansett Bay Campus, Narragansett, RI 02882, USA, miller@egr.uri.edu), Preston S. Wilson (The University of Texas, Mechanical Engineering Department, Austin, TX 78712, USA, pswilson@mail.utexas.edu), James F. Lynch (Woods Hole Oceanographic Institution, 98 Water Street, Bigelow 203A, MS-11, Woods Hole, MA 02543, USA, jlynch@whoi.edu), Arthur Newhall (Woods Hole Oceanographic Institution, 98 Water Street, Bigelow 203A, MS-11, Woods Hole, MA 02543, USA, anewhall@whoi.edu)
In the summer of 2006, Combustive Sound Sources (CSS) were deployed in shallow waters off New Jersey during the Shallow Water experiment (SW 06). The depth of these sources were $26 \mathrm{~m}$ in water depths of the order of $100 \mathrm{~m}$. CSS are low frequency broadband sound sources and suitable for modal dispersion based inversion. CSS data collected on various receivers (Single Hydrophone Receiving Units (SHRU), Vertical Line Array, etc.) will be used for the inversions. The inversions are based on an iterative scheme using a new time-frequency analysis technique (dispersion based short-time Fourier transform) in which each time-frequency tiling is adaptively rotated in the time-frequency plane, depending on the local wave dispersion. Data from various receivers will be used to investigate the spatial variation in compressional wave speed. Compressional wave attenuation will also be estimated using modal amplitude ratios. Inversion results will be validated by comparing the data with model predictions. [Work supported by the Office of Naval Research]

\section{Invited Paper}

2aAOb10. Tomographic and bottom geoacoustic inversions using Genetic Algorithms and a statistical characterization of the acoustic signal. Michael Taroudakis (University of Crete \& FORTH/IACM, Vassilika Vouton, P.O.Box 1385, 71110 Heraklion, Greece, taroud@iacm.forth.gr), Costas Smaragdakis (University of Crete, Dept of Mathematics, Knossou Ave, 71409 Heraklion, Greece, kesmarag@gmail.com)

The paper deals with the problem of estimation of the parameters of the water column and/or the sea bed, using measurements of the acoustic field due to a known source at a certain point in the water column. Following the work by Taroudakis et al. (JASA 119, 1396-1405 (2006)) the acoustic signal is characterized using the statistics of the wavelet sub-band coefficients, which obey a certain statistical law, described by an Alpha-Stable distribution. Thus, the signal observables are the set of the parameters of the appropriate distributions at the various levels of the signal decomposition. In this work an inversion procedure based on a Genetic Algorithm and the statistical characterization of the acoustic signal, is described. The quality of a certain population of candidate model parameters (properties of the water columns and/or the sea-bed) is evaluated using the Kullback-Leibler divergence (KLD) of the wavelet sub-band coefficient distributions, between the measured and simulated acoustic signals. Following an appropriate population regeneration procedure, the final population is described by an a-posteriori statistical distribution of the model parameters, indicating among the others their sensitivity in the inversion procedure. Very good inversion results have been observed in simulated shallow water environments.

\section{Contributed Paper}

\section{3:00}

2aAOb11. Dispersion curve estimation with partcile filters for geoacoustic inversion. Ivan Zorych (Department of Mathematical Sciences, New Jersey Institute of Technology, 323 M L King Blvd, Newark, NJ 07102, USA, zorych@gmail.com), Zoi-Heleni Michalopoulou (Department of Mathematical Sciences, New Jersey Institute of Technology, 323 M L King Blvd, Newark, NJ 07102, USA, michalop@njit.edu)

In this work we extend our particle filtering method for dispersion curve extraction from spectrograms of acoustic signals that have propagated in underwater environments, the goal being to obtain accurate representation of modal dispersion for geoacoustic inversion. The approach combines particle filtering with modeling of sound propagation in ocean environments to track dispersion curves of multimodal signals in noisy environments. In addition to providing connected modal "trajectories" that facilitate the computation of maximum a posteriori estimates of modal arrival times, the method provides posterior probability distributions for arrival times, quantifying errors that are then propagated into the geoacoustic inversion process. The method, thus, allows the calculation of posterior probability distributions of geoacoustic parameters. We present results from both synthetic and real data from the Gulf of Mexico experiment. [Work supported by ONR.] 


\section{Invited Paper}

\section{3:20}

2aAOb12. Geoacoustic characterization of the seafloor from a subbottom profiler applied to the BASE'07 experiment. Gwladys Theuillon (SHOM, 13 rue du Chatellier, CS 92803, 29228 Brest cedex 2, France, gwladys.theuillon@shom.fr), Yann Stephan (SHOM, 13 rue du Chatellier, CS 92803, 29228 Brest cedex 2, France, yann.stephan@shom.fr)

The most recent subbottom profilers present good performances in term of signal to noise ratio, resolution and penetration. These devices can thus be used to infer quantitatively the geoacoustic parameters of the seafloor. We have previously developed inversion methods which aim to estimate absorption, reflection, penetration, impedance contrast and micro-roughness in sediments from the SBP 120 subbottom profiler, manufactured by Kongsberg. These methods have been tested against real data and geoacoustic parameters derived from the SBP 120 are fully coherent with in situ measurements, which tends to confirm the possibility of seafloor characterization with subbottom profilers. In this work, the inversion methods are applied to a set of data acquired on the Malta Plateau during BASE'07 experiment. Geoacoustic parameters results are presented and discussed. They are in good agreement with the a priori knowledge of sediment properties in the area.

3:40-5:20 Posters

Lecture sessions will recess for presentation of poster papers on various topics in acoustics. See poster sessions for topics and abstracts.

\section{Invited Paper}

$$
5: 20
$$

2aAOb13. Bayesian model parameterization selection for seabed reflection-coefficient inversion. Jan Dettmer (University of Victoria, School of Earth and Ocean Sciences, Victoria, BC V8W 3P6, Canada, jand@ uvic.ca), Stan E. Dosso (University of Victoria, School of Earth and Ocean Sciences, Victoria, BC V8W 3P6, Canada, sdosso@uvic.ca), Charles W. Holland (Penn State University / Applied Research Lab, Applied Science Bldg., State College, PA 16804, USA, cwh10@psu.edu)

This paper considers Bayesian inversion of seabed reflection-coefficient data for multi-layer geoacoustic models; in particular, the important issue of determining appropriate model parameterizations (e.g., number of layers, sound speed and density variations within layers). A poor model parameterization can lead to unreasonable inversion results. In particular, under-parameterization can introduce theory error in the inversion, causing biased results. However, more complex models always fit the data better. Therefore, parameterization quality cannot be quantified only in terms of data misfit, and Occam's razor is commonly applied to prefer simple parameterizations. In a Bayesian framework, Occam's razor is inherently included through Bayesian evidence. Bayesian inversion can be associated with two levels of inference. The first level assumes a specific model parameterization, and quantifies the data information content. This paper focuses on the second level: using Bayesian evidence to compare different model parameterizations. To this end, Gibbs sampling is applied, including full error covariance estimation, to sample the posterior probability density (PPD) for various parameterizations. Bayesian evidence is then computed from the PPD samples by numerical integration (reverse importance sampling).

\section{Contributed Papers}

\section{5:40}

2aAOb14. Combined inversion of mid-frequency propagation and reverberation sonar data. Peter Louring Nielsen (NURC, Viale S. Bartolomeo 400, 19126 La Spezia, Italy, nielsen@ nurc.nato.int), Christopher H. Harrison (NATO Undersea Research Centre, Viale San Bartolomeo 400, 19138 La Spezia, Italy, harrison@nurc.nato.int)

Sonar performance predictions in shallow-water regions are strongly dependent on a good knowledge of the geoacoustic and scattering properties of the seabed. The bottom properties are probably the most difficult parameters to determine, but inversion of measured acoustic data to infer the geoacoustic and scattering properties is a feasible technique. One of these techniques relies on inverting: (1) propagation data to estimate local bottom properties and (2) long-range reverberation data providing effective bottom properties over larger areas. A matched-field inversion approach is applied to propagation and reverberation data received on a towed horizontal array during the BASE'04 experiment conducted on the Malta Plateau, Mediterranean Sea. A total of 30 transmissions along a 10-km track were used to capture eventual range-dependent bottom properties. The inversion algorithm is composed of efficient prediction tools which can provide environmental parameter estimates within tactical time frames for in-situ sonar performance predictions. The experimental set-up mimics a mid-frequency active sonar system using only the suite of sensors available on the vessel towing the sonar system; also known as the through-the-sensor technique. The impact on sonar performance utilizing the environmental characterization approach is shown for various experimental scenarios and seasons. [Research sponsored by NURC and the BOUNDARY Partners]. 


\section{6:00}

2aAOb15. Reverberation inversion for seabed properties. Stan E. Dosso (University of Victoria, School of Earth and Ocean Sciences, Victoria, BC V8W 3P6, Canada, sdosso@uvic.ca), Peter Louring Nielsen (NURC, Viale S. Bartolomeo 400, 19126 La Spezia, Italy, nielsen@nurc.nato.int), Christopher H. Harrison (NATO Undersea Research Centre, Viale San Bartolomeo 400, 19138 La Spezia, Italy, harrison @ nurc.nato.int)

This paper applies nonlinear inversion to reverberation and/or propagation data recorded on a towed horizontal array for geoacoustic and scattering properties of the seabed. A Bayesian inversion approach is applied to estimate properties of the posterior probability density, such as the maximum a posteriori (most-probable) model, 1-D and 2-D marginal probability distributions, and covariance/correlation matrices, providing optimal parameter estimates and quantifying parameter uncertainties and inter-relationships. Of particular interest is quantifying the information content of different combinations of data (e.g., reverberation or propagation data; joint inversion of reverberation and propagation data) to resolve seabed parameters, and understanding strong parameter inter-relationships (correlations) which, in some cases, act as the limiting factor in resolving geoacoustic/scattering parameters. The inversion is also applied to shallow-water reverberation and propagation data recorded in Mediterranean Sea.
$6: 20$

2aAOb16. Local bottom characterization using an autonomous underwater vehicle. Peter Louring Nielsen (NURC, Viale S. Bartolomeo 400, 19126 La Spezia, Italy, nielsen@nurc.nato.int), Charles W. Holland (Penn State University/Applied Research Lab, Applied Science Bldg., State College, PA 16804, USA, cwh10@psu.edu)

In the past decade the usage of autonomous underwater vehicles (AUV) to sample properties of the underwater environment has increased. The advantages of using platforms are their autonomy and that operations can be performed covertly. In 2007, the CLUTTER'07 experiment was conducted on the Malta Plateau with the main aim of characterizing the underwater environment. An AUV was deployed at a particular site on the Plateau to demonstrate the feasibility to infer bottom properties using such an autonomous platform. The AUV was equipped with 2 sound sources covering a frequency band $800-3500 \mathrm{~Hz}$, and the transmitted signals were acquired on a bottom moored vertical array. The mission of the AUV was to perform a linear track of $\sim 1000 \mathrm{~m}$ passing the vertical array as close as possible while transmitting every second from the sound sources. This experimental configuration is similar to the move-out or wide-angle reflection measurements. The received signals are inverted for geoacoustic properties using both matched-field techniques and processed for direct bottom reflection properties. The results obtained are compared to independent findings from different experiments using various types of equipment at different seasons in the same area. [Research sponsored by NURC, ONR OA321 and the CLUTTER Partners]

TUESDAY MORNING, 1 JULY 2008

ROOM 352B, 8:00 A.M. TO 2:40 P.M.

\title{
Session 2aBB
}

\section{Biomedical Ultrasound/Bioresponse to Vibration and Physical Acoustics: Ultrasound Contrast Agents for Imaging I}

\author{
Charles C. Church, Cochair \\ University of Mississippi, NCPA, 1 Coliseum Drive, University, MS 38677, USA \\ Ayache Bouakaz, Cochair \\ INSERM U930, 2, Bvd Tonnelle, Tours Cedex 9, 37044, France
}

Invited Paper

\begin{abstract}
8:00
2aBB1. Ultrasound and microbubble interaction. Nico De Jong (Erasmus MC, Dr Molewaterplein 50 room Ee2302, 3015GE Rotterdam, Netherlands, n.dejong@erasmusmc.nl)

Ultrasound contrast agent (UCA) bubble response to ultrasound (US) is still the subject of several studies, since it determines the power of medical diagnostic imaging modalities. Generally, bubbles are studied using optical or acoustical methods, both having their specific advantages, in an environment that mimic the clinical practice as good as possible (small-diameter capillaries, red blood cells, blood viscosity). Acoustical observations could provide a better insight in the acoustical scattering of bubbles, which determines the final efficacy of contrast-enhanced diagnostic US imaging, and are better suitable to determine the properties of populations of bubbles. Optical observations allow for easier characterization of fewer bubbles and a more detailed characterisation in case where bubbles vibrate non spherically, which is expected for bubbles nearby a wall or red blood cells. In this presentation, we show optical recordings of the various bubble responses with the ultrahigh speed Brandaris-128 camera, and discuss the clinical implications of our findings.
\end{abstract}




\section{Contributed Papers}

\section{8:20}

2aBB2. Nonlinear pulsing schemes for the detection of ultrasound contrast agents. Michalakis A. Averkiou (University of Cyprus/Dept. of Mech. Engineering, 75 Kallipoleos Str., 1678 Nicosia, Cyprus, maverk@ucy.ac.cy), Christophoros Mannaris (University of Cyprus/Dept. of Mech. Engineering, 75 Kallipoleos Str., 1678 Nicosia, Cyprus, mannaris@ucy.ac.cy), Matthew Bruce (SuperSonic Imagine, Les Jardins de la Duranne - Bât. E, 510, rue René Descartes, F - 13857 Aix-enProvence, France, matt.f.bruce@gmail.com), Jeffry Powers (Philips Medical Systems, 22100 Bothell Everett Hwy, Bothell, WA 98021, USA, jeff .powers@philips.com)

Ultrasound contrast agents are used in cardiology for the assessment of myocardial perfusion and in radiology for the detection and characterization of tumors. One widely used approach of imaging contrast agents is to use a low Mechanical Index (MI) nonlinear imaging technique to avoid bubble destruction and image both the macro- and micro-circulation in real-time. Various pulsing schemes are employed for the detection of nonlinear echoes from contrast microbubbles. The objective of this paper is to evaluate the various pulsing schemes for low $M I$ imaging of contrast microbubbles and better understand their similarities and differences. The pulsing schemes considered are pulse inversion, power modulation, and their combination. Emphasis is placed on identifying whether nonlinearity due to propagation in tissue may be discriminated from nonlinearity due to scattering from bubbles. Bubble destruction (use of high $M I$ ) and tissue motion were not considered in this work. The evaluation of the different pulsing schemes was performed with numerical simulations from well established theoretical models and experimental data from microbubbles in tissue phantoms and human tissues.

\section{8:40}

2aBB3. Contrast agent response to chirp reversal. Anthony Novell (INSERM U930, CHRU Bretonneau, 37044 Tours Cedex 9, France, anthony.novell@etu.univ-tours.fr), Sander Van Der Meer (Physics of Fluids, University of Twente, P.O. Box 217, 7500 AE Enschede, Netherlands, s.m.vandermeer@utwente.nl), Michel Versluis (Physics of Fluids, University of Twente, P.O. Box 217, 7500 AE Enschede, Netherlands, m.versluis@utwente.nl), Nico De Jong (Erasmus MC, Dr Molewaterplein 50 room Ee2302, 3015GE Rotterdam, Netherlands, n.dejong@erasmusmc.nl), Ayache Bouakaz (INSERM U930, 2, Bvd Tonnelle, 37044 Tours Cedex 9, France, bouakaz@med.univ-tours.fr)

We investigate an excitation approach for contrast agents based on chirps. This technique, named chirp reversal, consists in transmitting an up sweep frequency chirp (UPF) followed by a down sweep frequency chirp (DNF). Simulations using a modified Rayleigh-Plesset equation were carried out. Chirps with center frequencies from 1.4 MHz to $2 \mathrm{MHz}$, pressures from $50 \mathrm{kPa}$ to $200 \mathrm{kPa}$ and frequency bandwidths from $30 \%$ to $65 \%$ were considered. High speed optical observations and acoustical measurements were performed using individual contrast bubbles of radii from $1 \mu \mathrm{m}$ to 5 $\mu \mathrm{m}$ and a diluted solution of contrast agent respectively. Simulations showed differences between bubbles' oscillations following UPF and DNF chirps in terms of amplitude and duration. Maximal differences occurred for bubbles that were around $80 \%$ and $140 \%$ of the resonance size. Bubbles at resonance or far away from resonance provided identical responses to UPF and DNF chirps. Larger bandwidths and higher acoustic pressures accentuate further the difference between the UPF and DNF responses. These findings were confirmed through optical data and acoustical measurements. The results reveal the potential of chirp reversal for contrast agent detection.

\section{9:00}

2aBB4. Dual-frequency insonation of single microbubbles. Marcia Emmer (Erasmus MC, Ee2302, P.O. Box 2040, 3000 CA Rotterdam, Netherlands, m.emmer@erasmusmc.nl), Hendrik J. Vos (Biomedical Engineering, Erasmus MC, P.O. Box 2040, 3000 CA Rotterdam, Netherlands,
H.J.Vos@ErasmusMC.nl),Nico De Jong(Erasmus MC, Dr Molewaterplein 50 room Ee2302, 3015GE Rotterdam, Netherlands, n.dejong@erasmusmc .nl)

Radial modulation imaging is a new medical imaging technique based on dual-frequency insonation of ultrasound contrast agents. The difference in echo between a high frequency 'imaging' pulse transmitted at either the compression or rarefaction phase of a low frequency 'modulating' pulse is detected by regular correlation techniques. Little is however known about the contrast agent microbubble dynamics in a dual-frequency ultrasound field, which were investigated in this study. Using a high-speed camera system, the radial excursions of single phospholipid-coated microbubbles were recorded. The microbubbles were simultaneously insonified with 2.5 cycles pulse at $0.5 \mathrm{MHz}$ and $30 \mathrm{kPa}$ and a 32 cycles pulse at $3.75 \mathrm{MHz}$ and $80 \mathrm{kPa}$. The microbubbles studied had diameters ranging from $1.1-5.2 \mu \mathrm{m}$. Microbubbles smaller than $1.4 \mu \mathrm{m}$ frequently showed shrinkage. Microbubbles larger than $2.6 \mu \mathrm{m}$ showed low $(<8 \mathrm{~dB})$ or no amplitude modulation of the high frequency radial excursion. Microbubbles with diameters between 1.4 and $2.6 \mu \mathrm{m}$ showed high amplitude modulation (up to $25 \mathrm{~dB}$ ) and strong compression-only oscillation, which both may be explained by nonlinear shell properties. The observed behaviour is beneficial for the detection of contrast agents.

\section{9:20}

2aBB5. Sub-harmonic response from polymer-shelled contrast agents with a 40-MHz excitation. Jeffrey A. Ketterling (Frederic L. Lizzi Center for Biomedical Engineering, Riverside Research Institute, 156 William St., New York, NY 10038, USA, ketterling@ rrinyc.org), Jonathan Mamou (Riverside Research Institute, 156 William St., 9th Floor, New York, NY 10038, USA, mamou@ rrinyc.org)

There is a growing interest in using acoustic contrast agents with highfrequency ultrasound $(>15 \mathrm{MHz})$ in order to better visualize microcirculation. Experiments were performed with polycaprolactoneshelled agents (POINT Biomedical, San Carlos, CA) having mean diameters of $0.56,1.1$, and $3.4 \mu \mathrm{m}$. The agents were heavily diluted in filtered water and injected through a $200 \mu \mathrm{m}$ channel into the focal zone of a $40-\mathrm{MHz}$ transducer that had a focal length of $12 \mathrm{~mm}$ and an outer diameter of $6 \mathrm{~mm}$. Backscatter signals from single agents were digitized using tone bursts of 5 to 20 cycles at peak-negative pressures of 0.6 to $6.3 \mathrm{MPa}$. 1000 valid singlebubble backscatter events at each exposure condition were digitized and then analyzed for $20-\mathrm{MHz}$ subharmonic content. The data showed that the subharmonic response was initiated between 5 and 10 cycles and the likelihood of a subharmonic event increased as the number of cycles increased. A subharmonic backscatter response was most likely at 3.9 MPa for the $3.4 \mu \mathrm{m}$ agent and $1.7 \mathrm{MPa}$ for the 0.56 and $1.1 \mu \mathrm{m}$ agents. The increased pressure for subharmonic activity for larger agent was consistent with its larger size.

\section{9:40}

2aBB6. Combined optical and acoustical characterization of single ultrasound contrast agent microbubbles. Jeroen Sijl (Physics of Fluids, University of Twente, P.O. Box 217, 7500 AE Enschede, Netherlands, j.sijl@utwente.nl), Timo Rozendal (Physics of Fluids, University of Twente, P.O. Box 217, 7500 AE Enschede, Netherlands, t.rozendal@student.utwente.nl), Hendrik J. Vos (Biomedical Engineering, Erasmus MC, P.O. Box 2040, 3000 CA Rotterdam, Netherlands, H.J.Vos@ErasmusMC.nl), Benjamin Dollet (Physics of Fluids, University of Twente, P.O. Box 217, 7500 AE Enschede, Netherlands, benjamin.dollet@univ-rennes1.fr), Nico De Jong (Erasmus MC, Dr Molewaterplein 50 room Ee2302, 3015GE Rotterdam, Netherlands, n.dejong@erasmusmc.nl), Detlef Lohse (Physics of Fluids, University of Twente, P.O. Box 217, 7500 AE Enschede, Netherlands, d.lohse@utwente.nl), Michel Versluis (Physics of Fluids, University of Twente, P.O. Box 217, 7500 AE Enschede, Netherlands, m.versluis @utwente.nl)

Contrast enhancement in medical ultrasound imaging is provided by the non-linear characteristics of coated microbubbles used as Ultrasound Contrast Agents (UCA). Optical time-resolved observations of the UCA mi- 
crobubble dynamics have revealed new non-linear bubble behavior such as "compression only" and "thresholding" behavior. Up to now, the contributions of such behavior to the non-linear acoustic response of UCA microbubbles is not known. Theoretically, the sound emission of an oscillating microbubble is derived from the unsteady Bernoulli equation and mass conservation assuming incompressible and irrotational flow. An experimental validation of this relation between the radial dynamics and the sound emission of a microbubble is not straightforward. We present a combined optical and acoustical setup to characterize individual BR-14 (Bracco Research S.A., Geneva, Switzerland) UCA microbubbles. The bubbles were isolated in a capillary fiber by an active flow control. During insonation the radial response of the single microbubble was recorded with the Brandaris ultra high-speed camera while the resulting acoustic response was measured with an accurately calibrated sensitive transducer. The sound emission calculated from the measured radius-time curves gives excellent quantitative agreement with the directly measured sound emission for both the linearly oscillating microbubbles and bubbles displaying "compression-only" and "thresholding" behavior, which indeed resulted in a strong non-linear sound emission.

\title{
Invited Paper
}

10:00

\begin{abstract}
2aBB7. Secondary Bjerknes forces between ultrasound contrast agent microbubbles. Michel Versluis (Physics of Fluids, University of Twente, P.O. Box 217, 7500 AE Enschede, Netherlands, m.versluis@ utwente.nl), Valeria Garbin (Physics of Fluids, University of Twente, P.O. Box 217, 7500 AE Enschede, Netherlands, v.garbin@tnw.utwente.nl), Benjamin Dollet (Physics of Fluids, University of Twente, P.O. Box 217, 7500 AE Enschede, Netherlands, benjamin.dollet@univ-rennes1.fr), Leen Van Wijngaarden (Physics of Fluids, University of Twente, P.O. Box 217, 7500 AE Enschede, Netherlands, l.vanwijngaarden@tnw.utwente.nl), Nico De Jong (Erasmus MC, Dr Molewaterplein 50 room Ee2302, 3015GE Rotterdam, Netherlands, n.dejong@erasmusmc.nl), Detlef Lohse (Physics of Fluids, University of Twente, P.O. Box 217, 7500 AE Enschede, Netherlands, d.lohse@utwente.nl)

Acoustic radiation forces arise on UCA microbubbles from pressure gradients in the ultrasound pressure field. The ultrasound wave emitted by an oscillating UCA microbubble and experienced by a neighboring bubble results in the so-called secondary Bjerknes force. Typically, only the (time) averaged value of the secondary Bjerknes force is estimated from experiments. Here, the ability to resolve in time the radial dynamics of UCAs and the evolution of their relative distances, allows us to obtain a quantification of the instantaneous secondary Bjerknes force. Two bubbles were selected and confined within two separate optical traps and positioned away from the sample chamber wall prior to insonation. The radius-time curves and distance-time curves were then extracted from high-speed optical recordings. The camera fully resolved the alternating attractive-repulsive features of the secondary Bjerknes force in time. The effect of the time averaged secondary Bjerknes force results in a net attraction of the two bubbles, with a typical observed displacement of 1-2 $\mu \mathrm{m}$, with an instantaneous peak value of the secondary Bjerknes force of up to $10^{-6} \mathrm{~N}$. We predict the mutual interaction of the two coated bubbles in their translation with an accuracy better than $10 \%$.
\end{abstract}

\section{0:20-10:40 Break}

\section{Contributed Paper}

\section{0:40}

2aBB8. Mathematical models for contrast bubble dynamics. Charles C. Church (University of Mississippi, NCPA, 1 Coliseum Drive, University, MS 38677, USA, cchurch@olemiss.edu), John S. Allen (University of Hawaii-Manoa, Department of Mechanical Engineering, Holmes Hall Room 302, Honolulu, HI 96822, USA, alleniii@ hawaii.edu)

Theoretical investigation of the acoustic responses of albuminencapsulated microspheres began over fifteen years ago when Albunex, the first agent approved for clinical use in the US, was still in development. Since that time, the number of potential ultrasound contrast agents has grown considerably. Depending on the agent, the shell may comprise a layer of proteins, synthetic polymers, surfactants, or lipids with a thickness from a few $\mathrm{nm}$ to $500 \mathrm{~mm}$ and surrounding air, sulfur hexafluoride, perfluorocarbon, or other gas. A thorough understanding of the interaction between ultrasound pulses and contrast microbubbles is essential for the successful clinical application of a particular agent. In this talk, the behavior of various contrast agents will be discussed, and appropriate models for each will be described. The basis for each of these theories is a free bubble model "supplemented" by the effect of the encapsulating shell. The differences among these models lie primarily in their treatment of the encapsulating layer and, to some extent, the surrounding medium. Comparisons among models will include predictions of radial responses, thresholds for bubble destruction, and clinically significant acoustic properties (resonance frequency, scattering strength, nonlinearity, etc.). [Supported by NIH 2 RO1 EB000350-04A2 (CCC) and by NIH 2 G12RR003061-21 (JSA).]

\section{Invited Papers}

2aBB9. Ultrasound contrast agents: from concept to clinical use. François Tranquart (INSERM U930, 2, Bvd Tonnelle, 37044 Tours Cedex 9, France, tranquart@med.univ-tours.fr), Ayache Bouakaz (INSERM U930, 2, Bvd Tonnelle, 37044 Tours Cedex 9, France, bouakaz@med.univ-tours.fr), Aurore Bleuzen (INSERM U930, 2, Bvd Tonnelle, 37044 Tours Cedex 9, France, bleuzen@chu-tours.fr), Peggy Palanchon (INSERM U930, 2, Bvd Tonnelle, 37044 Tours Cedex 9, France, palanchon@med.univ-tours.fr), Jean-Michel Correas (Hopital Necker, 75015 Paris, France, jean-michel.correas@nck.ap-hop-paris.fr)

Contrast ultrasound imaging techniques are of interest for almost every clinical application in all organs including not only liver and kidney but also superficial organs and brain or lungs. This technique is mainly powerful in terms of lesion detection and characterization especially for liver with diagnostic value greater than $90 \%$. This was the case for all categories of lesions (with values nearly $100 \%$ for liver metastases, FNH) but the accuracy was slightly lower for HCC. Specific recommendations were established by EFSUMB for the use of contrast agents in liver, pancreas, kidney, trauma and brain imaging for a proper and safe use to improve diagnosis accuracy. On the other hand, contrast-enhanced ultrasound may be used in evaluating response to therapy. The follow-up of vascularization under specific targeted treatments offers the capacity to early diagnose positive or negative local response for an adaptation of therapeutic way 
before or in absence of tumour size changes. This non invasive method could be repeated without adverse events early after starting this therapy in order to depict local response or recurrence for a better adaptation of dose with a strong impact on cost and limitation of adverse events.

\section{1:20}

2aBB10. Dynamics of microbubbles targeted to surfaces: numerical and experimental modelling. Richard Manasseh (CSIRO, Fluid Dynamics Group, PO Box 56, Highett, VIC 3190 Melbourne, Australia, Richard.Manasseh@csiro.au), Edward Payne (University of Melbourne, Department of Mechanical \& Manufacturing Engineering, Parkville, VIC 3010 Melbourne, Australia, Edward.Payne@csiro.au), Andrew Ooi (University of Melbourne, Department of Mechanical \& Manufacturing Engineering, Parkville, VIC 3010 Melbourne, Australia, a.ooi@unimelb.edu.au)

Numerical calculations and illustrative experiments are presented on the volumetric oscillations of microbubbles on and near surfaces. There is a considerable theoretical and experimental literature on the acoustic interactions of bubbles. In the present study, the surface was represented by a mirror-image bubble and the nonlinear frequency response calculated by integrating acoustically coupled sets of Rayleigh-Plesset-like equations. A significant shift was found in the peak nonlinear response frequency of a bubble targeted onto a surface. This effect is increased when other bubbles are nearby on the surface. Owing to the asymmetric influence of the surface, experimental images were dominated by shape-mode instabilities, making optical determination of the peak nonlinear response frequency difficult. Moreover, it was found that even if bubbles are separated by only a small fraction of the sound wavelength, time delays owing to the finite speed of sound have a surprisingly significant influence. Calculations on the symmetric mode of mutual oscillation showed that the introduction of time delays significantly modified harmonics of the spectrum.

\section{1:40}

2aBB11. Echogenic liposomes for image-guided drug delivery. Christy K. Holland (University of Cincinnati, Biomedical Engineering, MSB, 231 Albert Sabin Way, Cincinnati, OH 45267-0586, USA, Christy.Holland@uc.edu), David D. McPherson (University of Texas Health Science Center, 6431 Fannin St., MSB 1.252, Houston, TX 77030, USA, David.D.McPherson@uth.tmc.edu)

Echogenic liposomes (ELIP) are under development to enable ultrasound-controlled drug delivery. Ultrasound-triggered release of hydrophilic and lipophilic agents was assessed from circulating ELIP in vitro using color Doppler ultrasound (6 MHz). Calcein, or recombinant tissue plasminogen activator (rt-PA), both hydrophilic agents, or papaverine, a lipophilic agent, were each loaded into ELIP and diluted in $0.5 \%$ bovine serum albumin. Ultrasound-triggered release of calcein, rt-PA or papaverine from ELIP was determined relative to detergent and untreated controls. Calcein concentration was measured by fluorimetry and release of rt-PA was assayed with a chromogenic substrate and a spectrophotometric method. Papaverine concentration was quantified by absorbance spectrophotometry and the amount of papaverine associated with P-ELIP was determined using a spin column filtration technique. Dynamic changes in echogenicity were assessed with low output B-mode ultrasound (0.04 MI) as mean digital intensity. Treatment with color Doppler ultrasound resulted in a statistically significant amount of calcein and rt-PA release from liposomes $(\mathrm{p}<0.01)$, but did not induce papaverine release $(\mathrm{p}>0.05)$. The differential efficiency of ultrasound-mediated pharmaceutical release from ELIP for water-soluble and lipid-soluble compounds suggests that water-soluble drugs are better candidates for the design and development of ultrasoundcontrolled drug delivery systems.

\section{Contributed Papers}

12:00

2aBB12. Ultrasound contrast agents in an in vivo murine melanoma model. François Tranquart (INSERM U930, 2, Bvd Tonnelle, 37044 Tours Cedex 9, France, tranquart@med.univ-tours.fr), Ayache Bouakaz (INSERM U930, 2, Bvd Tonnelle, 37044 Tours Cedex 9, France, bouakaz@med.univ-tours.fr), Sophie Serriere (INSERM U930, 2, Bvd Tonnelle, 37044 Tours Cedex 9, France, serrie_s@med.univ-tours.fr)

The purpose of the study was to test different types of microbubble contrast agents (SonoVue ${ }^{\circledR}$, Definity ${ }^{\circledR}$ and targeted microbubbles against vascular endothelial growth factor receptors (KDR, antiCD31)) to quantify angiogenesis. After SonoVue $₫$ and Definity $®$ injections, a strong, rapid and heterogeneous signal enhancement was detected in all tumors. According to the tumor size, the quantification of the perfused area revealed major interindividual variations. Three groups of animals bearing tumors, following SonoVue ${ }^{\circledR}$ administration, were arbitrarily constituted to compare various sonographic parameters such as AUC, mTT, etcœ. The tumoral size increase seemed negatively correlated to a decrease in all quantified parameters. Non linear acoustic signal from microbubbles targeted to the molecular site was determined by an ultrasound-based destruction-reperfusion scheme. In tumor-bearing mice, an increase of the retention time ( $>10$ minutes) was revealed following KDR and antiCD31 targeted microbubbles administrations when compared to the retention time of non targeted microbubbles. The amount of remaining targeted bubbles remains always lower than non specific agents. Results showed that quantitative contrast-enhanced ultrasound imaging could be an effective method for monitoring angiogenesis process in mice and so could be used for the follow-up of tumours under specific treatment.

\section{2:20}

2aBB13. Ultrasound contrast imaging of angiogenisis in a murine tumor model. Olivier Lucidarme (CHU Pitié-Salpétrière, 47-83 Boulevard de l'Hôpital, 75651 Paris, France, olivier.lucidarme@psl.aphp.fr), Laurent Taillade (Laboratoire d'Imagerie Paramétrique, 15 rue de l'Ecole de Médecine, 75006 Paris, France, laurenttaillade2@yahoo.fr), Aymeric Guibal (Laboratoire d'Imagerie Paramétrique, 15 rue de l'Ecole de Médecine, 75006 Paris, France, guibala@hotmail.com), Sebastien Mule (Lab d'Imagerie Fonctionnelle, 91 Boulevard de l'Hôpital, 75634 Paris, France, Sebastien.Mule@imed.jussieu.fr), Eva Comperat (CHU Pitié-Salpétrière, 47-83 Boulevard de l'Hôpital, 75651 Paris, France, eva.comperat@psl.aphp.fr), Yasmina Badachi (Laboratoire d'Imagerie Paramétrique, 15 rue de l'Ecole de Médecine, 75006 Paris, France, yasmina.badachi@gmail.com), Erwan Jouannot (Laboratoire d'Imagerie Paramétrique, 15 rue de l'Ecole de Médecine, 75006 Paris, France, Erwan.Jouannot@sanofi-aventis.com), Olivier Rixe (CHU PitiéSalpétrière, 47-83 Boulevard de l'Hôpital, 75651 Paris, France, olivier.rixe@psl.aphp.fr), Lori_Bridal (Laboratoire d'Imagerie Paramétrique, 15 rue de l'Ecole de Médecine, 75006 Paris, France, bridal @lip.bhdc.jussieu.fr)

Microvascularization modifications should precede tumor size-changes during anti-angiogenic therapy. We applied contrast functional ultrasound imaging (fUSI) to detect changes in Wilms tumors with anti-angiogenic treatment (Bevacizumab). Human Wilms tumor cells was grafted in left kidney of 32 mice. Once tumors had $>5 \mathrm{~mm}$ diameter, mice received : placebo, $\mathrm{N}=14$; Bevacizumab for 21days, $\mathrm{N}=11$; and Bevacizumab for 10days followed by placebo for 11 days, $\mathrm{N}=7$. On days $-1,+1,+9,+14$ and +21 
with respect to treatment start, fUSI was performed (CPS mode, SonoVue) Linear time intensity curves were obtained from regions in kidney cortex and matched-depth of tumor for first bolus passage and 50s following acoustic destruction of contrast. Excised tumor weight decreased with increased treatment duration: $3.7+/-1.8 \mathrm{~g}$ (placebo), $2.3+/-1.9 \mathrm{~g}$ (Bevacizumab10days, placebo-11days), 1.4+/-0.7 g (Bevacizumab-21 days) $[\mathrm{p}<0.05]$. Area under the bolus-passage curve (AUC) and the plateau intensity of the destruction-reperfusion were greater from $D+9$ to $D+21[p<0.04]$ in the placebo than Bevacizumab-21day. For the group treated during the first 10 days, fUSI values were comparable to those of the treated group until $\mathrm{D}+14$, then increased to become slightly superior to those of the placebo group by $\mathrm{D}+21$. Noninvasive fUSI demonstrated revascularization after suspension of anti-VEGF therapy.

12:40-1:40 Lunch Break

\section{Invited Paper}

\section{$1: 40$}

2aBB14. Violent cavitation from optically configured microbubble pairs. Paul A. Campbell (University of Dundee, Ewing 1-6, Carnegie Physics Laboratory, Main Campus, DD1 4HN Dundee, UK, p.a.campbell@dundee.ac.uk)

The mutual interaction between two cavitating microbubbles was investigated using a novel optical trapping arrangement. This approach facilitated development of arbitrary, stable, and initial spatial configurations for two-bubble systems. Critically, exercising optical control over such a binary system can effectively isolate it from resident bubble populations during insonation. This ensures that any early stage dynamical evolution of the system is dominated by the mutual interaction of the two bubbles in view, rather than any extraneous influence arising from 'cross-talk'. In circumstances where the bubbles are located far from a rigid boundary, we observed, using high speed microphotography at framing rates of over $1 \mathrm{MHz}$, that the action of secondary radiation forces leads to mutual bubble attraction often followed by the development of violent microjetting. Microbubble coalescence, with subsequent fragmentation could be observed, and the generation of short-lived anti-bubbles can often occur also. Finally, when cells are present as confluent monolayers on rigid substrates, the statistics for microbubble outcome and dynamics are modified somewhat. I will relate statistics to the parameter space that we interrogated, which included: inter-bubble spacing; relative bubble radii; and individual bubble stand-off parameters.

\section{Contributed Papers}

2:00

2aBB15. Nonlinear propagation in microbubble cloud, does it make the distal myocardium appear brighter or darker? Meng-Xing Tang (Imperial College London, Dept. of Bioengineering Engineering, South Kensington, SW7 2AZ London, UK, mengxing.tang@ic.ac.uk), Robert Eckersley (Imperial College London, Imaging Sciences Department, Hammersmith Campus, Du Cane Road, W12 0HS London, UK, r.eckersley@ic.ac.uk)

A number of questions remain in contrast ultrasound imaging regarding the complex interaction between bubbles, US and tissue. E.g. in contrast echocardiography for perfusion imaging, it is sometimes difficult to interpret the images for the myocardium distal to the probe. Due to US nonlinear propagation through the bubble-filled chamber, the distal part can appear artefactually brighter or darker in e.g. Pulse Inversion images. Although tissue at target is likely to increase in brightness in contrast specific images when nonlinear propagation happens, it is less clear for microbubbles. This work tries to gain better understanding of this by investigating how nonlinear propagation of ultrasound pulses can change the appearance of microbubbles and tissues in Pulse Inversion images by altering their acoustic response. A series of specifically designed simulations and experiments were performed. The results show that nonlinear propagation can have a significant impact on the appearance of tissue containing bubbles, and this varies with insonating frequency and pressure. These results suggest the mechanism involves a balance between a reduction in nonlinear bubble scattering after propagation against an increase in the nonlinear tissue scattering. Consequently the deeper tissues containing bubbles may appear brighter or darker depending on the relative contribution of these two effects.

\section{2:20}

2aBB16. Frequency dependence of kidney injury induced by contrast-aided diagnostic ultrasound. Douglas Miller (University of Michigan, Department of Radiology, Ann Arbor, MI 48109-0553, USA, douglm@umich.edu), Chunyan Dou (University of Michigan, Department of Radiology, Ann Arbor, MI 48109-0553, USA, chunyand@umich.edu), Roger C. Wiggins (University of Michigan, Department of Internal Medicine (Nephrology), Ann Arbor, MI 48109, USA, rwiggins@med.umich.edu)

Glomerular capillary hemorrhage (GCH) induced in rat kidneys by contrast aided diagnostic ultrasound was examined as a function of ultrasonic frequency. The right kidneys of anesthetized rats mounted in a water bath were exposed to image pulse sequences at $1 \mathrm{~s}$ intervals during intravenous infusion of diluted ultrasound contrast agent. Diagnostic ultrasound (DUS) scanners were utilized for exposure at 1.5, 2.5, 3.2, 5.0 and 7.4 MHz. A laboratory exposure system (LES) was used to simulate DUS exposure at 1.0, $1.5,2.25,3.5,5.0$ and $7.5 \mathrm{MHz}$ at relatively high peak rarefactional pressure amplitudes (PRPAs). The exposed kidneys were removed and fixed for histology. GCH was measured by counting glomeruli with blood in the urinary (Bowman's) space on histological sections. The percentage of GCH at a fixed exposure frequency showed a rapid rise with PRPA above an apparent threshold. The threshold values were approximately proportional to the ultrasound frequency, with proportionality constants of $0.5 \mathrm{MPa} / \mathrm{MHz}$ for DUS and 0.6 MPa/MHz for LES exposures. The increasing thresholds with increasing frequency limited the GCH effect for contrast aided DUS, and no GCH was seen for DUS at 5.0 or $7.4 \mathrm{MHz}$ for the highest available PRPAs. 


\title{
Session 2aEAa
}

\section{Engineering Acoustics, Underwater Acoustics, and ECUA: Sonar Transducer Design and Modeling II}

\author{
John B. Blottman, Cochair \\ Naval Undersea Warfare Center, Division Newport, 1176 Howell Street, Newport, RI 02841-1708, USA \\ Pascal Mosbah, Cochair \\ IEMN dpt ISEN, UMR CNRS 8520, Lille, France
}

\section{Invited Papers}

8:00

2aEAa1. Acoustic array interactions in the time domain. George W. Benthien (511 Savoy Street, San Diego, CA 92106, USA, benthien@cox.net)

In this paper I discuss the computation of acoustic array interactions in the time domain. Acoustic array interactions in the time domain are expressed in terms of mutual impulse response functions. The mutual impulse response between a pair of array elements is the acoustic force on the first element of the pair due to an acceleration impulse of the second with all other elements held rigid. The mutual response functions are calculated in the frequency domain using the boundary element program CHIEF and then Fourier transformed to the time domain. As the convergence in the frequency domain can be very slow, I introduce modified impulse response functions that converge much faster in the frequency domain and are simply related to the desired impulse response functions. This approach reduces the range of frequencies required in the CHIEF computations and gives better accuracy in the initial time period. Transient results are presented for an array of simple transducer elements. The mutual impulse response functions are combined with the transducer element equations to yield a system of differential-integral equations of delay type. A solution procedure for equations of this type is presented.

\section{$8: 20$}

2aEAa2. Exact model of Langevin transducers with internal losses. D. D. Ebenezer (Naval Physical and Oceanographic Laboratory, Thrikkakara, 682021 Kochi, India, tsonpol@vsnl.com), P. A. Nishamol (Naval Physical and Oceanographic Laboratory, Thrikkakara, 682021 Kochi, India, dd_ebenezer@yahoo.com)

An exact method is presented to analyze classical Langevin transducers with internal losses. The transducers consist of an axially polarized piezoelectric cylinder sandwiched between two elastic cylinders. All three cylinders are of the same diameter. Exact solutions to the exact equations of motion of the piezoelectric and elastic cylinders and the Gauss electrostatic condition are used. Complex piezoelectric and elastic coefficients are used to model internal losses. For each cylinder, the first set of solutions contains Bessel functions that form a complete set in the radial direction. The second and third sets contain trigonometric functions that form complete sets in the axial direction. They are used to represent fields that are symmetric and anti-symmetric with respect to the plane midway between the ends of the cylinder, respectively. The interface and boundary conditions are satisfied by using the orthogonal properties of the functions. Transducers with identical elastic cylinders at the ends as well as those with a light head mass and a heavy tail mass are analyzed. Numerical results are presented to illustrate the input electrical admittances of transducers. They are compared with those obtained using ATILA - a finite element package for the analysis of sonar transducers.

\section{8:40}

2aEAa3. Numerical acoustic modeling code applied to sonar transducers and arrays: review and perspectives. Gerard Vanderborck (Thales Underwater Systems SAS, Acoustic Department, 525 route des Dolines, BP 157, 06903 Sophia Antipolis Cedex, France, gerard.vanderborck@fr.thalesgroup.com)

In this paper a review based on several examples of Thales Underwater Systems (TUS) design and modeling of transducers will be presented: several examples like wide band free flooded ring flextensional and bender transducers, high frequency transducers with thermal analysis modeling included, will be discussed. An approach of the problem of the mutual impedance computation appearing in array modeling will be also presented. Tus used since several decades FEM - BEM codes to design acoustic transducers and arrays. The perspectives in transducer and array modeling will be indicated like the possibility to take into account the non-linearities in the material, the time dependent problem and the use of new kind of piezoelectric material will be also discussed. Specific aspect of single crystal transducers modeling will be presented. The increase of the computing power will also permit to take into account the complex problem of the interaction between transducers in an array (mutual impedances). We will conclude on complex ultra wide band antenna (based on strong transducers interactions) optimization and the associated modeling architecture issues. 
2aEAa4. Finite element analysis simulations of a piezoelectric cymbal actuator using atila software. Thomas Tremper (Micromechatronics Inc., 200 Innovation Blvd, Suite 155, State College, PA 16803, USA, ttremper@mmech.com), Alfredo Vazquez Carazo (Micromechatronics Inc., 200 Innovation Blvd, Suite 155, State College, PA 16803, USA, avc@mmech.com), Kenji Uchino (Micromechatronics Inc., 200 Innovation Blvd, Suite 155, State College, PA 16803, USA, kenjiuchino@mmech.com)

The piezoelectric ultrasonic cymbal actuator is a well known flextensional type actuator. Because of the actuator's geometry, the small movements of the piezoelectric ceramic are amplified by the flextensional structure. Simulation of this device was performed using Atila finite element software, which is specially suited for piezoelectric devices. This study will focus on some of the necessary parameters to accurately simulate cymbal actuators and other piezoelectric actuators. The simulations discussed will be compared to actual experimental data. Based on the experimental results the simulations will be reexamined and modifications will be made to increase the models accuracy. Employing this iterative process will promote increased accuracy in future simulations, which would therefore decrease development time and increase productivity.

\section{Contributed Papers}

\section{9:20}

2aEAa5. Underwater tensor sensors based on optical fiber bragg gratings. Francois M. Guillot (Georgia Institute of Technology, Mechanical Engineering, 771 Ferst Drive, Atlanta, GA 30332-0405, USA, francois.guillot@me.gatech.edu), David H. Trivett (Georgia Institute of Technology, Mechanical Engineering, 771 Ferst Drive, Atlanta, GA 303320405, USA, david.trivett@me.gatech.edu), Peter H. Rogers (Georgia Institute of Technology, Mechanical Engineering, 771 Ferst Drive, Atlanta, GA 30332-0405, USA, peter.rogers@me.gatech.edu)

This paper deals with the development a new type of low-noise underwater tensor sensor aimed at improving the performance and the design of directional arrays. The transducer can be configured either as a particle velocity sensor (dipole) or as fluid shear sensor (quadrupole). The sensing principle of the device relies on the interference signal from two Bragg gratings written on the same fiber, and illuminated by a tunable, narrowband light source. The gratings are a few centimeters apart, and they each reflect a portion of the incident light. The fiber is epoxied to two spacers separated by a small gap situated between the gratings. This assembly is then adhered to two plates connected by a hinge, which is located below the gap. One plate is held rigidly and the tip of the other (free) plate experiences transverse vibrations, when ensonified. These vibrations produce periodic gap length changes, which modulate the interference signal from the two gratings. The modulation is related to the amplitude of the sound wave and is monitored with a photodetector. A noise analysis will be presented and the performance of prototype sensors will be discussed.

\section{9:40}

2aEAa6. Beampattern optimization for a conformal projecting array of transducers with baffle effect and mutual coupling among elements. Yuanliang Ma (Institute of Acoustic Engineering, Northwestern Polytechnical University, 710072 Xi’an, China, ylma@nwpu.edu.cn), Zhengyao $\mathrm{He}$ (Institute of Acoustic Engineering, Northwestern Polytechnical University, 710072 Xi'an, China, hezhengyao@163.com)

Beampattern optimization for projecting arrays is much more challenging in comparison with receiving arrays, particularly for conformal projecting arrays. It is because the array elements have to work in resonance state for efficient projecting and the variation of radiation impedance has strong effect on the array performance. The radiation impedance for each element is determined by a lot of factors, i.e, the element position, baffle property, mutual coupling and velocity weighting vector of the array. Thus beampattern optimization for a projecting conformal array must take into account all these factors. One thing makes the topic more difficult that the mutual impedance for each element is changeable along with the weighting vector change. In this paper a global solution approach is formulated and a boundary element model combined with transducer equivalent circuits is developed. The driving voltage weighting vector is deduced through an optimization algorithm. Computer simulation together with experiments are conducted for a 14-element conformal array. The results agree well. The multiple beampatterns formed within a wide observation sector exhibit uniform beamwidth and low sidelobes. The source level for each beam is maximized in constraint of the maximum driving voltage of array elements being constant.

\section{0:00}

2aEAa7. Advanced Single Crystal Piezoelectric Transducers for Naval Sonar and Medical Ultrasound Applications. Wesley Hackenberger (TRS Technologies, Inc., 2820 East College Avenue, State College, PA 16801, USA, wes@ trstechnologies.com), Jun Luo (TRS Technologies, Inc., 2820 East College Avenue, State College, PA 16801, USA, jun@trstechnologies.com), Kevin Snook (TRS Technologies, Inc., 2820 East College Avenue, State College, PA 16801, USA, kevin@ trstechnologies .com)

Single crystal piezoelectrics have received much attention due to their very high piezoelectric and electromechanical coupling coefficients. Naval sonar and medical ultrasound transducers, fabricated from this material, exhibit unprecedented increases in bandwidth, source level, and sensitivity compared to ceramic based devices. In this paper we report many new developments in the growth and manufacture of single crystals. These include increased diameter $\left(>3^{\prime \prime}\right)$ crystal boules, improved compositional uniformity, increased thermal stability, and minimized surface damage. Manufacturing improvements are being applied to a broad range of crystal transducer applications. For navy sonar systems, crystals are resulting in very high bandwidths $(>100 \%)$ for transducer sizes that are 1/2 the resonating length and $1 / 4$ the weight of ceramic counterparts with equivalent (or often lower) source level. Manufacturing improvements such as high tensile strength, achieved by precision polishing crystal surfaces, are enabling new transducer operational regimes not possible with ceramic. In particular, single crystal 1-3 composite projectors show promise for achieving high source level in a compact, integrated system designs. Single crystals are also enabling new applications in medical ultrasound including very broadband and extremely high frequency transducers. Further improvements are expected in the near future.

\section{0:20-10:40 Break}

\section{0:40}

2aEAa8. Design of a phase array ultrasonic sensor using vibration decoupled concept. Chia-Yu Lin (National Taiwan University, Rm.433, Institute of Applied Mechanics, No.1, Sec.4, Roosevelt Rd., 10617 Taipei, Taiwan, cylinx@mems.iam.ntu.edu.tw), Chih-Chiang Cheng (National Taiwan University, Rm.433, Institute of Applied Mechanics, No.1, Sec.4, Roosevelt Rd., 10617 Taipei, Taiwan, cccheng@nbm.ntu .edu.tw), Wen-Jong Wu (Department of Engineering Science and Ocean Engineering, National Taiwan University, Rm.433, Institute of Applied Mechanics, No.1, Sec.4, Roosevelt Rd., 10617 Taipei, Taiwan, wjwu@ntu.edu.tw), Chuin-Shan Chen (Department of Civil Engineering, National Taiwan University, Rm.433, Institute of Applied Mechanics, No.1, Sec.4, Roosevelt Rd., 10617 Taipei, Taiwan, dchen@ntu.edu.tw), Jay Shieh (Department of Material Science and Engineering, National Taiwan University, Rm.433, Institute of Applied Mechanics, No.1, Sec.4, Roosevelt Rd., 10617 Taipei, Taiwan, jayshieh@ntu.edu.tw), Chih-Kung Lee (National Taiwan University, Rm.433, Institute of Applied Mechanics, No.1, Sec.4, Roosevelt Rd., 10617 Taipei, Taiwan, cklee@mems.iam.ntu .edu.tw)

Phase array ultrasonic sensors have been used widely to generate higher directional radiating patterns in which piezoelectric units are distributed 
sparsely in space. In this study, we present a novel design of a phase array ultrasonic sensor based on the concept of vibration decoupling. Decoupling is achieved by careful design of source aperture, and such design allows piezoelectric units tightly located in the same structure. The phase array sensor is designed herein as a cylinder with a dumbbell shape groove to decouple vibration, and finite element analysis is used to optimize the design. Two piezoelectric discs are adhered on the bottom plate of the sensor whereby desirable wave generation and detection are controlled adaptively. By electrical steering, the sensor thus operates as a dipole mode. Finally, prototypes of the sensor are made and experiments are conducted to verify simulation results.

\section{1:00}

2aEAa9. Nonlinear multifrequency transmitter for seafloor characterization. Lucilla Di Marcoberardino (Institut Jean le Rond D’Alembert, UMR 7190, 2 Place de la Gare de Ceinture, 78210 Saint Cyr L'Ecole, France, lucilladm@hotmail.com), Jacques Marchal (Institut Jean le Rond D'Alembert, UMR 7190, 2 Place de la Gare de Ceinture, 78210 Saint Cyr L'Ecole, France, jmarchal@ccr.jussieu.fr), Pierre Cervenka (Institut Jean le Rond D'Alembert, UMR 7190, 2 Place de la Gare de Ceinture, 78210 Saint Cyr L’Ecole, France, cvk@ccr.jussieu.fr)

In the underwater context, it is known that the frequency diversity provides essential information to derive the nature of the seafloor. This presentation deals with a new concept based on a transmitter that generates simultaneously several harmonic frequencies. Our final objective is to assert the feasibility of a multi-frequency tool whose desirable characteristics could be specified for applications such as detection of sunken oil slicks, sediment characterization, or surveys before cable or pipe laying. The acoustic beams are generated through the harmonic components of a shock wave radiated by an antenna driven at a high level. The source is unique in time and space so that the multi-frequency responses are inherently perfectly matched. A numerical model based on a generalized KZK equation has been developed to estimate the saturated fields. Measurements of the first harmonic fields obtained in our outdoor tank facility are compared with simulations.

11:20

2aEAa10. Modeling the acoustic radiation force in piezoelectrically driven micro fluidic chambers using ATILA. Karl Fisher (Lawrence Livermore National Laboratory, 7000 E Avenue, Brentwood, CA 94513, USA, fisher34@1lnl.gov)

A procedure is demonstrated to quantitatively evaluate the acoustic radiation forces in micro fluidic particle manipulation chambers. Typical estimates of the acoustic pressure and the acoustic radiation force are based on an analytical solution for a simple 1-dimensional standing wave pattern. The complexities of a typical micro fluidic channel limit the usefulness of this approach. By leveraging finite element approaches, and a generalized equation for the acoustic radiation force, channel designs can be investigated in two- and three-dimensions. Calculations and experimental observations are in good agreement. This work was performed under the auspices of the U.S. Department of Energy by University of California, Lawrence Livermore National Laboratory under Contract W-7405-Eng-48.

\section{1:40}

2aEAa11. Equivalent circuit models derived from finite element models using structural dynamics techniques. Julien Bernard (Thales Underwater Systems, 525 route des Dolines BP 157 Valbonne Parc d'Activités de Sophia Antipolis, 06903 Sophia Antipolis Cedex, France, julien.bernard@fr .thalesgroup.com)

Electroacoustic transducers can be divided into an active part, the driver, and a passive part, the structure. The driver ensures electromechanical transduction, while the structure performs various mechanical and acoustical functions, such as support, shock and pressure protection, and impedance transformation. For design purposes, one often needs an equivalent circuit model which gives a relationship between the acoustic characteristics of the overall device and that of its components. Transducer equivalent circuits are usually either physical or modal. Physical equivalent circuits lend themselves to the treatment of a transducer as an assembly, but in general yield frequency dependant parameters. Modal equivalent circuits are more adequate for resonant transducers, but describe transducers as a whole. This works shows how these two types of equivalent circuits can be obtained from a full finite element model by using common structural dynamics techniques: substructuring and modal expansion. It also shows that a third, hybrid type of equivalent circuit can be obtained by using a component mode synthesis technique derived from the Craig-Bampton method. This hybrid equivalent circuit combines the advantages of physical and modal equivalent circuits, enabling to express transducer modal parameters in terms of driver and structure modal parameters.

\section{2:00}

2aEAa12. Acoustic field calculation for a compact barrel-stave flextensional transducer array. Zhengyao He (Institute of Acoustic Engineering, Northwestern Polytechnical University, 710072 Xi'an, China, hezhengyao@163.com), Chao Sun (Institute of Acoustic Engineering, Northwestern Polytechnical University, 710072 Xi'an, China, csun@nwpu .edu.cn)

The boundary element method together with the finite element method is used to calculate the radiated acoustic field of a compact array of barrelstave flextensional transducers. At first, the surface vibration displacement distribution of one barrel-stave transducer is obtained by the finite element method using the commercial software ANSYS. The calculation results are then imported into the boundary element calculation software SYSNOISE. At the frequency of $1400 \mathrm{~Hz}$, the radiated acoustic field and radiation impedance are calculated by the boundary element method for a planar array which is composed of three identical barrel-stave flextensional transducers uniformly distributed on a circle with spacing much less than half wavelength. The calculation results show that the mutual interactions among elements are significant for the compact array. The mutual radiation resistance between two transducers is close to the self-radiation resistance of the transducers. And the transmitting source level of the 3-element array is $8.7 \mathrm{~dB}$ higher than that of one transducer if the surface vibration velocities of the transducers in the array are the same as that of one transducer. The proposed technique can be used to predict the performance of a transmitting transducer array at the stage of preliminary design.

\section{2:20}

2aEAa13. Finite Element Modeling of 2-D Transducer Arrays. Hind Mestouri (ISEN Brest (Institut Supérieur de l'Electronique et du Numérique), 20, rue Cuirassé Bretagne, C.S. 42807, 29228 Brest, France, hind.mestouri@isen.fr), Alain Loussert (ISEN Brest (Institut Supérieur de l'Electronique et du Numérique), 20, rue Cuirassé Bretagne, C.S. 42807, 29228 Brest, France, alain.loussert@isen.fr), Gilles Keryer (ISEN Brest (Institut Supérieur de l'Electronique et du Numérique), 20, rue Cuirassé Bretagne, C.S. 42807, 29228 Brest, France, gilles.keryer@isen.fr)

Active sonar detects objects underwater by sending out sound waves in pulses, scientists could measure the time it takes these pulses to travel through the water, reflect off of an object, and return to the ship. There are a number of factors limiting the performance of low frequency transducer arrays for active sonar systems including the crosstalk and housing interactions which affects the directivity pattern and the sensitivity. Many important issues in transducer arrays design, such as crosstalk, cannot be accurately studied using analytic method due to the complexity of the partial differential equations involved. Finite element method is the only appropriate way to gain more detailed information. In this paper, a 2-D finite element model is constructed to analyze the crosstalk and structure interactions, using ATILA code and GiD graphical interface, of transducer arrays, consists of six active piezoceramic bars, and developed for shallow water use. The directivity pattern strongly depends on the transducer size versus wavelength, each transducer elements interacts directly with its neighbour and indirectly with the other elements, and the radiated acoustic power may considerably vary from one element to another. It is shown that crosstalk and housing interactions can be reduced through the use of different interelement materials and housing characteristics. 


\title{
Session 2aEAb
}

\section{Engineering Acoustics: Acoustic Evaluation I}

\author{
David Brown, Chair \\ BTech Acoustics, LLC and Universtity of Massachusetts Dartmouth, ATMC, Electro-Acoustics Research Laboratory and ECE \\ Dept., 151 Martine St, Fall River, MA 02723, USA
}

\section{Contributed Papers}

\section{8:00}

2aEAb1. Measurement of Sound Velocity in Water Using Optical Probe and Acoustical Holography. Takeshi Ohbuchi (University of Tsukuba, 1-1-1 Tennoudai, 305-8573 Tsukuba, Japan, ohbuchi@aclab.esys.tsukuba.ac.jp), Koichi Mizutani (Tsukuba Univ., Tsukuba Science City, 305-8573 Ibaraki, Japan, mizutani@esys.tsukuba.ac.jp), Naoto Wakatsuki (Tsukuba Univ., Tsukuba Science City, 305-8573 Ibaraki, Japan, wakatuki@iit.tsukuba.ac.jp), Hiroyuki Masuyama (Toba National College of Maritime Technology, 1-1 Ikegami-cho, 517-8501 Toba, Japan, masuyama@ toba-cmt.ac.jp)

We propose a method for a determination of a three-dimensional sound velocity using optical computerized tomography (O-CT) and near-field acoustical holography (NAH) for measuring a phase object. Ultrasonic waves affect a phase of the light passing through radiated sound fields. The zeroth order diffraction light including sound pressure information is acquired by an avalanche photodiode. Eighteen projections are acquired by rotational scanning, and each projection along the optical axis is obtained by single linear scanning and electronically quadrature-detected as the complex amplitude. The complex sound fields are reconstructed by O-CT in a region of $40 \times 40 \mathrm{~mm}^{2}$. Then the sound field on another plane is propagated using $\mathrm{NAH}$ from the acquired sound fields, and the sound field on the same plane is reconstructed by O-CT. Comparing the phase of propagated and reconstructed sound fields in wavenumber domain, we can obtain the sound velocity in three dimensional space in a region of $30 \times 30 \times 10 \mathrm{~mm}^{3}$. The experimental results are in agreement with the reference value measured by another mean.

\section{8:20}

2aEAb2. Influence of a load on the nonlinear behavior of a piezoelectric rod under high sinusoidal voltages. Denis Parenthoine (Lussi, Université F Rabelais de Tours, 40, rue de la Chocolaterie, 41000 Blois, France, parenthoine_denis@yahoo.fr), Lionel Haumesser (Lussi, Université F Rabelais de Tours, 40, rue de la Chocolaterie, 41000 Blois, France, lionel.haumesser@univ-tours.fr), François Vander Meulen (Lussi, Université F Rabelais de Tours, 40, rue de la Chocolaterie, 41000 Blois, France, vandermeulen@univ-tours.fr), Louis-Pascal Tran-Hhuu-Hue (Lussi, Université F Rabelais de Tours, 40, rue de la Chocolaterie, 41000 Blois, France, tran@univ-tours.fr)

At high levels of excitation, appear, in piezoelectric devices, nonlinear phenomena which can adversely affect the quality of the applications to which they are devoted. It is, in particular, true in non-destructive evaluation, where the generation of harmonics in the ultrasonic tranducer can make inacurrate measurements in materials. In a previous work, third-order constants in a piezoceramic rod of PZT-21 under high sinusoidal electric fields have been evaluted from the analysis of second harmonic generation in the mechanical displacement. All these measurements have been performed under condition of complete free stress. In order to allow a better understanding of the nonlinear behavior of the transducer, various loads, are, now, applied at one end of the piezoceramic rod. Velocity masurements performed by a laser probe at the free end of the active element provides then informations about the nonlinear behavior of the system. Influence of load on the linear and nonlinear behavior of the piezoelectric rod is then studied in the case of an aluminium bar, and in the case of more nonlinear materials. Results are compared both experimentally and theoritically in loaded and unloaded configurations.

\section{8:40}

2aEAb3. Uncertainty of Acoustical Material Characteristic Measurements. Samir N. Gerges (Federal University of Santa Catarina (UFSC), Campus Universitario - Trindade, 88040-900 Florianopolis, Brazil, samir@emc.ufsc.br), Peter K. Giesbrecht (Federal University of Santa Catarina (UFSC), Campus Universitario - Trindade, 88040-900 Florianopolis,Brazil, samir@emc.ufsc.br)

The experimental determination of the acoustic properties of sound absorption materials depends on numerous factors. The accuracy level in the determination of each property depends, for example, on the measurement apparatus and the type of material. The objective of this work is to present a study about the main sources of uncertainty in the measurement of properties such as sound absorption coefficient, acoustic impedance, flow resistivity and porosity. The uncertainties of the measurement apparatus of each property are quantified. The uncertainty of the materials heterogeneity is also assessed and its individual contribution highlighted. Important aspects of the Standards ASTM C 522 and ISO 10534-2, which present, respectively, recommendations and a procedure for flow resistivity and sound absorption measurements, are discussed. The methodology to calculate the uncertainty of each property is discussed with emphasis on the relevancy of each uncertainty source.

\section{9:00}

2aEAb4. Design and implementation of a three-dimensional seven microphone vector intensity probe with low and high frequency compensations. Khalid Miah (University of Texas, P.O. Box 7155, Austin, TX 78713, USA, miah@mail.utexas.edu), Elmer Hixson (Univ. of Texas, ECE Dept., 1 University Station 0803, Austin, TX 78712-0240, USA, ehixson@mail.utexas.edu)

A seven microphone vector intensity probe has been designed and implemented in this report with low and high frequency compensations. Measurements from plane wave tube and anechoic chamber are used for overall system calibration with corrected gain and phase mismatch errors. Measured and calculated intensity level from both plane wave tube and anechoic room settings showed close agreement with each other in comparison to separate low and high frequency components of the array. Particle velocity vectors and pressures determined for calculating intensity were also used to calculate sound power and total energy density components of various sources. This microphone array vector intensity probe is then used to explore various sound source identification and localization problems.

\section{9:20}

2aEAb5. Application of contrast optimisation autofocus to flexible ultrasonic arrays for non-destructive testing. Alan J. Hunter (University of Bristol, Queen's Building, University Walk, BS8 1SY Bristol, UK, a.hunter@bristol.ac.uk), Bruce W. Drinkwater (University of Bristol, Queen's Building, University Walk, BS8 1SY Bristol, UK, 
b.drinkwater@bristol.ac.uk),Paul D. Wilcox(University of Bristol, Queen's Building, University Walk, BS8 1SY Bristol, UK, p.wilcox@bristol.ac.uk)

Flexible ultrasonic arrays are used for imaging within objects with complicated geometries for non-destructive testing and evaluation (NDT/NDE), e.g., thick-walled pipes, weld caps, etc. We consider the application of autofocus techniques (routinely used in synthetic aperture sonar (SAS)) to this engineering problem. A flexible ultrasonic array is functionally similar to a wide-beam, stripmap SAS with a single transmitter and receiver. Unfortunately, there are few autofocus algorithms available for this configuration. Popular algorithms, such as echo/image correlation, PGA, DPCA, etc., are better suited for use with a narrow-beam or multiple-receiver SAS. However, contrast optimisation is a more general technique that is well suited to the single transmitter/receiver geometry. In this presentation, we describe our implementation of contrast optimisation autofocus and show experimental results using a flexible array prototype.

\section{9:40}

2aEAb6. Inspection of complex components with flexible phased array transducer. Olivier Casula (CEA/LIST, bat 611, 91191 Gif sur Yvette, France, olivier.casula@cea.fr), Gwenael Toullelan (CEA/LIST, bat 611, 91191 Gif sur Yvette, France, gwenael.toullelan@cea.fr), Philippe Dumas (ZA, rue des Savourots, 70190 Voray-sur-l’Ognon, France, philippe .dumas@imasonic.com)

Non destructive testing techniques using ultrasonic methods are often carried out in contact. But, the inspection performances are limited to regular surfaces. Thus, surface irregularities lead to thickness variations of the coupling layer that result in beam distortions and losses of sensitivity. In the context, CEA/LIST has designed flexible phased-array techniques for compensating the surface irregularities and fitting the surface. The independent piezoelectric elements composing the radiating surface are mechanically assembled to build an articulated structure. An embedded profilometer measures the local surface distortion allowing to compute the optimized delay laws and to master the characteristics of the focus beam. Those delay laws computed by the UT-acquisition system are applied in real-time to the piezoelectric elements. To evaluate inspection method capabilities, CEA/LIST develops a simulation software for non destructive testing, CIVA, able to simulate realistic configurations in particular with complex 2D and 3D applications. Matrix flexible phased-array probes have been designed and manufactured in collaboration with IMASONIC. This paper sums up examples of inspections in complex geometries where these flexible probes have been successfully used. Moreover, the data are reconstructed thanks to CIVA tools and allow to locate and size the flaw in the part.

\section{0:00-10:20 Break}

\section{0:20}

2aEAb7. Characterization of laminated glasses by means of an inversion method using Finite Elements. Jorge Frances Monllor (DFISTS. Univ. de Alicante, Carretera de Sant Vicent del Raspeig s/n, 03690 San Vicente del Raspeig, Spain, jfmonllor@ua.es), Jaime Ramis Soriano (DFISTS. Univ. de Alicante, Carretera de Sant Vicent del Raspeig s/n, 03690 San Vicente del Raspeig, Spain, jramis@ua.es), Jesús Alba Fernandez (Escola Politècnica Superior de Gandia, Universitat Politècnica de València, Crtra Natzaret-Oliva s/n, 46730 Gandia, Spain, jesalba@ fis.upv .es), Enrique E. Segovia Eulogio (Depto Ing. Construcción. Univ. de Alicante, Carretera de Sant Vicent del Raspeig s/n, 03690 San Vicente del Raspeig, Spain, Enrique.Gonzalo@ua.es), Jenaro Vera Guarinos (DFISTS Univ. de Alicante, Carretera de Sant Vicent del Raspeig s/n, 03690 San Vicente del Raspeig, Spain, jenaro@dfists.ua.es)

The experimental determination of elastic modules and loss factor in laminated glass components is of great interest in building acoustics since at present there are a lot of building systems using these types of devices. This makes it necessary to predict the transmission losses in a partition. Even though described in regulation, the process is not exempt from serious difficulties. In this work we present a method in order to obtain the parameters mentioned above for a laminated glass composed of a sequence of isotropic layers by solving the model-based inverse problem for frequency admittance experimentally obtained. The parameter that best describes the mechanical constants of material of the layers is obtained by minimizing the discrepancy between the real numerically and numerically predicted waveform. This is done using an iterative optimization. A sensitivity study of the parameters uncertainty is performed in order to establish the feasibility of this technique.

\section{0:40}

2aEAb8. Detection of Shallow Underground Buried Object Using Air Vibration Probe. Yuji Sato (Tsukuba Univ., Tsukuba Science City, 3058573 Ibaraki, Japan, yuji@aclab.esys.tsukuba.ac.jp), Tomohiro Okamura (Univ. of Tsukuba, 1-1-1 Tennodai, 305-8573 Tsukuba, Japan, mizutani@esys.tsukuba.ac.jp), Koichi Mizutani (Tsukuba Univ., Tsukuba Science City, 305-8573 Ibaraki, Japan, mizutani@esys.tsukuba.ac.jp), Naoto Wakatsuki (Tsukuba Univ., Tsukuba Science City, 305-8573 Ibaraki, Japan, wakatuki@iit.tsukuba.ac.jp)

An air vibration probe is a device to measure an acoustic impedance using acoustic delay line oscillation. The frequency is changed when the acoustic impedance is changed. If some objects are varied, the frequency is expected to be changed in the case of the shallow underground detection. The advantage of this device is that it can detect the underground object easily without any contacts or destructions. Two experiments were practiced to study the relation among the frequency, the space between the probe and the ground, and the depth of object. The space was changed with the buried objects in various depths. The frequency became higher when the space became wider. In addition, the frequency was changed more obviously when the space was narrower. The probe scanned the buried objects in the fixed space. The objects were buried in various depths. The frequency became high when the object was buried shallower. In conclusion, the air vibration probe can detect the buried object because the frequency became high when something was buried.

\section{1:00}

2aEAb9. Ultrasonic Airflow Meter in Greenhouse Using Acoustic Reflection against Wall. Ikumi Saito (Tsukuba Univ., Tsukuba Science City, 305-8573 Ibaraki, Japan, saitou@aclab.esys.tsukuba.ac.jp), Naoto Wakatsuki (Tsukuba Univ., Tsukuba Science City, 305-8573 Ibaraki, Japan, wakatuki@iit.tsukuba.ac.jp), Koichi Mizutani (Tsukuba Univ., Tsukuba Science City, 305-8573 Ibaraki, Japan, mizutani@esys.tsukuba.ac.jp), Limi Okushima (National Institute for Rural Engineering, 2-1-6 Kannondai, 3058609 Tsukuba, Japan, limi@affrc.go.jp)

In this paper, we described an ultrasonic airflow meter in a greenhouse using an acoustic reflection against a wall. The ultrasonic airflow meter is available for measuring the spatial mean wind velocity and direction, which consists of two sound probes and the wall of the greenhouse. Use of sound probes has advantage that the airflow accumulated along sound paths from a loudspeaker to a microphone is obtained in contrast to point measurements. The wind velocity and direction are calculated from time of flights (TOFs) of direct and reflected signals. The wind velocity and direction were measured in a greenhouse of $7.2 \mathrm{~m} \times 29.0 \mathrm{~m}$ under factitious winds generated from two large electric fans located at the one side of the greenhouse. In addition, we detected air convection generated from two pairs of fans. These were measured every 20 seconds for 120 minutes. Regarding the measurement; by the proposed airflow meter and a reference; by a conventional one, their mean wind velocities were 0.12 and $0.15(\mathrm{~m} / \mathrm{s})$, respectively. The presented airflow meter using the acoustic reflection against the wall is convenient to measure the wind velocity and direction in large-size facilities such as the greenhouse.

\section{$11: 20$}

2aEAb10. An alternative and industrial method using low frequency ultrasound enabling to measure quickly tortuosity and viscous characteristic length. François Fohr (Centre de Transfert de Technologie du Mans, 20, rue Thalès de Milet, 72000 Le Mans, France, ffohr@cttm-lemans.com), Damien Parmentier (Centre de Transfert de Technologie du Mans, 20, rue Thalès de Milet, 72000 Le Mans, France, 
dparmentier@cttm-lemans.com),Bernard R. Castagnede(Laboratoire d'Acoustique de l'Université du Maine, Avenue Olivier Messiaen, 72085 Le Mans, France, bernard.castagnede@univ-lemans.fr), Michel Henry (Laboratoire d'Acoustique de l'Université du Maine, Avenue Olivier Messiaen, 72085 Le Mans, France, michel.henry@univ-lemans.fr)

In the past, several different methods have been proposed in order to determine tortuosity and characteristic lengths, using various physical approaches. For instance, the BET (from S. Brunauer, P. Emmett and E. Teller) method used the adsorption of argon molecules within the surface of the pores to measure thermal characteristic length (or the so-called specific surface). Ultrasonic methods, during the two last decades, were efficiently implemented to measure these parameters, using large frequency bandwidth, different gazes including helium, and varying static pressures. The most standard and well-known method relies on phase spectrum measurements performed over short bursts, by plotting the inverse of the squared velocity as a function of the inverse of the square root of the angular frequency. Unfortunately, this method is quite sensitive on noise during the unwrapping procedure. We here propose a much simplier and efficient method, which is very robust with noisy signals, working at one single frequency, in most cases around $40 \mathrm{kHz}$. Measurements have been done onto a specific bench developped at CTTM, with some calibrated glass beads, enabling to recover the expected values of the physicalparameters. This method is particularly well suited for industrial and "on line" applications.

\section{1:40}

2aEAb11. A new impedance tube for large frequency band measurement of absorbing materials. Jean Christophe Le Roux (CTTM, 20, rue Thales de Milet, 72000 Le Mans, France, jcleroux@cttm-lemans.com), Jean-Pierre_Dalmont (Laboratoire d'Acoustique de l'Université du Maine, Avenue Olivier Messiaen, 72085 Le Mans, France, Jean-Pierre.Dalmont@univ-lemans.fr), Bruno Gazengel (Laboratoire d'Acoustique de l'Université du Maine, Avenue Olivier Messiaen, 72085 Le Mans, France, bruno.gazengel@univ-lemans.fr)

The standard two microphones technique does not allow the measurement of absorbing materials characteristics at low frequency. Moreover, to cover a range from 100 to $6000 \mathrm{~Hz}$ two experiments have to be done with two different sample diameters. By using a sensor with a known volume velocity source developed by the LAUM together with the CTTM, it is demonstrated that the impedance can be obtained from 10 to $6000 \mathrm{~Hz}$ by performing only one measurement with a single material sample. Results showing the behaviour of some materials at low frequency are presented. On the other hand a comparison is done with classical Kundt tube results.

\section{2:00}

2aEAb12. An experimental study of Sound Transmission Loss (STL) measurement techniques using an impedance tube. Behrooz Yousefzadeh (Univ. of Tehran, School of Mechanical Engineering, 1439957131 Tehran, Iran, behrooz.j.y@gmail.com), Mohammad Mahjoob (Univ. of Tehran, School of Mechanical Engineering, 1439957131 Tehran, Iran, mmahjoob@ut.ac.ir), Nader Mohammadi (Univ. of Tehran, School of Mechanical Engineering, 1439957131 Tehran, Iran, nmohamady@ut.ac.ir), Ali Shahsavari (Univ. of Tehran, School of Mechanical Engineering, 1439957131 Tehran, Iran, shervin.shahsavari@gmail .com)

A comparison between the two sound transmission loss (STL) measurement techniques using an impedance tube (i.e. two-load method and anechoic termination method) is presented. A modified B\&K type 4206 impedance tube has been designed and built. STL tests have been carried out for three homogeneous and isotropic materials with disk-type test samples of identical diameters and different thicknesses. In addition, the results have been compared with those of the classical and more reliable method of tworoom. For both methods, the effect of downstream (tube termination) boundary conditions have been completely studied. The two-load method yields results which matches with two-room measurements, especially when the two boundary conditions are considerably different. The anechoic termination method, on the other hand, is significantly dependant on the termination boundary conditions.

TUESDAY MORNING, 1 JULY 2008

AMPHI MAILLOT, 8:00 TO 10:40 A.M.

\title{
Session 2aMUa
}

\section{Musical Acoustics and Physical Acoustics: Brass Instrument Acoustics I}

\author{
Thomas Moore, Cochair \\ Rollins College, Department of Physics, Winter Park, FL 32789, USA \\ Joël Gilbert, Cochair \\ Laboratoire d'Acoustique de l'Université du Maine, Avenue Olivier Messiaen, Le Mans, 72085, France
}

\section{Invited Paper}

\section{8:00}

\begin{abstract}
2aMUa1. Does mass matter? Examining a concrete didjeridu. Noam Amir (Tel Aviv University, Dept. of Communications Disorders, Sheba Medical Center, 52621 Tel Hashomer, Israel, noama@post.tau.ac.il)

The influence of wall material on the acoustics of musical wind instruments has been debated widely. While this has been examined for some western instruments, it has been barely touched upon regarding the Australian didjeridu. This is very interesting, considering that didjeridus vary enormously in materials and dimensions. Indeed, musicians and manufacturers alike often have very definite opinions concerning the influence of material type and thickness on instrument quality. As a first step towards examining this issue, we conducted a blind test involving three cylindrical didjeridus of identical internal dimensions: all three shared a basic structure of identical plastic tubing. However, a cement casing was cast on the exterior of one of these, bringing its weight to 17 kilograms. The three
\end{abstract}


instruments were fixed to a stationary wooden frame, then covered so that only the mouthpieces were visible. 32 players of varying expertise were allowed to play each instrument for as long as they wished, and then asked to judge which instrument was different from the other two. Results indicate that the responses were random, regardless of player level. Further research will be necessary to show whether this holds for noncylindrical bores and different internal finishings.

\section{Contributed Paper}

8:20

2aMUa2. Relationships among subjective assessments and measured physical properties in the didjeridu. John Smith (University of New South Wales, Music Acoustics, School of Physics, NSW 2052 Sydney, Australia, john.smith@unsw.edu.au), Guillaume Rey (University of New South Wales, Music Acoustics, School of Physics, NSW 2052 Sydney, Australia, guimms@free.fr), Joe Wolfe (University of New South Wales, Music Acoustics, School of Physics, NSW 2052 Sydney, Australia, J.Wolfe@unsw .edu.au)

Traditional didjeridus are unusual and ancient lip-valve instruments with an irregular bore that is largely constructed by termites eating the interiors of small eucalypt trees. This produces instruments with a broad range of bore geometries with many details not immediately apparent to a player. They are therefore well-suited for examining relationships between subjective assessments by players and their measured physical properties. In this study, seven experienced players assessed 38 didjeridus that spanned a wide range of quality, pitch and geometry. A control group of 11 plastic cylindrical pipes were also studied. Eight subjective parameters (backpressure, clarity, resonance, loudness, overtones, vocals, speed, and overall quality) were assessed and the ranking of each instrument correlated with measurements of their geometry and acoustic input impedance spectrum. A strong result of these experiments was the finding that the ranked quality of a didjeridu correlated negatively with the magnitude of its acoustic input impedance, particularly in the frequency range from 1 to $2 \mathrm{kHz}$. Maxima in the impedance of the player's vocal tract would have a greater effect on instruments with a low impedance, thus favouring the production of the varying spectral peaks or formants in the sound envelope that characterise this instrument.

\section{Invited Papers}

\section{8:40}

2aMUa3. A psychoacoustical investigation into the effect of wall material on the sound produced by lip-reed instruments. James W. Whitehouse (Acoustics Research Group, DDEM, MCT Faculty, Open University, Walton Hall, MK7 6AA Milton Keynes, UK, j.w.whitehouse@open.ac.uk), David B. Sharp (Acoustics Research Group, DDEM, MCT Faculty, Open University, Walton Hall, MK7 6AA Milton Keynes, UK, d.sharp@open.ac.uk)

In order to investigate whether a lip-reed instrument's material of manufacture plays a significant role in determining the timbre of the notes it produces, tests have been conducted on five post horns. These post horns have identical geometry but are manufactured from different copper alloys. Using a laser Doppler vibrometer, the structural resonances of each instrument have been established and the wall vibrations induced in each instrument when artificially blown have been measured. In this paper, these measurements are compared with findings from a series of blindfold playing tests carried out using professional musicians and with listening tests comprising notes produced by the different instruments.

\section{9:00}

2aMUa4. More experimental evidence favouring the hypothesis of significant wall vibration influence on radiated horn sound. Wilfried Kausel (Inst. f. Wiener Klangstil, Univ. f. Music, Anton von Webernplatz 1, A-1030 Vienna, Austria, kausel@mdw.ac.at), Alexander Mayer (Inst. f. Wiener Klangstil, Univ. f. Music, Anton von Webernplatz 1, A-1030 Vienna, Austria, mayer@mdw.ac.at)

The question whether wall vibrations of wind instruments do or can affect the radiated sound has not been finally answered yet. Instrument makers and musicians make a strong claim that wall thickness, material and conditioning are crucial factors for sound quality and response of wind instruments, while acousticians rather tend to question that claim. Recent experiments on horns are presented favoring the hypothesis that wall vibrations do matter. Although horn sound in general appears to change quite significantly when wall vibrations, particularly those of the bell, are dampened during artificial playing, it becomes more and more evident that more than one single mechanism has to be considered to explain the whole phenomenon. Radiation of the bell as multi-pole will add directivity and strengthen higher harmonics while absorption by the wall will rather weaken them. Dynamic interaction between air column and oscillating bore profile theoretically depends on broken symmetry. Feedback of structural resonances to the oscillating lips can be avoided by decoupling the mouthpiece from the instrument. Experiments and statistical evaluation have been laid out in order to exclude or identify certain mechanisms as well as possible artifacts like changes of mouthpiece position, bore or acoustical environment caused by damping forces.

\section{9:20}

2aMUa5. Design and manufacturing of an artificial marine conch by bore optimisation. Jef Petiot (Ecole Centrale Nantes IRCCyN, 1 rue de la Noe, BP 92101, 44321 Nantes, France, jean-francois.petiot@irccyn.ec-nantes.fr), Francois Tavard (Ecole Centrale Nantes - IRCCyN, 1 rue de la Noe, BP 92101, 44321 Nantes, France, francois.tavard@eleves.ec-nantes.fr)

The marine conch is a traditional instrument of the brass family. The resonator is made of the inner shape of the shell, with which the lips of the player interact by the way of a hole pierced in the extremity of the shell. Several notes can be played with this instrument. Unfortunately, the marine conch has become very rare and expensive. In order to build an artificial conch (by injection moulding), we studied the acoustics property of a natural conch. This paper is dedicated to the description of the measurements we made on the conch, and to the presentation of the method used to design the bore. Firstly, the input impedance of the natural conch was measured. Secondly, the size of the bore of the natural conch was assessed by the way of pictures and image processing tools. An initial bore of the artificial 
conch was designed with a CAD software, by taking into account manufacturing constraints. We developed next an optimisation procedure to improve the harmonicity of the artificial conch. Finally, an artificial conch was next manufactured by rapid prototyping. As a result, we noticed that the artificial conch manufactured can clearly be used as an interesting musical instrument.

\section{Contributed Paper}

9:40

2aMUa6. A design strategy for brass instruments. Robert W. Pyle (S. E. Shires Co., 11 Holworthy Place, Cambridge, MA 02138, USA, rpyle @ post.harvard.edu)

This paper describes the processes being used to design a $\mathrm{C}$ trumpet. Since "one size fits all" is not a good approach for high-quality brass instruments, one of the goals is to create a basic design that, with minor variations, can be tailored to meet the needs of a variety of players. There are other goals, secondary to playing quality but nonetheless desirable. These include appearance and mechanical reliability ("fit and finish"), ease of manufacture (consistent quality at minimal possible cost), and ease of repair (accidents will happen, alas!). Existing instruments provide input data to the design process by way of physical dimensions, acoustic input impedance, and players' judgements. Computer modeling helps to predict the effect on intonation of changes of bore contour. Feedback from top professional players is essential to refine the design, especially the "black magic" aspects like brace placement and even the type of solder used to assemble the instrument.

\section{Invited Paper}

10:00

2aMUa7. Characterisation of brass musical instrument designs using the brassiness parameter. Arnold Myers (University of Edinburgh, Reid Concert Hall, Bristo Square, EH8 9AG Edinburgh, UK, am@ed.ac.uk)

Recent work has established a "brassiness" parameter as a measure of the support given over the sounding length of a brass instrument to non-linear propagation in sound production. This parameter is a function of the geometry (bore profile) of an instrument, and provides a useful means of relating the timbral characters to the designs of the various kinds of brass instrument. The geometries of over 1000 brass instruments belonging to museums worldwide and to individual musicians have been measured to determine the values of their brassiness parameter. Comparisons of these enable not only a more precise taxonomy but also provide a tool for studying the evolution of instrument design. This paper explores both the taxonomic analysis of the whole brasswind field and also gives examples of the use of brassiness in historical research.

\section{Contributed Paper}

10:20

2aMUa8. Influence of acoustic waveguides lengths on self-sustained oscillations: Theoretical prediction and experimental validation. Nicolas Ruty (Département Parole \& Cognition, GIPSA-lab, 46, avenue Félix Viallet, 38031 Grenoble Cedex, France, nicolas.ruty@gipsa-lab.inpg.fr), Xavier Pelorson (Département Parole \& Cognition, GIPSA-lab, 46, avenue Félix Viallet, 38031 Grenoble Cedex, France, pelorson@icp.inpg.fr), Annemie Van Hirtum (Département Parole \& Cognition, GIPSA-lab, 46, avenue Félix Viallet, 38031 Grenoble Cedex, France, annemie.vanhirtum@gipsa-lab.inpg.fr)

Human vocal folds and lips of brass instruments players produce selfsustained oscillations due to the interaction between airflow, acoustic waveguides and deformable tissues. This interaction is commonly modelled as a distributed one or two mass-spring system coupled with a simple airflow and acoustic description. This study focuses on the influence of the acoustic waveguide length on the resulting self-sustained oscillation characteristics, i.e. the minimum pressure required to sustain oscillations, the oscillation frequency. Both fixed and varying waveguide lengths are considered. Theoretical predictions with the simplified interaction model are compared to experimental data obtained with a deformable in-vitro replica suitable to produce self-sustained oscillations in presence of an upstream $(12,24$ or $32 \mathrm{~cm}$ ) and downstream (varying from 0 up to $235 \mathrm{~cm}$ ) acoustic waveguide. The current study shows the strong influence on the minimum pressure regardless the waveguide length. In addition the waveguide length is shown to impose the resonance frequency for waveguide length superior to $40 \mathrm{~cm}$. A rapid change in waveguide length introduces bifurcations between different oscillation regimes. 


\title{
Session 2aMUb
}

\section{Musical Acoustics: Interaction Between Instrument and Instrumentalist I}

\author{
Gary Scavone, Cochair \\ Schulich School of Music, McGill University, 555 Sherbrooke Street West, Montreal, QC H3A 1E3, Canada \\ Xavier Boutillon, Cochair \\ Laboratoire de Mécanique des Solides, Ecole Polytechnique, Palaiseau Cedex, 91128, France
}

\section{Invited Papers}

\section{1:00}

2aMUb1. Motor control in drumming: Influence of movement pattern on contact force and sound characteristics. Sofia Dahl (Institute of Music Physiology and Musicians' Medicine, Hanover University of Music and Drama, Hohenzollernstr. 47, 30161 Hannover, Germany, dr.sofia.dahl@gmail.com), Eckart Altenmüller (Institute of Music Physiology and Musicians' Medicine, Hanover University of Music and Drama, Hohenzollernstr. 47, 30161 Hannover, Germany, altenmueller@hmt-hannover.de)

Whereas wind instrumentalists and string players have a continuous control of the acoustic sound parameters during playing, a percussionists' direct contact with the instrument is limited to a few milliseconds. The player has no possibilities to adjust grip or dampening during the actual contact. Whatever timbre and sound level the player is aiming for therefore has to be integrated in the entire striking gesture. How can the player control the complex interaction between drumstick and drumhead? In order to investigate how the players' grip and striking gestures influence the sound characteristics of drum strokes we recorded movements, audio, contact time and contact force during drumming. Different instructions were given with the intention to influence how the player's grip controlling the drumstick. "Normal" strokes were allowed to freely rebound from the drumhead. For "controlled" strokes the player was asked to control the ending position of the drumstick, stopping it as close as possible to the drumhead after the stroke. Preliminary analysis showed that the instructions influenced contact force, contact time, and perceptual ratings of the strokes. Further results and implications will be discussed.

11:20

2aMUb2. Two dimensional finger-string interaction in the concert harp. Jean-Loic Le Carrou (Laboratoire d'Acoustique de l’Université du Maine, Avenue Olivier Messiaen, 72085 Le Mans, France, jean-loic.le_carrou@univ-lemans.fr), Elio Wahlen (LAUM, CNRS, Université du Maine, Lab. d'Acoustique Université du Maine, UMR CNRS 6613, 72085 Le Mans Cedex 9, France, elio@elio.de), Emmanuel Brasseur (LAUM, CNRS, Université du Maine, Lab. d’Acoustique Université du Maine, UMR CNRS 6613, 72085 Le Mans Cedex 9, France, emmanuel.brasseur@univ-lemans.fr), Joël Gilbert (Laboratoire d'Acoustique de 1’Université du Maine, Avenue Olivier Messiaen, 72085 Le Mans, France, joel.gilbert@univ-lemans.fr)

The sound of the concert harp partly depends on the way the string is plucked. The vibrating string is brought into a state of initial conditions by the finger-string interaction and then oscillates according to two transverse planes. In order to understand the plucking action of the concert harp, a one-dimensional model of this interaction has been developed in a previous paper [Le Carrou et al, Proc. ISMA (2007)]. The parameters of this model were deduced from measurements of the string's and finger's trajectories. The aim of the present paper is to extend this model to a more realistic one, including a two-transverse trajectory for each one of the interaction's elements. To do so, a special experiment with a high-speed camera, which films the interaction, is set up. Specific image processing, based on edges detection, helps to automatically track both objects' positions. The results show that the finger-string interaction takes place in two planes and permits us to obtain the parameters of the two-dimensional model for the finger-string interaction.

\section{1:40}

2aMUb3. The modern violin bow in off-string action. Knut Guettler (Norwegian Academy of Music, P.O.Box 5190 Majorstuen, 0302 Oslo, Norway, knut.guettler@nmh.no)

The convex camber of the modern Tourte-model bow permits off-string stroke techniques to be performed with resulting clean and crisp attacks. This paper discusses how different bow-, string-, and bowing parameters must combine in order for this to be achieved. Of particular interest are the geometrical changes imposed on the bow stick during bouncing strokes. A good spiccato bow is characterized by a stick that feels "lively", even when the bouncing is low or the hairs are not leaving the string at all. With lesser-quality bows the player has to work harder (on the lower-pitched double bass even sometimes synchronously "shake" the bow stick in the stringlength plane) in order to induce sufficient stick oscillation. Included in this study are measurements of finger action on bow sticks of different quality. 


\section{Contributed Paper}

12:00

2aMUb4. Observations on bow changes in violin performance. Matthias Demoucron (IRCAM, 1 Place Igor Stravinsky, 75004 Paris, France, demoucron@ircam.fr), Anders Askenfelt (Dept. of Speech, Music and Hearing, Royal Institute of Technology (KTH), Lindstedtsvägen 24, SE10044 Stockholm, Sweden, andersa@speech.kth.se), René E. Causse (IRCAM, 1 Place Igor Stravinsky, 75004 Paris, France, causse @ircam.fr)

Players of bowed instruments commonly separate notes by changing the direction of the bow motion ("bow changes"). The separation can be made more or less pronounced (detaché - portato - martellato). In contrast, long notes, requiring more than a full bow stroke, are played by making the bow changes as inaudible as possible. Acceptable bow changes require accurate control and coordination of a set of bowing parameters, in particular bow speed, bow force and bow-bridge distance. Long practice is required before optimal control is achieved. Detailed descriptions of basic bowing gestures such as bow changes are of great interest for various fields of violin-related studies, including realistic control of synthesis algorithms. We present recent measurements that provide an accurate description of the evolution of bow speed, acceleration and bow force during bow changes at the tip and the frog, respectively. Using these data for controlling a synthesis algorithm, we will discuss how modifications of the parameters influence the bow-string interaction and resulting string vibrations. The simulations are used to model efficient implementations of bow changes.

\section{Invited Paper}

$$
\text { 12:20 }
$$

2aMUb5. Extraction of lumped clarinet reed model parameters from numerically synthesised sound. Vasileios Chatziioannou (Sonic Arts Research Centre, Queen's University Belfast, BT7 1NN Belfast, UK, vchatziioannou01@qub.ac.uk), Maarten Van Walstijn (Sonic Arts Research Centre, Queen’s University Belfast, BT7 1NN Belfast, UK, m.vanwalstijn@qub.ac.uk)

Fluid dynamical analysis and time-domain modelling of a single reed-mouthpiece-lip system can be used to inform the formulation of a lumped model of the woodwind excitation mechanism. Coupling this lumped model to a model of the instrument bore enables computationally efficient generation of sustained oscillations, using a small number of physical parameters that define the instrument and the way the player controls them. As such, the embouchure of the player as well as the geometry of the system is taken into account. In this paper, an attempt is carried out to use the numerically generated sound as an input to an inversion algorithm for the reedmouthpiece-lip system. Assuming that the reed motion is proportional to the pressure difference across it, a relationship can be established between the pressure and the total flow inside the mouthpiece that allows a first estimation of the physical parameters using standard optimisation techniques. Currently we are undertaking efforts to apply the inversion to data measured under real playing conditions, i.e. effectively capturing player gesture information in the form of physical control parameters.

\section{2:40-1:40 Lunch Break}

\section{Contributed Paper}

\section{$1: 40$}

2aMUb6. How to play the first bar of Rhapsody in Blue. Jer-Ming Chen (University of New South Wales, Music Acoustics, School of Physics, NSW 2052 Sydney, Australia, jerming@phys.unsw.edu.au), John Smith (University of New South Wales, Music Acoustics, School of Physics, NSW 2052 Sydney, Australia, john.smith@unsw.edu.au), Joe Wolfe (University of New South Wales, Music Acoustics, School of Physics, NSW 2052 Sydney, Australia, J.Wolfe@unsw.edu.au)

The two-and-a-half octave glissando opening Gershwin's Rhapsody in Blue is one of the great icons of 20th century music and one of the best known bars in music. Expert clarinettists combine unusual fingerings with even more unusual configurations of their vocal tract to achieve a nearly continuous rise in pitch. Using a novel method [1], we incorporated an acoustic impedance measurement head within a clarinet mouthpiece, allowing us to study the player's vocal tract at various stages in the glissando. We measured and compared vocal tract impedance spectra with the corresponding clarinet impedance spectra for the fingering used at that pitch. Partially uncovering an open finger-hole raises the frequency of clarinet impedance peaks in the lower register, thereby allowing smooth increases in the playing pitch. In the upper register, however, resonances in the clarinettist's vocal tract are manipulated to be comparable with those in the clarinet for frequencies in this range. Thus the pitch in the higher section of the glissando is largely controlled by smoothly varying a resonance of the player's vocal tract. [1] Chen, JM. Smith, J. and Wolfe, J., (2008) 'Experienced saxophonists learn to tune their vocal tracts". Science, (in press).

\section{Invited Papers}

2aMUb7. Evaluating Vocal-Tract Influence in the Production of Saxophone Multiphonics. Gary Scavone (Schulich School of Music, McGill University, 555 Sherbrooke Street West, Montreal, QC H3A 1E3, Canada, gary@music.mcgill.ca), Antoine Lefebvre (Schulich School of Music, McGill University, 555 Sherbrooke Street West, Montreal, QC H3A 1E3, Canada, antoine.lefebvre2@mail.mcgill.ca), Andrey R. Da Silva (Schulich School of Music, McGill University, 555 Sherbrooke Street West, Montreal, QC H3A 1E3, Canada, andrey.dasilva@mail.mcgill.ca)

A new approach for the analysis of vocal-tract influence in single-reed woodwind instruments during performance was recently reported (Scavone et. al., 2008). Two types of vocal-tract influence were observed. When the downstream air column provides only weak support of a given note, players can use a strong and narrow-bandwidth upstream resonance to override the reed vibrations, such as when pitch bending or playing extended register notes. Performers can also use a more wide-bandwidth upstream resonance to affect subtle timbre variations when playing notes over the full range of the instrument. The research reported here addresses the performance of multiphonic tones, for which the results of the previously mentioned study were less conclusive. While it is clear that upstream influence is involved in the production of multiphonics, we are interested in determining whether performers must support a specific intermodulation component or a wider bandwidth range of components for proper production. The latest results of this research will be reported. 
2aMUb8. A study of flute control parameters. Benoit Fabre (Institut Jean Le Rond d'Alembert / LAM (UPMC / CNRS / Ministère Culture), 11, rue de Lourmel, 75015 Paris, France, fabreb@ccr.jussieu.fr), Nicolas Montgermont (Institut Jean Le Rond d'Alembert / LAM (UPMC / CNRS / Ministère Culture), 11, rue de Lourmel, 75015 Paris, France, montgermont@lam jussieu.fr), Patricio De La Cuadra (Centro de Investigación en Tecnologias de Audio (CITA), Universidad Católica de Chile, Alameda 340, Oficina 13, Casilla 114-D Santiago, Chile, pcuadra@uc.cl)

The sound of musical instruments played in self-sustained oscillations can be interpreted as a sounding transposition of the player's gesture. The playing of wind instruments requires expert control of the blowing that may be difficult to grasp and to measure because it induces only very little motion of the player, as opposed to string or keyboard instruments. In the case of instruments of the flute family, the player seems to control mainly the air jet velocity for mode selection. Flutes in which the air jet is formed between the lips also allow for a control of the total jet flow by the player, through lip adjustments. We present measurements carried on several players, in different playing conditions. The score includes technical exercises such as scales and short musical excerpts from the flute repertoire. Time evolutions of the control parameters are analysed, based on pressure and geometrical measurement, in the framework of the current knowledge on the sound production in flutes, such as jet instability. The control over different parameters will be discussed, both for basic technical exercises and in a musical melodical context.

\section{Contributed Paper}

\section{2:40}

2aMUb9. Acoustical analysis of timbral modulations on the flute as controlled by phonetic gestures. Maryse Lavoie (Laboratoire informatique, acoustique et musique, Faculté de musique, Université de Montréal, C.P. 6128, succursale Centre-Ville, Montréal, QC H3C 3J7, Canada, maryse.lavoie@umontreal.ca), Caroline Traube (Laboratoire informatique, acoustique et musique, Faculté de musique, Université de Montréal, C.P. 6128, succursale Centre-Ville, Montréal, QC H3C 3J7, Canada, caroline.traube@umontreal.ca), Marie-Hélène Breault (Observatoire international de la création et des cultures musicales (OICCM), Faculté de musique, Université de Montréal, C.P. 6128, succursale Centre-Ville, Montréal, QC H3C 3J7, Canada, mhbreault@yahoo.ca)
The purpose of this project is to study the control of timbre on the flute by varying articulatory parameters (i.e. embouchure) which correspond to distinct phonetic gestures. The main goal is to compare the production of a specific timbral modulation on the flute and its vocal reproduction (diphthong) by means of acoustical analyses of these sounds. The recordings of timbral modulations performed on the flute (e.g. by increasing the mouth opening) and reproduced vocally as diphthongs (e.g. [u] -> [a] transition) have been analyzed acoustically for intensity as well as for the first two formant trajectories. The results of the formant analyses as presented in an F1-F2 plane reveal the cardinal vowel triangle and confirm a correlation between the flute timbre modulations and the vocal diphthongs corresponding to the underlying phonetic gestures. In addition, a listening test has shown that flutists are able to perceive and recognize these timbral modulations from the sound alone.

\section{Invited Papers}

2aMUb10. Lip control of brass instruments. Donald M. Campbell (Edinburgh University, 4201 JCMB, Kings Buildings, Mayfield Road, EH9 3JZ Edinburgh, UK, d.m.campbell@ed.ac.uk)

Brass instruments are frequently described in the acoustics literature as "lip reed" instruments, emphasising the fact that the sounding mechanism of this instrumental class is flow modulation by the vibration of the player's lips. As well as providing the source of sound, the lips act as the major interface through which the performer controls the intonation, dynamics, articulation and timbre of the performance. Indeed, on instruments such as the natural trumpet and the alphorn, the player's lips form the only control interface. This paper reviews recent studies which have examined the vibrational properties of brass players' lips, the types of motion which they undergo when playing different instruments, and the nature of the interaction between the lips and the instrument. It also considers the extent to which current physical models of the lips are capable of encompassing these control processes.

\section{3:20}

2aMUb11. Vocal tract interactions in saxophone performance. Jer-Ming Chen (University of New South Wales, Music Acoustics, School of Physics, NSW 2052 Sydney, Australia, jerming@phys.unsw.edu.au), John Smith (University of New South Wales, Music Acoustics, School of Physics, NSW 2052 Sydney, Australia, john.smith@unsw.edu.au), Joe Wolfe (University of New South Wales, Music Acoustics, School of Physics, NSW 2052 Sydney, Australia, J.Wolfe@unsw.edu.au)

Although acousticians have debated the importance of the vocal tract in reed instrument performance, expert saxophonists report adjusting their vocal tract for advanced techniques including altissimo playing, subtone playing, bugling and multiphonics. Using a novel method [1], we incorporated an acoustic impedance head within a saxophone mouthpiece to study the vocal tract directly during playing. For fingerings above the first register, the operating peak in the saxophone's input impedance decreases with increasing pitch, falling to below $20 \mathrm{MPa} . \mathrm{m}^{-3}$ after 2.7 octaves, thus ending the standard range that is readily available to amateurs. Above this, in the altissimo, professional saxophonists produce peaks in the impedance of their tracts of about 20 to $40 \mathrm{MPa} . \mathrm{s}^{-3} \mathrm{~m}^{-3}$, which they tune to select the desired note. The crossover of the relative magnitudes of saxophone and tract impedance peaks coincides with the transition from standard to altissimo register. While professionals use the vocal tract thus for other extended effects, inexperienced players do not tune their tract resonances and are unable to produce advanced effects. [1] Chen, JM. Smith, J. and Wolfe, J., (2008) '’Experienced saxophonists learn to tune their vocal tracts". Science, (in press). 


\title{
Contributed Paper
}

2aMUb12. Reproducibility $\begin{array}{r}3: 40 \\ \text { of }\end{array}$ piano playing. Nicolas Principeaud (Laboratoire d'Acoustique Musicale, 11 rue de Lourmel, 75015 Paris, France, nicolas.principeaud@free.fr), Xavier Boutillon (Laboratoire de Mécanique des Solides, Ecole Polytechnique, 91128 Palaiseau Cedex, France, boutillon@1ms.polytechnique.fr)

Mechanically, piano playing consists in giving to the hammer a certain escapement velocity at a certain time $-t_{e}, v_{e}$ - by means of a traditional keyboard and piano action. Numerous replacement systems for the keyboard, action or even for the pianist's finger have been proposed. They implicitly raise the question of the accuracy with which the $\left(t_{e}, v_{e}\right)$ information must be coded or reconstructed. The precision with which pianists are able to reproduce their playing sheds some light on this question. At this end we have asked several concert pianists to play the same passage several times with the highest reproducibility level they could achieve. The experiment was done on a traditional grand piano equipped with the Yamaha Disklavier system, used as a measurement tool for the escapement velocity of the hammers. The beginnings of a fugue by Bach and a study by Debussy were recorded. Results show that the reproducibility varies highly from note to note and can be as good as approximately $1 \%$. Additional tests on auditors show that deviations on note amplitudes are not perceived unless they reach a level which turns out to be significantly higher than the reproducibility level achieved by the pianists.

TUESDAY MORNING, 1 JULY 2008

ROOM 250A, 8:00 A.M. TO 8:00 P.M.

\section{Session 2aNSa}

\section{Noise, Physical Acoustics, and EURONOISE: Aeroacoustics II}

\author{
Philip J. Morris, Cochair \\ Penn State University, 233C Hammond Building, University Park, PA 16802, USA \\ Christophe Bailly, Cochair \\ Ecole Centrale de Lyon, 36, avenue Guy de Collongue, LMFA, Ecully, 69134, France
}

\section{Contributed Papers}

\section{8:00}

2aNSa1. Source location prediction of subsonic isothermal jet flows. Juan Battaner-Moro (Institute of Sound and Vibration Research, University of Southampton, University Road, SO17 1BJ Southampton, UK, jpb@isvr.soton.ac.uk), Mahdi Azarpeyvand (Institute of Sound and Vibration Research, University of Southampton, University Road, SO17 1BJ Southampton, UK, ma@isvr.soton.ac.uk), Rod Self (Institute of Sound and Vibration Research, University of Southampton, University Road, SO17 1BJ Southampton, UK, rhs@isvr.soton.ac.uk)

The location of jet noise sources is a far from trivial problem that is of great importance for both understanding the noise production and radiation mechanisms and also for finding new jet noise reduction strategies. This paper presents comparisons of theoretical results with data for a number of jets. The theory used is based on the MGBK method but including a novel time scale based on the rate of energy transfer through the turbulent cascade. This new technique has been shown to give a number of advantages over existing models. The experimental results were obtained using the Polar Correlation Technique and were made at QinetiQ's Jet Noise Facility in the UK as part of the EU FP6 programme CoJeN. The high resolution jet noise images resulted from using a 64 microphone polar arc array set at two reference angles, namely 60 and 90 degrees to the jet axis. Comparisons with experimental data are made for coplanar and short cowl nozzles at different working conditions for predictions from different theoretical models. It is shown that the best agreement is obtained for the prediction methodology using the energy transfer rate timescale

\section{8:20}

2aNSa2. A hybrid method for jet noise predictions based on Large Eddy Simulation and Reynolds-Averaged Navier-Stokes simulations. Guillaume Bodard (Snecma Villaroche, Rond point René Ravaud, 77550 Moissy-Cramayel, France, guillaume.bodard@snecma.fr), Christophe Bailly (Ecole Centrale de Lyon, 36, avenue Guy de Collongue, LMFA, 69134 Ecully, France, christophe.bailly@ec-lyon.fr)

A hybrid jet noise prediction method combining steady and unsteady flow calculations is discussed. The main objective is to merge advantages of each technique to obtain a robust acoustic prediction tool, which will be able to correctly evaluate design and installation effects such as chevron nozzles or jet-pylon interaction for instance. The low-frequency component of acoustic spectra is computed using large-eddy simulations and the integral formulation derived by Ffowcs-Williams \& Hawkings (1969, Phil. Trans. Roy. Soc. Lond., vol. 264). The high-frequency component associated with fine scale turbulence is obtained thanks to Tam \& Auriault's mixing noise theory (1999, AIAA Journal, vol. 37) from Reynolds-Averaged NavierStokes simulations. The oral presentation will detail the two methods, the LES simulations for subsonic round single and coaxial jets, and the matching between the two approaches to get a complete picture of the acoustic spectra.

\section{8:40}

2aNSa3. Efficiency of optimized microjets on realistic nozzles. Alexandre Vuillemin (Snecma, Etablissement de Villaroche Sud, Rondpoint René Ravaud - Réau, 77550 Moissy-Cramayel, France, alexandre .vuillemin@snecma.fr)

Even if chevrons nozzles are an efficient way to reduce jet noise during take off, they also decrease performances during cruise. An innovative way to avoid this decrease is the use of active devices that could be switch off after take off, such as microjets nozzles. Snecma led test campaigns at Martel Facility (LEA/CEAT) to optimize microjets settings (geometric and thermodynamic parameters) and to characterize their efficiency on both 2D axisymmetric and chevrons nozzles. Acoustic and PIV measurements were done on a single stream hot jet with and without external flow, and on a double stream hot jet with pylon.

\section{9:00}

2aNSa4. Numerical investigation of the effect of nonlinear propagation distortion on helicopter noise. Penelope Menounou (University of Patras, Department of Mechanical and Aeronautical Engineering, Rion, 26504 Patras, Greece, menounou@mech.upatras.gr), Panagiotis 
Vitsas(University of Patras, Department of Mechanical and Aeronautical Engineering, Rion, 26504 Patras, Greece, pvitsas@upnet.gr)

Nonlinear propagation distortion causes energy to be shifted to the high frequency end of the spectrum. This leads to underestimation of the noise levels at high frequencies. The effect has been demonstrated in the case of aircraft noise, but less attention has been given to helicopters. In the present work, the effect of nonlinear propagation distortion on helicopter noise is demonstrated based on measured data for low-speed descent and numerical calculations that predict the noise level away from the helicopter with and without nonlinear effects. It is shown that (i) for some frequency bands the difference between linear and nonlinear calculations can be as high as $8 \mathrm{~dB}$, (ii) frequencies between 1000 and $3000 \mathrm{~Hz}$ are more affected, and (iii) the effect is highly directional depending on the receiver location around the helicopter. It is further shown that nonlinear effects manifest themselves differently than in the case of aircraft noise and that they depend on the specific helicopter noise mechanism. More specifically, the following helicopterspecific noise source types are investigated with regards to nonlinear effects: advancing Blade Vortex Interaction (BVI) noise vs retreating BVI noise, High Speed Impulsive noise, and BVI vs Very Impulsive noise.

\title{
Invited Papers
}

\section{9:20}

\begin{abstract}
2aNSa5. Recent developments in helicopter rotor noise prediction. Kenneth S. Brentner (Penn State University, 233C Hammond
\end{abstract} Building, University Park, PA 16802, USA, ksb16@psu.edu)

Prediction of discrete frequency noise for rotorcraft in steady flight has reached a high level of sophistication and understanding. The primary challenge in such rotor noise predictions is the accurate determination of the loading on and flow field around the blades. Although current rotor noise prediction tools have been demonstrated for steady flight conditions, the utility of rotorcraft comes from their unique ability to hover and maneuver. Such maneuvers often occur near the ground in close proximity to people. This paper describes an initial study to characterize maneuver noise. A maneuver noise prediction system has been developed, which consists of a flight simulation code, free-vortex wake code, and a maneuver noise prediction code, loosely coupled together. One of the key aspects of this system is the ability to investigate the additional noise caused during the transition from one flight state to another. Several maneuvers including turns, accelerations, and pop-up/popdown maneuvers were considered. Significant increases in the low-frequency noise can occur, depending on how aggressive the maneuver is performed. The status of advanced research on predicting acoustic scattering of the rotor noise by the aircraft and the first-principles prediction of rotor broadband noise will also be presented.

\section{9:40}

2aNSa6. Aeroacoustic research complex for aircraft source noise characterization. Micah Downing (Blue Ridge Research and Consulting, 13 1/2 W. Walnut St., Asheville, NC 28801, USA, micah.downing@blueridgeresearch.com), Robert McKinley (Air Force Research Laboratory, AFRL/RHCB, 2610 Seventh St., Bldg 441, Wright-Patterson AFB, OH 45433, USA, Robert.McKinley@wpafb.af.mil), John Hall (Air Force Research Laboratory, AFRL/RHCB, 2610 Seventh St., Bldg 441, WrightPatterson AFB, OH 45433, USA, John.Hall@wpafb.af.mil), Frank Mobley (Air Force Research Laboratory, AFRL/RHCB, 2610 Seventh St., Bldg 441, Wright-Patterson AFB, OH 45433, USA, Frank.Mobley@ wpafb.af.mil), Michael James (Blue Ridge Research and Consulting, 13 1/2 W. Walnut St., Asheville, NC 28801, USA, michael.james@ blueridgeresearch.com)

Aircraft noise has been traditionally measured with either a few ground-based microphones or a linear ground-plane array of microphones. These techniques capture one-dimensional and/or two-dimensional characteristics of aircraft flight noise. The US Air Force Research Laboratory has started the construction of a 3-dimensional measurement facility at White Sands Missile Range in New Mexico. This facility, the Aeroacoustic Research Complex (ARC), will allow aircraft to fly through the array, collecting fully 3D acoustic data. ARC is initially being developed in two phases The first phase includes two $91.4 \mathrm{~m}$ tall towers separated by $244 \mathrm{~m}$ and will focus on noise from rotary wing and UAV aircraft. The second phase will add two $366 \mathrm{~m}$ tall towers separated by $610 \mathrm{~m}$ and will focus on large and high performance fixed wing aircraft. This facility will allow more accurate characterization of in-flight noise directivity by providing synchronized 3-dimensional magnitude \& spectral acoustical signatures from $50+$ microphones. ARC responds to a critical need for validation of existing predictive acoustic models. Such models are used for aircraft design, survivability, nonlinear acoustic propagation research and assessing noise exposure to residents living adjacent to airfields.

\section{0:00-10:20 Break}

\section{Contributed Papers}

\section{0:20}

2aNSa7. Lateral directivity of aircraft noise. Walter Krebs (Empa, Ueberlandstrasse 128, 8600 Duebendorf, Switzerland, walter.krebs @empa.ch), Georg Thomann (Empa, Ueberlandstrasse 128, 8600 Duebendorf, Switzerland, georg.thomann@empa.ch)

A three-dimensional model was developed to characterise the directional sound emission of different aircraft. The model is based on spherical harmonics and defines the directional spectral sound pressure level at a reference distance. The parameters of the model are derived from acoustic measurements on real aircraft traffic. With the help of this model different physical effects on sound propagation and sound impact are analysed. Variations with respect to a rotational symmetric sound emission are outlined and compared to the engine installation corrections proposed in the revised Doc 29 3rd edition. In addition the influence of the ground effect on A-weighted sound levels is analysed for different receiver heights and ground impedances.

\section{0:40}

2aNSa8. Sonic booms, spectral analysis, and diffraction by buildings. Victor W. Sparrow (Penn State, Graduate Program in Acoustics, 201 Applied Science Bldg., University Park, PA 16802, USA, vws1@psu.edu)

An analysis has been performed upon conventional and low-amplitude $\mathrm{N}$-wave sonic boom data taken by NASA outside two houses in June 2006 and July 2007. The buildings were one-floor residences which were carefully instrumented with strategically-placed, multiple microphones. The incident sonic booms had a substantial variation in rise times, most likely due to atmospheric effects. Previous work using the June 2006 data [Sparrow, Klos, and Buehrle, J. Acoust. Soc. Am. 122 (5, Pt. 2) 3084] revealed maximum pressure loads near the ground and wall facing the incident boom. A new spectral analysis of the individual booms now confirms that the diffraction of sound over the house tops is substantially affected by the spectral content of each boom. These results indicate that the pressure loading of homes due to conventional or low-amplitude sonic boom will be affected by 
both the spectral content of each boom's rise phase and each house's exterior geometry. [Work supported by NASA.]

\section{1:00}

2aNSa9. Identification of aerodynamic sound sources: the key problem in noise control. Alexander Fedorchenko (Independent Researcher/Consultant, Dm. Ul'yanov Str. 27/12-1-50, 117449 Moscow, Russian Federation, fedorchenko@mail.com)

Despite the recent advances in the noise control technology, the key mechanisms of aerodynamic sound emission remained poorly studied. The "well-recognized" methods of TCAA did not lead to any breakthrough in this topical problem, and what seems most disappointing, the family of "acoustic analogies" is still used by many for definition of aerodynamic noise sources, though the sufficient set of mathematical proofs has been given by the author that this model is wrong. Experimental approaches aimed at localization of aerodynamic noise sources, including the method of acoustic imaging via using a microphone array, are considered, and their inherent limitations are pointed out. Unfortunately, no current experimental technique enables one to measure instantly all sound sources and sound disturbances inside the zone of generation, and so the latter is often regarded as a kind of "black box". The two-medium nonlinear theory of aerodynamic sound, based on the original decomposition of each flow variable into two components, for unsteady background flow and for acoustic field, has been created, that promotes better comprehension of the noise generation phenomena and opens new ways in flow/noise control. The main properties of this theory are now indicated in comparison with the most detrimental delusions originated from "acoustic analogies".

\section{Invited Paper}

\section{1:20}

2aNSa10. Direct aeroacoustic simulations based on domain decompositions. Jens Utzmann (University of Stuttgart, Institute for Aerodynamics and Gasdynamics, Pfaffenwaldring 21, 70569 Stuttgart, Germany, utzmann@iag.uni-stuttgart.de), Claus-Dieter Munz (University of Stuttgart, Institute for Aerodynamics and Gasdynamics, Pfaffenwaldring 21, 70569 Stuttgart, Germany, munz@iag.unistuttgart.de)

For CAA, an accurate and feasible direct simulation that considers both the generation of sound within the flow and its propagation into the far-field is hard to realize with one numerical method in a single computational domain. On the other hand, a direct approach contains automatically the interaction of the acoustic perturbations with the flow-field, a property which lacks the popular acoustic analogy models. The proposed method is basically a direct simulation, but it simplifies the problem that has to be solved for individual regions in the computational domain. The idea is to use a non-overlapping domain decomposition method where the equations, methods, grids and even the time steps are adapted to meet the local requirements. Inside the coupling framework, high-order solvers from different classes of methods are available: On unstructured grids, a reconstructed ADER finite volume method (ADER-FV) is used for linear and nonlinear problems, as well as a discontinuous Galerkin method. On structured grids, the ADER-FV and the ADER-FD method are efficiently implemented for nonlinear (FV) and linear (FV, FD) problems. These high-order accuracy methods ensure excellent wave propagation capabilities throughout the entire computational domain. In the subdomains, the Navier-Stokes, Euler and the linearized Euler equations are solved.

\section{Contributed Papers}

11:40

2aNSa11. Direct Noise Computation in subsonic and transonic flows. Frédéric Daude (LaMSID UMR EDF/CNRS, 1 avenue du Général de Gaulle, 92141 Clamart, France, frederic-externe.daude@edf.fr), Thomas Emmert (LaMSID UMR EDF/CNRS, 1 avenue du Général de Gaulle, 92141 Clamart, France, tommem@gmail.com), Philippe Lafon (EDF, 1, avenue du Général de Gaulle, 92141 Clamart, France, Philippe.lafon@edf.fr), Fabien Crouzet (EDF, 1, avenue du Général de Gaulle, 92141 Clamart, France, fabien.crouzet@edf.fr), Christophe Bailly (Ecole Centrale de Lyon, 36, avenue Guy de Collongue, LMFA, 69134 Ecully, France, christophe.bailly@ec-lyon.fr)

In order to model flow phenomena involving interactions between aerodynamics and acoustics, it is necessary to use Direct Noise Computation (DNC) instead of hybrid methods that are not suitable to take into account the feedback of acoustics on the flow. The methods that are now available in the field of Computational AeroAcoustics (CAA) allows us to deal with DNC in realistic configurations. The numerical code SAFARI (Simulation of Aeroacoustics in Fluids And with Resonance and Interactions) has been developed for this goal. The set of equations are the compressible 3-D NavierStokes equations. High order finite difference schemes are used. Multidomain capabilities are implemented in order to deal with complex geometries. Block decomposition is used in order to take advantage of parallel processing on large clusters Validation cases are presented: diffraction by a cylinder, shock tube. Results on realistic configurations are also shown: ducted cavity, transonic sudden enlargment, airfoil interactions.

\section{2:00}

2aNSa12. Asymptotic expressions for the directivity of round jets Ricardo E. Musafir (UFRJ / COPPE, Universidade Federal do Rio de Janeiro, 21941-972 Rio de Janeiro, Brazil, rem@serv.com.ufrj.br)

Expressions for the directivity of round jets, in the high and low frequency limits, are derived, based on solutions of Lilley's equations. Two different forms of the equation in what concerns source terms representation are considered, along with general point sources of the appropriate type (i.e., equivalent stress and force sources and also, when necessary, volume sources), which are assumed to be statistically axisymmetric. Effects of mean shear and temperature gradients are accounted for, as well as of source movement. The expressions are compared with earlier ones, based on a plug flow model. The differences in the resulting expressions due to the choice of source description, which are relevant for hot jets, are discussed and used to derive a form consistent with both representations. Comparison with experimental data is also presented.

\section{2:20}

2aNSa13. Investigations of roughness-generated TBL sound using coupled physical-computational experiments in conjunction with theoretical development. William Blake (Naval Surface Warfare Center (Ret.), 6905 Hillmead Road, Bethesda, MD 20817, USA, hydroacoustics@aol.com), Ki-Han Kim (Office of Naval Research, Randolph St., Arlington, VA 22202, USA, kihan.kim@navy.mil), Michael Goody (Naval Surface Warfare Center (Ret.), 6905 Hillmead Road, Bethesda, MD 20817, USA, michael.goody@navy.mil), Meng Wang (University of Notre Dame, Departmernt of Aerospace and Mechanical Engineering, Notre Dame, MD 46556, USA, m.wang@nd.edu), William J. Devenport (Virginia Tech, Aerospace and Ocean Engineering, 224E Randolph Hall, Blacksburg, VA 24061, USA, devenport@vt.edu), Stewart A. Glegg (Florida Atlantic University, Department of Ocean Engineering, Boca Raton, VA 33431, USA, glegg@oe.fau.edu)

Sound produced by turbulent-boundary layers (TBL) over rough walls is being studied in a series of physical-computational experiments. At issue is the development of a knowledge of how the wall elements generate flow dipoles which directly determines how the sound is described in terms of dependent variables. The considered mechanisms include dipoles at the 
roughness elements due to their shed wakes, distributed surface dipoles due to convecting turbulence impinging onto elements, Rayleigh-like scattering into sound of aerodynamic pressures of hydrodynamic wave numbers of flow above the roughness. The LES of rough-wall TBL consists of "numeri$\mathrm{cal}^{\prime \prime}$ experiments being used to isolate the separate mechanisms. These simulations are benchmarked with analysis and with matching physical experiments on rough wall patches in which identical geometries of wall roughness and identical Reynolds numbers are used. In the physical measurements, array-based measurements of the radiated sound are being used to characterize the directivity and magnitude of the sound and to relate the sound to aerodynamic wall pressure and to classical characteristics of the turbulent boundary layer. The LES produces comparison for both radiated sound and detailed flow structure around the roughness elements. This project is funded by the Office of Naval Research, Washington, D.C., USA.

12:40-2:00 Lunch Break

\section{Contributed Paper}

2:00

2aNSa14. Experimental investigation of sound from flow over a rough surface. Michael Goody (Naval Surface Warfare Center (Ret.), 6905 Hillmead Road, Bethesda, MD 20817, USA, michael.goody@navy.mil), Jason Anderson (Naval Surface Warfare Center (Ret.), 6905 Hillmead Road, Bethesda, MD 20817, USA, Jason.M.Anderson@navy.mil), Devin Stewart (Naval Surface Warfare Center (Ret.), 6905 Hillmead Road, Bethesda, MD 20817, USA, Devin.Stewart@navy.mil), William Blake (Naval Surface Warfare Center (Ret.), 6905 Hillmead Road, Bethesda, MD 20817, USA, hydroacoustics@aol.com)

Measurements of radiated noise and unsteady surface pressures have been carried out in order to better understand the mechanism for sound production from flow over a rough surface. In order to investigate scaling re- lationships, the flow speed, roughness height, geometry and element distribution were varied systematically. When considered in total, previous investigations do not present a consistent picture of the scaling behavior of roughness noise, or the underlying physical mechanism. They have reported roughness noise levels that scale on flow velocity, roughness height, and fetch area and have indicated that the sound production may be dipole or quadrupole in nature. Prevailing analytical models assume that both dipole and quadrupole sources are present. The scaling of roughness noise for large roughness height has not been investigated previously and is part of the current study. A recent developed scattering model (dipole) developed by Glegg et al is interrogated using detailed measurements of the roughness element height distribution, turbulent boundary layer properties, and array-based radiated sound levels.

\section{Invited Paper}

2aNSa15. On the sound generated by boundary-layer vorticity. Umberto Iemma (University Roma Tre, via vasca navale 79 , 00146 Rome, Italy, u.iemma@uniroma3.it), Luigi Morino (via vasca navale 79, 00146 Roma, Italy, 1.morino@uniroma3.it), Roberto Camussi (University Roma Tre, via vasca navale 79, 00146 Rome, Italy, camussi@uniroma3.it), Giovanni Caputi Gennaro (University Roma Tre, via vasca navale 79, 00146 Rome, Italy, gcaputi@uniroma3.it)

Turbulent boundary layers generate broadband noise as the effect of vortical-disturbances scattering into acoustic waves. The paper presents a formulation for evaluating of acoustic pressure in the field in terms of the transpiration velocity, here defined in terms of vorticity and closely related to Lighthill equivalent source. Specifically, the formulation used allows one to obtain, in the frequency domain, a matrix relationship between the transpiration velocity at a number of points on the body surface (those arising from boundaryelement discretization) and the pressure at given points in the irrotational region. From this, the relationship between the corresponding PDF is easily obtained using the Wiener-Khintchine theorem. The paper will include the general formulation, validating numerical results, and comparison with experimental data. The inverse problem, that is, determining the source intensity on the body surface from field noise (in particular, the invertibility of the operator), will be also addressed.

\section{Contributed Papers}

2:40

2aNSa16. A numerical study on multimode sound propagation in lined ducts and radiation to the far field. Rie Sugimoto (ISVR, University of Southampton, Highfield, SO17 1BJ Southampton, UK, rs@isvr.soton.ac.uk) , R Jeremy Astley (ISVR, University of Southampton, Highfield, SO17 1BJ Southampton, UK, rja@isvr.soton.ac.uk), Claire R. McAleer (ISVR, University of Southampton, Highfield, SO17 1BJ Southampton, UK, crm@soton.ac.uk), Iansteel Achunche (ISVR, University of Southampton, Highfield, SO17 1BJ Southampton, UK, ia1@isvr.soton.ac.uk)

In previous articles, the authors developed a hybrid scheme for analysing bypass duct noise, in which a numerical analysis using finite element method for in-duct propagation and an analytic radiation code with fully represented effects of bypass shear layer are coupled. Such procedure permits detailed study on the interaction between duct configurations, such as geometry and acoustic liner impedances, and modal propagation and attenuation, and also the effects on the radiation pattern, within practical timescale and at modest computational cost. The scheme has been applied to realistic aero-engine bypass ducts and has been integrated with an optimisation programme. The numerical results obtained so far have revealed that for ducts with acoustic liners highly attenuated modes are not necessarily those with high mode angles, which is contrary to general anticipation. The aim of the study in the current paper is to understand the physics behind this phe- nomenon and its effect on the radiation to the far field. Detailed investigation on modal attenuation is performed by using the hybrid scheme. The effect of modal power distribution at the duct exit on the directivity pattern of the radiated noise to the far field is also discussed.

\section{3:00}

2aNSa17. Modeling of sound propagation in nonuniform waveguides. Wenping Bi (Laboratoire d'Acoustique de l'Université du Maine, UMR CNRS 6613, AV. O. Messiaen, 72085 Le Mans, France, wenping.bi@univ-lemans.fr), Vincent Pagneux (Laboratoire d'Acoustique de l'Université du Maine, UMR CNRS 6613, AV. O. Messiaen, 72085 Le Mans, $\quad$ France, vincent.pagneux@univ-lemans.fr), Denis Lafarge (Laboratoire d'Acoustique de l'Université du Maine, Avenue Olivier Messiaen, 72085 Le Mans, France, denis.lafarge@univ-lemans.fr), Yves Aurégan (Laboratoire d'Acoustique de l'Université du Maine, Avenue Olivier Messiaen, 72085 Le Mans, France, yves.auregan@univ-lemans.fr)

Sound propagation in waveguides is modeled by a Multimodal Method. The waveguides geometries may involve bends, variable cross-sections, or their combinations. The waveguide boundaries may involve axially or circumferentially nonuniform impedance conditions or acoustically rigid 
conditions. Uniform flow may also be included for a simple uniform geometry. The pressure (displacement potential for uniform flow) is expanded in terms of the modes of acoustically rigid waveguides and an additional function that carries the information about the impedance boundary. The rigid waveguide modes and the additional function are known a priori so that calculations of the true modes of waveguides with impedance boundary, which are difficult, are avoided. By matching the pressure and axial velocity (displacement potential and axial derivative for uniform flow) at the interface between different axially uniform segments, scattering matrices are obtained for each individual segment; these are then combined to construct a global scattering matrix for multiple segments. After calculating the scattering matrix, the transmitted and reflected sound fields or intensities may be obtained for any kind of modal sources. The method allows modeling sound propagation in waveguides with axially and circumferentially nonuniform impedance boundaries up to dimensionless frequency $\mathrm{K}=70$ in just hours on a personal computer, which advantageously compares with other techniques.

\section{3:20}

2aNSa18. An Active Network Representation to Predict the Flow Noise Characteristics of Corrugated Ducts. Wim De Roeck (K.U.Leuven Dept. of Mechanical Engineering, Celestijnenlaan 300B - bus 2420, 3001
Heverlee, Belgium, wim.deroeck@mech.kuleuven.be),Vasilisa Solntseva (Andreev Acoustics Institute, Shvernik, 4, Moscow, 117036 Moscow, Russian Federation, mironov@akin.ru), Wim Desmet (K.U.Leuven Dept. of Mechanical Engineering, Celestijnenlaan 300B - bus 2420, 3001 Heverlee, Belgium, Wim.Desmet@mech.kuleuven.be)

In this paper, an active network representation is used to numerically predict and gain more insight in the internally generated flow noise sources in corrugated ducts (the active bi-port component) as well as to describe the acoustic transmission characteristics of this type of application (the passive bi-port components) in the presence of a non-uniform mean flow. For this purpose a numerical approach is chosen, using compressible Large Eddy Simulations (LES) to predict the noise generation mechanisms and Linearized Euler Equations (LEE) with a plane pulse excitation to obtain the transmission characteristics for these components. The accuracy of the active network component determination is increased by separating the aerodynamic and the acoustic fluctuating field of the LES using both an aerodynamic/acoustic splitting technique and multiple plane mode-matching strategies. In this way, a numerical method is proposed to analyze the flow-acoustic behavior of corrugated tubes, which can be generally used for all types of duct system applications.

3:40-5:20 Posters

Lecture sessions will recess for presentation of posters on various topics in acoustics. See poster sessions for topics and abstracts.

\section{Contributed Papers}

\section{$5: 20$}

2aNSa19. Validation of a hybrid method of aeroacoustic noise computation applied to internal flows. Mélanie Piellard (Delphi Thermal, Avenue de Luxembourg, 4940 Bascharage, Luxembourg, melanie.piellard@delphi.com), Christophe Bailly (Ecole Centrale de Lyon, 36, avenue Guy de Collongue, LMFA, 69134 Ecully, France, christophe .bailly@ec-lyon.fr)

A hybrid method of aeroacoustic noise computation based on Lighthill's acoustic analogy is first validated, and then applied to investigate the noise radiated by a low Mach number flow through a diaphragm in a duct. The simulation method is a two-step hybrid approach relying on Lighthill's acoustic analogy, assuming the decoupling of noise generation and propagation. The first step consists in an incompressible Large Eddy Simulation of the turbulent flow field, during which the Lighthill's source term is recorded. In the second step, a variational formulation of Lighthill's Acoustic Analogy using a finite element discretization is solved in the Fourier space. The validation of this method is briefly presented: a general validation is performed on the case of two corotating vortices in a medium at rest; the exit of turbulent structures from the computational domain is accounted for by a spatial filtering; and a study of spatial interpolation from the CFD mesh to the acoustic mesh shows an acceptable level of error. This method is applied to a three-dimensional diaphragm with low Mach number flow, showing good agreement with both experimental results and Direct Noise Computation performed by Gloerfelt \& Lafon (Computers \& Fluids, 2007).

\section{5:40}

2aNSa20. Numerical strategies for investigation of gust-airfoil interaction. Florent Margnat (Arts et Métiers Paris Tech - Sinumef Lab, 151 bd de 1'Hopital, 75013 Paris, France, florent.margnat@paris .ensam.fr), Thomas Le Garrec (Arts et Métiers Paris Tech - Sinumef Lab, 151 bd de l'Hopital, 75013 Paris, France, thomas.le-garrec@paris .ensam.fr), Djaafer Fedala (Lab. d'Energétique et de Mécanique des Fluides Interne, Arts et Métiers ParisTech, 151 boulevard de l'Hôpital, 75013
Paris, France, djaafer.fedala@paris.ensam.fr),Xavier Gloerfelt(Arts et Métiers Paris Tech - Sinumef Lab, 151 bd de l'Hopital, 75013 Paris, France, xavier.gloerfelt@paris.ensam.fr), Smaine Kouidri (LIMSI-CNRS, BP 133, 91403 Orsay Cedex, France, smaine.kouidri@limsi.fr)

The noise generated by the interaction between a gust and an airfoil in a uniform flow is investigated. This problematic is of major industrial interest, regarding fans, turbomachinery, or wind turbine applications. A twodimensional symmetric Joukowski-type airfoil is immersed without incidence in a flow at Mach number 0.5 , disturbed by a harmonic gust at $45^{\circ}$ of incidence (4th CAA Workshop on Benchmark Problems, 2004). Our methodology is first to perform a high-order direct resolution of Euler's equations of the disturbed flow over the airfoil and the associated acoustic emission, which is taken as a reference simulation. Second, the near aerodynamic field is simulated with Fluent 6.3 solver based on finite volume method with second-order schemes. The aerodynamic data thus obtained are used for far field acoustic prediction, based on Ffowcs Williams and Hawkings analogy. Finally, following another hybrid approach, the noise is predicted by using integral formulations with source field from the DNS. The aim of the study is to provide insight into the efficiency and validity of these numerical strategies commonly used. Comparisons with results of the CAA workshop are given, covering various wavenumbers values.

\section{6:00}

2aNSa21. A stochastic source model for turbulent noise prediction including sweeping time dynamics. Malte Siefert (German Aerospace Center, Lilienthalplatz 7, 38108 Braunschweig, Germany, malte.siefert@dlr.de), Roland Ewert (DLR/Institute of Aerodynamics and Flow Technology, Lilienthalplatz 7, 38108 Braunschweig, Germany, Roland .Ewert@dlr.de)

We extend a low-cost computational aeroacoustic approach by taking into account temporal effects of the modeled turbulent flow. As the noise production is determined by the turbulent frequency spectrum rather than the wavenumber spectrum, temporal properties of the turbulent flow play a role in many cases. We focus on the most dominant temporal effect, on sweeping, i. e. the advection of inertial range structures by the energy containing large scales. By introducing a feedback-mechanism of the turbulent field onto itself, we are able to incorporate this effect in the fast random particle method, which was successfully applied to different cases such as 
slat noise, jet noise and others. It is shown that sweeping reproduces important properties of spatio-temporal correlations of the turbulent flow, which are not grasp by most turbulence-models. The influence on the sound generation will be discussed for aeroacoustic simulations of jet and trailing edge noise.

\section{6:20}

2aNSa22. Aeroacoustic simulation based on linearized Euler equations and stochastic sound source modelling. Hervé Dechipre (Volkswagen AG, Brieffach 1777, $38436 \quad$ Wolfsburg, Germany, herve.dechipre@volkswagen.de), Michael Hartmann (Volkswagen AG, Brieffach 1777, 38436 Wolfsburg, Germany, michael.Hartmann2 @ volkswagen.de), Jan W. Delfs (DLR/Institute of Aerodynamics and Flow Technology, Lilienthalplatz 7, 38108 Braunschweig, Germany, jan.delfs@dlr.de), Roland Ewert (DLR/Institute of Aerodynamics and Flow Technology, Lilienthalplatz 7, 38108 Braunschweig, Germany, Roland .Ewert@dlr.de)

In order to continually improve passenger car acoustic comfort, effective methods are needed to simulate the noise generated by external flows as well as the noise produced by duct flows in HVAC systems. The present paper illustrates the use of two methods based on the Linearized Euler equations and derivatives thereof to compute the acoustic field. The two methods implemented in the DLR's aeroacoustic code PIANO are the introduction of a perturbation (vortex) in the flow and the stochastic sound source modelling by Ewert. The application considered by this study is the noise produced by a flat plate in a two-dimensional duct at a thickness related Reynolds number of 1300. Different sizes and edge shapes of the flat plate have been tested. The simulations show a good agreement of the results obtained by the two methods as well as the existence of a vortex street behind the plate corresponding to a Strouhal number of 0.18 . It could be shown computationally, that even in the absence of the classical Aeolian tone generation (pure broadband turbulence related trailing edge noise generation) resonance type phenomena occur in the duct. The computed modes for the different configurations show encouraging correlations with the Parker-type modes or resonance phenomena described by Koch.

\section{6:40}

2aNSa23. Active Perturbation on Vortex-Induced Acoustic Resonance. Li Cheng (Hong Kong Polytechnic University, Hung Hom, Hong Kong, Hong Kong, mmlcheng@polyu.edu.hk), Y Zhou (Hong Kong Polytechnic University, Hung Hom, Hong Kong, Hong Kong, mmyzhou@polyu.edu.hk) , Ming Ming Zhang (Hong Kong Polytechnic University, Hung Hom, Hong Kong, Hong Kong, mingming@jhu.edu)

Flow-induced acoustic resonance results from strong interactions between unsteady separated flows and the acoustic modes of a cavity. This work explores the feasibility of using piezoelectric-actuator-based perturbation technique in the control of vortex-induced noise through systematic experimental studies. A thick rectangular plate was used as the vortex generator and placed upstream of a cavity. Curved piezo-ceramic actuators were embedded in a slot on the top side of the plate to provide a perturbation to the flow. Uncontrolled flow-acoustic interaction was first examined to provide a baseline for comparison. Results show that noises induced by flow separation from the thick rectangular plate and the wall ahead of the cavity have very different critical flow velocities so that their effects can be well separated. Open-loop control tests indicate that vortex-induced acoustic resonance can be successfully controlled using the proposed technique. Analyses suggest that the convection of votices separated from the leading edge along the plate surface was accelerated by the surface perturbation which interacted vigorously with the formation of the dominant trailing edge vortex, thus weakening the vortex strength in the wake of the plate. This weakened vortex strength substantially alters the flow-acoustic interaction, resulting in a significant impairment of vortex-induced acoustic resonance. (Supported by Research Grants Council of HKSAR. PolyU 5132/07E)

\section{7:00}

2aNSa24. Sound amplification in a lined duct with flow: PIV measurements. David Marx (Laboratoire d'Etudes Aerodynamiques CNRS, Bat K, 40 avenue du recteur Pineau, 86022 Poitiers, France, david.marx@lea.univ-poitiers.fr), Yves_ Aurégan (Laboratoire d'Acoustique de l'Université du Maine, Avenue Olivier Messiaen, 72085 Le Mans, France, yves.auregan@univ-lemans.fr), Helène Bailliet (Laboratoire d'Etudes Aerodynamiques - CNRS, Bat K, 40 avenue du recteur Pineau, $86022 \quad$ Poitiers, France, helene.bailliet@lea .univ-poitiers.fr), Jean-Christophe Valière (Laboratoire d'Etudes Aérodynamiques (LEA), Université de Poitiers, ENSMA, CNRS, Bat K, 40 avenue du recteur Pineau, 86022 Poitiers, France, jean-christophe.valiere@lea.univpoitiers.fr)

An experimental investigation of the acoustic behavior of a liner in a rectangular channel with grazing flow has been conducted. The liner is a locally reacting structure. When increasing the velocity of the grazing flow the transmission coefficient increases at resonance frequency. The transmission coefficient can even become larger than 1 meaning that acoustical energy is produced by the liner. This amplification of the sound wave is accompanied by an increase in the stationary pressure drop induced by the liner (up to $300 \%$ ). This effect is attributed to a modification of the flow induced by the acoustic wave. Thus, the flow is measured using Particle Image Velocimetry (PIV) imaging technique and a comparison of velocity maps with and without sound amplification is performed. Some differences occur and will be presented.

\section{7:20}

2aNSa25. Acoustic PIV: Measurement of the acoustic particle velocity using synchronized PIV-technique. André Fischer (German Aerospace Center (DLR), Mueller-Breslau-Str. 8, 10623 Berlin, Germany, andre.fischer@dlr.de), Emilie Sauvage (Ecole Polytechnique de 1'Univerité d'Orléans, 8 rue Léonard de Vinci, 45072 Orléans, France, milie.sauvage@gmx.net), Ingo Roehle (German Aerospace Center (DLR), Mueller-Breslau-Str. 8, 10623 Berlin, Germany, ingo.roehle@dlr.de)

This paper outlines a technique for measuring the acoustic particle velocity and the flow field simultaneously by applying synchronized particle image velocimetry (PIV). As test set-up a squared acrylic glass chamber was chosen. One side of the test section is connected to a loudspeaker, which allows a sinusoidal excitation of the chamber. To point out constrains of this method the investigation includes an analysis of excitation amplitude and frequency as well as the effect of the mean flow magnitude. Therefore a small PC fan can be mounted inside the test section to produce an adjustable mean flow. It can be shown that for a low number of averaged images (80) reasonable results can be achieved up to a certain level of fan rotation speed. Beyond this level the turbulence sensitivity increases and more images are necessary for the calculations. However, the acoustic particle velocity can be computed in the presence of turbulent flow. The presented method called acoustic PIV is a non intrusive technique, applied successfully in measuring acoustic particle velocity fields over a wide range of conditions.

\section{$7: 40$}

2aNSa26. A finite element method for time harmonic acoustics in arbitrary flows. Anne-Sophie Bonnet- Ben Dhia (CNRS, ENSTA - 32 Boulevard Victor, 75015 Paris, France, bonnet@ensta.fr), Jean-Francois Mercier (CNRS, ENSTA - 32 Boulevard Victor, 75015 Paris, France, jmercier@ensta.fr), Florence Millot (CERFACS, 42 avenue Gaspard Coriolis, 31057 Toulouse, France, millot@cerfacs.fr), Sebastien Pernet (CERFACS, 42 avenue Gaspard Coriolis, 31057 Toulouse, France, pernet@cerfacs.fr)

The reduction of noise in aeronautics motivates an intensive research in aeroacoustics. In particular, there is a need for efficient tools to simulate acoustic propagation in a mean flow. We are interested here by solving the linearized problem in the frequency domain, by a finite element method able to take into account general geometries and flows. To our knowledge, only 
the potential case has been completely handled. Recently, a new approach has been developed and validated in the case of a parallel shear flow: it relies on a regularized formulation of Galbrun's equation, well-suited for a discretization by Lagrange finite elements, combined with Perfectly Matched Layers. A drawback of the method comes from the additional term of regularization, which requires the evaluation of an oscillating integral, coupling all degrees of freedom located on the same streamline. This difficulty can be avoided by replacing this non-local term by its Low-Mach approximation We show here how to extend this Low-Mach approach to the case of a non parallel flow. Numerical experiments are done. In the case of a potential mean flow, a good agreement with the exact approach is observed, even for quite large Mach numbers.

TUESDAY MORNING, 1 JULY 2008

ROOM 251, 8:00 TO 10:00 A.M.

\title{
Session 2aNSb
}

\section{Noise, Biomedical Ultrasound/Bioresponse to Vibration, ASA Committee on Standards, and EURONOISE: Session in Honor of Henning von Gierke}

\author{
Paul Schomer, Cochair \\ Schomer and Associates, Inc., 2117 Robert Drive, Champaign, IL 61821, USA
}

Brigitte Schulte-Fortkamp, Cochair

TU Berlin, Institute of Fluid Mechanics and Engineering Acoustics, Einsteinufer 25, Sekr. TA 7, Berlin, D-10587, Germany

\section{Invited Papers}

8:00

2aNSb1. Where do we stand on standards for noise? William Lang (Noise Control Foundation, 29 Hornbeck Ridge, Poughkeepsie, NY 12603, USA, lang1ww@gmail.com)

Almost 40 years ago, Henning von Gierke organized and chaired a symposium at the ASA Cleveland meeting with the above title. The chairs of nine writing groups of the ANSI-predecessor working on methods for the measurement and rating of noise presented summaries of their tasks and their progress in 1968. If a symposium on the same theme were to be held today, there would be reports from fifty-four working groups of ANSI Accredited Standards Committees on Acoustics (S1), Bioacoustics (S3), and Noise (S12). These committees are cornerstones of the ASA standards program. As the first ASA Standards Director, Henning revitalized the Society's standards program to become the productive organization it is today. The progress in standardization in America during the past four decades is a tribute to his foresight and participation. He also contributed to international standardization as the leader of the U.S. delegation to ISO TC/43 (Acoustics) and its subcommittee on noise (SC1), and for three decades he chaired ISO TC/108/SC4 on human exposure to mechanical shock and vibration. Trained as an engineer, Henning was a leader of national and international standardization efforts to relate the mechanical energy in sound waves to the responses of the human organism.

8:20

2aNSb2. Henning E. von Gierke and human biodynamics. Anthony J. Brammer (Ergonomic Technology Center, Univ. of Connecticut Health Center, 263, Farmington Ave., Farmington, CT 06030, USA, brammer@uchc.edu)

In addition to his well-known involvement in protecting humans from exposure to excessive noise, Dr. Henning E. von Gierke was deeply involved in protecting humans from exposure to excessive mechanical shocks, impacts and vibration. From exploring the limits of survivability during the landing phase of space and aircraft capsules to the more everyday issues of occupational exposure to vibration, he provided scientific insight into problems and leadership in developing solutions. While most of Henning's contributions are buried in past literature, the spirit of his efforts lives on in a review chapter published in Harris' Shock and Vibration Handbook that I have had the privilege to co-author with him through two editions, now extending back more than a decade. The challenge of encompassing the original content of his contribution while introducing the results of more recent research has, so far, yielded a composition that he has endorsed. Henning's involvement and interests in human biodynamics will be illustrated by examples taken from this work.

\section{8:40}

2aNSb3. Service to his fellow men. Richard McKinley (AFRL, Wright-Patterson Air Force Base, Dayton, OH 45433-7901, USA, richard.mckinley@wpafb.af.mil)

Henning Von Gierke's life of service followed in the footsteps of his family legacy. This presentation will focus on Henning's 50 years of research at the Air Force Research Laboratory at Wright-Patterson Air Force Base, Dayton, Ohio and the recollections of Henning gathered from $20+$ hours of video interviews. Henning's life and his research were dedicated to helping mankind. Beginning with his dissertation on noise produced by jet flow and continuing with the accomplishments of his division at the Air Force Research Laboratory his contributions to acoustics were outstanding. His contributions include the Air Force hearing conservation program, the EPA levels document, the BENOX report, the development of Bionics in the 1960's, car airbag research in the 60's, testing of the 
original 7 US astronauts, human limits of survival for impact, human limits of performance in vibration, pioneering work in hearing protector modeling, bone conduction, and microwave hearing, speech communication, as well as his extensive work in American National Standards and international standards. His contributions have in one way or another affected nearly everyone. He was a great researcher and a great mentor. He will be missed by his colleagues at the Air Force Research Laboratory and around the world.

\section{Contributed Paper}

\section{9:00}

2aNSb4. Henning von Gierke, leader, consensus builder, mentor, and friend. Alice H. Suter (Alice Suter \& Associates, 575 Dogwood Way, Ashland, OR 97520, USA, ahsuter@charter.net)

Over his long and illustrious career, Henning von Gierke has had an enormous effect on public policy in noise and vibration, not only because he was active in the field for more than half a century, but because of his intense dedication, his persistence, and his unique, almost legendary ability to build consensus. EPA's Office of Noise Abatement and Control could not possibly have produced its Criteria and Levels documents in response to the timeframe imposed by Congress without Henning's leadership. Those of us who were lucky enough to be mentored by him were able to accomplish more than we ever could have without his guidance. In addition to his invaluable assistance in my doctoral program, I knew him as ASA's Standards Director, then President of ASA, chairman of interagency task forces, chairman of several standards committees, and participant in numerous international activities. He was always willing to share the burden of work and to encourage the rest of us to forge ahead in an effort to make the world a quieter and healthier place.

\section{Invited Papers}

2aNSb5. Dr. Henning von Gierke-My mentor, everyone's mentor. Paul Schomer (Schomer and Associates, Inc., 2117 Robert Drive, Champaign, IL 61821, USA, schomer@SchomerAndAssociates.com)

Dr. Henning von Gierke was a leading force in acoustics for a majority of the 20th century. He led in human response to noise AND vibration and its assessment. He was a leading force in the US government and in the National Academy of Science (NAS) Committee on Hearing and Bioacoustics (CHABA). He was a leader in hearing assessment and conservation, and in Standards. And to many working in these various areas, he was a mentor, a confidant, and a friend. Mentoring did not stop at the doors of his laboratory, but rather, his mentoring extended to the world. He was a mentor to me in my first years after graduation when we developed the $\mathrm{C}$-weighted procedure for the assessment of large-amplitude impulse noise, and in the development of related Standards and NAS studies. He thrust me into ISO work and CHABA studies, and, later, he chose me to succeed him as ISO delegation chair in acoustics and noise. Not enough can be said about Henning's impact on noise and vibration research and policy in the USA and in the world, and on the expanded reach of Henning's influence through the many like me that he mentored.

\section{9:40}

2aNSb6. Henning von Gierke's continuing contribution: Underwater whole-body vibration. Sarah Gourlie (Applied Research Laboratories, The University of Texas, P.O. Box 8029, Austin, TX 78713-8029, USA, sarah.gourlie@gmail.com), Mark F. Hamilton (Applied Research Laboratories, The University of Texas, P.O. Box 8029, Austin, TX 78713-8029, USA, hamilton@mail.utexas.edu)

Henning von Gierke was a leader in the characterization of human response to sound and vibration and in the development of international standards in this area. One focus of his research was using a multidisciplinary approach to create biodynamic models for whole-body vibration with the aim of predicting and preventing injury in vehicles. The focus of this presentation is whole-body vibration underwater with the aim of predicting discomfort or possibly injury to divers from low-frequency sonar. The frequency range of interest is $40-80 \mathrm{~Hz}$, which encompasses the resonance of human lung. For this purpose, a biodynamic model developed by von Gierke to simulate thoracic, abdominal, and spinal responses to different vibrational excitations in air [H. E. von Gierke, J. Acoust. Soc. Am. 50, 1397 (1971)] is adapted for underwater conditions. It is assumed that the diver is neutrally buoyant and will therefore experience whole-body acceleration equal to the particle acceleration produced by the sound field in the absence of the diver. Emphasis is placed on determining the appropriate source distribution on the body as well as adjusting von Gierke's model to accommodate the decrease in lung compressibility with diver depth and mass loading on the chest. [Work supported by ONR.] 


\title{
Session 2aNSc
}

\section{Noise and EURONOISE: Physical and Psychophysical Evaluation of Vehicle Exterior Noise I}

\author{
Paul Donavan, Cochair \\ Illingworth \& Rodkin, Inc., 505 Petaluma Blvd. South, Petaluma, CA 94952, USA \\ Hugo Fastl, Cochair \\ AG Technische Akustik, MMK, TU München, Arcisstr. 21, München, 80333, Germany
}

Invited Papers

8:00

2aNSc1. Low speed exterior vehicle voise and the effect of pavement type. Paul Donavan (Illingworth \& Rodkin, Inc., 505 Petaluma Blvd. South, Petaluma, CA 94952, USA, pdonavan@illingworthrodkin.com)

For operating conditions of cruise and moderate acceleration, the exterior noise emission of light vehicles is typically dominated by tire/pavement noise at speeds of $50 \mathrm{~km} / \mathrm{h}$ or greater. At a test speed of $56 \mathrm{~km} / \mathrm{h}$, it has been found that pavement type can create a 10 $\mathrm{dB}$ or more variation in tire/pavement noise. This has significant implications for both community noise and vehicle noise emission testing. In this paper, the results tire/pavement noise measurements for over 40 different pavements in Europe and the United States are reported. These pavements include research surfaces, existing roadways, and ISO 10844 passby test surfaces. Measurements were conducted using an on-board sound intensity methodology that has been correlated to cruise-by noise levels. These results are discussed in terms of the revisions being considered for the newly revised ISO 362 passby test procedure and the ISO 10844 test surface specification. Additionally, a case history of community traffic noise reduction achieved by use of a quieter pavement is reviewed to demonstrate the importance of the pavement in low speed vehicle noise emissions.

\section{$8: 20$}

2aNSc2. Noise source mapping for trucks, part 1: development and design. Kenneth J. Plotkin (Wyle Laboratories Inc., 241 18th Street S., Suite 701, Arlington, VA 22202, USA, kenneth.plotkin@wylelabs.com), Yuriy Gurovich (University of Mississippi, NCPA, 1 Coliseum Drive, University, MS 38677, USA, yuriy.gurovich@wylelabs.com), William Blake (Naval Surface Warfare Center (Ret.), 6905 Hillmead Road, Bethesda, MD 20817, USA, hydroacoustics@aol.com), Paul Donavan (Illingworth \& Rodkin, Inc., 505 Petaluma Blvd. South, Petaluma, CA 94952, USA, pdonavan@illingworthrodkin.com)

Mapping and quantifying noise sources on trucks under actual operating conditions on the road are important for traffic noise modeling and mitigation. The purpose of this study is to develop a practical truck noise source localization technique using acoustic beamforming. An experimental $70+$ microphone elliptical array was designed and fabricated for truck testing. Beam-forming software was developed and implemented using a computerized data acquisition system. Proof-of-concept tests were performed at low-speed and high-speed truck testing facilities for a representative sample of trucks with widely different characteristics to validate the measurement system performance. The measurement system design parameters were verified experimentally, and certain improvements to the system were recommended for future implementation based on the field experience. The developed beam-forming measurement system provided adequate noise mapping and localization for various noise sources on trucks, stationary and moving with the speed up to $50 \mathrm{mph}$. The results of the proof-of-concept testing presented in an accompanying paper (Part 2) confirm that the developed microphone array, data acquisition system and beam-forming software performed generally as expected and required no major adjustments. This ongoing project is funded by the National Cooperative Highway Research Program of the Transportation Research Board of the National Academies, USA.

\section{8:40}

2aNSc3. Noise source mapping for trucks, Part 2: Experimental results. William Blake (Naval Surface Warfare Center (Ret.), 6905 Hillmead Road, Bethesda, MD 20817, USA, hydroacoustics@aol.com), Kenneth J. Plotkin (Wyle Laboratories Inc., 241 18th Street S., Suite 701, Arlington, VA 22202, USA, kenneth.plotkin@wylelabs.com), Yuriy Gurovich (University of Mississippi, NCPA, 1 Coliseum Drive, University, MS 38677, USA, yuriy.gurovich@wylelabs.com), Paul Donovan (505 Petaluma Blvd, South, Petaluma, VA 94952, USA, pdonovan@illingworthrodkin.com)

The elliptical array described in the previous paper (Part 1) was deployed road-side at a test track for measuring the sound emitted from trucks during passby. Measurements were made on various truck models in various operating states with and without trailers. Extensive spherical-source calibrations confirmed the array's beamforming at both on-normal and steering angles (to $45^{\circ}$ ); they also disclosed the magnitudes of ground reflection paths for localized sources at different elevations. In all cases, the array output was computationally inverted to produce two-dimensional spatial maps of source levels in the side profile of the truck. In the cases of stationary trucks, the source maps were correlated with simultaneously-obtained intensity maps. Comparisons verified that the arraybased source maps for the trucks ranked sound sources of disparate levels in the same order as did the sound power levels deduced from the sound intensity data. Acoustic source maps obtained during truck passbys were then used to provide time-histories and spatial distributions of sources and source paths from the engine, muffler, tires, and certain body components. This project is funded by the National Cooperative Highway Research Program of the Transportation Research Board of the National Academies, USA. 
2aNSc4. Vehicle exterior noise from the view point of new experts. Brigitte Schulte-Fortkamp (TU Berlin, Institute of Fluid Mechanics and Engineering Acoustics, Einsteinufer 25, Sekr. TA 7, D-10587 Berlin, Germany, brigitte.schulte-fortkamp@tu-berlin.de)

The combination of physical and psycho- acoustical measurements with scientific evaluation of perceptual responses to environmental noise, known as Soundscaping, is an essential method for assessing and actualizing environments. Empirically, the perception of sounds and their evaluation will be explored under the premise of combining human judgment and physical factors. In a recent Soundscape Project, environmental noise perception and evaluation in a defined urban area were investigated in detail. Classical measurements and open, narrative, but issue-centred interviews with residents were carried out, and those interviews allowed the interviewed persons to set their own focus on certain aspects concerning the development of a public space in Berlin. Method and procedure will be discussed in detail to introduce to the New Expert Concept. The Soundscape Project is a module of the Project "Nauener Platz - Remodelling for Young and Old" in the framework of the research program "Experimental Housing and Urban Development (ExWoSt)" of the "Federal Ministry of Transport, Building, and Urban Affairs (BMVBS)" by the "Federal Office for Building and Regional Planning $(\mathrm{BBR})$ ". It is related to the fields of research (ExWoSt) concerned with "Innovation of Urban Neighbourhoods for Families and the Elderly". The project executing organization is the Regional Office Berlin-Mitte.

\section{9:20}

2aNSc5. Significance of Psychoacoustic Aspects for the Evaluation of Vehicle Exterior Noise. Klaus Genuit (HEAD acoustics GmbH, Ebertstrasse 30a, 52134 Herzogenrath, Germany, klaus.genuit@head-acoustics.de)

For 25 years acoustic engineers have been using specific measurement technologies and analyses to determine sound quality of vehicle interior noise. However, with regard to exterior vehicle noise only standard measurements, e.g. the A-weighted sound pressure level (ISO 362), have been used and interpreted so far. Extensive knowledge and experiences concerning the evaluation of interior noise are still unutilized for an advanced assessment of vehicle exterior noise. Within EU research projects, such as SVEN or QCity, exterior noise was investigated, methods established and psychoacoustic analyses carried out. The relevance of vehicle exterior noise with respect to perceived product quality was underestimated for a long time. Today, manufacturers recognize the importance of exterior noise beyond the annoyance perspective. Vehicle exterior noise conveys an impression of product quality. By optimizing the product sound quality manufacturers can compete against others and meet increased requirements. Customers prefer vehicle sounds which match their expectations, which also applies to the exterior sound of a vehicle. The acoustical fingerprint of a vehicle - inside and outside - provides enormous marketing opportunities. This paper shows how the knowledge of sound quality analyses with regard to interior noise can be transferred to the evaluation of exterior vehicle noise.

\section{9:40}

2aNSc6. Rating the Dieselness of engine-sounds. Hugo Fastl (AG Technische Akustik, MMK, TU München, Arcisstr. 21, 80333 München, Germany, fastl@mmk.ei.tum.de), Bernhard Priewasser (AG Technische Akustik, MMK, TU München, Arcisstr. 21, 80333 München, Germany, bernhard@prie.de), Markus Fruhmann (AG Technische Akustik, MMK, TU München, Arcisstr. 21, 80333 München, Germany, Markus.Fruhmann@gmx.de), Herbert Finsterhölzl (BMW Group, Knorrstr. 147, 80788 München, Germany, herbert.finsterhoelzl@bmw.de)

Sounds of idling Diesel engines were rated according to their Dieselness with two different psychoacoustic methods. On the one hand, a yes/no procedure was used. Subjects were presented sounds of two seconds duration and had to answer by yes or no to the following question: Does this sound stem from a Diesel engine? Histograms were calculated and a hypothesis was put forward as follows: The more positive answers are given to a specific sound, the larger is the Dieselness of this sound. On the other hand, the method of paired comparison was used, applying a kind of Bradley-Terry-Luce (BTL) procedure. Results are discussed with respect to the psychophysical method used and possible differences in ratings by expert versus naive listeners. Finally, psychoacoustic data are compared to predictions of Dieselness calculated by four different algorithms.

\section{0:00}

2aNSc7. Perceptive temporal features of train passbys. Jonathan Terroir (Laboratoire MRTE - UCP, 5, mail Gay-Lussac, Neuville-sur-Oise, 95031 Cergy-Pontoise Cedex, France, jterroir@u-cergy.fr), Catherine Lavandier (Laboratoire MRTE - UCP, 5, mail Gay-Lussac, Neuville-sur-Oise, 95031 Cergy-Pontoise Cedex, France, catherine.lavandier@u-cergy.fr)

This work deals with characterization of temporal features linked to train passages which may act upon annoyance. Two varying parameters in the recordings have been selected: distance from the railway (7.5 meters, 50 and 100 meters) and train category (four types of French trains). A first series of psychoacoustic tests are carried out in order to mainly extract temporal aspects which could be important for sound perception. To avoid the level saliency, a preliminary equalization has been made on $\mathrm{L}_{\mathrm{A} 5}$. The selection of perceptive factors is carried out through a multidimensional analysis (INDSCAL). Dissimilarity tests highlight several dimensions: train category, slope of the level increase linked to train arrival and passage length. In addition to the dissimilarity between two stimuli estimation, subjects have to choose the one they prefer and explain their answers. The verbalization task helps illustrate multidimensional analysis axis. A second series of tests, designed with recordings varying on two independent parameters (the level and the slope of the temporal evolution) makes it possible to compare perceptive variations on temporal effects to loudness. 
2aNSc8. Synthesis of pass-by railway noise. Shafiquzzaman Khan (Kungliga Tekniska Hogsklan, Department of Aeronautical and Vehicle Engineering, SE - 10044 Stockholm, Sweden, Shafik@kth.se), Mohite Ulhas (Indian Institute of Technology Roorkee, Graduta Student, Dept. of Mech. \& Ind. Engg, 247667 Roorkee, India, goelvfme @iitr.ernet.in), Virendra Goel (Indian Institute of Technology Roorkee, Graduta Student, Dept. of Mech. \& Ind. Engg, 247667 Roorkee, India, goelvfme@iitr.ernet.in)

Pass-by railway noise is one of the main problems in the community. Typically pass-by railway noise is estimated using Leq or Lden in $\mathrm{dB}(\mathrm{A})$ and often these methods do not reflect the listener perceptual overview. It is therefore necessary to reduce the pass-by railway noise by synthesizing the noise characters into various segments. The segments could be based on the physical properties of rail vehicles like rolling, traction, pantograph, cooling system noise, etc. There are few other segments which are also based on the perceptual view of the pass-by railway noise like squeaking, rattling or beating (tadak, tadak). In this study only a few of the physical characteristics of the railway noises are examined. The idea of synthesization is to determine what segments in the noise characters are annoying. And thereby make a virtual pleasant pass-by railway sound. Several pass-by noises from Swedish rail vehicles were recorded using binaural technology according to ISO 3095. Recorded noise signals were then analyzed with the help of spectrogram analysis using Matlab. The results of the studies indicate that the rolling noise and broadband noise are most annoying for the long distance passenger rail vehicles.

TUESDAY MORNING, 1 JULY 2008

ROOM 251, 10:20 A.M. TO 4:00 P.M.

\title{
Session 2aNSd
}

\section{Noise and EURONOISE: Environmental Noise Mapping II}

\author{
Kenneth Cunefare, Cochair \\ Woodruff School of Mechanical Engineering, The Georgia Inst. of Technol., Atlanta, GA 30332-0405, USA \\ Gaetano Licitra, Cochair \\ ARPAT - Dept. Firenze, Via Porpora, 22, Firenze, 50144, Italy
}

Invited Papers

10:20

2aNSd1. Managing Risk by Utilising an Integrated Approach to Quality Assurance During Strategic Noise Mapping. Simon J. Shilton (Acustica Ltd, Trident One, Styal Road, M22 5XB Manchester, UK, simon.shilton@acustica.co.uk), Alan Štimac (DARH2 Acoustics \& Civil Eng. Ltd., Ljubicin prolaz 3, HR-10430 Samobor, Croatia, alan@ darh2.hr), James Trow (Hepworth Acoustics Ltd, 5 Bankside, Crosfield Street, WA1 1UP Warrington, UK, james.trow @ hepworth-acoustics.co.uk), Nathan Archer (Hepworth Acoustics Ltd, 5 Bankside, Crosfield Street, WA1 1UP Warrington, UK, nathan.archer@hepworth-acoustics.co.uk), Vincent Hii Jiu Ta (Hepworth Acoustics Ltd, 5 Bankside, Crosfield Street, WA1 1UP Warrington, UK, vincent.hii@hepworth-acoustics.co.uk), Nigel Jones (Extrium Ltd, Calverley House, 55 Calverley Road, TN1 2TU Tunbridge Wells, UK, nigel.jones@extrium.co.uk)

With the proclamation of the Directive on Environmental Noise (2002/49), the process of noise mapping and action planning has begun around Europe. As the development of strategic noise maps is arguably a new experience for many end-users, the first round of noise mapping could potentially lead to uncertainties within many aspects of the process. Noise maps represent a baseline for the implementation of noise management systems and any systematic errors within the noise maps could reduce the effective implementation of the whole management system. The extensive range of data inputs required in strategic noise mapping are usually obtained from different data sources or base datasets and as such, cataloguing with the use of metadata is key. The implementation of a quality assurance system is imperative to maintain consistency between technicians and within multi-disciplinary teams. It is also essential to ensure the control of processes and the ability to review inputs, intermediaries and deliverables. A similar approach may also be applied to calculation and post processing of noise levels. This paper presents collective experience of the implementation of quality assurance procedures used in several EU countries during the successful completion of projects within the first round of mapping.

10:40

2aNSd2. A methodology for mapping neighbourhood impacts. Ronny Klaeboe (Institute of Transport Economics, Gaustadalleen 21, 0349 Oslo, Norway, rk@toi.no), Erik Engelien (Statistics Norway, Oterveien 23, N-2225, 2225 Kongsvinger, Norway, erik.engelien@ssb.no), Margrete Steinnes (Statistics Norway, Oterveien 23, N-2225, 2225 Kongsvinger, Norway, margrete.steinnes @ssb.no)

A methodology for the spatial analysis of environmental impacts in urban areas and social gradients is presented. The methodology is computational intensive and requires access to geographically located data at the dwelling level. Neighbourhood information is harvested to provide contextual information, and a spatial smoothing technique used to provide powerful visualisations of residential and neighbourhood impacts. By focussing on impacts rather than exposures, the construction of accumulative non-monetarised and monetarised indicators of impacts such as disability adjusted life years (DALY) and health costs is facilitated. Since the residential dwelling location is, or can be made, the focal point for social, dwelling, environment, neighbourhood, and transportation related indicators, a rich network of information in a uniform format is available for construction of novel indicators. By focussing on neighbourhoods rather 
than individual dwellings, privacy is enhanced while maintaining useful level of detail for involving the public and politicians in local planning. By combining information on social status and environmental exposures, questions on whether transportation related impacts are distributed fairly and whether social inequity is increased or decreases as a result of a particular measure can be addressed. The methodology is not applicable to rural areas and low probability events.

\section{1:00}

2aNSd3. Noise emissions at intersections: comparing microscopic and macroscopic traffic simulation approaches. Bert De Coensel (Ghent University - Department of Information Technology, Sint-Pietersnieuwstraat 41, 9000 Ghent, Belgium, bert.decoensel@intec.ugent.be), Dick Botteldooren (University Ghent - Department Information Technology, Sint-Pietersnieuwstraat 41, 9000 Gent, Belgium, dick.botteldooren@intec.ugent.be), Luc Dekoninck (Ghent University - Department of Information Technology, Sint-Pietersnieuwstraat 41, 9000 Ghent, Belgium, luc.dekoninck@intec.ugent.be), Dominique Gillis (Ghent University - Department of Civil Engineering, Krijgslaan 281 S8, 9000 Ghent, Belgium, dominique.gillis@ugent.be), Dirk Lauwers (Ghent University - Department of Civil Engineering, Krijgslaan 281 S8, 9000 Ghent, Belgium, dirk.lauwers@ugent.be)

Environmental noise mapping often involves the use of traffic simulation software. In most cases, traffic flows are simulated in a macroscopic way, considering only traffic flow parameters averaged over road segments. This approach does not allow to correctly account for the typical deceleration and acceleration patterns of traffic at intersections, which influence local noise emissions. Microscopic traffic simulation models, which model each vehicle individually, incorporate these dynamic effects. However, they require much larger amounts of input data for calibration, and are therefore less suited to map large areas. In this paper, we investigate the possible influence of the choice of simulation detail on simulated traffic flow parameters and, as a consequence, on noise emissions near intersections. As a case study, several types of road intersections under various traffic demand and traffic composition conditions are considered. Simulation results are obtained using a microscopic (Paramics) as well as a mesoscopic (MaDAM) and a macroscopic (OmniTRANS) traffic simulation model, and predicted traffic flow parameters and noise emission values are compared between these approaches.

\section{Contributed Papers}

$11: 20$

2aNSd4. Environmental Noise Directive - results of noise mapping in Germany. Matthias Hintzsche (Federal Environment Agency, Woerlitzer Platz 1, 06844 Dessau-Rosslau, Germany, matthias.hintzsche@uba.de)

The Environmental Noise Directive intends the development of a concept to avoid, prevent or reduce on a prioritised basis the harmful effects, including annoyance, due to exposure to environmental noise and to inform the public on environmental noise and its effects. In a first step noise maps were made for all agglomerations and for all major roads, major railways and major airports. This noise maps are the basics for noise action plans. These plans have the target to prevent and to reduce environmental noise where necessary to preserve environmental noise quality where it is good. The results of the first round for noise mapping in Germany will be presented.

\section{$11: 40$}

2aNSd5. Noise mapping of public roads in Norway: New calculation tool based on the Nord2000 Road Engineering Method. Ingunn Milford (Norwegian Public Roads Administration, Brynsengfaret 6A, P.O .Box 8142 Dep., N-0033 Oslo, Norway, ingunn.milford@vegvesen.no)

To comply with requirements in the Directive 2002/49/EC on environmental noise (END) and to national guidelines on land use and transport noise, the Norwegian Public Roads Administration has developed the calculation tool called NorStoy, which is composed of two main modules: 1. A calculation module with the Nord2000 Road Engineering Method algorithms. 2. An ArcGIS module to arrange the input data, delimit the calculation area, locate the calculation points and report the results. Nord2000 Road Engineering Model was completed in 2006, in cooperation with the Nordic countries. The model has very much in common with the coming joint European Harmonoise model. The challenge in using these models is to obtain an acceptable calculation time. To prevent NorStoy from stopping every time insufficient data occurs, we use default values based on either a typical mean value or the nearest registered value. At this time preparing input data is quite time consuming. We are able to produce the noise maps for the EC directive, but NorStoy is not yet an efficient tool for noise mapping in general. The development of NorStoy is still in progress.

\section{2:00}

2aNSd6. The national noise map of the Netherlands $A$ trend in exposure since 1990. Rik Van Haaren (DHV, P.O. box 1132, 3800BC Utrecht, Netherlands, rik.vanhaaren@dhv.com)

Noise maps according to the environmental noise directive have been provided by several actors in the Netherlands. The results of the noise maps show the significance of each of the source types, as well as the distribution within and outside agglomerations. In the Netherlands 2.7 million people are exposed to noise levels above $55 \mathrm{~dB}(\mathrm{~A})$. In some cases up to 4000 people are exposed to level higher than $75 \mathrm{~dB}(\mathrm{~A})$. In the night period 2.3 million people are exposed to noise levels higher than $50 \mathrm{~dB}(\mathrm{~A})$. Road noise is the dominant source for annoyance and sleep disturbance. Within agglomerations road noise is responsible for $88 \%$ of the highly annoyed population. Major roads, outside agglomerations, contribute less than $10 \%$ to the total annoyance due to road traffic. Railways are a second source in producing annoyance and sleep disturbance. 57\% of the high annoyance due to railways is produced outside agglomerations. The results of the noise maps for road traffic noise have been compared with results for a selection of cities in 1990. The results show that the total number of exposed people has increased. However, the exposition to the higher bands has decreased.

\section{2:20}

2aNSd7. Noise Mapping and Noise Scoring - Software Techniques and Result Presentation. Wolfgang Probst (DataKustik GmbH, Gewerbering 5,86926 Greifenberg, Germany, wolfgang.probst@ datakustik.com)

Noise scoring is based on noise level maps and on exposure distribution. Different techniques can be used to develop the areal distribution of these values and to present them as coloured maps. Especially more complex ratings that are not only based on an exposure level and on population densities need relatively complex procedures to develop them from noise maps. It must be taken into account that there are two important steps in this procedure: One is the calculation of the noise score itself and the other is the presentation as coloured map to detect and focus on Hot Spots. Different techniques have been developed, were integrated in the software and have been applied in the frame of the QCity project. The consequences of different parameter settings are presented and discussed. The second step is the assignment of colours to a given scale of result values and even this simple process must be handled carefully to transport the needed information. 


\section{Contributed Papers}

\section{2:00}

2aNSd8. The Milan agglomeration Strategic Noise Map. Giovanni Zambon (Department of Environmental Sciences of the University of Milano - Bicocca, Piazza della Scienza, 1, 20126 Milan, Italy, giovanni.zambon@unimib.it), Simone Radaelli (Department of Environmental Sciences of the University of Milano - Bicocca, Piazza della Scienza, 1, 20126 Milan, Italy, s.radaelli@unimib.it)

The aim of this paper is to describe the procedure used for the Milan agglomeration Strategic Noise Mapping, as defined by Environmental Noise Directive (2002/49/EC). City of Milan is a complex case study because of the presence of a plurality of sources: road traffic, railway traffic (also tram traffic) and aircraft noise (Linate airport). The assessment method is based on the guidelines contained in the "Good Practice Guide for Strategic Noise Mapping and the Production of Associated Data on Noise Exposure" (WGAEN). The study of noise generated from several sources was carried out with the integrated use of both numerical models and Geographic Information Systems (GIS). Numerical models allow us to estimate of noise levels in large areas near to a specific noise source and GIS enable the efficient acquisition, management and elaboration of geo-referenced data representative of territory, sources and buildings. By means of the study of specific GIS application it was possible to predict the global noise exposure for overall sources, by overlapping the noise maps of the single noise sources.

\section{2:20}

2aNSd9. Budapest Noise Mapping Project I. - Experiences. András Muntag (Enviroplus Kft., Telepy u. 3., 1096 Budapest, Hungary, muntaga@zajan.hu), Mihály Berndt (Enviroplus Kft., Telepy u. 3., 1096 Budapest, Hungary, berndt@mgx.hu)

The Hungarian capital Budapest, and their agglomeration have recently finished their first strategic noise map. According to the Hungarian noise regulations and to the European Directive 2002/49/EC, this is a legal obligation. The main part of the costs was supported by the EU. The preparation of the strategic noise maps based on a computer model and describing the present noise situation of the municipalities, should be seen as the first step to build a true noise information and management system, based on the computer model initially developed. The main parameters of the project were the planned area $1100 \mathrm{qkm}$, number of dwellings 440 thousand, length of the mapped roads ca. $2000 \mathrm{~km}$ This paper based on the experience of 23 municipal noise maps - Budapest and other 22 settlements in the agglomeration. We successfully finished the project on time - the results (all maps) can be found on the internet-page: http://terkep.budapest .hu/website/zajterkep The first paper deals with the technical experiences of the project.

\section{2:40}

2aNSd10. Budapest Noise Mapping Project II. - Results. Mihály Berndt (Enviroplus Kft., Telepy u. 3., 1096 Budapest, Hungary, berndt@mgx.hu), András Muntag (Enviroplus Kft., Telepy u. 3., 1096 Budapest, Hungary, muntaga@zajan.hu)

The Hungarian capital Budapest, and their agglomeration have recently finished their first strategic noise map. According to the Hungarian noise regulations and to the European Directive 2002/49/EC, this is a legal obligation. The main part of the costs was supported by the EU. The preparation of the strategic noise maps based on a computer model and describing the present noise situation of the municipalities, should be seen as the first step to build a true noise information and management system, based on the computer model initially developed. The main parameters of the project were the planned area $1100 \mathrm{qkm}$, number of dwellings 440 thousand, length of the mapped roads ca. $2000 \mathrm{~km}$ This paper is based on the experience of 23 municipal noise maps - Budapest and other 22 settlements in the agglomeration. We successfully finished the project on time - the results (all maps) can be found on the internet-page: http://terkep.budapest .hu/website/zajterkep The second paper shows the results of the project.

\section{3:00}

2aNSd11. The Port of Livorno noise mapping experience. Mario Morretta (Sintesis - Enginering and Consulting S.r.l., via Martin Luther King, 15, 57128 Livorno, Italy, mario.morretta@ sintesis.toscana.it), Andrea Iacoponi (ARPAT - Dipartimento di Livorno, via Marradi, 114, 57128 Livorno, Italy, a.iacoponi@arpat.toscana.it), Fabrizio Dolinich (Sintesis Enginering and Consulting S.r.l., via Martin Luther King, 15, 57128 Livorno, Italy, fabrizio.dolinich@gmail.com)

Livorno Municipality and its seaport are requested to produce the strategic noise map according to the European Directive only in 2012. However Livorno Port Authority, following the principle of environmental sustainability and in coherence with its EMAS status, started the investigation about the interaction between the noise generated by port activities and the nearby city. This analysis has been carried out within the Life funded project NoMEPorts, whose goal is to develop and provide tools and expertise to European ports for implementing their strategic noise maps, by means of a collaborative mapping of 8 Port Authority technical representatives from all around Europe. The work presented outlines in detail the process followed in performing the noise mapping of the Livorno port area and the port-city interface area, focusing in the problems encountered in the data collection, in port noise model building steps and in the consequent solutions adopted. The paper also talks about the result obtained by showing the noise maps in six detail levels and reports about the action plan proposed. As a result this experience emphasises the importance of noise maps as a powerful tool for decision-makers in port planning.

\section{3:20}

2aNSd12. Strategic Noise Mapping of the City of Ljubljana, Slovenia. Alan Štimac (DARH2 Acoustics \& Civil Eng. Ltd., Ljubicin prolaz 3, HR10430 Samobor, Croatia, alan@darh2.hr), Aleš Globevnik (A-PROJEKT Natasa Kepe-Globevnik s.p., Vinarje 110b, SI-2000 Maribor, Slovenia, aprojekt@siol.net), Dinko Stipanicev (DARH2 Acoustics \& Civil Eng. Ltd., Ljubicin prolaz 3, HR-10430 Samobor, Croatia, dinko@darh2.hr), Lilijana Kuhelj (Environmental Agency of the Republic of Slovenia, Vojkova 1B, SI-1000 Ljubljana, Slovenia, lilijana.kuhelj@gov .si)

Currently Ljubljana is the only agglomeration according to the definition of the directive 2002/49/EC in Slovenia. For this agglomeration, separate noise maps not only for road and rail traffic but also for industrial sources were made. As for the first phase of preparation there is no precise methodology stated for representing industrial noise sources in strategic noise maps regarding END and following the vision of other EU countries, only industrial plants classified as IPPC installations were taken into consideration of noise mapping. Short term noise measurements around industrial plants conducted according to ISO 1996 during previous three years were used as reverse engineering techniques for the determination of sound power levels of industrial plants. Elaboration of road and rail traffic noise maps required development of perfect acoustical model with 3D terrain modelling including all possible obstacles (barriers, walls, bridges, viaducts, etc.). For calibration of noise maps more then 30 measurements were performed following the standard DIN 45642. During all stages of elaborating noise maps, quality assurance procedures were performed with a view to estimate accuracy of the results. Strategic noise maps of the agglomeration performed at municipal level of Ljubljana will be used mainly for drawing up action plans and spatial planning of areas with different noise levels allowed. 


\section{3:40}

2aNSd13. Mixed Industry and Traffic Noise Mapping. Céline Boutin (Acouphen Environnement, Campus de la DOUA, 66, BD Niels Bohr, BP 52132, 69603 Villeurbanne, France, celine.boutin @acouphen-environnement.com), Alexis Bigot (Acouphen Environnement, Campus de la DOUA, 66, BD Niels Bohr, BP 52132, 69603 Villeurbanne, France, alexis.bigot@ acouphen-environnement.com)

The authors have a large experience on Noise Mapping of both transportation sources and industrial plants. This paper provides examples of
Noise Maps for Communities of different sizes with road, railway and industrial sources. The difficulties to estimate Noise Emission from industrial sites are discussed. Recommandations are provided. A Methodology to create the Industry data base for a Noise Map with a large number of industrial sites in the territory is presented. It is shown how Noise Maps can be an attractive tool to be used in the Environmental Management System of the Plant Manager to communicate on the Plant environmental integration and Noise Abatement Plan.

TUESDAY MORNING, 1 JULY 2008

ROOM 252A, 11:00 A.M. TO 3:20 P.M.

\section{Session 2aNSe}

\section{Noise and EURONOISE: General Topics in Noise I}

\section{Contributed Papers}

11:00

2aNSe1. Comparison of International Standards for Measuring Sound Power in Tool-Machines. Jose A. Ballesteros (Universidad de Castilla-La Mancha, Campus Universitario, 16071 Cuenca, Spain, Josea.Ballesteros@uclm.es), Marcos D. Fernandez (Universidad de Castilla-La Mancha, Campus Universitario, 16071 Cuenca, Spain, Marcos.Fernandez@uclm.es), Samuel Quintana (Universidad de Castilla-La Mancha, Campus Universitario, 16071 Cuenca, Spain, Samuel.Quintana@uclm.es), Ivan Suarez (Universidad de Castilla-La Mancha, Campus Universitario, 16071 Cuenca, Spain, ivan.suarezcasal@gmail.com), Laura Rodriguez (Universidad de Castilla-La Mancha, Campus Universitario, 16071 Cuenca, Spain, Laura .Rodriguez@uclm.es)

Industrial noise is one of the most important contaminant agents in the industrialised countries due to its effects on health. Sound power is used in order to characterize the noise emitted by the machines because it does not depend on aspects like place or distance of the measurement. These measurements are handled by international standards like UNE-EN ISO 374x and UNE-EN ISO 9614-x. The advantages and disadvantages of each one of these standards have been evaluated after making a representative number of measurements in several types of tool-machines and then, a set of recommendations have been derived to choose the best standard depending on the machine that is going to be measured.

\section{1:20}

2aNSe2. Activated Resonance Systems as Silencers and Sound Absorbers. Philip Leistner (Fraunhofer IBP, Nobelstrasse 12, 70569 Stuttgart, Germany, philip.leistner@ibp.fraunhofer.de)

A great variety of classical resonance systems is used for sound absorption and attenuation in technical systems. Apart from other requirements such as minimum size, high reliability and low costs the acoustic efficiency can be fulfilled in a wide range. However, the performance is limited and focused on a certain frequency band. These restrictions can significantly be overcome by activation. A very clear system comprises a membrane (mass) and a rear air volume (spring) which can be assembled by using a conventional loudspeaker in front of a cassette. Even the 'passive' system is causing a respectable sound attenuation also at low frequencies. The activation is based on a microphone close to the membrane the signal (according to the exciting sound pressure) of which is linearly amplified and fed back to the loudspeaker. A lot of details have to be considered prior to the real applicability of such an activated resonator. But the remarkable benefits can be documented for numerous practical scenarios. Theoretical and experimental results are based on a sophisticated model of the resonant structure and its acoustic environment. Additionally, several practical challenges, e.g. the presences of turbulent air flow or high temperatures, can be solved by using specific modifications.

\section{1:40}

2aNSe3. Sound radiation of the end of cylindrical duct application on industrial stacks. Thierry Simoneau (Acoustique \& Conseil, 17-19 rue des Grandes Terres, 92508 Rueil-Malmaison, France, ts@acoustique-conseil .com)

In order to determine the sound field emitted by the top end of an industrial stack, a study of the acoustical radiation of unbaffled semi-infinite cylindrical duct ends was conducted. Firstly, the main publications on the subject were studied. A calculation model of the radiation directivity of the end of a chimney, based on H. Levine and J. Schwinger's results for the fundamental mode and on the Geometrical Theory of Diffraction for the modes of superior order, was established. In order to validate the model, measurements on scaled model were made in an anechoic chamber. Tallying between theoretical and experimental results is very good, especially at low frequencies, where the number of radiating modes is low. Moreover, measurements on a real size industrial site have been made. They also show satisfying tallying between the theory and experimentation.

\section{2:00}

2aNSe4. Austrian investigation on the influence of sound leakage in noise reducing devices. Marco Conter (Arsenal Research, Giefinggasse 2, 1210 Vienna, Austria, marco.conter@arsenal.ac.at), Manfred Haider (Arsenal Research, Giefinggasse 2, 1210 Vienna, Austria, manfred .haider@arsenal.ac.at)

Noise barriers are the most widely used means for road traffic noise abatement. Sound absorption and sound insulation are the key properties for noise barrier elements. The standard method of determining these properties by reverberation room measurements has recently been complemented by an in-situ method following CEN/TS 1793-5, also known as Adrienne method. This method allows flexible assessment of the acoustic performance of noise barriers in almost arbitrary places by means of mobile measurement equipment. Using the possibility to perform in-situ measurements the authors have investigated the presence of sound leakage due to structural imperfections which limit the sound insulation performance of noise barriers. This paper summarizes the results of Austrian research on different kinds of barriers regarding the difference in sound insulation between measurements carried out in front of the supporting post (where leakage is likely to occur) and in the middle of the barrier. A statistical correlation of the data was also performed. 


\section{$12: 20$}

2aNSe5. A study of twenty-one cases of low-frequency noise complaints. Christian Sejer Pedersen (Acoustics, Aalborg University, Fredrik Bajers Vej 7 B5, 9220 Aalborg Ø, Denmark, cp@acoustics .aau.dk), Henrik Møller (Acoustics, Aalborg University, Fredrik Bajers Vej 7 B5, 9220 Aalborg Ø, Denmark, hm@acoustics.aau.dk), Kerstin Persson Waye (Dept. of Environ. Medicine, The Sahlgrenska Acad. of Gothenburg Univ., Box 414, 40530 Gothenburg, Sweden, kerstin.persson-waye@amm .gu.se)

From 203 cases of low-frequency complaints a random selection of twenty-one previously unsolved cases were investigated. The main aim of the investigation was to answer the question whether the annoyance is caused by an external physical sound or by a physically non-existing sound, i.e. low-frequency tinnitus. Noise recordings were made in the homes of the complainants, and the complainants were exposed to these in blind test listening experiments. Furthermore, the low-frequency hearing function of the complainants was investigated, and characteristics of the annoying sound was matched. The results showed that some of the complainants are annoyed by a physical sound $(20-180 \mathrm{~Hz})$, while others suffer from low-frequency tinnitus (perceived frequency 40-100 Hz). Physical sound at frequencies below $20 \mathrm{~Hz}$ (infrasound) is not responsible for the annoyance - or at all audible - in any of the investigated cases, and none of the complainants has extraordinary hearing sensitivity at low frequencies. For comparable cases of low-frequency noise complaints in general, it is anticipated that physical sound is responsible in a substantial part of the cases, while low-frequency tinnitus is responsible in another substantial part of the cases.

\section{2:40-2:00 Lunch Break}

\section{Contributed Papers}

\section{2:00}

2aNSe6. Measuring low-frequency noise indoors. Steffen Pedersen (Acoustics, Aalborg University, Fredrik Bajers Vej 7 B5, 9220 Aalborg Ø, Denmark, stp@acoustics.aau.dk), Henrik Møller (Acoustics, Aalborg University, Fredrik Bajers Vej 7 B5, 9220 Aalborg Ø, Denmark, hm@acoustics.aau.dk), Kerstin Persson Waye (Dept. of Environ. Medicine, The Sahlgrenska Acad. of Gothenburg Univ., Box 414, 40530 Gothenburg, Sweden, kerstin.persson-waye@ amm.gu.se)

At low frequencies, the sound pressure level may vary $20-30 \mathrm{~dB}$ in a room due to standing waves. For assessment of annoyance, mainly areas with the highest occurring levels are relevant, since persons present in such areas are not helped by the existence of lower levels in other areas. The level that is exceeded in $10 \%$ of the volume of a room $L_{10}$ is proposed as a rational and objective target for a measurement method. In Sweden and Denmark rules exist for measuring low-frequency noise indoors. The performance of these procedures was investigated in three rooms. The results from the Swedish method were close to the $L_{10}$ target, but, due to a doubtful use of $\mathrm{C}$-weighting in the scanning, it may give too low results in case of complex sounds. The Danish method was found to have a high risk of giving results substantially below the target, unless complainants can precisely appoint measurement positions, where the sound is loudest/most annoying which they often cannot. An alternative method using measurements in four three-dimensional corners of the room is proposed. This easy and straightforward method seems to give reliable results close to the proposed target.

\section{2:20}

2aNSe7. The use of communication device in background noise. Esko Toppila (Finnish Institute of Occupational Health, P.O:Box 486, 33101 Tampere, Finland, esko.toppila@ttl.fi), Pekka Airre (FIOH, P.O.Box 486, 33101 Tampere, Finland, erkko.airo@ttl.fi), Pekka Olkinuora (FIOH, Topeliuksenkatu 41, 00250 Helsinki, Finland, pekka.olkinuora@ttl.fi)

Communication devices are used more and more often in industrial premises. We have evaluated how the users experience these device in a call center $(\mathrm{N}=41)$, in a low noise warehouse $(\mathrm{N}=67)$ and in a noisy $(\mathrm{L}>80 \mathrm{~dB})$ warehouse $(\mathrm{N}=25)$. For all subjects the same questionnaire was sent. The questionnaire contained questions were about the quality of communications device and self-evaluated hearing symptoms. The self-evaluated speech intelligibility was lowest in the high background environments. Still in the call center five persons and five in the silent warehouse had great difficulties with speech intelligibility. All these persons evaluated that they have always difficulties when communicating with people. In the noisy environments this relationship was not found. The need to rise the voice was highest in the warehouses ad did not depend on the background noise. Tinnitus was more frequent among process industry workers. The results suggests that lowered hearing function cause problems in low background noise. The selfevaluated nuisance depends on the complexity of the communication. The quality of the communication channel may also play a vital role in the communication problems especially for workers with hearing problems.

\section{2:40}

2aNSe8. Phase spectral processing for improved time-domain soft microphone based noise estimation. Ioannis Paraskevas (T.E.I. Piraeus / Department of Electronics, 250, Thivon str., GR-12244 Athens-Aigaleo, Greece, paraskevas@env.aegean.gr), Maria Rangoussi (T.E.I. Piraeus / Department of Electronics, 250, Thivon str., GR-12244 Athens-Aigaleo, Greece, mariar@teipir.gr), Stylianos M. Potirakis (T.E.I. Piraeus / Department of Electronics, 250, Thivon str., GR-12244 Athens-Aigaleo, Greece, spoti@teipir.gr), Stylianos Savvaidis (T.E.I. Piraeus / Department of Electronics, 250, Thivon str., GR-12244 Athens-Aigaleo, Greece, ssavaid@teipir $. \mathrm{gr})$

'Soft Microphones' (SM) constitute a cost-effective, yet quality alternative to the multiple microphones measurement, in applications related to noise mapping. SM offer a solution of great interest to real field applications, e.g., industrial plants. The SM approach, proposed in previous work of the authors, is based on the estimation of the noise signal and the calculation of noise levels over a set of points within the space of interest. To this end, a novel, frequency domain method was introduced and verified in a real field, textile plant experiment, with satisfactory results. However, in order to expand the use of SM from accurate noise mapping to a full Active Noise Control application, it is necessary to obtain accurate noise signal estimates in the time rather than the frequency domain. Further research into the deconvolution step of the proposed method reveals that discontinuities, appearing across the phase spectrum of the estimated signals, cause ambiguities that affect the deconvolution process. We propose here the use of the Hartley transform phase spectrum, which conveys fewer discontinuities as compared to its Fourier Transform counterpart, while it allows for a discontinuities compensation scheme. Experimental results verify that phase spectrum preprocessing provides accurate time domain signal estimates.

\section{3:00}

2aNSe9. Research into the improvement of the management of helicopter noise in the UK. David C. Waddington (Acoustics Research Centre, School of Computing, Science \& Engineering, University of Salford, M5 4WT Salford, UK, d.c.waddington@salford.ac.uk), Paul Kendrick (Acoustics Research Centre, School of Computing, Science \& Engineering, University of Salford, M5 4WT Salford, UK, p.kendrick@salford.ac.uk), Geoff Kerry (Acoustics Research Centre, School of Computing, Science \& Engineering, University of Salford, M5 4WT Salford, UK, g.kerry@salford.ac.uk), Matthew Muirhead (QinetiQ Ltd, Cody Technology Park, Ively Road, GU14 OLX Farnborough, UK, mmuirhead@qinetiq.com), Ray Browne (QinetiQ Ltd, Cody Technology Park, Ively Road, GU14 0LX Farnborough, UK, rwbrowne@ qinetiq.com)

Helicopter noise has a negative impact on the quality of life for many people. Effected populations are not just those living close to heliports, but include those exposed to noise from helicopters used by emergency services, the military, and commercial companies. One problem identified in the UK is that it is often difficult to complain about helicopter noise, since it is un- 
clear which organisation is responsible for dealing with the complaint. Consequently, the Department for Environment, Food and Rural Affairs (Defra, UK) has commissioned research to summarise the following: i. the nature and extent of the concern about helicopter noise in the UK. ii. rules and regulations governing operations. iii. existing procedures for handling complaints. This stage of the project will produce a detailed report into the improvement of the management of helicopter noise. Also to be produced is a short non-technical guide including the means of redress for perceived disturbance. This paper will report on the findings of this project. [Work funded by Defra, UK]

TUESDAY MORNING, 1 JULY 2008

AMPHI HAVANE, 8:00 A.M. TO 12:40 P.M.

\title{
Session 2aPAa
}

\section{Physical Acoustics: Acoustics of Porous Media II}

\author{
Walter Lauriks, Cochair \\ Lab. ATF, Katholieke Universiteit Leuven, Celestijnenlaan 200D, Leuven, B-3001, Belgium
}

Keith Attenborough, Cochair

Open University, Department of Design, Development, Materials and Environment, Walton Hall, Milton Keynes, MK7 6AA, UK

\section{Contributed Papers}

\section{8:00}

2aPAa1. Sensitivity analysis and non linear parameters estimation of porous materials from normal sound coefficient absorption measurements. Mohammed Garoum (Ecole Supérieure de Technologie de Salé, Laboratoire Energétique Matériaux et Environnement, Avenue du Prince Héritier, BP 227, 10000 Salé, Morocco, garoum1@yahoo.fr), Rachida Idchabani (Ecole Supérieure de Technologie de Salé, Avenue du Prince Héritier, BP 227, Salé, Morocco, idchabani@mcinet.gov.ma), Mohammed Tajayouti (Ecole Supérieure de Technologie de Salé, Avenue du Prince Héritier, BP 227, Salé, Morocco, mtajayouti@gmail.com), Mohammed Rhachi (Ecole Supérieure de Technologie de Salé, Laboratoire Energétique Matériaux et Environnement, Avenue du Prince Héritier, BP 227, 10000 Salé, Morocco, mrhachi@hotmail.com), Antonio Moreno (Instituto de Acústica, CSIC, 144 Callé Serrano, 28006 Madrid, Spain, amoreno@ia.cetef.csic.es)

In literature, various models have been proposed in order to predict characteristic impedance $\mathrm{Zc}$ and wave number kc of porous materials. To use these models their involved nonacoustical parameters (i. e. flow resistivity, tortuosity porosityœ) are usually measured. Unfortunately measurements require specialised equipments and are often difficult on loose porous materials leading most often to erroneous values. Moreover, the inverse estimation of theses parameters from experimental data is not an easy task as believed. This is due to the non linearity of models and the presence of some parameters with weak and/or linearly dependent influences. In this work an approach based on the tradeoff between magnitude and linear independence of the sensitivity matrix components is presented in order to achieve an efficient ranking of parameters according to their inherent ease of estimation. Next this approach is associated to Genetic Algorithms to minimize the least squares norm between measured and modelled normal sound absorption coefficient. For five widely used models in literatures, the application of the proposed inverse estimation procedure on loose granular cork and vegetable fibres, shows that, unlike classical gradient approaches methods, better estimation has been obtained.

\section{8:20}

2aPAa2. Characterization of an acoustic ceramic liner in a subsonic flow by mean of Laser Doppler Velocimetry. Romain Pethieu (ONERA, 2, av. Edouard Belin - BP 4025 - Cedex 4, 31055 Toulouse, France, romain .pethieu@onecert.fr)

As aircraft traffic constantly increases, serious efforts are made to reduce engine noise. Among them, the design of high performance absorbing materials (liners) placed on the nacelle's internal walls is an exciting challenge. Optimizing these media requires to know the mechanisms of acoustical propagation near them in the conditions encountered in jet engines: high speed hot shear flows. Particular interest is being granted to the influence of the grazing flow in contact with the liners. This is due to its known effects on the liners characteristics: efficiency of absorption and frequency resonance. This influence is remarkable for those configurations based on perforated plates. Therefore, a testing method based on non-intrusive measurements has been developed at ONERA. This technique consists in measuring acoustic velocities by laser Doppler velocimetry to determine acoustic quantities under grazing turbulent high-speed flow, thanks to an EulerianLagrangian description of the perturbations. Experimental activity is performed on a honeycomb material, via the achievement of acoustical pressure and intensity fields, for different Mach numbers. Moreover, these measurements bring into evidence a coupling between acoustical modes and hydrodynamic modes (from Kelvin-Helmholtz instability) which modify the material acoustical behaviour.

\section{8:40}

2aPAa3. The effect of mechanical elasticity on the surface impedance of a organic/inorganic composite aerogel. Winny Dong (California State Polytechnic University, 3801 West Temple Avenue, Chemical and Materials Engineering, Pomona, CA 91768, USA, winnydong@csupomona.edu), Wayland Dong (Veneklasen Associates, 1711 Sixteenth Street, Santa Monica, CA 90404, USA, wdong@veneklasen.com), Tanya Faltens (California State Polytechnic University, 3801 West Temple Avenue, Chemical and Materials Engineering, Pomona, CA 91768, USA, tafaltens@csupomona.edu), Elizabeth Scott (California State Polytechnic University, 3801 West Temple Avenue, Chemical and Materials Engineering, Pomona, CA 91768, USA, escott@csupomona.edu), Travis Thompson (California State Polytechnic University, 3801 West Temple Avenue, Chemical and Materials Engineering, Pomona, CA 91768, USA, trthompson@csupomona.edu)

Previous reports on the acoustical properties of monolithic silica aerogels have indicated that although the material's high porosity, pore tortuosity, and surface area contribute to a low sound velocity in the material, the rigid matrix of the aerogel exhibit a high reflection coefficient. Work by Forest et al. and others have shown that using granules instead of silica aerogel monoliths, the acoustical properties can be significantly enhanced. We report the acoustic properties of a polydimethylsilicate (PDMS)/silica composite aerogel that has both high surface area and a mechanically elastic matrix. Both monoliths and granules are studied. The surface impedance (at normal incidence) as a function of open porosity, pore size distribution, surface area, elasticity, and granule size will be reported. 


\section{9:00}

2aPAa4. Numerical modeling of transient poroelastic waves in the low frequency range. Guillaume Chiavassa (Ecole Centrale Marseille MSNM-GP, Technopole de Chateau-Gombert, 13013 Marseille, France, guillaume.chiavassa@ec-marseille.fr), Bruno Lombard (Laboratoire de Mécanique et d'Acoustique LMA, 31 chemin Joseph Aiguier, 13402 Marseille, France, lombard@1ma.cnrs-mrs.fr), Joel Piraux (Laboratoire de Mécanique et d'Acoustique LMA, 31 chemin Joseph Aiguier, 13402 Marseille, France, piraux@1ma.cnrs-mrs.fr)

A numerical method is proposed to simulate the propagation of transient poroelastic waves across heterogeneous media, in the low frequency range. A velocity-stress formulation of Biot's equations is followed, leading to a first-order differential system. The latter is splitted in two parts: a propagative one discretized by a fourth-order ADER scheme, and a diffusive one solved analytically. Near sources and interfaces, a space-time mesh refinement is implemented to capture the small scales of evolution of the diffusive slow compressional wave. Lastly, an immersed interface method is implemented to accurately model the jump conditions at interfaces between the different media. Numerical experiments in one and two dimensions are shown, with porous/porous or fluid/porous interfaces. Comparisons with analytical solutions confirm the efficiency of the approach. [1] G. Chiavassa, B. Lombard, J. Piraux, Numerical modeling of 1-D transient poroelastic waves in the low-frequency range, soumis au J. Comput. Appl. Math., (2007), disponible sur http://hal.archives-ouvertes.fr/hal-00193103/fr/

\section{9:20}

2aPAa5. On the scattering of a plane wave by porous sound-absorbing strip. Alex De Bruijn (Independent Acoustical Consultant, Schonenvaardersstraat 18014, 7418 CC Deventer, Netherlands, alex.bruijn@planet.nl)

The analysis concerning the scattering of a plane wave by a porous sound-absorbing strip embedded in an infinite sound-hard surface is most relevant for the evaluation of measurements with respect to absorbing materials tested in a reverberation room. This diffraction phenomenon around the edges leads to an additional sound absorption, the so-called edge effect. This extra absorption can be analyzed and computed - among other wellknown methods - by using a spatial Fourier field expansion just on the absorbing strip. The field in the space above the reflecting surface can be put into a contour integral of an angular spectrum in the complex plane or -in other words- a superposition of plane waves with complex angles of incidence. Matching both field representations leads to the solution of the unknown coefficients of the Fourier expansion and hence to the amplitudes of the total diffracted field. It would be interesting to compare these results with diffraction data obtained via an alternative technique: the FiniteDifference Time-Domain method using the direct Euler equations, also within the porous sound-absorbing material. The paper will show the data for the field amplitudes obtained by both methods and will discuss the agreement and differences.

\section{9:40}

2aPAa6. Analysis of porous plate/water layered structures by means of the transition terms method. Ferroudja Belhocine (LOMC FRE CNRS 3102, Université du Havre, Place Robert Schuman, 76610 le Havre, France, ferroudja.belhocine@univ-lehavre.fr), Serge Derible (LOMC FRE CNRS 3102, Université du Havre, Place Robert Schuman, 76610 le Havre, France, serge.derible@univ-lehavre.fr), Cole Franklin (1241 East dyer road, Santa Ana, CA 92672, USA, colesfranklin@yahoo.com)

This paper is devoted to the study of water-saturated porous plate/water layered structures by means of the transition terms defined from the reflection and transmission coefficients $\mathrm{R}$ and $\mathrm{T}$ as follows: $\mathrm{TT}_{\text {sym }}=(1-(\mathrm{R}+\mathrm{T})) /(2 \mathrm{i}), \mathrm{TT}_{\text {asym }}=(1-(\mathrm{R}-\mathrm{T})) /(2 \mathrm{i})$. They are the eigenvalues of the scattering matrix of the water-immersed structure and are directly connected with its symmetric or antisymmetric vibrations. The $\mathrm{N}$ porous plates associated in our structures obey Biot's theory which is first used to calculate the reflection and transmission coefficients of a unique water-saturated plate. An induction on $\mathrm{N}$ process allows to find the reflection and transmission coefficients of a given $\mathrm{N}$ plate/water-layer structure. The plates used in the experiments at normal incidence are $5 \mathrm{~mm}$ thick. The reflection and transmission coefficients of sets of 1,2,3, and 4 water immersed plates, separated from each other by a $1 \mathrm{~cm}$ water gap, are measured thanks to two wideband transducers with central frequency $0.5 \mathrm{MHz}$. There are good agreements between the calculated and experimental transition terms which exhibit the symmetric and antisymmetric resonances of the structures. They obey the Breit-Wigner resonant form which characteristics can be obtained

\section{0:00}

2aPAa7. On the adsorption-desorption relaxation time of carbon in very narrow ducts. Timothy J. Mellow (Nokia, Nokia House, Summit Avenue, GU14 0NG Farnborough, UK, tim.mellow@nokia.com), Olga Umnova (University of Salford, Acoustics Research Centre, Newton Building, M5 4WT Salford, UK, o.umnova@salford.ac.uk), Konstantinos Drossos (University of Southampton: Institute of Sound and Vibration Research, University Road, Highfield, SO17 1BJ Southampton, UK, kd806@soton.ac.uk), Keith Holland (University of Southampton: Institute of Sound and Vibration Research, University Road, Highfield, SO17 1BJ Southampton, UK, krh@isvr.soton.ac.uk), Andrew Flewitt (University of Cambridge: Centre for Advanced Photonics and Electronics, 9, JJ Thomson Ave., CB3 0FA Cambridge, UK, ajf@eng.cam.ac.uk), Leo Kärkkäinen (Nokia Research Center, Itämerenkatu 11 - 13, 00180 Helsinki, Finland, leo.m.karkkainen@nokia.com)

Loudspeakers generally have boxes to prevent rear wave cancellation at low frequencies. However, the stiffness of the air in a small box reduces the diaphragm's excursion at low frequencies. Hence the size of the box is generally a compromise between low frequency performance and practicality. Activated carbon has been found to increase the apparent size of a given box through adsorption of the air molecules when the pressure increases and likewise desorption when it decreases. However, the exact viscous effects in the granular structure are difficult to model. Thus it is impossible determine the high frequency limit due to the natural adsorption/desorption relaxation time in the absence of viscous losses. In this study, a tube model is presented which takes into viscous and thermal losses with boundary slip together with adsorption. Impedance measurements are performed on an array of 12 million holes, each 2 micrometers in diameter, etched in a $0.5 \mathrm{~mm}$ thick silicon wafer so that the viscous and thermal losses can be verified against the model without adsorption. Impedance measurements are then performed on an array of holes coated with graphite in order to create an activated carbonlike structure, thus enabling the adsorption/desorption relaxation time to be evaluated.

\section{0:20}

2aPAa8. Acoustic pulse attenuation and transmission in rigid porous media: Experimental investigation and numerical simulations. Diego Turo (University of Salford, Acoustics Research Centre, Newton Building, M5 4WT Salford, UK, d.turo@pgr.salford.ac.uk), Olga Umnova (University of Salford, Acoustics Research Centre, Newton Building, M5 4WT Salford, UK, o.umnova@salford.ac.uk)

This paper presents an investigation on acoustic pulse attenuation and transmission in rigid porous media. Transmission measurements of acoustic pulses through porous material with a rigid frame were performed using an appropriate standing wave tube. A vertical tube was built to make measurements on granular materials. The influence of pulse duration, layer thickness and material microstructure on the transmission has been investigated. Numerical time domain simulations based on two different semi-empirical models with one and two viscous relaxation times, respectively, were performed to compare with existing data. First, material microstructure and pulse durations were adapted to investigate the influence of viscous and inertial effects on pulse propagation separately. The simultaneous contribution of both viscous and thermal effects was then investigated. In the finite difference time domain (FDTD) model, different approaches are used to correctly simulate the pulse transmission through a porous layer. Accuracy and computational time required for the numerical methodologies have been compared and their advantages and drawbacks applied to this particular case shown. 


\section{0:40-11:00 Break}

\section{1:00}

2aPAa9. On the influence of the micro-geometry on sound propagation through periodic array of cylinders. Rodolfo Venegas (University of Salford, Acoustics Research Centre, Newton Building, M5 4WT Salford, UK, R.G.VenegasCastillo@pgr.salford.ac.uk), Olga Umnova (University of Salford, Acoustics Research Centre, Newton Building, M5 4WT Salford, UK, o.umnova@salford.ac.uk)

Sound propagation in rigid porous media has been widely studied by using macroscopical models. These models make use of averaged quantities in which the microscopic details of the porous media geometry are represented by macroscopical parameters and, in a certain way, the influence of the microscopic geometry is not directly identified. In this paper, homogenization theory and finite element method are used for solving the full microscopic dynamic flow and dynamic heat problems for a porous medium modelled as an idealized geometry consisting of a periodic array of cylinders. Different cross-section shapes of the cylinders (circular, ellipsoidal and square crosssection shapes) and a wide range of porosity values are considered. The influence of the microscopic features of the porous media on dynamic permeability and dynamic compressibility is also studied.

\section{1:20}

2aPAa10. Acoustic emission before avalanches in granular media Vincent Gibiat (Université Paul Sabatier, PHASE, 118, route de Narbonne, 31062 Toulouse cedex 9, France, gibiat@cict.fr), Eric Plazza (Université Paul Sabatier, PHASE, 118, route de Narbonne, 31062 Toulouse cedex 9, France, ericvpp@hotmail.com), Pierre De Guibert (Université Paul Sabatier, PHASE, 118, route de Narbonne, 31062 Toulouse cedex 9, France, dguibert@cict.fr)

Avalanches of granular media are mainly characterized by the observation and the measurement of the main angles of avalanche corresponding first to the movement of isolated beads and to the whole movement of a great part of the grains. These characterisations do not give any information about the rearrangements of the grains inside the layer of granular beads. As any movement of a grain produces a deformation of the structure it is quite normal to expect for a sound that will propagate inside the granular medium. We present an experimental study of the precursors of avalanches on spherical granular glass beads and silica aerogels in powder (size of grains less than 80 micrometers). Acoustic emission has been recorded with two piezoelectric transducers placed on the lower part of the material layer. Our results show clearly that before any movement on the upper part of the beads layer, so for an angle less than the first angle of avalanche, movements inside the material produce pulsed sounds that can be recorded. Theses vibrating events are occurring more and more when the angle is increasing until the first angle of avalanche where acoustic emission becomes intense.

\section{1:40}

2aPAa11. On heterogeneous blankets: Analytical solution for the interaction between masses and poro-elastic layers. Kamal Idrisi (Virginia Tech, Mechanical Engineering, 143 Durham 0238, Blacksburg, VA 24061, USA, idrisi@vt.edu), Andreas Wagner (Virginia Tech, Mechanical Engineering, 143 Durham 0238, Blacksburg, VA 24061, USA, andreasw@vt.edu), Marty Johnson (Virginia Tech, Mechanical Engineering, 143 Durham 0238, Blacksburg, VA 24061, USA, martyj@vt.edu), David Bartylla (Virginia Tech, Mechanical Engineering, 143 Durham 0238, Blacksburg, VA 24061, USA, bartylla@vt.edu)

There has been substantial research over the last five decades on control of aircraft cabin noise. One new passive approach is the heterogeneous (HG) blanket where a traditional acoustic blanket treatment is altered by adding mass inhomogeneities into the poro-elastic/viscoelastic layers. These masses act like distributed vibration absorbers and can be used to reduce vibration and sound transmission by targeting modes of the fuselage. The natural frequency of a mass inhomogeneity is determined by the mass itself and by the effective stiffness of the porous layer due to the mass/poro interaction. An experimental 1st order approach to predict the effective stiffness based on the shape of the mass inhomogeneities is reviewed. An analytical model for poro-elastic media proposed by Allard et al. was simplified for low frequencies and is used to validate and extend the 1st order approach. It is shown that the effective stiffness depends on mass shape, the foam thickness and material constants such as the modulus of elasticity and Poisson's ratio. Furthermore the model can be used to calculate the stress and displacements fields in the blanket in order to give further insight into the behaviour of the HG blankets.

\section{2:00}

2aPAa12. Influence of boundary slip on the acoustical properties of microfibrous absorbents. Olga Umnova (University of Salford, Acoustics Research Centre, Newton Building, M5 4WT Salford, UK, o.umnova@salford.ac.uk), David Tsiklauri (University of Salford, Acoustics Research Centre, Newton Building, M5 4WT Salford, UK, d.tsiklauri@salford.ac.uk), Rodolfo Venegas (University of Salford, Acoustics Research Centre, Newton Building, M5 4WT Salford, UK, R.G .VenegasCastillo@pgr.salford.ac.uk)

In the past decades a variety of new highly porous materials with unusually small pores have been manufactured. In aerogels, for instance, pores can be less than $20 \mathrm{~nm}$ in diameter. The conventional models have to be modified when applied to describe acoustical properties of those materials. The non-slip condition on a pore surface is no longer valid and needs to be replaced by the Knudsen boundary condition. In attempt to provide an insight into the behaviour of microfibrous materials, an analytical model has been developed, which accounts for the boundary slip in a medium consisting of rigid parallel fibres assuming different directions of sound propagation with respect to fibres. It has been shown that the presence of the boundary slip leads to a significant change in model predictions. For instance, in a material with fibre radius $80 \mathrm{~nm}$ and $95 \%$ porosity the sound speed decreases and attenuation increases by more than $20 \%$ compared to the values obtained assuming no boundary slip. The effect is stronger for smaller size fibres, lower porosity values and for sound propagating parallel to fibres. Numerical computations have been performed to simulate oscillatory flow around the cylindrical fibres assuming Knudsen boundary conditions and the results have been compared with the analytical model predictions

\section{2:20}

2aPAa13. Time-Resolved Spectroscopy of Water confined in Vycor. Riccardo Cucini (European Lab. for Non-Linear Spectroscopy (LENS), Univ. di Firenze, via Nello Carrara 1, 50019 Sesto Fiorentino (Fi), Italy, cucini@lens.unifi.it), Andrea Taschin (European Lab. for Non-Linear Spectroscopy (LENS), Univ. di Firenze, via Nello Carrara 1, 50019 Sesto Fiorentino (Fi), Italy, taschin@lens.unifi.it), Paolo Bartolini (European Lab. for Non-Linear Spectroscopy (LENS), Univ. di Firenze, via Nello Carrara 1, 50019 Sesto Fiorentino (Fi), Italy, bart@lens.unifi.it), Renato Torre (European Lab. for Non-Linear Spectroscopy (LENS), Univ. di Firenze, via Nello Carrara 1, 50019 Sesto Fiorentino (Fi), Italy, torre@lens .unifi.it)

We present measurements on water confined in Vycor 7930 by heterodyne-detected transient grating experiments (HD-TG) [1]. We studied the acoustic wave propagation, the thermal diffusion and the liquid viscous flow through the pores as a function of temperature $\left(-15-90{ }^{\circ} \mathrm{C}\right)$ and of the exchanged q-vector $\left(0.63-2.5 \mu \mathrm{m}^{-1}\right)$. The data show interesting aspects due partly to the confinement effects and partly to the peculiarities of bulk water. Acoustic results are compared with the predictions of the Biot theory finding a good agreement only for the sound velocity data, while the predictions for the acoustic wave attenuation is poor as just reported in [2]. Our technique is able to induce a thermal grating inside the sample. The analysis of the data requires an extension of the Biot theory, that takes into account the temperature variations. Surprisingly, our data show that the acoustic oscillation are not affected by the temperature. The induced thermal grating is also used to analyze the viscous flow of water inside the pores and the thermal diffusion. [1] Time-resolved spectroscopy of complex liquids, edited by Torre R. (Springer, New York) 2008 [2] A. Taschin, R. Cucini, P. Bartolini, R. Torre, Europhys. Lett., in press. 


\title{
Session 2aPAb
}

\section{Physical Acoustics: Diffraction of Waves on Periodical Structures: Acoustic, Ultrasonic, and Acousto-Optical Diffraction Phenomena I}

\author{
Nico F. Declercq, Cochair \\ Georgia Tech Lorraine - G.W. Woodruff School of ME, UMI Georgia Tech - CNRS 2958, 2 rue Marconi, Metz, 57070, France
}

Nataliya Polikarpova, Cochair

M.V. Lomonosov Moscow State University, Department of Physics, Moscow, 119991, Russian Federation

\section{Invited Papers}

8:00

\begin{abstract}
2aPAb1. Spectral instruments based on acousto-optical tunable filters: advantages and prospects. Vladislav Pustovoit (Scientific Technological Center of Unique Instrumentation of RAS, ul.Butlerova, 15, 117342 Moscow, Russian Federation, np@ckbup.dol.ru), Vitold Pozhar (Scientific Technological Center of Unique Instrumentation of RAS, ul.Butlerova, 15, 117342 Moscow, Russian Federation, aoslab@ckbup.dol.ru)
\end{abstract}

A review of modern spectral techniques and instruments using acousto-optical tunable filters (AOTF) is presented. New approaches for spectrometers and spectral systems development are considered. The following instruments are described: a series of compact spectrometers and spectrophotometers of ultra-violet, visible, and infra-red ranges for Raman, fluorescence, and absorption spectroscopy for various applications, including out-of-door measurements; spectroradiometers for environment monitoring (open air, Earth surface, subsurface and depth seawater), specialised spectral equipment for industrial monitoring (microelectronics, alcoholometry), imaging spectral devices, time-resolve fluorescent spectrometers, and instruments for differential spectroscopy. Also some fundamental problems of AOTF-based spectroscopy are considered: spectrogram correction of instrument function distortions, ultimate spectral resolution of different methods, modulation techniques for instrument function synthesizing, optimization of measurement procedures, including adaptive algorithms. Basic trends of the past 30 years of AOTF-based spectroscopy development are formulated. The most promising ideas are discussed.

8:20

2aPAb2. Advances in acousto-optic devices based on frequency intermodulations suppression. Valeriy V. Proklov (IRE RAS, 11 Mokhovaya str., build.7, 125009 Moscow, Russian Federation, proklov@mail.cplire.ru), Sergey Antonov (IRE RAS, 11 Mokhovaya str., build.7, 125009 Moscow, Russian Federation, olga-ant@yandex.ru), Yury Rezvov (IRE RAS, 11 Mokhovaya str., build.7, 125009 Moscow, Russian Federation, rezvov@newmsk.tula.net), Alexander Vainer (IRE RAS, 11 Mokhovaya str., build.7, 125009 Moscow, Russian Federation, sawashi@yandex.ru)

The limitative influence of the frequency intermodulation effect on the most important parameters in a big family of acousto-optic (AO) devices is well known. The proposed presentation deals with new principles and related technical tools to perform significant suppression of the inherent intermodulation effects within broadband AO devices. The different AO interaction mechanisms in solids offering the intermodulations decrease have been considered. On this basis there were theoretically established and experimentally verified facilities to design a few types of advanced AO devices for a wideband signal processing with the extended spurious free dynamic range, as well as for a flexible laser beam forming with extremely high efficiency and etc.

\section{Contributed Paper}

\section{8:40}

2aPAb3. Periodically non-homogeneous acoustic beams and their application in acousto-optics. Vladimir I. Balakshy (Dept. of Physics, M.V.Lomonosov Moscow State Univ., Vorobyevy Gory, Bldg. 1, 119991 Moscow, Russian Federation, balakshy@phys.msu.ru), Bogumil B. Linde (Inst. of Exper. Physics, Univ. of Gdańsk, Ul. Wita Stwosza, Bldg. 57, 80-952 Gdańsk, Poland, fizbl@univ.gda.pl)

Light diffraction by ultrasonic waves is of great interest in the view of both fundamental physics and diverse applications. Acousto-optic devices are used for modulation of optical wave parameters, for optical information processing in real time, etc. In the given research, basic attention has been focused on the analysis of characteristics of anisotropic acousto-optic cells in which acoustic waves are excited by means of a phased array of transducers. Current technology makes it possible to create easily such transducers with antiphase excitation of adjacent elements of the array. The directional diagram of this composite transducer consists of several lobes whose spatial orientation varies with frequency. This peculiarity results in changing the Bragg condition. In the case of the anisotropic acousto-optic diffraction, every branch of the Bragg angle frequency dependence is split into several curves. This opens up new possibilities for optimization of acousto-optic devices. In this work, we have analyzed amplitude, frequency and angular characteristics of the phased transducer cells depending on crystal cut and transducer parameters. In particular, it has been shown that, in spite of a noticeable phase mismatch between interacting waves, the diffraction efficiency can approach $100 \%$. 


\section{Invited Paper}

\section{9:00}

2aPAb4. Differential properties of acousto-optical tunable filters in phase-modulation mode. Vitold Pozhar (Scientific Technological Center of Unique Instrumentation of RAS, ul.Butlerova, 15, 117342 Moscow, Russian Federation, aoslab@ckbup.dol.ru), Vladislav Pustovoit (Scientific Technological Center of Unique Instrumentation of RAS, ul.Butlerova, 15, 117342 Moscow, Russian Federation, np@ckbup.dol.ru), Sergey Beryoza (Scientific Technological Center of Unique Instrumentation of RAS, ul.Butlerova, 15, 117342 Moscow, Russian Federation, bruce_lee_22@mail.ru)

Tailoring the transmission function of acousto-optical tunable filters (AOTF) via acoustic waves control is one of the most challenging tasks, which is promising for optical spectroscopy applications. In our early work it was theoretically predicted and experimentally demonstrated that collinear AOTF with fast periodic phase manipulation exhibits differential properties, in particular capability of detection spectrum derivatives. In the report, the form of the instrument function of such AOTF was measured with use of Ne-lamp linear spectrum. Comparison to theoretically calculated function shows rather good agreement. Also the form of window of equivalent differential filter was determined and proved to be quite similar to AOTF classical window. Advantages of using this differential detection technique are considered. Its applicability to fluorescence spectroscopy is discussed.

\section{Contributed Papers}

\section{9:20}

2aPAb5. Quasicollinear acoustooptic tunable filters based on KDP single crystal. Vladimir Molchanov (Technological State Univ., Moscow Steel and Alloys Institute, Leninsky prospect, 4, 119049 Moscow, Russian Federation, v_molchanov@smtp.ru), Sergei Chizhikov (Technological State Univ., Moscow Steel and Alloys Institute, Leninsky prospect, 4, 119049 Moscow, Russian Federation, aocenter@mail.ru), Oleg Makarov (Molecular Technology GmbH, Rudower Chaussee 29-31, 12489 Berlin, Germany, moltech@mt-berlin.com)

The paper is devoted to the theoretical and experimental investigation of acoustooptical tunable filters, based on quasicollinear geometry of lightsound interaction in KDP single crystal. This geometry uses the effect of acoustic anisotropy in KDP, as well as peculiarities of acoustic wave reflections from the free boundary of KDP crystal. The mathematical approach for determination of optical and consructional characteristics of the filters is elaborated. Different types of quasicollinear filters are considered. The filters are intended for UV-VIS regions of optical radiation. The experimental acoustooptical filter was designed and investigated. Typical tunable spectral band is $220-500 \mathrm{~nm}$, the spectral resolution doesn't exceed $0.1 \mathrm{~nm}$ in UV region of spectra. The experimental data are in a good agreement with the predicted one. This research is supported by RFBR grants \# 07-02-12238 and \# 07-02-01195.

\section{9:40-10:00 Break}

\section{0:00}

2aPAb6. Surface profile investigations by means of acousto-optic technique. Boris S. Gurevich (Scientific Instruments, Rizhsky prospekt 26, $190103 \quad$ St. Petersburg, Russian Federation, bgurevich@mail.ru), Valentine V. Shapovalov (Intelligent Software Systems, Nevsky pr. 190, 193317 St. Petersburg, Russian Federation, Shapovalov@incomsys.ru), Sergey V. Andreyev (Scientific Instruments, Rizhsky prospekt 26, 190103 St. Petersburg, Russian Federation, svan51@mail.ru), Andrey V. Belyaev (Institute for analytical instrumentation RAS, Rizhsky pr. 26, 190103 St. Petersburg, Russian Federation, bgurevich@mail.ru), Ilya A. Kolesov (Intelligent Software Systems, Nevsky pr. 190, 193317 St. Petersburg, Russian Federation, ilkak@mail.ru)

Acousto-optic tunable filters (AOTF) represent a very strong tool in different kinds of investigations where high rate light wavelength variations are required. The rough surfaces profile investigation is among the problems which can be solved partially by this way. Our investigations have been connected with use of AOTF and lens components with strong chromatic aberration. One of the problems was to choose the z-distance resolving power criterion. The proposed criterion is defined by the admissible probability to miss information unit regarding the object characterization in z-distance. In our experiments we used the specially elaborated AOTF based on tellurium dioxide crystal with transmission bandwidth of several nm, depending on the central wavelength position. Also we have used the lenses fabricated with tellurium dioxide because this material provides high chromatic aberrations. The results of experiments are discussed from the point of view of the AOTF possibilities to increase the z-distance resolving power in comparison with existing systems. The $90 \%$-probability distinguishing of defocusing while electric frequency variation of $200 \mathrm{kHz}$, can be considered as serious advantage. The device improvement can be attained by means of the noise level decreasing to the level taking place for electric frequency of $94 \mathrm{MHz}$.

\section{0:20}

2aPAb7. Transformation of Acoustic Modes in Case of Arbitrary Reflection in Acousto-Optic Crystals. Nataliya Polikarpova (M.V. Lomonosov Moscow State University, Department of Physics, Leninskie gory 1 bldg. 2, Division of Oscillations, 119991 Moscow, Russian Federation, polikarp@phys.msu.ru), Vitaly Voloshinov (M.V. Lomonosov Moscow State University, Faculty of Physics, Vorob'evy Gory, MSU, 119991 Moscow, Russian Federation, volosh@phys.msu.ru), Nico F. Declercq (Georgia Tech Lorraine - G.W. Woodruff School of ME, UMI Georgia Tech - CNRS 2958, 2 rue Marconi, 57070 Metz, France, nico .declercq@me.gatech.edu)

The phenomenon of unusual reflection of plane elastic waves is examined in the crystalline compounds of tellurium and mercury. It is predicted and registered in experiments that energy flow of one of the reflected waves may propagate practically in a back direction with respect to an incident energy flow. This phenomenon is observed in the materials with a strong anisotropy of elastic properties. In the case of inclined incidence, the reflection may take place strictly in the back direction with respect to the intrinsic energy flow. Moreover, all incident elastic energy may be reflected in a form of a back elastic wave. The analysis was carried out for all exisiting cases of inclined incidence in the crystals. It was proved that relative intensity of the unusually reflected wave might be close to a unit in a wide variety of incidence and cut angles in the crystals. A comparative analysis of the effect in new anisotropic materials and in the well-known crystals has been carried out. Since the crystals under investigation possess relatively high magnitudes of acousto-optic figure of merit, e.g. paratellurite and calomel, principal results of the research are of significance in development of new generations of acousto-optic devices.

\section{0:40}

2aPAb8. Optimization of KDP-based acousto-optic imaging filters. Konstantin B. Yushkov (Moscow State University, Phisical Faculty, Leninskie Gory, 119991 Moscow, Russian Federation, protonoa@mail.ru)

Acousto-optic devices can perform spatial and spectral tunable filtration of light with the use of anisotropic Bragg diffraction. In the near UV range 
of spectrum, KDP single crystals provide the highest acousto-optic figure of merit among uniaxial crystals. The report is devoted to the problem of optimization of the filter parameters in order to maximize angle aperture of the instruments. It was found that the maximum angle aperture of wide angle tunable acousto-optic filters is equal to 3 degrees in air at output of the KDP crystals. This magnitude of the angle aperture is limited by a narrow deflection angle of light in KDP that is due to a relatively low birefringence of the material. The investigation showed that the diffraction efficiency of light depends on the dimensions of the acousto-optic cell, i.e. linear aperture and length of a piezoelectric transducer. A discussion on trade-off between transmission coefficient and the spatial resolution in the filter is presented in the report.

\section{1:00}

2aPAb9. Acousto-optic collinear diffraction of arbitrary polarized light. Sergey Mantsevich (Dept. of Physics, M.V.Lomonosov Moscow State Univ., Vorobyevy Gory, Bldg. 1, 119991 Moscow, Russian Federation, manboxx@mail.ru), Vladimir I. Balakshy (Dept. of Physics, M.V
.Lomonosov Moscow State Univ., Vorobyevy Gory, Bldg. 1, 119991 Moscow, Russian Federation, balakshy@phys.msu.ru)

Acousto-optic collinear diffraction of light is a type of light scattering interesting as a physical phenomenon and important from practical point of view. This type of diffraction is used in tunable acousto-optic filters. In this case, the incident light has to be linearly polarized along one of the acoustooptic crystal anisotropy axes. If the incident light is not primordially polarized, a polarizer should be disposed at the input of the acousto-optic cell. Unfortunately, this leads to loss of half the light power. The present work is devoted to theoretical and experimental investigation of collinear acoustooptic interaction peculiarities that arise when the incident optical beam is not polarized. It is shown that the output diffraction spectrum in the general case contains four components which have different polarization and frequency. Beating of these components leads to modulation of light intensity passed through the output analyzer. In this work, dependences of modulation components amplitudes on frequency and power of the acoustic wave are examined for different polarizer and analyzer orientations.

\section{Invited Paper}

\section{1:20}

2aPAb10. Ultrasonic diffraction grating spectroscopy: particle size measurements and investigation of the inertial model for attenuation. Margaret Greenwood (Pacific Northwest National Laboratory, P. O. Box 999, Mailstop K5-26, Richland, Wa, WA 99352, USA, margaret.greenwood@pnl.gov)

The experimental setup consists of a unit with a grating machined on the surface of the unit. Send and receive transducers are placed at equal angles to the grating surface that is in contact with a slurry. The transmitted beam of spectral order $\mathrm{m}=1$ reaches an angle of $90^{\circ}$ at the so-called critical frequency, where it becomes an evanescent wave that interacts with particles in the slurry. As a result of this interaction, the signal in the receive transducer exhibits a dependence upon particle size of the slurry. Data will be presented for slurries of polystyrene spheres, ranging in size from $45 \mu \mathrm{m}$ to $467 \mu \mathrm{m}$, as a function of the volume fraction. The velocity of sound is also determined from the critical frequency. The distributions will be compared with the viscous and inertial models. The results show that larger particle sizes can be understood in terms of the inertial model, while the smaller sizes can be explained as a result of scattering. Also, arguments will be presented to show that this technique can probe the inertial model, without the simultaneous observation of scattering effects. Such studies can improve the theoretical interpretation of standard attenuation measurements.

\section{Contributed Paper}

11:40

2aPAb11. Non-reciprocity of acousto-optic interaction at high acoustic frequencies. Yury Dobrolenskiy (M.V. Lomonosov Moscow State University, Faculty of Physics, Vorob'evy Gory, MSU, 119991 Moscow, Russian Federation, dobrolenskiy@phys.msu.ru), Vitaly Voloshinov (M.V. Lomonosov Moscow State University, Faculty of Physics, Vorob'evy Gory, MSU, 119991 Moscow, Russian Federation, volosh@phys.msu.ru), Yury Zyuryukin (Saratov State Technical University, Politechnicheskaja str., 77, 410054 Saratov, Russian Federation, phys@sstu.ru)

Modern acousto-optic (AO) tunable filters have reached the values of spectral resolution exceeding 104 and operate with acoustic frequencies up to a few gigahertzes. At such high frequencies and such narrow bandwidths, there appear effects so far neglected. Among them there is non-reciprocity of
AO interaction. It consists in the fact that acoustic frequency of light diffraction by ultrasound is different when optic beams propagate in directions opposite to each other. In the present research, the new effect has been studied theoretically and experimentally for the example of collinear AO diffraction. Theoretical analysis and calculations related to particular AO materials have shown that the effect is essential at acoustic frequencies about $1 \mathrm{GHz}$ and higher. The value of the non-reciprocal shift of acoustic frequency can be as high as the frequency bandwidth of the filter. The effect has also been registered and investigated experimentally in a sample of lithium niobate crystal. The experimental data has totally confirmed the theoretical analysis. Therefore, it has been proved that the non-reciprocal effect influences the parameters of $\mathrm{AO}$ diffraction and, consequently, operation of AO filters at high frequencies. On the other hand, one can design devices, e.g. directional couples, exactly based on the non-reciprocity.

\section{Invited Paper}

\section{2:00}

2aPAb12. Critical cone channelling in directly bonded wafers. Evgeny Twerdowski (Institute of Experimental Physics II, University of Leipzig, Linnéstr. 5, 04103 Leipzig, Germany, twerdowski@ physik.uni-leipzig.de), Moritz Von Buttlar (Institute of Experimental Physics II, University of Leipzig, Linnéstr. 5, 04103 Leipzig, Germany, vbuttlar@physik.uni-leipzig.de), Reinhold Wannemacher (Institute of Experimental Physics II, University of Leipzig, Linnéstr. 5, 04103 Leipzig, Germany, wannemacher@physik.uni-leipzig.de), Wolfgang Grill (Institute of Experimental Physics II, University of Leipzig, Linnéstr. 5, 04103 Leipzig, Germany, grill@physik.uni-leipzig.de)

Directly bonded semiconductor wafers have been investigated using ultrasonic transmission tomography and imaging of the phonon focusing patterns at ultrasonic frequencies. Beside of total disbonds, several bonded wafers contained defects that are fully transparent to normally incident waves of longitudinal polarization, and are fully opaque to those of transverse polarization. These defects, which are due to slip boundary conditions at the wafer-wafer interface, generate an additional acoustic mode by mode conversion at the interface. The additional mode is clearly observable in the experimental phonon focusing patterns and is indicative of the critical cone 
channelling phenomenon, which is caused by the generation of pseudo-surface and head waves at weakly bonded solid-solid interfaces. The effect is also expected to be present in the case of weakly-bonded isotropic materials. Since the strong pseudo-surface wave resonance exists only in the case of boundary conditions that allow for the relative displacement of the two adjoining media along the interface, the critical cone channelling effect can serve as a measure of the type and quality of the bond.

\section{Contributed Papers}

$12: 20$

2aPAb13. Sound waves in 3D periodic granular materials. Orion Mouraille (UTwente, Multi Scale Mechanics, P.O.Box 217, 7500 AE Enschede, $\quad$ Netherlands, o.j.p.mouraille@utwente.nl), Stefan Luding (UTwente, Multi Scale Mechanics, P.O.Box 217, 7500 AE Enschede, Netherlands, s.luding@utwente.nl)

The sound propagation mechanisms inside dense granular matter, even for periodical structures, are challenging the attempts to describe it because of the discrete nature of the material. Phenomena like dissipation, scattering, and dispersion are hard to predict based on the material state and/or properties and vice-versa. We propose here a simulation method using dynamic discrete elements in order to get more insight in this problem. The small perturbation created on one side of a dense, static regular packing of grains is examined during its propagation and when it arrives at the opposite side. Both longitudinal and shear perturbation are studied and an interesting wave-acceleration is observed [1]. Moreover the rotational degree of freedom permits to observe the role of rotations in the wave propagation. The control of the inter-particle forces like, contact potential, cohesion and friction make possible to observe the effect of these micro-parameters on the macro-behavior (at the wave scale). Long term goal is to predict the largescale macroscopic material behavior from the microscopic structure and material parameters. [1] O. Mouraille, W A Mulder and S. Luding: Sound wave acceleration in granular materials, J. Stat. Mech. (2006) P07023.

\section{$12: 40$}

2aPAb14. Bragg light diffraction in nontransparent crystals. Farkhad Akhmedzhanov (Navoi State Mine Institute, 27a Yuzhnaya Street, 210100 Navoi, Uzbekistan, farkhad2@yahoo.com)

As is well known, in absolutely transparent crystals an acoustooptical interaction is lacking. In this connection's acoustooptical investigations are presented greatest interest in optical range, in which the crystal is not transparent. In order to carry out similar experiments, it is necessary to use the crystal with a small light absorption coefficient at applied wavelength. The main problem is selection of an appropriate buffer crystal that the light beam can penetrate into sample by Bragg angle at required frequency. At present work the acoustooptical properties of Si crystal were investigated at light wavelength $632.8 \mathrm{~nm}$. Dy $\mathrm{S}_{3}$ crystals were used as a buffer sample. It was detected the diffracted light intensity is much more powerful in comparison with that for $\mathrm{LiNbO}_{3}$ crystals. Acoustooptical efficiency M2 (it is defined the acoustic-optical quality of material) has been calculated from the values of optic coefficients and elastic constants for appropriate directions of sound and light. The results of executed investigations have shown the possibility of determination of acoustooptical properties of nontransparent crystals by Bragg diffraction method. At that rate, the very high intensity of diffracted light can be obtained in comparison with that in transparent crystals.

1:00-2:00 Lunch Break

\section{Invited Paper}

\section{2:00}

2aPAb15. Theory of resonant acoustic transmission through subwavelength apertures. Johan Christensen (Universidad Autónoma de Madrid, Departamento de Física Teórica de la Materia Condensada, Facultad de Ciencias, C-V, 28049 Madrid, Spain, johan.christensen@uam.es), Luis Martín-Moreno (Universidad de Zaragoza, Departamento de Física de la Materia Condensada, Instituto de Ciencia de Materiales de Aragón, C/ Pedro Cerbuna 12, 50009 Zaragoza, Spain, 1mm@unizar.es), Francisco Jose García-Vidal (Universidad Autónoma de Madrid, Departamento de Física Teórica de la Materia Condensada, Facultad de Ciencias, C-V, 28049 Madrid, Spain, fj.garcia@uam.es)

The discovery of the phenomenon of extraordinary optical transmission through a two-dimensional array of subwavelength holes in a metallic film has opened a new line of research within optics. The key role played by surface plasmons in transferring light efficiently from the input side of the metal film to the output region was soon realized. This fundamental knowledge enabled extension of this surface-plasmon ability to achieve extraordinary optical transmission and strong collimation of light in a single hole surrounded by a finite periodic array of indentations. Here, we show how these ideas developed for electromagnetic radiation can be transferred to other classical waves such as acoustic waves.

\section{Contributed Papers}

\section{2:20}

2aPAb16. Theoretical and experimental ultrasonic investigation of a thin plate with regions of different periodic double-corrugations. Sarah W. Herbison (Georgia Tech Lorraine - G.W. Woodruff School of ME, UMI Georgia Tech - CNRS 2958, 2 rue Marconi, 57070 Metz, France, sherbison@gatech.edu), Nico F. Declercq (Georgia Tech Lorraine - G.W Woodruff School of ME, UMI Georgia Tech - CNRS 2958, 2 rue Marconi, 57070 Metz, France, nico.declercq@me.gatech.edu)

In order to provide a qualitative measurement of the depth of periodic double-corrugations existing on both sides of a thin plate, theoretical and experimental ultrasonic techniques have been applied. The aesthetic galvanized steel sheets under investigation contain many regions of periodic double-corrugations, each region with a different corrugation depth. An ultrasonic technique is investigated as a means to improve existing optical testing techniques in quality and speed. Theoretical simulations using the theory of diffraction from periodically corrugated surfaces have been employed to obtain reflection spectra to facilitate qualitative observations regarding the depth of the corrugation in the different regions. Experiments to obtain reflection spectra correlate well with the theoretical simulations, and show the application of ultrasound to the qualitative measurement of the corrugation depth. Because the wavelengths necessary to make the investigations produce Lamb waves, some difficulty lies in the generation of diffracted bulk waves and Lamb waves. The research shows that correlations between theory and experiments exist, but they can only be obtained after thorough simulations that do not produce easy to apply rules of thumb. 


\section{2:40}

2aPAb17. BEM analysis of plane waves scattered from periodic surfaces. Mahesh Bansal (Institute of Technical Acoustics, Tech. Univ. Berlin, Einsteinufer 25, 10587 Berlin, Germany, mbansal.iitk @ gmail.com), Wolfgang Ahnert (Ahnert Feistel Media Group, Arkonastr. 45-49, 13189 Berlin, Germany, wahnert@ada-acousticdesign.de), Stefan Feistel (Ahnert Feistel Media Group, Arkonastr. 45-49, 13189 Berlin, Germany, sfeistel@afmg.eu)

Periodic surfaces like stairs, seats and repetitive designs on walls are very common and integral part of room acoustics. Effective simulation of the sound field inside enclosures requires the investigation of scattering from such periodic surfaces. In this work, we perform BEM analysis to calculate the scattering coefficients of different samples. For comparison purposes we also introduce a simple point-source based model to calculate the scattered wave fronts. Both approaches are implemented in a computational tool called EASE Scatterer. The incident plane waves are considered at various angles and scattered waves computed in both models are then compared with the measured data. It is found that while the point-source model can give reasonable asymptotic results, the BEM model matches with the measurement data significantly better in quantity and quality. Moreover, a study by varying the number of periods for the given sample is also performed.

\section{3:00}

2aPAb18. Diffraction phenomena associated with a composite plate containing an interior periodically corrugated interface. Sarah W. Herbison (Georgia Tech Lorraine - G.W. Woodruff School of ME, UMI Georgia Tech - CNRS 2958, 2 rue Marconi, 57070 Metz, France, sherbison@gatech.edu), Nico F. Declercq (Georgia Tech Lorraine - G.W. Woodruff School of ME, UMI Georgia Tech - CNRS 2958, 2 rue Marconi, 57070 Metz, France, nico.declercq@me.gatech.edu)

The interest in the study and applications of phononic crystals has naturally lead to the investigation of other novel periodic structures. The present work examines the case of a plate constructed of two solid layers of differing elastic properties separated by a periodically corrugated interface. It is shown how the dispersion curves are influenced by the internal corrugated interface and how they evolve as a function of the magnitude of this corrugation. Internal diffraction effects alter the dispersion properties and thus have an important effect on the composite when it is used as an acoustic filter. These effects are also important for the transmission and reflection of sound when the composite is used as a panel or when it is the intention to generate Lamb waves to investigate the composite plate nondestructively.

3:20

2aPAb19. Multiple scattering of acoustic waves from two transversely isotropic cylinders. Sina Sodagar (Faculty of Mechanical Engineering, K. N. Toosi University of Technology, Pardis St., Molla Sadra Ave., Vanak Sq., Postal code 1999143344, 16579 Tehran, Iran, ssodagar@alborz.kntu.ac.ir), Farhang Honarvar (Faculty of Mechanical Engineering, K. N. Toosi University of Technology, Pardis St., Molla Sadra Ave., Vanak Sq., Postal code 1999143344, 16579 Tehran, Iran, honarvar @ mie.utoronto.ca), Anthony N. Sinclair (Department of Mechanical Engineering, University of Toronto, 5 King's College Road, Toronto, ON M5S 1A4, Canada, sinclair@mie.utoronto.ca)

The study of the interaction of acoustic waves with cylindrical structures has numerous applications including the ultrasonic nondestructive testing of materials. The scattered pressure field from a submerged cylinder contains valuable information about its physical properties. Scattering of acoustic waves from single cylindrical components including solid cylinders, shells, and multilayered cylinders has been of interest during the past two decades. These studies include theoretical modeling, numerical calculations, and experimental measurements of the scattered field of these objects. The more complex problem of multiple scattering from a grating of cylindrical components has also been considered during the past few years. These studies usually deal with either rigid or isotropic cylindrical objects. In this paper, the mathematical modeling for the scattering of plane acoustic waves from two adjacent infinite anisotropic solid cylinders will be presented. The type of anisotropy considered is transverse isotropy (hexagonal symmetry). The mathematical model accounts for the effects of the scattered filed of each cylinder on the total resultant pressure field. Numerical calculations are used to verify the validity of the developed mathematical model.

3:40-5:20 Posters

Lecture sessions will recess for presentation of poster papers on various topics in acoustics. See poster sessions for topics and abstracts.

\section{Contributed Papers}

\section{$5: 20$}

2aPAb20. Phononic crystals in the diffraction regime. Vincent Laude (Institut FEMTO-ST/CNRS, 32 avenue de l'Observatoire, $25044 \mathrm{Be}-$ sançon cedex, France, vincent.laude@femto-st.fr), Sarah Benchabane (Institut FEMTO-ST/CNRS, 32 avenue de l'Observatoire, 25044 Besançon cedex, France, sarah.benchabane@femto-st.fr), Abdelkrim Khelif (Institut FEMTO-ST/CNRS, 32 avenue de l'Observatoire, 25044 Besançon cedex, France, abdelkrim.khelif@femto-st.fr)

Phononic crystals are periodic composite materials exhibiting amazing wave propagation properties. In many works, complete band gaps are being looked for, i.e. the materials constituting the phononic crystal and its lattice arrangement are chosen such that propagation for all waves within a prescribed frequency range is forbidden. In other studies, the phononic crystal is considered a metamaterial, the anisotropic spatial dispersion of which can be tuned, and in which negative refraction can even be observed under certain circumstances. Such effects are usually considered in the sub-diffraction regime, i.e. below some critical onset frequency. In this work, we specifically examine phononic crystals in the diffraction regime. Indeed, the boundaries of a finite size phononic crystal embedded in a host propagation medium can be viewed as diffraction gratings, as we show. We will specifically consider two cases: two-dimensional phononic crystals composed of steel rods in water, and two-dimensional phononic crystals for surface acoustic waves achieved by etching cylindrical holes in a solid substrate.

\section{$5: 40$}

2aPAb21. Methods to investigate the possibilities of using a three element periodic structure to suppress the transmission of energy in an elastic tube. Ole Holst-Jensen (Minus10dB, Stokrosevej 29, 8330 Beder, Denmark, ole.holst@minus10db.dk), Sergey Sorokin (Univ. of Aalborg, Institute for mechanical engineering, Pontoppidanstraede 101, 9220 Aalborg East, Denmark, svs@ime.aau.dk)

In industrial applications, such as pump and compressor systems, pipe vibrations can exceed an acceptable level. Periodically spaced masses added to the piping are an appropriate method of attenuation, and investigations to develop valid prediction models and measurement techniques has been initiated in the Danish Makunet network. The present investigation concern the effect of three masses attached periodically to a pipe of small diameter. The pipe is small enough that it can be treated as a beam. The masses are eccentric to the center of the beam, to achieve a large change in the moment of inertia by the added elements. The theoretical model is formulated as a system of boundary equations, which describe propagation of flexural, axial and torsion waves within each segment of a tube between periodic elements. An exact solution of this system is obtained and the power transfer is dramatically reduced in some frequency 'stop bands' regardless the excitation conditions. Transfer impedance measurements between the force input and the acceleration on selected positions are used to find the insertion loss due to the application of the periodic elements, showing good agreement with theory. 


\section{6:00}

2aPAb22. Diffraction influence on the SAW tag characteristics. Boris V. Sveshnikov (Lebedev Research Center in Physics, 23, Lenin Av., 119991 Moscow, Russian Federation, bvs@ieee.org)

The diffraction of acoustic beams may influence notably on the responses of the SAW based RFID systems. In order to prevent such an influence one cannot increase the tag acoustic aperture too much because of ohmic loss in a transponder. On the other hand, usually the tag length should be large enough. There is a very specific and unique feature of the acoustoelectric scattering of SAW beam on the coding sequence of the reflective metal electrodes placed on a piezoelectric substrate. The point is that the SAW itself is formed by two coupled sub-systems, namely: elastic displacements and electric field. Both of them are scattered on every discontinuity because of two kinds of the boundary condition perturbation: electric shorting beneath electrodes and mass-loading effect. A uniform metal electrode scatters differently from each other the "elastic" and "electric" subsystems with non-uniform wave-front caused by diffraction. The closed form expressions are found, allowing us to describe consistently the mentioned phenomena. Their numerical analysis has been added by the analytical one in case of parabolic approximation of the crystal slowness curve. The synthesis algorithm of SAW tags with high capacity is proposed taking into account the diffraction effect in a natural way.

\section{6:20}

2aPAb23. Frequency-domain diffraction for edges of arbitrary length with efficient numerical integration. Peter Svensson (Norwegian Univ. of Science and Technology, O.S. Bragstads plass 2B, Dept. of Electronics and Telecommunications, NO-7491 Trondheim, Norway, svensson@iet.ntnu.no), Paul Calamia (Rensselaer Polytechnic Institute, Greene Bldg., 110 8th St., Troy, NY 12180, USA, calamp@rpi.edu)

Frequency-domain edge diffraction from an infinite wedge insonified by a point source has been widely studied, with various analytical solutions dating back nearly a century. In this talk we present an alternative frequency- domain solution which can be used for finite as well as infinite edges. The expression, given as a line integral along the diffracting edge, is derived from an analytical time-domain expression presented in [U. P. Svensson et al., J. Acoust. Soc. Am. 106, 2331-2344 (1999)]. The new formulation is shown to be equivalent to an exact, analytical, contour-integral formulation for the infinite wedge via a variable transformation. Results for various finite and infinite edge cases will be presented, and numerical integration using an efficient quadrature method for highly oscillatory integrals will also be discussed.

TUESDAY MORNING, 1 JULY 2008

ROOM 352A, 8:00 A.M. TO 7:00 P.M.

\title{
Session 2aPAc
}

\section{Physical Acoustics: Outdoor Sound Propagation and Uncertainties I}

\author{
Keith Wilson, Cochair \\ U.S. Army Cold Regions Research and Engineering Laboratory, 72 Lyme Road, Hanover, NH 03755-1290, USA
}

Michel C. Berengier, Cochair

Laboratoire Central des Ponts et Chaussées, Centre de Nantes - Route de Bouaye, BP 4129, Bouguenais cedex, 44341, France

\section{Invited Papers}

\section{8:00}

2aPAc1. Expected mean in an environmental noise measurement and its related uncertainty. Marco Paviotti (European Commission, via e. fermi, 1, 21020 Ispra, Italy, marco.paviotti@jrc.it), Stylianos Kephalopoulos (European Commission, via e. fermi, 1, 21020 Ispra, Italy, Stylianos.Kephalopoulos@jrc.it)

In the context of the implementation of the Environmental Noise Directive 2002/49/EC a study on noise measurement uncertainty was developed. In performing any noise measurement average, there might be assumptions over the distribution and independency of the samples. In a concrete example dealing with environmental noise, this might be the case of a series of measurements of a constant noise source like an industrial plant or a fluctuating noise source like road traffic. Using a series of 1 day Leq in the first case, or a series of 15 minutes samples in the second case, the average of these values is usually considered as the expected mean, however, the error caused by the specific selection of the samples is not evaluated. Statistically speaking, before establishing an average value, at least the lognormal distribution of the samples and the effect of adding-up several uncertainties should be evaluated. This article will focus on the formulas to be used and will discuss differences in assessing the expected mean for normally distributed values, or for lognormally distributed, and will suggest an approach to properly add-up all uncertainties related to an environmental noise measurement campaign. 
2aPAc2. Criterion to select meteorological factors to evaluate uncertainties in sound propagation. Panu Maijala (VTT, Tekniikankatu 1, 33101 Tampere, Finland, Panu.Maijala@vtt.fi)

Most of the environmental factors have some effect on sound propagation outdoors. Many of these factors can be properly implemented to a sound propagation model. However, it is not easy to handle sound scattering due to the turbulence, and at the same time, the turbulence is the most important source of uncertainties. Concurrently with the studies of turbulence models we have developed a concept to get an estimate of the excess attenuation using a state-of-the-art physical model and to evaluate the uncertainties using a statistical model. This statistical model is based on two years continuous measurements using extensive acoustical and meteorological measurement facilities and producing over 100 factors hourly. Many meteorological factors had a strong and significant correlation with the excess attenuation, but between each other too. To avoid instable model due to the collinearity many factors were abandoned. In this paper the criterion and methods to select best explaining factors and to form this statistical model are considered.

\section{8:40}

2aPAc3. Methodological and metrological benchmarks for determining experimental values of meteorological parameters. Benoit Gauvreau (Laboratoire Central des Ponts et Chaussées, Centre de Nantes - Route de Bouaye, BP 4129, 44341 Bouguenais cedex, France, benoit.gauvreau@1cpc.fr)

Outdoor sound propagation is largely influenced by meteorological conditions: mean refraction, intermittency and atmospheric turbulence. Thus, acoustic engineers and scientists have to rigorously characterize those propagation conditions through several parameters, both for numerical predictions (input data) and for operational reports (impact studies). One of these influent parameters is the well-known (and widely used) effective vertical sound speed gradient, which depends on spatial and temporal mean values of wind and temperature between source and receiver. However, the experimental characterization of wind and temperature (and next sound speed) vertical profiles remains delicate: it requires accurate meteorological devices and adapted measuring methods. At this purpose, an experimental campaign has been carried in 2007 on a perfectly flat and open ground, in order to quantify the confidence, uncertainty and spreading of such parameter values for different time scales. This campaign involved different meteorological devices: instrumented towers, classical sensors, 3D sonic anemometers, etc. The benchmark results will be presented and next analysed in terms of metrological and methodological requirements for acousticians in charge of environmental studies for standard organizations and/or scientific institutes.

\section{9:00}

2aPAc4. Sources and potential influences of uncertainty in ground impedance measurements and estimations. Shahram Taherzadeh (The Open University, Faculty of Mathematics, Computing and Technology, Walton Hall, MK7 6AA Milton Keynes, UK, s.taherzadeh@open.ac.uk), Keith Attenborough (Open University, Department of Design, Development, Materials and Environment, Walton Hall, MK7 6AA Milton Keynes, UK, Keith.Attenborough@ioa.org.uk)

Knowledge of acoustic impedance of ground surface is essential for determining noise levels outdoors. At distances up to a few hundred metres from the source ground effect may be the dominant factor. The short-range measurement of sound level spectra from an omni-directional source has been used as a standard method to deduce the acoustic impedance spectrum of ground surfaces (e.g. ANSI S1.18, 1999). However, there remain a number of uncertainties in such measurement methods. For example, at low frequencies the difficulties in fitting theoretical or phenomenological models to short range data may result is a large uncertainty in predictions of sound levels at larger distances. Another source of uncertainty is the variability of the acoustic impedance of apparently uniform ground. Another potential influence on predictions of long term equivalent noise levels is seasonal variation. This paper discusses the extent and potential influences of such uncertainties in measured and deduced ground impedance spectra

\section{9:20}

2aPAc5. Facing the challenge of calculating outdoor sound propagation using a 3D multi domain approach based on linear euler equations. Fabrice Junker (EDF, 1, avenue du Général de Gaulle, 92141 Clamart, France, fabrice.junker@edf.fr), Fabien Crouzet (EDF, 1, avenue du Général de Gaulle, 92141 Clamart, France, fabien.crouzet@edf.fr), Philippe Lafon (EDF, 1, avenue du Général de Gaulle, 92141 Clamart, France, Philippe.lafon@edf.fr)

Solving the Linear Euler Equations (LEE) is the reference method that allows to take into account all the phenomena involved in Outdoor Sound Propagation. However, the huge size of the problems to be treated is still a great limitation to the practical application of this method. The concept of multi domain computations associated with the use of massively parallel computers now pushes the limits away. The code SAFARI, recently developed by EDF, solves LEE with high order numerical schemes on structured grids. To deal with complex and large geometries, a multi domain approach is used. The computational domain is composed of several partially overlapping grids (overset grids). Computations are parallelized by domain decomposition to be runned on cluster facilities. The presentation means to show the capability of SAFARI to deal with propagation over realistic 3D domains. The strategy used to carry out the calculations is detailed. The new perspectives of this kind of method are finally given. 
2aPAc6. Computing the parameter sensitivities of outdoor sound propagation in a random environment. Chris L. Pettit (U.S. Naval Academy, Aerospace Engineering Dept., 590 Holloway Rd., MS 11-B, Annapolis, MD 21402, USA, petitcl@usna.edu), Keith Wilson (U.S. Army Cold Regions Research and Engineering Laboratory, 72 Lyme Road, Hanover, 03755-1290, USA, D.Keith.Wilson @erdc.usace.army.mil)

Computational forecasts of near-ground sound levels are compromised by uncertainty (e.g., randomness) and error (e.g., grid resolution) in the atmosphere and terrain representations, and by simplified or incorrect physics. For an incompletely known environment, a model's predictive power cannot be assessed without first quantifying the sensitivity of its forecasts to the full range of every parameter. Knowledge of these sensitivities throughout the spatial domain also is essential for effectively investing data-gathering resources to support sound propagation forecasts. Sensitivity analysis therefore is central to raising the relevance of computational acoustics in practical applications. These considerations should motivate practitioners of computational acoustics to adopt a consistent framework for sensitivity and uncertainty analyses. Topics to be discussed include: (1) standard uncertainty taxonomies in computational mechanics, (2) why uncertainty about a parameter should be distinguished from sensitivity of a model to that parameter, (3) sources of uncertainty in the near-ground acoustics, (4) a sampling-based sensitivity analysis framework that facilitates estimating typical and extreme values of sensitivities at each point in the spatial domain (i.e., full-field sensitivities), (5) factors to be aware of when applying sensitivity analysis to forecasts of near-ground sound propagation, and (6) ways of representing sensitivity estimates to facilitate insight.

\section{0:00-10:20 Break}

\section{$10: 20$}

2aPAc7. Field experiments on the influences of wind speed and direction on outdoor sound propagation over flat ground. Takatoshi Yokota (Kobayasi Institute of Physical Research, 3-20-41 Higashi-Motomachi Kokubunji, 185-0022 Tokyo, Japan, t-yokota@kobayasi-riken.or.jp), Koichi Makino (Kobayasi Institute of Physical Research, 3-20-41 Higashi-Motomachi Kokubunji, 185-0022 Tokyo, Japan, makino@kobayasi-riken.or.jp), Kohei Yamamoto (Kobayasi Institute of Physical Research, 3-20-41 HigashiMotomachi Kokubunji, 185-0022 Tokyo, Japan, yamamoto@kobayasi-riken.or.jp), Yasuaki Okada (Faculty of Science and Technology, Meijo University, 1-501 Shiogamaguchi Tempaku-ku, 468-8502 Nagoya, Japan, okada@ccmfs.meijo-u.ac.jp), Koichi Yoshihisa (Faculty of Science and Technology, Meijo University, 1-501 Shiogamaguchi Tempaku-ku, 468-8502 Nagoya, Japan, yosihisa@ccmfs .meijo-u.ac.jp)

The influences of wind on outdoor sound propagation are investigated both by field experiments and numerical simulations. At first, measurements of sound propagation at a distance of $160 \mathrm{~m}$ were carried out under various wind speed and direction. The relationship between vector wind speed and the variation of sound pressure level has been examined not only for the up/down wind conditions but also for the cross wind conditions. Secondly, the short term sound fluctuations were investigated by the use of a high energy impulsive sound generator and a loud speaker as sound sources. Sound propagation up to $300 \mathrm{~m}$ was examined with frequency range from $16 \mathrm{~Hz}$ to $4 \mathrm{kHz}$ octave band. Finally, the sound speed profiles in the measurement field were estimated by two methods; one was based on the time interval during the sound propagation from the source to the receivers, the other was based on the wind speed at height of $0.6 \mathrm{~m}$, $1.2 \mathrm{~m}, 2.4 \mathrm{~m}$ and $4.8 \mathrm{~m}$, and the temperature. By using those sound speed profiles, the excess attenuation was calculated by the PE method. The measured and the calculated values have been compared and the methods to estimate the influences due to wind condition have been discussed.

\section{0:40}

2aPAc8. Variation in measured sound level as a function of propagation environment and distance. Michelle E. Swearingen (Norwegian Defense Research Establishment/US Army, Postboks 25, 2027 Kjeller, Norway, michelle.swearingen@ffi.no), Morten Huseby (Norwegian Defense Research Establishment/US Army, Postboks 25, 2027 Kjeller, Norway, Morten.Huseby@ffi.no), Michael J. White (US Army Engineer Research and Development Center, 2902 Newmark Drive, Champaign, IL 61826, USA, Michael.J.White @usace.army.mil)

The propagation environment exerts a large influence on the range of received levels of impulsive events. This talk focuses on the variation in excess attenuation over durations of less than approximately 15 minutes. Data are presented for greatly different measurement distances $(25 \mathrm{~m}$ to $7 \mathrm{~km}$ ) and propagation environments (sparse vegetation to forested), illustrating the effects of distance and terrain cover on sounds from a propane cannon and an artillery source. Over sparse vegetation $7 \mathrm{~km}$ from an artillery source, the received CSEL varied $11 \mathrm{~dB}$ within a 12-minute duration. In measurements up to approximately $300 \mathrm{~m}$ from the source, variation in received level (both peak and SEL) was less than $1 \mathrm{~dB}$ within the forest, and much more in the open. The control of the forest canopy on the micrometeorology seems to explain the effect.

\section{1:00}

2aPAc9. The revision of the French method for road traffic noise prediction. Guillaume Dutilleux (Lab. Régional des Ponts et Chaussées, 11, rue Jean Mentelin, BP 9, 67035 Strasbourg Cedex 2, France, Guillaume.Dutilleux@equipement.gouv.fr), Jérôme Defrance (CSTB, 24 rue Joseph Fourier, 38400 Saint-Martin-d'Hères, France, jerome.defrance@cstb.fr), Benoit Gauvreau (Laboratoire Central des Ponts et Chaussées, Centre de Nantes - Route de Bouaye, BP 4129, 44341 Bouguenais cedex, France, benoit.gauvreau@lcpc.fr), Francis Besnard (Lab. Reg. Régional des Ponts et Chaussées de l'Est Parisien, Rue de l'égalité prolongée, BP 34, 93352 Le Bourget, France, francis.besnard@equipement.gouv.fr)

A revision of the French method for road traffic noise prediction (NMPB-Routes-2008) has been released. The major principles of NMPB-Routes-2008 are outlined. The most important modifications regarding the source are the reduction of its height and the introduction of two different spectra. In this complete revision, the main change is the replacement of the ISO 9613-2 - based ground attenuation formula in downward conditions by the formula for ground attenuation in homogeneous conditions of NMPB-Routes-1996 
with corrected heights in order to take into account the mean curvature of rays (refraction) and its spreading (turbulence). The revised NMPB adds an attenuation term for an occasional cutting embankment. Regarding diffraction the $\Delta$ dif formula is now suitable for low height barriers. The validation of the revised NMPB with respect to experiment is presented. It is based on measurement campaigns on 6 sites with complex geometries and shows that the predicted noise levels obtained from the revised NMPB are significantly closer to experimental results than in the case of the original method.

11:20

2aPAc10. Long range propagation of high speed train noise: Sound level variations before and after the pass-bys. Benjamin Cotte (Ecole Centrale de Lyon, LMFA, UMR CNRS 5509, Université de Lyon, Bât. KCA, 36 Avenue Guy de Collongue, 69134 Lyon, France, benjamin.cotte@ec-lyon.fr), Philippe Blanc-Benon (Ecole Centrale de Lyon, LMFA, UMR CNRS 5509, Ecully, 69134 Lyon, France, Philippe.Blanc-Benon@ec-lyon.fr), Franck Poisson (SNCF DIR, 45, rue de Londres, 75379 Paris, France, franck.poisson@sncf.fr), Cora Cremezi-Charlet (UIC, 16,rue J. Rey, F-75015 Paris, France, cremezi@uic.asso.fr)

It has been observed relatively frequently that high speed train (TGV) noise could be heard tens of seconds before or after the actual train pass-bys. This study is aimed at characterizing this phenomenon and the conditions in which it occurs, both experimentally and numerically. Acoustic measurements of TGV pass-bys have been performed under controlled conditions. A relatively strong wind was blowing from the South during the experiment. The measurements show that the TGV noise can be heard before the pass-bys when the TGV was coming from the South (same wind and train directions), and after the pass-bys when the TGV was coming from the North (opposite wind and train directions). This noise is relatively low in frequency (around $400 \mathrm{~Hz}$ ), and corresponds to propagation distances that can exceed $1 \mathrm{~km}$. Levels associated with this phenomenon can vary significantly over short time intervals (5-10 minutes), which raises the issue of the representativeness of TGV measurements at long ranges. It will be shown using numerical prediction methods (parabolic equation in the frequency domain, linearized Euler equations in the time domain) that these acoustic variations are mostly due to variations in the meteorological conditions between the pass-bys.

\section{1:40}

2aPAc11. A model based monitoring system for aircraft noise. Frank Van Den Berg (TNO - Science and Industry, Stieltjesweg 1, 2628 CK Delft, Netherlands, Frank.vandenBerg@tno.nl), Arno R. Eisses (TNO, Stieltjesweg 1, P.O.Box 155, 2600 AD Delft, Netherlands, Arno.Eisses@tno.nl), Pieter J. Van Beek (TNO, Stieltjesweg 1, P.O.Box 155, 2600 AD Delft, Netherlands, Pieter.vanBeek @tno.nl)

A new approach for an airport noise monitoring system is presented that comprises not only a number of measuring stations, but also a dedicated sound propagation model and an aircraft noise emission model. This approach enables estimation of noise levels in the whole area around the airport and not only at the location of the measuring stations. An additional advantage is that the locations of the measuring stations can be chosen more freely. Traditionally the measuring stations must be located near residential areas where the influence of other noise sources (such as cars or wind) cannot be eliminated. Better locations can be found that yield more reliable data and therefore more reliable noise levels. To update the sound propagation model frequently, the measuring stations not only measure noise levels, but also other parameters, like temperature and wind profile. The sound emission of the aircraft is derived from a directional aircraft noise emission model. A tool was developed to visualize the power of combining data and models in this model-based monitoring system. The use of models opens possibilities for interesting applications such as i) short term noise forecasts, ii) scenario studies with various aircraft distributions and iii) enforcement of noise limits.

\section{Contributed Papers}

12:00

2aPAc12. The variability of acoustical turbulence in the atmospheric boundary layer. Sylvain Cheinet (ISL, 5 Rue du General Cassagnou, BP 70034, 68301 Saint-Louis, France, sylvain.cheinet@isl.eu)

Outside sound propagation is affected by small-scale turbulence in the atmospheric boundary layer through refractive processes. These effects modulate the performance of acoustical sensors, and are at the basis of the atmospheric remote sensing by sodar. Previous studies have documented the impact of a mean turbulence profile or a statistical distribution of turbulence. In this study, we use a high-resolution atmospheric model called LES to reveal the spatial and temporal variability of the small-scale turbulence. First, we introduce some standard results on the role of various eddy sizes on acoustical propagation. Second, we discuss the methodology to derive the acoustical refractive index fluctuations from LES data. Third, we analyze the LES prediction in the case of a fair-weather boundary layer, emphasizing the impact of large-scale convective organization. Finally, we discuss some challenges in attempting to predict the acoustical turbulence from presentday weather forecasts.
12:20

2aPAc13. Sound propagation in areas with a complex meteorology: a meteorological-acoustical model. Frits Van Der Eerden (TNO - Science and Industry, Stieltjesweg 1, 2628 CK Delft, Netherlands, frits.vandereerden@tno.nl), Frank Van Den Berg (TNO - Science and Industry, Stieltjesweg 1, 2628 CK Delft, Netherlands, Frank.vandenBerg@tno .nl)

Long range sound propagation is largely affected by the vertical wind and temperature gradients. These gradients are more complicated in areas where the meteorology can be complex, such as: coastal areas, islands, and lake districts. Furthermore, the gradients usually vary as a function of the horizontal distance, for instance at water-land crossings near a coast or a lake. As a result the sound propagation in these areas cannot be calculated with "standard" acoustic models, such as the ISO 9613 or Harmonoise engineering model. By using an advanced meso-scale meteorological model, which is using large-scale weather forecast data, and an acoustical model, that incorporates horizontal and vertical meteorological variations, long range sound propagation in such complex areas is calculated. In this paper 
we present two applications of this hybrid meteorological-acoustical calculation scheme: i) propagation of impulse noise in a coastal area, and ii) propagation of industrial noise in a rural area with a lake. For the coastal area sound level contours have been calculated as a function of the meteorology for a complete year. For the lake area the effect of the lake on the sound propagation is demonstrated.

\section{2:40-2:00 Lunch Break}

\section{Contributed Papers}

\section{2:00}

2aPAc14. Horizontal wave number spectra of atmospheric acoustic fields in range-dependent environments. Kenneth E Gilbert (National Center for Physical Acoustics, University of Mississippi, University, MS 38677, USA, kgilbert@olemiss.edu), Xiao Di (National Center for Physical Acoustics, University of Mississippi, University, MS 38677, USA, xiaodi@olemiss.edu), Santosh Parakkal (National Center for Physical Acoustics, University of Mississippi, University, MS 38677, USA, sparakka @ olemiss.edu)

The parabolic equation (PE) is a powerful method for computing sound propagation in range-dependent environments. However, the PE gives only the total field, with no information on the horizontal wave number spectrum of the acoustic field. In many situations, e.g., for propagation over irregular terrain, the horizontal wave number spectrum is valuable for analyzing the modal content of the acoustic field. For example, one might want to know the strength of the surface wave component at different ranges. A spectral decomposition method developed by Gilbert and Evans for ocean acoustics is applied here to atmospheric propagation over irregular terrain. This paper shows that for a PE field $p(z)$ at range $r$, the horizontal wave number spectrum $\phi(\kappa, z)$ can be obtained directly and efficiently by solving a onedimensional elliptic equation that uses the field $p(z)$ as a source term. The derivation of the spectral decomposition equation is given along with a numerical method for solving it. Examples are presented and discussed for propagation over irregular terrain. [Research supported by the U. S. Army TACOM-ARDEC at Picatinny Arsenal, New Jersey]

\section{2:20}

2aPAc15. Numerical modeling of sonic boom propagation from hypersonic aircraft. Alexandra Loubeau (Institut Jean Le Rond d'Alembert, Université Pierre et Marie Curie, Boites 161 et 162, 4 place Jussieu, 75252 Paris Cedex 05, France, loubeau@1mm.jussieu.fr), François Coulouvrat (Institut Jean Le Rond d'Alembert, Université Pierre et Marie Curie, Boites 161 et 162, 4 place Jussieu, 75252 Paris Cedex 05, France, coulouvr@ccr.jussieu.fr)

A numerical study of sonic boom propagation from hypersonic aircraft is performed including the effects of nonlinearity, atmospheric absorption and dispersion, and atmospheric stratification. A second-order split-step algorithm, which alternates application of nonlinearity in the time domain and complex absorption in the frequency domain, allows for a faster convergence of results with fewer range steps than with conventional first-order algorithms. Nonlinearity is calculated using the potential, the integral of the pressure, as proposed by Burgers and later applied to sonic booms by Hayes et al. This method, an alternative to Landau's law of equal areas, efficiently locates the shock position by selecting the maximum potential in multivalued regions. Definition of atmospheric absorption at high altitudes is important for modeling the propagation of sonic booms from hypersonic aircraft. Some aspects of an extended absorption model by Sutherland and Bass are adopted, therefore, which extend absorption predictions above the $20 \mathrm{~km}$ limit of the current ISO and ANSI standards. The study is completed using the meteorological conditions at two locations, Le Havre, France and Edwards Air Force Base, CA, USA, over the course of a year. [Work supported by European Union through ATLLAS AST5-CT-2006-030729, meteorological data provided by ECMWF.]

\section{2:40}

2aPAc16. Road noise: characterization and estimation of uncertainty due to meteorological effects. David Ecotiere (Lab. Régional des Ponts et Chaussées, 11, rue Jean Mentelin, BP 9, 67035 Strasbourg Cedex 2, France, david.ecotiere@equipement.gouv.fr)

Meteorological effects can lead to important temporal fluctuations of the sound level in outdoor sound propagation. These fluctuations lead to sound level uncertainties that are rarely estimated. A method based on the coupling of a model of sound propagation and a temporal micrometeorological model is used to quantify sound level fluctuations only due to meteorological fluctuations, over a very long period (typ. 10-30 years). Some statistical analysis are presented: diary or seasonal fluctuations, influence of the duration of observation on the accuracy of the estimation of a LAeq. A semi-analytical method is also proposed for characterizing the uncertainty of the sound level of a distribution of punctual sources. An application to a road noise source is presented.

\section{3:00}

2aPAc17. A numerical study of sound propagation over urban canyons. Martin Schiff (Division of Applied Acoustics, Chalmers University of Technology, 41296 Göteborg, Sweden, schiff@student.chalmers.se), Maarten Hornikx (Applied Acoustics, Chalmers University of Technology, Sven Hultins Gata 8a, SE-41296 Gothenburg, Sweden, maarten.hornikx@chalmers.se), Jens Forssén (Division of Applied Acoustics, Chalmers University of Technology, 41296 Göteborg, Sweden, jens .forssen@chalmers.se)

Because quiet areas in dense urban environments are important, there is high interest in propagation to areas shielded from direct road traffic noise. Sound levels in shielded areas are strongly influenced by distant sources, so intermediate propagation factors such as metrology, screening, and intermediate canyons must therefore be addressed in a realistic propagation model. A numerical investigation of sound propagation across the open tops of intermediate urban canyons has been performed, using the Parabolic Equation and Equivalent Sources methods. Results have been collected for various canyon geometries, and the influence of multiple canyons, canyon/rooftop absorption, variable rooftop height, and correlated versus uncorrelated source models has been investigated. By characterizing the "insertion loss" of canyons intermediate to the source and receiver, the influence of these intermediate canyons could be addressed simply, without the overhead of a detailed numerical calculation.

\section{3:20}

2aPAc18. A validation test for the Acoustic Footprint Mission Planning System. Doru Velea (QinetiQ North America, Technology Solutions Group, 12030 Sunrise Valley Dr., Suite 400, Reston, VA 20191, USA, dvelea@plansys.com), Troy D. Schultz (Wyle Laboratories Inc., 241 18th Street S., Suite 701, Arlington, VA 22202, USA, troy.schultz@wylelabs.com), Kenneth J. Plotkin (Wyle Laboratories Inc., 241 18th Street S., Suite 701, Arlington, VA 22202, USA, kenneth.plotkin@wylelabs.com), Andy Rogers (QinetiQ North America, Technology Solutions Group, 12030 Sunrise Valley Dr., Suite 400, Reston, VA 20191, USA, arogers@plansys.com)

QinetiQ North America, Technology Solutions Group (QNA) and Wyle Laboratories have integrated two mature, proprietary technologies into a prototype Acoustic Footprint Mission Planning System. Wyle's Noise Model Simulation (NMSim) uses validated linear noise propagation routines in combination with ray-tracing algorithms based on weather data provided by QNA's Precision Airdrop System (WindPADS) to predict the time his- 
tory of spectral noise levels from a flying aircraft at any number of userdefined receptor locations. Through interpolation over a ground-based receptor grid, the impact of noise propagated through a stratified atmosphere may be quantified in terms of audibility at any point inside the acoustic footprint. Weather data for the NMSim ray-tracing routines is extracted from the WindPADS three-dimensional forecast of wind, pressure, temperature and humidity in the atmosphere as a function of time. The three-dimensional field is the result of physical and dynamic assimilation of forecast and measured atmospheric data, with wind-forcing by the underlying terrain. This paper describes the results of a validation test performed at Yuma Proving Ground, AZ. It is shown that the noise propagation model predictions agree satisfactorily with the measurements of a C-130 cargo plane. [Work supported by Natick Soldier Center.]

3:40-5:20 Posters

Lecture sessions will recess for presentation of poster papers on various topics in acoustics. See poster sessions for topics and abstracts.

\section{Contributed Papers}

\begin{abstract}
5:20
2aPAc19. Modal probabilistic analysis. Christophe Heinkele (ENTPE/DGCB, Rue Maurice Audin, 69518 Vaulx-en-Velin, France, heinkele@entpe.fr), Claude-Henri Lamarque (rue Maurice Audin, 69100 Vaulx-en-Velin, France, lamarque@entpe.fr)

In this paper, we first recall a method for estimating the numerical Probability Density Function (PDF) with Parzen-Rosenblatt estimators of experimental data (like the sound absorption coefficient with the Kundt's tube). Then we focus on the model of Mikki which is used as a predictive tool. We are interested then in inversing the model and in identifying the 3 parameters (q, $\sigma, \phi)$ of Mikki's model. But we want to go further and to identify from the estimated PDF directly the PDF of the 3 parameters. We explain first when it is possible, then we give some examples to illustrate the method. This work allows for evaluating the robustness of a model from experimental data.
\end{abstract}

\section{$5: 40$}

2aPAc20. An experimental evaluation of a new approach to aircraft noise modelling. Foort De Roo (TNO Science and Industry, Stieljesweg 1, 2628CK Delft, Netherlands, foort.deroo@tno.nl), Erik Salomons (TNO Science and Industry, Stieljesweg 1, 2628CK Delft, Netherlands, erik .salomons@tno.nl)

Common engineering models for aircraft noise, such as INM, yield noise levels by interpolation of Noise Power Distance (NPD) tables. In the European project Imagine (2004 2006), a different approach was proposed: the source is characterized by an emission spectrum and the received noise spectrum is calculated by subtracting the propagation attenuation spectrum from this emission spectrum. This is the usual approach for noise mapping of most noise sources. The aircraft emission spectrum is a function of (downward) emission direction, so each aircraft is represented by a hemisphere of emission spectra. This has been described by Butikofer in Acta Acustica 93 (2007). As hemisphere emission data are not yet available for all aircraft types, a 'reverse engineering' scheme was developed within Imagine to derive first order estimates of hemispheres from NPD tables. To gain experience with this approach, we have performed an experiment near Amsterdam airport. Various types of data were collected for a set of aircraft departures, including noise data at eleven positions and flight data. The Imagine approach was used to calculate noise contours, and noise spectra at the eleven positions. The differences between measured and calculated spectra may be used as a basis for improving the first order estimates of the hemispheres.

\section{6:00}

2aPAc21. Sound propagation in a street canyon: A study by modal decomposition. Adrien Pelat (Laboratoire d'Acoustique de l'Université du Maine, Avenue Olivier Messiaen, 72085 Le Mans, France, adrien.pelat.etu@univ-lemans.fr), Simon Félix (Laboratoire d'Acoustique de l'Université du Maine, Avenue Olivier Messiaen, 72085 Le Mans, France, simon.felix@univ-lemans.fr), Vincent Pagneux (Laboratoire d'Acoustique de l'Université du Maine, UMR CNRS 6613, AV. O. Messiaen, 72085 Le Mans, France, vincent.pagneux@univ-lemans.fr), Christophe
Ayrault(LAUM, CNRS, Université du Maine, Av. O. Messiaen, 72085 Le Mans, $\quad$ France, christophe.ayrault@univ-lemans.fr), Olivier Richoux (Laboratoire d'Acoustique de l'Université du Maine, Avenue Olivier Messiaen, 72085 Le Mans, France, olivier.richoux@univ-lemans.fr)

An urban, U-shaped, street canyon being considered as an open waveguide in which the sound may propagate, one is interested in a multimodal approach to describe the sound propagation within. The key point in such a multimodal formulation is the choice of the basis of local transversal modes on which the acoustic field is projected. For a classical waveguide, with a simple and bounded cross-section, a complete orthogonal basis can be analytically obtained. The case of an open waveguide is more difficult, since no such a basis can be exhibited. However, an open resonator, as displays for example the U-shaped cross-section of a street, presents resonant modes with complex eigen frequencies, owing to radiative losses. This work first presents how to numerically obtain these modes and, then, how they can be used as a basis for the modal decomposition of the sound field in a street canyon. Results are compared with experimental measurements on a scale model.

\section{6:20}

2aPAc22. Acoustical waves propagation in rough walls street. Bertrand Lihoreau (Laboratoire d'Acoustique de l'Université du Maine, Avenue Olivier Messiaen, 72085 Le Mans, France, bertrand.lihoreau@univ-lemans.fr), Simon_ Félix (Laboratoire d'Acoustique de l'Université du Maine, Avenue Olivier Messiaen, 72085 Le Mans, France, simon.felix@univ-lemans.fr), Claude Depollier (Laboratoire d'Acoustique de 1'Université du Maine, Avenue Olivier Messiaen, 72085 Le Mans, France, claude.depollier@univ-lemans.fr)

We present a method for the numerical calculation of a sound wave propagating in a two dimensional rough-sided street. Is this situation, the wave is subject to multiple scattering at the two boundaries. The propagation is governed by a parabolic equation. We show that the amplitude of a pulse may be expressed as the fractional Fourier transform of the incident pulse, and that the order of the transformation is related to the distance between the source and the point of observation. Numerical simulations are proposed as illustration of this model and are compared to experimental results.

\section{6:40}

2aPAc23. A high-density infrasound array of particle velocity sensors in the Netherlands. Arnout Tim Van Zon (Royal Netherlands Meteorological Institute (KNMI), PO Box 201, 3730 AE De Bilt, Netherlands, zon@knmi.nl), Laeslo G. Evers (Royal Netherlands Meteorological Institute (KNMI), PO Box 201, 3730 AE De Bilt, Netherlands, evers@knmi.nl)

A small aperture, High-Density Infrasound Array (HDIA) is being operated by the Royal Netherlands Meteorological Institute (KNMI). This 80 sensor array is part of the geophysical application within LOFAR, i.e. an astronomical low-frequency array in the Netherlands. HDIA occupies an area of 100 by 100 meter, so about the size of a noise reducer used in infrasound arrays for verification purposes. HDIA will be able to estimate the wind-noise correlation length, which has implications for noise reducer design. Of the 80 instruments, 74 are Microflowns. These measure particle velocity and have a directional sensitivity. If two of them are closely spaced, then the azimuth of the incoming sound wave can be calculated from the 
amplitude ratio. The field setup has 37 elements, each containing a pair of Microflowns. Six elements also have a pressure microphone. We will present the first results of using the vector properties of the particle velocity to estimate the direction of arrival (DOA) of events. These will be compared with DOA-estimates from beamforming of 1) the pressure data, 2) the particle velocity data and 3) pressure data of a nearby 6-element microbarometer array. Furthermore, the observed correlation length of wind will be discussed.

TUESDAY MORNING, 1 JULY 2008

ROOM 362/363, 8:00 A.M. TO 1:20 P.M.

\title{
Session 2aPAd
}

\section{Physical Acoustics: Photoacoustics I}

\author{
Gerald Diebold, Cochair \\ Department of Chemistry, Brown University, Providence, Rhode Island 02912, USA \\ Christ Glorieux, Cochair \\ Lab. ATF, Katholieke Universiteit Leuven, Celestijnenlaan 200D, Leuven, B-3001, Belgium
}

\section{Invited Papers}

\section{8:00}

\begin{abstract}
2aPAd1. On the optical generation and detection of high frequency ultrasounds: thermal and non-thermal processes. Pascal Ruello (LPEC/UMR 6087/CNRS/Université du Maine, Avenue Olivier Messiaen, 72085 Le Mans Cedex 09, France, pascal.ruello@univ-lemans.fr), Philippe Babilotte (LPEC/UMR 6087/CNRS/Université du Maine, Avenue Olivier Messiaen, 72085 Le Mans Cedex 09, France, philippe.babilotte.etu@univ-lemans.fr), Shuo Zhang (INSP - UMR 7588 CNRS \& Université Pierre et Marie Curie, 140 Rue de Lourmel, 75015 Paris, France, Shuo.Zhang @insp.jussieu.fr), Denis Mounier (LPEC/UMR 6087/CNRS/Université du Maine, Avenue Olivier Messiaen, 72085 Le Mans Cedex 09, France, denis.mounier@univ-lemans.fr), Jean-Marc Breteau (LPEC/UMR 6087/CNRS/Université du Maine, Avenue Olivier Messiaen, 72085 Le Mans Cedex 09, France, jean-marc.breteau@univ-lemans.fr), Mathieu Edely (LPEC/UMR 6087/CNRS/Université du Maine, Avenue Olivier Messiaen, 72085 Le Mans Cedex 09, France, mathieu.edely@univ-lemans.fr), Patrick Laffez (Université François Rabelais, Tours, IUT Blois, 6, place Jean Jaures, 41029 Blois, France, patrick.laffez@univ-tours.fr), Alain Bulou (LPEC/UMR 6087/CNRS/Université du Maine, Avenue Olivier Messiaen, 72085 Le Mans Cedex 09, France, alain.bulou@univ-lemans.fr), Bernard Perrin (INSP - UMR 7588 CNRS \& Université Pierre et Marie Curie, 140 Rue de Lourmel, 75015 Paris, France, bernard.perrin@insp.jussieu.fr), Vitali Gusev (LPEC/UMR 6087/CNRS/Université du Maine, Avenue Olivier Messiaen, 72085 Le Mans Cedex 09, France, vitali.goussev@univ-lemans.fr)
\end{abstract}

We report on the results of the generation and detection by femtosecond laser pulses of the acoustic waves at frequencies of tens to hundreds $\mathrm{GHz}$ in semiconductors and in oxides compounds exhibiting phase transition. We focus first on the generation mechanisms involved to achieve opto-acoustic transformations. Particular attention will be paid to the cases where classical thermal effects (thermoelastic coupling) drive the mechanism of generation of acoustic phonons and those where non-thermal effects become significant and sometimes dominant sources of acoustic phonons field. In the latter cases, we will especially focus on the phonons generation based on photo-induced modifications of microscopic internal electric fields (potential deformation) and also on the use of photoexcited carriers dynamics (carriers recombination) as a tuning parameter of the photo-generated ultrasounds spectrum. Secondly, we give the examples demonstrating that the choice of optical frequency for ultrasound detection influences not only the amplitude of the detected signal but provides an opportunity to detect separately high or low frequencies in the spectrum of ultrasounds. This study was supported by CPER and ANR project No. BLAN06-3-136284.

8:20

2aPAd2. Optical detection of longitudinal and shear acoustic waves with laser picosecond acoustics. Osamu Matsuda (Division of Applied Physics, Graduate School of Engineering, Hokkaido University, 0608628 Sapporo, Japan, omatsuda@eng.hokudai.ac.jp), Oliver B. Wright (Division of Applied Physics, Graduate School of Engineering, Hokkaido University, 0608628 Sapporo, Japan, olly@eng.hokudai.ac.jp), David H. Hurley (Materials Characterization Department, Idaho National Laboratory, P.O.Box 1625, Idaho Falls, ID 83415-2209, USA, David.Hurley@inl.gov), Vitali Gusev (LPEC/UMR 6087/CNRS/Université du Maine, Avenue Olivier Messiaen, 72085 Le Mans Cedex 09, France, vitali.goussev@univ-lemans.fr), Ken’Ichi Shimizu (University Chemical Laboratory, Keio University, 4-1-1 Hiyoshi, Kohoku-ku, 223-8251 Kanagawa, Japan, shimizuk@econ.keio.ac.jp)

The absorption of picosecond light pulses in a medium can generate sub-THz acoustic waves. These cause a transient optical reflectance change that can be monitored by delayed probe light pulses. This technique, termed laser picosecond acoustics, can be used for the nondestructive evaluation of the physical properties of thin films and substrates. This paper describes a general method for quantitative analysis of such reflectance changes. It is applicable to multiple anisotropic layers that may be opaque or transparent. Longitudinal or shear acoustic waves propagating along the stacking direction of the multilayers modulate the dielectric permittivity anisotropically and inhomogeneously through the photoelastic effect, through local rotation, or through the surface and interface displacements. We describe how the optical reflectance for obliquely incident probe light can be calculated for the modulated medium. 
We then demonstrate the method with reference to experimental results for a sample consisting of a silica film on a zinc substrate in which both longitudinal and shear acoustic waves are generated and detected. The analysis yields the film thickness, sound velocity, and photoelastic tensor components, for example. The method is also applicable to various light scattering problems involving the inhomogeneous modulation of optical properties such as in photothermal experiments.

\section{Contributed Papers}

8:40

2aPAd3. Experimental studies of generation and propagation of high frequency acoustic waves in various solid materials using ultraviolet picosecond laser pulses. Eirini Tzianaki (Department of Electronics, Technological Educational Institute of Crete, Romanou 3, 73133 Chania, Greece,i.tzianaki@mail.chania.teicrete.gr), Michael Tatarakis (Department of Electronics, Technological Educational Institute of Crete, Romanou 3, $73133 \quad$ Chania, Greece, m.tatarakis@chania.teicrete.gr), Makis Bakarezos (Department of Music Technology and Acoustics, Technological Educational Institute of Crete, 1 E. Daskalaki Str., 74100 Rethymnon, Greece, bakarezos@stef.teicrete.gr), Maria Elefteriou (Department of Music Technology and Acoustics, Technological Educational Institute of Crete, 1 E. Daskalaki Str., 74100 Rethymnon, Greece, marel@physics.uoc.gr), Nektarios Papadogiannis (Department of Music Technology and Acoustics, Technological Educational Institute of Crete, 1 E. Daskalaki Str., 74100 Rethymnon, Greece, npapadogiannis@stef.teicrete.gr), Spyros Kazianis (Department of Physics, University of Ioannina, 45110 Ioannina, Greece, skaziannis@in.gr), Costas Kosmidis (Department of Physics, University of Ioannina, 45110 Ioannina, Greece, kkosmidis@uoi.gr), Andreas Lyras (Department of Physics, University of Ioannina, 45110 Ioannina, Greece, alyras@uoi.gr)

The generation of high frequency acoustic waves by picosecond laser pulses in the ultraviolet region and their detection by optical interferometric schemes, is presented. The two main acoustical modes, longitudinal and shear are clearly apparent in the time resolved spectra of solid materials, for various absorbing energies, extending from the thermoelastic to the ablative regime. The ultraviolet light is strongly absorbed by insulator materials like Pyrex and thus strong elastic waves are produced. From the time separation of the longitudinal waves we have deduced values for the speed of sound in various materials and of different thickness, that are in very good agreement with those reported in the literature. Also the time bandwidth of the sound waves is measured and significant differences, originating from different sample thickness, are apparent.

\section{9:00}

2aPAd4. Effect of photoacoustic generation mechanisms on transient grating measurements of thin films on silicon. Alexei A. Maznev (Department of Applied Physics, Hokkaido University, Kita 13 Nishi 8, Kita-ku, 060-8628 Sapporo, Japan, alexei.maznev@gmail.com)

Laser induced transient gratings technique is widely used for noncontact measurements of surface acoustic waves (SAWs). One major application of such measurements is characterization of thin films used in microelectronics. A peculiar phenomenon has been observed in transient gratings measurements of thin films on a silicon substrate: if the film is transparent, there is a systematic discrepancy between the measured and expected SAW velocity values that cannot be accounted for by instrumental errors. However, as soon as the sample is coated by an opaque metal film, the discrepancy disappears. In this presentation, we will show that the origin of the effect is in the competition of thermal and electronic mechanisms of the optical generation of sound in silicon. We will also describe another effect of the same origin observed on very thin semi-transparent metal films: at a certain film thickness, the SAW signal vanishes as the thermal expansion of the film and the contraction of silicon caused by photoexcited carriers cancel each other.

$$
\text { 9:20 }
$$

2aPAd5. Thickness measurement of submicron metal coatings on transparent substrate by laser optoacoustic method. Ivan Pelivanov (International Laser Center of Moscow State University, Vorob'yovy Gory, 1, 119992 Moscow, Russian Federation, pelivanov@ilc.edu.ru), Daria Kopylova (International Laser Center of Moscow State University, Vorob'yovy Gory, 1, 119992 Moscow, Russian Federation, dskopylova@mail.ru), Nataliya Podimova (Moscow State University, MSU, 1, building 2, GSP-2, Leninskiye Gory, 119992 Moscow, Russian Federation, npodymova@mail.ru), Alexander Karabutov (Moscow State University, MSU, 1, building 2, GSP-2, Leninskiye Gory, 119992 Moscow, Russian Federation, akarabutov@gmail.com)

New nondestructive method for measurement of the thickness of submicron metal coatings on a transparent dielectric substrate is developed. Theoretical background includes the consecutive solution of the thermal and acoustic problems. The experimental part is based on the measurement of frequency dependence of the laser-ultrasound excitation efficiency on the metal coating thickness for the system where the coating is covered by a transparent liquid. Three chrome coatings of various thicknesses $(0.2,0.3$ and 0.6 micrometers) deposited on a quartz substrate were tested experimentally. A rough estimate of their thickness was obtained from the sputtering time. Two different experimental modes were used: forward mode (laser pulses irradiate the metal film through the substrate, excited acoustical transients are detected in the liquid) and backward mode (both laser irradiation of the film and detection of excited acoustical transients are performed in the liquid). Spectral dependencies of the thermo-optical transformation efficiency were calculated analytically and obtained experimentally for both signal detection modes. The values of the film thicknesses were determined by the least squares fitting of the theoretical curves to experimental data. It is demonstrated, that the developed optoacoustic method can be used for metal coatings thickness measurement in the range of $50 \mathrm{~nm}-5$ microns with inaccuracy of $50 \mathrm{~nm}$.

\section{9:40-10:00 Break}

\section{Invited Papers}

\section{0:00}

2aPAd6. Acoustic phenomena in porous media studied by transient grating spectroscopy: a critical test of the Biot theory. Riccardo Cucini (European Lab. for Non-Linear Spectroscopy (LENS), Univ. di Firenze, via Nello Carrara 1, 50019 Sesto Fiorentino (Fi), Italy, cucini@lens.unifi.it), Andrea Taschin (European Lab. for Non-Linear Spectroscopy (LENS), Univ. di Firenze, via Nello Carrara 1, 50019 Sesto Fiorentino (Fi), Italy, taschin@lens.unifi.it), Paolo Bartolini (European Lab. for Non-Linear Spectroscopy (LENS), Univ. di Firenze, via Nello Carrara 1, 50019 Sesto Fiorentino (Fi), Italy, bart@ lens.unifi.it), Renato Torre (European Lab. for Non-Linear Spectroscopy (LENS), Univ. di Firenze, via Nello Carrara 1, 50019 Sesto Fiorentino (Fi), Italy, torre@lens.unifi.it)

The propagation of sound in a porous solid filled by a liquid can be described by a phenomenological model introduced by M. A. Biot, that is still extensively used to predict the essential acoustic properties of a wide variety of porous media. Nevertheless testing of the Biot theory at ultra/hypersonic frequencies and in porous media with nanometric scale heterogeneities remains an open issue. We 
studied the propagation of acoustic waves in two liquid-filled porous glasses by heterodyne detected transient grating experiments [1]. A test of the Biot theory is presented under two new regimes, namely, for a new frequency range up to $1.3 \mathrm{GHz}$ and for porous media with nanometric scale heterogeneities [2]. We show that the Biot theory describes the sound velocity data correctly, but does not account for the acoustic attenuation. We suggest that the acoustic damping is mainly due to the dissipation mechanisms intrinsic of the matrix and the liquid which are not accounted for in the theory. [1] Time-resolved spectroscopy of complex liquids, edited by Torre R. (Springer, New York) 2008. [2] A. Taschin, R. Cucini, P. Bartolini, R. Torre, Europhys. Lett. In press.

\section{0:20}

2aPAd7. Laser ultrasonic study of Lamb wave propagation in different film loading configurations. Xiaodong Xu (Modern Acoustics, Institute of Acoustics, Nanjing University, 210093 Nanjing, China, xdxu@nju.edu.cn), G. Shkerdin (Laboratorium voor Akoestiek en Thermische Fysica - Departement Natuurkunde en Sterrenkunde - Katholieke Universiteit Leuven, Celestijnenlaan 200D, B-3001 Leuven, Belgium, shkerdin@yahoo.com), Christ Glorieux (Lab. ATF, Katholieke Universiteit Leuven, Celestijnenlaan 200D, B-3001 Leuven, Belgium, christ.glorieux@ fys.kuleuven.be)

A study is performed concerning the propagation Lamb waves in technologically and scientifically interesting configurations. In a first configuration a thin film was loaded with different liquids on its two sides. Three experimental laser ultrasonic configurations, line excitation with scanning detection, grating excitation with single point detection, and grating excitation with scanning probe beam, were implemented. All experimental results were found to be consistent between experimental methods and with theoretical predictions. The configuration can be implemented in monitoring applications where elastic properties of liquids that are kept in containers can be determined via the outside container wall. In a second configuration laser excited and detected Lamb waves in a rubber-metal bi-layer were used to determine the highly damped elastic properties of rubber.

\section{Contributed Papers}

\section{0:40}

2aPAd8. Zero-group velocity modes and local vibrations of an elastic plate. Claire Prada (Laboratoire Ondes et Acoustique, ESPCI, Université Paris 7, CNRS, 10 rue Vauquelin, 75005 Paris, France, claire.prada-julia@espci.fr), Dominique Clorennec (Laboratoire Ondes et Acoustique, ESPCI, Université Paris 7, CNRS, 10 rue Vauquelin, 75005 Paris, France, dominique.clorennec@espci.fr), Daniel Royer (Laboratoire Ondes et Acoustique, ESPCI, Université Paris 7, CNRS, 10 rue Vauquelin, 75005 Paris, France, daniel.royer@espci.fr)

Elastic plates or cylinders can support guided modes with zero group velocity $(\mathrm{ZGV})$ at a nonzero value of the wave number. The conditions required for the existence of ZGV Lamb modes in isotropic plates are discussed. It is shown that these modes appear in a range of Poisson's ratio over the value for which the cut-off frequency curves of modes belonging to the same family intercept, i.e for a bulk wave velocity ratio equal to a rational number. An interpretation of this phenomenon in terms of a strong repulsion between a pair of modes having a different parity in the vicinity of the cutoff frequencies is given. Using laser-based ultrasonic techniques, we experimentally investigate some properties of these ZGV Lamb modes: resonance, backward wave propagation, interference between backward and forward waves. Experiments performed with materials of various Poisson's ratio demonstrate that the resonance spectrum of an unloaded elastic plate, locally excited by a laser pulse, is dominated by the ZGV Lamb modes. From these local resonance frequencies, thickness variations can be measured accurately and material properties like the Poisson's ratio, bulk acoustic wave velocities or material attenuation can be determined without any mechanical contact.

\section{1:00}

2aPAd9. Mechanical contacts probed with picosecond ultrasonics. Thomas Dehoux (Division of Applied Physics, Graduate School of Engineering, Hokkaido University, 060-8628 Sapporo, Japan, dehoux@eng.hokudai.ac.jp), Motonobu Tomoda (Division of Applied Physics, Graduate School of Engineering, Hokkaido University, 060-8628 Sapporo, Japan, mtomoda@eng.hokudai.ac.jp), Oliver B. Wright (Division of Applied Physics, Graduate School of Engineering, Hokkaido University, 0608628 Sapporo, Japan, olly@eng.hokudai.ac.jp)

We investigate the contact between a thin metal film and a ball bearing indented to nanometre depths using picosecond ultrasonics. The area of contact is acoustically imaged to micron spatial resolution using $\mathrm{GHz}$ acoustic pulses produced by ultrashort pulsed optical excitation of the film through a transparent substrate. In particular, acoustic echoes are detected through transient optical reflectance changes that are monitored by probe optical pulses. In this way we image the acoustic reflection coefficient at the interface between the film and the indenter. In addition, by imaging the arrival time of the acoustic echoes we determine the penetration profile of the indenter to nanometre precision. Furthermore, imaging the transient thermoreflectance gives a different means for measuring the contact area through spatial variations in thermal diffusion. We thus demonstrate that picosecond ultrasonics and thermoreflectance provide powerful tools for the non-contact evaluation of mechanical contacts. These techniques could be applied to the in situ characterization of contact interfaces between machine elements.

\section{Invited Paper}

\section{1:20}

2aPAd10. Photoacoustic imaging and laser-ultrasonics using Fourier domain reconstruction methods. Peter Burgholzer (Upper Austrian Research, Hafenstr. 47, 4020 Linz, Austria, peter.burgholzer@uar.at), Thomas Berer (Upper Austrian Research, Hafenstr. 47, 4020 Linz, Austria, thomas.berer@uar.at), Bernhard Reitinger (Upper Austrian Research, Hafenstr. 47, 4020 Linz, Austria, bernhard.reitinger@uar.at), Robert Nuster (Karl-Franzens-Universität Graz, Universitätsplatz 5, 8010 Graz, Austria, ro.nuster@uni-graz.at), Günther Paltauf (Karl-Franzens-Universität Graz, Universitätsplatz 5, 8010 Graz, Austria, guenther.paltauf @uni-graz.at)

Laser-ultrasonics as well as photoacoustic imaging use optically generated acoustic waves detected at the sample surface to image its interior. In laser-ultrasonics a laser pulse is absorbed at the sample surface generating an ultrasound pulse that propagates into the sample, is subsequently reflected at internal structures, and finally detected at the surface by an interferometer. In photoacoustic imaging ultrasound is generated by heating of light-absorbing structures inside of an optical semitransparent sample. The goal in photoacoustic imaging is to recover the spatial distribution of the absorbed energy density inside the sample from the acoustic pressure signals measured outside the sample (photoacoustic inverse problem). Fourier reconstruction is based on the decomposition into plane waves and is a fast and efficient method used in photoacoustic imaging. Interpolation is needed when signal Fourier components are mapped to 
source Fourier components. We have shown that the synthetic aperture focusing technique (SAFT) in frequency domain, which needs no interpolation, and the Fourier reconstruction method are mathematically equivalent if the step size of the spatial discretization goes to zero. Both imaging methods are compared using simulated data and measurement data acquired with our interferometer set-up. This work has been supported by the Austrian Science Fund (FWF), project P18172-N02 and project L418-N20.

\section{Contributed Papers}

\section{1:40}

2aPAd11. Stroboscopic interferometric full-field imaging of laser-induced surface acoustic waves. Bart Sarens (Laboratorium voor Akoestiek en Thermische Fysica - Departement Natuurkunde en Sterrenkunde - Katholieke Universiteit Leuven, Celestijnenlaan 200D, B-3001 Leuven, Belgium, bart.sarens@fys.kuleuven.be), Osamu Matsuda (Division of Applied Physics, Graduate School of Engineering, Hokkaido University, 0608628 Sapporo, Japan, omatsuda@eng.hokudai.ac.jp), Xiaodong Xu (Modern Acoustics, Institute of Acoustics, Nanjing University, 210093 Nanjing, China, $\quad x d x u @ n j u . e d u . c n)$, Georgios Kalogiannakis (Laboratorium voor Akoestiek en Thermische Fysica - Departement Natuurkunde en Sterrenkunde - Katholieke Universiteit Leuven, Celestijnenlaan 200D, B-3001 Leuven, Belgium, Georgios.Kalogiannakis@vub.ac.be), Robbe Salenbien (Lab. ATF, Katholieke Universiteit Leuven, Celestijnenlaan 200D, B-3001 Leuven, Belgium, robbe.salenbien@fys.kuleuven.be), Renaud Côte (Lab. ATF, Katholieke Universiteit Leuven, Celestijnenlaan 200D, B-3001 Leuven, Belgium, renaud.cote@fys.kuleuven.be), Christ Glorieux (Lab. ATF, Katholieke Universiteit Leuven, Celestijnenlaan 200D, B-3001 Leuven, Belgium, christ .glorieux@fys.kuleuven.be)

Surface acoustic waves (SAW) have the interesting property that they specifically interact with surface and sub-surface regions, rendering them suitable for non-contact investigation of sub-surface properties and heterogeneities. Transmission, reflection and diffraction effects of SAW propagation are analysed in order to reveal information on the region over which they propagate. Laser excitation allows to efficiently excite SAW with short wavelengths, enhancing both the lateral and the depth resolution. Typically information on the waves is collected by scanning a part of the surface of interest with a fast laser probe, using the surface displacement or slope as a real time witness of the wave field. Here we present results obtained by a full field imaging technique [1], in which the sample is repetitively excited by a pulsed pump laser, and the wave displacement field is stroboscopically gathered by illuminating the sample surface with an electronically delayed pulsed probe laser, whose displacement induced optical path variations are visualized by interferometrically analyzing it. In this work, the technique is applied on different samples, varying the geometry of the pump laser beam pattern, the interferometric configuration, and the heterogeneities of the sample. [1] "Phase mask-based interferometer: operation principle, performance, and application to thermo-elastic phenomena", C. Glorieux, J.D. Beers, E.H. Bentefour, K. Van de Rostyne and K.A. Nelson, Rev. Sci. Instrum. 75(9), 2906-2920 (2004).

\section{2:00}

2aPAd12. Laser-ultrasonic defectoscope with focused transducer Alexander Karabutov (Moscow State University, MSU, 1, building 2, GSP-2, Leninskiye Gory, 119992 Moscow, Russian Federation, akarabutov@gmail.com), Nataliya Podimova (Moscow State University, MSU, 1, building 2, GSP-2, Leninskiye Gory, 119992 Moscow, Russian Federation,npodymova@mail.ru)

The novel focused laser-ultrasonic transducer for ultrasonic microscopy of constructive materials is presented. Short and sharp ultrasonic pulse is produced by optoacoustic effect - excitation of ultrasound by absorption of nanosecond laser pulse in special layer. Absorbing layer is integrated with the wide-band ultrasonic receiving system into common unit - laserultrasonic transducer. Ultrasound beam is focused by acoustical lens with relatively low numerical aperture (convergence angle $-15^{\circ}$, focal distance $20 \mathrm{~mm}$ ). An acoustic impedance of the lens matches that of the laserultrasonic transducer. The duration of the irradiated ultrasonic pulse was $\sim 0.1 \mu \mathrm{s}$. The focused laser-ultrasonic transducer was mounted on 3-D linear translation system. The object under control and the transducer was placed in a tank with distilled water. The transducer was moved across the object with $2.5 \mu \mathrm{m}$ step. The caustic of the probe ultrasonic beam was analyses by sliding the transducer across sharp beard of a knife. The reflected ultrasonic signal was recorded by 12-bit ADC. The diameter of the focal area was 0.53 $\mathrm{mm}$. The length of caustic of ultrasonic was $28 \mathrm{~mm}$. The amplitude of the probe ultrasonic pulse was $\sim 20 \mathrm{kPa}$ at laser energy $0.1 \mathrm{~mJ}$. The dynamic range of ultrasonic trace measurement exceeded $90 \mathrm{~dB}$.

\section{2:20}

2aPAd13. Acoustic waves generated by a laser point pulse in a micrometric fiber. Damien Segur (LMP, UMR CNRS 5469, Université Bordeaux I, 351, cours de la Libération, 33405 Talence, France, d.segur@1mp.u-bordeaux1.fr), Alexander Shuvalov (LMP, UMR CNRS 5469, Université Bordeaux I, 351, cours de la Libération, 33405 Talence, France, a.shuvalov@1mp.u-bordeaux1.fr), Yong Dong Pan (Institute of Acoustics, Tongji University, 200092 Shangai, China, ypan@mail.tongji.edu.cn), Nikolay Chigarev (LPEC/UMR 6087/CNRS/Université du Maine, Avenue Olivier Messiaen, 72085 Le Mans Cedex 09, France, Nikolay.Chigarev@univ-lemans.fr), Clément Rossignol (LMP, UMR CNRS 5469, Université Bordeaux I, 351, cours de la Libération, 33405 Talence, France, c.rossignol@1mp.u-bordeaux1.fr), Bertrand Audoin (LMP, UMR CNRS 5469, Université Bordeaux I, 351, cours de la Libération, 33405 Talence, France, b.audoin@1mp.u-bordeaux1.fr)

Having emerged in the 1980s, the laser ultrasonics technique with its non-contact generation and detection process overpasses the difficulties of coupling piezoelectric transducers with curved surfaces. To date, the authors [1] have been interested in acoustic generation for cylinders opaque at a given laser wavelength and for the acoustic source located at the cylinder surface. In this presentation, assuming point focusing of the laser pulses, we propose a three-dimensional (3D) semi-analytical model for acoustic waves generation and propagation in a partly transparent isotropic cylinder. First, the radial displacement at any position on the free surface is derived, in a 3D Fourier domain, for an inner point source. The response to a volume-source distribution along a radius is obtained as a convolution of the above Green function with the corresponding source distribution caused by optical absorption. Three inverse transforms are then applied to obtain the radial displacement at the cylinder surface. Picosecond ultrasonics experiments are performed on different micrometric fibers and compared with calculated waveforms for different optical absorptive properties. References [1] Y.D. Pan, C. Rossignol and B. Audoin, Appl. Phys. Lett. 82, 4379 (2003).

\section{2:40}

2aPAd14. Simulations of Thermally Induced Photoacoustic Wave Propagation Using a Pseudospectral Time-Domain Method. Yae-Lin Sheu (Dept. Electrical Engineering, National Taiwan University, No.1, Sec. 4, Roosevelt Road, 106 Taipei, Taiwan, b88901147@ntu.edu.tw), Pai-Chi Li (Institute of Biomedical Electronics and Bioinformatics, National Taiwan University, No.1, Sec. 4, Roosevelt Road, 106 Taipei, Taiwan, paichi@cc.ee .ntu.edu.tw)

Physical models used to evaluate thermally induced photoacoustic waves in biomedical applications are mostly approximations based on certain hypotheses, such as the thermal and stress confinements, for the sake of obtaining analytical results. On the other hand, using numerical methods to solve the general photoacoustic wave equations gives detailed information of wave phenomena without making as many assumptions. The photoacoustic wave generated by thermal expansion involves the heat conduction theorem and the state, continuity, and Navier-Stokes equations. In this study a numerical approach was developed in $2.5 \mathrm{D}$ axis-symmetric cylindrical coordinates using a pseudospectral time-domain (PSTD) scheme. The method is efficient for large scale simulations in that only two grids for the smallest 
wavelength are required, where in conventional methods $10 \sim 20$ grids are typically needed. The numerical techniques include Berenger's perfectly matched layers (PMLs) for free wave simulations, and linear-perturbation analytical solutions are used to validate the simulation results. The numeri- cal results using 2 grids for the minimum wavelength in simulation domain agree with theory to within an error of $7 \times 10^{-3}$ in the absolute differences. On the other hand, conventional methods such as finite-difference timedomain method requiring 10 grids result in an error of $1.3 \times 10^{-3}$.

\title{
Invited Paper
}

\section{1:00}

2aPAd15. Laser ultrasonic study of Lamb wave propagation in different film loading configurations. Xiaodong Xu (Modern Acoustics, Institute of Acoustics, Nanjing University, 210093 Nanjing, China, xdxu@nju.edu.cn), G. Shkerdin (Laboratorium voor Akoestiek en Thermische Fysica - Departement Natuurkunde en Sterrenkunde - Katholieke Universiteit Leuven, Celestijnenlaan 200D, B-3001 Leuven, Belgium, shkerdin@yahoo.com), Christ Glorieux (Lab. ATF, Katholieke Universiteit Leuven, Celestijnenlaan 200D, B-3001 Leuven, Belgium, christ.glorieux@fys.kuleuven.be)

A study is performed concerning the propagation Lamb waves in technologically and scientifically interesting configurations. In a first configuration a thin film was loaded with different liquids on its two sides. Three experimental laser ultrasonic configurations, line excitation with scanning detection, grating excitation with single point detection, and grating excitation with scanning probe beam, were implemented. All experimental results were found to be consistent between experimental methods and with theoretical predictions. The configuration can be implemented in monitoring applications where elastic properties of liquids that are kept in containers can be determined via the outside container wall. In a second configuration laser excited and detected Lamb waves in a rubber-metal bi-layer were used to determine the highly damped elastic properties of rubber.

TUESDAY MORNING, 1 JULY 2008

ROOM 241, 8:00 A.M. TO 8:00 P.M.

\section{Session 2aPPa}

\section{Psychological and Physiological Acoustics and ASA Committee on Standards: Applications of Psychoacoustics I}

\author{
Patricia Davies, Cochair \\ Ray W. Herrick Lab., School of Mechanical Engineering, Purdue Univ., 140 S. Martin Jischke Drive, \\ West Lafayette, IN 47907-2031, USA \\ Hugo Fastl, Cochair \\ AG Technische Akustik, MMK, TU München, Arcisstr. 21, München, 80333, Germany
}

\section{Invited Papers}

\section{8:00}

2aPPa1. Determination of filtering parameters for dichotic-listening binaural hearing aids. Yôiti Suzuki (R.I.E.C., Tohoku University, 2-1, Katahira, Aoba-ku, 980-8577 Sendai, Japan, yoh@ais.riec.tohoku.ac.jp), Atsunobu Murase (Panasonic Shikoku Electronics Co., Ltd, 600, Saedo-cho, Tsuzuki-ku, 224-8539 Yokohama, Japan, murase.atsunobu@jp.panasonic.com), Motokuni Itoh (Matsushita Electric Industrial Co., Ltd, 600, Saedo-cho, Tsuzuki-ku, 224-8539 Yokohama, Japan, ito.gempo@jp.panasonic.com), Shuichi Sakamoto (R.I.E.C., Tohoku University, 2-1, Katahira, Aoba-ku, 980-8577 Sendai, Japan, saka @ ais.riec.tohoku.ac.jp)

Sensorineural hearing-impaired people have difficulty in hearing sounds not only because of the increased hearing threshold, but also because of their reduced dynamic range of hearing (loudness recruitment), as well as the large and extensive masking resulting from the reduced frequency selectivity, especially masking by which middle-frequency and high-frequency components are masked by the intense low-frequency component, the so-called upward spread of masking. Dichotic listening, listening to complementary filtered speech signals given to the two ears, has been proposed to cope with these problems. We introduce a dichotic listening technique for binaural hearing aids and the relationship between the effect of this technique and auditory characteristics of hearing-impaired people. The results of listening tests imply that some relationship exists between the width of the auditory filter and the effect of this technique. Moreover, intelligibility test results obtained using the low-frequency-boosted sounds suggest that this dichotic listening reduced the upward spread of masking. We are applying this technique to hearing aid systems and have begun evaluating the performance of this hearing aid. 
2aPPa2. Binaural auralization based on spherical-harmonics beamforming. Wookeun Song (Brüel \& Kjær Sound \& Vibration Measurement A/S, Skodsborgvej 307, DK-2850 Nærum, Denmark, wksong@bksv.com), Wolfgang Ellermeier (Institut für Psychologie, Technische Universität Darmstadt, Alexanderstraße 10, D-64283 Darmstadt, Germany, ellermeier@psychologie .tu-darmstadt.de), Jørgen Hald (Brüel \& Kjær Sound \& Vibration Measurement A/S, Skodsborgvej 307, DK-2850 Nærum, Denmark, JHALD@bksv.com)

The binaural auralization of a 3D sound field using spherical-harmonics beamforming (SHB) techniques was investigated and compared with the traditional method using a dummy head. The new procedure was verified by comparing simulated room impulse responses with directly measured ones both monaurally and binaurally. The objective comparisons show that there is good agreement in the frequency range between 0.1 to $6.4 \mathrm{kHz}$. Psychoacoustic attributes of multi-channel reproduced sounds were measured in a listening experiment to validate the method subjectively. The results show that subjective ratings of the width, spaciousness and preference of different audio reproduction modes auralized based on SHB were not significantly different from those obtained for dummy head measurements. Thus binaural synthesis using SHB may be a useful tool to reproduce a 3D sound field binaurally while saving considerably on measurement time because head rotation can be simulated based on a single recording.

\section{$8: 40$}

2aPPa3. Simulation of wave field synthesis. Florian Völk (AG Technische Akustik, MMK, TU München, Arcisstr. 21, 80333 München, Germany, florian.voelk@mytum.de), Josef Konradl (AG Technische Akustik, MMK, TU München, Arcisstr. 21, 80333 München, Germany, koj@mmk.ei.tum.de), Hugo Fastl (AG Technische Akustik, MMK, TU München, Arcisstr. 21, 80333 München, Germany, fastl@mmk.ei.tum.de)

Wave field synthesis utilizes a large number of loudspeakers to generate a desired wave field. It therefore is necessary to drive each speaker with an independent signal, which requires as many amplifier and soundcard channels as there are loudspeakers. These enormeous hardware costs make research and development very expensive and time consuming. Additionally, different rooms influence the wave field synthesis arrays in different ways. For this reason a simulation technique is of advantage which permits the evaluation of the perceived properties of arbitrary wave field synthesis configurations without the need to physically construct them. This paper proposes a simulation system capable to simulate wave field synthesis systems in different rooms based on physical measurements of one loudspeaker in each room. The techniques used are presented, and possiblities as well as limits of the system are discussed.

\section{9:00}

2aPPa4. The next generation of artificial heads. Janina Fels (Institute of Technical Acoustics, RWTH Aachen University, Neustr. 50, 52056 Aachen, Germany, Janina.Fels@akustik.rwth-aachen.de), Michael Vorlaender (Institute of Technical Acoustics, RWTH Aachen University, Neustr. 50, 52056 Aachen, Germany, mvo@akustik.rwth-aachen.de)

Standardised artificial heads are vital means when it comes to describing the binaural transmission from the sound field into the ear canal or rather the eardrum. In recent years numerous fields of application were created ranging from room acoustics, to product sound design or telecommunications, all based on the well-known KEMAR standard IEC TR 959. In the meantime, however, it has become a well-known fact, that a) specific artificial heads with natural heads or replicas in hearing experiments are superior to standard artificial heads as far as the quality of spatial hearing is concerned and that b) the standardised heads do not comply with the dimensions of an average population (the standard heads are too small). In this contribution first of all today's situation will be assessed and then a possible way will be outlined that could lead to a new future artificial head standard. This includes an adequate match with adult population for various continents, and also new approaches such as children-size artificial heads to measure and fit hearing aids or for new measurement techniques for classroom acoustics.

\section{9:20}

2aPPa5. Auditory memory and evaluation of environmental sounds. Sonoko Kuwano (Graduate School of Human Sciences, Osaka University, 1-2 Yamadaoka, 565-0871 Suita, Osaka, Japan, kuwano@see.eng.osaka-u.ac.jp), Seiichiro Namba (2-7-5-604 Obana, 666-0015 Kawanishi, Hyogo, Japan, QZW00041@nifty.com), Tohru Kato (Otemon Gakuin University, 2-1-15 Nishiai, 5678502 Ibaraki, Osaka, Japan, tkatou@ res.otemon.ac.jp)

A series of experiments were conducted concerning the memory of environmental sounds. Twelve kinds of sound were presented with soft background noise of about 6 min to participants. They were asked to recall or recognize the sound sources and to judge the loudness of the recalled or recognized sounds some period after they listened to the sounds. The recalled or recognized loudness was examined in relation to the length of the period between the presentation of sounds and the judgment of loudness, the method to measure the memory, LAeq of each sound source, etc. The results suggest that it is possible to judge the loudness of the memorized sounds and that the judgments seems reliable. 
2aPPa6. Comparison of subjective impression of copy machine noise between Japanese, American and German participants. Tatsuya Furukawa (Ricoh Co., Ltd., 16-1 Shinei-cho, Tsuzuki-ku, 224-0035 Yokohama, Japan, furukawa@rdc.ricoh.co.jp), Osamu Takehira (Ricoh Co., Ltd., 16-1 Shinei-cho, Tsuzuki-ku, 224-0035 Yokohama, Japan, osamu.takehira@nts.ricoh.co.jp), Masaki Nagamiya (Ricoh Co., Ltd., 16-1 Shinei-cho, Tsuzuki-ku, 224-0035 Yokohama, Japan, masaki.nagamiya@nts.ricoh.co.jp), Sonoko Kuwano (Graduate School of Human Sciences, Osaka University, 1-2 Yamadaoka, 565-0871 Suita, Osaka, Japan, kuwano@see.eng.osaka-u.ac.jp), Seiichoro Namba (Graduate School of Human Sciences, Osaka University, 1-2 Yamadaoka, 5650871 Suita, Osaka, Japan, namba.seiichiro@nifty.com), Hugo Fastl (AG Technische Akustik, MMK, TU München, Arcisstr. 21, 80333 München, Germany, fastl@mmk.ei.tum.de)

Psychological evaluations of copy machine noise are in progress to lower acoustical noise and to avoid disturbing the office environment. Psychological experiments were made for Japanese, American and German participants to examine cultural differences in sensitivity to copy machine noise using the Semantic Differential. Seventeen copy machine and laser printer sounds were used for the experiments. The results were analyzed statistically. The experiments revealed that, just as with Japanese, Americans and Germans were mostly sensitive to "sound pressure levels." However, the second influential factor for Japanese participants was "impulsiveness" but "sharpness" for Americans and Germans. Evaluation formulas for "pleasing" noise using physical values were decided for each country. This suggests that copy machine noise will be evaluated without psychological experiments. The information gathered will possibly be used to adapt copy machine noise to levels that are comfortable to users in different countries.

\section{0:00}

2aPPa7. Applications of psychoacoustics to information technology products. Willem M. Beltman (Intel Corporation, $2111 \mathrm{NE}$ 25th Avenue, M/S JF2-86, Hillsboro, OR 97124, USA, willem.m.beltman@intel.com), Rina A. Doherty (Intel Corporation, 5200 NE Elam Young Parkway, Hillsboro, OR 97124, USA, rina.a.doherty@intel.com), Eric Salskov (University of Twente, Dept. Mechanical Engineering, P.O. Box 217, 7500 AE Enschede, Netherlands, eric.salskov@intel.com), Philip J. Corriveau (University of Twente, Dept. Mechanical Engineering, P.O. Box 217, 7500 AE Enschede, Netherlands, philip.j.corriveau@intel.com), Doug Gabel (Intel Corporation, 2111 NE 25th Avenue, M/S JF2-86, Hillsboro, OR 97124, USA, doug.gabel@intel.com), Eric Baugh (Intel Corporation, 15400 NW Greenbrier Parkway, Beaverton, OR 97006, USA, eric.baugh@intel.com)

Emerging usage models for computing devices require low acoustic noise, for example in home entertainment systems. Studies have shown that not only the overall level, but also the psychoacoustic aspects matter. This paper provides an overview of testing techniques that are used in the information technology industry and outlines two specific case studies. First, an extensive subjective psychoacoustic study was designed and conducted in multiple geographies to determine the aspects of sound that best describe the annoyance to sound from information technology products in a home type environment. Over 200 participants in four countries participated in this carefully controlled experiment and rated typical steady state sounds on a 5 point annoyance scale. The relevant sound quality metrics were extracted and geographical variations quantified. Second, in a paired comparison study the influence of modulation on annoyance was investigated by superimposing different frequency and amplitude modulated sounds onto a baseline sound. The results indicate that modulation can have a significant effect on subjective perception.

\section{0:20-10:40 Break}

\section{0:40}

2aPPa8. Identification of transient events from a hard disk drive using non-stationary loudness. Dave Ali (Western Digital, 5863 Rue Ferrari, San Jose, CA 95127, USA, dave.ali@wdc.com)

The Hard Disk Drive (HDD) industry has been pushed so much to lower its limits of acoustic emissions by OEMs and customers that the levels of most HDDs flirt with the threshold of hearing and in some cases go below. With these much reduced levels come different problems and complaints from customers, namely transient events; latching, de-latching, spin up, spin down, etc. The difference in amplitude and duration of these events typically stand out to the human ear above the steady state nature of the HDD during idle and sometimes when it's active. This paper shows a method of characterizing these events with confidence to help in designing a better product for the industry.

\section{1:00}

2aPPa9. Automotive tire/road sound quality. Gabriella Cerrato Jay (Sound Answers Inc, 4856 Alton Drive, Suite 100, Troy, MI 48085, USA, gabriella.cerratojay@soundanswers.net), Todd Freeman (Sound Answers Inc, 4856 Alton Drive, Suite 100, Troy, MI 48085, USA, todd.freeman@soundanswers.net), Chris Raglin (Cooper Tire \& Rubber Company, 701 Lima Avenue, Findlay, OH 45840, USA, caraglin@ coopertire.com), Timothy Carson (IAC North America, 47785 W Anchor CT, Plymouth, MI 48170, USA, tcarson@iacna.com)

Tire/road noise and sound quality are increasingly important factors for customer satisfaction. As vehicle interior sound levels decrease, the noise from tires and their interaction with the road become more noticeable. Both tire and vehicle manufacturers need to assess as early as possible the impact of any tire-vehicle combination on perceived interior sound quality. In this paper, we describe two projects in which psychoacoustics concepts were applied to help, on one hand, tire manufacturers to screen for designs likely to generate poor sound quality in vehicle, and, on the other hand, vehicle manufacturers to measure the impact of road noise and interior acoustic treatment on speech transmission in the cabin. In both projects, the activities were driven by the understanding of the psychoacoustic features of tire/road noise and by the need to improve customer satisfaction. 


\section{Contributed Paper}

11:20

2aPPa10. Acoustical aspects of travel comfort in the aircraft cabin. Ingo Baumann (Oldenburg University, Institute of Physics - Acoustics, Carl-von-Ossietzky Str. 9-11, 26111 Oldenburg, Germany, ingo @ aku.physik.uni-oldenburg.de), Sandra Buss (Oldenburg University, Institute of Physics - Acoustics, Carl-von-Ossietzky Str. 9-11, 26111 Oldenburg, Germany, sandra@aku.physik.uni-oldenburg.de), Nils Freese (Oldenburg University, Institute of Physics - Acoustics, Carl-von-Ossietzky Str. 9-11, 26111 Oldenburg, Germany, nils_f@aku.physik.uni-oldenburg.de), Volker Mellert (Oldenburg University, Institute for Physics, 26111 Oldenburg, Germany, volker.mellert@uni-oldenburg.de), Reinhard Weber (Oldenburg University, Institute of Physics - Acoustics, Carl-von-Ossietzky Str. 9-11, 26111 Oldenburg, Germany, Reinhard.Weber@uni-oldenburg.de)
In several European projects tests were conducted during real flights and in aircraft simulators in order to identify important parameters of travel comfort. Physical environmental, physiological and questionnaire data were measured and investigated with respect to human perception. Besides numerous intrinsic quantities affecting health, well-being and awareness of ambience defined measurable environmental parameters influence flight and cabin crews' as well as passengers' perception, psychology and physiology. Sound and vibration are relevant environmental parameters with impact on passenger and crew. Other important influence is caused by e.g. air quality, pressure, local climate. Statistical analysis of the collected data reveals significant correlations between environment and human response for selected groups of test persons. A specific acoustic comfort is not well defined but part of common well-being and comfort. The demand for a general human response model is discussed, which relates ambience and perception.

\section{Invited Papers}

\section{1:40}

2aPPa11. The influence of speed bumps on perceived annoyance. Anna Preis (Institute of Acoustics, Adam Mickiewicz University, Unultowska 85, 61-614 Poznan, Poland, apraton@amu.edu.pl), Tomasz Kaczmarek (Institute of Acoustics, Adam Mickiewicz University, Unultowska 85, 61-614 Poznan, Poland, tomek@spl.ia.amu.edu.pl), Barbara Griefahn (Institute for Occupational Physiology, Ardeystr. 67, 44139 Dortmund, Germany, griefahn@ifado.de), Truls Gjestland (O.S. Bragstads plass 2, N-7464 Trondheim, Norway, Truls.Gjestland@sintef.no)

Recently, several attempts to use speed bumps as a noise reduction method have been made. Objective analyses of the effect of speed bumps on noise have been shown to result in a rather small reduction of noise. In the present paper the influence of speed bumps on perceived annoyance is investigated. The annoyance rating of a situation in which a passenger car approaches with constant velocity, then decelerates, crosses the bump, accelerates, and then recedes at a constant speed was compared with a car pass-by at a constant velocity without a bump. Three different velocities were analyzed: 40,50 and $60 \mathrm{~km} / \mathrm{h}$, and two types of driving conditions: normal, and aggressive. Listeners judged their annoyance for all the investigated scenarios using the ICBEN scale (0-10) for annoyance assessment. Objective analyses showed a significant reduction of LAeqT in the bump situation for all tested velocities, and for both driving conditions. The results of this psychoacoustic experiment show no effect of the bump on annoyance rating for normal driving conditions. However, in aggressive driving conditions the bump resulted in a significant increase in annoyance. In the light of these results, speed bumps cannot be considered as a noise reduction method.

\section{2:00}

2aPPa12. The Adequate Sound Levels for Acoustic Signs for Visually Impaired in the Sound Environment with Ambient Musics from shops. Katsuya Yamauchi (Faculty of Engineering, Nagasaki University, 1-14 Bunkyo-machi, 852-8521 Nagasaki, Japan, yamauchi@cis.nagasaki-u.ac.jp), Koji Nagahata (Faculty of Symbiotic Systems Science, Fukushima University, 1 Kanayagawa, 9601296 Fukushima, Japan, nagahata@sss.fukushima-u.ac.jp), Mari Ueda (Faculty of Human-Environment, Kyushu University, 6-19-1 Hakozaki, Higashik-ku, 812-8581 Fukuoka, Japan, mari-u@gsd.design.kyushu-u.ac.jp), Shin-Ichiro Iwamiya (Kyushu University, 4-9-1 Shiobaru, Minami-ku, 815-8540 Fukuoka, Japan, iwamiya@design.kyushu-u.ac.jp)

Providing auditory signs for the visually impaired is one of the most effective ways to support their orientation and mobility. Although the use of such sounds by the visually impaired has been revealed qualitatively, the acoustical properties of ideal sound-designs for them have not known sufficiently, even a basic property such as the sound level of these sounds. On the other hand, the advertising sounds from shops such as ambient musics are the one of the typical sounds in the Japanese downtown. These sounds also disturb their sound information listening and make their mobility difficult. Our previous studies revealed the adequate sound levels of acoustic signs under the road traffic noise environment. This study discusses the the effect of the ambient musics from shops on the adequate sound levels of acoustic signs through the psychoacoustical experiment. The results showed that the difference between the adequate sound level of acoustic signs and the environmental noise levels are larger than that under the road traffic noise environment. This trend is thought to be attributed to the frequency characteristics of the acoustical signs and the environmental noise.

12:20

2aPPa13. An algorithm modelling the Irrelevant Sound Effect (ISE). Sabine J. Schlittmeier (Work, Environmental and Health Psychology, Catholic University of Eichstaett-Ingolstadt, Ostenstr. 26-28, 85072 Eichstaett, Germany, sabine.schlittmeier@ku-eichstaett.de), Tobias Weissgerber (AG Technische Akustik, MMK, TU München, Arcisstr. 21, 80333 München, Germany, tobias@p-weissgerber.de), Stefan Kerber (AG Technische Akustik, MMK, TU München, Arcisstr. 21, 80333 München, Germany, stefan@ihr.mrc.ac.uk), Hugo Fastl (AG Technische Akustik, MMK, TU München, Arcisstr. 21, 80333 München, Germany, fastl@mmk.ei.tum.de), Juergen Hellbrueck (Work, Environmental and Health Psychology, Catholic University of Eichstaett-Ingolstadt, Ostenstr. 26-28, 85072 Eichstaett, Germany, juergen.hellbrueck@ku-eichstaett.de)

Verbal short-term memory capacity is reduced significantly during certain background sounds. Remembering a series of digits is significantly impaired by speech or music with prominent staccato passages whereas, for example, music with prominent legato passages does not disturb performance in comparison to silence. This so-called Irrelevant Sound Effect (ISE) occurs although the background sounds are irrelevant with respect to the digit sequences to be remembered. Until now, a multitude of cognitive psychological experiments explored the ISE and collected behavioral performance data during different sound conditions. The talk presents an algo- 
rithm which models performance data in ISE experiments, i.e. the detrimental impact of background sounds on memory performance. The data base of this algorithm is about 50 background sounds and corresponding performance data, which have been collected in cognitive psychological experiments at the KU Eichstaett-Ingolstadt. The algorithm is based on the instrumental measuring of the hearing sensation fluctuation strength and is able to reconstruct the performance results in about $90 \%$ of cases within the interquartile ranges. The algorithm will be discussed within the scope of cognitive short-term memory models, which claim to explain the ISE and with respect to practical implications.

\section{2:40-2:00 Lunch Break}

\section{Invited Papers}

2aPPa14. Fusion and masking threshold of a tone in narrowband noise. Hans Hansen (IRCAM - Sound Perception \& Design, 1 place Igor Strawinsky, 75004 Paris, France, Hans_Hansen@gmx.de), Reinhard Weber (Oldenburg University, Institute of Physics Acoustics, Carl-von-Ossietzky Str. 9-11, 26111 Oldenburg, Germany, Reinhard.Weber@uni-oldenburg.de)

When comparing different pitch phenomena, it is not clear whether the expression pitch strength points towards a unique perceptive phenomenon or object. As many studies concentrate on one special phenomenon, the question, what is actually judged, seems only implicitly answered. Kubovy and Van Valkenburg (2001) define a perceptual object as "that what is susceptible to figure-ground segregation". Pitch plays a major role in these grouping processes. In this context the judgment of pitch strength refers to two perceptual cases. The first one is the pitch strength of a tone-in-noise, i.e. the case where the pitch is linked to a separate contour within the narrowband noise (NBN), while the second is the pitch strength of tonal noise. Here, the noise evokes a pitch sensation that is not related to a separate object. In order to explore the transition from case $/ 1$ tone-in-noise to case $/ 2$ tonal noise, the identification threshold hearing a tone in NBN centered on the tone is determined in a lab experiment. This segregation or fusion threshold is compared to the measured masking threshold for center frequencies $250-4000 \mathrm{~Hz}$ octave-wise at $60 \mathrm{~dB}$ SPL noise level. The bandwith of the NBN is varied from $50-250 \mathrm{~Hz}$ accordingly.

\section{$2: 20$}

2aPPa15. Are absolute thresholds and loudness judgements influenced by different colours? Daniel Menzel (AG Technische Akustik, MMK, TU München, Arcisstr. 21, 80333 München, Germany, menzel@tum.de), Elias Faccinelli (AG Technische Akustik, MMK, TU München, Arcisstr. 21, 80333 München, Germany, fac@mmk.ei.tum.de), Hugo Fastl (AG Technische Akustik, MMK, TU München, Arcisstr. 21, 80333 München, Germany, fastl@mmk.ei.tum.de)

Previous experiments showed that images of differently colored trains and sports cars can influence the loudness ratings given by subjects via free magnitude estimation. Red vehicles caused subjects to rate the loudness of simultaneously presented train or car sounds higher relative to green vehicles. To investigate whether these loudness differences correspond to shifts in absolute threshold, subject's threshold in quiet was measured via Békésy-tracking while viewing red, green, and neutral color patches. Also, the influence of color on the loudness of broadband noise was measured using a method of adjustment, in which subjects had to adjust the level of a test sound until it was perceived as loud as a reference sound. In both cases, no influence of color on either absolute threshold or loudness perception measured via adjustment could be found. These results support the hypothesis that, compared to previously used methods like magnitude estimation, methods that require subjects to concentrate on the auditory stimulus do not seem to be as applicable for measurements of audio-visual interactions.

\section{2:40}

2aPPa16. An efficient masking model for audio coding exploiting spectro-temporal masking. Steven Van De Par (Philips Research Europe, Digital Signal Processing (MS WO02), High Tech Campus 36, 5656 AE Eindhoven, Netherlands, steven.van.de.par@philips.com), Jeroen Koppens (Philips Research Europe, Digital Signal Processing (MS WO02), High Tech Campus 36, 5656 AE Eindhoven, Netherlands, jeroen.koppens@philips.com), Armin Kohlrausch (Philips Research Europe, Digital Signal Processing (MS WO02), High Tech Campus 36, 5656 AE Eindhoven, Netherlands, armin.kohlrausch@philips.com), Werner Oomen (Philips Research Europe, Digital Signal Processing (MS WO02), High Tech Campus 36, 5656 AE Eindhoven, Netherlands, werner .oomen@philips.com)

Perceptual audio coding achieves part of its coding efficiency by spectrally shaping the quantization noise such that it is masked by the audio signal to be encoded. In order to determine how much quantization noise is allowed within each frequency band and time interval a masking model is used to predict a masking curve specifying the maximally allowed quantization noise level within each frequency band. In most audio coders only spectral masking properties of the audio signal are used. The model by Dau et al. [J. Acoust. Soc. Am. 99, Vol. 3615, 1996] provides an interesting approach to also model temporal masking. Since this model operates as an artificial observer it only predicts whether the quantization noise is audible or not in the presence of the audio signal. In order to determine the most efficient quantization noise shape, the encoder needs to iteratively adapt the noise shape and evaluate each option with the model. This implies a highly computational complex encoding algorithm. In this contribution we will present an efficient masking model based on the Dau et al. model that only requires a single evaluation of the input signal to determine the maximally allowed noise level within each frequency band. 
2aPPa17. Factor analyses of critical-band-filtered speech of British English and Japanese. Kazuo Ueda (Perceptual Psychology Unit, Kyushu University, 4-9-1 Shiobaru, Minami-ku, 815-8540 Fukuoka, Japan, ueda@ design.kyushu-u.ac.jp), Yoshitaka Nakajima (Perceptual Psychology Unit, Kyushu University, 4-9-1 Shiobaru, Minami-ku, 815-8540 Fukuoka, Japan, nakajima@design.kyushu-u .ac.jp)

Two-hundred sentences of British English and Japanese, each uttered by 10 native speakers ( 5 females and 5 males) in each language, were analyzed through 20 bands of critical band filters. Smoothed power fluctuations derived from the filters were submitted to principal component analyses followed by varimax rotation. The first three factors explained $34-37 \%$ of variance. One of the factors exhibited two peaks along frequency axis in the standardized scores and two of the factors showed one peak for each. These three factors divided the whole frequency range of speech sound into four bands. The structure of the factors and frequency bands was essentially the same across the two languages. These frequency bands can be used for speech perception in general, because intelligible noise-vocoded speech sounds can be synthesized with the frequency bands.

\section{Contributed Paper}

\section{3:20}

2aPPa18. A Structuralistic Approach to Acoustic-Auditory Functions of Meaning. Ute Jekosch (Chair of Communication Acoustics, TU Dresden, Helmholtzstr. 10, 01069 Dresden, Germany, ute.jekosch@tu-dresden.de), Ercan Altinsoy (Chair of Communication Acoustics, TU Dresden, Helmholtzstr. 10, 01069 Dresden, Germany, ercan.altinsoy@ias.et.tu-dresden.de), Sebastian Merchel (Chair of Communication Acoustics, TU Dresden, Helmholtzstr. 10, 01069 Dresden, Germany, sebastian.merchel@tu-dresden.de)

In this paper we introduce a methodology of semio-acoustics to get information on how human listeners associate meaning to acoustic-auditory events. We concentrate on identifying cues in the auditory stream listeners base the association of meaning on as well on modelling major characteristics of the reference system of meaning. The methodology we use is closely related to structuralism, an approach that has its origins in semiotics. In principle, structuralism differentiates between creating functions, carrying functions and changing functions of systems of meaning. We concentrate on carrying and changing functions here using the following procedure: a signcarrier (in our case an acoustic-auditory event the association of meaning is based on) is decomposed into sub-units. By a minimal pair analysis we investigate carrying and changing functions of acoustic-auditory features with regard to the associated meaning. We will introduce the methodology used and discuss first results of a pilot study.

3:40-5:20 Posters

Lecture sessions will recess for presentation of poster papers on various topics in acoustics. See poster sessions for topics and abstracts.

\section{Contributed Papers}

\section{$5: 20$}

2aPPa19. Optimization of a dual recognition tasks for speech quality assessment. Virginie Durin (France Télécom, 2 avenue Pierre Marzin, 22300 Lannion, France, virginie.durin@orange-ftgroup.com), Laetitia Gros (France Télécom, 2 avenue Pierre Marzin, 22300 Lannion, France, laetitia.gros@orange-ftgroup.com)

This paper deals with perceptive test methodologies to assess speech quality of telecommunication systems. Faced with drawbacks of typical methodologies recommended by ITU-T, a new way to assess speech quality is investigated. The new approach requires collecting reaction times and performances when subjects are achieving tasks involving degraded speech signals; it is shown that reactions times lengthen and performances decrease in a specific task when quality is impaired. The proposed task is a dual task with a digit recognition memory task and a letter recognition task. Three different quality levels are applied to audio signals describing digits and letters. Different experimental designs are examined to reinforce the effect of speech quality on performances and reaction times. The results show significant differences of performances and reaction times between the three quality levels, depending on the experimental design.

\section{$5: 40$}

2aPPa20. Electronic pass-through hearing protection and directional hearing restoration integrated in a helmet. Wouter $\mathrm{K}$. Vos (TNO, Kampweg 5, 3769ZG Soesterberg, Netherlands, wouter.vos@tno.nl), Adelbert W. Bronkhorst (TNO, Kampweg 5, 3769ZG Soesterberg, Netherlands, adelbert.bronkhorst@tno.nl), Jan A. Verhave (TNO, Kampweg 5, 3769ZG Soesterberg, Netherlands, jan.verhave@tno.nl)

Compared to standard earplugs, electronic pass-through earplugs provide better sound localisation. Provided that the bandwidth is sufficiently wide and the earplugs do not change the shape of the pinnae. However, when a helmet is worn that partially or completely covers the ears, the directional hearing capability is diminished. We attempt to restore directional hearing when wearing a helmet by attaching a microphone array to the helmet. The signals from the microphone array are filtered with Finite Impulse Response (FIR) filters to recreate an individual or generic open-ear Head Related Transfer Function (HRTF). The filters are designed by minimisation of an error measure in the frequency domain. The error measure incorporates both the log magnitude and the phase differences between the original and the recreated HRTF. The global minimum is found using modern optimisation techniques like Particle Swarm Optimisation (PSO) or Differential Evolution (DE). The total system is evaluated with subject experiments. Participants have to localise sounds and rate the quality of sounds. Independent variables are the number of microphones that should be used and their positions on the helmet.

\section{6:00}

2aPPa21. How many psycho-acoustic attributes are needed? Torben Holm Pedersen (Delta Acoustics \& SenseLab, Venlighedsvej 4, 2970 Hørsholm, Denmark, thp@delta.dk), Nick Zacharov (Delta Acoustics \& SenseLab, Venlighedsvej 4, 2970 Hørsholm, Denmark, nvz@delta.dk)

Sounds may be characterized by objective perceptive attributes (for which there may exist physical metrics) or by subjective (affective or connotative) attributes. This paper will deal with the perceptive attributes. Within product sound quality the metrics for classical the psycho-acoustic attributes (loudness, sharpness, roughness and fluctuation strength) -maybe supplemented with tone and impulse prominence- are often used as the only attributes to characterize the sounds. But are these 4-6 attributes or dimensions sufficient to characterize a sound? Within room acoustics and reproduced sound many other attributes are used and in the language around 100 direct sound describing words may be found. This paper will give an overview over attributes used within different acoustic areas. The latter part of the paper will discuss the role of sensory evaluation methods as a means to systematically developing attributes for the objective qualification and quantification of sound characteristics. 


\section{6:20}

2aPPa22. Noise perception of wall-hung gas boilers. Patrick Chevret (01dB-Metravib, 200 Chemin des Ormeaux, 69578 Limonest Cedex, France, patrick.chevret@01db-metravib.com), Anne Coulon (CETIAT, Domaine Scientifique de la Doua, 25 avenue des Arts, BP 2042, F-69603 Villeurbanne cedex, France, anne.coulon@cetiat.fr), François Bessac (CETIAT, Domaine Scientifique de la Doua, 25 avenue des Arts, BP 2042, F-69603 Villeurbanne cedex, France, francois.bessac@cetiat.fr), Etienne Parizet (Laboratoire Vibrations Acoustique, Insa Lyon, 25 bis, av. J. Capelle, 69621 Villeurbanne Cedex, France, etienne.parizet@insa-lyon.fr)
The aim of this study is to assess the quality image for domestic wallhung gas-fired boilers, based on their noise. Nine boilers were recorded using an acoustic manikin in a hemi-anechoic room, for different operating conditions. Two of these operating conditions (maximum heat input, hot water tapping) were first studied. Five-second sequences were presented (through headphones) to sixty listeners using the mixed assessment method allowing for the comparison between signals. Analyses showed several assessment strategies: according to listeners, the relevant noise parameters could be the loudness, the sharpness or the presence of tonal components. A second experimental phase focused on tonal components by artificially modifying some sounds to offer a relevant perceptive indicator. The results of this second experiment will also be presented.

\section{Invited Papers}

\section{6:40}

2aPPa23. Methodological aspects in the determination of the auditory filters and critical band at low and mid-frequencies. Carlos A. Jurado (Acoustics, Aalborg University, Fredrik Bajers Vej 7 B5, 9220 Aalborg Ø, Denmark, cjo@es.aau.dk), Henrik Møller (Acoustics, Aalborg University, Fredrik Bajers Vej 7 B5, 9220 Aalborg Ø, Denmark, hm@ acoustics.aau.dk), Christian Sejer Pedersen (Acoustics, Aalborg University, Fredrik Bajers Vej 7 B5, 9220 Aalborg Ø, Denmark, cp@acoustics.aau.dk)

In order to evaluate loudness or audibility of complex sounds, knowledge of the auditory filter characteristics is necessary. At low frequencies, where both the threshold of hearing and dynamic range become considerably frequency dependent, care must be taken to account for this both in the psycho-acoustical model and the methodological approach. To account for variation in hearing sensitivity at low frequencies, equal loudness contours have been used to weight the stimuli accordingly. At mid and high frequencies, threshold of hearing curves have been used. These stimuli weightings can be applied before or after the experiment, normally being applied afterwards. Due to the non-linear characteristics of the cochlear amplifier, it is arguable whether post-experimental weighting is a proper approach, or whether at low frequencies there will be any difference between pre or post stimuli weighting. Listening experiments are then to be performed to test possible differences in pre or post filtering the stimuli. The most appropriate approach will then be discussed. Measurements will be done at low and mid frequencies. To obtain accurate auditory filter estimates, individual ELC or threshold curves will be determined. Methods such as the notched-noise method and the classical band-widening approach will be tested with these conditions.

\section{7:00}

2aPPa24. Effect of recording/playback technique and experimental method on assessments of noise. Emine Çelik-Christensen (Aalborg University / Rockwool International A/S, Rockwool International, Building Knowledge Center, Hovedgaden 584, Entrance C, 2640 Hedehusene, Denmark, emine.christensen@ rockwool.com), Kerstin Persson Waye (Dept. of Environ. Medicine, The Sahlgrenska Acad. of Gothenburg Univ., Box 414, 40530 Gothenburg, Sweden, kerstin.persson-waye@amm.gu.se), Henrik Møller (Acoustics, Aalborg University, Fredrik Bajers Vej 7 B5, 9220 Aalborg Ø, Denmark, hm@acoustics.aau.dk)

The study investigated possible effects of recording/playback technique and experimental method on assessments of annoyance, loudness and unpleasantness. A possible effect of exposure duration was also studied. Sounds were recorded with two different techniques: monophonic and binaural (dummy-head technique). In addition, they were reproduced with three different techniques: monophonic recordings presented through a loudspeaker system, binaural recordings presented through closed (circum-aural) and completely open (free-of-the-ear headphones. The study adopted three psychometric methods for collecting responses from test-subjects. Fifty-four subjects participated, and three types of sounds were used: everyday restaurant sound, road traffic sound and ventilation sound dominated by low frequencies. Each sound was played back at three different levels. The results show that there is no significant main effect of recording and playback technique for any of the three perceptual attributes; however, significant interactions between techniques and sounds were found. Since the effect of recording and playback technique differs depending on sound, this finding is of importance for future design of experiments and interpretation of results. The results also show that long-term annoyance and unpleasantness are poorly predicted by short-duration methods.

\section{Contributed Papers}

$7: 20$

2aPPa25. Relation between the overall unpleasantness of a long duration sound and the one of its event6: application to a delivery truck. Emilie Geissner (Laboratoire Vibrations Acoustique - INSA Lyon, 25 bis avenue Jean Capelle, Bâtiment Saint-Exupéry, F-69621 Villeurbanne cedex, France, emilie.geissner@insa-lyon.fr), Etienne Parizet (Laboratoire Vibrations Acoustique, Insa Lyon, 25 bis, av. J. Capelle, 69621 Villeurbanne Cedex, France, etienne.parizet@insa-lyon.fr)

The goal of this study was to investigate the link between the unpleasantness assessment of an unstationary long duration sound composed of several distinct sound events and the corresponding judgments of each of those events. For that purpose, a sound sequence of a delivery truck was evaluated by 16 listeners during a test in laboratory: first, subjects had to continuously quantify the perceived unpleasantness of the sequence by moving a sliding cursor along a five levels graduated scale and then give a global rating by using the same scale. In a second step, listeners had to express their overall judgment of unpleasantness for eight samples of the delivery sequence. As previously shown for loudness by Kuwano and Namba (1985), the global rating of the unpleasantness of long sound could not be estimated by the arithmetic mean of the continuous assessment. It also appeared that the overall judgment corresponds to the arithmetic mean on the local values of unpleasantness of each main sound event. This last result was similar to the conclusions of Hellbrück et al. (2001) for the loudness scaling of traffic noise.

\section{$7: 40$}

2aPPa26. Just noticeable differences of loudness and sharpness for earth moving machines. Francesca Pedrielli (CNR-Imamoter, via Canal Bianco 28, 44100 Ferrara, Italy, f.pedrielli@imamoter.cnr.it), Eleonora 
Carletti(CNR-Imamoter, via Canal Bianco 28, 44100 Ferrara, Italy, e.carletti@imamoter.cnr.it), Camilla Casazza (CNR-Imamoter, via Canal Bianco 28, 44100 Ferrara, Italy, c.casazza@imamoter.cnr.it)

This paper describes some results of a research concerning the improvement of the noise climate at the operator station of construction machines during real working conditions. Binaural noise signals were previously recorded and then used in subjective listening tests aimed at identifying the set of acoustic and psychoacoustic parameters which affect the auditory perception of these signals with respect to the annoyance sensation. Results showed that loudness and sharpness are the parameters best correlated to the annoyance. In order to verify the efficacy of some noise control solutions in improving the operator comfort conditions, the next necessary step is to build a specific metrics able to show the minimum differences in these parameters which are subjectively perceived. This paper describes the results of specific listening tests carried out in order to evaluate the differential thresholds of loudness and sharpness by the Method of Limits. The test was repeated at two different signal presentation levels. The loudness value of each original stimulus was varied by interval steps of 0,3 sone, while the Sharpness by interval steps of 0,02 acum. At this moment in time, the data analysis is still in progress and the ultimate results will be presented in the manuscript.

\title{
Session 2aPPb
}

\section{Psychological and Physiological Acoustics: Auditory Perception and Signal Processing by Prostheses I}

\author{
Huanping Dai, Cochair
}

Univ. of Arizona, Speech, Language and Hearing Science, Tucson, AZ 85721, USA

\author{
Birger Kollmeier, Cochair \\ Universität Oldenburg, Medizinische Physik, Carl-von-Ossietzky Str. 9-11, Oldenburg, 26111, Germany
}

\section{Invited Papers}

\begin{abstract}
8:00
2aPPb1. Advantages and disadvantages of fast and slow compression in hearing aids. Brian Moore (University of Cambridge, Department of Experimental Psychology, Downing Street, CB2 3EB Cambridge, UK, bcjm@cam.ac.uk)

Compression is used in hearing aids to compensate for the effects of loudness recruitment. However, there is no consensus about the "best" compression speed. The theoretical advantages and disadvantages of slow and fast compression will be discussed. Studies comparing the relative merits of slow and fast compression have led to a great variety of outcomes. It is argued, following the work of Gatehouse and colleagues, that this is partly the result of a failure to consider individual differences and the auditory ecology of each individual. It is argued that listening in the dips of a fluctuating background sound, such as a competing talker, depends on the ability to process the temporal fine structure (TFS) of sounds, as represented in patterns of phase locking in auditory neurons. For people with a good ability to process TFS, fast compression can amplify sounds in the dips, increasing the effectiveness of dip listening. However, for people with a poor ability to process TFS, envelope cues may be critical for speech intelligibility and fast compression may disrupt such cues. It is proposed that a test of the ability to process TFS might be useful for selecting compression speed for an individual.
\end{abstract}

8:20

2aPPb2. Dynamic compression in hearing aids based on an auditory model. Volker Hohmann (Carl von Ossietzky Universität Oldenburg, Ammerländer Heerstraße 114-118, 26111 Oldenburg, Germany, volker.hohmann@uni-oldenburg.de)

A multichannel dynamic compression algorithm is proposed that uses a novel nonlinear auditory filterbank which aims at effectively describing the basilar membrane (BM) response to arbitrary signals. It is based on a linear Gammatone filterbank, a subsequent instantaneous compression stage and a frequency-synthesis stage. In order to model the linear response to off-frequency tones and suppression effects from signals below the characteristic frequency (low-side suppression) the compression characteristics is controlled in each filter band by the deviation of the current sub-band instantaneous frequency from the band's center frequency. If the deviation is small, on-frequency components are prominent, and full gain and compression is applied. If the deviation is large, off-frequency components are prominent, and gain and compression is reduced. Simulations of responses to sinusoids as a function of frequency and level at a fixed BM place (i.e., within a fixed filter band) and for all filter bands (i.e., BM excitation patterns) show good correspondence with psychoacoustical excitation pattern models. Responses to two-tone stimuli quantitatively simulate psychoacoustical two-tone suppression. Likewise, nonlinear growth of simultaneous masking is quantitatively modeled. First results show that speech reception in modulated noise may be improved by the system for a subgroup of hearing-impaired subjects.

\section{8:40}

2aPPb3. Speech perception in fluctuating noise with signals compensated for hearing loss. Joost M. Festen (VU University Medical Center, de Boelelaan 1117, 1081 HV Amsterdam, Netherlands, jm.festen@vumc.nl)

For speech reception in noise normal-hearing listeners gain from masker modulations up to about $12 \mathrm{~dB}$ depending on rate, duty cycle, and depth of the modulations. Listeners with sensorineural hearing loss need a better signal-to-noise ratio to improve signal quality as a compensation for their auditory deficits. Generally, a larger compensation is needed for fluctuating interferences leading to 
reduction or even absence of release from masking for modulated maskers. With the Speech Intelligibility Index adapted for modulated maskers, SIImod [Rhebergen and Versfeld, J. Acoust. Soc. Am. 117, 2181-92 (2005)] it is shown that these elevated thresholds are needed to compensate not only reduced hearing sensitivity but also impaired auditory and non-auditory processing. After frequencydependent compensation for hearing loss as offered by a hearing aid, more of the speech is presented at impaired frequency regions. As a consequence the effect of a hearing aid on speech intelligibility will be less than predicted by SIImod. Speech reception thresholds in noise are affected by peripheral spectro-temporal processing (bottom-up) and by cognitive processing capabilities, like working memory (top-down). Effects of cognitive processing on speech perception are found especially in fluctuating noise as this masker presents a more complex and demanding environment than steady noise.

\section{9:00}

2aPPb4. Signal processing algorithms for speech in fluctuating noise. Peggy Nelson (University of Minnesota, 164 Pillsbury Drive SE, Minneapolis, MN 55455, USA, peggynelson@umn.edu), Janet Rutledge (University of Maryland Baltimore County, 1000 Hilltop Circle, Baltimore, MD 21250, USA, jrutledge@umbc.edu), Juan Carlos Tejero-Calado (University of Malaga, Boulevar Louis Pasteur 35, Campus de Teatinos - Complejo Tecnologico, 29071 Malaga, Spain, jctejero@uma.es)

Fluctuating background noise is a significant problem for listeners with sensorineural hearing loss (SNHL). Data indicate that fluctuating noise significantly affects both speech understanding and satisfaction with hearing aids. Listeners with SNHL do not take advantage of momentary dips in the noise and thus to not experience release from masking in fluctuating noise, as normal-hearing listeners do. Our results have shown that listeners with SNHL obtain about half of the masking release of their normal-hearing counterparts, and that this masking release is generally related to the audibility of the speech in the noise dips. Current slow-acting amplitude compression is based on the level of the background noise and does not improve the audibility of speech in the dips of fluctuating noise. Fast-acting multiband compression can improve audibility but may produce unwanted artifacts. Fast-acting algorithms based on spectral peaks shows potential for maximizing the audibility of speech in fluctuating noise to improve speech intelligibility, with limited artifact. Portions of this work are supported by the University of Minnesota and Starkey Laboratories.

\section{9:20}

2aPPb5. Signal processing in hearing aids: results of the HEARCOM project. Jan Wouters (ExpORL, Dept. Neurosciences, K.U. Leuven, Herestraat 49 bus 721, O. \& N2, B-3000 Leuven, Belgium, jan.wouters@med.kuleuven.be), Heleen Luts (ExpORL, Dept. Neurosciences, K.U. Leuven, Herestraat 49 bus 721, O. \& N2, B-3000 Leuven, Belgium, heleen.luts@med.kuleuven.be), Koen Eneman (ExpORL, Dept. Neurosciences, K.U. Leuven, Herestraat 49 bus 721, O. \& N2, B-3000 Leuven, Belgium, koen.eneman@med.kuleuven.be), Ann Spriet (ESAT/SISTA, K.U. Leuven, Kasteelpark Arenberg 10, B-3001 Leuven, Belgium, ann.spriet@esat.kuleuven.be), Marc Moonen (ESAT/SISTA, K.U. Leuven, Kasteelpark Arenberg 10, B-3001 Leuven, Belgium, marc.moonen@esat.kuleuven.be), Michael Büchler (University Hospital, $8091 \quad$ Zürich, Switzerland, michael.buechler@usz.ch), Norbert Dillier (University Hospital, 8091 Zürich, Switzerland, norbert.dillier@usz.ch), Wouter A. Dreschler (AMC, Clinical and Experimental Audiology, 1105 Amsterdam, Netherlands, w.a.dreschler@amc.uva.nl), Matthias Froehlich (Siemens Medical Solutions SAT, 91058 Erlangen, Germany, Matthias.froehlich@ siemens.com), Giso Grimm (Carl von Ossietzky Universität Oldenburg, Ammerländer Heerstraße 114-118, 26111 Oldenburg, Germany, giso.grimm@vegri.net), Volker Hohmann (Carl von Ossietzky Universität Oldenburg, Ammerländer Heerstraße 114-118, 26111 Oldenburg, Germany, volker.hohmann@uni-oldenburg.de), Rolph Houben (AMC, KNO-Audiologie, 1105 Amsterdam, Netherlands, a.c.houben@amc.uva.nl), Arne Leijon (KTH, Royal Institute of Technology, 10044 Stockholm, Sweden, arne.leijon@ee.kth.se), Anthony Lombard (University of Erlangen, 91058 Erlangen, Germany, lombard@nt.e-technik.uni-erlangen.de), Dirk Mauler (Ruhr University of Bochum, 44780 Bochum, Germany, dirk.mauler@ruhr-uni-bochum.de), Henning Puder (Siemens Medical Solutions SAT, 91058 Erlangen, Germany, henning.puder@siemens.com), Michael Schulte (Hoerzentrum Oldenburg, 26129 Oldenburg, Germany, M.Schulte@hoerzentrum-oldenburg.de), M Vormann (Hoerzentrum Oldenburg, Hoerzentrum Oldenburg, 26129 Oldenburg, Germany, matthias.vormann@web.de)

Digital hearing aids of today allow the application of advanced signal processing strategies. In recent years a number of promising signal processing approaches have been designed and developed. However, most of these different evolutions have been evaluated only in a limited way. Within the framework of the HEARCOM EU-research project a number of signal enhancement techniques have been further developed and evaluated based on a representative set of real-life recordings and physical performance measures. Different auditory profiles, representing common categories of hearing aid users, have been taken into account. A selection of 5 of these signal enhancement techniques (single-channel noise suppression, blind source separation, dereverberation, multi-microphone adaptive processing, feedback reduction) has been implemented on a single common hard- and software test platform, the Master Hearing Aid (MHA). These signal processing strategies have been evaluated perceptually based on speech reception thresholds, listening effort and preference rating, at 5 different test-sites for a number of speech-and-noise listening scenarios. Fifty normal hearing subjects and 100 hearing aid users according to 2 auditory profiles, took part in this study.

\section{9:40}

2aPPb6. Model-based objective assessment of noise reduction systems for hearing aids. Birger Kollmeier (Universität Oldenburg, Medizinische Physik, Carl-von-Ossietzky Str. 9-11, 26111 Oldenburg, Germany, birger.kollmeier@uni-oldenburg.de), Rainer Huber (Kompetenzzentrum HörTech, Marie-Curie-Str. 2, 26129 Oldenburg, Germany, Rainer.Huber@HoerTech.de), Thomas Rohdenburg (Universität Oldenburg, Medizinische Physik, Carl-von-Ossietzky Str. 9-11, 26111 Oldenburg, Germany, thomas.rohdenburg@uni-oldenburg.de), Rainer Beutelmann (Universität Oldenburg, Medizinische Physik, Carl-von-Ossietzky Str. 9-11, 26111 Oldenburg, Germany, rainer.beutelmann@uni-oldenburg.de), Volker Hohmann (Carl von Ossietzky Universität Oldenburg, Ammerländer Heerstraße 114-118, 26111 Oldenburg, Germany, volker.hohmann@uni-oldenburg.de)

Since the ultimative goal of hearing-aid development is the (subjective) judgment of the individual hearing-impaired listener, timeconsuming tests with the end user are indispensable. However, time- and effort-saving objective methods to assess the potential benefit of different versions and parameter sets of hearing aid algorithms are gaining importance. This contribution reviews perception-modelbased approaches to predict the hearing-impaired judgement and speech reception performance achieved with various noise reduction 
schemes. The perceptual similarity measure PSM evaluates the similarity between a tested condition and an "ideal" reference condition not on the physical level, but rather on the perceptual level at the output of a perception model for the individual hearing-impaired listener. The binaural extention of the SII approach uses a binaural preprocessing stage followed by a speech intelligibility index (SII)based prediction scheme capable of predicting the relative benefit of binaural signal presentation and signal enhancement in complex spatial signal and noise source configurations. Both model-based schemes can be combined to assess the effect of noise reduction algorithms (such as adaptive beamformers) and to optimize their respective performance for different acoustical situations.

\section{Contributed Papers}

10:00

2aPPb7. Quantifying and modeling the acoustic effects of compression on speech in noise. Koenraad S. Rhebergen (AMC - Dept. of Clinical and Experimental Audiology, AMC, Clinical and Experimental Audiology, 1105 Amsterdam, Netherlands, k.s.rhebergen@amc.uva.nl), Niek J. Versfeld (AMC - Dept. of Clinical and Experimental Audiology, Meibergdreef 9, 1105AZ Amsterdam, Netherlands, n.j.versveld@amc .uva.nl), Wouter A. Dreschler (AMC, Clinical and Experimental Audiology, 1105 Amsterdam, Netherlands, w.a.dreschler@amc.uva.nl)

In this presentation a method is proposed that is able to separate a speech signal out of a noise signal after processing of the signal through widedynamic-range compression (WDRC). This technique reconstructs the speech signal and noise signal sample by sample separately using the gain factor of the WDRC, and can be used to quantify the acoustic effects of WDRC in noise. It will be shown that this technique is more accurate than a frequently used inversion technique, because the method is not affected by phase shifts that introduce distortion products in the reconstructed speech signal. As a result, the acoustic effects of WDRC can be measured more accurately. In addition, this reconstruction method allows modeling the speech intelligibility after non-linear signal processing in the Speech Intelligibility Index. With the aid of Speech Reception Threshold data it will be shown that this approach can give a good account for most existing data.

\section{0:20}

2aPPb8. Acoustical frequency discrimination and pitch matching in bimodal and hybrid hearing. Uwe Baumann (Univ. of. Frankfurt -
ZHNO - Audiologische Akustik, Theodor-Stern-Kai 7, Haus 8D, 60590 Frankfurt a.M., Germany, uwe.baumann@kgu.de), Tobias Rader (Univ. of. Frankfurt - ZHNO - Audiologische Akustik, Theodor-Stern-Kai 7, Haus 8D, 60590 Frankfurt a.M., Germany, tobias.rader@kgu.de), Silke Helbig (Univ. of. Frankfurt - ZHNO - Audiologische Akustik, Theodor-Stern-Kai 7, Haus 8D, 60590 Frankfurt a.M., Germany, silke.helbig@kgu.de), Wolfgang Gstöttner (Univ. of. Frankfurt - ZHNO - Audiologische Akustik, TheodorStern-Kai 7, Haus 8D, 60590 Frankfurt a.M., Germany, a.jan@em.unifrankfurt.de)

Frequency discrimination and pitch matching of implantees using combined electric and acoustic stimulation in either the same ear (EAS) or the opposite ear (bimodal condition) was assessed by means of adaptive procedures. EAS patients received either the MED-EL standard electrode or the recently introduced FLEX design with reduced diameter. Acoustic JNDF in EAS patients ranged from close to normal to grossly abnormal compared to a group of matched SNHL listeners. The median JNDF was $7.1 \%$ in the SNHL and $7.5 \%$ in the EAS group. There was no statistically significant difference in terms of JNDF between both groups of listeners. Frequency mapping was studied by means of an adjustment method where subjects were instructed to control the pitch of an acoustically presented sinusoid in reference to electrical stimulation. The findings demonstrate that the insertion of an intra-cochlear electrode does not significantly hamper the average frequency discrimination ability in EAS patients.

\section{Invited Paper}

\section{0:40}

2aPPb9. Combining hearing aids and cochlear implants to solve the cocktail party problem. Fan-Gang Zeng (University of California Irvine, 364 Med Surge II, Irvine, CA 92697, USA, fzeng@uci.edu)

The cocktail party problem refers to the difficulty in speech recognition in noise that a hearing-impaired listener must face in daily life. Combining a hearing aid with a cochlear implant can provide complementary information that may have a great potential to solve this problem. On one hand, a hearing aid may provide low-frequency temporal fine structure cues that are not conveyed by a cochlear implant. On the other hand, a cochlear implant can provide high-frequency temporal envelope cues that are not effectively delivered by a hearing aid. This talk will provide psychophysical and speech recognition evidence for combining hearing aids and cochlear implants to solve the cocktail party problem. One interesting finding along this line of research is that in many important functional tasks, the hearing aid and cochlear implant combination provides a more effective solution than bilateral cochlear implants. Another interesting finding is that the fundamental frequency cue alone can significantly improve speech perception in noise, especially when the noise is a competing voice. The latter finding suggests that combining a tactile aid and a cochlear implant can potentially achieve the same benefit as combining a hearing aid and a cochlear implant in patients with no residual acoustic hearing. 


\title{
Session 2aPPc
}

\section{Psychological and Physiological Acoustics: Binaural Perception by Hearing-Aid Wearers}

\author{
Sridhar Kalluri, Cochair \\ Starkey Hearing Research Center, 2150 Shattuck Ave, Suite 408, Berkeley, CA 94704, USA \\ Michael A. Akeroyd, Cochair \\ MRC Institute of Hearing Research, Glasgow Royal Infirmary, 16 Alexandra Parade, Glasgow, G31 2ER, UK \\ Invited Paper \\ 11:20
}

2aPPc1. Binaural function and its benefits for wearers of hearing aids. Sridhar Kalluri (Starkey Hearing Research Center, 2150 Shattuck Ave, Suite 408, Berkeley, CA 94704, USA, sridhar_kalluri@ starkey.com), Brent Edwards (Starkey Hearing Research Center, 2150 Shattuck Ave, Suite 408, Berkeley, CA 94704, USA, brent_edwards@starkey.com)

Hearing-aid design has been predicated traditionally on improving the reception of speech in background noise, a goal that has, at least in part, been successfully attained. Despite the advances, hearing-aid wearers continue to have difficulty in complex acoustic environments and in auditory tasks where normal-hearing listeners benefit greatly from binaural hearing. In order to improve hearing-aid wearers' performance in such challenging conditions, recent research has begun examining the effects of hearing aids on binaural perception. Interest in the topic is heightened by the rapidly approaching prospect of bilateral hearing aids that communicate wirelessly and thereby allow implementation of more complex signal-processing algorithms than currently possible. This talk will review binaural function and its benefits, with particular emphasis on the aspects that hearing aids can affect. The talk will also speculate on which hearing-aid algorithms can affect binaural perception.

\section{Contributed Paper}

\section{1:40}

2aPPc2. Auditory localization with linear and compression hearing aids. Helen J. Simon (Smith-Kettlewell Eye Research Institute, 2318 Fillmore St., San Francisco, CA, CA 94115, USA, helen@ski.org), E. William Yund (VAMC, 150 Muir Road, Martinez, CA 94553, USA, yund@ebire.org), Harry Levitt (Advanced Hearing Concepts, PO Box 610, Bodega Bay, CA 94923, USA, harrylevitt@earthlink.net)

The question of how well hearing-impaired individuals can localize sound (with or without amplification) is still not fully resolved. This study was designed to compare sound localization with two types of hearing-aid (HA) processing, wide dynamic range multichannel compression (WDRMCC) and linear amplification (LA) with compression limiting, during the first 32 weeks of HA use. HAs from two different manufacturers were included to compare different digital signal processing implementations, (1) fast Fourier transform (FFT), necessitating a 10 ms delay, and (2) non-FFT signal processing with a shorter time delay $(r 1 \mathrm{~ms})$. We found an initial degradation of sound localization, relative to original unaided performance, for both WDRMCC and LA in both FTT and non-FTT platforms. We found no difference between WDRMCC and LA processing. However, sound localization with non-FFT platform improved consistently throughout 32 weeks of HA use and was better than the original unaided measurements at 16 and 32 weeks. In contrast, localization with the FFT platform showed no consistent change throughout the 32 -week test period and remained inferior to original unaided performance. The continuing localization problems present for the FFT, but not the non-FFT, implementations of LA and WDRMCC may be due to its 10 -ms processing delay.

\section{Invited Papers}

\section{2:00}

2aPPc3. Effects of noise type and location on binaural benefit in asymmetric directional fittings. Benjamin Hornsby (Vanderbilt University, Room 8310 Medical Center East, South Tower, 1215 21st Ave. South, Nashville, TN 37232-8242, USA, ben.hornsby @ vanderbilt.edu)

The benefits of bilateral directional processing for improving speech understanding in noise are well documented. However, these fittings are not universally accepted by hearing aid wearers. Research suggests that an asymmetric fitting (omnidirectional in one ear/directional in the other) may provide benefit in noise comparable to symmetric directional fittings (directional in both ears). This study evaluated factors that may affect the relative benefit provided by an asymmetric directional fitting. Specific factors evaluated included noise configuration, reverberation and noise type. Twenty individuals with mild-moderate SNHL participated. Aided speech understanding in cafeteria babble was assessed in bilateral omnidirectional and directional modes and in an asymmetric mode in four (4) different noise configurations. Measures were made in both an anechoic and reverberant (RT $\sim 620 \mathrm{~ms}$ ). In a second experiment the effects of noise type were evaluated by comparing performance in symmetric and asymmetric modes in both steady state noise and cafeteria babble. Results suggest that noise configuration has a significant effect on the relative benefit provided by asymmetric fittings. No significant differences due to noise type were observed. Individual differences in bilateral directional benefit also appear to have a significant effect on the reduction in benefit resulting from an asymmetric fitting. 
2aPPc4. Speech-in-noise enhancement and sound localization with improved binaural hearing instruments. Jan Wouters (ExpORL, Dept. Neurosciences, K.U. Leuven, Herestraat 49 bus 721, O. \& N2, B-3000 Leuven, Belgium, jan.wouters @med.kuleuven.be), Simon Doclo (ESAT/SISTA, K.U. Leuven, Kasteelpark Arenberg 10, B-3001 Leuven, Belgium, simon.doclo@esat.kuleuven.be), Marc Moonen (ESAT/SISTA, K.U. Leuven, Kasteelpark Arenberg 10, B-3001 Leuven, Belgium, marc.moonen@esat.kuleuven.be), Tim Van Den Bogaert (ExpORL, Dept. Neurosciences, K.U. Leuven, Herestraat 49 bus 721, O. \& N2, B-3000 Leuven, Belgium, tim.vandenbogaert@med.kuleuven.be)

Multi-microphone noise reduction schemes have become standard in commercial hearing aids and cochlear implants. Recent studies with bilateral hearing aids have shown that common adaptive directional microphone systems tend to distort localization cues, leading to inappropriate and reduced spatial awareness for bilateral hearing aid users. Here we show that binaural multi-microphone signal processing based on multi-channel Wiener filter (MWF) are capable of combining noise reduction with the preservation of directional hearing. Physical simulations and perceptual results from 10 listeners have been studied for different noise source scenarios, in different reverberant conditions, and for a number of signal processing schemes using up to 4 microphone inputs ( 2 each side). An overview is given of the localization performance and the speech reception benefits in these different listening conditions for the different noise reduction strategies. An adaptive directional microphone system (ADM) is used as a reference system. Signal processing based on MWF does, unlike ADM, provides a combination of noise reduction and preservation of spatial awareness. Moreover, in some conditions it even offers an improved spatial release from masking. The MWF outperforms the ADM in terms of localization and noise reduction if signals are not arriving from the most forward field of view.

\section{2:40-2:00 Lunch Break}

\section{Invited Papers}

2:00

2aPPc5. Interaction between stimulus and compression type in precedence situations with hearing aids. Bernhard U. Seeber (MRC Institute of Hearing Research, Science Rd / University Park, NG7 2RD Nottingham, UK, seeber@ihr.mrc.ac.uk), Cheryl Eiler (Starkey Hearing Research Center, 2150 Shattuck Ave, Suite 408, Berkeley, CA 94704, USA, cheryl_eiler@starkey.com), Sridhar Kalluri (Starkey Hearing Research Center, 2150 Shattuck Ave, Suite 408, Berkeley, CA 94704, USA, sridhar_kalluri@starkey.com), Ervin R. Hafter (University of California, Department of Psychology, 3210 Tolman Hall, Berkeley, CA, CA 94720-1650, USA, hafter@berkeley.edu), Brent Edwards (Starkey Hearing Research Center, 2150 Shattuck Ave, Suite 408, Berkeley, CA 94704, USA, brent_edwards@starkey.com)

Multiband compression in hearing aids has the potential to interfere with binaural perception by altering binaural cues. We compared binaural precedence in patients fitted with compression hearing aids or linear hearing aids and tested one month later with a variety of stimuli. In an open field environment simulated in an anechoic chamber, subjects localized the direction of a leading sound accompanied by a lagging copy played from a different direction. Precedence, defined by a strong influence of the first sound, was found in $6 / 7$ of the subjects when the stimulus was a sentence, but in only $4 / 7$ when it was a high-pass or a wideband burst of noise. The fact that increasing the noise-bandwidth to include low frequencies did not produce precedence in the two patients who had shown it with a sentence may indicate a need for them to accumulate information through the successive pseudo onsets in the speech-envelope. The choice of amplification, compressive or linear, did not significantly affect precedence for any sound, except for one subject who showed weak influence of compression with the high-pass noise. We conclude that although compression might alter interaural level cues, for most, especially with long sounds, localization dominance appears unimpaired.

$$
\text { 2:20 }
$$

2aPPc6. Effect of hearing aids on distance perception. Michael A. Akeroyd (MRC Institute of Hearing Research, Glasgow Royal Infirmary, 16 Alexandra Parade, G31 2ER Glasgow, UK, maa@ihr.gla.ac.uk)

The two primary auditory cues to distance in rooms are the overall level of the sounds received by the listener and the ratio of the level of the direct sound to the reverberant sounds. In previous work we have demonstrated that hearing-impaired listeners showed no overall deficits in the ability to use the overall-level cue, but they did have deficits in the ability to use the direct-to-reverberant cue [M. Akeroyd, S. Gatehouse, and J. Blaschke, J. Acoust. Soc. Am., 121, 1077-1089 (2007)]. These deficits would be expected to contribute to the auditory disability suffered by the listeners. But both of these auditory cues are level cues, as they require some measurement of intensity, and so they would be expected to be affected adversely by the amplitude compression found in most modern hearing aids. Initial results from an experiment measuring the just-noticeable difference (JND) for changes in distance (at $2 \mathrm{~m}$ and $5 \mathrm{~m}$ ) with experienced hearing-aid users suggest, however, that their JNDs are no different to those of unaided but impaired listeners. If confirmed by the final results, this surprising result may be interpretable if listeners have acclimatized to the effects of their aids on level.

$$
\text { 2:40 }
$$

2aPPc7. Binaural hearing abilities of bilaterally fitted hearing aid users assessed using objective and subjective outcome measures. Thomas Behrens (Eriksholm Research Centre, Oticon A/S, 243 Kongevejen, 3070 Snekkersten, Denmark, tbs@oticon.dk), Tobias Neher (Eriksholm Research Centre, Oticon A/S, 243 Kongevejen, 3070 Snekkersten, Denmark, ton@oticon .dk)

Aided spatial hearing in the hearing impaired remains a rather sparsely explored topic. Therefore we do not know enough about the ability of the hearing impaired to exploit auditory cues for spatial hearing. In an attempt to advance our knowledge the following 
experiment was set up. A group of 21 experienced hearing aid users took part in a test using modern completely in the canal hearing aids in the field for at least 7 weeks. After acclimatization to the devices they were tested on a number of outcome measures. These included spatial unmasking, an interview administered selection of questions from the Speech Spatial and Qualities of hearing scale, and a baseline speech in noise measure. Spatial unmasking was assessed using three concurrent female talkers, with the target talker always presented directly ahead and the maskers presented either at $+/$ - 50 degrees or both at 180 degrees. This presentation will provide results from the study described above along with possible relations to auditory and non-auditory predictors of spatial hearing performance.

\title{
Contributed Paper
}

3:00

2aPPc8. The effect of binaural processing techniques on speech quality ratings of assistive listening devices in different room acoustics conditions. Johan Odelius (Luleå University of Technology, Dept Human Work Sciences, Div of Sound and Vibration, SE-97187 Luleå, Sweden, johan.odelius@1tu.se), Örjan Johansson (Luleå University of Technology, Dept Human Work Sciences, Div of Sound and Vibration, SE-97187 Luleå, Sweden, orjan.johansson@ltu.se)

External microphone systems, referred to as assistive listening devices (ALD), are used in classrooms for hearing impaired students. The objective is to investigate the effect of binaural processing techniques in different room acoustic conditions. A listening experiment was conducted with 10 normal hearing adults. Response variables were judgements of clarity, pleas- antness, listening effort and overall speech quality. Design variables were binaural processing, room acoustics and ALD bandwidth. Stimuli were generated using the room acoustic modelling software CATT Acoustic. Three speech sources, two male voices and one female voice, were placed at a table in the centre of a room and one Brown noise source was placed in one corner of the room. Microphones were placed $0.5 \mathrm{~m}$ in front of each speech source. Target source was a random choice of one of the two male voices. The binaural processing was utilized by a simple HRTF filtering. Depending on the angle to the source from a fictitious listening position at the table, corresponding interaural time difference (ITD) and the interaural level difference (ILD) was applied to the signal. Stimuli were presented by loudspeakers using cross-talk cancellation. The hypothesis is that binaural processing will give a significant improvement in speech quality.

TUESDAY MORNING, 1 JULY 2008

ROOM 242B, 8:00 TO 10:40 A.M.

\author{
Session 2aSAa
}

\section{Structural Acoustics and Vibration and EURONOISE: Vibration and Radiation from Complex Structural Systems II}

\author{
David Feit, Cochair \\ Applied Physical Sciences Corp., Ste. 300, 2 State St., New London, CT 06320, USA \\ Jean-Louis Guyader, Cochair \\ INSA de Lyon - LVA, Bâtiment St. Exupéry, 25 bis avenue Jean Capelle, Villeurbanne Cedex, F-69621, France
}

\section{Contributed Papers}

\begin{abstract}
8:00
2aSAa1. On the comparison of symmetric and unsymmetric formulations for experimental vibro-acoustic modal analysis. Morvan Ouisse (FEMTO-ST UMR CNRS, 23 chemin de l'Epitaphe, 25000 Besançon, France, morvan.ouisse@univ-fcomte.fr), Emmanuel Foltete (FEMTO-ST Applied Mechanics, 24 chemin de l'épitaphe, 25000 Besançon, France, emmanuel.foltete@univ-fcomte.fr)
\end{abstract}

The classical u-p formulation for vibro-acoustic problems is very convenient for experimental vibro-acoustic modal analysis since the physical variables are directly those which are measured by operators. In this particular context, the objective is to identify from experimental measurements a reduced model which has the same behaviour as the measured one. The complex mode shapes which are identified using this technique must satisfy a properness condition. When they do not verify it, they should be modified to be able to represent the behaviour of a physical system. Some techniques have been proposed in order to develop a strategy to obtain the modified eigenshapes, but this is a quite difficult point because of the unsymmetric topology of the equations. In this paper, a symmetric formulation is used in order to be able to directly apply the classical methodology which has been developed for structural modal analysis to obtain the physical reduced system. The methodology is described and compared with the u-p formulation, in terms of efficiency and precision, in particular when some absorbing devices are considered. All results are first presented on an ideal numerical test-case, and applications on experimental data are finally shown.

\section{8:20}

2aSAa2. Numerical modeling of Panphonics's G1 flat loudspeaker. Krisztián Gulyás (Budapest University of Technology and Economics, BME Dept. of Telecommunications, Magyar tudósok körútja 2, H-1117 Budapest, Hungary, gulyas@ hit.bme.hu), Fülöp Augusztinovicz (Budapest University of Technology and Economics, BME Dept. of Telecommunications, Magyar tudósok körútja 2, H-1117 Budapest, Hungary, fulop@ hit.bme .hu)

The basic idea behind AABC (Active Acoustic Barrier Control) is to reduce the sound radiation of a structure acoustically without influencing the vibration behavior of the structure. The large surface acoustic polymer material actuator/sensor systems are primarily meant to form the actuator/sensor system for the $\mathrm{AABC}$ concept. The main component of this package is a special active device: the rEMA - revised Elastic Mass Actuator based on the Panphonics' G1 panel loudspeaker element. This paper deals with the modeling and the low-frequency performance issues of the G1 flat loudspeaker. The aim was to create an accurate mathematical model to understand the operational principles of the loudspeaker, and then this model was used to optimize its low-frequency performance. Due to the special 
structure and the optimization tasks, a new numerical method was developed to model special multi-layer coupled vibro-acoustics systems based on the Finite Difference and Boundary Element Method. The investigation focuses on the mechanical behavior of the panel and describes the sound radiation properties also. The performed work was a part of the research of the project InMAR (Intelligent Materials for Active Noise Reduction) which was funded by the European Union.

\section{$8: 40$}

2aSAa3. Metrology and prediction for integrating a subsystem on a vehicle: Application to a fan system attached to the front end of a car. Marie-Hélène Moulet (CEVAA, Technopole du Madrillet, 2 Rue Joseph Fourier, 76800 Saint Etienne du Rouvray, France, mh.moulet@cevaa.com), Saul Mapagha (CEVAA, Technopole du Madrillet, 2 Rue Joseph Fourier, 76800 Saint Etienne du Rouvray, France, s.mapagha@cevaa.com), Vincent Martin (Institut Jean Le Rond d'Alembert, UMR CNRS 7190, UPMC, 2 Place de la Gare de Ceinture, 78210 Saint-Cyr 1'Ecole, France, vmartin@ccr.jussieu.fr)

The car industry must satisfy physical acoustic and vibratory objectives in order to comply with safety and comfort norms. Among others, efforts entering the chassis should be limited. When a host structure is excited by a vibratory system, called subsystem, it also vibrates and may radiate an acoustic field. Usually, the subsystem is first tested on a bench and the question is then to deduce the efforts entering the chassis from those entering the test bench. Globalising notions of impedance already made evident via deformable structure configurations are used presently through measurements in a technological configuration, the complexity of which does not allow modelling. The case of a fan system attached to the front end of a car is under study. Some metrological conclusions, as well as more dedicated ones concerning the fan system itself, will be given. Finally, on the calculation and prediction sides, expected properties that are not satisfied (such as symmetry in measured matrices) are seen to be of no great consequence in the present case.

\section{9:00}

2aSAa4. Numerical study of the transition to chaos in nonlinear forced vibrations of plates. Cédric Camier (ENSTA, Chemin de la Hunière, $91761 \quad$ Palaiseau cedex, France, cedric.camier@ensta.fr), Cyril Touze (ENSTA, Chemin de la Hunière, 91761 Palaiseau cedex, France, cyril.touze@ensta.fr), Olivier Thomas (CNAM, 2, rue Conté, 75003 Paris, France, olivier.thomas@cnam.fr), Stefan Bilbao (University of Edinburgh, Room 7306B, JCMB, King's Bldgs., Mayfield Rd., EH9 3JZ Edinburgh, UK, sbilbao@staffmail.ed.ac.uk)

Geometrically nonlinear vibrations of free edge circular plates subjected to a harmonic excitation are discussed. Particularly, transition from periodic to chaotic motion is observed when increasing the amplitude of the forcing. The present work is devoted to reproduce numerically these highly nonlinear behaviours. The temporal integration of such dynamics, including instabilities and chaotic regimes, is not straight forward because a stiff problem with a very large number of dofs is at hand. Consequently, numerical instabilities are observed when typical Runge-Kutta schemes are applied. To settle the matter, two methods have been tested and compared. They both rely on a modal approach applied to the von Kármán's model for large amplitude vibrations of plate. For the first one, the energies of the plates are expressed at the continuous level. The Hamiltonian of the system is then derived and discretized using the eigenmodes. The Hamiltonian formulation ensures the conservation of energy. An implicit time discrete scheme is then chosen to approximate the equations of motion. For the second one, the Gear's BDF method, implemented in the IMSL Fortran library, is used to integrate the nonlinear oscillator equations.

\section{9:20}

2aSAa5. Numerical investigations into the squeal propensity of a railway disc brake. Olivier Chiello (INRETS, Transport and Environment Lab., 25 avenue François Mitterrand, 69675 Bron cedex, France, olivier.chiello@inrets.fr), Xavier Lorang (SNCF, Innovative \& Research Dept., 45 rue de Londres, 75379 Paris, France, xavier.lorang@sncf.fr)

This paper comes within the scope of a research program concerning with the reduction of the squeal noise generated by high power railway disc brakes. It focuses on the numerical results provided by a finite element model of the brake including unilateral contact and Coulomb friction at the disc/pad interfaces. In particular, the dynamic stability of the sliding equilibrium is investigated by performing a complex eigenvalue analysis of the linearized equations verified by the structural displacement fields. Complex eigenvalues and complex modes are used to estimate the squeal propensity of the brake in a given frequency range. The effect of various mechanical and geometrical parameters is studied in order to better understand the mechanism leading to the system instability.

\section{9:40}

2aSAa6. Three dimensional orthogonality of the Lamb modes in layered plates of elastic and viscoelastic materials and their implementation to the far field evaluation. Dmitry Zakharov (LMP, UMR CNRS 5469, Université Bordeaux I, 351, cours de la Libération, 33405 Talence, France, dmitrii.zakharov@gmail.com)

The 3D guided waves in the linearly viscoelastic laminates are considered. On the plate surfaces any of the homogeneous boundary conditions are allowed, e.g., the Lamb waves, waves in clamped plates, etc. are taken into account. The fundamental property of these waves is their generalized orthogonality, which is deduced and discussed. The applications of the orthogonality relations for solving some particular boundary value problems are suggested. A method for the exact calculation of the far field caused by an acoustic source of a finite size is suggested. The only restriction is that the distance required must exceed the longitudinal radius of the source. The obtained results can be used for evaluating the fields radiated by ultrasonic transducers of arbitrary aperture and by other realistic sources.

\section{0:00}

2aSAa7. Sound Radiation of a Large Truck Oil Pan: Estimation and Experimental Investigation. Olaf Heintze (German Aerospace Center (DLR), Institute of Composite Structures and Adaptive Systems, Lilienthalplatz 7, 38108 Braunschweig, Germany, olaf.heintze@dlr.de), Volker Wittstock (Physikalisch-Technische Bundesanstalt, Bundesallee 100, 38116 Braunschweig, Germany, volker.wittstock@ptb.de), Carl Fredrik Hartung (Volvo Technology Corporation, Götaverksgatan 10, 40508 Göteborg, Sweden, carl.fredrik.hartung@volvo.com)

The oil pan of large diesel engine trucks has been identified as a significant contributor to their external noise radiation. This undesired broadband noise is caused by the oil pan's structural vibration and can not be treated effectively by passive measures especially in the low frequency regime up to $500 \mathrm{~Hz}$. In order to address this challenge, an active structural acoustic control system consisting of structural sensors and actuators is suitable to alter the oil pans vibrations in a sound reducing manner. A first step is however to classify the broadband sound radiation such that it allows for a proper and efficient sound power estimation resulting from structural measurements. Therefore, an acoustical model was set up based on a geometrical surface scan of a serial production large truck oil pan mounted in a laboratory test stand. This model served for the numerical computation of a set of principle velocity patterns contributing independently to the active sound power, where its hybrid estimation has been performed employing additionally the measured structural response of the oil pan assembly due to a shaker excitation. Finally, the sound power radiation of the test stand has been measured in a reverberation room to validate this hybrid estimation. 


\section{0:20}

2aSAa8. Sound propagation on a high pressure gas pipe. Rolf Schirmacher (Müller-BBM, Robert-Koch-Straße 11, 82110 Planegg, Germany, Rolf.Schirmacher@MuellerBBM.de), Robert Baars (M+P raadgevende ingenieurs B.V., Visserstraat 50, 1431 GJ Aalsmeer, Netherlands, robertbaars@mp.nl)

At a two stage high pressure gas compressor with intermediate cooler, the cooler radiate a tone at the compressor rotational frequency of approx.
$160 \mathrm{~Hz}$. For the design of noise reduction devices, the mechanism of sound propagation in between the compressor an the cooler on a DN 400 steel pipe with $24 \mathrm{~mm}$ wall thickness and 160 Bar internal gas pressure was to be determined. By non-invasive vibration measurements on the pipe, the dominance of the fluid borne sound (natural gas) over the structure borne sound was found. Later, pressure measurements in the pipe clearly approved this result. The concepts upon which the measurements and data evaluation were based (propagating modes of coupled system, wave separation, etc) as well as the results are presentend.

TUESDAY MORNING, 1 JULY 2008

ROOM 243, 8:00 A.M. TO 12:20 P.M.

\title{
Session 2aSAb
}

\section{Structural Acoustics and Vibration and EURONOISE: General Topics in Structural Acoustics and Vibration II}

\author{
Wolfgang Kropp, Cochair \\ Chalmers University of Technology, Division of Applied Acoustics, Gothenburg, SE-41296, Sweden \\ Sean $\mathrm{Wu}$, Cochair \\ Wayne State University, 5050 Anthony Wayne Dr., Detroit, MI 48202, USA
}

\section{Contributed Papers}

\begin{abstract}
8:00
2aSAb1. Wavelet-based data processing for comparative study of noncontact measurement techniques for vibroacoustics. Dan Borza (National Institute of Applied Sciences of Rouen, INSA Rouen, LMR, Ave l'Universite, BP8 76800 Rouen, France, dan.borza @insa-rouen.fr), Ioana Nistea (Institut National des Sciences Appliquées de Rouen, BP8 avenue de l'Université, 76801 Saint-Etienne du Rouvray, France, ioana.nistea@insa-rouen.fr)

In vibration analysis of structures complex measurement information is required in order to perform modal analysis, therefore the choice of the measurement technique to be applied is essential. Non contact measurements are preferred to classical transducer based methods, mainly due to the absence of influence upon the structure under test. The various techniques available today in vibroacoustics produce results which are quite different in terms of spatial and temporal resolution or measured quantities and therefore a choice has to be made of the experimental tool best adapted for different fields of research (acoustics, mechanical structures, dynamics). In this paper, we present a comparative study of several optical, acoustical and numerical techniques for vibration measurement or simulation, namely Digital Speckle Interferometry, Laser Doppler Vibrometry and a FE model. The tests were made for the free and the forced vibrations of a highly dumped, non-metallic plate. In the data processing stage, discrete wavelet decomposition has been applied on the experimental data in order to match up the spatial maps of vibration amplitudes.
\end{abstract}

\section{8:20}

2aSAb2. Analysis of the acoustic signals backscattered by a tube using the time-frequency representations. Mustapha Laaboubi (Ibn Zohr University, FS Agadir, 80000 Agadir, Morocco, laaboubi @ gmail.com), Elhoucien Aassif (Ibn Zohr University, FS Agadir, 80000 Agadir, Morocco, aassif@hotmail.com), Rachid Latif (ESSI - ENSA, BP 1136, Ibn Zohr University, 80000 Agadir, Morocco, latif@ensa-agadir.ac.ma), Gerard Maze (LAUE, Université du Havre, Place Robert Schuman, F-76610 Le Havre, France, gerard.maze
@univ-lehavre.fr),Dominique Decultot(LOMC FRE 3102 CNRS Groupes Ondes Acoustiques, Université du Havre (IUT), Place Robert Schuman, 76610 Le Havre, France, dominique.decultot@univ-lehavre.fr), Ali Moudden (Ibn Zohr University, FS Agadir, 80000 Agadir, Morocco, ali_moudden@yahoo.fr), Abdelilah Dariouchy (LMTI, univirsté ibn zohr Faculté des Sciences LMTI bp 8106, 80000 Agadir, Morocco, abdelilah _dariouchy@yahoo.fr)

The normal excitation of a tube immersed in water by the acoustic plane wave, circumferential waves are generated inside the shell. These circumferential waves, standing form stationary waves on the circumference of the tube for some frequencies. These stationary waves, constituting resonances of the tube which are perfectly visible on the backscattered spectrum. Moreover, the studies carried out on the diffusion of a plane acoustic wave by target were based primarily on the use of the monodimensional methods (Temporal domain and/or frequencial domain). To exceed the disadvantages of these methods, in this work, we used the time-frequency representations such as the Short-Term Fourier Transform (STFT), Wigner-Ville Distribution (WVD) and Wavelet Transform method. These representations are applied to a theoretical signal backscattered by a tube of aluminium, copper and steel with radii ratio $\mathrm{b} / \mathrm{a}=0.95$ ( $\mathrm{a}$ is the external radius, and $\mathrm{b}$ the internal radius). From the time-frequency images obtained we have visualized the dispersion of circumferential waves (S0, A1, S1,œ) and identified these different waves. This analysis permits to compare between these timefrequency representations. And also we have compared between the cut-off frequencies of circumferential waves obtained from these representations and those computed by the proper modes theory of the vibration.

\section{8:40}

2aSAb3. Weak radiator design using dimples. D. Chih-Chun Cheng (National Chung Cheng University, Dept. of Mechanical Engineering, 160, San-Hsing, Ming-Hsiung, 621 Chia-Yi, Taiwan, imeccc@ccu.edu.tw), Wen-Nan Cheng (National Chung Cheng University, Dept. of Mechanical Engineering, 160, San-Hsing, Ming-Hsiung, 621 ChiaYi, Taiwan, zzs1003@hotmail.com), Cary H. Koopmann (Pennsylvania 
State University, Dept. of Mechanical and Nuclear Engineering, 157 Hammond Building, University Park, PA 16802, USA, ghk@engr.psu.edu)

A design method for achieving minimum radiation of sound from a beam by creating cylindrical dimples on its surface is presented. Two strategies of determining the dimple size and its location on the beam are presented. The first is based on the optimization method, in which the dimple size and its location are the design variables. The design variables that minimize the sound power are obtained directly using an optimization subroutine. The second is to synthesize the beam's weak radiator mode using a set of dimples, in which the mode shape of the dimpled beam is a close fit to the beam's weak radiator mode. As a comparison in sound power reduction between these strategies, numerical results for a simply supported beam in a rigid baffle excited by a harmonic force are presented.

\section{9:00}

2aSAb4. Estimation for vibration mode of membrane by NAH method Nabilah Ibrahim (Shibaura Institute of Technology, 3-7-5, Toyosu, Koto-ku, 135-8548 Tokyo, Japan, m107068@ sic.shibaura-it.ac.jp), Hideo Shibayama (Shibaura Institute of Technology, 3-7-5, Toyosu, Koto-ku, 135$8548 \quad$ Tokyo, Japan, sibayama@ sic.shibaura-it.ac.jp), Toru Itakura (Shibaura Institute of Technology, 3-7-5, Toyosu, Koto-ku, 1358548 Tokyo, Japan,m106009@ sic.shibaura-it.ac.jp)

Near Field Acoustic Holography (NAH) is the method of measuring sound information on the radiant surface in the near field as a hologram. By use the technique, we can visualize the vibration of the radiant surface. Thus, we can easily understand the object of the acoustic conditions in acoustic image processing. We made the measurement on one side of the drum using array microphone while the driven source is attached to the other side. In addition, the measurement is not touched on the vibrating membrane surface. This paper describes the estimated results of the vibration mode on the vibration membrane of a drum. And this is possible by apply it to the vibration controlling field for detecting the sound structure on the membrane.

\section{9:20}

2aSAb5. Computing high frequency vibrations of polygonal plates by the Image Source Method. Jacques Cuenca (LAUM, CNRS, Université du Maine, Lab. d'Acoustique Université du Maine, UMR CNRS 6613, 72085 Le Mans Cedex 9, France, Jacques.Cuenca.etu@univ-lemans.fr), François_ Gautier (Laboratoire d'Acoustique de l'Université du Maine, Avenue Olivier Messiaen, 72085 Le Mans, France, francois.gautier@univ-lemans.fr), Laurent Simon (LAUM, CNRS, Université du Maine, Lab. d'Acoustique Université du Maine, UMR CNRS 6613, 72085 Le Mans Cedex 9, France, Laurent.Simon@univ-lemans .fr)

The aim of this paper is to show that the Image Source Method (ISM) can be used for predicting medium and high frequency vibrations of arbitrarily shaped polygonal plates with controllable precision. Modeling the vibrations of polygonal plates by ISM consists in superposing the contributions of elementary image sources, which allows the plate boundary conditions to be satisfied. For particular plate shapes leading to a spatially periodic pattern of image sources, it is shown that ISM allows the exact computation of the Green's function by means of a Poisson summation formula. For arbitrarily polygonal plates, a criterion for truncating the image source generation process is examined and used as a control parameter for the precision of the estimations. The given examples show that the results are in good agreement with exact analytical solutions for simple plate shapes and with results obtained by the finite element method for more complex shapes. The convergence towards reference solutions as the number of image sources is increased is investigated. The main advantage of the method is that the accuracy is improved with structural damping and with frequency for a given number of image sources.

\section{9:40}

2aSAb6. Estimation of received acoustic levels in the near-field of a ship. Christopher Barber (Penn State University, PO Box 30, State College, PA 16804-0030, USA, cbarber@psu.edu)

Measured acoustic levels at the face of a receiver in close proximity to a ship in shallow water can differ substantially from the levels predicted by applying standard propagation models to a source level determined from a far-field radiated noise measurement. At distances typical of mine-ship engagements, the dimensions of the source (ship) can be large with respect to range, ocean depth and acoustic wavelength, and the ship cannot be approximated as a single point source, nor is the receiver necessarily located outside the acoustic near field. Direct free-field measurements of ship radiated noise at short range are problematic, so that the received acoustic level in the nearfield of a ship must be estimated from other available information. Options for generating such estimates are presented, including a hybrid approach combining empirically-derived transfer functions, a virtual distributedsource representation of the ship, and in-situ hull vibration measurements. The potential for computational methods such as BEM - FEM and measurements such as Nearfield Acoustic Holography to provide an improved understanding of the vibration sources, structural responses, radiation mechanisms and propagation paths associated with the radiated noise field of realistic ship structures is also explored. (Work sponsored by ONR Code $331)$.

\section{0:00}

2aSAb7. Some notes on the sound reduction index of pax cabins panels on cruise ships. Marco Biot (DINMA, University of Trieste, Via Valerio, 10, 34100 Trieste, Italy, biot@units.it), Francesco De Lorenzo (Fincantieri SpA, Passeggio S. Andrea, 6, 34100 Trieste, Italy, Francesco.Delorenzo @fincantieri.it)

The issue of comfort of passenger ships has became in the last years of paramount importance; in this context the noise and vibration control plays a leading role. The concept of comfort on board is subjective and it is impossible to define it with simple formula. On the other hand, a few number of significant parameters is commonly used to characterize the level of comfort on board of ships, and the sound insulation index in one of the most important. As known, it has a paramount importance in defining the contractual comfort on board of ships. The report is related with the problem of setting up the sound insulation index for pax cabins, specifically in relation to the nature and characteristics of the panels between cabins.

\section{0:20-10:40 Break}

\section{0:40}

2aSAb8. Acoustic and vibration measurements of NASA spacesuits. Durand R. Begault (NASA Ames Research Center, Mail Stop 262-2, NASA ARC, Moffett Field, CA 94035, USA, Durand.R.Begault@nasa.gov), James L. Hieronymus (NASA Ames Research Center, Mail Stop 262-2, NASA ARC, Moffett Field, CA 94035, USA, JimH@riacs.edu), Bernard D. Adelstein (NASA Ames Research Center, Mail Stop 262-2, NASA ARC, Moffett Field, CA 94035, USA, Bernard.D.Adelstein@ nasa.gov)

Research was conducted to examine interactions between acoustic signals and mechanical factors relevant to the design of improved auditory displays and noise mitigation for future NASA Spacesuits. Data were collected at NASA Johnson Space Center for the current Mark III advanced prototype surface suit during walking, walking with arm motion, and while seated. Measurements were made using multiple microphones (both head mounted and helmet mounted) and a tri-axial accelerometer to evaluate the effects of suit-borne vibration on overall background noise. Footfall impacts were found to create temporary depolarization of condenser microphone signals, and microphone positioning had a strong effect on signal-noise ratio, partially due to the effect of the helmet enclosure. The results indicate noise mitigation and signal conditioning strategies for improving lunar suit audio voice communications under extra-vehicular (EVA) conditions. Funded by a directed research program of NASA's Space Human Factors Engineering project and by NASA-JSC's Constellation EVA system audio group. 


\section{1:00}

2aSAb9. Influence of material properties on sound radiation for flat loudspeakers. Jaime Ramis Soriano (DFISTS. Univ. de Alicante, Carretera de Sant Vicent del Raspeig s/n, 03690 San Vicente del Raspeig, Spain, jramis@ua.es), Jesús Alba Fernandez (Escola Politècnica Superior de Gandia, Universitat Politècnica de València, Crtra Natzaret-Oliva s/n, 46730 Gandia, Spain, jesalba@fis.upv.es), Jorge Frances Monllor

(DFISTS. Univ. de Alicante, Carretera de Sant Vicent del Raspeig s/n, 03690 San Vicente del Raspeig, Spain, jfmonllor@ua.es), Jorge P. Arenas Bermudez (Universidad Austral de Chile, Institute of Acoustics, 567 Valdivia, Chile, jorgep.arenas@gmail.com)

This work aims to analyze numerically, analytically and experimentally, the influence on sound power radiated, frequency response, and directionality of different materials in flat loudspeakers. Concerned experimental setup, the excitation force consists in a shaker which his mechanical characteristics are completely known. Finite Element Method has been used to simulate this configuration. The analytical model used couples the electrical, mechanical and acoustical system of the panel. The parameters of the panel material which we are focused are elastic modulus and loss factor. The analytical formalism serves as the basis to study the structural and vibrational behaviour of the system that also is analyzed with experimental results. The results obtained from different materials and different excitation position serves as basis for optimum configuration in this type of speakers.

\section{$11: 20$}

2aSAb10. Acoustical Effectiveness of Damping Coating. Raymond Fischer (Noise Control Engineering Inc, 799 Middlesex Tnpk, Billerica, MA 01821, USA, rayf@noise-control.com), Leonid Boroditsky (Noise Control Engineering Inc, 799 Middlesex Tnpk, Billerica, MA 01821, USA, leob@noise-control.com)

Damping coatings have again become popular in the shipbuilding industry to reduce onboard and underwater noise. Understanding of the actual effectiveness of damping tiles applied to ship structures is important for noise prediction during the design stage and for optimizing noise control. The commonly held opinion that energy loss is proportional to loss factor values is not confirmed by tests conducted on actual structures. This paper discusses differences between loss factors measured on the Oberst beam and on two-dimensional real structures. The paper shows how loss factor change may influence transmission loss, radiation efficiency and acceptance. The results of loss factor measurements in different conditions including waterloaded media are demonstrated. influences of resonant and non- resonant modes on damping tile effectiveness is discussed. Corrections for SEA noise prediction algorithms, taking into account results of this research, are proposed.

\section{1:40}

2aSAb11. On the prediction of absorption coefficient of porous materials with Finite Elements. Sabine Langer (TU Braunschweig, Institute of Applied Mechanics, Spielmannstr. 11, 38106 Braunschweig, Germany, s.langer@tu-bs.de), Meike Wulkau (TU Braunschweig, Institute of Applied Mechanics, Spielmannstr. 11, 38106 Braunschweig, Germany, m.wulkau@tu-bs.de)

The absorption coefficient gives the relation between incited and reflected sound power on a surface and is a measure for the damping properties of materials. Experimentally it can be investigated by using the impedance tube for perpendicular incitation and the echo chamber in the case of a diffuse sound field as is known. To optimize the sound absorption properties of materials in the pre-prototype stage, numerical simulation can support the design of materials. A detailed finite element simulation based on Biots Theory for poroelastic-media is used to predict the absorption coefficient of materials with open-pored surfaces. Prospects and limits of this strategy are discussed.

\section{2:00}

2aSAb12. Ground-borne vibration case studies in residential constructions. James Perry (Cerami \& Associates, 404 Fifth Avenue, New York, NY 10018, USA, jperry@ ceramiassociates.com)

Case studies are examined for several new high-rise residential and hotel buildings proposed for construction over or near underground and surface transit systems. Baseline vibration measurements were taken on grade and existing building structures and projected against standard functional vibration criteria (ANSI S3.29 / ISO 2631 2). Through post-construction testing and client feedback, the vibration and structure-borne noise impacts were field verified. Study results offer insight into the correlation between these vibration criteria and the subjective human perceptions of various sensitivities. Further items of discussion include the relative effects of various architectural constructions, building types, and applications as well as mitigating sound and vibration controls. 


\title{
Session 2aSAc
}

\section{Structural Acoustics and Vibration and EURONOISE: Source Characterization in Structure Borne Noise Problems I}

\author{
Evan Davis, Cochair \\ The Boeing Company, P.O. Box 3707, Seattle, WA 98124, USA \\ Charles Pezerat, Cochair \\ Laboratoire Vibrations Acoustique - INSA Lyon, 25 bis avenue Jean Capelle, Bâtiment Saint-Exupéry, Villeurbanne cedex, \\ F-69621, France
}

Invited Papers

11:00

2aSAc1. Structure-borne sound transmission from machines into ribbed structures. Barry M. Gibbs (University of Liverpool, School of Architecture, Abercromby Square, L693BX Liverpool, UK, bmg@liv.ac.uk), Andreas R. Mayr (Stuttgart University of Applied Sciences, Schellingstrasse 24, 70174 Stuttgart, Germany, andreas.mayr@hft-stuttgart.de)

The total structure-borne sound power of an installed machine is a function of the source activity and mobility, and the receiver mobility, at each contact and for each component of excitation. The data and computational requirements for prediction therefore are large. Manufacturers view their products as single entities and desire corresponding single values of source strength. A laboratory reception plate measurement procedure has been proposed which yields single equivalent values of source strength and source mobility. The source data, in combination with an estimate of the single equivalent value of receiver mobility, yields the approximate total installed power. The accuracy of the estimate is dependent on the spatial variation in contact conditions over the connections. In addition, phase information has been lost in the simplification. Case studies are described for multiple contact sources on a non-homogeneous plate (a timber-joist floor) where the approximate estimates of structure-borne power are compared with exact values obtained from full mobility formulations.

11:20

2aSAc2. Time domain identification of loads on plate-like structures using an array of acoustic velocity sensors. Quentin Leclere (Laboratoire Vibrations Acoustique - INSA Lyon, 25 bis avenue Jean Capelle, Bâtiment Saint-Exupéry, F-69621 Villeurbanne cedex, France, quentin.leclere@insa-lyon.fr), Charles Pezerat (Laboratoire Vibrations Acoustique - INSA Lyon, 25 bis avenue Jean Capelle, Bâtiment Saint-Exupéry, F-69621 Villeurbanne cedex, France, charles.pezerat@insa-lyon.fr)

The FAT (Force Analysis Technique) method has been developped to identify loads on beams or plates from the knowledge of their flexural displacements. The method is based on a local discretisation of the differential operator of the studied structure : all derivatives of the displacement field are assessed at a given point from a finite difference scheme. The estimation of the operator gives as an output the value of the force distribution. Up to now, applications of the FAT method have been made in the frequency domain, scanning the studied structure with accelerometers or with a laser vibrometer, and using phase references to get the phase relation between different points. The aim of the present study is to show that the FAT method allows to identify loads in the time domain. This operation requires the simultaneous measurement of at least 13 points on the plate, that can be realized without contact using an array of acoustic velocity sensors in the very near field of the plate. The method has been applied on a plate excited by an acoustic diffuse field. The identified force distribution is compared to the parietal acoustic pressure measured in the reverberant room.

\section{1:40}

2aSAc3. Measurements of the bending moment at boundaries of a structure. Simon Chesne (LaMCoS - INSA-Lyon - CNRS UMR5259, 18-20, rue des Sciences, Bâtiment Jean d'Alembert, F-69621 Villeurbanne, France, simon.chesne@insa-lyon.fr), Baptiste Chomette (LaMCoS - INSA-lyon - CNRS UMR5259, 18-20, rue des Sciences, Bâtiment Jean d'Alembert, F-69621 Villeurbanne, France, baptiste.chomette@insa-lyon.fr), Charles Pezerat (Laboratoire Vibrations Acoustique - INSA Lyon, 25 bis avenue Jean Capelle, Bâtiment Saint-Exupéry, F-69621 Villeurbanne cedex, France, charles.pezerat@insa-lyon.fr)

In the vibration transmission process, the part due to the moment excitation is often neglected, because of the difficulty to measure them, even if these terms are sometimes important. Indeed, several studies show that the influence of the moment in structure borne power transmission can be higher than simple force. Moment measurement or identification is an old problem especially at boundaries. It has been mainly investigated in mobility methods. In fact, bending moment expression can be seen as spatial derivatives of displacements. These derivatives can be approximated from measured displacements but two major difficulties appear: derivatives are highly sensitive to measurement errors and the usual methods used to obtain them (finite differences, modal approach, etc.) are not well adapted at boundary points. In this paper, three different moment identification methods are investigated where the considered structure is a beam. Both of these approaches are already developed by authors, the third method is based on the use of particular mechanical and electric setups of piezoelectric patches. These 3 methods are numerically and experimentally implemented allowing us to compare and discuss on results. 
2aSAc4. Characterisation of structure borne sound sources from measurement in-situ. Andrew Elliott (University of Salford, Acoustic Research Centre, Newton Building, M5 4WT Salford, UK, a.s.elliott@pgr.salford.ac.uk), Andy T. Moorhouse (University of Salford, Acoustic Research Centre, Newton Building, M5 4WT Salford, UK, a.t.moorhouse@ salford.ac.uk)

In-situ source characterisation methods are those which involve measurements made whilst source and receiver are coupled as they would be in a real installation. Potentially in-situ source characterisation may account for the physical reality lost in the "black box" approach. There are other potential benefits such as ease of measurement. In this work a structure borne sound source is characterised using in-situ measurements of blocked force and coupled mobility. Promising results from the method have been presented previously. Further to this, an extension of the method allowing the use remote measurement positions has been developed. Using reciprocity, the extended method will further ease measurement for situations where access poses a problem. The extended method is outlined and some preliminary validation results are presented.

$12: 20$

2aSAc5. General model of a structure-borne sound source and its application to shock vibration. Yuri Bobrovnitskii (Mechanical Engineering Research Institute, 4, M. Kharitonievky Str., 101990 Moscow, Russian Federation, yuri@imash.ac.ru), Tatiana Tomilina (Mechanical Engineering Research Institute, 4, M. Kharitonievky Str., 101990 Moscow, Russian Federation, tatiana@imash .ac.ru)

One of the well-known models of structure-borne sound sources is described by the quantities defined at the source-receiving structure interface: by the matrix of the source impedances or mobilities and by the vector of the blocked forces or free velocities. Together with the receiver impedances or mobilities it allows one to predict the vibration field in the receiver and to develop and examine candidates for source characterization. In this paper, it is shown analytically that this model is valid when the sound is generated inside the source by a kinematic excitation as well as by force excitation or their combination and, thus, it represents a rather general model with wide range of applications. Most attention is paid to its peculiarities and limitations when applied to shock sources. Results are illustrated by the data measured in the shock tests of some scientific space instruments.

TUESDAY MORNING, 1 JULY 2008

ROOM 240, 8:20 TO 10:40 A.M.

\title{
Session 2aSCa
}

\section{Speech Communication: Speech Technology II}

\author{
Indranil Dutta, Chair \\ Rice University, Center for the Study of Languages, MS \#36, 6100 Main St., Houston, TX 77005, USA
}

\section{Contributed Papers}

8:20

2aSCa1. Evaluation of acoustic environments using deteriorated speech sound. Yoshiki Nagatani (Nara Medical University, 840 Shijo-cho, 634-8522 Kashihara, Japan, naramed-u@nagatani.ne.jp), Takefumi Sakaguchi (Nara Medical University, 840 Shijo-cho, 634-8522 Kashihara, Japan, t-saka@naramed-u.ac.jp), Hiroshi Hosoi (Nara Medical University, 840 Shijo-cho, 634-8522 Kashihara, Japan, hosoi@naramed-u.ac.jp)

Aged or hearing-impaired people require better acoustical environments for higher QOL. Many methods of evaluating the quality of speech in acoustical environments have been developed. However, since they mainly focus on quality of speech in bad conditions (e.g. environments with huge noise or long reverberation), they are not suited for evaluations in generic environments such as normal houses or public facilities for aged people. For instance, the scores of D value (deutlichkeit) or speech transmission index (STI) are too high and not sensitive enough to refer to such environments. The intelligibility test using normal speech sounds cannot clarify the differences between different room environments because the intelligibility scores reach almost 100 percent in ordinary room environments. Therefore, we proposed a new evaluation method using deteriorated speech sounds. In this method, signal-processed speech sounds are presented to trial subjects under target sound environments. In this study, Japanese monosyllabic speech sounds convoluted by the impulse responses of room reverberations were presented through a headphone in order to simulate the architectural acous- tic environments. As a result, it was shown that this new method could detect the small difference of sound environments, which the conventional methods could hardly evaluate.

\section{8:40}

2aSCa2. Measurement of Speech Intelligibility Using Low Level Output - Threshold Efficient S/N Ratios. Christos Nestoras (London South Bank University, FESBE, Borough Road, SE1 0AA London, UK, nestorasc@gmail.com), Stephen Dance (London South Bank University, FESBE, Borough Road, SE1 0AA London, UK, dances@1sbu.ac.uk)

Excessive background noise levels or perhaps annoyance due to a high test signal level are the main reasons for having a low signal to noise ratio $(\mathrm{S} / \mathrm{N})$ or low level output during a measurement session. A significant error, in this sense, can be introduced in the resulting data since the measurement technique requires, among others, a minimum $\mathrm{S} / \mathrm{N}$ ratio for an accurate result. A validation is presented of low level output intelligibility measurements in an attempt to establish a point of reference for the verification of data accuracy for a given space. An indication of the functions taking place in this respect could be obtained through practical experimentation. Results are reported here. 


\section{9:00}

2aSCa3. Vowels recognition using mellin transform and plp-based feature extraction. Mahdi Jamaati (Technical University of Shahrood, 12345 Shahrood, Iran, mahdi.jamaati@gmail.com), Hossein Marvi (Technical University of Shahrood, 12345 Shahrood, Iran, h_marvi@shahroodut.ac.ir), Milad Lankarany (Technical University of Shahrood, 12345 Shahrood, Iran, milad.lankarany@gmail.com)

Feature extraction for speech recognition is a subject of majer interest today. Different feature have been investigated in speech recognition systems. The scale transform is a particular restriction of the Mellin transform. The key property of the scale transform is the scale invariance .The mel frequency(MFCC) and perceptual linear predicate (PLP) have usually reported to have yielded good performance. In this paper, a new method is presented which combines feature extracted from mellin transform with the plp features. To evaluate the performance of the proposed features the comparative tests with the lpc features, MFCC and PLP were performed on the vowels based speech recognition system. Preliminary experiment show that this approach posses promissing result.

\section{9:20}

2aSCa4. A system for automatic detection and correction of detuned singing. Michał Lech (Gdansk University of Technology, Multimedia Systems Department, 11/12 Gabriela Narutowicza Street, 80-952 Gdansk, Poland, mlech@sound.eti.pg.gda.pl), Bozena Kostek (Gdansk University of Technology, Multimedia Systems Department, 11/12 Gabriela Narutowicza Street, 80-952 Gdansk, Poland, bozenka@ sound.eti.pg.gda.pl)

The aim of the paper is to show a system engineered for automatic detection and correction of detuned singing. For this purpose, existing methods of fundamental frequency detection and pitch correction are reviewed. In addition, main characteristics of some existing detuning systems are presented. As algorithms for fundamental frequencies detection and pitch correction, the fast autocorrelation and HPS (Harmonic Product Spectrum), and the modified phase vocoder and PSOLA (Pitch-Synchronous Overlap-Add) are chosen and examined. Four possible combinations of the algorithms are reviewed not only in the context of fundamental frequency detection and pitch shifting correctness but also with regard to the quality of the resulting singing signal. Experiments are performed on both male and female singing samples consisting of a variety of tones and various articulations. Basing on the obtained results, it is concluded that the HPS and PSOLA algorithms are the optimum choice as means to correct detuned singing. In addition, listening tests are performed in order to confirm objective measurements of pitch detection and correction. The system is implemented in JAVA. Conclusions are drawn and proposals of improvements are provided.

\section{9:40}

2aSCa5. Spectrotemporal and f0 evidence for a theory of enhancement. Indranil Dutta (Rice University, Center for the Study of Languages, MS \#36, 6100 Main St., Houston, TX 77005, USA, indranil.dutta@rice.edu)

According to Keyser \& Stevens, 2006, apart from universal features that are utilized to calculate motoric instructions, there is a second parallel and language-specific process called enhancement, where additional motoric instructions are processed for the enhancement of the saliency of features that are in danger of being obliterated. The "Standard View" on the distinction between voiced stops (VS) and voiced aspirated stops (VAS) in Hindi proposes that the breathy-murmured release following VAS is sufficient to make the contrast between the VS and VAS (Ladefoged \& Maddieson, 1996). Hence, audible distinctions between VAS and VS during closure (CD/VLT) are not relevant in maintaining the contrast. Results from our studies show that CD is a relevant cue in making a distinction between aspirated and unaspirated stops. Spectral intensity measures show that speakers employ different glottal configurations to obtain the breathy/modal voicing contrast. VLT durations are inversely correlated with f0, such that longer
VLT for VAS leads to further lowering of $f_{0}$. This evidence suggests that $f_{0}$ perturbations, differences in the durations of closure, and nature and extent of aspiration, all contribute in making the four-way stop contrast possible in Hindi, in support of a theory of enhancement.

\section{0:00}

2aSCa6. A Statistical Prosodic Model for Voice Conversion. Jan Schwarz (Institute for Circuit and System Theory, Christian-AlbrechtsUniversity of Kiel, Kaiserstrasse 2, 24143 Kiel, Germany, js@tf.uni-kiel.de), Ulrich Heute (Institute for Circuit and System Theory, Christian-Albrechts-University of Kiel, Kaiserstrasse 2, 24143 Kiel, Germany,uh@tf.uni-kiel.de)

Nowadays Text-to-Speech (TTS) systems adapt the output voice to the user and the corresponding application. The aim is a personalisation. Thereby, the user is set into familiar surroundings, increasing the TTS acceptance. For example, an e-mail client that may read the incoming messages with the synthesised voice sounding like that of the sender. Such a personalised TTS system is costly; so, voice-conversion (VC) techniques are used to save resources. VC transforms the voice of a "source speaker" in such a way that the converted voice sounds like that of another "'target speaker'. This voice sounds only natural, if it includes all features relevant for the true target voice. Here, a main problem is the mapping of the prosody which is one of the essential features. This contribution introduces a statistical prosodic model for voice conversion. It is based on Gaussian-Mixture Models (GMM), trained for the pitch and the duration of diphones. To ensure sufficient data for the GMM training, seven diphone classes are separated as related to the international phonetic alphabet. The suitability for VC as well as limitations, necessary extensions (stress) and problems are pointed out.

\section{0:20}

2aSCa7. Modelling acoustic parameters of prosody for read and acted-speech synthesis. Milan Rusko (Institute of Informatics of the Slovak Academy of Sciences, Dubravska cesta 9, 84507 Bratislava, Slovakia, milan.rusko@savba.sk), Marián Trnka (Institute of Informatics of the Slovak Academy of Sciences, Dubravska cesta 9, 84507 Bratislava, Slovakia, trnka@savba.sk), Sakhia Darjaa (Institute of Informatics of the Slovak Academy of Sciences, Dubravska cesta 9, 84507 Bratislava, Slovakia, utrrsach@savba.sk), Richard Kováč (Institute of Informatics of the Slovak Academy of Sciences, Dubravska cesta 9, 84507 Bratislava, Slovakia, richard.kovac@savba.sk), Juraj Hamar (Philosophical Faculty, Comenius University, Gondova 2,81801 Bratislava, Slovakia, juraj.hamar@chello.sk)

The prosody model is one of the most important parts of every speech synthesizer, influencing mainly its naturalness. The intonation contour and phoneme lengths (together with speech quality) bear a great deal of extralinguistic and paralinguistic information contained in the synthesized speech. The features reflecting personality, mood and emotions of the speaker are in strong interaction with those reflecting speech styles. Anyway the appropriate choose of prosody model and training material can make it possible to create special model for every speaking style. The paper presents our approach to modelling of acoustic parameters of prosody in two different speech styles in Slovak. Our model is based on Classification and regression trees (CARTs). It uses independent CART for phoneme lengths and three CARTs for fundamental frequency (F0) at the beginning, centre, and end of every syllable. Two hours of read speech were used for training a model of read speech. The recordings of a puppet player were used to train a model of acted speech. The models were implemented in the Kempelen 2.2 unit selection Slovak speech synthesizer. The listening tests have shown that the models are capable of modelling significant amount of the differences of the two speaking styles. 


\title{
Session 2aSCb
}

\section{Speech Communication: How do Physical and Motor Knowledge Matter to Speech Perception?}

\author{
Carol A. Fowler, Cochair \\ Haskins Laboratories, 300 George St, Suite 900, New Haven, CT 06511, USA \\ Mikko Sams, Cochair \\ Helsinki University of Technology, Lab. of Computational Engineering, Cognitive Science and Technology, Otakaari 5A, 02150 \\ Espoo ('Magnet house'), Finland
}

\section{Invited Papers}

\section{8:20}

2aSCb1. Action-based multisensory integration in striking events. Bruno L. Giordano (Centre for Interdisciplinary Research in Music Media \& Technology (CIRMMT) - Schulich School of Music - McGill Univ., 555 Sherbrooke Street West, Montreal, QC H3A1E3, Canada, bruno.giordano@music.mcgill.ca), Stephen McAdams (Centre for Interdisciplinary Research in Music Media \& Technology (CIRMMT) - Schulich School of Music - McGill Univ., 555 Sherbrooke Street West, Montreal, QC H3A1E3, Canada, smc@music.mcgill.ca), Paolo Crosato (Dept. of Information Engineering, Univ. of Padova, Via G. Gradenigo 6/A, 35131 Padova, Italy, jimbobaus@virgilio.it), Federico Avanzini (Dept. of Information Engineering, Univ. of Padova, Via G. Gradenigo 6/A, 35131 Padova, Italy, avanzini@dei.unipd.it), Carmine Casciato (Centre for Interdisciplinary Research in Music Media \& Technology (CIRMMT) - Schulich School of Music - McGill Univ., 555 Sherbrooke Street West, Montreal, QC H3A1E3, Canada, casciato@music.mcgill.ca), Stephen Sinclair (Centre for Interdisciplinary Research in Music Media \& Technology (CIRMMT) Schulich School of Music - McGill Univ., 555 Sherbrooke Street West, Montreal, QC H3A1E3, Canada, sinclair@music.mcgill.ca), Marcelo M. Wanderley (Centre for Interdisciplinary Research in Music Media \& Technology (CIRMMT) - Schulich School of Music - McGill Univ., 555 Sherbrooke Street West, Montreal, QC H3A1E3, Canada, marcelo.wanderley@mcgill .ca)

Many theories of multisensory integration focus on action-independent perception. This approach has limited ecological validity, because under everyday conditions we frequently act upon multisensory events through motor behavior. We present a novel methodology for the study of multisensory integration in action-directed perception, focusing on striking events. Observers repeatedly hit a virtual object with a target striking velocity, and are presented with various types of sensory information about the striking event: auditory and/or haptic and/or visual. For each of the experimental trials, they initially receive feedback on the tracking of the target striking velocity. In a second phase, feedback is eliminated. In a third phase, the sensory properties of the striking event are changed. We quantify the extent to which a variation in each of the sensory modalities disrupts performance in the tracking of the target striking velocity. Multisensory dominance hierarchies are inferred from the comparison of the patterns of performance disruption in unimodal and multisensory contexts.

\section{$8: 40$}

2aSCb2. Amodal specification of talker-specific motor behavior. Lawrence D. Rosenblum (University of California, Riverside, Department of Psychology, 900 University Ave., Riverside, CA 92521, USA, rosenblu@citrus.ucr.edu)

There is substantial evidence that the speech perception function incorporates information for articulatory motor behavior. Research over the last 15 years has shown that speech perception also uses information for talker-specific motor behavior. Interestingly, this research reveals that talker information can facilitate speech perception whether it is apprehended through auditory or visual speech (lipread) means. The multimodal nature of these effects could mean that the talker-specific properties used by the system are amodal and motoric-or gestural-in nature. Evidence for this proposition-that talker-specific facilitation of speech is based on amodal specification of gestural style-will be discussed. This evidence includes findings that isolated talker-specific phonetic information, available both optically and acoustically, can be informative about talker identity to the degree that it supports cross-modal talker matching. Other evidence shows that the talker familiarity gained through one modality can facilitate perception of speech in the other. Finally, recent evidence reveals that speech perceivers will align to (inadvertently imitate) talker-specific properties of utterances they are asked to shadow, whether that speech is presented auditorily or visually. These results suggest that some of the 'motor knowledge' bearing on speech perception takes the form of talker-specific gestural properties, amodally specified. 
2aSCb3. Cross-modal synchrony perception reveals aspects of categorical perception. Armin Kohlrausch (Philips Research Europe, Digital Signal Processing (MS WO02), High Tech Campus 36, 5656 AE Eindhoven, Netherlands, armin.kohlrausch@philips.com), Rob L. Van Eijk (Technische Universiteit Eindhoven, Human-Technology Interaction, P.O. Box 513, 5600 MB Eindhoven, Netherlands, r.l.j.v.eijk@tue.nl), Steven Van De Par (Philips Research Europe, Digital Signal Processing (MS WO02), High Tech Campus 36, 5656 AE Eindhoven, Netherlands, steven.van.de.par@philips.com), James F. Juola (University of Kansas, Department of Psychology, 1415 Jayhawk Blvd., Lawrence, KS KS 66045, USA, juolas@ku.edu), Michael Vitevich (University of Kansas, Department of Psychology, 1415 Jayhawk Blvd., Lawrence, KS KS 66045, USA, mvitevit@ku.edu)

The phenomenon of "categorical perception" has played an important role in speech research. When a specific (combination of) feature(s) of a speech stimulus is varied along a physical dimension, categorical perception is reflected by two observations: (1) the percept of the sound changes abruptly from one category to another, e.g., from "ba" to "da" to "ga" for changes in formant transition frequencies, and (2) physical changes of a given amount lead to more easily perceivable differences for stimuli close to a category boundary, compared to stimuli in the center of a category. In this talk, I present data about audio-visual synchrony perception, which indicate that perceived synchrony also reveals properties of categorical perception. Depending on the physical delay between the auditory and visual component, the percept changes from "audio first," to "synchronous" to "video first." When measuring sensitivity to changes in audio-visual delays, we observe that the thresholds are small for stimuli at the transitions between the earlier determined categories, while they are larger for stimuli within a category.

\section{9:20}

2aSCb4. Do we perceive articulatory gestures when we listen to speech? Pierre Divenyi (VA Northern Calif. Health Care Syst. and East Bay Inst. for Research and Education, Bldg. R4, 150 Muir Rd., Martinez, CA 94553, USA, pdivenyi@ebire.org), Adam Lammert (VA Northern Calif. Health Care Syst. and East Bay Inst. for Research and Education, Bldg. R4, 150 Muir Rd., Martinez, CA 94553, USA, alammert@ebire.org)

Articulatory synthesis methods, classic and contemporary, have demonstrated that it is possible to generate speech from an ensemble of functions derived from articulatory gestures. Such gesture-to-waveform transforms suggest that, inversely, the speech signal should be also decomposable into the same set of gesture, or gesture-like, functions. These functions vary slowly in time and their association with the speech waveform (words as well as sentences) can be established by machine learning algorithms. In a recent study at our laboratory, listeners were asked to type the word or the sentence they heard, with speech (degraded in diverse ways) as the stimulus. The subjects' responses were synthesized, time-aligned with the stimulus, and decomposed into a set of eight gestures, as specified by the Haskins Laboratories TADA system (http://www.haskins.yale.edu/tada_download/index.html). When the running distance between input and response gesture functions is calculated, results indicate a significant degree of gesture information transmitted even during severely degraded speech segments, suggesting that the perceptual system may track speech via underlying functions similar to gestures. Epochs at which this running distance estimate fails, i.e., exceeds a certain threshold, may be considered to signal periods during which insufficient bottom-up information had to be supplemented using higher-order linguistic knowledge.

\section{9:40}

2aSCb5. Hearing the tongue and lips of vowel gestures: A new differential paradigm. Jean-Luc Schwartz (ICP-GIPSA, INPG, 46 Av. Félix Viallet, 38031 Grenoble, France, schwartz@icp.inpg.fr), Nathalie Vallée (ICP-GIPSA, INPG, 46 Av. Félix Viallet, 38031 Grenoble, France, vallee@icp.inpg.fr), Sonia Kandel (LPNC, Université Pierre Mendès-France - BP 47, 38040 Grenoble, France, Sonia.Kandel@upmf-grenoble.fr)

It is an old question to know to what extent a listener can recover the articulatory dimensions of a speaker's gesture. In the case of vocalic configurations, a number of experiments have been done on expert phoneticians, showing that vowel height can be reasonably well estimated from the sound, but the front-back and lip rounding dimensions are much less well recovered. However, almost nothing has been done on naive listeners, due to the difficulty to perform absolute estimations in the lack of explicit phonetic knowledge. In the past years, we have developed an original paradigm, exploiting differential rather than absolute estimations. We show that French listeners, even naïve, are able to discriminate to a certain extent which vowel in a given pair has a higher vs. lower or more front vs. more back tongue position, or more or less rounded lips. From these data, we have elaborated an algorithm enabling to estimate what are the internal representations of vowel height, frontness and rounding, and correlated these representations with acoustic parameters, F1, F2-F1 and F'2 in Barks appearing to play a key role in the auditory recovery of these three motor dimensions.

\section{0:00}

2aSCb6. Mathematical Evidence For Motor Theories of Speech Perception. Gordon Ramsay (Haskins Laboratories, 300 George Street, New Haven, CT 06511, USA, ramsay@ haskins.yale.edu)

Theories of speech perception have often proposed, controversially, that recovery of phonological information from the acoustic signal necessarily requires implicit knowledge of the physical processes underlying speech production. Most of the evidence supporting this hypothesis has been derived empirically from experiments in speech perception. In this paper, we show that many of the basic ideas underlying motor theories of speech perception can also be derived mathematically from first principles, using classic results in stochastic nonlinear filtering theory. Two key results, the Duncan-Mortenson-Zakai and Fujisaki-Kallianpur-Kunita theorems, show that the optimal state estimator for any partially-observed nonlinear stochastic dynamical system always takes the form of a "matched filter", which is itself a nonlinear stochastic dynamical system, the structure of which mimics, and resonates with, the structure of the original. Interpreting this in the context of speech perception, recovery of phonological information from sound produced by a human vocal tract necessarily involves construction of an internal model of the processes implicated in speech production. A key prediction, which we explore, is that any such model need not reproduce all the details of these processes, but is only required to predict the lawful conditional correlation between gesture and sound. 


\title{
Session 2aSCc
}

\section{Speech Communication: Speech Recognition in Noisy Environments}

\author{
Olivier Siohan, Cochair \\ Advanced Large Vocabulary Speech Recognition, IBM Watson Research Center, Yorktown, NY 10598, USA \\ Georges Linares, Cochair \\ Université d'Avignon et des Pays de Vaucluse, Laboratoire Informatique d'Avignon, 339, chemin des Meinajaries, Agroparc BP \\ 1228, 84911 Avignon Cedex 9, France
}

\section{Contributed Paper}

\section{0:40}

2aSCc1. Speech recognition with body-conducted speech using differential acceleration. Masashi Nakayama (Hiroshima City University / National Institute of Advanced Industrial Science and Technology, 3-4-1 Ozuka-Higashi, Asa-Minami-Ku, 731-3194 Hiroshima, Japan, m.nakayama @ aist.go.jp), Shunsuke Ishimitsu (Hiroshima City University / National Institute of Advanced Industrial Science and Technology, 3-4-1 OzukaHigashi, Asa-Minami-Ku, 731-3194 Hiroshima, Japan, ishimitu @ hiroshima-cu.ac.jp), Seiji Nakagawa (National Institute of Advanced Industrial Science and Technology (AIST), 1-8-31 Midorigaoka, 563-8577 Ikeda, Osaka, Japan, s-nakagawa@aist.go.jp)

Speech-recognition rates decrease in noisy environments. The bodyconducted speech, conducted in solids such as body and skins, has a noise- robust characteristics and can be served for recognition systems even in 98 dBSPL-noise (-20 dBSNR) environments. However, the body-conduction could not capture high frequency sounds. Conventional methods for the improvement in sound quality of body-conducted speeches needs both speeches and body-conducted speeches. In this paper, a new body-conducted speech retrieval technique in sound quality without a speech signal itself is proposed. First, high-frequency components in the body-conducted speech were emphasized using differential acceleration. Second, conventional noise reduction method was adopted to make a clear body-conducted speech from a retrieval speech which contains constant noise. The recognition experiments using the proposed method showed that it improved recognition rate in all speakers.

\section{Invited Paper}

\section{1:00}

2aSCc2. Exploiting confidence measures for missing data speech recognition. Christophe Cerisara (LORIA UMR 7503, Campus Scientifique, 54506 Vandoeuvre-lès-Nancy, France, Christophe.Cerisara@loria.fr)

\begin{abstract}
Automatic speech recognition in highly non-stationary noise, for instance with a competing speaker or background music, is an extremely challenging and still unsolved problem. Missing data recognition is a robust approach that is well adapted to this kind of noise. A standard missing data technique consists in marginalizing out, from the observation likelihoods computed during decoding, the contribution of the spectro-temporal fragments that are dominated by noise. However, such an approach can hardly be applied to advanced parameterization domains that do not separate speech from noise frequencies, such as the cepstrum or ETSI AFE. We propose in this work to extend this technique to such parameterization domains, and not only to spectrographic-like front-ends as it was the case before. This is realized by masking the observations that favor erroneous decoding paths, instead of masking the features that are dominated by noise. These new missing data "masks" are now estimated based on speech recognition confidence measures, which can be considered as indicators of the reliability of decoding paths. A first version of this robust algorithm is evaluated on the French broadcast news ESTER corpus.
\end{abstract}

\section{Contributed Paper}

11:20

2aSCc3. An MTF-based blind restoration of temporal power envelopes as a front-end processor for automatic speech recognition systems in reverberant environments. Xugang Lu (Japan Advanced Institute of Science and Technology, 1-1, Asahidai, Nomi, 923-1292 Sendai, Japan, xugang@jaist.ac.jp), Masashi Unoki (JAIST, 1-1 Asahidai, 923-1292 Nomi, Japan, unoki@jaist.ac.jp), Masato Akagi (Japan Advanced Institute of Science and Technology, 1-1, Asahidai, Nomi, 923-1292 Sendai, Japan, akagi@jaist.ac.jp)

To reduce speech degradation in reverberant environments, we previously proposed a modulation transfer function (MTF) based method for speech dereverberation. It is based on the MTF relation that the sub-band temporal power envelope of reverberant speech can be represented as the convolution between temporal power envelopes of clean speech and the room impulse response. Therefore, the sub-band power envelope of clean speech can be estimated using inverse MTF filtering without measuring the room impulse response. We tested the effectiveness of this method as a front-end for automatic speech recognition (ASR) in both artificial and real reverberant environments. Reverberant speech signals were created by simple convolution of clean speech (AURORA-2J) and artificially-produced or real room impulse responses. The relative spectral filtering of the auditory-power-spectrum based method was used as a baseline. Compared with the baseline, our proposed method had $36.64 \%$ and $21.68 \%$ improvements in error reduction rate for artificial reverberant environments (reverberation times from 0.2 to $2.0 \mathrm{~s}$ ) and real reverberant environments (43 reverberant impulse responses), respectively. These results indicate that our proposed method can be used as a robust front-end for ASR. [Work supported by a Grant-in-Aid for Science Research from the Japanese Ministry of Education (No. 18680017).] 


\section{Invited Paper}

\section{1:40}

2aSCc4. Front-end processing of a distant-talking speech interface for control of an interactive TV system. Maurizio Omologo (Fondazione Bruno Kessler - IRST, Via Sommarive, 18, Povo, 38050 Trento, Italy, omologo@ fbk.eu)

This work addresses a research activity being conducted for the development of a user-friendly interface for the access to a virtual smart assistant enabling the interaction with TV-related digital devices and infotainment services. In the given scenario, the users can speak in a natural and comfortable way, not encumbered by any hand-held or head-mounted microphone. The environment is typically a living room, equipped with digital TV, Hi-Fi audio devices, etc., and populated by a group of people (e.g., family members). Among the most challenging issues involved in this scenario are a multi-microphone front-end for an effective processing of the given acoustic scene, an Acoustic Echo Cancellation (AEC) component to compensate the sound produced by loudspeakers, and a multi-modal distanttalking spoken dialogue system. As far as the front-end is concerned, multiple speaker localization, speech activity detection, speaker identification, and speech recognition will have to perform accurately even when AEC is applied to the given microphone array. The paper aims to present preliminary results of this research, which is being conducted under the European Project DICIT.

\section{Contributed Paper}

\section{2:00}

2aSCc5. Speaker adaptation combined with missing data reconstruction. Ulpu Remes (Adaptive Informatics Research Centre, Helsinki University of Technology, P.O. Box 5400, 02015 Espoo, Finland, ulpu.remes@tkk.fi), Kalle J. Palomäki (Adaptive Informatics Research Centre, Helsinki University of Technology, P.O. Box 5400, 02015 Espoo, Finland, kalle.palomaki@tkk.fi), Mikko Kurimo (Adaptive Informatics Research Centre, Helsinki University of Technology, P.O. Box 5400, 02015 Espoo, Finland, mikko.kurimo@tkk.fi)

Missing data methods offer an attractive framework for automatic speech recognition (ASR) under time-varying and unpredictable noise conditions. The motivation for the methods comes from the human speech perception and auditory scene analysis, studies on which have suggested that the more intense sound components in an auditory scene dominate the neu- ral response to the scene. The missing data methods developed for ASR divide the noisy speech signal to speech and noise dominated regions. Speech components in the noise dominated regions are viewed as missing but may be reconstructed based on the observed components. Missing data methods have performed well in previous studies with noise corrupted speech but have not become a standard in ASR. Especially considering ASR on large vocabularies, the methods have a disadvantage: it is not straightforward to combine them with common speaker adaptation methods such as constrained maximum likelihood linear regression (CMLLR). In this work, we investigate efficient ways to combine missing data reconstruction with speaker adaptation in large vocabulary ASR task with speech data recorded in noisy real-world environments. The experiments show that speaker adaptation combined with the missing data reconstruction can improve the missing data approach in noisy large vocabulary ASR.

\section{Invited Paper}

2aSCc6. An efficient frame selection approach to variable frame rate analysis for noise robust speech recognition. Zheng-Hua Tan (Department of Electronic Systems, Aalborg University, Niels Jernes Vej 12, 9220 Aalborg, Denmark, zt@es.aau.dk), B $ø$ rge Lindberg (Department of Electronic Systems, Aalborg University, Niels Jernes Vej 12, 9220 Aalborg, Denmark, bli@es.aau.dk)

This paper presents a low-complexity, effective variable frame rate (VFR) analysis method that conducts frame selection on the basis of a posteriori signal-to-noise ratio (SNR) weighted energy distance. It has two characteristics. First, energy distance (instead of cepstral distance) is used to make it computationally efficient and thus enable a finer granularity in search as compared with cepstral distance criterion. Secondly, SNR weighting is used to emphasize the reliable regions in noisy speech signals. In terms of frame selection, it is experimentally found that the method is able to assign a higher frame rate to fast changing events such as consonants, a lower frame rate to steady regions like vowels and no frames to silence, even for very low SNR signals. The VFR method is applied to speech recognition in noisy environments to improve noise robustness. Being a method that takes effect in the time-domain, it is moreover combined with spectral- and cepstral-domain techniques to gain further improvement. Experiments are conducted on the Aurora 2 database, which is the TI digits database artificially distorted by adding different noises, and very encouraging results are obtained. 


\title{
Session 2aSCd
}

\section{Speech Communication: Speech Perception I}

\author{
Mirjam Broersma, Chair \\ Radboud University Nijmegen, PO Box 9104, Nijmegen, 6500 HE, Netherlands
}

\section{Contributed Papers}

\begin{abstract}
11:00
2aSCd1. Phonological specificity of vowels and consonants in 20-month-olds' word representations. Anders Højen (Univ. of Southern Denmark, Dept. Lang. Communication, Campus Vej 55, 5230 Odense, Denmark, anders@andershojen.dk), Thomas O. Madsen (Univ. of Southern Denmark, Dept. Lang. Communication, Campus Vej 55, 5230 Odense, Denmark, tom@language.sdu.dk), Werner Vach (Univ. of Southern Denmark, Dept. Lang. Communication, Campus Vej 55, 5230 Odense, Denmark, werner.v@stat.sdu.dk), Torkil Østerbye (Univ. of Southern Denmark, Dept. Lang. Communication, Campus Vej 55, 5230 Odense, Denmark, oesterbye@sdu.dk), Karina F. Christensen (Univ. of Southern Denmark, Dept. Lang. Communication, Campus Vej 55, 5230 Odense, Denmark, kf@language.sdu.dk), Hans Basbøll (Univ. of Southern Denmark, Dept. Lang. Communication, Campus Vej 55, 5230 Odense, Denmark, hba@language.sdu.dk), Sueli Caporali (Univ. of Southern Denmark, Dept. Lang. Communication, Campus Vej 55, 5230 Odense, Denmark, s.caporali@widex.com), Dorthe Bleses (Univ. of Southern Denmark, Dept. Lang. Communication, Campus Vej 55, 5230 Odense, Denmark, bleses @ sdu.dk)
\end{abstract}

At the onset of word learning around the age of 12 months, infants are highly capable of perceiving native phonological distinctions. However, required to make word-object associations, infants sometimes fail to perceive certain phonological distinctions. The present study used an inter-modal preferential looking technique to examine Danish 20-month-olds' sensitivity to mispronunciations involving either a vowel or a consonant in familiar words. Each mispronounced word could be "reconstructed" to either of two familiar Danish words. By way of example, the English nonword "bock" can be turned into either "rock" or "book" depending on whether the mispronunciation is perceived to be in the first consonant or in the vowel. The results so far indicate that the infants were not differentially sensitive to vowel vs. consonant mispronunciations. This result, in turn, suggests that vowels and consonants do not differ in constraining lexical access to familiar words in Danish 20-month-olds.

\section{1:20}

2aSCd2. Perceptual asymmetries induced by category learning. Laurent Bonnasse-Gahot (CAMS-EHESS, 54 bd Raspail, 75270 Paris Cedex 06, France, lbg@ehess.fr)

This paper takes an information-theoretic approach to study the perceptual consequences of the neural encoding of categories (e.g. vowels). We focus on two well-known psychophysical phenomena: categorical perception, characterized by greater cross-category than within-category discrimination, and perceptual magnet effect, stating that perceptual space is wrapped around prototypical instances of a given category, leading to a better ability to discriminate stimuli near non-prototypical exemplars of a category than near prototypical ones. Introducing a perceptual distance based on the Kullback-Leibler distance between the patterns of activity evoked by two stimuli, we show that both categorical perception and prototypical effects emerge from maximizing information contained in the neural representation about a set of categories. We discuss the relations between these two psychophysical phenomena and show that they go in hand with another kind of asymmetric effect. The discriminability between a category prototype and a non-prototype is not symmetric, depending on which stimuli serves as a referent. Non-prototypical stimuli are judged closer to prototypical exem- plars than the reverse. Quantitative and qualitative comparisons with experimental data and previous theoretical work are presented and discussed.

\section{1:40}

2aSCd3. Order effects and peripherality: a cross-linguistic perceptual study using an [i]-[e] articulatory continuum. Charalampos Karypidis (UMR 7018, CNRS / Univ. Paris III, 19, rue des Bernardins, $75005 \quad$ Paris, France, ch_karypidis@yahoo.com), Antonia Colazo-Simon (UMR 7018, CNRS / Univ. Paris III, 19, rue des Bernardins, 75005 Paris, France, simonantonia@hotmail.com), Angelica V. Costagliola (UMR 7018, CNRS / Univ. Paris III, 19, rue des Bernardins, 75005 Paris, France, angelicacostagliola@yahoo.it), Cirineu Cecote Stein (UMR 7018, CNRS / Univ. Paris III, 19, rue des Bernardins, 75005 Paris, France, cirineustein@uol.com.br), Gilles Guglielmi (ARP / UFRL Univ. Paris VII - Denis Diderot, 30, rue du Chateau des Rentiers, 75013 Paris, France, gillesgug@yahoo.fr)

This paper attempted to provide further evidence on whether peripherality is a universal bias triggering stimulus order effects. Participants from four linguistic systems - Brazilian Portuguese, Salentinian Italian, French and Spanish - participated in the study. In Experiment 1, listeners identified as /i/ or /e/ the ten stimuli of a continuum prepared with an articulatory model. Experiment 2 consisted in an AX 'same-different' discrimination task including pairs of stimuli differing in one or two steps along the continuum. Discrimination was better when the more peripheral stimulus was presented second in a pair. However, these order effects were found mainly within the /i/ category and only for Italian and French. Experiment 3 was a replication of Experiment 2, except that two modifications were introduced: a. identical pairs were added, and b. one- and two-step pairs were separately examined. Order effects were again found but, this time, they covered a wider area of the continuum. Peripherality alone was not able to account for order effects. The role of focalization was later discussed.

\section{2:00}

2aSCd4. Perception of an infrequent assimilation: Labial-to-alveolar assimilation in German. Holger Mitterer (Max Planck Institute for Psycholinguistics, Wundtlaan 1, 6525 XD Nijmegen, Netherlands, holger .mitterer@mpi.nl)

In German (and many languages) the alveolar nasal $/ \mathrm{n} / \mathrm{can}$ assimilate to $[\mathrm{m}]$ if followed by a labial (e.g. 'in Berlin' $\leftarrow$ "i $[\mathrm{m}]$ Berlin"). In a German spontaneous speech corpus (KielCorpus), one also finds a few cases in which an $/ \mathrm{m} /$ followed by an alveolar consonant surfaces as [n] (e.g., 'samstag' $\leftarrow$ "sa[n]stag", Engl. Saturday). Four experiments investigated whether there is a similar pre-lexical compensation process for these labial-toalveolar assimilations as previous research uncovered for alveolar-to-labial assimilations. This turns out to be the case: German and Dutch listeners-the latter potentially unfamiliar with this type of assimilation-tend to perceive the assimilated [n] in "sanstag" as /n/ if presented in isolation ("an"), but as $/ \mathrm{m} /$ if presented with minimal context ("ansta"). For German listeners, this context effect is larger if they hear the complete word "sanstag", introducing an additional lexical bias to perceive the $[\mathrm{n}]$ as $/ \mathrm{m} /$. Finally, phonetic detail such as transitions of the [s] fricative pole are also important: If the assimilated "an" is spliced into another/s/-initial syllable by the same speaker, the context effect is reduced. Perceiving infrequent assimilations seems therefore similar to perceiving frequent assimilations. 
12:20

2aSCd5. Nonnative listeners prefer perceptual cues they know from their L1: Dutch listeners use vowel duration less than English listeners for English final /v/-/f/. Mirjam Broersma (Radboud University Nijmegen, PO Box 9104, 6500 HE Nijmegen, Netherlands, mirjam .broersma@mpi.nl)

Two 2AFC experiments investigated Dutch and English listeners' use of preceding vowel duration for the English nonword-final /v/-/f/ contrast. Like English, Dutch has a /v/-/f/ contrast, but unlike English, Dutch has no final /v/. Dutch listeners therefore have no native language experience with the use of preceding vowel duration as a final voicing cue. Previous research showed that Dutch listeners used preceding vowel duration less than English listeners when it was invariable in the experiment (Broersma, 2005, JASA, 117, 3890-3901). The present results show that they also used it less when it was varied. An 11-step fricative voicing continuum from a natural /v/ to a natural /f/ and a 7-step vowel duration continuum from a long (originally /v/-preceding) to a short (originally /f/-preceding) vowel were created. In Experiment 1, all steps of the fricative voicing continuum were combined with one long and one short vowel. In Experiment 2, all steps of the vowel duration continuum were combined with the /v/ and /f/ endpoints and with one ambiguous fricative. In both experiments, Dutch listeners used fricative voicing more and vowel duration less than English listeners did. Thus, the nonnative listeners especially used the perceptual cues they knew from their native language.

TUESDAY MORNING, 1 JULY 2008

ROOM 343, 8:00 TO 10:20 A.M.

\title{
Session 2aSPa
}

\section{Signal Processing in Acoustics, Physical Acoustics, Biomedical Ultrasound/Bioresponse to Vibration, and Underwater Acoustics: Overview of Time Reversal in Acoustics I}

\author{
David Chambers, Cochair \\ Lawrence Livermore Natl. Lab., L-333, 7000 East Ave., Livermore, CA 94550, USA \\ Claire Prada, Cochair \\ Laboratoire Ondes et Acoustique, ESPCI, Université Paris 7, CNRS, 10 rue Vauquelin, Paris, 75005, France
}

\section{Invited Papers}

\section{8:00}

2aSPa1. An overview of time-reversal acoustics. Mathias Fink (Laboratoire Ondes et Acoustique, ESPCI, Université Paris 7 , CNRS, 10 rue Vauquelin, 75005 Paris, France, mathias.fink@espci.fr)

Time-reversal invariance is a very powerful concept in physics. In the field of acoustics where time reversal invariance occurs, time-reversal experiments may be achieved simply with arrays of transmit-receive transducers, allowing an incident wave field to be sampled, recorded, time-reversed and re-emitted. Time reversal mirrors (TRMs) may be used to study random media and chaotic reverberating structures. Common to these complex media is a remarkable robustness exemplified by observations that the more complex the medium between the probe source and the TRM, the sharper the focus. TRMs open the way to new signal processings that interest imaging, detection, telecommunications and therapy. Time reversal mirrors have plenty of applications including ultrasonic therapy and medical imaging, non destructive testing, telecommunications, underwater acoustics, seismology, sound control, home automation. An overview of these fields will be presented.

2aSPa2. Ocean acoustic time reversal. William A. Kuperman (MPL, Scripps Institution of Oceanography, University of California, San Diego, La Jolla, CA 92093-0238, USA, wkuperman@ucsd.edu)

A series of ocean acoustic time experiments have been jointly conducted in the last ten years by the NATO Undersea Research Centre and the Marine Physical Laboratory. The experiments demonstrated a robustness of the ocean acoustic time reversal mirror process similar to that found in more controlled, ultrasonic laboratory experiments. Results from these experiments have pointed to potential applications to SONAR signal processing methods and to acoustics communications. In addition, the experimental procedures themselves have yielded data that provide further understanding of the ocean acoustic environment and the increased stability of time reversal process over one way propagation in a fluctuating medium. 


\title{
Contributed Paper
}

\section{8:40}

2aSPa3. 2004 M6.0 Parkfield earthquake characterization using Time Reversal. Carene Larmat (EES-11 (Geophysics) - Los Alamos National Laboratory, MS D443, Los Alamos, NM 87545, USA, carene@lanl.gov), Paul A. Johnson (EES-11 (Geophysics) - Los Alamos National Laboratory, MS D443, Los Alamos, NM 87545, USA, paj@lanl.gov), Lianjie Huang (EES-11 (Geophysics) - Los Alamos National Laboratory, MS D443, Los Alamos, NM 87545, USA, ljh@lanl.gov)

Time reversal has proved to be a robust source location method in acoustics and is now being developed for a number of seismic applications. One problem of particular interest is locating sources where the signal-to-noise ratio is small. These include small earthquakes $(<$ M5.5) or atypical seismic sources with a small seismic energy radiation (e.g., tremor, slow earthquakes). Time reversal has been shown to be very robust and work in the presence of poor data, low signal to noise ratio, etc. We present a prototype study showing the power of time reversal, using seismic data from the 2004 M6.0 Parkfield earthquake, which is the world's best recorded event to date and thus one of the most studied. The back-propagation of recorded seismic data in a 3D Earth velocity model is numerically carried out. We show that the reconstructed reverse wave-field exhibits clear focusing at the source point but also displays a four-lobe radiation pattern for each type of rebroadcast waves (body, surface), which is consistent with the known source mechanism: a right-lateral strike slip along the almost-vertical San Andrea fault.

\section{Invited Paper}

\begin{abstract}
9:00
2aSPa4. Time reversal and subwalength focal spot. Julien De Rosny (Laboratoire Ondes et Acoustique, ESPCI, Université Paris 7, CNRS, 10 rue Vauquelin, 75005 Paris, France, julien.derosny@espci.fr), Arnaud Tourin (Laboratoire Ondes et Acoustique, ESPCI, Université Paris 7, CNRS, 10 rue Vauquelin, 75005 Paris, France, arnaud.tourin@espci.fr), Geoffroy Lerosey (Laboratoire Ondes et Acoustique, ESPCI, Université Paris 7, CNRS, 10 rue Vauquelin, 75005 Paris, France, geoffroy.lerosey@espci.fr), Mathias Fink (Laboratoire Ondes et Acoustique, ESPCI, Université Paris 7, CNRS, 10 rue Vauquelin, 75005 Paris, France, mathias.fink@espci.fr)

When a wave is time-reversed inside a homogeneous medium, the focal spot width at best equals half a wavelength. This limit comes from the loss of evanescent wave during propagation. An analysis of the time reversed field in terms of the Green's function formalism shows that in order to get a finer spot, not only the field has to be time reversed but also the initial source. In such a case, an acoustic sink is obtained. Experimental results are presented. Then we show two methods to obtain subwavelength focal spot without time-reversed source. The first method consists of setting the time reversal mirror in the near field of the initial source. Despite the evanescent wave transmission, we will see that subwavelength focusing is only observed for a special time-reversal mirror. The second method consists of surrounding the initial source by many scatterers. In such a case, the evanescent waves emitted by the initial source are converted into propagating ones. During the time reversal step, back-conversion occurs that leads to a sub wavelength focal spot. Thanks to this principle, a focal spot of a thirtieth of a wavelength has been reported in the case an electromagnetic experiment.
\end{abstract}

\section{Contributed Papers}

9:20

2aSPa5. Model-based time reversal method for photoacoustic imaging of heterogeneous media. Peter Burgholzer (Upper Austrian Research, Hafenstr. 47, 4020 Linz, Austria, peter.burgholzer@uar.at), Hubert Gruen (Upper Austrian Research, Hafenstr. 47, 4020 Linz, Austria, huber.gruen@uar.at), Robert Nuster (Karl-Franzens-Universität Graz, Universitätsplatz 5, 8010 Graz, Austria, ro.nuster@uni-graz.at), Günther Paltauf (Karl-Franzens-Universität Graz, Universitätsplatz 5, 8010 Graz, Austria, guenther.paltauf@uni-graz.at), Markus Haltmeier (University of Innsbruck, Technikerst. 21a/2, 6020 Innsbruck, Austria, markus.haltmeier @uibk.ac.at)

In photoacoustic (also called optoacoustic or thermoacoustic) tomography acoustic pressure waves are generated by illumination of a semitransparent sample with pulsed electromagnetic radiation. Subsequently the waves propagate towards the detection surface enclosing the sample. The inverse problem consists of reconstructing the initial pressure sources from those measurements. In certain applications of photoacoustic imaging one has to deal with media with spatially varying sound velocity, e.g. bones in soft tissue. Image reconstruction without any compensation of this effect leads to a poor image quality. It is therefore essential to develop reconstruction algorithms that take spatially varying sound velocity into account and are able to reveal small structures in acoustically heterogeneous media. A model-based time reversal reconstruction method is presented that is capable of reconstructing the initial pressure distribution despite variations of sound speed. This reconstruction method calculates the time reversed field directly with a second order embedded boundary method by retransmitting the measured pressure on the detector positions in reversed temporal order. Numerical simulations and experiments with phantoms consisting of areas with spatially varying sound velocity are presented.

\section{9:40}

2aSPa6. The effects of transducers on the time reversal process in solids. Brian E. Anderson (EES-11 (Geophysics) - Los Alamos National Laboratory, MS D443, Los Alamos, NM 87545, USA, bea@lanl.gov), Michele Griffa (EES-11 (Geophysics) - Los Alamos National Laboratory, MS D443, Los Alamos, NM 87545, USA, mgriffa@lanl.gov), Paul A. Johnson (EES-11 (Geophysics) - Los Alamos National Laboratory, MS D443, Los Alamos, NM 87545, USA, paj@lanl .gov)

Every experimental implementation of Time Reversal (TR) involves the use of transducers to convert wave motion, whether mechanical or acoustical, into electrical signals, and vice versa. Practical considerations of transducers are not included in the basic theory of time reversal, which is based on idealized point-like sources. These considerations include temporal ring down at a narrowband transducer resonance, the finite size of the transducer giving rise to directivity, and the impedance contrast between the transducer and the medium. The effects of these considerations on the TR process will be characterized by presenting data from various TR experiments.

\section{0:00}

2aSPa7. Estimation of guided waves from cross-correlations of diffuse Wavefields for passive Structural Health Monitoring. Adelaide Duroux (Georgia Institute of Technology, School of Mechanical Engineering, 771 Ferst Drive, NW, Atlanta, GA 30332-0405, USA, adelaide.duroux@gatech.edu), Karim G. Sabra (Georgia Institute of Technology, School of Mechanical Engineering, 771 Ferst Drive, NW, Atlanta, GA 30332-0405, USA, karim.sabra@me.gatech.edu), Massimo 
Ruzzene(Georgia Institute of Technology, School of Aerospace Engineering, 270 Ferst Drive, Atlanta, GA 30332-0150, USA, massimo .ruzzene@ae.gatech.edu), Vin Sharma (Georgia Institute of Technology, School of Aerospace Engineering, 270 Ferst Drive, Atlanta, GA 30332-0150, USA, vin.sharma@millenniumdynamics.com), James Ayers (Georgia Institute of Technology, School of Aerospace Engineering, 270 Ferst Drive, Atlanta, GA 30332-0150, USA, jayers3@gatech.edu)

Recent theoretical and experimental studies in a wide range of applications (ultrasonics, underwater acoustics, seismicœ) have demonstrated that Green's functions (impulse responses) can be extracted from crosscorrelation of diffuse fields using only passive sensors. The technique, whose validity is supported by a physical argument based on time-reversal invariance, effectively uses a correlation process between the point source and points located in the focal zone. Indeed, the coherent noise source distributions can be considered as a time-reversal mirror and the crosscorrelation operations gives the field measured at one receiver after refocusing on the other receiver. Passive-only reconstruction of coherent Lamb waves $(80-200 \mathrm{kHz})$ in an aluminum plate and thickness comparable to aircraft fuselage and wing panels will be presented. In particular, the influence of the noise source characteristics (location, frequency spectrum) on the signal-to-noise ratio the emerging coherent waveform will be investigated using a scanning laser Doppler velocimeter. This study suggests the potential for a structural health monitoring method for aircraft panels based on passive ultrasound imaging reconstructed from diffuse fields.

Session $2 \mathrm{aSPb}$

\title{
Signal Processing in Acoustics, Biomedical Ultrasound/Bioresponse to Vibration, and Underwater Acoustics: Time Reversal Methods for Array Imaging and Signal Processing I
}

\author{
David Chambers, Cochair
}

Lawrence Livermore Natl. Lab., L-333, 7000 East Ave., Livermore, CA 94550, USA

Claire Prada, Cochair

Laboratoire Ondes et Acoustique, ESPCI, Université Paris 7, CNRS, 10 rue Vauquelin, Paris, 75005, France

\section{Invited Papers}

\section{0:40}

2aSPb1. The detection and characterization of buried resonant targets by iterative, single-channel time reversal. Ronald A. Roy (Boston University, Dept. of Aerosp. and Mech. Eng., 110 Cummington St., Boston, MA 02215, USA, ronroy@bu.edu), Zachary J. Waters (Boston University, Dept. of Aerosp. and Mech. Eng., 110 Cummington St., Boston, MA 02215, USA, zjwaters@bu.edu), Benjamin R. Dzikowicz (Naval Surface Warfare Center, Panama City Division, Code HS-11, 100 Vernon Ave., Panama City, FL 32407, USA, benjamin.dzikowicz@navy.mil), R. Glynn Holt (Boston University, Dept. of Aerosp. and Mech. Eng., 110 Cummington St., Boston, MA 02215, USA, rgholt@bu.edu)

A technique for detecting buried resonant targets is described in which enhanced signal-to-noise ratio and convergence to a narrowband signal is achieved using the iterative time reversal of backscattered echo returns. [Waters et al., J. Acoust. Soc. Am. 122, 3023 (2007).] The center frequency of the converged-upon signal is typically a resonance frequency of the target and thus could be used for classification and identification purposes. This technique could offer a straightforward means for enhancing target return levels in a noisy or cluttered environment using existing SONAR systems. The procedure consists of exciting the transducer with a broadband pulse, digitizing the echo return windowed about the target, reversing the data stream in time, and using this signal as the source waveform for the next interrogation pulse. We report results derived from a multi-pronged investigation that includes numerical modeling, high-frequency $(100 \mathrm{kHz}-2 \mathrm{MHz})$ scaled tank experiments employing both free-field and buried targets, and mid-frequency $(20 \mathrm{kHz}$ $-200 \mathrm{kHz}$ ) buried target experiments run in a shallow pond. [Work supported by The Office of Naval Research and the Center for Subsurface Sensing and Imaging Systems (NSF ERC Award No. EEC-9986821).]

\section{1:00}

2aSPb2. Green's function estimation in speckle using the FDORT method. Jean-Luc Robert (Philips Research, 345 Scarborough Road, Briarcliff Manor, NY 10510, USA, jean-luc.robert@philips.com), Mathias Fink (Laboratoire Ondes et Acoustique, ESPCI, Université Paris 7, CNRS, 10 rue Vauquelin, 75005 Paris, France, mathias.fink@espci.fr)

The FDORT method (French acronym for Decomposition Of the Time Reversal Operator using Focused beams) is a Time Reversal based method that can detect point-scatterers in a heterogeneous medium and extract their Green's function. It is particularly useful when focusing in a heterogeneous medium. In this presentation, the theory of the FDORT method is generalized to random media (speckle), and it is shown that it is possible to extract Green's functions from speckle signal using this method. Therefore it is possible to achieve a good focusing even if no point scatterers are present. Moreover, a link is made between FDORT and the Van Cittert Zernike theorem. We deduce from this interpretation that the normalized first eigenvalue of the Focused Time Reversal Operator is a well-known 
focusing criterion. The concept of an equivalent virtual object is introduced, that allows the random problem to be replaced by an equivalent deterministic problem and leads to an intuitive understanding of FDORT in speckle. Applications to aberration correction are presented. The reduction of the variance of the Green's function estimate is discussed.

\section{Contributed Papers}

\section{1:20}

2aSPb3. Separation of single and multiple scatterring: Application to the ultrasonic detection of a target embedded in a diffusive medium. Alexandre Aubry (Laboratoire Ondes et Acoustique, ESPCI, Université Paris 7, CNRS, 10 rue Vauquelin, 75005 Paris, France, alexandre.aubry@espci.fr), Arnaud Derode (Laboratoire Ondes et Acoustique, ESPCI, Université Paris 7, CNRS, 10 rue Vauquelin, 75005 Paris, France, arnaud.derode@ujf-grenoble.fr)

We present a new imaging technique dedicated to the detection of a target embedded in a strongly scattering medium. Classical imaging techniques such as echography fail in this kind of configuration because of multiply scattered echoes and aberration distorsions. The experimental set up consists in an array of programmable transducers placed in front of a collection of steel rods randomly distributed. The slab thickness is three times the mean free path. Behind this strongly diffusive slab, we set an echogene steel cylinder that we want to detect and localize. The impulse responses between each couple of transducers are measured and form the interelement matrix. Our technique separates the single-scattered echoes from the multiple scattering background. This is possible because of a deterministic coherence of single-scattering signals along the antidiagonals of the array response matrix, whatever the distribution of scatterers. Once this operation is performed, the detection of the target is achieved by applying the DORT method (French acronym for decomposition of the time reversal operator). The quality of detection is assessed theoretically with Random Matrix Theory and shown to be, by far, better than what is obtained with echography and the classical DORT method.

\section{1:40}

2aSPb4. Time reversal processing to forward scattering waves of underwater targets. Yoshiaki Tsurugaya (NEC @Corp., 1-10 @ Nissincho, Fuchu, 183-8501 Tokyo, Japan, y-tsurugaya @bp.jp.nec.com), Toshiaki Kikuchi (National Defence Academy, 39-21 Uhoyabe 4-chome, Yokosuka, 238-0024 Kanagawa, Japan, ADS01881@nifty.com), Koichi Mizutani (Tsukuba Univ., Tsukuba Science City, 305-8573 Ibaraki, Japan, mizutani@esys.tsukuba.ac.jp)

This paper presents the detection of underwater targets using a time reversal. When a target exists between a sound source and a time reversal array in shallow water, the time reversal array receive the sound waves from the sound source and the waves scattering by the target. If the time reversal processing to them is carried out and they are re-transmitted from the time reversal array, it will be thought that they are converged at the position of the sound source and the target. However, since the waves converging at the sound source have a high level, the waves converging at the target position are usually masked by the high level sounds. Then, we cannot observe the waves converged at the target. We eliminate only the high level sounds from the sound fields. In each array element, the signals in case of non target are subtracted from the signals including the target. As a result of subtraction, the components of the scattering wave by the target are left on the array elements. The time reversal fields of the scattering wave are constructed by radiating the components of the scattered waves from each element again.

\section{2:00}

2aSPb5. Super-resolution imaging of active sound and vibrational sources using a time-reversal sink. Eric Bavu (Univ. de Sherbrooke, Mechanical Engineering Depart., 2500 Boulevard de l'Université, Sherbrooke, QC J1K 2R1, Canada, eric.bavu@usherbrooke.ca), Alain Berry (Univ. de Sherbrooke, Mechanical Engineering Depart., 2500 Boulevard de l'Université, Sherbrooke, QC J1K 2R1, Canada, alain.berry @ usherbrooke.ca), Jean-Dominique Polack (Institut Jean le Rond
d'Alembert, Laboratoire d'Acoustique Musicale, 11, rue de Lourmel, 75015 Paris, France, polack@ccr.jussieu.fr), Vincent Gibiat (Université Paul Sabatier, PHASE, 118, route de Narbonne, 31062 Toulouse cedex 9, France, gibiat@cict.fr), Charles Besnainou (Institut Jean le Rond d'Alembert, Laboratoire d'Acoustique Musicale, 11, rue de Lourmel, 75015 Paris, France, chbesnai@ccr.jussieu.fr)

Theory and experiments of super-resolution focusing using a timereversal sink have been investigated in high-frequency regime [Rosny and Fink, Phys. Rev. Lett. 89] and in audible range [Bavu, Besnainou, Gibiat, Rosny and Fink, Act. Acoust., 93]. This technique, generalized to the case acoustic and vibrational imaging of active sources, allows super-resolution imaging and provides a new method of characterization of active sources in a known background medium. This imaging technique involves a measurement in the background medium using an array, and the simulation of the backpropagating-field in a fictive medium. An ideal numerical time-reversal sink (NumTRAS) is then used to refine results and obtain high-contrast, high-resolution imaging of initial sources. The algorithm has been validated in parallel supercomputer simulations, in both vibrational and acoustics fields and has been used to detect active vibrational sources in a clamped Mindlin plate and active sound sources in an anechoic room. All results show high-resolution imaging capabilities when compared with classical time-reversal backpropagation. NumTRAS provides an alternative to other imaging and source detection techniques, such as acoustic holography and beamforming. Beyond the applications of acoustic and vibrational nondestructive evaluation of industrial structures, NumTRAS has applications in evaluation of musical structures and is being tested to detect and characterize moving sources.

\section{2:20}

2aSPb6. Invariants of the time reversal operator for an elastic target in a water waveguide. Franck D. Philippe (Laboratoire Ondes et Acoustique, ESPCI, Université Paris 7, CNRS, 10 rue Vauquelin, 75005 Paris, France, franck.philippe@espci.fr), Claire Prada (Laboratoire Ondes et Acoustique, ESPCI, Université Paris 7, CNRS, 10 rue Vauquelin, 75005 Paris, France, claire.prada-julia@espci.fr), Julien De Rosny (Laboratoire Ondes et Acoustique, ESPCI, Université Paris 7, CNRS, 10 rue Vauquelin, 75005 Paris, France, julien.derosny@espci.fr), Jean-Gabriel Minonzio (Laboratoire Ondes et Acoustique, ESPCI, Université Paris 7, CNRS, 10 rue Vauquelin, 75005 Paris, France, jean-gabriel.minonzio@espci.fr), Mathias Fink (Laboratoire Ondes et Acoustique, ESPCI, Université Paris 7, CNRS, 10 rue Vauquelin, 75005 Paris, France, mathias.fink@espci.fr)

Detection and characterization of a target in shallow water is an active field of research. In general, in a waveguide, the dispersion is such that the frequency signature of a target cannot be extracted from a single backscatterd signal unless the waveguide properties as well as the target's position are known (Mignerey et al., JASA 1992 and Yang et al., JASA 1994). We propose to apply the Decomposition of the Time-Reversal Operator method to recover the target's signature in an unknown waveguide. Using a modal theory, we show that provided the target is far from the boundaries of the guide the first singular value of the time reversal operator is proportional to its signature. Using the same approach, the second singular value is shown to be proportional to the second derivative of the angular dependant form function which is a relevant parameter for target identification. Ultrasonic laboratory experiments are presented that confirm these theoretical results. 


\title{
Session 2aUW
}

\section{Underwater Acoustics and ECUA: Fifty Years of Progress in Sonar Acoustic Research: The Role of NURC/SACLANTCEN}

\author{
Henrik Schmidt, Cochair \\ MIT, 77 Massachusetts Ave, 5-204, Cambridge, MA 02139, USA \\ Finn B. Jensen, Cochair \\ NATO Undersea Research Centre, Viale San Bartolomeo 400, La Spezia, 19126, Italy
}

8:40-9:00 Director's Welcome

Invited Papers

9:00

2aUW1. Saclant ASW Research Centre contributions to underwater acoustics during the first sixteen years of its existence: Personal records. Robert Laval (1 rue Emile Duclaux, 75015 Paris, France, robert-laval@wanadoo.fr)

In less than one year the NATO Undersea Research Centre, previously called Saclant ASW Research Centre, will celebrate its 50th anniversary. I had the great privilege to be one of the first scientists joining the Centre in 1959 and to work there until 1975. The main purpose of the research conducted in underwater acoustics was to reach a physical understanding of the different processes of sound propagation, including multipath effects, transformations introduced by a multilayered bottom and random scattering by the volume inhomogeneities and by the surface and bottom roughness. Experiments at sea first conducted from the research ships Aragonese and then Maria Paolina used explosives charges and active sonars FM pulses as sound sources. The digital analysis equipment, which had been designed to record, process and facilitate the interpretation of the received signals, had no equivalent at the time. The Centre has been a very active platform of intercommunications for most of the civilian and military organizations, which were involved in this discipline. The result of this continuous exchange of ideas has facilitated the harmonization of the research programs in the different NATO countries and has greatly contributed to the creation of a large international research community in underwater acoustics.

9:20

2aUW2. Shallow water environmental acoustics at NURC/SACLANTCEN. William A. Kuperman (MPL, Scripps Institution of Oceanography, University of California, San Diego, La Jolla, CA 92093-0238, USA, wkuperman@ucsd.edu)

The Centre has a long tradition of studying shallow water acoustics. The research has been experimental and theoretical, the latter effort often involving either using or developing models. Examples include innovations such as the concept of the optimum frequency of an acoustic channel, first measured and then precisely explained with modeling, to, for a recent example, the utilization of ocean ambient noise based on its special properties. Consequently, the Centre has continued to remain at the leading-edge for a half century. The common goals at the Centre that provided the impetus for the progression of research are presented. Then a brief historical review of a selection of the contributions the Centre has made in shallow water acoustics is given.

\section{9:40}

2aUW3. Seafloor studies at NURC/SACLANTCEN: The first 50 years (1959-2008). Michael Richardson (Naval Research Laboratory, Marine Geosciences Division, Stennis Space Center, MS 39529-5004, USA, Mike.Richardson@nrlssc.navy.mil)

Characterization of the seafloor has always been one of the central research themes at NURC/SACLANTCEN. These studies, reviewed in this presentation, generally supported acoustic measurement and modeling programs. Motivation for bottom-interacting acoustic research began with the deep-water ASW programs of the 1960s and 1970s (long-range propagation, reflection, and bottom loss measurements) and slowly evolved into support for high-frequency, shallow-water MCM programs of the 1990s and 2000s (acoustic propagation within sediments, penetration into and scattering from the sediment surface). Seafloor characterization has included scales appropriate for plate tectonics to sub-mm scale sediment microstructure used to statistically characterize sediment heterogeneity for high-frequency acoustic studies. Although collection and analysis of sediment cores has been the dominate methods of seafloor characterization, direct in situ measurements and remote acoustic characterization has often been used. Seafloor studies were first rate, cutting edge research as demonstrated by quality and quantity of peer-reviewed publications by NURC/SACLANTCEN scientists and their post-SACLANTCEN research careers. The excellent engineering department made possible the development of unique acoustic and seafloor sampling equipment. Ship support for seafloor studies has always been a NURC/SACLANTCEN advantage that has attracted many scientists to multi-national, multi-institutional experiments and symposia. 
2aUW4. My personal perspective of sonar research at the NATO Undersea Research Centre. Peter Wille (Kitzeberger Strasse 31, 24226 Heikendorf, Germany, p.c.wille@ t-online.de)

As the first German Director of the NATO Undersea Research Centre I was contemporary to the fall of the Berlin Wall in November 1989 and the unification of Germany in October 1990, the breathtaking termination of the Cold War without bloodshed. NATO's Undersea Research Centre, thirty years of age when the Berlin Wall fell, has been a key player and focal point of a most important and demanding field of defense research. It has been exemplary in combining customer oriented applied research on sonar concepts up to system demonstrators with basic research to understand, to model and to predict the variability of the ocean environment, the most difficult challenge of successful surveillance and reconnaissance. Some unique capabilities enable the Centre to serve the nations and the NATO commands: Above all, the ability to attract first class scientists to work together at the Centre for a few years and then return to their home countries, forming an ever growing international network of intense scientific cooperation. The advanced sea trial related technology and data treatment realized by a capable and flexible technical staff is no less decisive. The many high-ranking visitors, scientific, military and political underline the international standing of the Centre.

\section{0:20-10:40 Break}

\section{0:40}

2aUW5. 19 years in the SCNR for NURC: A recollection. Leif Bjørnø (UltraTech Holding, Stendiget 19, DK-2630 Taastrup, Denmark, prof.lb@mail.dk)

I joined the SCNR for NURC by January 1st 1980 and left it again by the end of 1998. This period, on which I will concentrate my talk, includes the toughest period of the "Cold War" up to 1989 and the period after, when money to NURC became sparse and new ways of funding had to be found. This presentation will emphasize some of the highlights in NURC's contributions to Underwater Acoustics, ASW, MCM and to engineering aspects of underwater research. These highlights include for instance towed arrays, underwater acoustic modelling, MILOC, the MCM and the REA programmes, time-reverse underwater acoustics, operations research and engineering of underwater instruments. These important contributions over 19 years were supervised by 10 chairmen of the SCNR and by 6 directors, all putting their special fingerprint, closely related to their personality, on the course of NURC's development. A few humoristic features and experiences from the 19 years will briefly be referred to.

11:00

2aUW6. The Centre: It's Impact on Ocean Science---Past, Present and Future. David Bradley (Pennsylvania State University, Post Office Box 30, State College, PA 16804-0030, USA, dlb25@psu.edu)

The Centre (NATO Undersea Research Centre) was formed in 1959, in response to the (then) clear Soviet Union expansion of submarine operations and consequent threat to the NATO Alliance. It began as an experiment to provide a "water leveling" of ASW capability to the Nations of the Alliance. The combination of multi-national staff, on site for 3-5 years for the most part; together with the resident staff, has made this "experiment" an incredible success. The contributions to this success come in two parts: Specific work and scientific progress on site at the Centre and equally importantly, continued international collaboration upon return to one's Nation. The primary purpose of this presentation is to provide some "data" to illustrate both the impact of the Centre on Ocean Science and it's role within the Alliance. Given the rather specific circumstances that lead to the creation of the Centre, the question should be (and is) asked: "Should the experiment end?" The simple answer is: "NO!" The Centre is poised to have an even more critical international role in the future: The knowledge and stewardship of vital marine resources.

\section{1:20}

2aUW7. NURC/SACLANTCEN contributions to sonar signal processing and ASW systems research. Douglas Abraham (CausaSci LLC, PO Box 5892, Arlington, VA 22205, USA, abrahad@ieee.org)

The NATO Undersea Research Centre (NURC) was established in 1959 as the SACLANT ASW Research Centre and tasked with providing scientific research and technical analysis in the area of antisubmarine warfare (ASW) to NATO nations. This presentation will review NURC's contributions to sonar acoustic research in the areas of sonar signal processing and ASW systems. These contributions span at-sea testing and experimentation of novel sonar system concepts to algorithm development and theoretical advances in sonar signal processing. The diversity of expertise across fields such as oceanography, acoustics, signal processing, systems, and operations research brought by international scientists into the cauldron of a small, focused research centre with world-class technicians, engineers, and at-sea capabilities produced innovative, interdisciplinary research. [Supported by the Office of Naval Research Code 321US under contract number N0001407C0092]

\section{1:40}

2aUW8. NURC/SACLANTCEN milestone experiments toward solving inverse problems in ocean acoustics. Jean-Pierre Hermand (Université libre de Bruxelles (U.L.B.) - Environmental hydroacoustics lab, av. Franklin D. Roosevelt 50, CP 194/5, 1050 Bruxelles, Belgium, jhermand@ulb.ac.be)

This paper reviews milestone experiments conducted by Saclant Undersea Research Centre to support the development and validation of techniques for obtaining and taking into account environmental information in sonar. The experiments brought together underwater acousticians, geophysicists and oceanographers with the aim to collect comprehensive acoustic and environmental groundtruth data. Environmental-adaptive signal processing was first demonstrated in a deep water area (WESTSARDINIA'89\&90). From ducted propagation measurements, a model-based matched filter (MBMF) receiver that fully incorporates the physics of wave propagation determined a source range, depth and Doppler. For environmental inversion the broadband extension of matched-field processing 
was investigated in a shallow water area south of Elba island (YELLOWSHARK'94\&95). Geoacoustic properties of the sea bottom were determined by finding the best fit between predicted and observed sound fields at multiple frequencies using genetic search algorithms. The same inversion results were obtained by MBMF processing of broadband linearly-frequency-modulated signals enabling the use of an array of a few hydrophones instead of a dense and large vertical array. This motivated the development of drifting acoustic buoys which were tested successfully over the southern continental shelf off Marettimo island, Sicily (ENVERSE'97\&98). A recent experiment demonstrated an integrated concept of Rapid Environmental Assessment (MREA/BP'07) using sparse arrays of hydrophones and pressure/temperature sensors, hand-deployed from small vessels.

TUESDAY AFTERNOON, 1 JULY 2008

ROOM 202/203, 2:00 TO 6:40 P.M.

\title{
Session 2pAAa
}

\section{Architectural Acoustics and ASA Committee on Standards: Comparison of US and European Standards in Building/Room Acoustics I}

\author{
Kenneth P. Roy, Cochair \\ Armstrong Building Products, 2500 Columbia Ave, Lancaster, PA 17603, USA \\ Eddy Gerretsen, Cochair \\ TNO Science and Industry, Stieljesweg 1, Delft, 2628CK, Netherlands
}

2pAAa1. Building acoustics standards in the USA - ASTM and ISO. Sean D. Browne (Armstrong World Industries, 2500 Columbia Avenue, Lancaster, PA 17604, USA, sdbrowne@armstrong.com), Kenneth P. Roy (Armstrong Building Products, 2500 Columbia Ave, Lancaster, PA 17603, USA, kproy@armstrong.com)

In the US most architectural design and acoustical performance specifications are based on the ASTM International standards for Construction/Building \& Environmental Acoustics. Those standards are developed as a responsibility of ASTM International committee E 33 on Building \& Environmental Acoustics. The US is of course a participating member in the development of ISO standards through the American National Standards Institute (ANSI), and this participation relative to standards in architectural acoustics is within ISO TC 43 SC2 which deals with building acoustics. The actual ISO standard development and voting responsibilities in the US rests with the ANSI Technical Advisory Group (TAG) to ISO TC43 SC2 which is delegated to ASTM International committee E 33.06 International Standards. This paper will present the process whereby international participation is involved in the development of both ASTM and ISO standards from the US perspective.

\section{2:20}

2pAAa2. Prediction models for building performance - European need and world wide use. Eddy Gerretsen (TNO Science and Industry, Stieljesweg 1, 2628CK Delft, Netherlands, eddy.gerretsen@tno.nl)

The development of the unified European market made it necessary to create CE-marking to indicate a certain performance of products. For building products the performance had to enable the fulfilment of essential requirements by buildings, constructed with those products. This made it essential for acoustic requirements to standardize predictions models: the link between acoustic product performance and building performance. In the mean time all six parts of that standard (EN 12354) have been published and are used. Some parts have also been published as ISO standard (ISO 15712) indicating the wider interest in the subject. To be of use in Europe and elsewhere it is important that all types of building structures are covered and indeed work is going on to extend the models to lighter building elements, even more common outside Europe than within. Furthermore, a very important aspect of prediction models is the input data, hence an increased need of standards to determine product performance in an appropriate way. This is even more an item of world wide interest. So the existing standards, the current developments for improvement and the identified need for product standards will be addressed.

\section{2:40}

2pAAa3. Comparison of ASTM and ISO sound absorption test methods. Marsha S. Bischel (Armstrong Building Products, 2500 Columbia Ave, Lancaster, PA 17603, USA, msbischel@armstrong.com), Kenneth P. Roy (Armstrong Building Products, 2500 Columbia Ave, Lancaster, PA 17603, USA, kproy@armstrong.com), Joan V. Greenslade (Armstrong Building Products, 2500 Columbia Ave, Lancaster, PA 17603, USA, jvgreenslade@armstrong.com)

A series of planned experiments has been conducted in which the differences between ASTM C423 and ISO 354 have been compared. These experiments focused on the differences in the two test methods, with the goal of identifying and understanding differences in the ultimate test results. A variety of acoustical ceiling tiles were tested in the same NVLAP-certified facility, generating data that cover a broad range of acoustical performance. The factors examined were: the effect of sample size on absorption; the effect of 
plenum depth on measured absorption; and the differences between the SAA and $\alpha_{\mathrm{w}}$ curves resulting from the two test methods. The results of these experiments will then be used to identify areas in which additional research is needed if harmonization of the two standards is to occur.

\title{
Contributed Papers
}

3:00

2pAAa4. Evaluation of standards for transmission loss tests. Matthew Cassidy (Queen's University Belfast, School of Mechanical and Aerospace Engineering, Ashby Building, Stranmillis Road, BT9 5AH Belfast, UK, mcassidy06@qub.ac.uk), Richard K. Cooper (Queen’s University Belfast, School of Mechanical and Aerospace Engineering, Ashby Building, Stranmillis Road, BT9 5AH Belfast, UK, R.Cooper@qub.ac.uk), Richard Gault (Queen's University Belfast, School of Mechanical and Aerospace Engineering, Ashby Building, Stranmillis Road, BT9 5AH Belfast, UK, r.gault@qub.ac.uk), Jian Wang (Queen's University Belfast, School of Mechanical and Aerospace Engineering, Ashby Building, Stranmillis Road, BT9 5AH Belfast, UK, j.wang@qub.ac.uk)

FG Wilson, a generator set manufacturer in Larne, UK, (part of the CAT Electric Power Division), has an acoustics facility for obtaining noise emission of current products, future developments and research. A wall between a hemi-anechoic chamber and a reverberation room contains an aperture which is used for testing canopy panels to determine their sound transmission loss. Two different standard procedures for transmission loss testing are compared; the J1400, and ISO 15186. Tests were carried out on both to determine which is most feasible for the company to use. The J1400 is a variation of the ASTM E90 which uses the same method but is specifically for a reverberation room to hemi-anechoic chamber. A known 'limp' material, chosen to be lead, is used to obtain a transmission loss correction. ISO 15186 is based on a sound intensity method. A microphone on the source side recorded the sound pressure levels, and an intensity mapping was done on the receiving side, from which transmission loss was calculated. Two tests were completed; a lead sheet, and a steel plate. Both standards showed an acceptable accuracy in relation to the mass law.

\section{3:20}

2pAAa5. Evaluation of Metrics for Field Airborne Sound Isolation. Wayland Dong (Veneklasen Associates, 1711 Sixteenth Street, Santa Monica, CA 90404, USA, wdong@veneklasen.com), John Loverde (Veneklasen Associates, 1711 Sixteenth Street, Santa Monica, CA 90404, USA, jloverde@veneklasen.com)

ASTM E336 and ISO 140 define several metrics describing airborne sound isolation for field measurements of wall and floor/ceiling assemblies. The current standards include provisions for the measurements of an apparent sound power reduction (ASTC and $\mathrm{R}^{\prime}{ }_{\mathrm{w}}$ ), a non-normalized noise reduction (NIC and $\mathrm{D}_{\mathrm{n}, \mathrm{w}}$ ), and a normalized noise reduction (NNIC and $\mathrm{D}_{\mathrm{nT}, \mathrm{w}}$ ). In a previous paper [LoVerde and Dong, J. Acoust. Soc. Am. 119, 3219 (2006)], the relationships between these metrics were reviewed. The desired metric for isolation in residential uses is discussed, specifically whether it should be based on sound power and related to the performance of the partition system (ASTC and $\mathrm{R}^{\prime}{ }_{\mathrm{w}}$ ), or based on sound pressure and related to the acoustic isolation between spaces (NNIC or NIC or ISO equivalents). The analysis indicates that the sound pressure-based metric is preferred. Since power and pressure-based airborne sound metrics have small differences in field tested assemblies in most cases, it appears engineers have assumed that ASTC (and $\mathrm{R}_{\mathrm{w}}$ ) correlates with isolation. Field tests where this assumption does not hold are presented. In these cases, the power metric provides an inaccurate assessment of the acoustic isolation and can lead to engineering evaluations inconsistent with actual performance.

3:40-5:20 Posters

Lecture sessions will recess for presentation of poster papers on various topics in acoustics. See poster sessions for topics and abstracts.

\section{Invited Papers}

\author{
$5: 20$
}

2pAAa6. The adaptation of the façade sound insulation requirements in the Belgian standard. Bart Ingelaere (BBRI, rue du Lombard, 42, B-1000 Brussel, Belgium, bart.ingelaere@bbri.be), Lieven De Geetere (Belgian Building Research Institute, Lombardstraat 42, B-1000 Brussel, Belgium, lieven.de.geetere@bbri.be), Gerrit Vermeir (Lab. ATF, Katholieke Universiteit Leuven, Celestijnenlaan 200D, B-3001 Leuven, Belgium, Gerrit.Vermeir@ bwk.kuleuven.be), Manuel Vandamme (BBRI, rue du Lombard, 42, B-1000 Brussel, Belgium, manuel.vandamme@bbri.be)

In a previous paper, a draft of the new Belgian requirements for the sound insulation in dwellings in the prNBN S01-400-1 was presented. During the public enquiry, there was protest of the architectural associations against the façade sound requirements and this part of the draft had to be revised. Architects complained that it would become almost impossible to build even smaller projects without the help of acoustical engineers, what would increase building costs. The building market for dwellings is mainly a liberal market of an individual architect working for a single family, and only the intervention of a structural engineer is usual. The façade requirements are expressed as a function of the outdoor noise and concern the finished building. Architects found the relation of these requirements with the performance of the façade elements as can be calculated by EN 12 354-3:2000 too complicated. A simplified approach was developed and got the approval of the building sector and will be detailed in the presentation. Due to federal law on "the obligatory use of good craftsmanship in building", requirements in standards in Belgium are obligatory. The new standard will be of application from the 15th of February 2008.

\section{$5: 40$}

2pAAa7. Latinoamerican perspective of acoustical standards - the Chilean approach. Kenneth P. Roy (Armstrong Building Products, 2500 Columbia Ave, Lancaster, PA 17603, USA, kproy@armstrong.com), Micaelina Campos (Armstrong Building Products, 2500 Columbia Ave, Lancaster, PA 17603, USA, mcampos@armstrong.com)

A review will be made of current acoustical standards in several countries of Latinoamérica: Brasil, Mexico, Costa Rica, Argentina, Chile, and Perú. Federal standards will be discussed relative to typical end user approaches to meeting requirements. Additionally, press articles warning of health concerns related to acoustics will be presented. Most Latinoamerican acoustical standards include measure- 
ments of exterior noise, but do not mention interior noise requirements as mandatory. Brazilian standards establish overall maximum noise levels for different areas, but do not mention acoustical testing of materials. However, this is changing as a multi-professional team in Mexico is working to definition the acoustical coefficients required for ceilings and walls. And, this team is also working to establish levels of acoustical comfort in different office spaces. Most countries in Latinoamerica have federal institutions that deliver technical standards for the building industry. In Chile, we have the "Instituto Nacional de Normalizacion"(National Standards Institute), and they base their standards on the ISO standards. In 2006 they have delivered many acoustical standards and regulations, including those on transmission loss for private housing including social housing parameters. They are planning to work on standards for Healthcare and School segments.

\title{
6:00
}

2pAAa8. A special procedure to guarantee sound isolation in dwellings. Wilhelmus Beentjes (Lichtveld Buis \& Partners, Postbus 1475, 3430 BL Nieuwegein, Netherlands, beentjes@lbp.nl)

Brochures of projects for newly build dwellings and apartments create many expectations about the various qualities of the new buildings. This also concerns the quality of the sound isolation. But what happens if the quality is not as one expected and one cannot come to an agreement with the contractor? Where can one get one's rights. In the Netherlands we therefore have the Guarantee Institute Housing ( In Dutch GIW). This organisation guarantees that the building fulfills the requirements of the Dutch Building Decree. In this paper the procedure will be explained in a few cases. The topics can vary from airborne- and impact sound insulation between buildings, the sound insulation of facades to the sound levels produced by domestic installations. What are the possibilities to improve the sound isolation if the measured values do not fulfill the guaranteed values?

\section{Contributed Paper}

\section{$6: 20$}

2pAAa9. Analysis and comparison of acoustic insulation in dwellings and buildings, recently build. Leonardo Meza (Pontificia Universidad Católica de Chile, Av. Vicuña Mackena 4860, Macul, 7820436 Santiago, Chile,1mezam@uc.cl)

At this work the acoustic quality of new dwellings is analyzed from acoustic insulating measurements taken in both single-family dwellings and buildings. Through this work is intended to conduct a case study in order to know the acoustic quality of dwellings built after the year 2001, which fulfill the in force regulations called Basic Standards for Building. Therefore, acoustic insulation tests where realized in-situ in sitting rooms and bedrooms of the dwellings. We performed a total of 30 trials according to the ISO standard measure 140-5. This work is part of an investigation that will continue with the implementation of the Basic Document HR Code Technical Construction in Spain, which recently entered into force, so that this future will be how to compare the real benefits obtained with the implementation of this new regulation, to make a new campaign measures in dwellings built fulfilling this new regulation.

TUESDAY AFTERNOON, 1 JULY 2008

ROOM 242B, 2:00 TO 3:40 P.M.

\section{Session 2pAAb}

\section{Architectural Acoustics, Musical Acoustics, Physical Acoustics, and Noise: Acoustics of Opera Houses II}

\author{
Robin Glosemeyer Petrone, Cochair \\ 918 16th St., Apt 3, Santa Monica, CA 90403, USA \\ Roberto Pompoli, Cochair \\ Engineering Dept., Univ. of Ferrara, Via Saragat, 1, Ferrara, 44100, Italy
}

\section{Contributed Papers}

\section{2:00}

2pAAb1. Sound-focusing effects in the plan of horse-shoe shaped opera theatres. Gino Iannace (Built Environment Control Laboratory Ri.A.S., Second University of Naples, Abazia di S. Lorenzo, 81031 Aversa, Italy, gino.iannace@unina2.it), Elvira Ianniello (DIMEC - University of Salerno, via Ponte don Melillo, 84084 Fisciano, Italy, gino.iannace@unina2.it)

An acoustical feature reported specifically for Italian-style opera houses is the non uniformity of early sound in the stalls. The curvature of the lower part of the lateral walls produces a sort of partial wave-guiding effect, like the well known "whispering gallery" effect, that is responsible of the concentration of early sound energy at seats located back in the stalls when the sound source radiates from the foreground of the stage floor. As much of the global room impression depends on the early part of the impulse response it can be supposed that the perceived sound quality of singers' voice and instrumentalists' music performing on the stage will be much different if the listener seats in the last rows with respect to different locations in the stalls. This paper reports an objective analysis on this subject based on measurements carried out in the stalls of a baroque-type theatre. Relevant computer simulations were also implemented.

$$
\text { 2:20 }
$$

2pAAb2. Opera Performance in a Broadway Roadhouse. Dawn Schuette (Threshold Acoustics, 53 W. Jackson Blvd, Suite 1734, Chicago, IL 60604, USA, dschuette@ thresholdacoustics.com)

The John A. Williams Theatre at The Cobb Energy Centre for the Performing Arts is a facility that was designed to accommodate touring, large- 
scale musical theatre productions, such as presentations of off-Broadway shows, as its primary revenue source. The 2750 -seat space has recently received rave reviews, not just for its amplified music capabilities but for nonamplified opera presented by the Atlanta Opera, the first resident company in the facility. This paper discusses how the room form as well as fixed and adjustable acoustic elements in the space have allowed it to be successful for its various uses being conscious of its construction budget limitations. [Work completed while author was employed as a Principal Consultant at Kirkegaard Associates.]

\section{2:40}

2pAAb3. Acoustics of a selection of famous 18th century opera houses: Versailles, Markgräfliches, Drottningholm, Schweitzingen. Alban A. Bassuet (Arup Acoustics, 155 avenue of the americas, New York, NY 10013, USA, alban.bassuet@arup.com)

This paper presents the analysis of the acoustical measurements conducted in 8 renowned baroque Opera Houses located in France and Germany. Still in authentic condition to their time of usage, and incorporating the historical changing scenery system (shutter and groove), these Houses have undergone little renovations. The acoustical analysis investigates the performances of these great Houses using energy parameters, balance between stage and orchestra parameters, 3D intensity plots, new spatial distribution parameters and timbre frequency analysis. The objective of the study is to deduce (for the Constellation Project) design targets for a medium size Opera House against these benchmarks and also to revisit the use of the lateral changing scenery system as used in the Baroque era, as an original scenery design concept for a new House. The paper presents the unique qualities of the rooms, illustrates some of acoustical benefits of the 18th century Opera House design characteristics, discusses some differences in the design approach between the Italian and the French traditions and proposes new perspectives for modern Opera Houses design.

\section{3:00}

2pAAb4. The Ellie Caulkins Opera House: A study in simplified predictive modeling. Robert Mahoney (Robert F Mahoney \& Associates, 310 Balsam Avenue, Boulder, CO 80304, USA, rfm@rfma.com)

Recent advances in predictive modeling and experience in understanding and interpreting the significance of the data provided by such models have given rise to a spate of wonderful new performance spaces. However the models - whether computer models or physical scale models - demand an investment in time, money and organizational resources that are not always available due to the funding and political climate surrounding some projects. In order to secure public funding for the Ellie Caulkins Opera House in Denver, CO USA, the architectural design team had to combine elements of modeling from both the recent past and decades ago in order to present to public authorities a definitive and detailed design and an equally firm estimate of construction costs quickly and with little investment. This paper discusses how the predictive techniques of the last half-century were synthesized to provide a confident estimate of the proposed design's acoustical character as well as definitive construction information.

\section{3:20}

2pAAb5. Opera House Acoustics - Case Studies of Renovations. Mark Holden (JaffeHolden, 114a Washington St, Norwalk, CT 06854, USA, mholden@jaffeholden.com)

In the Unites States, there has been a rapid increase in the number and type of perfomance halls built and renovated in the last 10 years. New Opera Houses are few, however three significant Opera Houses have been renovated by our firm, including the Seattle Opera House, The Houston Grand Opera House, and the Kennedy Center Opera House in Washington DC, home of the National Opera. This paper will discuss, compare and contrast approaches to pit design, wall shaping and ceiling shaping, materials for surfaces and orientation and distribution of acoustic volume. Design calculations and completed measurement data, where applicable, will be presented.

TUESDAY AFTERNOON, 1 JULY 2008

ROOM 243, 2:00 TO 7:00 P.M.

\title{
Session 2pAAc
}

\section{Architectural Acoustics and Noise: Acoustics and Privacy in Healthcare Facilities II: Emerging Research Around the World I}

\author{
William J. Cavanaugh, Cochair \\ Cavanaugh Tocci Associates, Inc., 327F Boston Post Road, Sudbury, MA 01776, USA \\ Kerstin Persson Waye, Cochair \\ Dept. of Environmental Medicine, The Sahlgrenska Academy of Gothenburg Univ., Box 414, Gothenburg, 405 30, Sweden
}

Invited Papers

2:00

2pAAc1. AIA/FGI interim acoustical guidelines for health care facilities. Gregory C. Tocci (Cavanaugh Tocci Associates, Inc., 327F Boston Post Road, Sudbury, MA 01776, USA, gtocci@cavtocci.com), William J. Cavanaugh (Cavanaugh Tocci Associates, Inc., 327F Boston Post Road, Sudbury, MA 01776, USA, wcavanaugh@cavtocci.com)

Currently, noise is one of, if not the most prominent complaint of hospital care by patients and staff. In response to a growing concern for sound in hospitals and other types of healthcare facilities, the Facility Guidelines Institute (FGI) of the American Institute of Architects (AIA) has asked for assistance in incorporating acoustical guidelines into their Guidelines for the Design and Construction of Health Care Facilities. A group of 40 acoustical consultants and persons from the architectural and medical professions were assembled to develop the resulting 32-page document entitled "Interim Sound and Vibration Design Guidelines for Hospital and Healthcare Facilities." The document divides the variety of health care facility acoustical design issues into 6 sections as follows 1) site exterior noise, 2) interior finishes, 3) room noise levels, 4) sound isolation and speech privacy, 5) electroacoustics and alarms, and 6) building vibration. The presentation will provide an overview of the Interim Guides as they are expected to be adopted into the 2010 issue of the AIA/FGI Guidelines for the Design and Construction of Health Care Facilities. 
2pAAc2. Relating the hospital sound environment to occupant psychological and physiological response. Kerstin Persson Waye (Dept. of Environ. Medicine, The Sahlgrenska Acad. of Gothenburg Univ., Box 414, 40530 Gothenburg, Sweden, kerstin.persson-waye@amm.gu.se), Erica Ryherd (Woodruff School of Mechanical Engineering, Georgia Institute of Technology, Atlanta, GA 30332-0405, USA, erica.ryherd@me.gatech.edu), Berit Lindahl (School of Health and Sciences, Borås University College, 50190 Borås, Sweden, berit.lindahl@hb.se), Ingegerd Bergbom (Institute of Health and Caring Sciences, The Sahlgrenska Academy at Göteborg University, PO Box 457, 40530 Göteborg, Sweden, ingegerd.bergbom@fhs.gu.se)

Examining the relationship between specific acoustical characteristics of noise and occupant response is a fundamental step in determining the current hospital soundscape. Hospitals should be conducive to patient recovery and safety as well as employee health and productivity. Therefore, the sound environment that occupants are exposed to should be attended to from a health standpoint. Previous research on hospital patients has indicated negative effects of hospital sounds such as sleep disturbance, cardiovascular response, increased incidence of rehospitalization, extended hospital stay, and increased dosages of pain medication. There is some evidence that overall levels of hospital noise may impact staff mental efficiency, short-term memory and stress. A series of studies are currently being conducted by the authors to evaluate the modern hospital soundscape and the associated psycho-physiological responses of occupants, incorporating both quantitative and qualitative approaches. One phase in which detailed sound measurements, staff evaluations, and patient physiological measurements were conducted in a general intensive care unit will be highlighted, focusing also on methodological aspects related to physiological measurements. This phase also includes evaluation of patient ICU delirium, a condition that may negatively impact patient health and length of stay in the hospital. [Work supported by ASA and Swedish FAS].

\section{2:40}

2pAAc3. Quantifying the noise environment: effects of the wearer's voice on body-mounted noise dosimeter measurements. Steve Ryherd (Chalmers Rm. Acoust. Group, Dept. of Appl. Acoust., Chalmers Univ. of Technology, 41296 Gothenburg, Sweden, srryherd@arpeggioacoustics.com), Kerstin Persson Waye (Dept. of Environ. Medicine, The Sahlgrenska Acad. of Gothenburg Univ., Box 414, 40530 Gothenburg, Sweden, kerstin.persson-waye@amm.gu.se), Mendel Kleiner (Chalmers Rm. Acoust. Group, Dept. of Appl. Acoust., Chalmers Univ. of Technology, 41296 Gothenburg, Sweden, mendel.kleiner@chalmers.se), Erica Ryherd (Woodruff School of Mechanical Engineering, Georgia Institute of Technology, Atlanta, GA 30332-0405, USA, erica.ryherd@me.gatech.edu)

The purpose of this project was to investigate the effects of the wearer's voice as a dominant sound source on body-mounted noise dosimeters in medium-level acoustic environments. Noise dosimeters provide a convenient method of quantifying an occupant's acoustical exposure. In recent years, interest in personal noise exposure has expanded beyond a necessary safety measure used in industrial settings to be used as a means of documenting a person's exposure to sound pressure levels in lower level environments like hospitals and schools. This study quantifies the contribution of the wearer's voice to the dosimeter measurement in sixteen different office conditions. Statistical analysis was used to determine the effects of the following experimental variables on the measured levels: 1) the type of room in which the measurement is taken, 2) the type of background noise present, 3) the level of background noise. Preliminary statistical results suggest significant main effects of the experimental variables with no significant interaction effects. The statistical effect of the subject's hearing level was also considered. The results of this study can improve the interpretation of dosimeter measurements in medium-level environments and may provide further motivation for alternate methods of quantifying sound pressure levels associated with body-mounted measurements.

\section{3:00}

2pAAc4. Acoustics in the Environment of Care: An Interdisciplinary Harvard Medical School Research Project. Joanne Solet (Harvard Medical School, 25 Shattuck Street, Boston, MA 02115, USA, joanne_solet@hms.harvard.edu), Andrew Carballeira (Cavanaugh Tocci Associates, Inc., 327F Boston Post Road, Sudbury, MA 02421, USA, acarballeira@cavtocci.com), Orfeu Buxton (Harvard Medical School, 25 Shattuck Street, Boston, MA 02115, USA, orfeu@hms.harvard.edu), Grace Wyshak (Harvard Medical School, 25 Shattuck Street, Boston, MA 02115, USA, unknown@unknown.com)

Noise in hospitals and healthcare facilities is a growing concern. Anticipating the wave of aging baby-boomers, the US healthcare industry is committed to significant construction and renovation over the next decade. New construction guidelines from the American Institute of Architects/Facility Guidelines Institute and the LEED-based "Green Guide for Healthcare" recognize improved acoustical conditions as vital to quality of care quality. To set these specific standards for acoustical performance in healthcare facilities, clinical evidence is needed on the impact of the acoustical environment on patients. An interdisciplinary team of Harvard Medical School faculty and acoustical engineers, in concert with public policy professionals, have designed and implemented a simulation methodology to test acoustic disruption of sleep in human subjects. This research collaboration uses soundscapes derived from recordings made in real healthcare environments, and then quantifies the human response to them. The methodology also enables virtual design changes with corresponding subject exposure to acoustically improved environments. Studies of the human sleep response using this methodology are providing objective evidence for establishing and validating design and construction standards to integrate improved acoustics with healthcare facility design.

3:20

2pAAc5. Speech privacy in healthcare buildings:review of early studies and current procedures for analysis. William J. Cavanaugh (Cavanaugh Tocci Associates, Inc., 327F Boston Post Road, Sudbury, MA 01776, USA, wcavanaugh@cavtocci.com), Gregory C. Tocci (Cavanaugh Tocci Associates, Inc., 327F Boston Post Road, Sudbury, MA 01776, USA, gtocci@ cavtocci.com)

Studies on speech privacy problems in buildings, including healthcare facilities, since the mid-1950s, have convincingly shown that a person's sense of acoustical privacy is directly related to the intelligability of the intruding speech in adjacent spaces [Cavanaugh et al, "Speech Privacy in Buildings" J. Acoust. Soc. Am. 34, 475-492 (1962)]. Both in laboratory and in real-world settings people feel they have adequate "confidential" privacy when the articulation index (AI) of the intruding speech is 0.05 or less. Higher values of AI, up to 
about 0.15 , are acceptable for less demanding tasks, i.e. "normal" or "everyday" privacy. The analysis procedure quantifies each of the significant variables involved and compares summations of these metrics with response data from prior case histories both to predict and, in the case of existing situations, to evaluate speech privacy conditions. In the mid-1960s independent review of the analysis method showed that sufficient accuracy is preserved if A-scale metrics are used in place ofmore cumbersome frequency-based metrics. later in the 1960s the method was successfully applied to open plan spaces although achieving confidential levels of speech privacy in such spaces proved difficult. Lessons leared in applying this relatively simple analysis method over the years are discussed.

3:40-5:20 Posters

Lecture sessions will recess for presentation of poster papers on various topics in acoustics. See poster sessions for topics and abstracts.

\title{
Invited Papers
}

\author{
$5: 20$
}

2pAAc6. New Measurement Procedure and Rating Categories for Speech Privacy of Closed Rooms. John S. Bradley (National Research Council, 1200 Montreal Rd, Ottawa, ON K1A 0R6, Canada, john.bradley@nrc-cnrc.gc.ca), Brad N. Gover (National Research Council, 1200 Montreal Rd, Ottawa, ON K1A 0R6, Canada, Brad.Gover@nrc-cnrc.gc.ca)

A procedure for assessing the speech privacy of enclosed rooms is described based on measured level differences between roomaverage levels in the room and levels at spot receivers $0.25 \mathrm{~m}$ from the outside boundaries of the room. The procedure is not sensitive to the acoustics of the receiving space, assesses conditions at more sensitive listening positions, and can describe variations in speech privacy due to weaker elements such as doors. The arithmetic average of the level differences over speech frequencies from 160 to $5 \mathrm{k}$ $\mathrm{Hz}$ is used to determine uniform-weighted signal-to-noise ratios found to indicate the audibility and intelligibility of transmitted speech (J. Acoust. Soc. Am. 116 (6) 3480-3490 (2004)). The degree of speech privacy is related to the speech levels in the room, the measured transmission characteristics and ambient noise levels outside the room. The probability of transmitted speech being audible or intelligible to eavesdroppers is related to the likelihood of louder speech in the meeting room. The measured statistics of speech levels in a large number of meeting rooms have been used to describe categories of speech privacy of rooms ranging from minimal privacy to situations where transmitted speech would be very rarely audible.

\section{5:40}

2pAAc7. Evaluation of Speech Privacy Index and Noise Isolation Class rating of medical examination rooms and comparison with predictions. Steve Pettyjohn (The Acoustics \& Vibration Group, Inc., 5700 Broadway, Sacramento, CA 95820-1852, USA, spettyjohn@acousticsandvibration.com)

The Health Insurance Portability and Accountability Act of 1996 (HIPAA) set speech privacy requirements for medical related information without defining speech privacy for all situations. Areas of special concern for medical organizations are examination rooms because of the need to exchange information between patient and care giver. These rooms are usually constructed in groups with up to three walls common to other examination rooms and a fourth wall next to a corridor. Each adjacency can pose special problems because of construction and position of the equipment in the room. Medical groups are attempting to design and build examination rooms to achieve what they perceive as acceptable levels of privacy while staying within budget and waiting for definitions and requirements. This study provides results of NIC and SPI tests of examination rooms for two medical groups using several different designs. A comparison is given between measured and predicted values based on wall heights and construction details. The wall designs ranged from standard products and construction to employing specialized products, but with only partial height walls. All rooms employed acoustical tile ceilings rather than hard lids. Construction details were found to be the major factor affecting SPI.

\section{Contributed Papers}

\section{6:00}

2pAAc8. Prediction of speech privacy between rooms. Jukka S. Keränen (Finnish Institute of Occupational Health, Lemminkäisenkatu 14-18 B, $20520 \quad$ Turku, Finland, jukka.keranen@ttl.fi), Petra Virjonen (Finnish Institute of Occupational Health, Lemminkäisenkatu 14-18 B, 20520 Turku, Finland, petra.virjonen@gmail.com), Valtteri O. Hongisto (Finnish Institute of Occupational Health, Lemminkäisenkatu 14-18 B, 20520 Turku, Finland, valtteri.hongisto@ttl.fi)

The main purpose of sound insulation is usually to provide an appropriate level of speech privacy between rooms. However, the desired privacy is not always reached if, e.g. the background noise level of receiving room has not been considered or rooms are very small. This is often the case in dwellings and office rooms. Thus, sound reduction index does not correlate well with speech privacy. The aim of this study is to present a simple and validated model to predict the speech privacy between two arbitrary rooms when basic room and wall parameters are known. Speech privacy was described using Speech Transmission Index, STI. STI can be determined when the speech effort, room dimensions, early decay times of the rooms and background noise level of the reception room are known. The model was validated in several cases. The model is useful when economic optimization is appreciated. It can lead to major reductions or increments of conventional recommendations for $\mathrm{R}_{\mathrm{w}}$ value, especially, when room dimensions are unusual or the rooms have special purposes. The optimization is very easy using a web-based software.

\section{$6: 20$}

2pAAc9. A systematic review on the influence of noise on patients' sleep in the Intensive Care Units. Hui Xie (School of Architecture, University of Sheffield, Western Bank, S10 2TN Sheffield, UK, h.xie@sheffield.ac.uk), Jian Kang (School of Architecture, University of Sheffield, Western Bank, S10 2TN Sheffield, UK, j.kang@sheffield.ac.uk)

Noise level in the Intensive Care Units (ICU) often ranges far above the recommended standards. Patients in the ICUs are usually critically ill, making them more susceptible to the unfavorable environment in the hospital. Several objective and subjective methods have been used for the sleep measurement in previous intensive care studies, which showed that the sleep of ICU patients is remarkably and extraordinarily disrupted. While a number of 
factors may affect sleep in the ICUs, many earlier studies reported that noise is one of the most common causes for disturbed nocturnal sleep among ICU patients, although some other studies also suggested that noise in the hospital environment is not the most frequent cause of patients' awakenings.
Moreover, patients would become accustomed to noise accompanied with their hospital stay. In this paper, a systematic review is given to the influence of noise on patients' sleep in the ICUs, and some strategies to improve the sleep quality are also discussed.

\title{
Invited Paper
}

6:40

2pAAc10. Vibration effects in healthcare facilities. Anthony Nash (Charles M. Salter Associates, 130 Sutter, 5th Floor, San Francisco, CA 94104, USA, anthony.nash@cmsalter.com)

In the USA, the principal rating system for "green" buildings is named, "Leadership in Energy and Environmental Design" (LEED). There is now a proposed LEED rating system for healthcare facilities as described in a "pilot draft" document that was released for public comment in November 2007. This document, in turn, cites the 2006 AIA/AHA Draft Interim Sound and Vibration Design Guidelines. These AIA/AHA Guidelines focus on both the human perceptual effects of vibration as well as the control of structure-borne noise. This paper addresses another aspect of vibration in healthcare facilities - the effects of vibration upon sensitive diagnostic equipment installed on floors above grade. Such equipment may include magnetic resonance imagers, surgical microscopes, and computed tomography (CT) scanners, some of which do not have specific vibration limits provided by the manufacturer. The obvious need for attaining low-level building vibration may conflict with other design constraints imposed on modern healthcare facilities as architects and engineers strive for lightweight floor bays having widely-spaced support columns. This paper reviews the generic vibration design criteria found in the AIA/AHA Guidelines and discusses them in light of several case studies.

TUESDAY AFTERNOON, 1 JULY 2008

P2-B, LEVEL 2, 3:40 TO 5:20 P.M.

\section{Session 2pAAd}

\section{Architectural Acoustics and Noise: Acoustics of Open-Plan Spaces II (Poster Session)}

\author{
Kenneth P. Roy, Cochair \\ Armstrong Building Products \\ Valtteri Hongisto, Cochair \\ Finnish Institute of Occupational Health
}

\begin{abstract}
All posters will be on display from 3:40 p.m. to 5:20 p.m. To allow contributors an opportunity to see other posters, contributors of odd-numbered papers will be at their posters from 3:40 p.m. to 4:30 p.m. and contributors of even-numbered papers will be at their posters from 4:30 p.m. to 5:20 p.m.
\end{abstract}

\section{Contributed Papers}

2pAAd1. Acoustic Design for Open Plan Spaces. Björn Hellström (ÅF-Ingemansson AB, Box 47321, SE-100 74 Stockholm, Sweden, Bjorn.Hellstrom@afconsult.com), Erling Nilsson (Saint-Gobain Ecophon AB, Box 500, SE-260 61 Hyllinge, Sweden, erling.nilsson @ecophon.se), Björn Berthelsen (Saint-Gobain Ecophon AB, Box 500, SE26061 Hyllinge, Sweden, bjorn.berthelsen@ecophon.se)

In an ongoing Nordic cooperation project (Nordic Innovation Center Sound Design of Open-Plan Offices) the acoustical conditions in open-plan spaces are investigated (see also the adjacent paper Room Acoustical Measures for Open Plan Spaces). The project's starting point is that acoustic qualities in terms of communication, orientation, comfort and privacy are crucial for open-plan spaces. Therefore, beside acoustic parameters, the aim is to develop methodologies in order to measure and specify such qualities. Beside site analyses, one major methodological tool concerns the development of a questionnaire, intended to be utilized as a compliment to acoustic measurements, and thereby function as a tool for design measures of large open-plan offices. Its purpose is to aid acousticians as well as architects and designers to identify different types of acoustic design criteria. The questionnaire covers not only the perception of the sound environment, but also psychosocial criteria, architectural design and the furnishing of the workplace. These qualitative criteria will be discussed and suitable parameters for design and evaluation of open-plan spaces will be suggested.

2pAAd2. Adaptive sound masking. Robert Chanaud (Secure Sound, 3044 Chaco Circle, Prescott, AZ 86305, USA, 3044sound@ cableone .net), Ronald Zuydervliet (STCSound, Drususlaan 20, 6932NS Westervoort, Netherlands, ronald@STIsound.com)

Because activity sound levels vary widely during the day in open offices, fixed level sound masking spectra do not keep employees free of distractions. Programmed level control, time scheduling that permits hourly changes in level, is considerably better, but requires pre-knowledge of the activity. An adaptive masking system detects and responds to activity sound, both human and nonhuman, to provide constancy of privacy as opposed to constancy of masking level. These systems use several sound detectors in an area, average the sound level from them, and develop a running table of percentile levels with a given sensitivity. Two percentile levels (L10 and L99) are used to separate the masking from the activity sound. The system acts to continually keep the difference between them constant. The advantage of adaptive masking will be shown by comparison with other methods. The distraction potential will be discussed in terms of $\mathrm{dB}$-minutes. The overall design of these systems will be discussed and examples of experience with existing systems will be given. 


\title{
Session 2pAAf
}

\section{Architectural Acoustics, Musical Acoustics, Physical Acoustics, and Noise: Acoustics of Opera Houses III (Poster Session)}

\author{
Robin Glosemeyer Petrone, Cochair \\ Roberto Pompoli, Cochair \\ Engineering Dept. - Univ. of Ferrara
}

\begin{abstract}
All posters will be on display from 3:40 p.m. to 5:20 p.m. To allow contributors an opportunity to see other posters, contributors of odd-numbered papers will be at their posters from 3:40 p.m. to 4:30 p.m. and contributors of even-numbered papers will be at their posters from 4:30 p.m. to 5:20 p.m.
\end{abstract}

\section{Contributed Papers}

2pAAf1. Diagnosis of an historical performance hall: case study. Maud Serra (Peutz \& Associés, 10 rue des Messageries, F75010 Paris, France, m.serra@peutz.fr)

The Opera of Vichy is an historical Italian style performance hall. Initially solely devoted to opera performance, the economical trends have pushed towards concert and theatre. A first major refurbishment was performed in the 1990s and prior to that a diagnosis of that hall was performed in order to characterize the acoustics of the facility prior to actual work being carried out. While the historical preservation constraints did not allow for much acoustical work to be performed, the hall proved to be quite decent. However, in order to improve the intelligibility for theatre purposes, a new diagnosis was recently carried out so as to support the relevant computer simulations. After a brief reminder of the historical background of the project, this paper submits the diagnosis procedure that was followed, as well as its main conclusions regarding the fitness of the hall for the various performances. It turns out that such a diagnosis can be a convincing tool in order to help both the design team and the end user understand the implications - and the limitations too!- of the existing facility and its possible developments.

2pAAf2. Acoustics of new and renovated Russian opera houses: Decade 1998-2007. Michael Lannie (MNIIP, Acoustic Lab., 2-nd Brestskaja Str, 29a, 123056 Moscow, Russian Federation, mlannie@mail.ru)

Four new opera houses have been built in Russia and two existing opera houses have been fully renovated during the last 10 years. New chairs were installed and new materials were used on the inner surfaces of the renovated theatres. All the opera houses are not very large and the biggest one has 1200 seats. The plan of the two theatres has a horse-shoe form that is typical for old classic opera houses. The other three theatres have plans which are close to rectangular and one renovated theatre has a semicircular form of the back walls behind the stalls and the large balcony. The subject of the presentation is the acoustic design of these theatres. The results of the acoustic measurements which have been done in the halls are going to be discussed as well. Acoustics of the recently opened fully renovated Moscow academic musical theatre for 1100 seats is going to be presented in a more substantial way.

2pAAf3. The influence of the stage layout on the acoustics of the auditorium of the Grand Theatre in Poznan. Anna Sygulska (Poznan University of Technology, ul. Nieszawska 13C, 60-965 Poznan, Poland, annasygulska@wp.pl)

The paper concerns the influence of the stage layout on the acoustic properties of the auditorium of the Grand Theatre in Poznan. An experimental investigation and a numerical calculation were carried out. During the experimental investigation three cases were analyzed. Firstly, the stage was prepared for a concert (acoustical shell), secondly in had sets for the "Wedding of Figaro", and thirdly it was empty. The reverberation time and the acoustic pressure distribution were analyzed. The research was done by the interrupted noise method and by the true impulsive sources (pistol shots). The modified ray method was used in numerical calculations of acoustic field parameters. It turned out that the light weight structures (materials used in construction of stage sets and acoustical shell) had only decorative function and their influence on the acoustics of the auditorium opera theatre is small. Moreover, the investigation proved that the distribution of the sound pressure level in the auditorium is asymmetrical. It is because of the geometrical asymmetry of the stage (only one side wing). An acoustical correction of the stage was suggested i.e. applying of slided acoustics walls to separate the stage from the background and the side wing.

2pAAf4. Resonant cavities and acoustics vases in Italian Opera Houses; the "Teatro Principal" of Valencia and the eighteenth century treatises about theatres. Arturo Barba Sevillano (Grup d'Acustica Arquitectònica, Ambiental i Industrial, E.T.S.I.I, Univ. Politécnica de Valencia, Camino de Vera, s/n, 46022 Valencia, Spain, arbarse@doctor.upv.es), Alicia Giménez (Grup d'Acustica Arquitectònica, Ambiental i Industrial, E.T.S.I.I, Univ. Politécnica de Valencia, Camino de Vera, s/n, 46022 Valencia, Spain, agimenez@fis.upv.es), Radu Lacatis (Grup d'Acustica Arquitectònica, Ambiental i Industrial, E.T.S.I.I, Univ. Politécnica de Valencia, Camino de Vera, s/n, 46022 Valencia, Spain, rala1@doctor.upv.es), Rosa Cibrián (Facultad de Medicina, Univ. de Valencia, Av. Blasco Ibáñez, 15, 46010 Valencia, Spain, rosa.m.cibrian@uv.es)

This paper describes the research job carried out at Polytechnic University of Valencia -Spain- about resonant cavities placed under the orchestra pits of Italian Opera Houses, and others acoustics mechanisms like this one. The Teatro Principal of Valencia was built following the project developed by the Italian architect Felipe Fontana in 1774 and it opened its doors in 1832. Fifteen years ago, in the last architectonic intervention in this theatre, almost a hundred of acoustic vases were found in one of those resonant cavities. What acoustics role they accomplish? How they "functioned"? This was the starting point to study this kind of artifices, throughout the eighteenth century European treatises about theatres and in our Teatro Principal.

2pAAf5. CARMEN $®$ in the Norwich Theatre Royal, UK. Christophe Rougier (CSTB, 24, Rue Joseph Fourier, 38400 Saint Martin D'Hères, France, christophe.rougier@cstb.fr), Isabelle Schmich (CSTB, 24, Rue Joseph Fourier, 38400 Saint Martin D'Hères, France, 
isabelle.schmich@cstb.fr),Paul Chervin(CSTB, 24, Rue Joseph Fourier 38400 Saint Martin D’Hères, France, paul.chervin@cstb.fr), Paul Gillieron (Paul Gillieron Acoustic Design, 130 Brixton Hill, SW2 1RS London, UK,paul@pgacoustics.org)

Since 1999 the CARMEN@ system has been installed in several halls in Europe. The last installation took place in November 2007 at the Norwich Theatre Royal. This classical stage venue has been completely refurbished to become a multipurpose hall with 1280 seats and a single very deep balcony. To fulfil the different acoustic requirements for each performance type (from speech drama to symphonic music) it was decided to install an electroacoustic Reverberation Enhancement System. CARMEN@ is a regenerative one which is based upon the virtual wall principle. It can increase the Reverberation Time (RT) of a hall up to $100 \%$ and adapt the acoustics to each performance. The installed system has been tuned with 6 configurations: Theatre, Chamber Music, Opera, Concerto, Symphony, and Chorus. The RT of the empty hall now ranges from 1.1s without CARMEN ${ }^{\circledR}$ to $2.2 \mathrm{~s}$ in the Chorus preset. A brief description of the CARMEN ${ }^{\circledR}$ principle will be presented first. Then the tuning of the different configurations in the hall will be described. Empty hall measurements have been done and the measured acoustic criteria will be analysed. The deep balcony of the Norwich Theatre Royal separates it into two slightly coupled volumes. This particularity will be discussed.
2pAAf6. Italian opera house Mantua's Teatro Scientifico acoustical parameters measurements' comparison between Neumann KU 100 and Schoeps KFM 6 binaural recording systems. Franco Policardi (University, DIENCA Dept. Facoltà di Ingegneria, Viale Risorgimento 2, 40136 Bologna, Italy, franz@nemail.it), Ryota Shimokura (AIST, 1-8-31 Midorigaoka, Ikeda, 563-8577 Osaka, Japan, ryota.shimokura@aist.go.jp), Marco C. Consumi (University, DIENCA Dept. Facoltà di Ingegneria, Viale Risorgimento 2, 40136 Bologna, Italy, marco.cesare@mail.ing.unibo.it), Alessandro Cocchi (University, DIENCA Dept. Facoltà di Ingegneria, Viale Risorgimento 2, 40136 Bologna, Italy, alessandro.cocchi@mail.ing.unibo.it)

From the dawning of modern acoustics, researchers tryed to develop useful measurement techniques to describe acoustical field. Sound field measurement improvement's first endeavours implementing microphone capsule miniaturization technology date back to the thirtyes of the past century. Long human perception reproduction path through recording tecnique came to the attempt to position transducers similarly to the human natural hearing system, frequently obtaining as alluring results as difficult comparison among them and reapply. This paper describes processes, first results and comparison between as much as possible identical acoustical measurement sessions in Mantua's Teatro Scientifico opera house using Neumann KU 100 and Schoeps KFM 6 binaural recording systems at the same time.

TUESDAY AFTERNOON, 1 JULY 2008

P2-B, LEVEL 2, 3:40 TO 5:20 P.M.

\title{
Session 2pAAg
}

\section{Architectural Acoustics: Acoustics of Concert Halls II (Poster Session)}

\author{
Takayuki Hidaka, Cochair \\ Takenaka $R \& D$ Institute \\ Daniel Commins, Cochair \\ Commins Acoustics Workshop
}

\begin{abstract}
All posters will be on display from 3:40 p.m. to 5:20 p.m. To allow contributors an opportunity to see other posters, contributors of odd-numbered papers will be at their posters from 3:40 p.m. to 4:30 p.m. and contributors of even-numbered papers will be at their posters from 4:30 p.m. to 5:20 p.m.
\end{abstract}

\section{Contributed Papers}

2pAAg1. Measuring absorption coefficients of open ceilings in multi-purpose halls. Yong Hee Kim (Hanyang University, Department of Architectural Engineering, 133-791 Seoul, Republic of Korea, kimyonghee @ gmail.com), Hye Mi Lee (Hanyang University, 133-791 Seoul, Republic of Korea, ymihoo@daum.net), Jin Yong Jeon (Hanyang University, Department of Architectural Engineering, 133-791 Seoul, Republic of Korea, jyjeon@hanyang.ac.kr)

This paper investigates the acoustical characteristics of open ceilings in multi-purpose halls using a 1:25 scale model. A steel truss, duct system, catwalk and opening were selected for the major components of open ceilings from a field survey of 17 existing halls. Absorption characteristics of the equipped ceilings with the 1:25 scale model were measured in a reverberation chamber according to ISO 354. It was found that different volume and materials of the ceiling spaces cause the composite decay characteristics to be similar to those of coupled room effects. Results showed that the absorption coefficients of an empty ceiling with $50 \%$ opening and $6 \mathrm{~m}$ of depth are 0.2-0.3 above $500 \mathrm{~Hz}$. Adding steel trusses to the ceiling increases the absorption coefficient by 0.1 at $125-2,000 \mathrm{~Hz}$; ducts and catwalks with steel trusses yield an increase in the absorption coefficient at high frequencies. The average absorption coefficients of the various composite ceiling structures were 0.19 to 0.61 .

2pAAg2. Singers' preferences for acoustical characteristics of concert halls. Kathleen Stetson (Rensselaer Polytechnic Institute, Greene Bldg., 110 8th St., Troy, NY 12180, USA, kstetson@post.harvard.edu), Jonas Braasch (Rensselaer Polytechnic Institute, Greene Bldg., 110 8th St., Troy, NY 12180, USA, braasj@rpi.edu)

It is the goal of acousticians aiding in concert hall design to create an ideal acoustical environment for experiencing music. To this end, parameters have been developed to quantify the listener's experience in a hall, and while many contribute positively toward listener satisfaction, they only tell part of the story. In reality, the experience of a listener is dependent on the quality of the performance occurring in the hall in addition to the acoustics; even great performers can only perform their best when in comfortable environments conducive to their art. Yet only one widely used parameter, 
Stage Support, considers a performer's acoustical needs, and this metric only applies to instrumentalists playing in ensemble. This study examines the unexplored case of a solo singer in a concert hall, augmenting the little existing research on this subject as a first step toward a new parameter. Subjective responses from a survey given to a number of experienced singers and the preferences of singers for acoustical aspects of various virtual concert halls, auralized real-time as the singers sang, are examined. The limitations in using typical acoustical measurement techniques to capture data for voice self-perception are discussed.

2pAAg3. The Temporal and Spectral characteristics of Gamelan Sunda Music. I Gde Nyoman Merthayasa (Institute of Technology Bandung, Dept. of Engineering Physics - ITB, Jln. Ganesha no. 10, 40132 Bandung, Indonesia, ignmerth@tf.itb.ac.id), Bayu Pratomo (Institute of Technology Bandung, Dept. of Engineering Physics - ITB, Jln. Ganesha no. 10, 40132 Bandung, Indonesia, rah_220@yahoo.com)

In order to design a dedicated Concert hall for Gamelan Sunda, first of all, it is important to determine the temporal and spectral parameters of Gamelan Sunda Music (Degung Musics). Gamelan Sunda's music compositions are strongly affected by the sound envelope and timbre. The fundamental frequencies of instrument keys also affect the musical scale, notes placings and the communication between the musical ensembles. The acoustical parameters of the anechoic studio recorded sound waves has been analyzed to determine the acoustical parameters. The sound envelopement factors have been compared with the note configuration in the musical composition, in order to analyze the influence of the sound envelopement to the music composition. In addition, the effective duration of the autocorrelation has also been analyzed to determine the preffered reverberation time in the proposed Concert hall for Gamelan Sunda.

2pAAg4. Diffuser design for both auditorium and stage acoustics in concert halls. Jae Ho Kim (Hanyang University, 133-791 Seoul, Republic of Korea, nosaer4@gmail.com), Yong Hee Kim (Hanyang University, Department of Architectural Engineering, 133-791 Seoul, Republic of Korea, kimyonghee@gmail.com), Jin Yong Jeon (Hanyang University, Department of Architectural Engineering, 133-791 Seoul, Republic of Korea, jyjeon@hanyang.ac.kr)

This study investigates the effect of the shapes and the locations of diffusers on acoustical characteristics, both in the audience area and on the stage, using a 1:50 scale model. Random-incidence scattering coefficients of the diffusers were measured in a reverberation chamber together with the RT, EDT, C80, G and ST1 of the model hall. The results show that an appropriate design of diffusers in halls affects the sound field of both the auditorium and the stage of the hall. It was also found that the effective number of surface reflections at a receiver position actually quantifies the sound diffusion. Through instillation of omni-directional diffusers close to stage walls, the number of reflected rays increases not only in the audience area but also in the stage area.

2pAAg5. New (stage) parameter for conductor's acoustics? Elisabeth W. Van Den Braak (DHV Building and Industry, Larixplein 1, 5616 VB Eindhoven, Netherlands, bertie.vandenbraak@dhv.com), Laurentius C. Van Luxemburg (LeVeL Acoustics BV, De Rondom 10, 5612 AP Eindhoven, Netherlands, l.c.j.v.luxemburg@tue.nl)

During fine-tuning of the stage acoustics of the main auditorium of Casa da Musica, designed by the Office for Metropolitan Architecture, in Porto (Portugal) we were confronted with conflicting comments with respect to the acoustic effect of different canopy positions. The well known stage parameters, ST and CS, did not point out a difference in results for the different canopy positions, while the conductor's experience did. So we carried out several measurements and tried to find a parameter that could clarify the contradictory experiences of the musicians and the conductor. We needed a parameter that correlated with the 'transversely support over the stage' instead of or additional to the known parameters. It was also not clear to what degree the commonly used parameters describe the acoustics at the conductor's position. This study aims at proposing a new (stage) parameter as well as trying to find out more about the 'conductor's acoustics'. In the main auditorium $(\mathrm{CdM})$ the newly defined parameter seems to describe the musi- cians' and conductor's experiences on the hearing 'ensemble' on stage even better than the known stage parameters. The same measurements have been carried out in other halls in order to check the validity of this new parameter.

2pAAg6. On the interaction between double basses and the stage floor. Knut Guettler (Norwegian Academy of Music, P.O.Box 5190 Majorstuen, 0302 Oslo, Norway, knut.guettler@nmh.no), Anders Askenfelt (Dept. of Speech, Music and Hearing, Royal Institute of Technology (KTH), Lindstedtsvägen 24, SE-100 44 Stockholm, Sweden, andersa@speech.kth.se), Anders Buen (Brekke \& Strand akustikk as, Hovfaret 17, 0275 Oslo, Norway, Anders.Buen@bs-akustikk.no)

Double bassists unanimously claim the importance of a compliant stage floor for producing a warm and nuanced orchestra sound. However, in the limited number of reports studying the stage floor's contribution to radiated sound no clear conclusion has been reached. The present study, based on measurements of three concert halls and three double basses, points at some measurable features that should be considered when trying to settle the question: (1) With a compliant floor the velocity transfer between the bass bridge and floor is often higher than $0 \mathrm{~dB}$ in the low-frequency range. In these cases the bass largely acts as a mass (viewed from the end pin) while the floor acts like a spring. (2) The floor properties affect the bridge mobility in the low-frequency range. (3) Below the Helmholtz resonance, around 60 $\mathrm{Hz}$, the radiation of the bass corpus falls about $40 \mathrm{~dB}$ within one octave while the ratio between the input power at the bridge and the power transferred to the floor via the end pin has been observed to boost from 3 to $40 \%$ in the same range. (4) The effect of a compliant floor may be more pronounced for the player than for the audience.

2pAAg7. Historical and chronological evolution of the concert hall acoustics parameters. Radu Lacatis (Grup d'Acustica Arquitectònica, Ambiental i Industrial, E.T.S.I.I, Univ. Politécnica de Valencia, Camino de Vera, s/n, 46022 Valencia, Spain, rala1@doctor.upv.es), Alicia Giménez (Grup d'Acustica Arquitectònica, Ambiental i Industrial, E.T.S.I.I, Univ. Politécnica de Valencia, Camino de Vera, s/n, 46022 Valencia, Spain, agimenez@fis.upv.es), Arturo Barba Sevillano (Grup d'Acustica Arquitectònica, Ambiental i Industrial, E.T.S.I.I, Univ. Politécnica de Valencia, Camino de Vera, s/n, 46022 Valencia, Spain, arbarse@doctor.upv.es), Salvador Cerdá (Grup d'Acustica Arquitectònica, Ambiental i Industrial, E.T.S.I.I, Univ. Politécnica de Valencia, Camino de Vera, s/n, 46022 Valencia, Spain, salcerjo@mat.upv.es), José Romero (Grup d'Acustica Arquitectònica, Ambiental i Industrial, E.T.S.I.I, Univ. Politécnica de Valencia, Camino de Vera, s/n, 46022 Valencia, Spain, romerof@fis.upv.es), Rosa Cibrián (Facultad de Medicina, Univ. de Valencia, Av. Blasco Ibáñez, 15, 46010 Valencia, Spain, rosa.m.cibrian@uv.es)

The present paper examines the historical evolution of the concert halls acoustic parameters, in the international field. From the beginnings of twentieth century, the acoustic parameters have had a continuous evolution, covering more and more aspects of the concert hall acoustics qualification. Since the bibliography about these is fragmented, we performed in this article, a global and updated presentation on the chronology and the evolution of the studied parameters. Considering the great variety of the same, we presents the appearance of each parameter, located in decades, who has proposed them and, where is possible, indicates articles or publications where they have been presented for the first time. Thus, the historical presentation of the majority of the acoustic parameters of concert halls, it optimizes and can be useful for future research as a central platform in a bibliographical study. The presentation of the same is also grouped according to quality criteria.

2pAAg8. Designing a desirable medium-sized multipurpose hall using ODEON. Jason Duty (Charles M Salter Associates, Inc., 130 Sutter St, Suite 500, San Francisco, CA 94104, USA, jason.duty@ cmsalter.com), Hari V. Savitala (Charles M Salter Associates, Inc., 130 Sutter St, Suite 500, San Francisco, CA 94104, USA, hari.savitala@cmsalter.com)

This presentation focuses on using the room acoustics program ODEON for modeling a desirable medium-sized multipurpose hall (400-600 seats). A sampling of medium-sized halls was taken and impulse response measurements were conducted with drapes deployed and retracted. In each hall, the 
same configurations were modeled in ODEON. Acoustical parameters, such as T20, T30, C50, C80, were used to check the agreement of the model to the measurements. A systematic approach was then used to adjust model parameters to match the real-world measurements. These results and modifications are presented to better understand how to accurately model and design medium-sized multipurpose halls.

2pAAg9. The acoustical design of rehearsal rooms for orchestra and choir in the Teatro Lirico in Cagliari. Andrea Farnetani (Engineering Dept. - Univ. of Ferrara, Via Saragat, 1, 44100 Ferrara, Italy, andrea.farnetani@unife.it), Nicola Prodi (Engineering Dept. - Univ. of Ferrara, Via Saragat, 1, 44100 Ferrara, Italy, nicola.prodi@unife.it), Roberto Pompoli (Engineering Dept. - Univ. of Ferrara, Via Saragat, 1, 44100 Ferrara, Italy, roberto.pompoli@unife.it)

The acoustical conditions of rehearsal rooms are of primal importance during the training process of an orchestra or a choir. For this reason these spaces should be specifically designed to allow the musicians to clearly ear themselves and each other. At the same time an appropriate sound level should be maintained to avoid extensive exposure to high levels, which is a risk seldom lamented among musicians. Notwithstanding the peculiar role of these rooms in the musical production process, their acoustical requirements are still not sufficiently clarified. In particular the most effective parameters to employ and the best ranges to adopt need further investigations. This work reports the final acoustical optimization of the orchestra and choir rehearsal rooms in the Teatro Lirico in Cagliari (Italy). The comparison between the acoustical parameters before and after the refurbishment will be shown and discussed, with a focus on the difference between the sound level generated by the performer himself and by the others.

2pAAg10. The influence of the source directivity on the measurement uncertainty. Renzo Vitale (Institute of Technical Acoustics, Templergraben 55, 52056 Aachen, Germany, renzo.vitale@akustik .rwth-aachen.de), Ingo Witew (Institute of Technical Acoustics, Templergraben 55, 52056 Aachen, Germany, Ingo.Witew@akustik.rwth-aachen.de)

Modelling can play a key role in assessing the measurement uncertainties, as specified in the "Guide to the expression of Uncertainty in Measurements" (GUM). A model is presented that is used to quantify the influence of the source directivity on the measurement uncertainty. On the grounds of image sources and radiosity methods the propagation of sound in rooms is simulated. In this model the characteristics of dodecahedron sound sources are implemented with their statistical properties and characteristics. In Monte Carlo Simulations these two core concepts are combined and the results are compared with measurement results. This comparison allows a quality assessment of the model. In a conclusion the input quantities that affect the measurement uncertainty that is introduced by the source directivity are identified.
2pAAg11. Establishment a methodology for an objective-subjective acoustic analysis in a multifunctional hall: Paraninfo of the Polytechnic University of Valencia. Radu Lacatis (Grup d'Acustica Arquitectònica, Ambiental i Industrial, E.T.S.I.I, Univ. Politécnica de Valencia, Camino de Vera, s/n, 46022 Valencia, Spain, rala1@doctor.upv.es), Alicia Giménez (Grup d'Acustica Arquitectònica, Ambiental i Industrial, E.T.S.I.I, Univ. Politécnica de Valencia, Camino de Vera, s/n, 46022 Valencia, Spain, agimenez@fis.upv.es), Salvador Cerdá (Grup d'Acustica Arquitectònica, Ambiental i Industrial, E.T.S.I.I, Univ. Politécnica de Valencia, Camino de Vera, s/n, 46022 Valencia, Spain, salcerjo@mat.upv.es), Rosa Cibrián (Facultad de Medicina, Univ. de Valencia, Av. Blasco Ibáñez, 15, 46010 Valencia, Spain, rosa.m.cibrian@uv.es), José Romero (Grup d'Acustica Arquitectònica, Ambiental i Industrial, E.T.S.I.I, Univ. Politécnica de Valencia, Camino de Vera, s/n, 46022 Valencia, Spain, romerof@fis.upv.es), Arturo Barba Sevillano (Grup d'Acustica Arquitectònica, Ambiental i Industrial, E.T.S.I.I, Univ. Politécnica de Valencia, Camino de Vera, s/n, 46022 Valencia, Spain, arbarse@doctor.upv.es)

This study presents a methodology for the acoustic analysis, from a comprehensive study of a multifunctional hall, of the Polytechnic University of Valencia, as a pilot hall in a Spanish Coordinated Project. The acoustic parameters studied were those who the most acoustics experts consider of interest. In the objective measures were use the equipment and methods conforming to ISO 3382 . The questionnaire used to analyze the subjective response has been patented in Spain, by the research group that developed the project. The correlation between objective and subjective results, along with the accurate simulation of the hall provides a valid tool for design, restoration and improvements the enclosures where the sound quality is preferred.

2pAAg12. Relationship between room shape and acoustics of rectangular concert halls. Andrzej K. Klosak (Institute of Building Materials and Structures, Faculty of Civil Eng., Cracow University of Technology, Warszawska 24, 31-155 Cracow, Poland, andrzej.klosak @ pk.edu.pl), Anders C. Gade (Dept. of Acoustic Technology, Technical University of Denmark, Building 352, DK 2800 Lyngby, Denmark, acg @ oersted.dtu.dk)

Extensive acoustics computer simulations have been made using Odeon computer simulation software. In 24 rectangular rooms representing "shoebox" type concert halls with volumes of $8000 \mathrm{~m}^{3}, 12000 \mathrm{~m}^{3}$ and $16000 \mathrm{~m}^{3}$ from 300 to 850 measurements positions have been analysed. Only room averaged objective measures are considered here, in particular Clarity $\left(\mathrm{C}_{80}\right)$, Strength $(\mathrm{G})$ and Early Lateral Energy Fraction $\left(\mathrm{LF}_{80}\right)$. Results from simulations have been compared with regression models created based on real hall measurements. In general, simulated results of $\mathrm{C}_{80}$ and $\mathrm{G}$ are found to be in good agreement with regression models. Divergences are found in $\mathrm{LF}_{80}$ behaviour; these have been associated with influence of proportions of rectangular halls. Updated formula for predicting of $\mathrm{LF}_{80}$ in rectangular halls has been proposed, which takes into the account both width and length of hall. 


\title{
Session 2pAAh
}

\section{Architectural Acoustics and Noise: Low Frequency Absorption: Mechanisms, Measurement Methods and Application III (Poster Session)}

\author{
Peter D'Antonio, Cochair \\ RPG Diffusor Systems, Inc. \\ Christian Nocke, Cochair \\ Akustikbüro Oldenburg
}

\begin{abstract}
All posters will be on display from 3:40 p.m. to 5:20 p.m. To allow contributors an opportunity to see other posters, contributors of odd-numbered papers will be at their posters from 3:40 p.m. to 4:30 p.m. and contributors of even-numbered papers will be at their posters from 4:30 p.m. to 5:20 p.m.
\end{abstract}

\section{Contributed Papers}

2pAAh1. Sound absorption control at low frequencies in the new symphonic concert Hall of VillaPrado Valladolid. Victoria Chavez Thielemann (University of Chile \& Xu Acoustique, 17, rue Danton, 94270 Le Kremlin Bicêtre, France, v.chavez@yahoo.fr)

In large concert halls for symphonic orchestras the sound absorption control at low frequencies is a compromise between acoustics and architecture. In general the architectural approach is imposed over acoustical wills. In this way the acoustician must adapt his work to the architectural choice of finishing materials. Commonly is the case of the wood material's choice (usage of thin wood panels in medium density), whose architectural expression is related to orchestra instruments and to the visual warmth. Today the acoustical trend is to obtain in concert halls the appropriate acoustical "warmth". As convention, this acoustical quality in a concert hall is related to the bass sound spotlessness, and an appropriate strength of the bass tones arriving to the audience. In this context we will present the solutions and constructive compromises realised in the auditorium of Villa Prado in the way to obtain the desired warmness. This concert hall has a "shoe box" shape, an audience capacity of 1720 seats and it was made completely - walls, ceiling - in wood panels (architect: R. Bofiil, acoustician: XuAcoustique, inaugurated in April 2007, Spain).

2pAAh2. Signal analysis in the sound absorption measurement procedure: The importance of time subtraction and reference surface corrections. Paolo Guidorzi (DIENCA - Univ. of Bologna, Viale Risorgimento 2, 40136 Bologna, Italy, paolo.guidorzi@ mail.ing.unibo.it), Massimo Garai (DIENCA - Univ. of Bologna, Viale Risorgimento 2, 40136 Bologna, Italy, massimo.garai@mail.ing.unibo.it)

The sound absorption coefficient measurement (following ISO 13472-1 normative) of highly absorbing materials, which are tipically employed in room acoustic correction, presents some inherent difficulties. These materials present highly sound absorption coefficient indeed, but usually low sound insulation index and are installed over highly reflective surfaces. This leads to some measurement problems, partially solved by means of the reference surface method. In this work some measurement examples on the same materials but with different contour conditions are analyzed and the improvements on the results, due to reference surface normalization and time domain subtraction of free field response, are discussed. 


\title{
Session 2pAAi
}

\section{Architectural Acoustics and ASA Committee on Standards: Comparison of US and European Standards in Building/Room Acoustics II (Poster Session)}

\author{
Kenneth P. Roy, Cochair \\ Armstrong Building Products \\ Eddy Gerretsen, Cochair \\ TNO Science and Industry
}

\begin{abstract}
All posters will be on display from 3:40 p.m. to 5:20 p.m. To allow contributors an opportunity to see other posters, contributors of odd-numbered papers will be at their posters from 3:40 p.m. to 4:30 p.m. and contributors of even-numbered papers will be at their posters from 4:30 p.m. to 5:20 p.m.
\end{abstract}

\section{Contributed Paper}

2pAAi1. Sound Insulation Requirements in Hospitals: Comparisons and case studies. Simone Secchi (Univ. of Florence, Via San Niccolò 89/a, $50125 \quad$ Florence, Italy, simone.secchi@taed.unifi.it), Gianfranco Cellai (Univ. of Florence, Via San Niccolò 89/a, 50125 Florence, Italy, gianfranco.cellai@unifi.it), Elisa Nannipieri (Univ. of Florence, Via San Niccolò 89/a, 50125 Florence, Italy, elisa.nannipieri@taed.unifi.it), Giorgio Raffellini (Univ. of Florence, Via San Niccolò 89/a, 50125 Florence, Italy, giorgio@studioraff.it)

Italian and EU regulations for sound insulation of hospitals are very hard to be satisfied. Especially with reference to sound insulation from outside noise, the simultaneous presence of important sound sources (refrigerating units, fans and others) and of sensitive receivers (the patients) imposes high requirements for façade sound insulation. Moreover, the necessity to assure high values of natural lighting in interiors imposes the use of large windows on façades. By using the tabular estimation model defined by annex B of the European Norm 14351 for the calculation of the performance of windows and the model of EN 12354-3 for the performance of façades, it is easy to show that the limiting values defined by the Italian Law can be achieved only with a perfect realisation of the building construction. In the paper the comparison between EU regulations for hospitals is shown. Moreover, different case studies of hospital buildings are analysed either with a theoretical approach or with measures carried out in site. 


\title{
Session 2pAAj
}

\section{Architectural Acoustics and Musical Acoustics: Surround Sound Acoustics II (Poster Session)}

\author{
Anthony Hoover, Cochair \\ McKay Conant Hoover Inc. \\ Alexander Case, Cochair \\ Fermata Audio and Acoustics \\ Etienne Corteel, Cochair \\ Sonic Emotion
}

\begin{abstract}
All posters will be on display from 3:40 p.m. to 5:20 p.m. To allow contributors an opportunity to see other posters, contributors of odd-numbered papers will be at their posters from 3:40 p.m. to 4:30 p.m. and contributors of even-numbered papers will be at their posters from 4:30 p.m. to 5:20 p.m.
\end{abstract}

\section{Contributed Papers}

2pAAj1. Directivity control with Wave Field Synthesis: implications for live music performances. Terence Caulkins (Arup, 155 Ave of the Americas, New York, NY 10013, USA, terence.caulkins@arup.com), Etienne Corteel (Sonic Emotion, Eichweg, 6, CH-8154 Oberglatt, Switzerland, etienne.corteel@sonicemotion.com), Joseph Sanson (IRCAM, 1 Place Igor Stravinsky, 75004 Paris, France, joseph.sanson@ircam.fr), Olivier Warusfel (IRCAM, 1 Place Igor Stravinsky, 75004 Paris, France, Olivier .Warusfel@ircam.fr)

Loudspeaker room interaction has a large impact on the perception of surround sound, especially in the context of "mixed concert", in which a loudspeaker system is used to synthesize virtual instruments that share the acoustic space of the stage with real musicians. Loudspeakers excite the listening environment in a way that differs from real instruments since, by design, they tend to exhibit constant directivity characteristics as a function of frequency. Moreover, real instruments exhibit radiation patterns that vary according to pitch, fingering and expressive movements. We present an approach based on Wave Field Synthesis which enables a control of radiation properties of the loudspeaker system which can be dynamically monitored in real time. Applications and potential perceptual impacts are discussed, focusing on the possibility of using directivity control to vary the excitation of the concert hall in order to monitor early reflections level and direct/reverberant energy ratio associated to virtual sources.

2pAAj2. Acoustic Radiation Properties of Dynamic Loudspeaker Arrays versus Multiactuator Panels for Wave Field Synthesis. Basilio Pueo (University of Alicante, Signals, Systems and Telecommunications, Cta San Vicente del Raspeig s/n, E-03690 Alicante, Spain, basilio@ua.es) , José J. Lopez (Tech. Univ. of Valencia, Camino de Vera S/N, 46021 Valencia, Spain, jjlopez@dcom.upv.es)

Wave Field Synthesis (WFS) is a spatial sound rendering technique that generates a true sound field using loudspeaker arrays. Dynamic loudspeaker arrays, that were used since the beginning of WFS, is the technology employed in most prototypes. Alternatively, Distributed Mode Loudspeakers can be used to build arrays for WFS, also known as Multiactuator Panels (MAPs). For that purpose, multiple vibrating exciters are attached to a single flat panel of a light and stiff material, creating bending waves that are then radiated as sound fields. An analysis of the radiation properties and spatial performance of such loudspeaker arrays for WFS reproduction is presented in this paper. Wave fields have been interpreted in the wavenumber domain, where the source radiation is decomposed into plane waves for arbitrary angles of incidence. Then, measurement and analysis of the radiation per- formance, evaluation of the spatial aliasing frequency and associated sampling artefacts for both linear loudspeaker arrays are addressed. In addition, parameters that can modify spatial aliasing artifacts, such as truncation effects caused by the array geometry or array directivity are also discussed. Simulations and experiment results are discussed through several case studies, comparing dynamic loudspeaker arrays and MAPs in WFS operation.

2pAAj3. Acoustical design of multichannel audio listening environments. Timothy Gulsrud (Kirkegaard Associates, 954 Pearl Street, Boulder, CO 80302, USA, tgulsrud@kirkegaard.com)

The acoustical design of spaces for surround sound music production raises issues concerning room size and shape, loudspeaker placement, and placement of sound-absorbing and sound-diffusing finishes that require different solutions than for 2-channel stereo listening environments. Design and construction of a new room for multichannel audio, rather than renovating a room originally conceived for a 2-channel stereo loudspeaker system, presents an opportunity to address the issues from first principles. This paper presents a case study of the design, construction, and commissioning of a new audio control room for multichannel classical music recording, and discusses unique solutions to the critical issues for these listening environments. The use of a computer model as a design tool and in situ measurements in the completed room are also discussed.

2pAAj4. Effects of practical loudspeaker characteristics on virtual acoustic imaging systems. Xiaojun Qiu (Key Laboratory of Modern Acoustics and Institute of Acoustics, Nanjing University, 210093 Nanjing, China, xjqiu@nju.edu.cn), Michael Vorlaender (Institute of Technical Acoustics, RWTH Aachen University, Neustr. 50, 52056 Aachen, Germany, mvo@akustik.rwth-aachen.de)

Loudspeakers used in virtual acoustic imaging systems are usually considered as monopoles in the models; however, it is only an approximation at low frequency range, and practical loudspeaker boxes behave complex directivity in mid to high frequency range and almost only radiate in the front direction above a certain frequency. Some studies have taken into account of the frequency response characteristics of the loudspeakers in virtual acoustic imaging systems, and others have considered their directivity characteristics separately. This paper presents a complete model of a virtual acoustics imaging system which takes account of both the temporal and spatial characteristics of the sound sources, and the effects of practical loudspeaker characteristics on virtual acoustic imaging systems are studied. 


\title{
Session 2pAAk
}

\section{Architectural Acoustics: Case Studies and Design Approaches}

\author{
Bryon Harrison, Cochair \\ 124 South Boulevard, Oak Park, IL, 60302 USA \\ Witew Jugo, Cochair \\ Institut für Technische Akustik, RWTH Aachen University Neustrasse 50, 52066 Aachen, Germany
}

\section{Contributed Papers}

\begin{abstract}
$5: 20$
2pAAk1. Towards a classification of the "sonic character" of the architectural space. Fausto E. Rodriguez-Manzo (Departamento de Procesos y Tecnicas, CyAD, Universidad Autonoma MetropolitanaAzcapotzalco, Av. San Pablo 180, Edificio H-PB, Col. Reynosa Tamaulipas, Delegacion Azcapotzalco, 02200 Mexico, D.F., Mexico, rfme@correo.azc .uam.mx)

Today it is well-known that an architectural space can be compared with a musical instrument. One of the main characteristics of a musical instrument is its timbre, which makes unique the sound produced by it. We can understand this timbre as the sound quality of the instrument that is produced due to the specific physical characteristics of the instrument. In an architectural space these concepts of timbre and sound quality could be applied through the concept of "sonic character" of the space. The "sonic character" of the architectural space is the sound personality of the space due to the specific physical or architectural characteristics of it. Understanding this concept in architecture could be of great importance to architectural design purposes since this could be the way by which sound can be embodied in an integral way of designing architectural spaces. This paper presents a proposal for the classification of the "sonic character" of the architectural space as an important step of a research towards a better understanding of the need of taking into account the sound as an essential component of the architectural space. The presentation includes also some architectural space examples to improve the understanding of this important concept.
\end{abstract}

\section{5:40}

2pAAk2. High Environmental Quality (HQE), the GIAc/ADEME approach. Eric Gaucher (Acoustique \& Conseil, 17-19 rue des Grandes Terres, 92508 Rueil-Malmaison, France, eg@ acoustique-conseil.com)

The "Haute Qualité Environnementale" HQE concept is increasingly important for French building. The French acoustics consultants association, GIAc, has been working, with the ADEME's support in order to define acoustically a building that aims to be HQE. The approach is to come back to the analysis of a building acoustics situation: the activity developed in a room generates a sound which can disturb activities held in surrounding rooms. In return, this very activity requires quietness to be held in adequate conditions. If we define rules allowing quantification of this aggressivity and sensitivity, we can deduce the insulation criteria corresponding to the quality we are looking for. The main advantage of this method is the homogeneity it induces on the different criteria, which were usually defined independently one from the other. Many present situations show that the equilibrium between acoustic criteria is at least as important as their absolute values. Even though this approach has not been included in the QEB reference book, it can be used to validate the absolute values, to extend the qualification to other kinds of rooms, and also to adapt objective criteria to different situations.

\section{6:00}

2pAAk3. Auditory sketching as a tool in architectural design education. Tim Frank (Georgia Institute of Technology, College of Architecture, Atlanta, GA 30332-0155, USA, timothy.frank@coa.gatech.edu), Selen Okcu (Georgia Institute of Technology, College of Architecture, Atlanta, GA 30332-0155, USA, okcuse @yahoo.com)

Architectural spaces are perceived through embodied experiences that are made up of both visual and non-visual stimuli. However, the conventional means that a designer uses to depict non -visual stimuli such as aural phenomena contain a visual bias. We have introduced a new aural drawing system called, "Auditory Sketching" as a way for design students to visualize the auditory fields that structure one's perception of architectural space. The Auditory Sketching procedure requires that the design student first mediate aural perceptions linguistically through meticulous observation during which they are encouraged to define aural qualities within the space such as notes, tones, clusters, bands, and pitches that would be difficult to capture using a graphic system of notation. Using this written language as a guide for graphic expression, they explore different ways of expressing these time and space notations with ink pens; creating an auditory system of 'tones' by building up marks in different ways: hatching, layering up, scribbling, etc. The eventual translation of aural perception into graphic patterns is crucial because designers need to visualize the sound in order to consider it a significant medium of architecture that they can define and shape.

\section{Invited Paper}

2pAAk4. Concepts \& Methods in Architectural Acoustics from the Classical Period to the Enlightenment. Adam Di Angelo (Rensselaer Polytechnic Institute, Greene Bldg., 110 8th St., Troy, NY 12180, USA, diangelo@secondstation.com), Paul Calamia (Rensselaer Polytechnic Institute, Greene Bldg., 110 8th St., Troy, NY 12180, USA, calamp@rpi.edu)

Herein is presented a critical examination of the early concepts in architectural acoustics in the western world. This study suggests that the historical extent of architectural acoustics is much more sophisticated than is generally considered. The presentation focuses on specific contributors and their discoveries in the nascent art of architectural acoustics. Among those studied are Marcus Vitruvius Pollio, the ancient Roman architect responsible for the tome De Architectura; German polymath Athanasius Kircher who delicately balanced empirical science with magic to explain acoustical phenomena in his two books, the Phornugia Nova and the encyclopedic Musurgia 
Universalis; and English architects-cum-scientists Sir Samuel Morland and Sir Robert Hooke whose wide and varied research often entered into acoustics. In the works of these gentlemen are elegant, precocious, and sometimes absurd revelations on the science of sound and surface.

\title{
Contributed Paper
}

\section{6:40}

2pAAk5. Acoustic characterization of rehabilitated cloisters. Antonio P. Carvalho (FEUP - Laboratory of Acoustics, R. Dr. Roberto Frias, P-4200465 Porto, Portugal, carvalho@fe.up.pt), Silvia R. Vilela (FEUP - Laboratory of Acoustics, R. Dr. Roberto Frias, P-4200-465 Porto, Portugal, ec01199@fe.up.pt)

This paper presents the results of field measurements in eight rehabilitated cloisters of old monasteries in Portugal (length: 20 to $35 \mathrm{~m}$ and height:
3.3 to $6.3 \mathrm{~m}$ ) regarding their acoustic behavior to two objective parameters: RT and RASTI. The goal is to characterize the acoustic effect of the rehabilitation done on theses spaces to adapt them to new uses. All these cloisters had recently their galleries' openings to the central yard closed with glass panels. Simple formulas were obtained that relate among the objective acoustic parameters and between the acoustic and architectural parameters. This paper also presents the variation on the RASTI and RT average values resulting of the rehabilitation work done on these buildings.

TUESDAY AFTERNOON, 1 JULY 2008

ROOM 342B, 2:20 TO 7:00 P.M.

\section{Session 2pABa}

\section{Animal Bioacoustics, Underwater Acoustics, Acoustical Oceanography, Signal Processing in Acoustics, and ECUA: Animal Sonar Systems I}

\author{
James A. Simmons, Cochair \\ Brown University, Department of Neuroscience, Box G-LN, Providence, RI 02912, USA \\ Michel Andre, Cochair \\ Laboratori d'Aplicacions Bioacústiques, Universitat Politècnica de Catalunya, avda. Rambla Exposició s/n, Vilanova i la \\ Geltrú, 08800, Spain
}

Invited Paper

2:20

2pABa1. The echolocating bat controls the direction and distance of its acoustic gaze. Cynthia Moss (University of Maryland, Department of Psychology, Biology-Psychology 2123M, College Park, MD 20742, USA, cmoss@psyc.umd.edu), Kaushik Ghose (University of Maryland, Department of Psychology, Biology-Psychology 2123M, College Park, MD 20742, USA, kghose@umd.edu), Annemarie Surlykke (University of Southern Denmark, Institute of Biology, Campusvej 55, -DK-5230 Odense, Denmark, ams@biology.sdu.dk)

Echolocation is an active sensing system that depends upon the dynamic interplay between auditory information processing and adaptive motor control. The echolocating bat produces ultrasonic vocalizations and uses information contained in the returning echoes to build a three-dimensional auditory representation of its surroundings. The timing, bandwidth, and duration of echolocation signals used to probe the environment directly impact the information available to the bat's acoustic imaging system. In turn, the bat's auditory representation of space guides its actions--ear movements, head aim, flight path, and the features of subsequent sonar vocalizations. This talk will summarize the bat's adaptive vocal behavior as it engaged in complex spatial tasks. The bat's 3D flight path was recorded with high-speed stereo IR video; its sonar signals were recorded with a microphone array that permitted reconstruction of the emission pattern. The sonar emission pattern of the big brown bat, Eptesicus fuscus, is directional, but broad enough to collect echo information from objects within a 60-90 deg cone, which would enable simultaneous inspection of objects in the frontal plane. Here, we report that bats encountering a complex environment shifted the direction and distance of their sonar gaze to inspect closely spaced obstacles and targets sequentially. 


\section{Contributed Papers}

\section{2:40}

2pABa2. Simulating bat sonar using vector sound intensity. Robert Hickling (Sonometrics Inc., 8306 Huntington Road, Huntington Woods, MI 48070-1643, USA, sonometrics@ comcast.net)

In general bats use ultrasound to determine their surroundings and to detect flying insects. Generally the beam width of ultrasonic signals emitted by bats is too wide to distinguish closely-spaced objects and the receiving pattern of bats' ears is similar (J. A. Simmons, "Directionality of biosonar broadcasts and reception by the ears", Tutorial Lecture, ASA Spring Meeting, 2002). To locate an object requires knowing both distance and direction. Distance can be readily determined using time-of-flight. How a bat determines direction is less clear. In this presentation, an ultrasonic device is demonstrated that measures both distance and direction (R. Hickling, "Method and Apparatus for Echolocation" US Patent No. 7,054,226, May 2006). This uses a combination of a pulsed ultrasonic source and a vector sound-intensity probe. The probe has four ultrasonic receivers at the vertices of a regular tetrahedron and determines direction from the sound-intensity vector, using finite-difference approximations and the cross-spectral formulation. The frequencies of the echoes received by the probe have to be heterodyned down to lower frequencies for accuracy in the finite-difference approximations. Echolocation data using the device are presented.

2pABa3. An autocorrelation model of bat sonar. Lutz Wiegrebe (Biocenter, University of Munich, Großhaderner Str. 2, 82152 Munich, Germany, lutzw@1mu.de)

Their sonar system allows echolocating bats to navigate with high skill through a complex, three-dimensional environment at high speed and low light. The auditory analysis of the echoes of their ultrasonic sounds requires a detailed comparison of the emission and echoes. Here an auditory model of bat sonar is introduced and evaluated against a set of psychophysical phantom-target, echo-acoustic experiments. The model consists of a relatively detailed simulation of auditory peripheral processing in the bat, Phyl- lostomus discolor, followed by a functional module consisting of a strobed, normalised, autocorrelation in each frequency channel. The model output is accumulated in a sonar image buffer. The model evaluation is based on the comparison of the image-buffer contents generated in individually simulated psychophysical trials. The model provides reasonably good predictions for both temporal and spectral behavioural sonar processing in terms of sonar delay, roughness, and phase sensitivity and in terms of sensitivity to the temporal separations in two-front targets and the classification of spectrally divergent phantom targets.

$3: 20$

2pABa4. Object-oriented echo perception and cortical representation in the bat Phyllostomus discolor. Uwe Firzlaff (Biocenter of the LudwigMaximilians University of Munich, Großhaderner Str. 2, 82152 PlaneggMartinsried, Germany, firzlaff@zi.biologie.uni-muenchen.de)

Echolocating bats can identify three-dimensional objects exclusively through the analysis of acoustic echoes of their ultrasonic emissions. However, objects of the same structure can differ in size and the auditory system must achieve a size-invariant, normalized object representation for reliable object recognition. In this talk both the neural representation in the auditory cortex and the behavioral classification of echoes of complex virtual objects that vary in object size are described. Electrophysiological experiments revealed a population of units in the auditory cortex of the bat Phyllostomus discolor which showed an object-size invariant response (14/109 units, $13 \%)$. These units respond preferentially to echoes from objects in which echo duration (encoding object depth) and echo amplitude (encoding object surface area) co-varies in a meaningful manner. The electrophysiological results are corroborated by the results of a phantom-target playback experiment, in which it is shown that P. discolor spontaneously classifies most scaled versions of objects according to trained standards. These results indicate that at the level of the bat's auditory cortex, an object-oriented rather than a stimulus-parameter oriented representation of echoes is achieved. This work was supported by the Volkswagen Foundation (I/79782)

3:40-5:00 Posters

All sessions will recess for presentation of poster papers on various topics in acoustics. See poster sessions for topics and abstracts.

\section{Invited Papers}

\section{5:00}

2pABa5. Absolute target range and target range profile in separate computational compartments: Synthetic aperture echolocation. James A. Simmons (Brown University, Department of Neuroscience, Box G-LN, Providence, RI 02912, USA, james _simmons@brown.edu)

Echolocating big brown bats emit wideband (20-100 kHz) FM sounds and perceive target range from echo delay with an accuracy determined by echo relative bandwidth $(\mathrm{Q})$. They distinguish among target shapes by echo spectra, but they perceive shape in terms of distances to different parts (glints), or the target's range profile. Shape resolution based on glint delay differences extracted from target interference spectra is in fractions of millimeters. The bat's flight velocity is about $5 \mathrm{~m} / \mathrm{s}$, so that echo delay changes continuously during production of broadcasts and then again during reception of echoes, smearing and displacing delay estimates for target ranging by several hundred microseconds. Previous workers have identified a particular distance at which smearing is minimal, but it is not known whether this "distance of focus" has significance for the bat. However, perception of target shape is based on a spectral representation that is only slightly distorted by the bat's motion, and it is kept in separate computational path from absolute delay until after absolute delay is determined, whereupon it is attached to absolute delay to place the target's image in an approximate spatial position. The bat's system achieves synthetic aperture imaging of shape while avoiding motion-related blurring.

$$
5: 20
$$

2pABa6. Vocal motor control in horseshoe bats - a bottom-up approach. Kota Kobayasi (UCLA, Dept. Physiol. Sci., Box 951606, Los Angeles, CA 90095, USA, kobayash@ucla.edu), Steffen Hage (UCLA, Dept. Physiol. Sci., Box 951606, Los Angeles, CA 90095, USA, hage@ucla.edu), Jie Ma (UCLA, Dept. Physiol. Sci., Box 951606, Los Angeles, CA 90095, USA, majie@ucla.edu), Walter Metzner (UCLA, Dept. Physiol. Sci., Box 951606, Los Angeles, CA 90095, USA, metzner@ucla.edu)

Most animals constantly adjust the spectrotemporal composition of their vocalizations depending on the information content intended to be conveyed by these signals. Whereas most studies focus on the sensory processing of spectrotemporal features of vocalizations in various vertebrates, it is still widely unknown how vocal motor structures in the brain adjust these call parameters. We used 
echolocation and acoustic communication behaviors in horseshoe bats to analyze the neurobiological and biomechanical basis for the motor control of their vocalizations. Results from pharmacological studies of the vocal motor nucleus, the nucleus ambiguus, lead us to developed a novel model for call frequency control by vocal motor neurons. Neurophysiological recordings combined with pharmacological manipulations at the single cell level in spontaneously vocalizing bats substantiated this model. Biomechanical properties of the larynx were also found to aid in call frequency control: Altering tracheal air flow in an isolated larynx preparation resulted in two distinct frequency bands that were non-harmonically related and reflected the main frequency components of echolocation pulses and communication signals, respectively. In addition, changes in air pressure revealed transitions from periodic to chaotic that occurred within a single signal cycle, giving rise to spectral features observed in horseshoe bat communication signals.

\section{$5: 40$}

2pABa7. Echolocation-like pulse emission for communication by Japanese horseshoe bats, Rhinolophus ferrumequinum nippon. Hiroshi Riquimaroux (Faculty of Engineering, Doshisha Univ., 1-3 Miyakodani Tatara, 610-0321 Kyotanabe, Japan, hrikimar@mail.doshisha.ac.jp), Ryota Shimazawa (Faculty of Engineering, Doshisha Univ., 1-3 Miyakodani Tatara, 610-0321 Kyotanabe, Japan, bte0060@mail4.doshisha.ac.jp), Shizuko Hiryu (Faculty of Engineering, Doshisha Univ., 1-3 Miyakodani Tatara, 6100321 Kyotanabe, Japan, shiryu@mail.doshisha.ac.jp)

It has been reported that the bats communicate with conspecifics by various types of vocalizations, which are different from echolocation pulses. However, we have found pulses used for communication, which were emitted when a bat walked to another individual, appeared to be very similar to those for echolocation. Data have shown two evidences. First, the bats never approached another one without emitting pulses. Second, the bats emitted echolocation-like pulses whose CF2 frequency sequentially changed during the approaching behavior. Such pulses were never emitted by an isolated bat. The changes in CF2 frequency were clearly different from Doppler-shift compensation. If this frequency shift was caused by Doppler-shift compensation, the bat should be moving as fast as 1-5 $\mathrm{m} / \mathrm{s}$ in the cage. Thus, we suggest that these CF2 frequency shifts may play an important role for communication during the approaching behavior to a conspecific animal. [The research supported by a grant to RCAST at Doshisha Univ. from MEXT and by the Innovative Cluster Creation Project promoted by MEXT.]

\section{Contributed Paper}

\section{6:00}

2pABa8. A model of range discrimination of multiple objects by using the linear period modulation signal. Ikuo Matsuo (Tohoku Gakuin University, Tenjinzawa 2-1-1, Izumi-ku, 9813193 Sendai, Japan, matsuo@cs tohoku-gakuin.ac.jp)

Using the echolocation, bats can capture moving objects in 3D space. The big brown bats, Eptesicus fuscus, emit the linear period modulation sound and can identify objects with an accuracy of less than a millimeter. The delay separation of three or more closely spaced objects can be determined through analysis of the echo spectrum. However, delay times cannot be properly correlated with objects using only the echo spectrum because the sequence of delay separations cannot be determined without information on temporal changes in the interference pattern of the echoes. We previously proposed the model to determine delays of multiple objects from the echoes by using the linear frequency modulation sound. In order to extract the temporal changes, Gaussian chirplets with a carrier frequency compatible with bat emission sweep rates were used. In this paper, we examined the effect of the window's shape which was changed to extract the temporal changes dependent on delays of multiple objects by using the linear period modulation sound.

\section{Invited Paper}

2pABa9. Acoustic focussing: how flying bats control spatial distribution of Doppler-ranging errors by signal sweep rate. Marc W. Holderied (School of Biological Sciences, University of Bristol, Woodland Road, BS8 1UG Bristol, UK, marc.holderied@bristol .ac.uk)

Echolocating bats obtain three-dimensional images of their surroundings in complete darkness by emitting sonar signals and evaluating returning echoes. When flying close to objects, bats risk collision and therefore depend on the accuracy of images - particularly in the perceived distance of obstacles, which is coded by the time delay between call and echo. Yet, during flight, such accuracy is perturbed first because bats call and receive echoes at different positions and second because echoes are modified by Doppler shifts. The spatial distribution of such ranging errors is range dependent - objects at one particular distance from the bat have zero ranging errors, while ranging-errors increase for closer or more distant objects. Interestingly, this distance of zero ranging error depends on signal design, in particular sweep rate. By adjusting signal design flying bats could shift this distance adaptively to their target of interested. Because this has similarities with focusing (i.e. accommodation) in vision, this distance is called distance of focus (DOF). We will present examples for actual distances of focus of different bat species in different behavioural contexts, such as search flight, obstacle avoidance and target approach. DOF gives a novel perspective to the adaptive relevance of frequency modulated sonar signals. 


\title{
Contributed Paper
}

\section{6:40}

2pABa10. Passive acoustic localization techniques of Eastern Pacific grey whales. Jennifer L. Wladichuk (University of Bath, Dept. Mechanical Engineering, Claverton Down, BA2 7AY Bath, UK, jlw23 @ bath.ac.uk), William M. Megill (University of Bath, Dept. Mechanical Engineering, Claverton Down, BA2 7AY Bath, UK, enswmm@bath.ac.uk), Philippe Blondel (University of Bath, Department of Physics, Claverton Down, BA2 7AY Bath, UK, pyspb@bath.ac.uk)

Eastern Pacific grey whales (Eschrichtius robustus) apparently do not actively echolocate, yet still they thrive in shallow water environments where visibility is much reduced. Along their migration route and in their feeding grounds these whales are exposed to high levels of ambient noise, highlyturbid waters and many underwater obstacles. To test possible passive acoustic localization mechanisms (e.g. Acoustic Daylight Imaging and Passive Synthetic Aperture), we made extensive acoustic measurements during comprehensive field studies of these whales on their summer feeding grounds in British Columbia (Canada). In combination with visual observations of the whales and their behaviours, we investigated the acoustical sources available to the whales when navigating within a feeding bay. First, we measured ambient noise levels to construct the acoustic landscape around the whales. Second, we investigated how sound is altered when objects such as kelp beds and rocks are present. We also measured acoustic changes induced by direct, controlled modifications of the near-shore environment. The aim of this research is to understand how grey whales might be finding their way around, and what impact, if any, increased levels of ambient noise might have on the whales' ability to find food and navigate within the feeding grounds.

TUESDAY AFTERNOON, 1 JULY 2008

P3-C, LEVEL 3, 3:40 TO 5:20 P.M.

Session 2pABb

\section{Animal Bioacoustics and ECUA: Animal Bioacoustic Censusing II (Poster Session)}

\author{
Marie Roch, Cochair \\ San Diego State University \\ Cédric Gervaise, Cochair \\ E3I2 - EA3876
}

\begin{abstract}
All posters will be on display from 3:40 p.m. to 5:20 p.m. To allow contributors an opportunity to see other posters, contributors of odd-numbered papers will be at their posters from 3:40 p.m. to 4:30 p.m. and contributors of even-numbered papers will be at their posters from 4:30 p.m. to 5:20 p.m.
\end{abstract}

\section{Contributed Papers}

2pABb1. Acoustic detection of beaked whales from autonomous recording buoys. Antón Arias (Departamento de Zoología y Antropología Física, Facultad de Biología, Universidad Complutense de Madrid, 28040 Madrid, Spain, amarias@estumail.ucm.es), Mark Johnson (Woods Hole Oceanographic Institution, Applied Ocean Physics \& Engineering Dept., Woods Hole, MA 02543, USA, majohnson@whoi.edu), Natacha Aguilar Soto (Univ. de La Laguna, Department of Animal Biology, 38256 La Laguna (Tenerife), Spain, naguilar@ull.es), Peter T. Madsen (University of Aarhus, Biological Sciences, Zoophysiology, C. F. Møllers Allé, Building 1131, DK-8000 Aarhus, Denmark, peter.madsen@biology.au.dk), Peter Tyack (Woods Hole Oceanographic Institution, Applied Ocean Physics \& Engineering Dept., Woods Hole, MA 02543, USA, ptyack@whoi.edu), Bertel Møhl (University of Aarhus, Biological Sciences, Zoophysiology, C. F. Møllers Allé, Building 1131, DK-8000 Aarhus, Denmark, bertel.moehl@biology.au.dk)

Beaked whales are among the least known cetaceans but may be especially vulnerable to mid-frequency sonars. Effective implementation of passive acoustic monitoring (PAM) of beaked whale vocalizations may help both to study the biology of these elusive species and to mitigate human impacts. Here we report results of a feasibility study of PAM performed off El Hierro (Canary Islands) where there are coastal resident populations of two species of beaked whales. An acoustic recording system, comprising a GPS-equipped buoy with an archival acoustic tag (Dtag) suspended $200 \mathrm{~m}$ below, was deployed in an area with visual coverage from land. The DTAG recorded broadband acoustic data sampled at $96 \mathrm{kHz}$ along with timing and position information from the GPS. The buoy was deployed on 6 days and clicks were clearly detectable in all recordings. A majority of these were confirmed to be beaked whale clicks by comparison against sounds previously recorded from the same species. Visual sightings were then compared against acoustic detections to develop a methodology for estimating the probability of detection as a function of distance and group size. Results demonstrate the effectiveness of sonobuoy-type PAM systems for beaked whales and the possibility for low-cost tests of acoustic detection models.

2pABb2. Automated real-time detection of cetaceans at the Station ALOHA Cabled Observatory. Julie N. Oswald (Univ. of Hawaii, P.O. Box 1106, Kailua, HI 96734, USA, oswald.jn@gmail.com), Whitlow W. Au (Univ. of Hawaii, P.O. Box 1106, Kailua, HI 96734, USA, wau@ hawaii.edu), Fred K. Duennebier (Univ. of Hawaii, Dept. of Geology and Geophysics, 2525 Correa Rd., Honolulu, 96822, USA, fred@soest .hawaii.edu)

Hawaiian waters support a wide variety of cetacean species, however, little is known about their occurrence in offshore waters. A cabled ocean bottom observatory located at Station ALOHA (Station ALOHA Cabled Observatory - ACO) provides a unique opportunity to study the occurrence of cetaceans in real-time and over long time scales at a deep ocean research site located $100 \mathrm{~km}$ north of Oahu. A retired electro-optical telecommunications cable provides power and broadband Ethernet communications capability to 
the ACO, allowing real-time continuous acoustic monitoring. The ACO has been operational since February 2007 and includes a hydrophone with a bandwidth of $0.01 \mathrm{~Hz}$ to $40 \mathrm{kHz}$. Automated methods for detection and identification of cetacean calls have been developed and data are processed in nearly real-time. Several cetacean species have been detected, including: dolphins and humpback, minke, fin, and sperm whales. The relationship between the occurrence of different species and changes in the environment has been explored using near-monthly shipboard oceanographic observations collected at Station ALOHA. In addition, seasonal trends in the occurrence of minke whales, a species that has been especially difficult to study using visual methods due to their cryptic behavior, have been examined.

2pABb3. Validating passive acoustic monitoring data loggers by visual observations. Line A. Kyhn (National Environmental Research Institute, University of Aarhus, Frederiksborgvej399, DK-4000 Roskilde, Denmark, lky@dmu.dk), Jakob Tougaard (National Environmental Research Institute, University of Aarhus, Frederiksborgvej399, DK-4000 Roskilde, Denmark, jat@dmu.dk), Mats Amundin (Kolmardens Djurpark \& Biology Dep., Inst. of Physics, Chemistry and Biology, Linkoping University, Kolmardens Djurpark, SE-61892 Kolmarden, Sweden, MATS.AMUNDIN @ KOLMARDEN.COM), Joanna Stenback (Kolmardens Djurpark \& Biology Dep., Inst. of Physics, Chemistry and Biology, Linkoping University, Kolmardens Djurpark, SE-61892 Kolmarden, Sweden, JOANNA .STENBACK@GMAIL.COM), Jonas Teilmann (National Environmental Research Institute, University of Aarhus, Frederiksborgvej399, DK-4000 Roskilde, Denmark, jte@dmu.dk), Daniel Wennerberg (Kolmardens Djurpark \& Biology Dep., Inst. of Physics, Chemistry and Biology, Linkoping University, Kolmardens Djurpark, SE-61892 Kolmarden, Sweden, daniel .wennerberg@gmail.com)

The use of passive acoustic monitoring (PAM) is increasingly used as a monitoring tool in e.g environmental impact assessments. However, very few studies have focused on validating PAM data against independent observations which is critical in order to compare data from different studies and areas, and over time. In August 2007 we tested two types of odontocete PAM equipment: version 5 T-PODs (Chelonia, U.K.) and PCL's (Aquatec, Lochborough, UK) by comparing acoustic detections to visual theodolite tracings of wild harbour porpoises. Eight T-PODs with known detection thresholds and four PCLs were mounted in four clusters. Detection thresholds of the T-PODs varied from 117 to $125 \mathrm{~dB}$ re $1 \mu$ Papp. Based on visual tracks of 28 porpoises it is shown that detection ranges correlated inversely with detection threshold. However, PAM-detection also strongly depended on the angle between the swim track of porpoises and the line to the data logger. Some porpoises were not detected despite swimming within $20 \mathrm{~m}$ of the nearest data logger. It was not possible to distinguish between recordings from one and more than one animal on the PAM-recordings, despite that the number of animals was known from the corresponding tracks. All in all, the results showed good correspondence between PAM recordings and visual tracings, as well as among PAM recordings.

2pABb4. Data Handling of a Perennial Acoustic Observatory in the Antarctic Ocean. Lars Kindermann (Alfred Wegener Institute, P.O. Box 120161, 27515 Bremerhaven, Germany, lars.kindermann@awi.de), Holger
Klinck(Alfred Wegener Institute, P.O. Box 120161, 27515 Bremerhaven, Germany, holger.klinck@awi.de), Ilse Catharina Van Opzeeland (Alfred Wegener Institute, P.O. Box 120161, 27515 Bremerhaven, Germany, Ilse .Van.Opzeeland@awi.de)

In December 2005, 4 broadband hydrophones were deployed through boreholes under the $100 \mathrm{~m}$ thick floating ice shelf near Atka Bay, Antarctica. Since then, more than 10,000 hours of underwater sounds have been recorded, containing marine mammal vocalizations and the unique background sounds generated by ice and the occasional ship. These records from this pristine region are analyzed in terms of the local noise budget, the behaviour of marine mammals and anthropogenic impacts. The observatory is energetically self-sufficient and connected by a $15 \mathrm{~km}$ WLAN link to the year-round manned German Neumayer Base, where a leased satellite line allows live streaming of highly compressed audio to http://icecast.awi.de. While the recording device is capable of four channels / $192 \mathrm{kHz} / 24 \mathrm{Bit}$, this cannot be transferred continuously through the WLAN and satellite link, so a multi-stage automatic buffering and selection scheme is implemented to allow for both, continuous coverage and high quality recordings of selected events. About two terabytes per year are automatically streamed into the petabyte data storage of the World Data Centre for Marine Environmental Data, hosted by AWI, where they are analysed and prepared for publishing under an open access license.

2 pABb5. Development of a prototype electronic tag for studying the migratory behaviour of marine species. Prajas John (Department of Electronics, Cochin University of Science \& Technology, Thrikkakara, 682 022 Ernakulam, India, prajas@cusat.ac.in), Jinto George (Department of Electronics, Cochin University of Science \& Technology, Thrikkakara, 682 022 Ernakulam, India, jintokg@cusat.ac.in), Supriya M. Hariharan (Department of Electronics, Cochin University of Science \& Technology, Thrikkakara, 682022 Ernakulam, India, supriya @ cusat.ac.in), Saseendran Pillai (Department of Electronics, Cochin University of Science \& Technology, Thrikkakara, 682022 Ernakulam, India, prspillai@cusat.ac.in)

Archival electronic tags can be used as standalone data loggers for sampling the ocean for gathering the environmental parameters and studying the migratory patterns of marine species, identifying their feeding and spawning grounds, etc. A prototype archival electronic tag for monitoring the ocean parameters like temperature, pressure and light intensity has been developed. A digital temperature sensor is used to sample the temperature from the tag's surroundings, while a micro machined piezoresistive silicon digital pressure sensor, which is capable of measuring absolute pressure levels upto 14 bars, provides the depth information. One of the important parameters to be measured is the geolocation of the species, which is computed from the ambient light intensities recorded by the digital light sensor in the tag. These parameters can be sampled and recorded in the memory at preset time intervals, as set at the time of deployment of the tag. This minuaturised tag provides the temperature data with 13 bit resolution, while the pressure and light intensity values have 15 bit resolutions. When used in fisheries studies, the size of the device has to be miniaturised, so that by way of attaching such devices, the normal behaviour of the species remain unaffected. 


\title{
Session 2pABc
}

\section{Animal Bioacoustics, Noise, and ECUA: Anthropogenic Noise Effects on Animals II (Poster Session)}

\author{
Michael Stocker, Cochair \\ Ocean Conservation Research \\ Jonathan Gordon, Cochair \\ Sea Mammal Research Unit
}

\begin{abstract}
All posters will be on display from 3:40 p.m. to 5:20 p.m. To allow contributors an opportunity to see other posters, contributors of odd-numbered papers will be at their posters from 3:40 p.m. to 4:30 p.m. and contributors of even-numbered papers will be at their posters from 4:30 p.m. to 5:20 p.m.
\end{abstract}

\section{Contributed Papers}

2pABc1. Discovery of Sound in the Sea website: Educational and media resources. Kathleen Vigness Raposa (Marine Acoustics, Inc., 809 Aquidneck Ave., Middletown, RI 02842, USA, kathleen.vigness@marineacoustics.com), Gail Scowcroft (University of Rhode Island, Graduate School of Oceanography, Narragansett, RI 02882, USA, gailscow@gso.uri.edu)

The scientific community and the public have become increasingly aware of, and concerned about, underwater sound. There is growing interest in learning about sources and uses of underwater sound, and potential effects of sound on the marine environment. Underlying this interest, however, is a need to provide scientific information at a level appropriate for the general public and for educational and media professionals that communicate with the public. The "Discovery of Sound in the Sea" website (http://www.dosits .org) provides scientific content introducing the physical science of underwater sound and how people and animals use sound to accomplish various tasks. In addition, it has recently been expanded to include three major resource sections: (1) Media Resources, (2) Teacher Resources, and (3) Student Resources. The Media Resources include a Facts \& Myths quiz, Frequently Asked Questions, and PDF reprints of a tri-fold pamphlet and a 12page educational brochure highlighting the content of the website. The Teacher and Student Resources include structured tutorials, educational games, and PowerPoint presentations. These resource sections provide easy, efficient access to timely information on the science of underwater sound and the current state of knowledge of the effects of underwater sound on marine mammals and fishes.

2pABc2. The effects of noise emanating from an adjoining architectural space on beluga whale audition in an aquarium exhibit. Katie Starke (University of Connecticut, 70 Pinney Hill Rd., \#95, Willington, CT 06179, USA, kathryn.starke@uconn.edu), Peter Scheifele (University of Cincinnati, French-East 345A, Cincinnati, OH 45221, USA, scheifpr@UCMAIL.UC.EDU)

Aquaria must have regulations regarding the intensity levels of anthropogenic noise that make their way into their captive belugas' tank, thus affecting the animals' ability to hear and vocalize naturally. My research focuses on the sound transmission across the acrylic and cement boundary that separates the beluga tank from the ballroom in the Georgia Aquarium. This ballroom hosts many social events which are often accompanied by high levels of noise that cross the barrier into the animal enclosure. I focus on the intensity range of 90-100dBA and experiment with a variety of frequencies, specifically those that are included in the range of beluga hearing. Though the data will be specific to the Georgia Aquarium, the results will apply to all aquaria that keep beluga whales, as high intensity sound will always travel through the exhibit boundaries and affect the animals living on the other side. 


\title{
Session 2pABd
}

\section{Animal Bioacoustics, Underwater Acoustics, Acoustical Oceanography, Signal Processing in Acoustics, and ECUA: Animal Sonar Systems II (Poster Session)}

\author{
James Simmons, Cochair \\ Brown University \\ Michel Andre, Cochair \\ Laboratori d'Aplicacions Bioacústiques, Universitat Politècnica de Catalunya
}

\begin{abstract}
All posters will be on display from 3:40 p.m. to 5:20 p.m. To allow contributors an opportunity to see other posters, contributors of odd-numbered papers will be at their posters from 3:40 p.m. to 4:30 p.m. and contributors of even-numbered papers will be at their posters from 4:30 p.m. to 5:20 p.m.
\end{abstract}

\section{Contributed Paper}

2pABd1. An area sensitive to apparent acoustic motion in the auditory cortex of the bat Phyllostomus discolor. Susanne Hoffmann (Biocenter of the Ludwig-Maximilians University of Munich, Großhaderner Str. 2 , 82152 Planegg-Martinsried, Germany, hoffmann@zi.biologie .uni-muenchen.de), Uwe Firzlaff (Biocenter of the Ludwig-Maximilians University of Munich, Großhaderner Str. 2, 82152 Planegg-Martinsried, Germany, firzlaff@zi.biologie.uni-muenchen.de), Gerd Schuller (Biocenter of the Ludwig-Maximilians University of Munich, Großhaderner Str. 2, 82152 Planegg-Martinsried, Germany, gschuller@1mu.de)

The processing of apparent acoustic motion was investigated in neurons in the auditory cortex of anaesthetized bats (Phyllostomus discolor). Apparent motion in the horizontal plane was generated by presenting pairs of pure tones with different interaural intensity differences (IIDs) and temporal separations, i.e. inter-pulse intervals (IPIs). Thus the spatial extent, motion direction and velocity of motion changed for each stimulus pair. A complete stimulus matrix consisted of 81 IID-combinations in the range between -40 to $+40 \mathrm{~dB}$ IID and was randomly presented via earphones with ten repetitions for up to five different IPIs ( 6.25 to $150 \mathrm{~ms})$. Thirty percent (71) of the 236 extracellularly recorded cortical single cells or small neuronal clusters showed facilitatory responses to acoustic motion compared to static stimulation and were classified as motion sensitive. With decreasing IPI, all motion sensitive neurons changed their azimuthal receptive field in size or spatial position. Twenty two percent (15) of them preferred small movements in the frontal area at very short IPIs. Most interestingly, the motion sensitive neurons were almost exclusively (97 \%) found in the dorsal area of the caudal part of the auditory cortex indicating that this cortical area is specifically involved in the processing of acoustic motion. 


\title{
Session 2pAOa
}

\section{Acoustical Oceanography and ECUA: Marine Ecosystem Acoustics III (Poster Session)}

\author{
Kenneth Foote, Cochair \\ Woods Hole Oceanographic Institution \\ Olav Godoe, Cochair \\ Institute of Marine Research
}

\begin{abstract}
All posters will be on display from 3:40 p.m. to 5:20 p.m. To allow contributors an opportunity to see other posters, contributors of odd-numbered papers will be at their posters from 3:40 p.m. to 4:30 p.m. and contributors of even-numbered papers will be at their posters from 4:30 p.m. to 5:20 p.m.
\end{abstract}

\section{Contributed Papers}

2pAOa1. An acoustic finite-element model to study sonar interactions with marine mammals. Gonzalo R. Feijoo (Woods Hole Oceanographic Institution, Woods Hole, MA 02543, USA, gfeijoo@whoi.edu), Kenneth G. Foote (Woods Hole Oceanographic Institution, Woods Hole, MA 02543, USA, kfoote@whoi.edu)

A computer model based on the finite-element method (FEM) is being developed to study the interaction of sonar signals with marine mammals. This model solves the Helmholtz equation in a computational box that includes the animal and the surrounding medium, water. The FEM code has been validated with analytical solutions for scattering of a plane wave by a fluid sphere over a range of parameters and frequencies of interest. The same FEM code has been applied to a 142-cm-long specimen of the common dolphin (Delphinus delphis); internal pressure and displacement fields have been computed. The animal is represented in the computer model by a set of tissue groups whose acoustic properties, density and sound speed, are taken from the literature. The geometry of each tissue group was constructed from segmented computerized tomography images. Results are presented for harmonic signals in the $1-10 \mathrm{kHz}$ frequency range. [Work supported by NOPP through ONR award N000140710992.]

2pAOa2. Mid-frequency pelagic imaging multi-beam sonar. Christopher D. Jones (Applied Physics Laboratory, University of Washington, 1013 NE 40th Street, Seattle, WA 98105, USA, cjones@ apl.washington .edu)

A unique circular array multi-beam sonar is used to investigate volumetric imaging of pelagic marine environments. Imaging of a radial volume of the ocean up to a kilometer in range and at $8-12 \mathrm{kHz}$ will be discussed, focusing mainly on the imaging of large aggregations of fish in shallow water. Several imaging geometries are presented including backscatter imaging in a shallow water waveguide used to resolve the two-dimensional horizontal structure of large fish schools, and the autonomous deployment of the sonar in a fixed location to create a time series of images and observations of variability over a 24 hour period. The potential for new imaging geometries will be explored including vertical profiling to create volumetric images of different pelagic zones in the ocean. Operational issues related to specific scientific question, and signal processing issues related to circular arrays will be discussed. Preliminary field data will be presented.

2pAOa3. Using multi-angle reflections to enhance ecosystem characterization. Jules S. Jaffe (Scripps Institution of Oceanography, Marine Physical Laboratory, 8820 Shellback Way, La Jolla, CA 92093, USA,
jules@mpl.ucsd.edu),Olav R. Godo(Institute of Marine Research, PO Box 1870, 5817 Bergen, Norway, olav.rune.godoe@imr.no), Hector Pena (Institute of Marine Research, PO Box 1870, 5817 Bergen, Norway, hector.pena@imr.no), Ben Maurer (Scripps Institution of Oceanography, Marine Physical Laboratory, 8820 Shellback Way, La Jolla, CA 92093 , USA, bmaurer@ucsd.edu)

Improved knowledge about target tilt can be used to improve acoustic estimates of abundance and to gain insights into the behavioural patterns of exploited marine species. This is because tilt angle strongly affects backscatter, especially at high frequencies. In order to estimate tilt, a dualtransducer system for synchronous observation of marine organisms at two angles (0o and 9o) at $38 \mathrm{kHz}$ was tested. Data was characterized via the "angle response"; the area backscatter from one transducer divided by the sum of both. This metric was computed for layers of small planktonic organisms as well as fish. While the angle response is almost negligible for small organisms at night and slightly in favour of the tilted transducer during day, there is a significant angle response from fish in layers. A theoretical model supports the idea that this effect is due swimbladder tilt. Backscatter from 13 schools of horse mackerel was also analyzed. Although no statistically significant difference was observed, the variability indicate that the angle response is highly dynamic and dependent on the actual behaviour of the school at time of observation. Further studies are planned to improve upon data collection as well as the theoretical aspects of interpretation.

2pAOa4. Application of multiple angle acoustic scatter to remote fish classification. Paul L. Roberts (University of California San Diego, Electrical and Computer Engineering Department, 9500 Gilman Dr., La Jolla, CA 92093, USA, plrobert@ucsd.edu), Jules S. Jaffe (Scripps Institution of Oceanography, Marine Physical Laboratory, 8820 Shellback Way, La Jolla, CA 92093, USA, jules@mpl.ucsd.edu)

Observation of broadband acoustic scatter from fish at multiple angles has the potential to offer advantages for remote classification in cases where the species exhibit differences in size and or body shape, and animal orientation is unknown or difficult to estimate. However, the total angular coverage of the observations, available bandwidth, and the inter- and intra-species variability in size can significantly influence classification accuracy. These parameters are investigated using laboratory data collected from ten different fish species using a linear array of receivers and a single transmitter with an effective angular coverage of up to 25 degrees with a 3 degree sampling increment. Results indicate that when the species exhibit significant differences in length, even two observations covering only 10 degrees are sufficient to obtain an 80 percent improvement in classification accuracy over a single observation angle. This holds true for a wide range of animal 
orientations. As inter-species size similarity increases, and intra-species size variability increases, more observations covering a larger range of angles are required to maintain a similar improvement in classification accuracy.

2pAOa5. Simultaneous acoustic observations of turbulence and zooplankton. Tetjana Ross (Dalhousie University, Department of Oceanography, 1355 Oxford Street, Halifax, NS B3H 4JI, Canada, tetjana.ross@dal.ca), Isabelle Gaboury (Jasco Research, Suite 432 - 1496 Lower Water Street, Halifax, NS B3J 1R9, Canada, isabelle.gaboury@gmail.com), Rolf Lueck (Rockland Scientific International Inc., 520 Dupplin Road, Victoria, BC V8Z 1C1, Canada, rolf @ rocklandscientific.com)

Models and laboratory experiments show that zooplankton may locate food more easily in turbulent waters, but whether plankton seek or avoid turbulence in the ocean is an open question. It is difficult to measure turbulence and plankton simultaneously and with the necessary spatial resolution using traditional methods (nets and airfoil shear sensors). Acoustics is commonly used to survey zooplankton abundance and recent studies have shown that stratified turbulence can also be a significant source of sound scatter This may seem like more of a complication than a boon for those aiming to use acoustics to observe plankton in turbulence. We present acoustic data, however, that show that at least under certain circumstances, zooplankton and turbulence can be observed simultaneously with a single $307 \mathrm{kHz}$ sounder. The different natures of the two targets (discrete targets versus a volume effect) allow them to be distinguished. The key is sampling the same targets at multiple ranges and having low enough concentrations that there is no more than one animal within the beam at the closest ranges.

2pAOa6. Potential influence of shells on multibeam backscatter imagery within the Te Matuku Marine Reserve, New Zealand Alexandre C. Schimel (Univ. of Waikato - Coastal Marine Group, Ruakura Satellite Campus, PB3105 Hamilton, New Zealand, alex.schimel@gmail.com), David Johnson (MetOcean Solutions Ltd, 3/17 Nobs Line, PO Box 441 New Plymouth, New Zealand, d.johnson@metocean.co.nz), Terry Healy (Univ. of Waikato - Coastal Marine Group, Ruakura Satellite Campus, PB3105 Hamilton, New Zealand, t.healy@waikato.ac.nz), Peter J. McComb (MetOcean Solutions Ltd, 3/17 Nobs Line, PO Box 441 New Plymouth, New Zealand, p.mccomb@metocean.co.nz), Brett Beamsley (MetOcean Solutions Ltd, 3/17 Nobs Line, PO Box 441 New Plymouth, New Zealand, b.beamsley@metocean.co.nz), Dirk Immenga (Univ. of Waikato - Coasta Marine Group, Ruakura Satellite Campus, PB3105 Hamilton, New Zealand, d.immenga@waikato.ac.nz)

Backscatter imagery from a KONGSBERG EM3000 multibeam echosounder $(300 \mathrm{kHz})$ has been analyzed to investigate its potential for benthic habitat mapping. A MATLAB code has been developed to process both bathymetry and coregistered echo-strength, which includes a simple correction to remove the typical multibeam echo strength swathe contrast introduced by the backscatter incident-angle dependence. Although the correction algorithm does completely remove the effect, it is sufficient to generate maps of suitable quality for further analysis. The processing code was applied to a dataset from a survey performed on two sites in the Tamaki Strait, New Zealand, in August 2007, including a section of the Te Matuku marine reserve. Within the marine reserve, a $400 \mathrm{~m} 2$ area displays a strong reflectivity that contrasts with its surroundings while a past ground-truth data collection shows the full area is dominated by mud with only some differences in shell fragments distribution. Hypotheses to explain the origin of this reflectivity contrast are formulated and verified against past sediment and benthic survey results with emphasis on the potential influence of shells on multibeam backscatter. The potential of multibeam surveying for detecting the coverage of shells patches in benthic habitat mapping is discussed.

2pAOa7. Acoustic methods for water mass delineation in coastal marine ecosystems. John Proni (AOML/NOAA, 4301 Rickenbacker Cswy, Miami, FL 33149, USA, john.proni@noaa.gov), Jack Stamates (AOML/NOAA, 4301 Rickenbacker Cswy, Miami, FL 33149, USA,Jack.Stamates@noaa.gov), Thomas Carsey (AOML/NOAA, 4301 Rickenbacker Cswy, Miami, FL 33149, USA, thomas.p.carsey@noaa.gov), Jia-Zhong Zhang (AOML/NOAA, 4301 Rickenbacker Cswy, Miami, FL 33149, USA, Jia-Zhong.Zhang@noaa.gov), Chris Sinigalliano (AOML/NOAA, 4301 Rickenbacker Cswy, Miami, FL 33149, USA, Christopher.Sinigalliano@noaa.gov), Kevin Sullivan(AOML/NOAA, 4301 Rickenbacker Cswy, Miami, FL 33149, USA, Kevin.Sullivan@noaa .gov)

Acoustical methods play an important role in the in the study of nutrient sources for coral reef ecosystems in the south Florida coastal ocean. Many nutrients released into the coastal ocean are released in distinct water masses such as inlet discharge plumes, wastewater outfall discharge plumes, and hurricane-induced re-suspensions. Using water column acoustic backscatter profiles, obtained via either ship-borne instrumentation or in-situ instrumentation, multidimensional images of nutrient bearing water masses are derived. Said images are of assistance in the design of chemical and biological sampling efforts. Examples of water mass imaging will be presented as well as the use of such images in the design of water quality sampling programs. Using a combination of acoustic backscatter, Doppler current profilers, nutrient (nitrate, nitrite, silica, phosphate, ammonia) samples and microbiological (fecal, protozoa, viruses) samples, flux estimates of said quantities may be made for inlet discharges. Results will be presented for a south Florida inlet having an average net seaward discharge of approximately 200 million gallons per day. Selected acoustic images of treated waste water effluent plumes will be presented and compared with plume spatial distributions inferred from conservative plume tracer studies.

2pAOa8. Acoustic data from platforms of opportunity as fuel for ecosystem models. Nils Olav Handegard (Institute of Marine Research, C. Sundtsgt 64, Nordnes, 5817 Bergen, Norway, nilsolav@imr.no), Olav Rune Godoe (Institute of Marine Research, C. Sundtsgt 64, Nordnes, 5817 Bergen, Norway, olavrune@imr.no), Patrick Lehodey (Collecte Localisation Satellites, 8-10, rue Hermès, Parc Technologique du Canal, 31520 Ramonville Saint-Agne, France, plehodey@cls.fr)

Echo sounders are a widely used tool for observing marine ecosystems. Traditionally, rigid designed surveys are used to integrate biomass to a global estimate, which is coupled to traditional stock assessment models. More recently, the focus has shifted from single species assessment to an ecosystem approach, taking into account ecosystem function and dynamics. As a response, several ecosystem models have been developed. The data requirements for these models are different than for the traditional models, and new sensors and platforms have been developed as a response to this need. However, examples on how to integrate these observations with these new models are scarce. We propose a state-space observation-space approach, and compare the results from a vertically structured eco-system model with observations from an echo sounder positioned at the Mid Atlantic ridge. We argue that this approach is less vulnerable to assumptions in the data analysis process, and discuss adequate observations regimes for these models. 


\title{
Session 2pAOb
}

\section{Acoustical Oceanography and ECUA: Acoustical Oceanography of Polar Environments II (Poster Session)}

\author{
Peter N. Mikhalevsky, Cochair \\ Science Applications International Corporation \\ Hanne Sagen, Cochair \\ Nansen Environmental and Remote Sensing Center
}

\begin{abstract}
All posters will be on display from 3:40 p.m. to 5:20 p.m. To allow contributors an opportunity to see other posters, contributors of odd-numbered papers will be at their posters from 3:40 p.m. to 4:30 p.m. and contributors of even-numbered papers will be at their posters from 4:30 p.m. to 5:20 p.m.
\end{abstract}

\section{Contributed Papers}

2pAOb1. The factors that influence the design of an underwater acoustic modem for Arctic missions. Andrew M. Smerdon (Aquatec Group Ltd, High Street, RG27 8NY Hartley Wintney, UK, asmerdon@aquatecgroup .com)

As summer Arctic sea ice cover continues to reduce, there is a growing need to monitor the ice-ocean interface to aid in understanding and quantifying Arctic climate change. As part of the DAMOCLES EU 6th Framework programme, several subsea elements of a new Arctic Ocean observing system will be deployed in the Arctic Ocean during 2008. To ensure that the observing system is effective, it is essential that measured data from the ocean environment be delivered in a timely fashion for assimilation with data sets from the ice surface and atmosphere. As several of the observing platforms are drifting freely in the water below the ice, there is a need to communicate data to the surface using underwater acoustic techniques. We discuss the factors that influence the specification of an acoustic modem to achieve this data transfer. These include development of standards for data transfer and storage processes, standby power considerations, logistics of establishing a communication link, and under-ice acoustics.

2pAOb2. High-frequency broadband acoustic scattering for investigating double- diffusive convection. Tetjana Ross (Dalhousie University, Department of Oceanography, 1355 Oxford Street, Halifax, NS B3H 4JI, Canada, tetjana.ross@dal.ca), Andone Lavery (Woods Hole Oceanographic Institution, Applied Ocean Physics \& Engineering Department, 98 Water Street, MS \#11, Woods Hole, MA 02543, USA, alavery@whoi.edu)

Polar regions, with their supercooled and relatively fresh surface water, are highly susceptible to the diffusive regime of double-diffusive convection (DDDC). The fluxes associated with DDDC can play a significant role in their heat/buoyancy budgets. The use of high-frequency acoustics as a tool to map the extent and evolution of DDDC in the ocean is explored through a series of laboratory $200-300 \mathrm{kHz}$ broadband acoustic backscattering measurements. Pulse compression signal processing allows centimeter-scale interface thicknesses to be rapidly and remotely measured, and the evolution, and ultimate merging, of multiple centimeter-scale interfaces to be observed. Combining the acoustically measured interface thicknesses with knowledge of the relatively-constant temperatures within the surrounding layers allows the estimation of DDDC fluxes. Thus, broadband acoustics offers a rapid and remote method to infer fluxes, without the need for timeconsuming microstructure measurements, suggesting that this technique could benefit field studies of DDDC. Using simple models to extrapolate to the thermohaline steps typically found associated with DDDC in polar regions, diffusive-convection interfaces are predicted to scatter at measurable levels in many areas. Narrowband (120 and $200 \mathrm{kHz})$ acoustic backscattering observations of two scattering layers in the Western Antarctic Peninsula support this prediction.
2pAOb3. Acoustic contributions to marine ecosystem studies. Andrzej J. Orlowski (Sea Fisheries Institute in Gdynia, Kollataja 1, 81-332 Gdynia, Poland, orlov@mir.gdynia.pl)

Since the early 1970s, acoustic data collected in a form of calibrated measurements of integrated echo energy have been applied by the Sea Fishery Institute in Gdynia to observe the relationships among fish distribution and marine environment. Measurements were collected primarily with scientific echo sounders operating at $38 \mathrm{kHz}, 24 \mathrm{~h}$ a day, and stored in the form of standardized intervals of sailed distance and depth for comparison with values of selected environmental parameters, measured concurrently. Acoustic, biological, and hydrological data were transferred to the complex data base, enabling spatial correlation and four-dimensional analyses of relationships characterising a wide range of the marine organism behaviour. Selected methods and standards of comparison are described to explain how to improve the recognition of relationships between three-dimensional spatial environmental gradients and fish distribution. Results of several case studies, including the influence of hydrologic and seabed characteristics, illustrate the practical application and validity of the methods. Particular attention is given to indicators of the dependence of local fish biomass density on temperature structure in the sea and diel cycles of fish behaviour. Animations of time-dependent processes, modelled on the collected data, will be included as a new tool for marine ecosystem analysis.

2pAOb4. Estimation of macrophytes using single-beam and multibeam echosounding for environmental monitoring of arctic fjords (Kongsfjord, West Svalbard Island). Aleksandra Kruss (Institute of Oceanology Polish Academy of Sciences, Powstancow Warszawy 55, 81712 Sopot, Poland, kruss@iopan.gda.pl), Philippe Blondel (University of Bath, Department of Physics, Claverton Down, BA2 7AY Bath, UK, pyspb@bath.ac.uk), Jaroslaw Tegowski (Institute of Oceanology Polish Academy of Sciences, Powstancow Warszawy 55, 81-712 Sopot, Poland, tegowski@iopan.gda.pl), Jozef Wiktor (Institute of Oceanology Polish Academy of Sciences, Powstancow Warszawy 55, 81-712 Sopot, Poland, wiktor@iopan.gda.pl), Agnieszka Tatarek (Institute of Oceanology Polish Academy of Sciences, Powstancow Warszawy 55, 81-712 Sopot, Poland, derianna@iopan.gda.pl)

This paper presents results of a study on the spatial distribution and biomass of macrophytobentos in a fjord of Arctic Svalbard. Kongsfjord represents a periglacial environment with intense morphodynamic processes and rapidly progressing changes in the biotic environment, making it one of the most promising areas to research climate impact on ecosystems. The main objective was to provide an acoustic tool for the evaluation of benthic habitats. The 2007 field survey included systematic, co-registered, singlebeam and multibeam echosounder measurements. Acoustic observations were verified by biological samplings and observations for the classification 
algorithm development and verification. Analyses of acoustic signals scattered on bottoms covered by algae indicate the good quality of the data recorded, providing a map of phytobenthos distribution and biomass estimation in Kongsfjord. The algorithms designed and tested for processing single- and multibeam data allow extracting the morphological forms of the bottom and determine the areas covered by algae. This survey was the first instance where a multibeam imager was used to map macrophytes in an Arctic environment, in a wide variety of depths and ranges. Comparing the two types of echosounders was a great opportunity to understand the visualisation of macrophytes by multibeam systems and their identification.

TUESDAY AFTERNOON, 1 JULY 2008

P3-C, LEVEL 3, 3:40 TO 5:20 P.M.

\title{
Session 2pAOc
}

\section{Acoustical Oceanography, Underwater Acoustics, Signal Processing in Acoustics, and ECUA: Geoacoustic Characterization of the Ocean Bottom and Geoacoustic Inversion II (Poster Session)}

\author{
Peter Gerstoft, Cochair \\ Marine Physical Laboratory, Scripps Institute of Oceanography \\ Dag Tollefsen, Cochair \\ Norwegian Defence Research Establishment (FFI)
}

\begin{abstract}
All posters will be on display from 3:40 p.m. to 5:20 p.m. To allow contributors an opportunity to see other posters, contributors of odd-numbered papers will be at their posters from 3:40 p.m. to 4:30 p.m. and contributors of even-numbered papers will be at their posters from 4:30 p.m. to 5:20 p.m.
\end{abstract}

\section{Contributed Papers}

2pAOc1. Extracting Green's functions from noise correlation of SW06 data. Laura Brooks (Marine Physical Laboratory, Scripps Institute of Oceanography, 8602 La Jolla Shores Drive, La Jolla, CA 92093-0238, USA, lbrook02@gmail.com), Peter Gerstoft (Marine Physical Laboratory, Scripps Institute of Oceanography, 8602 La Jolla Shores Drive, La Jolla, CA 92093-0238, USA, gerstoft@ucsd.edu)

Ambient noise was recorded on an L-shaped array during the SW06 (Shallow Water 2006) sea trials. The data were cross-correlated in order to approximate the Green's function, and subsequently the acoustic travel time, between hydrophone pairs. Examination of the individual noise spectra and their mutual coherence revealed that the coherently propagating noise is dominated by frequencies less than $100 \mathrm{~Hz}$. Both time and frequency domain preprocessing techniques, and their effect upon the resulting correlation, were investigated. Times corresponding to the envelope peak of the noise cross-correlation time-derivative are in agreement with both the expected direct, and surface reflected, inter-hydrophone travel times.

2pAOc2. Single parameter description of seafloors for shallow oceans. Adrian D. Jones (Defence Science and Technology Organisation, P.O Box 1500, 5111 Edinburgh, Australia, bearjones@chariot.net.au), Graham J. Day (Defence Science and Technology Organisation, P.O Box 1500, 5111 Edinburgh, Australia, Graham.Day@dsto.defence.gov.au), Paul A. Clarke (Defence Science and Technology Organisation, P.O Box 1500, 5111 Edinburgh, Australia, Paul.Clarke@dsto.defence.gov.au)

For a shallow ocean, sound transmission beyond short range is dominated by seafloor interactions at small grazing angles, for which the loss in $\mathrm{dB}$ on each bottom reflection may be approximated by a function $\mathrm{F} \mathrm{dB} / \mathrm{ra}-$ dian which is linear with grazing angle. Acoustic inversion techniques have been shown to be able to obtain the value of $\mathrm{F}$ for a particular frequency. The suitability of this single parameter $\mathrm{F}$ as a seafloor descriptor has now been studied for an extensive range of seafloor types. It is shown that the phase-incoherent transmission loss (TL) obtained using the parameter F is not greatly different to TL predicted using complete knowledge of a particular seafloor, for a shallow ocean. Further, if the phase angle for a seafloor reflection is linked to the parameter $\mathrm{F}$ via a simple approximation, the phase- coherent properties of the shallow water interference field may be estimated to an accuracy which is surprising. This paper reviews the relevant theory and presents comparisons between TL predicted using full geoacoustic parameters versus TL based on the single parameter, for both uniform halfspace and layered seafloor types.

2pAOc3. On some possibilities and properties of matched-field geoacoustic inversion in shallow water. Alexander Zharikov (M.V. Lomonosov Moscow State University, Faculty of Physics, Alexander Zharikov, ul. Simonovskiy val 14, kv. 58, 115088 Moscow, Russian Federation, alexandr-j@yandex.ru), Pavel Kravchun (Moscow State University, Faculty of Physics, Department of Acoustics, Box 15, 125130 Moscow, Russian Federation, gedackt@mail.ru)

The aim of this paper is to estimate possibilities of matched-field geoacoustic inversion (MFI) in shallow water, and to recommend optimal arrangements of signal source and hydrophone array in variety of particular environments. We assumed ocean to be range independent with bottom consisting of homogeneous liquid layers. Sound fields were calculated as superposition of normal modes and continuous spectrum for tonal point source and vertical line array. MFI based on Bartlett processor was used. Possibilities of MFI were characterized by MFI penetration depth, sensitivity to various bottom parameters and non-uniqueness of inverted data. These characteristics were analysed as functions of frequency and the source depth and range for different values of sound attenuation in sediments and for various sound-speed profiles. To estimate possibilities of MFI in real ocean conditions, influence of array tilt and inadequacy of geoacoustic model were analysed. The influence of continuous spectrum was also discussed. Among the major results, optimal source ranges corresponding to the maximum penetration depth of MFI were calculated, and high influence of attenuation in sediments on possibilities of MFI was revealed.

2pAOc4. A mixin algorithm for geoacoustic inversion. Hefeng Dong (Dept. of Electron. \& Teleco., Norwegian Univ. of Science and Technology, NO-7491 Trondheim, Norway, dong@iet.ntnu.no), Arne Johannes 
Jenssen(Norwegian Defense Research Establishment, NO-3191 Horten, Norway, Arne-Johannes.Jenssen@ffi.no)

Shear wave velocities in the sediment can be inverted by using the dispersion curves of interface wave. It can be done by use of an algorithm based on singular value decomposition (SVD). This method can only invert the shear wave velocities and requires knowledge of the thicknesses and densities of the sediment layers. This paper presents a new algorithm for inversion of geoacoustic parameters based on dispersion curves that we have coined "mixin inversion". Mixin inversion combines SVD and global search using genetic algorithm (GA). The GA searches the depths and densities by doing a shear wave velocity inversion with SVD for each of its parameter values. This algorithm can be applied on cases where the depths, densities and shear wave velocities of the sediments are unknown. The advantage is that it is faster than pure GA since the search space is much smaller and it can be applied on cases where pure SVD inversion fails because necessary information about depths and densities is not known.

TUESDAY AFTERNOON, 1 JULY 2008

ROOM 352B, 2:40 TO 7:00 P.M.

\title{
Session 2pBBa
}

\section{Biomedical Ultrasound/Bioresponse to Vibration: Ultrasound Contrast Agents for Therapy I}

\author{
Tyrone Porter, Cochair \\ Boston University, 110 Cummington Street, Boston, MA 02215, USA \\ Eleanor Stride, Cochair \\ University College London, Department of Mechanical Engineering, Torrington Place, London, WC1E 7JE, UK
}

\section{Invited Papers}

2pBBa1. Acceleration of ultrasonic tissue heating by microbubble agent. Shin-Ichiro Umemura (Tohoku University, Aoba 6-605, Aramaki, Aoba-ku, 980-8579 Sendai, Japan, sumemura@ecei.tohoku.ac.jp), Shin Yoshizawa (Tohoku University, Aoba 6-6-05, Aramaki, Aoba-ku, 980-8579 Sendai, Japan, syoshi@ecei.tohoku.ac.jp), Kazuaki Sasaki (Tokyo University of Agriculture and Technology, 3-5-8 Saiwai-cho, Fuchu, 183-0054 Tokyo, Japan, skazuaki@cc.tuat.ac.jp), Ken-Ichi Kawabata (Hitachi Central Research Laboratory, 1-280, Higashi-Koigakubo, Kokubunji, 185-8601 Tokyo, Japan, kenichi.kawabata.ap@ hitachi.com)

Ultrasound in the existence of microbubbles, whether they have been generated by acoustic cavitation or administered into the body, is known to induce bioeffects normally through mechanical or sonochemical mechanisms. Recently, it is reported that ultrasonic tissue heating can be accelerated several times by an administered microbubble agent. A microbubble, subjected to ultrasonic pressurein the frequency range of its resonanance converts the acoustic energy to heat through its volume oscillation. The acoustic power converted to heat by a microbubble through viscous heating was numerically calculated, and it was predicted that tissue ultrasonic absorption would be doubled if a microbubble agent is delivered to the tissue at a concentration in the order of 100 microbubbles $/ \mathrm{mg}$. Exteriorized murine kidneys were exposed to focused ultrasound at $3.2 \mathrm{MHz}$ in degassed saline and the tissue temperature change was measured. Optison at a dose of $0.2 \mathrm{ml} / \mathrm{kg}$ multiplied the temperature elevation induced by ultrasonic exposure by several times. In order to use this effect to treat a deep-seated tumor, microbubbles should be somehow delivered to the tumor selectively. A novel method of selective delivery, in which nano liquid particles are administered and converted into microbubbles by ultrasonic stimulation, will also be discussed.

\section{3:00}

2pBBa2. Ultrasound contrast agents pushing drug delivery: high speed optical observations. Annemieke Van Wamel (Erasmus MC, Dr Molewaterplein 50 room Ee2302, 3015GE Rotterdam, Netherlands, Annemieke@ieee.org), Klazina Kooiman (Erasmus MC, Dr Molewaterplein 50 room Ee2302, 3015GE Rotterdam, Netherlands, k.kooiman@erasmusmc.nl), Nico De Jong (Erasmus MC, Dr Molewaterplein 50 room Ee2302, 3015GE Rotterdam, Netherlands, n.dejong@erasmusmc.nl)

Ultrasound-activated-microbubbles can cause transient non-lethal sonoporation of cells. Studies of influx of cell-membrane impermeable-dye, genetic materials, and nanoparticles have confirmed that the action of ultrasound-microbubbles on the cell-membrane is to alter both the cell-permeability. The sonoporation action of ultrasound-contrast-agent on cells lies in the fact that microbubbles oscillate while irradiated with ultrasound resulting in a mechanical-deformation of the cells. Oscillation microbubbles nearby cells have been optically recorded using a microscope and a high-speed camera. This camera is able to record the MHz oscillation microbubbles and therefore the interaction between oscillation microbubbles and cells. Micro-mechanical forces generated by the oscillating microbubble may also be used to locally 'push' the drugs into the cells. Ultrasound-activated-microbubbles are able to transiently increase the vascular-endothelial-layer permeability. These features may be used for future ultrasound-guided drug-delivery-systems. Next to increasing the drugs internalization, microbubbles can also be used as drug/particle carrier. Drug-loaded contrast-agents can be intentionally ruptured by diagnostic ultrasound. Using microbubbles to carry drugs to targets sites and rupturing the microbubbles by localized ultrasound energy, will result in a high local concentration of drugs. High-speed-optical-recordings of ultrasound-contrast-agent induced cell and vascular-endothelial-layer permeability will be demonstrated. Furthermore, recordings of ultrasound-controlled drugdelivery using pay-loaded ultrasound-contrast-agents will be shown. 
2pBBa3. Characterization and Optimization of Ultrasound-induced Molecular Delivery In Vivo. Elisa Konofagou (Dept. of Biomedical Engineering, Columbia Univ., 351 Engineering Terrace MC 8904, 1210 Amsterdam Ave., New York, NY 10027, USA, ek2191@columbia.edu), James Choi (Dept. of Biomedical Engineering, Columbia Univ., 351 Engineering Terrace MC 8904, 1210 Amsterdam Ave., New York, NY 10027, USA, jjc2132@ columbia.edu), Shougang Wang (Dept. of Biomedical Engineering, Columbia Univ., 351 Engineering Terrace MC 8904, 1210 Amsterdam Ave., New York, NY 10027, USA, sw2401@ columbia.edu), Barclay Morrison (Dept. of Biomedical Engineering, Columbia Univ., 351 Engineering Terrace MC 8904, 1210 Amsterdam Ave., New York, NY 10027, USA, bm2119@columbia.edu), Mark Borden (Dept. of Biomedical Engineering, Columbia Univ., 351 Engineering Terrace MC 8904, 1210 Amsterdam Ave., New York, NY 10027, USA, mb2910@columbia.edu)

Current treatments of neurological and neurodegenerative diseases are limited due to the lack of a truly noninvasive, transient, and regionally selective brain drug delivery method. The brain is particularly difficult to deliver drugs to because of the blood-brain barrier (BBB). Over the past few years, we have been developing methods that combine Focused Ultrasound (FUS) and microbubbles in order to noninvasively, locally and transiently open the BBB so as to treat neurodegenerative diseases. In this paper, we will focus on the characterization of the type of molecular delivery that can be induced through the opened BBB. More specifically, we willl characterize important properties of the BBB opening such as its size and permeability using fluorescence and MR imaging techniques, respectively. The role of the microbubble type, size and concentration on the BBB diffusion properties, its reversibility and the pressure threshold for the opening will also be described in vivo. Finally, results will be shown in both non-transgenic (normal) and transgenic (Alzheimer's) mice in order to determine the variability of the properties of the opened BBB in the presence and absence of disease.

3:40-5:20 Posters

Lecture sessions will recess for presentation of poster papers on various topics in acoustics. See poster sessions for topics and abstracts.

\section{Invited Papers}

2pBBa4. Echogenic liposomes for vasoactive gas delivery and inhibition of intima hyperplasia in atheroproliferative disease. Huang Shaoling Huang (MSB 1.246, 6431 Fannin St., Houston, TX 77030, USA, Shaoling.Huang@uth.tmc.edu), David D. McPherson (University of Texas Health Science Center, 6431 Fannin St., MSB 1.252, Houston, TX 77030, USA, David.D.McPherson@uth.tmc.edu), Christy K. Holland (University of Cincinnati, Biomedical Engineering, MSB, 231 Albert Sabin Way, Cincinnati, OH 45267-0586, USA, Christy.Holland@uc.edu)

Our group has developed targeted echogenic liposomes (ELIP) for targeted ultrasound enhancement of atheroma as well as targeted therapeutic delivery. Nitric oxide (NO) has potent biological activities but is too labile for in-vivo vascular delivery. We have developed a methodology to entrap NO, cause local prolonged release and retain NO effect. NO-loaded ELIP were injected into the common carotid artery after balloon injury. Fourteen days later, the carotids were removed. Administration of NO-ELIP resulted in $51 \pm 6 \%$ inhibition of intimal thickening when compared with controls. Using a $6.0 \mathrm{MHz}$ clinical Doppler diagnostic ultrasound system, we (Smith et al) have identified the optimal pressure threshold (MI 0.08) for NO diffusion from ELIP. Using these parameters we have enhanced vascular permeability of drugs and stem cells to help stabilize atherosclerotic plaques. We have developed a novel method for encapsulating NO into ELIP demonstrating their capacity for NO delivery, inhibition of intimal hyperplasia and controlled gas diffusion with ultrasound. This methodology provides a new approach for delivering a variety of bioactive gases to target tissues, which otherwise would have negligible effects when administered systemically.

2pBBa5. Echogenic liposomes for molecular targeted therapeutic delivery. David D. McPherson (University of Texas Health Science Center, 6431 Fannin St., MSB 1.252, Houston, TX 77030, USA, David.D.McPherson@uth.tmc.edu), Shaoling Huang (University of Texas Health Science Center, 6431 Fannin St., MSB 1.252, Houston, TX 77030, USA, Shaoling.Huang@uth.tmc.edu), Christy K. Holland (University of Cincinnati, Biomedical Engineering, MSB, 231 Albert Sabin Way, Cincinnati, OH 45267-0586, USA, Christy.Holland@uc.edu)

Our group has developed targeted echogenic liposomes (ELIP) that can entrap therapeutics and release them at the target site. A key therapeutic is t-PA (tissue plasminogen activator). We have demonstrated entrapment and release following ultrasound application with t-PA effect on clot dissolution. We have demonstrated that t-PA can act as a targeting agent. Thus this drug can be both a targeting agent and a lytic agent minimizing the development of complicated formulations required for targeting and lysis. We have entrapped glitazones and demonstrated release following ultrasound application with drug effect by suppressing vascular hyperplasia. We have demonstrated that ultrasound with our ELIP can enhance delivery of agents into all areas of the atheroma, including the intima, media, the loose and dense adventitia. Heretofore these regions close to the arterial media have been impenetrable using standard intravenous drug therapy. These projects are helping to develop our echogenic immunoliposomes to; optimize targeted contrast delivery into vascular beds that are poorly penetrable by standard imaging agents; and optimize local release and concentration of therapeutics at the site of interest. This will ultimately help to direct therapeutic delivery, increase local effect, while minimizing the systemic effects of drugs and genes. 


\section{Contributed Papers}

\section{6:00}

2pBBa6. Enhancement of microbubble mediated gene delivery by simultaneous exposure to ultrasonic and magnetic fields. Eleanor Stride (University College London, Department of Mechanical Engineering, Torrington Place, WC1E 7JE London, UK, e_stride@meng.ucl.ac.uk), Colin Porter (The Institute of Cancer Research, Gene Therapy Group, Section of Cell and Molecular Biology, 237 Fulham Road, SW3 6JB London, UK, colin.porter@icr.ac.uk), Ana-Garcia Prieto (University College London, London Centre for Nanotechnology, 17-19 Gordon Street, WC1H 0AH London, UK, a.prieto@ucl.ac.uk), Quentin Pankhurst (University College London, London Centre for Nanotechnology, 17-19 Gordon Street, WC1H 0AH London, UK, q.pankhurst@ucl.ac.uk)

It has been shown in previous studies that ultrasound mediated gene delivery can be greatly enhanced by the presence of contrast agent microbubbles and that transfection efficiency is highly dependent upon both bubble/cell separation and the ultrasound field parameters. It has also been shown that gene delivery can be promoted by exposing cells to a magnetic field in the presence of DNA conjugated to magnetic nanoparticles. The aim of this work was to investigate whether it was possible to combine the advantages of both these techniques. It was found that transfection of Chinese hamster ovary cells by naked plasmid DNA was enhanced by simultaneous exposure to ultrasound (40-cycle sinusoidal pulses, centre frequency 0.5 $3 \mathrm{MHz}$, peak negative pressure $0.25-1.5 \mathrm{MPa}$, repetition frequency $1 \mathrm{kHz}$ for $10 \mathrm{~s})$ and a uniform magnetic field $(3-5 \mathrm{Nm}-3)$ in the presence of two different microbubble/nanoparticle preparations. The first consisted of phospholipid-coated microbubbles mixed with micelles containing magnetic nanoparticles; the second of microbubbles which were themselves magnetically active. These preparations were found to be much more effective than either magnetic micelles or phospholipid-coated microbubbles alone. The mechanisms underlying these observations in terms of microbubble dynamics, the sensitivity to the ultrasound parameters and their significance for potential therapeutic applications will be discussed.

\section{6:20}

2pBBa7. Acoustic properties of multifunctional nano/microbubbles used in ultrasonography and ultrasound-mediated chemotherapy. Natalia Rapoport (University of Utah, 72 S. Central Campus Dr. room 2646, Department of Bioengineering, Salt Lake City, UT 84112, USA, natasha.rapoport@utah.edu), Kwon-Ho Nam (University of Utah, 72 S. Central Campus Dr. room 2646, Department of Bioengineering, Salt Lake City, UT 84112, USA, kwonho@gmail.com), Zhonggao Gao (University of Utah, 72 S. Central Campus Dr. room 2646, Department of Bioengineering, Salt Lake City, UT 84112, USA, zggao@yahoo.com), Douglas A. Cristensen (University of Utah, 72 S. Central Campus Dr. room 2646, Department of Bioengineering, Salt Lake City, UT 84112, USA, christen@ee.utah.edu), Anne M. Kennedy (University of Utah, 72 S. Central Campus Dr. room 2646, Department of Bioengineering, Salt Lake City, UT 84112, USA, Anne.Kennedy@hsc.utah.edu)

Multifunctional nano/microbubbles were developed that combine properties of drug carriers, ultrasound imaging contrast agents, and enhancers of ultrasound-mediated drug delivery. At room temperature, the formulations comprised perfluoropentane (PFP) nanodroplets stabilized by biodegradable diblock copolymers. The nanodroplets converted into nano/microbubbles upon heating or ultrasound irradiation. The acoustic properties of the droplets and corresponding bubbles depended on the type of the bubble stabilizing copolymer. Two different copolymers were tested, poly(ethylene oxide)co-poly(L-lactide) (PEG-PLLA) and poly(ethylene oxide)-copolycaprolactone (PEG-PCL). For the same quantitative composition, the PEG-PLLA-stabilized bubbles demonstrated higher inertial cavitation threshold and coalescence propensity than PEG-PCL-stabilized bubbles. Nanobubble tendency to coalescence is advantageous for using PEG-PLLA bubbles as tumor-targeted contrast agents.On the other hand, a lower cavitation threshold for PEG-PCL-stabilized bubbles is useful for ultrasoundmediated drug delivery. The drug doxorubicin (DOX) was localized in the bubble walls; it was released from the bubbles in response to sonication by therapeutic ultrasound, which resulted in a significant degree of drug tumortargeting and effective tumor chemotherapy.

\section{6:40}

2pBBa8. Trans-blood-brain barrier delivery of compounds at pharmacologically relevant molecular weights in the hippocampus of mice using Focused Ultrasound. James Choi (Dept. of Biomedical Engineering, Columbia Univ., 351 Engineering Terrace MC 8904, 1210 Amsterdam Ave., New York, NY 10027, USA, jjc2132@ columbia.edu), Shougang Wang (Dept. of Biomedical Engineering, Columbia Univ., 351 Engineering Terrace MC 8904, 1210 Amsterdam Ave., New York, NY 10027, USA, sw2401@columbia.edu), Yao-Sheng Tung (Dept. of Biomedical Engineering, Columbia Univ., 351 Engineering Terrace MC 8904, 1210 Amsterdam Ave., New York, NY 10027, USA, yt2235@columbia.edu), Barclay Morrison (Dept. of Biomedical Engineering, Columbia Univ., 351 Engineering Terrace MC 8904, 1210 Amsterdam Ave., New York, NY 10027, USA,bm2119@columbia.edu), Elisa Konofagou (Dept. of Biomedical Engineering, Columbia Univ., 351 Engineering Terrace MC 8904, 1210 Amsterdam Ave., New York, NY 10027, USA, ek2191@ columbia.edu)

Molecular engineering has recently contributed immensely to the development of neuropharmaceuticals. However, most of these compounds are greater than $400 \mathrm{Da}$, which are too large to traverse the brain's natural defense, the blood-brain barrier (BBB). In this study, molecular delivery at pharmacologically relevant molecular weights through a Focused Ultrasound (FUS) induced BBB opening was investigated. The left hippocampus of mice $(n=13)$ was sonicated (frequency: $1.525 \mathrm{MHz}$, pressure: $0.64 \mathrm{MPa}$, duty cycle: $20 \%$, duration: 1 -min) in vivo through the intact skin and skull

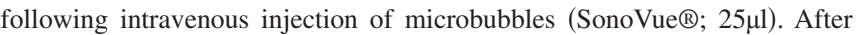
sonication, otherwise BBB-impermeable fluorescent-tagged dextrans at various molecular weights were administered intravenously. Ex vivo fluorescent microscopy determined BBB opening by a significant increase in fluorescence in the left over the right hippocampus. The threshold for the molecular weights allowed through the hippocampal BBB was between $70 \mathrm{k}$ and 2,000k Da. Smaller compounds (i.e., 3k Da) were more spatially uniformly deposited throughout the hippocampus than larger (i.e., 70k Da) compounds. In conclusion, dextrans at neuropharmaceutically-relevant sizes were deposited in the left hippocampus of mice. This study thus demonstrates that FUSinduced BBB opening could allow neuropharmaceuticals previously impermeable to the BBB, such as inhibitors and antibodies, to target tissue they were designed to treat. 


\title{
Session 2pBBb
}

\section{Biomedical Ultrasound/Bioresponse to Vibration: Ultrasound Contrast Agents for Therapy II (Poster Session)}

\author{
Tyrone Porter, Cochair \\ Boston University \\ Eleanor Stride, Cochair \\ University College London, Department of Mechanical Engineering, Torrington Place, London WC/E 7JE, UK
}

\begin{abstract}
All posters will be on display from 3:40 p.m. to 5:20 p.m. To allow contributors an opportunity to see other posters, contributors of odd-numbered papers will be at their posters from 3:40 p.m. to 4:30 p.m. and contributors of even-numbered papers will be at their posters from 4:30 p.m. to 5:20 p.m.
\end{abstract}

\section{Contributed Papers}

2pBBb1. Comparison of Spectral and Temporal Criteria for Inertial Cavitation Collapse. Mathieu Santin (Laboratoire d'Imagerie Paramétrique, 15 rue de l'Ecole de Médecine, 75006 Paris, France, santin@lip.bhdc.jussieu.fr), Alexander Haak (University of Illinois at Urbana-Champaign, Beckman Institute, 405 N Mathews, Urbana, IL 61801, USA, ahaak@uiuc.edu), Lori Bridal (Laboratoire d'Imagerie Paramétrique, 15 rue de l'Ecole de Médecine, 75006 Paris, France, bridal@lip.bhdc.jussieu.fr), William D. O’Brien, Jr. (University of Illinois at Urbana-Champaign, Beckman Institute, $405 \mathrm{~N}$ Mathews, Urbana, IL 61801, USA,wdo@uiuc.edu)

Dilute solutions of Optison and Definity were studied using a passive cavitation detector $(\mathrm{PCD})$ with a $2.8-\mathrm{MHz}$ transmitter and $13-\mathrm{MHz}$ receiver. The dilution was such that each received signal should, on average, arise from a single microbubble. Several hundred microbubble responses were acquired at each of three rarefactional pressures $(1.6+/-0.2,2.0+/ 0.2$ and $2.4+/-0.2 \mathrm{MPa}$ ). Each microbubble response was grouped with signals presenting post-excitation emissions (100\% Inertial Cavitation-Collapse) or those with no evidence of post-excitation emission (0\% IC-Collapse). For each incident pressure, we compared discrimination of $100 \%$ and $0 \%$ ICCollapse groups with peak-voltage, broadband noise (12-17.6 MHz) and power at the fundamental, $2^{\text {nd }}, 3^{\text {rd }}$, and $4^{\text {th }}$ harmonic peaks. In addition to increased peak-voltage and broadband noise, spectra from 100\% ICCollapse groups consistently presented increased $2^{\text {nd }}, 3^{\text {rd }}$ and $4^{\text {th }}$ harmonics compared to the $0 \%$ IC-Collapse group. Throughout the studied pressure range, best separation between $0 \%$ and $100 \%$ IC-Collapse groups was obtained with peak-voltage $(4.7+/-1.8 \mathrm{~dB})$, broadband noise $(4.4+/-1.8 \mathrm{~dB})$ and $4^{\text {th }}$ harmonic $(5.6+/-2.2 \mathrm{~dB})$ for Optison. For Definity, all harmonics $\left(2^{\text {nd }}\right.$ to $4^{\text {th }}$ ) increased strongly for the $100 \%$ IC-Collapse group (on the order of 6 $\mathrm{dB})$ as well as peak-voltage $(5.3+/-1.2 \mathrm{~dB})$ and broadband noise $(5.8+/$ $2 \mathrm{~dB}$ ). Results should contribute to relating PCD criteria and IC activity.

2pBBb2. Extraction of green fluorescent proteins with sonoporation Kadija Kaddur (INSERM U930, 2, Bvd Tonnelle, 37044 Tours Cedex 9, France, $\quad$ kadija.kaddur@etu.univ-tours.fr), Anthony Delalande (CNRS-UPR4301, Centre de Biophysique Moléculaire, 45071 Orléans Cedex 2, France, anthony.delalande@cnrs-orleans.fr), François Tranquart (INSERM U930, 2, Bvd Tonnelle, 37044 Tours Cedex 9, France, tranquart@med.univ-tours.fr), Patrick Midoux (CNRS-UPR4301, Centre de Biophysique Moléculaire, 45071 Orléans Cedex 2, France, patrick.midoux@cnrs-orleans.fr), Chantal Pichon (CNRS-UPR4301, Centre de Biophysique Moléculaire, 45071 Orléans Cedex 2, France,
pichon@cnrs-orleans.fr),Ayache Bouakaz(INSERM U930, 2, Bvd Tonnelle, 37044 Tours Cedex 9, France, bouakaz@med.univ-tours.fr)

The mechanism of permeabilization with sonoporation is not understood but the recurrent hypothesis assumes the formation of pores in the cell membrane. The aim of our study is to comfort this assumption by investigating whether ultrasound (US) and microbubbles could also facilitate outward transport of molecules across the plasma membrane through these probable pore formations. Stably transfected Hela-GFP cells were used and insonified with a $1 \mathrm{MHz}$ unfocused transducer in presence of BR14 microbubbles (Bracco Research, Geneva) at different acoustic parameters. The percentage of Hela-GFP cells and the mean cell fluorescence intensity (FI) were measured by flow cytometry to evaluate the GFP release. While US alone did not affect the cells, the addition of BR14 microbubbles induced a significant decrease of FI and the percentage of Hela-GFP cells. A reduction of more than $50 \%$ of FI and GFP + cells was achieved between $400-600$ $\mathrm{kPa}$ and $40-75 \%$ of duty cycle during 2 minutes insonation. However a progressive recovery of Hela-GFP cells percentage and FI post-insonation has been observed which indicates a viable situation of Hela-GFP permeabilized cells allowing a restoration of GFP cell content. These results comfort pore formation hypothesis allowing transmembrane transport following molecular concentration gradient.

2pBBb3. Blood-brain barrier disruption using a diagnostic scanner and Definity in mice. Kristin Frinkley (Biomedical Engineering, Duke Univ., 136 Hudson Hall, Durham, NC 27708, USA, kdf2@duke.edu), Gabriel Howles-Banerji (Center for In Vivo Microscopy, Duke Univ., 141D Bryan Res Bldg., Durham, NC 27710, USA, gabriel.howles@duke.edu), Yi Qi (Center for In Vivo Microscopy, Duke Univ., 141D Bryan Res Bldg., Durham, NC 27710, USA, yi.qi@duke.edu) , G. Allan Johnson (Center for In Vivo Microscopy, Duke Univ., 141D Bryan Res Bldg., Durham, NC 27710, USA, gaj@orion.duhs .duke.edu), Kathryn Nightingale (Biomedical Engineering, Duke Univ., 136 Hudson Hall, Durham, NC 27708, USA, kathy.nightingale@duke.edu)

Objective: To quantify localized disruption of the blood-brain barrier (BBB) in a murine model using focused, diagnostic ultrasound (at higher frequencies than previously studied) and contrast agent and to explore the optimal parameters for opening without causing irreversible damage. Methods: Definity (US contrast) and Magnevist (MR contrast) were injected before a custom ultrasound transmission sequence. The volume of tissue over which the BBB disruption allowed Magnevist to enter the brain was quantified by the contrast in T1-weighted MR images. Results: Preliminary results suggest increased BBB opening with decreased time between Definity injection and insonification. A range of typical diagnostic frequencies (e.g. 5-8 MHz) has shown BBB disruption, with maximum contrast occur- 
ring at 5.7 MHz. Increasing pressure had an apparent threshold for visible opening, while increasing $\mathrm{F} / \#$ led to a larger region of $\mathrm{BBB}$ opening and increasing duty cycle produced more contrast. Histology showed blood cell extravasation from $\mathrm{B}-$ mode imaging $(\mathrm{MI}=1.5$, duty cycle $=0.4 \%)$, but no damage was noted after the low-pressure, custom sequences. Conclusions: This study has shown the ability of a diagnostic ultrasound system and contrast agent to open the BBB for nanometer-scale particles (possibly drugs) and examined the effects of various parameters on this opening.

TUESDAY AFTERNOON, 1 JULY 2008

P3-B, LEVEL 3, 3:40 TO 5:20 P.M.

\title{
Session 2pBBc
}

\section{Biomedical Ultrasound/Bioresponse to Vibration and Physical Acoustics: Ultrasound Contrast Agents for Imaging II (Poster Session)}

\author{
Charles C. Church, Cochair \\ University of Mississippi \\ Ayache Bouakaz, Cochair \\ INSERM U930
}

\begin{abstract}
All posters will be on display from 3:40 p.m. to 5:20 p.m. To allow contributors an opportunity to see other posters, contributors of odd-numbered papers will be at their posters from 3:40 p.m. to 4:30 p.m. and contributors of even-numbered papers will be at their posters from 4:30 p.m. to 5:20 p.m.
\end{abstract}

\section{Contributed Papers}

2pBBc1. Influence of a rigid boundary on the resonance of an ultrasound contrast agent. Marie-Christine Pauzin (CNRS - LMA, 31 Chemin Joseph Aiguier, 13009 Marseille, France, pauzin@1ma.cnrs-mrs.fr), Serge Mensah (Laboratory for Mechanics and Acoustics CNRS, 31 chemin Joseph Aiguier, 13009 Marseille, France, mensah@1ma.cnrs-mrs.fr), Jean-Pierre Lefebvre (Laboratory for Mechanics and Acoustics CNRS, 31 chemin Joseph Aiguier, 13009 Marseille, France, lefebvre@1ma.cnrs-mrs.fr)

Recent experimental studies showed that the behavior of an Ultrasound Contrast Agent (UCA) is strongly modified when located close or targeted to a vessel wall. Asymmetrical oscillations can occur and the frequency response differs from those of free UCA. To design new techniques for diagnostic or therapeutic applications with UCA, it is important to have a better knowledge of the acoustic behavior and the dynamics of UCA in vessels. We used a finite element model (FEM) to focus on how the resonance frequency is affected by a boundary. A FEM had been first developed with COMSOL Multiphysics to calculate the pressure field scattered by two types of spherical objects (air microbubble or UCA with a viscoelastic shell) immersed in an unbounded liquid. The results obtained with the FEM being coherent with analytical models, a rigid boundary was then added to study its influence on the frequency responses of both objects. We observed a decrease of the resonance frequency of both objects when they are close to the boundary. This behavior is in good agreement with experimental results found in literature. The model has been used to perform parametrical studies (distance to the wall, UCA radius, shell parameter...).

2pBBc2. Simulation of the oscillation dynamics and translational motion of ultrasound contrast agents. Alexander Teterev (Belarus State University, 4, Nezavisimosti Ave., 220030 Minsk, Belarus, Teterev@bsu.by), Larisa Rudak (Belarus State University, 4, Nezavisimosti Ave., $220030 \quad$ Minsk, Belarus, rudak@bsu.by), Natalia Misuchenko (Belarus State University, 4, Nezavisimosti Ave., 220030 Minsk, Belarus, misuchenko@bsu.by)

A numerical model has been developed to simulate the oscillatory dynamics and the translational motion of both free and encapsulated bubbles in an ultrasound field. The model allows for the compressibility of the sur- rounding liquid and the bubble shell, which makes it possible to model the bubble dynamics at high acoustic pressures. Simulations can be carried out either by solving Rayleigh-Plesset-type equations, or in the hydrodynamic approximation taking into account the rheological properties of the liquid and the shell, or by a combined method. In the latter case, the gas pressure at the bubble surface, which is found from the gas-dynamic problem, is used as an input parameter for Rayleigh-Plesset-type equations at every time layer. The resulting radius of the bubble is then used as the boundary condition for the gas-dynamic equations. The model is a handy and flexible tool to investigate the effect of different factors on the oscillatory dynamics of the bubble, such as the thickness of the bubble shell, the rheological properties of the shell, and the translational displacement of the bubble. Using the developed model, the resonance frequency and translational displacement of contrast agents were numerically estimated.

2pBBc3. Improvement of the GMM-AR classification of multiframe contrast ultrasound images using gaussian filter. Bilal Ghazal (Univ. of Balamand Lebanon and Univ. of Claude Bernard France, Main Road Abdel Halim Ghazal bldg Kab-Elias Bekaa, 00961 Kab-elias, Lebanon, bilalghazal00@hotmail.com), Maha Khachab (Univ. of Balamand, Biomedical Dept., El Koura, 00961 Tripoli, Lebanon, maha.khachab@balamand.edu.lb), Denis Friboulet (Université de Lyon, 43 boulevard du 11 Novembre 1918, 69622 Villeurbanne, France, denis.friboulet@creatis.insa-lyon.fr), Chafic Mokbel (Univ. of Balamand, Biomedical Dept., El Koura, 00961 Tripoli, Lebanon, chafic.mokbel@balamand.edu.lb), Christian Cachard (Université de Lyon, 43 boulevard du 11 Novembre 1918, 69622 Villeurbanne, France, christian .cachard@creatis.insa-lyon.fr)

Despite the use of contrast agents that enhance the visualization of vascular zones, the backscattered signals from the contrast agent and tissue are still close which prevents the direct wide ultrasonic use in diagnosis. Thus, it was necessary to implement image-processing techniques that enhance the contrast echo and have the capability of classification. We have applied a new approach based on the autoregressive model where an image of prediction errors is calculated in the first phase. Then, a Gaussian filter is applied in order to model well afterward both agent and tissue behaviors by a Gaussian mixture model. The Agent to Tissue Ratio (ATR)factor and Fisher cri- 
terion are adopted to compare the performance of this method with existing techniques as the harmonic and B mode techniques. The experiments conducted have shown the advantages of our proposed approach where an increasing of ATR and Fisher are recorded. In fact, our ATR attains $19.20 \mathrm{~dB}$ which represents a good improvement in comparison with B mode $(9.50 \mathrm{~dB})$ and Harmonic technique $(12.13 \mathrm{~dB})$. Whereas Fisher, the parameter of classification feasibility, it reaches 2.01 which matches an excellent amelioration with respect the mentioning techniques with 0.97 and 1.00 respectively.

2pBBc4. Finite difference time domain numerical simulation of ultrasonic propagation in coated contrast agents. Galaz Belfor (Université Paris 6, Laboratoire d'Imagerie Paramétrique, 15, rue de l'Ecole de Médecine, 75006 Paris, France, bgalaz@fisica.usach.cl), Guillaume Haiat (CNRS, Laboratoire de Recherches Orthopédiques, 10, Avenue de Verdun, 75010 Paris, France, haiat@univ-paris12.fr), Taulier Nicolas (Université Paris 6, Laboratoire d'Imagerie Paramétrique, 15, rue de l'Ecole de Médecine, 75006 Paris, France, Nicolas.Taulier@lip.bhdc.jussieu.fr), Urbach Wladimir (Université Paris 6, Laboratoire d'Imagerie Paramétrique, 15, rue de l'Ecole de Médecine, 75006 Paris, France, urbach@1ps.ens.fr)

Acoustic wave propagation in solutions of ultrasound contrast agents (UCA) designed for in situ drug delivery remains unclear due to the complex interaction between ultrasound and the coated particles (mode conversion, scattering, multiple reflections). To address this problem, twodimensional Finite Difference Time Domain numerical simulations of ultrasound transmission and reflection were performed in solutions of an aqueous suspension of spheres made of fluid surrounded by a polymeric capsule. Simulations were made for particle concentrations equivalent ranging from 2 to $87 \mathrm{mg} / \mathrm{ml}$. For each concentration 15 aqueous solutions with randomly located particles were considered. The speed of sound $\mathrm{c}$, the attenuation coefficient and the integrated backscattered coefficient were computed from the transmitted and backscattered signals. The values of $\mathrm{c}$ decrease from $1498 \mathrm{~m} / \mathrm{s}$ to $1432 \mathrm{~m} / \mathrm{s}$ when the concentration increases, in good agreement with an effective medium model. The attenuation coefficient at $5 \mathrm{MHz}$ is an increasing function of the concentration, from 0.05 to $1.2 \mathrm{~dB} / \mathrm{cm}$. The integrated backscattered coefficient increases with the concentration for low concentration values but decreases for high concentration (with a maximum value for a concentration of about $50 \mathrm{mg} / \mathrm{ml}$ ). Our numerical simulations successfully mimic experimental results of ultrasound propagation in solutions of UCA.

2pBBc5. Photoacoustical evaluation of thermal response of microbubbles. Angelo Biagioni (Sapienza Università di Roma, Via A. Scarpa, 14, 00161 Rome, Italy, angelo.biagioni@uniroma1.it), Adriano Alippi (Sapienza Università di Roma, Via A. Scarpa, 14, 00161 Rome, Italy, adriano.alippi@uniroma1.it), Andrea Bettucci (Sapienza Università di Roma, Via A. Scarpa, 14, 00161 Rome, Italy, andrea.bettucci @uniroma1.it), Annunziata D’Orazio (Sapienza Università di Roma, Via A. Scarpa, 14, 00161 Rome, Italy, annunziata.dorazio @ uniroma1.it), Massimo Germano (Sapienza Università di Roma, Via A. Scarpa, 14, 00161 Rome, Italy, massimo.germano@uniroma1.it), Daniele Passeri (Sapienza, Università di Roma, Via A. Scarpa, 14, 00161 Rome, Italy, daniele.passeri@uniroma1.it)

Photoacoustic technique has emerged as a tool for the study of liquid, gel, suspension behaviour and has been recently employed in a number of new biomedical applications. In the present paper, a new photoacoustic sensor is presented which was designed and realized to analysing photoacoustic signals from solution filled with microbubbles, usually used as contrast agents in echotomography imaging. The device is a closed cell where photothermal expansion in acqueous solution samples causes the deflection of a thin membrane closing a short pipe $0.7 \mathrm{~mm}$ by radius. The overall set up acts as a Helmoltz resonator, where the solution in the pipe is the mass oscillating under the driving force produced by the alternating expansion of the solution in the cell body. Displacement of the membrane was measured using a laser probe interferometer, whereas photoacoustic signal was generated by chopping a laser light beam impinging into the solution through a glass window in the cell. The response of the cell filled with shelled microbubble solution was investigated with respect to water behaviour, at the cell resonance frequency and for different temperatures.

2pBBc6. Frequency response of bubble pulsations in tubes with arbitrary wall impedance. Todd A. Hay (Applied Research Laboratories, The University of Texas, P.O. Box 8029, Austin, TX 78713-8029, USA, haymaker@mail.utexas.edu), Theresa Y. Cheung (Applied Research Laboratories, The University of Texas, P.O. Box 8029, Austin, TX 78713-8029, USA, cheung.ty@gmail.com), Mark F. Hamilton (Applied Research Laboratories, The University of Texas, P.O. Box 8029, Austin, TX 78713-8029, USA, hamilton@mail.utexas.edu), Yurii A. Ilinskii (Applied Research Laboratories, The University of Texas, P.O. Box 8029, Austin, TX 787138029, USA, yura@arlut.utexas.edu)

A model is presented for the linear pulsation of a small bubble in a tube with locally reactive walls but with otherwise arbitrary wall impedance. The model is based on the normal mode expansion of the Green's function presented by Morse and Ingard [Theoretical Acoustics (McGraw-Hill, 1968), Eq. (9.2.10)]. The specific case of a cylindrical tube is considered. For a bubble that is located in the center of the tube and that is small compared with both tube diameter and wavelength, the radiation impedance on the bubble is given by a summation of the normal modes evaluated in the center of the tube. From the radiation impedance, the frequency response of the bubble to an applied sound field is obtained. For tube walls that are either rigid of pressure release, the solution agrees with the frequency response calculated using the method of images for a square tube having the same cross-sectional area. In tubes with hard walls the resonance frequency decreases as tube radius decreases because of radiation damping. In tubes with very soft walls the radiation damping is negligible below the cutoff frequency of the lowest mode, and the resonance frequency increases slightly as tube radius is decreased.

2pBBc7. New theoretical models for lipid-shelled ultrasound contrast agents. Alexander A. Doinikov (Belarus State University, 11 Bobruiskaya Street, 220030 Minsk, Belarus, doinikov@bsu.by)

Analysis of existing theoretical models for lipid-coated contrast agents is presented. A general theoretical approach to the development of zerothickness encapsulation models, allowing the testing of different rheological laws for encapsulation, is proposed. Based on available experimental data, analysis of the rheological behavior of encapsulating lipid shells is made. The problems of the existing models, such as the dependence of shell parameters on the initial bubble radius and the "compression-only" behavior, are discussed. Physical explanations for the observed effects and new theoretical models for their simulation are offered. In particular, it is shown that the inclusion of nonlinear shell viscosity allows one to model the "compression-only" behavior. It is also very important to select an appropriate rheological law describing the dependence of the shell viscosity on the shear rate. A correct choice reduces considerably the spread of the experimentally estimated values of the shell parameters and makes them look more ordered. Whereas with an inappropriate rheological law, the experimental data may look rather chaotic.

2pBBc8. Non-linear extensions to shell models of ultrasound contrast agents: theory and experiment. Nikos A. Pelekasis (Dept. Mechanical Engineering, University of Thessaly, Pedion Areos, 38334 Volos, Greece, pel@uth.gr), Kostas Tsiglifis (Dept. Mechanical Engineering, University of Thessaly, Pedion Areos, 38334 Volos, Greece, kotsigl@uth.gr), Benjamin Dollet (Physics of Fluids, University of Twente, P.O. Box 217, 7500 AE Enschede, Netherlands, benjamin.dollet@univ-rennes1.fr), Nico De Jong (Erasmus MC, Dr Molewaterplein 50 room Ee2302, 3015GE Rotterdam, Netherlands, n.dejong@erasmusmc.nl), Detlef Lohse (Physics of Fluids, University of Twente, P.O. Box 217, 7500 AE Enschede, Netherlands, d.lohse@utwente.nl), Michel Versluis (Physics of Fluids, University of Twente, P.O. Box 217, 7500 AE Enschede, Netherlands, m.versluis @utwente.nl)

The standard UCA models, characterized by the area dilatation modulus and viscosity of the encapsulating membrane, are extended in order to account for deviations from linear material behaviour and for non-spherical 
pulsations. The former effect is captured by introducing a parameter that measures the degree of membrane softness, whereas the latter is modelled by introducing a bending elasticity. Lipid shells exhibit the phenomenon of 'thresholding behavior' where excursions in the relative area dilatation are measured only beyond a critical sound amplitude. Treating the lipid shell as strain softening reproduces this non-linear behaviour which can be further optimized by controlling the shell softness parameter. Such a tuning can be used to estimate the degree of softness for the contrast agent BR-14. Stabil- ity analysis allowing for shape distortions provides an estimate for the bending elasticity that reproduces the experimentally observed threshold for growth of surface modes. Phase diagrams are constructed for BR-14, based on high-speed optical observations and stability analysis. Both experiment and numerical analysis exhibit a smaller variation between the stability threshold of subsequent shape modes in the case of UCA's, as compared to free bubbles. Numerical simulations reproduce the experimentally observed saturation of growing shape modes for pre-stressed UCA's.

TUESDAY AFTERNOON, 1 JULY 2008

P3-B, LEVEL 3, 3:40 TO 5:20 P.M.

\title{
Session 2pBBd
}

\section{Biomedical Ultrasound/Bioresponse to Vibration and Engineering Acoustics: High-Intensity Focused Ultrasound Metrology and Standards II (Poster Session)}

\author{
Peter Kaczkowski, Cochair \\ Center for Industrial and Medical Ultrasound, Applied Physics Lab. \\ Gail Ter Haar, Cochair \\ Institute of Cancer Research
}

\begin{abstract}
All posters will be on display from 3:40 p.m. to 5:20 p.m. To allow contributors an opportunity to see other posters, contributors of odd-numbered papers will be at their posters from 3:40 p.m. to 4:30 p.m. and contributors of even-numbered papers will be at their posters from 4:30 p.m. to 5:20 p.m.
\end{abstract}

\section{Contributed Papers}

2pBBd1. Simultaneous measurement of pressure and temperature in a focused ultrasound field with a fiber optic hydrophone. Michael S. Canney (Center for Industrial and Medical Ultrasound, Applied Physics Lab., University of Washington, 1013 NE 40th St., Seattle, WA 98105, USA, mcanney@u.washington.edu), Michael R. Bailey (Center for Industrial and Medical Ultrasound, Applied Physics Lab., University of Washington, 1013 NE 40th St., Seattle, WA 98105, USA, bailey@apl.washington.edu), Vera A. Khokhlova (Center for Industrial and Medical Ultrasound, Applied Physics Lab., University of Washington, 1013 NE 40th St., Seattle, WA 98105, USA, vera@acs366.phys.msu.ru), Oleg A. Sapozhnikov (Center for Industrial and Medical Ultrasound, Applied Physics Lab., University of Washington, 1013 NE 40th St., Seattle, WA 98105, USA, oleg@acs366.phys.msu.ru), Lawrence A. Crum (Center for Industrial and Medical Ultrasound, Applied Physics Lab., University of Washington, 1013 NE 40th St., Seattle, WA 98105, USA, lac@apl.washington.edu)

The characterization of high intensity focused ultrasound (HIFU) fields is important for both clinical treatment planning as well as for regulation of HIFU medical devices. In previous work, we have used a $100-\mu \mathrm{m}$ fiber optic probe hydrophone (FOPH) to measure pressure waveforms from a $2-\mathrm{MHz}$ HIFU source with 42-mm aperture and 44-mm focal length. The formation of shock waves with peak positive pressure of up to 80-MPa were measured and modeled in transparent tissue-mimicking gel phantoms and boiling was achieved in milliseconds [Canney MS, et al., J. Acoust. Soc. Am., 120:3110 (2006)]. In this work, the FOPH was also used to measure temperature changes in tissue phantoms from HIFU at peak focal intensities of 5000$20,000 \mathrm{~W} / \mathrm{cm}^{2}$. Temperature measurements were obtained by first low-pass filtering the voltage signal measured from the FOPH to remove the acoustic part of the measurement. Then, calibration of voltage to temperature was performed using results from a separate calibration experiment. Experimental measurements were compared with numerical modeling using a KZKtype model for acoustic propagation coupled with a heat transfer model. In summary, temperatures of $100^{\circ} \mathrm{C}$ were measured at the $\mathrm{HIFU}$ focus in milliseconds, in agreement with modeling [Work supported by NIH DK43881, NSBRI SMS00402, and RFBR.]

2pBBd2. Visualization of transducer fields using a two-dimensional acousto-optic sensor. Charles Pergantis (US Army Research Laboratory, Aberdeen Proving Ground, Abredeen, MD 21005-5069, USA, cpergan@arl.army.mil), Jaswinder Sandhu (Santec Systems, Inc., 716 South Milwaukee Ave., Wheeling, IL 60090, USA, j-sandhu@santecsystems.com), Witold Popek (Santec Systems, Inc., 716 South Milwaukee Ave., Wheeling, IL 60090, USA, w-popek@ santecsystems .com)

In this paper we will report on the use of a novel two-dimensional acousto-optic (2D AO) sensor as a simple, fast and cost-effective method of mapping transducer fields, which could be useful for quality control and quality assurance $(\mathrm{QC} / \mathrm{QA})$ of diagnostic and therapeutic transducers. This capability could be particularly useful for monitoring power output of therapeutic transducers prior to administering ultrasound based thermo-therapy treatment. It may also provide a quick tool for determining beam position and other transducer field characteristics such as propagation angle, beam diameter, divergence, and cross-sectional uniformity. Current standard transducer field mapping practices requires point-by-point scanning over a ball reflector or a hydrophone to map the transducer field. This approach is tedious, requiring hours of scanning time to generate the full cross-sectional and axial field distributions. The 2D AO system could overcome some of the drawbacks of current conventional scanning methodologies.

2pBBd3. Uncertainty Evaluation of Effective Radiation Area of Ultrasound Transducers: Preliminary Results of Inmetro's Laboratory of Ultrasound. Andre V. Alvarenga (Laboratory of Ultrasound - Inmetro, Av. N. Sra. das Gracas, 50, predio 1 - Xerem, 25250-020 Duque de Caxias - Rio de Janeiro, Brazil, avalvarenga@inmetro.gov.br), Rodrigo Costa-Felix (Laboratory of Ultrasound - Inmetro, Av. N. Sra. das Gracas, 
50, predio 1 - Xerem, 25250-020 Duque de Caxias - Rio de Janeiro, Brazil, rpfelix@inmetro.gov.br)

According to the "Guide to the Expression of Uncertainty of Measurement" (GUM), on calculation of the uncertainty of a given measurement it is necessary to take into account two types of uncertainty: Type A and Type B. Type A uncertainty is obtained from the statistical analysis of a series of observations, while Type B comes from sources that can not be evaluated considering statistical analysis, but can be obtained from previous measurements, knowledge on the behaviour of the measuring equipment, manufacturer's specifications, and data from certificates or handbooks.
Herein, the Effective Radiation Area (ERA) of ultrasound transducers has been estimated at Inmetro's Laboratory of Ultrasound using an acoustic pressure field mapping system. ERA calculation protocol developed was based on Standard IEC 61689. Besides, the Type A uncertainty was estimated from 4 repetitions of the full procedure for the determination of ERA, and Type B one was estimated from the mathematical model for ERA calculation, obtained based on IEC 61689 and the GUM. Initial tests using US transducer of $1.0 \mathrm{MHz}$ and $2.25 \mathrm{MHz}$ indicated an expanded uncertainty inferior to $7.0 \%$. This preliminary result encourages further research and development in order to increases frequency range to be assessed.

TUESDAY AFTERNOON, 1 JULY 2008

P3-B, LEVEL 3, 3:40 TO 5:20 P.M.

\title{
Session 2pBBe
}

\section{Biomedical Ultrasound/Bioresponse to Vibration: High-Intensity Focused Ultrasound II (Poster Session)}

\author{
Lawrence Crum, Cochair \\ Center for Industrial and Medical Ultrasound, Applied Physics Lab. \\ Jean Yves Chapelon, Cochair \\ INSERM, U556
}

\begin{abstract}
All posters will be on display from 3:40 p.m. to 5:20 p.m. To allow contributors an opportunity to see other posters, contributors of odd-numbered papers will be at their posters from 3:40 p.m. to 4:30 p.m. and contributors of even-numbered papers will be at their posters from 4:30 p.m. to 5:20 p.m.
\end{abstract}

\section{Contributed Papers}

2pBBe1. High intensity ultrasound activation of coagulation factors. Stuart B. Mitchell (Applied Physics Laboratory, University of Washington, 1013 NE 40th Street, Seattle, WA 98105, USA, sbmitch@u .washington.edu), Noemie C. Juaire (Center for Industrial and Medical U1trasound, Applied Physics Lab., University of Washington, 1013 NE 40th St., Seattle, WA 98105, USA, noemiej@apl.washington.edu), Yak-Nam Wang (Applied Physics Laboratory, University of Washington, 1013 NE 40th Street, Seattle, WA 98105, USA, ynwang@apl.washington.edu)

Although it has been demonstrated that High Intensity Focused Ultrasound (HIFU) can induce vascular cauterization, acoustic hemostasis technology has not been successful in making the transition from proof-ofconcept to clinical settings. One reason for this lack of acceptance is the limited understanding of the fundamental mechanisms involved in ultrasound-vessel and ultrasound-blood interactions. The aim of this research was to investigate the hematological and biochemical mechanisms which are influenced by HIFU induced coagulation. HIFU was used to induce coagulation in an in vitro hematological flow system and in animal models. The flow circuit and in vivo arteries were instrumented with flow probes and thermocouples in the treatment region. Physiological parameters were recorded throughout the in vivo experiments. Blood samples were drawn for analysis prior to, during, and immediately following each HIFU treatment. Clotting time, complete blood count, and biochemical analysis were performed immediately and citrated samples were immediately centrifuged and frozen for future analysis. Results indicate that HIFU can change local and systemic levels of thombin/anti-thrombin complex (TAT) and tissue plasminogen activator (tPA), as well as induce changes in activated clotting time (ACT). These results indicate that HIFU can induce coagulation via the coagulation cascades (TAT) and that normal hematological response to thrombus formation is unaffected.
2pBBe2. Dynamical focusing method for high-intensity ultrasound applications. Andrey Rybianets (Ultrashape Ltd., New Industrial Park, POB 80, 20692 Yoqneam, Israel, arybyanets@gmail.com)

New method for direct synthesis of dynamically focused acoustic field suitable for HIFU applications is proposed. The method is based on the continuous cyclic generation of different focal patterns at optimal repetition frequency by simultaneous applying of " $\mathrm{M}$ " different frequency signals to " $\mathrm{N}$ " sectors of spherically shaped sectored transducer array. The HIFU transducer arrays comprising a spherical piezoceramic cap with back electrode divided circumferentially into " $\mathrm{N}$ " regular or specially shaped sectors were designed and tested. The sectors were simultaneously powered by " $\mathrm{M}^{\prime \prime}(\mathrm{M} \leqslant$ N) sinus or burst drive signals with different frequencies from $6 \mathrm{~dB}$ bandwidth of the transducer. Calculation and modeling of acoustic field patterns for different array configurations and frequencies sets were performed. Acoustic pressure in focal planes was measured in water using calibrated hydrophone and 3D acoustic scanning system. In vitro experiments on different tissues confirming the advantages of dynamical focusing method were performed. The benefits of the method are creating bigger treated tissue volumes and enhancement of the cavitational, mechanical or thermal influences due to coincidence of the repetition frequency with a specific resonance/ relaxation times for tissue and/or for cavitation "cloud". Applications of the method for ultrasonic therapy, hyperthermia, and body aesthetics were considered.

2pBBe3. Delivery of antibacterial-nanoparticles into dentinal tubules with high intensity focused ultrasound. Siew Wan Fong (Institute of High Performance Computing, 1 Science Park Road, \#01-01 The Capricorn, 117528 Singapore, Singapore, fongsw@ihpc.a-star.edu.sg), Annie Shrestha (Department of Restorative Dentistry, Faculty of Dentistry, 
National University of Singapore, 5 Lower Kent Ridge road, 119074 Singapore, Singapore, g0600129@nus.edu.sg), Zhilong Shi (Department of Chemical and Biomolecular Engineering, National University of Singapore, 10 Kent Ridge Crescent, 119260 Singapore, Singapore, chesz@nus.edu.sg) , Boo Cheong Khoo (National University of Singapore, Dept of Mechanical Engineering, 10 Kent Ridge Crescent, 119260 Singapore, Singapore, mpekbc@nus.edu.sg), Anil Kishen (Department of Restorative Dentistry, Faculty of Dentistry, National University of Singapore, 5 Lower Kent Ridge road, 119074 Singapore, Singapore, rsdak@nus.edu.sg)

High-Intensity-Focused-Ultrasound (HIFU) when applied clinically often result in the formation of cavitation bubbles in the nearby fluid or tissue which collapse with high-speed jets and can be utilized for particle delivery. Bacteria residing in anatomical complexities and dentinal tubules resist conventional disinfection procedures during root canal treatment. This study aims is to evaluate the efficacy of jet flow produced by collapsing cavitations to push antibacterial nanoparticles into the areas inaccessible to conventional treatment. Eight dentin blocks of $8 \times 3 \times 1.5 \mathrm{~mm}^{3}$ were prepared from the root region of freshly extracted single rooted tooth samples and divided into two groups: (1) Control and (2) Nanoparticles. The samples were placed in sterile deionized water and nanoparticles suspension $(1 \mathrm{mg} / \mathrm{ml})$ for groups 1 and 2 respectively. HIFU at $27 \mathrm{kHz}$ for two minutes was employed. The samples were then sectioned to expose the dentinal tubules and viewed under field emission scanning electron microscopy and Energy Dispersive $\mathrm{X}$-ray analysis to ascertain the depth of penetration of the nanoparticles. The findings from this study suggested that HIFU treatment resulted in the significant penetration and coating of the dentinal tubules with nanoparticles. HIFU can be used as a potential tool for the delivery of antibacterial nanoparticles to disinfect the root canals.

2pBBe4. The effect of high intensity focussed ultrasound (HIFU) on pH responsive PEGylated micelles. Moira J. Smith (Chemical Engineering Department, Cambridge University, New Museums Site, CB2 3RA Cambridge, UK, mjs224@cam.ac.uk), Mark E. Eccleston (Chemical Engineering Department, Cambridge University, New Museums Site, CB2 3RA Cambridge, UK, mark.eccleston@ vivamer.com), Nigel K. Slater (Chemical Engineering Department, Cambridge University, New Museums Site, CB2 3RA Cambridge, UK, nigel_slater@cheng.cam.ac.uk)

The effect of HIFU on $\mathrm{pH}$ responsive PEGylated micelles was investigated. Micelles can be used as drug carrier vehicles reducing unwanted drug toxicity. HIFU is able to release drugs from the circulating micelles, as well as improving intracellular uptake of both micelleencapsulated and free drugs non-invasively. Large molecules generally enter cells by endocytosis. Endosomes gradually become acidic and fuse with enzyme containing lysosomes degrading the endosomal contents and preventing them from reaching their intracellular target. Using $\mathrm{pH}$ responsive polymers enables endosomes to be distrupted, releasing their contents into the cytoplasm before degradation occurs. Addition of poly(ethylene glycol), referred to as PEGylation, prolongs circulatory half-life and reduces degradation within the bloodstream. HIFU did enable release of encapsulated molecules from the modified micelles, and the micelles were taken up by H69 human carcinoma cells in vitro. Further work will investigate optimization of the micelles to maximize encapsulated drug release. The combined approach of using both $\mathrm{pH}$ responsive PEGylated micelles and HIFU to deliver drugs would provide more accurate targeting of therapies allowing higher therapeutic doses to be administered, reduce unwanted side effects and give patients a higher quality of life.

2pBBe5. The interaction of microbubbles with high intensity pulsed ultrasound. Siew Wan Fong (Institute of High Performance Computing, 1 Science Park Road, \#01-01 The Capricorn, 117528 Singapore, Singapore, fongsw@ihpc.a-star.edu.sg), Evert Klaseboer (Institute of High Performance Computing, 1 Science Park Road, \#01-01 The Capricorn, 117528 Singapore, Singapore, evert@ihpc.a-star.edu.sg), Boo Cheong
Khoo(National University of Singapore, Dept of Mechanical Engineering, 10 Kent Ridge Crescent, 119260 Singapore, Singapore, mpekbc@nus.edu .sg)

High intensity pulsed ultrasound when interacting with microbubble contrast agents is potentially useful for biomedical applications such as drug delivery, cancer treatment and tissue ablation. To establish a fundamental understanding of the interaction between a microbubble with a sound wave, numerical simulations are performed using the Boundary Element Method (BEM). Bubble dynamics in terms of shape changes in time, maximum bubble radius obtained, jet velocity and translational movement of the bubble center is studied. The effect of varying ultrasound intensity and initial bubble size is examined as well. One important result is the determination of the conditions under which a clear high speed jet will be formed in the microbubble when it is hit by a specific sound wave. The jet is crucial to the success of the biomedical applications mentioned. Also, it is shown that one cycle of ultrasound consisting of a single negative part followed by a single compressive part would be the optimized wave form because collateral damage by re-expanding remnants of a collapsed microbubble by the subsequent negative pressure wave is prevented. The BEM model has greater computational efficiency in terms of speed and storage space over other full domain methods because only surface meshing is needed.

2pBBe6. A passive array technique for cavitation mapping during HIFU treatment. Miklós Gyöngy (University of Oxford, Medical Engineering Unit, 43 Banbury Road, OX2 6PE Oxford, UK, miklos.gyongy@jesus.ox.ac.uk), Manish Arora (University of Oxford, Medical Engineering Unit, 43 Banbury Road, OX2 6PE Oxford, UK, manish.arora@eng.ox.ac.uk), Alison Noble (University of Oxford, Wolfson Medical Vision Laboratory, Department of Engineering, OX13PJ Oxford, UK, noble@ robots.ox.ac.uk), Constantin C. Coussios (University of Oxford, Medical Engineering Unit, 43 Banbury Road, OX2 6PE Oxford, UK, constantin.coussios@eng.ox.ac.uk)

During exposure of tissue to HIFU, cavitation has been shown to enhance the rate of heat deposition in tissue and could provide an effective means of treatment monitoring. However, cavitation activity occurring prefocally or growing unstable around the focal region is known to impede safe and effective treatment delivery. A combined computational and experimental approach is being applied to develop a passive array-based technique for mapping cavitation activity in real-time, using a novel cross-correlation algorithm to combine single-channel information received by an imaging array co-axial to the HIFU transducer. The cavitation maps produced in this manner were validated using two single-element $15-\mathrm{MHz}$ focused PCDs during continuous-wave insonation of a polyacrylamide-based tissue mimicking material by 1.1 MHz HIFU. Initial experiments have shown bubble cloud movement towards the HIFU transducer, in agreement with previously reported observations. Future work will involve applications of advanced imaging techniques to achieve a high spatiotemporal resolution, which is essential because of the unstable nature of cavitation activity and the requirement that treatment be well localized.

2pBBe7. Quantitative assessment of thermal dose using photographic measurements of tissue discoloration. Gavriel Speyer (Center for Industrial and Medical Ultrasound, Applied Physics Lab., University of Washington, 1013 NE 40th St., Seattle, WA 98105, USA, gavriel@u .washington.edu), Peter Kaczkowski (Center for Industrial and Medical Ultrasound, Applied Physics Lab., University of Washington, 1013 NE 40th St., Seattle, WA 98105, USA, peter@apl.washington.edu), Andrew Brayman (Center for Industrial and Medical Ultrasound, Applied Physics Lab., University of Washington, 1013 NE 40th St., Seattle, WA 98105, USA, brayman@apl.washington.edu), Marilee Andrew (Center for Industrial and Medical Ultrasound, Applied Physics Lab., University of Washington, 1013 NE 40th St., Seattle, WA 98105, USA, marilee@apl.washington .edu), Steven Kargl (Applied Physics Laboratory, University of Washington, 1013 NE 40th Street, Seattle, WA 98105, USA, kargl@troutmask 
.apl.washington.edu),Lawrence A. Crum(Center for Industrial and Medical Ultrasound, Applied Physics Lab., University of Washington, 1013 NE 40th St., Seattle, WA 98105, USA, lac@apl.washington.edu)

High Intensity Focused Ultrasound (HIFU) is rapidly gaining widespread clinical use in China, and is undergoing regulatory evaluation in Europe and the US for many target diseases. Nevertheless, tools for therapy planning, monitoring, and assessment remain at a rudimentary level. In particular, measurement of thermal dose in tissues exposed with HIFU has not been sufficiently quantitative to make detailed comparisons with numerical simulations, required for validation of therapy planning models. Indeed, model validation is complicated by high sensitivity of the results to small changes in parameter values and by the general difficulty of performing geometrical registration with sufficient precision to meaningfully compare millimeter scale features typical of HIFU lesions. Our work uses photographic measurement of visible tissue discoloration so that it can be used to accurately and rapidly quantify HIFU-induced bioeffects at scales of several centimeters for comparison with the prior therapy plan. Precise comparison between nonlinear acoustic simulation and macroscopic lesion data indicates that a newly defined "blanching index" is nearly linearly proportional to the logarithm of predicted thermal dose over a very wide range of exposure, including well below the 240 minute (at 43 degrees) necrotic threshold up to about 10,000 minutes.

TUESDAY AFTERNOON, 1 JULY 2008

P3-B, LEVEL 3, 3:40 TO 5:20 P.M.

\title{
Session 2pBBf
}

\section{Biomedical Ultrasound/Bioresponse to Vibration: Quantitative Ultrasound Methods for Diagnosis and Therapy II (Poster Session)}

\author{
Jonathan Mamou, Cochair \\ Riverside Research Institute \\ Geneviève Berger, Cochair \\ LIP, CNRS, Paris, France
}

\begin{abstract}
All posters will be on display from 3:40 p.m. to 5:20 p.m. To allow contributors an opportunity to see other posters, contributors of odd-numbered papers will be at their posters from 3:40 p.m. to 4:30 p.m. and contributors of even-numbered papers will be at their posters from 4:30 p.m. to 5:20 p.m.
\end{abstract}

\section{Contributed Papers}

2pBBf1. Liver fibrosis identification by spectral slope of the backscattering curve. Mahmoud Meziri (Univ Badji Mokhtar Annaba, Bp 12 Sidi- Amar Wilaya Annaba, 23000 Annaba, Algeria, mmahmouddz@yahoo.fr), Wagner C. Pereira (Biomedical Engineering Program - COPPE, Federal University of Rio de Janeiro, CEP. 21.941-972 Rio de Janeiro, Brazil, wagner@peb.ufrj.br), Naceur Tiah (Univ Badji Mokhtar Annaba, Bp 12 Sidi- Amar Wilaya Annaba, 23000 Annaba, Algeria, nac_tiah@yahoo.fr), Naamane Remita (Univ 20 Aout 55 Skikda, BP 24, 21000 Skikda, Algeria, n.remita@yahoo.fr), Bouzid Boudjema (Univ 20 Aout 55 Skikda, BP 24, 21000 Skikda, Algeria, boudjema_b@yahoo.fr), Pascal Laugier (Université Paris 6, Laboratoire d'Imagerie Paramétrique, 15, rue de l'Ecole de Médecine, 75006 Paris, France, laugier@lip.bhdc.jussieu.fr)

Ultrasonic tissue characterization is primarily based on radio-frequency (RF) signals. Different studies have demonstrated that the RF signals are closely linked to tissue structures. The processing of these signals using spectral methods has shown the possibility of deriving quantitative parameters (attenuation and backscattered coefficients) and also of providing a means to estimate the elementary properties of tissue (scatterer size, concentration, periodicity) by evaluating spectral parameters (slope, intercept etc). We have estimated the spectral slope of the average backscattering curve to test its potential in the discrimination of fibrosis stages (F0, F1, F2, F3, and F4, METAVIR scale) from 20 in-vitro human liver samples, insonified at $20 \mathrm{MHz}$. The slope estimations were $(\mathrm{dB} / \mathrm{MHz}): 0.95,0.24(\mathrm{~F} 0)$, $1.15,0.43(\mathrm{~F} 1), 1.20,0.26(\mathrm{~F} 3)$ and 1.07,0.33 (F4). The KolmogorovSmirnov test $(\mathrm{p}>0.05)$ indicated that the slope alone cannot discriminate all fibrosis groups (as well as the other mentioned parameters). When associated to integrated backscattered coefficient, discriminant analysis has correctly classified $80 \%$ of liver samples $(\mathrm{p}<0.0000)$. The misclassification resulted from some F0 samples grouped as F4 or vice-versa, which agrees with our previous results that suggested some parameter overlapping for normal and cirrhotic hepatic tissue. Nevertheless, combination of these parameters can help the diagnosis of liver fibrosis.

2pBBf2. Ultrasonic method to define human serum blood total protein and protein fractions. Anna Klemina (Dept. of Acoustics, Radiophysical Faculty, Nizhny Novgorod State University, 23 Gagarin Ave., 603950 Nizhny Novgorod, Russian Federation, klemina@rf.unn.ru)

Research of ultrasonic characteristics of biological liquids for purposes of medical diagnostics is the important task. Ultrasonic method to define total protein and protein fractions - albumin, $\alpha 1-, \alpha 2-, \beta-, \gamma$ - globulin's of human serum blood is discussed. This method is based on the precise measurements of velocity and absorption of ultrasonic waves in serum blood and its modified solutions under different temperatures from range (15-40)0C. The measurements of ultrasonic velocity and absorption in samples serum blood fulfils by means of method ultrasonic interferometer constant length or resonator method. The precision of relative measurements of velocity by means of this method is $10-5$ and absorption is $10-3$, temperature stability is the order of 10-3 0C. Protein fractions define by means of decision of linear system of equations where in the character of unknown components consider these protein fractions. Comparison of data of different patient obtained by traditional electrophoreses method with data of ultrasonic method shows very high correlation of all protein fractions and total protein. This work was supported by RFBR and Leading Scientific Schools grants.

2pBBf3. Evaluation of performance in some ultrasonic procedures for non-invasive thermal estimation into hyperthermia phantoms. Ivonne Bazan (CINVESTAV, Dpto. Ingen. Electrica, Mexico DF, Mexico, 
ibazan@cinvestav.mx),Antonio Ramos(Instituto de Acustica CSIC, Serrano 144, $28006 \quad$ Madrid, Spain, aramos@ia.cetef.csic.es), Monica Vazquez (UNAM, IIMAS, Mexico DF, Mexico, moni260577 @ yahoo.com.mx), Arturo Vera (CINVESTAV, Dpto. Ingen. Electrica, Mexico DF, Mexico, arvera@cinvestav.mx), Lorenzo Leija (CINVESTAV, Dpto. Ingen. Electrica, Mexico DF, Mexico, 1leija@cinvestav.mx)

To dispose of a precise and non-invasive temperature measurement over the treated area becomes very important during hyperthermia treatments, in order to make possible an optimization of their healing effects. A performance analysis of some previously reported ultrasonic techniques, which were proposed for non-invasive temperature estimation, is made in this work over phantoms mimicking human tissues. A first technique is based on discrete scattering modeling for tissue characterization and spectral analysis of frequency distributions related to average scatters spacing [R. Seip \& E.S. Ebbini]. Other technique uses certain relations between tissue temperature changes and time-shifts in echoes due to thermally induced sound velocity changes and expansions [R. Maass-Moreno \& C.A. Damianou]. And finally, the third procedure analyzed here, is a recently proposed alternative, based on phase demodulation processing, to estimate the indirect effects of echoes time-shifts in the phase domain [M. Vázquez, A. Ramos, et al.]. The three options are analyzed for the same simulated \& measured multi-pulse echo patterns, looking for detecting possible advantages and inconveniences in each case. Finally, some improvement paths over the basic estimation methods are also explored.

2pBBf4. Sensitivity study of the acoustic nonlinearity parameter for measuring temperatures during High Intensity Focused Ultrasound treatment. Koen W. Van Dongen (Lab. of Acoustical Imaging and Sound Control, Faculty of Applied Sciences, Delft Univ. of Technology, PO Box 5046, 2600 GA Delft, Netherlands, K.W.A.vanDongen@tudelft.nl), Martin D. Verweij (Lab. of Electromagnetic Research, Faculty of Electrical Engineering, Mathematics and Computer Science, Delft Univ. of Technology, Mekelweg 4, 2628 CD Delft, Netherlands, m.d.verweij@tudelft.nl)

The aim of High Intensity Focused Ultrasound (HIFU) is to locally increase the temperature in a body. For an adequate application of the treatment it is important to measure non-invasively the temperature profiles in the heated region. Most efficiently, this is done with the same modality as being used for heating. Consequently, the preferred measuring method should rely on the temperature dependence of an acoustic medium parameter. The goal of this study is to determine which parameter is most sensitive to temperature changes. To find the most suitable parameter, the temperature dependence of the speed of sound (SOS), the density of mass and the acoustic nonlinearity parameter B/A are compared for water. The temperature dependence of the SOS and the mass density are obtained by interpolating values found in literature. Since measured values of the B/A parameter are only known for a few coarsely distributed temperature values, it has been synthesised from a two-dimensional function describing the SOS versus temperature and pressure. It turns out that the $\mathrm{B} / \mathrm{A}$ parameter is far more sensitive for temperature changes than the other parameters. Comparison with measured $\mathrm{B} / \mathrm{A}$ values confirms the idea that nonlinear acoustics is a favourite candidate to measure temperature profiles.

2pBBf5. The scattering of sound by a moving sphere in a stratified liquid. Jason Bach (Georgia Tech Lorraine - G.W. Woodruff School of ME, UMI Georgia Tech - CNRS 2958, 2 rue Marconi, 57070 Metz, France, jsbach82@yahoo.com), David Ku (Georgia Tech Lorraine - G.W. Woodruff School of ME, UMI Georgia Tech - CNRS 2958, 2 rue Marconi, 57070 Metz, France, david.ku@me.gatech.edu), Nico F. Declercq (Georgia Tech Lorraine - G.W. Woodruff School of ME, UMI Georgia Tech - CNRS 2958, 2 rue Marconi, 57070 Metz, France, nico.declercq@me.gatech.edu)

The interaction of sound with a sphere and the Doppler shift caused by scatterers in motion are well described in the literature. A numerical study is performed on the interaction of sound with a moving sphere and with multiple moving spheres. The study shows how the reflected sound fields are influenced by the relative motion and relative position of the spheres. The aim of the research is to discover efficient acoustic methods to detect and characterize moving spheres at different levels of complexity determined by the number of spheres, their relative velocities and the velocity of the sur- rounding liquid. At a first stage the interaction of sound with a single sphere in a stratified fluid flow is investigated. The research is performed in the framework of the use of ultrasound for biomedical applications.

2pBBf6. Two coupling media method for ultrasonic velocity measurement in biological tissues. Jérôme Mathieu (IES - Univ. Montpellier 2, place Eugène Bataillon, cc 082, 34095 Montpellier Cedex 5, France, mathieu@lain.univ-montp2.fr), Audrey Leydier (IES - Univ. Montpellier 2, place Eugène Bataillon, cc 082, 34095 Montpellier Cedex 5, France, leydier@lain.univ-montp2.fr)

Measuring the ultrasonic velocity in soft materials, especially in biological tissues, is never easy. One of the main problems is that the thickness of the sample in test is badly defined. From time-of-flight measurement method uncertainties in the thickness induce important uncertainties in experimental results. This causes that the ultrasonic velocity is often a poor criteria whereas it could be a good one. This paper brings a new method to measure the ultrasonic velocity in soft materials without knowing the sample thickness The main idea of this method is to use two different coupling media for making two relative time-of-flight measurements. The comparison of these two measurements gives the ultrasonic velocity in the sample in test. Furthermore, this comparison can give the local sample thickness of the sample. The uncertainty calculi show up that the effect on the thickness uncertainties is drastically reduced by using this new ultrasound method. This method is validated on reference samples, in silicon. Before concluding, some experimental results obtained with mouse skin samples are presented.

2pBBf7. Ultrasound elasticity assessment of in vivo human achilles tendons during a quick-release exercise. Marielle Defontaine (LUSSI, 10 Bd Tonellé, 37032 Tours, France, defontai@med.univ-tours.fr), Mourad Bentahar (ENSIM - LAUM, Université du Maine, rue Aristote, 72085 Le Mans, France, Mourad.Bentahar@univ-lemans.fr), Francis Canon (CNRS UMR6600, Centre de Recherche de Royallieu - UTC, 60200 Compiègne, France, francis.canon@utc.fr)

An ultrasound (US) axial transmission technique has been developed to assess in vivo real time tendon biomechanical properties during calibrated exercises. The US propagation velocity changes are indeed related to the tendon visco-elasticity variations. We propose to monitor the propagation velocity changes during a very short period of time corresponding to the tendon/muscle complex release just after a sub-Maximal Voluntary isometric Contraction (MVC). This measurement is repeated for several sub-MVC amplitudes. The propagation velocity variations, linearly related to the torque measurements, allow to estimate a stiffness index, essentially due to the Series Elastic Component of the Achilles tendon. This study has been performed in collaboration with the UMR 6600 (UTC, France), and we have used the Ergometer they developed a few years ago. The US device is composed of a $1.8 \mathrm{MHz}$ US probe (1 emitter, 20 receivers), and an electronic module (Althaïs Technologies, France) allowing real time and synchronous acquisitions of US RF signals and auxiliary inputs (Torque, angular position). Four healthy subjects were asked to develop several submaximal voluntary isometric contractions, and the sole was suddenly released (sub MVC to relaxed state, $2 \mathrm{~s}, 2 \mathrm{kHz}$ ). This protocol was repeated two times with foot and probe repositioning.

2pBBf8. Carotid Inter-adventitial Distance based Vascular Age and Heart Disease Risk Estimation from B mode Ultrasound. Jayaraj Joseph (Indian Institute of Technology, ESB 313 ; Dept. of Electrical Engineering, IIT Madras, 600036 Chennai, India, jayaraj85@yahoo .com), Kishore Kumar (Indian Institute of Technology, ESB 313 ; Dept. of Electrical Engineering, IIT Madras, 600036 Chennai, India, kishorekumar015@yahoo.co.in), V Jayashankar (Indian Institute of Technology, ESB 313 ; Dept. of Electrical Engineering, IIT Madras, 600036 Chennai, India, jshankar@ee.iitm.ac.in)

A method to estimate the risk of heart disease in a person, based on diagnostic ultrasound is proposed here. The "vascular age" obtained from carotid ultrasound is very commonly used as a measure of cardiac health. Existing methods of vascular age estimation make use of the Carotid Intima Media Thickness (CIMT) measurements to arrive at an estimate of the vascular age. Recent work has demonstrated that the carotid inter adventitial 
distance $(\mathrm{CAD})$ has a direct relation with the risk of coronary heart disease in persons. It has also been demonstrated that CAD correlates better to a number of risk factors accounting for heart disease than the CIMT. Hence, we propose a method to estimate vascular age based on CAD. B mode images of the carotid artery are analyzed to find out the carotid inter adventitial distance. The vascular age is estimated from carotid diameter using a statistical model developed based on the Atherosclerosis Risk In Communities Limited access database. This vascular age is then used to modify standard risk assessment procedures like the Framingham risk equation to give a better estimate of coronary heart disease risk. A comparison of the method with the CIMT based method is also performed.

2pBBf9. Effect of inclination of articular surface on ultrasonic surface reflection and surface roughness in diagnosing cartilage degeneration Erna Kaleva (University of Kuopio, Department of Physics, POB 1627, FI70211 Kuopio, Finland, erna.kaleva@uku.fi), Simo Saarakkala (University of Kuopio, Department of Physics, POB 1627, FI-70211 Kuopio, Finland, simo.saarakkala@uku.fi), Juha Töyräs (Kuopio University Hospital, POB 1777, 70211 Kuopio, Finland, Juha.Toyras@kuh.fi)

High-frequency Quantitative Ultrasound Imaging is a potential method for detecting early osteoarthrotic changes in articular cartilage. However, uncontrolled inclination of the ultrasound transducer or the curvature of the cartilage surface can jeopardize the reliability of the method. Visually intact and mechanically degraded osteochondral bovine patellae samples were imaged using a scanning acoustic microscope equipped with a $50 \mathrm{MHz}$ ultrasound transducer. The surfaces of the sample and transducer were adjusted to a known relative inclination in three sequential scans (0,2 and 5 degrees) Surface reflection was evaluated in time and frequency domains and surface roughness in time domain as a function of the inclination. Inclination of the surface of the sample had a greater effect on the reflection than on the roughness value. Despite the inclination-induced error, the degraded sample could be distinguished from the intact one at all inclinations with both the reflection and roughness parameters. However, as the inclination affected the reflection parameters significantly more, their reliability at greater inclinations is questionable. Furthermore, the proportion of scattering vs. reflection may change as a function of inclination. Provided that the amount of scattering varies against cartilage surface fibrillation, this can affect the reliability of ultrasound diagnostics significantly.

2pBBf10. Acoustothermometrical control during hyperthemia of biological objects. Andrej Anosov (Institute for Radio-Engineering and Electronics of the RAS, Starosadskij per, 8, 101000 Moscow, Russian Federation, aanosov@atom.ru), Aleksandr Kazanskij (Institute for RadioEngineering and Electronics of the RAS, Starosadskij per, 8, 101000 Moscow, Russian Federation, kazansky@ihome.ru), Anton Sharakshane (Institute for Radio-Engineering and Electronics of the RAS, Starosadskij per, 8, 101000 Moscow, Russian Federation, iva@hotmail.ru)

A number of acoustothermometrical experiments modelling hyperthermia of biological objects was carried out. After that the first preliminary acoustothermometrical measurement of laser hyperthermia of human tissues was conducted. In all experiments the changes of the thermal acoustic radiation intensity in 1.4-2.2 $\mathrm{MHz}$ region were controlled during both the heating and the cooling of the object (about 8-10 minutes) with the help of set of acoustothermometers. As model objects we used the beef liver and glycerin water solution in which plasticine was placed. We reconstructed the 2-D inner temperature distribution by using acoustothermometrical experimental data, i.e. we calculated position, size and temperature of thermal source. The object surface temperature was controlled with the help of IRthermograph. Acoustothermometrical measurements were carried out during laser hyperthemia of mammary and thyroid glands for seven patients. Obtained data allowed us to estimate inner temperature changes which were a result of laser hyperthemia.

2pBBf11. Measurement of dynamic shear modulus in soft solids using laser vibrometry. Maria Y. Izosimova (Center for Industrial and Medical Ultrasound, Applied Physics Lab., University of Washington, 1013 NE 40th
St., Seattle, WA 98105, USA, maria.izossimova@mail.ru),Alexandr I. Korobov (Dept. of Acoustics, Physics Faculty, M.V. Lomonosov Moscow State University, Leninskie gory 1, 119991 Moscow, Russian Federation, akor@acs465a-1.phys.msu.ru), Ekaterina V. Prokhorova (Center for Industrial and Medical Ultrasound, Applied Physics Lab., University of Washington, 1013 NE 40th St., Seattle, WA 98105, USA, e-v-p@rambler.ru), Oleg A. Sapozhnikov (Center for Industrial and Medical Ultrasound, Applied Physics Lab., University of Washington, 1013 NE 40th St., Seattle, WA 98105, USA, oleg@acs366.phys.msu.ru)

Shear modulus is an important property of biological tissue and can be imaged for diagnostic purposes. A related application involves the acquisition of precise shear modulus measurements in excised soft tissue. Because the mechanical properties of tissue are frequency-dependent, it is important to develop methods that characterize the elasticity of soft solids at various frequencies. Here, two methods of measuring shear modulus are presented that employ laser vibrometry. At low frequencies $(1-10 \mathrm{~Hz})$, the tissue sample is fixed between plates so that the displacement of one plate induces a shear strain. Vibrometer measurements of the plate displacement define the induced strain so that shear modulus can be deduced if the applied shear force is known. At higher frequencies $(0.1-10 \mathrm{kHz})$, the sample lays flat on a surface and an impulsive force is applied to the exposed surface. Using the vibrometer to measure displacements along the exposed surface enables estimation of the surface wave speed and the implied shear modulus. To demonstrate the capabilities of these techniques, experiments were performed using plastisol tissue phantom samples. These experiments indicated a shear modulus that was $50 \%$ greater at high frequencies $(300 \mathrm{~Hz})$ than at low frequencies $(10 \mathrm{~Hz})$. Work supported by RFBR.

2pBBf12. Applying Genetic Algorithms to Optimise Breast Ultrasound Images Segmentation Procedure Based on Morphological Operators. Andre V. Alvarenga (Laboratory of Ultrasound - Inmetro, Av. N. Sra. das Gracas, 50, predio 1 - Xerem, 25250-020 Duque de Caxias - Rio de Janeiro, Brazil, avalvarenga@inmetro.gov.br), Wagner C. Pereira (Biomedical Engineering Program - COPPE, Federal University of Rio de Janeiro, CEP. 21 .941-972 Rio de Janeiro, Brazil, wagner@peb.ufrj.br), Antonio F. Infantosi (Biomedical Engineering Program - COPPE, Federal University of Rio de Janeiro, CEP. 21.941-972 Rio de Janeiro, Brazil, afci@peb.ufrj.br), Carolina M. Azevedo (Brazilian National Cancer Institute - INCa, Praça da Cruz Vermelha, 23, 20230-130 Rio de Janeiro, Brazil, azevedocma@bol.com.br)

Ultrasound (US) image segmentation is a complex problem due its textural nature. To address this difficulty, different segmentation procedures have been described to support radiologists in recognising abnormal USimage areas. This work proposes a procedure, based on Genetic Algorithms (GA) and Morphological Operators (MO), to segment breast tumours on ultrasound images. Each chromosome represents a complete image processing sequence, composed of MOs and structuring elements (SE). The GA generates 500 chromosomes of 93 genes, where the three first genes summed indicate the number of performed operations, the following 45 genes represent the operations' sequence, and the last 45, the SE-disk diameter (in pixel). The best segmentation sequence (best chromosome) is selected using an objective function, based on the weighted sum of the number of false-positive and false-negative pixels. The gold standards are the tumour contours depicted by an experienced radiologist. Twenty images are used to train the GA procedure, and the best chromosome tested with 40 different images. The performance of the procedure is assessed using the overlap ratio among the obtained segmentation and the gold standard. All tested images present an area overlap ratio superior to $70 \%$. This initial result encourages us to go further by increasing the number of images.

2pBBf13. Measuring physiological properties of lymphoedemous tissues by ultrasound: theoretical foundations. Paul E. Barbone (Boston University, 110 Cummington St, Boston, MA 02215, USA, 
barbone@bu.edu),Ricardo Leiderman(Federal University of Rio de Janeiro, Program of Mechanical Engineering, Ilha do Fundo, 21945-970, P.B. 68509 Rio de Janeiro, Brazil, leider@mecanica.coppe.ufrj.br), Jeff Bamber (Institute of Cancer Research, 15 Cotswold Road, Belmont, Sutton, SM2 5NG Surrey, UK, jeff.bamber@icr.ac.uk), Gearoid Berry (Institute of Cancer Research, 15 Cotswold Road, Belmont, Sutton, SM2 5NG Surrey, UK, gearoid.berry@icr.ac.uk), Assad A. Oberai (Rensselaer Polytechnic Institute, Mechanical, Aerospace and Nuclear Engineering, 5048 JEC, 110 8th Street, Troy, NY 12180, USA, oberaa@rpi.edu), Yixiao Zhang (Rensselaer Polytechnic Institute, Mechanical, Aerospace and Nuclear Engineering, 5048 JEC, 110 8th Street, Troy, NY 12180, USA, zhangy12@rpi.edu)

Roughly one in four breast cancer survivors report some degree of arm oedema. Lymphoedema is a build-up of excess lymph fluids in the tissues. Persistent lymphoedema leads to pain, diminished limb function, increased risk of infection, soft tissue fibrosis, and severe cases can be grossly disfiguring. From a mechanics perspective, the lymphoedemous tissue may be thought of as a two phase composite, consisting of both fluid and solid phases. Here we discuss the use of composites mixture theory to model the mechanics of lymphoedemous tissues. By treating the tissue as a fluid-solid composite, rules-of-mixtures may be used to estimate the effective moduli in terms of the properties of the individual components and their respective volume fractions in these two states. The mechanical properties of the tissue may be measured in vivo using a generalization of the methods of ultrasound elasticity imaging. We discuss how the measured "effective stiffness" depends upon whether the tissue is drained or undrained, and how ultrasound may be used to measure these properties. Thus we explore the possibility of evaluating volume fractions and component properties of the individual tissue phases from ultrasound elasticity imaging.
2pBBf14. Multilayered non-invasive temperature estimation from backscattered ultrasound. Cesar A. Teixeira (University of Algarve, Faculty of Sciences and Technology, Campus de Gambelas, 8005-139 Faro, Portugal, cteixei@gmail.com), Maria Graça Ruano (University of Algarve, Faculty of Sciences and Technology, Campus de Gambelas, 8005-139 Faro, Portugal, mruano@ualg.pt), António Ruano (University of Algarve, Faculty of Sciences and Technology, Campus de Gambelas, 8005-139 Faro, Portugal, aruano@ualg.pt), Wagner C. Pereira (Biomedical Engineering Program - COPPE, Federal University of Rio de Janeiro, CEP. 21.941-972 Rio de Janeiro, Brazil, wagner@peb.ufrj.br)

A major drawback on the application of thermal therapies is the lack of reliable knowledge about temperature in the region under treatment. The most attractive approach is non-invasive temperature estimation (NITE) strategies and, among them, techniques based on backscattered ultrasound (BSU) are a convenient choice for its portability and simplicity. Using BSU some methods have been proposed for temperature estimation basically for homogeneous media, and simple operating situations. More complex scenarios can be explored by non-linear approaches. In this work, a methodology based on neural networks is presented for temperature estimation in a multilayer phantom. A three-layer gel-based phantom was heated at eight intensities, between 0.30 and $2.0 \mathrm{~W} / \mathrm{cm}^{2}$ (at $1 \mathrm{MHz}$ ) for 15 minutes. Temperature and BSU signals were acquired from five spatial-points at each 10 seconds. Temporal echo-shifts, induced by temperature change, were computed from the BSU signals, and used as model's input information. Neural models were developed to estimate temperature at four intensities. Then models were validated in data including all the intensities. The best model presents a maximum absolute error of $0.43{ }^{\circ} \mathrm{C}$ for untrained situations. To the best of our knowledge this is the first NITE approach in multilayered media.

TUESDAY AFTERNOON, 1 JULY 2008

ROOM 353, 2:00 TO 7:00 P.M.

\title{
Session 2pEAa
}

\section{Engineering Acoustics: Silicon Microphones}

\author{
James West, Cochair \\ Johns Hopkins University, Department of Electrical Engineering, 3400 North Charles Street, Baltimore, MD 21218, USA \\ Gerhard Sessler, Cochair \\ Darmstadt University of Technology, Merckstrasse 25, 64283 Darmstadt, Germany
}

Invited Paper

2:00

2pEAa1. Overcoming Scaling Problems in Miniaturized Silicon Microphones. Sung B. Lee (Knowles Acoustics, 1151 Maplewood Dr, Itasca, IL 60143, USA, sung.lee@knowles.com), David E. Schafer (Knowles Acoustics, 1151 Maplewood Dr, Itasca, IL 60143, USA, dave.schafer@knowles.com), Peter V. Loeppert (Knowles Acoustics, 1151 Maplewood Dr, Itasca, IL 60143, USA, pete .loeppert@knowles.com)

In 2008 it is projected that global demand for mobile devices will reach 1.2 billion units. Compared to electronic gadgets from 2002 when 430 million cell phones were sold world wide, these devices are smaller, have more features, and will generally be priced lower. In order to keep up with designers and manufacturers who relentlessly pursue efficient use of space at lower cost, it is required that component manufacturers pursue the same goal. For a microphone manufacturer like Knowles, this led to the introduction of world's 
first commercialized surface-mountable microphone, the SiSonic ${ }^{\mathrm{TM}}$ microphone, in 2002. MEMS technology is used to manufacture the complex miniaturized components used in SiSonic ${ }^{\mathrm{TM}}$ microphone. Straightforward miniaturization of microphone diaphragms made of silicon, a common material in MEMS technology, instead of traditional diaphragm materials leads to poor performing microphones. To take advantage of MEMS technology without sacrificing microphone performance we developed the free plate technology. In this presentation we discuss the use of free plate technology to overcome difficulties related to miniaturization in MEMS.

\section{Contributed Paper}

\section{$2: 20$}

2pEAa2. Silicon microphones: a commercial success? and what comes next? Stephen C. Thompson (Applied Reearch Laboratory, The Pensylvania State University, P.O. Box 30, State College, PA 16804, USA, sct12 @psu.edu)

The first silicon microphone commercial product was introduced to the market in late 2005. As of early 2008 the cumulative production is well over a half billion units and the production rates continue to rise. To date the acoustical performance of these microphones is only as good as that of the of the lowest priced miniature electret microphones. Yet acoustical systems manufacturers often prefer silicon microphones because they are compatible with automated insertion and wave soldering assembly methods. Silicon microphones are also more environmentally stable in sensitivity than are electrets which may be important in matched microphone applications. Current research activities are investigating methods to reduce the internal noise improve matching tolerances, and further reduce manufacturing costs. This paper will review the current state of silicon microphones in commercial manufacture, and will survey some of the areas of current research in an attempt to suggest some possible directions of future market development.

\section{Invited Paper}

2pEAa3. Optimization of miniaturized silicon microphones using a two-wafer approach. Jianmin Miao (Nanyang Technological University, School of Mechanical and Aerospace Engineering, 50 Nanyang Avenue, 639798 Singapore, Singapore, mjmmiao@ntu .edu.sg), Chee Wee Tan (Nanyang Technological University, School of Mechanical and Aerospace Engineering, 50 Nanyang Avenue, 639798 Singapore, Singapore, cheewee@ntu.edu.sg), Zhihong Wang (Nanyang Technological University, School of Mechanical and Aerospace Engineering, 50 Nanyang Avenue, 639798 Singapore, Singapore, ezhwang@ntu.edu.sg)

A two-wafer concept is proposed for the fabrication of silicon microphones with emphasis on deep reactive ion etching and wafer bonding techniques. For miniaturized sensor structures with an air gap of 1-2 microns, the viscous damping effect dominates the dissipation mechanism, which can have an adverse influence on the microphone performance, namely frequency response characteristic and mechanical-thermal noise. Therefore, an optimum microphone performance has its origin in a well-designed backplate structure. A silicon backplate with carefully placed acoustic slots and holes is attached to a silicon nitride/metal-based diaphragm. An impediment to achieve high sensitivity is the residual stress that is presented in the diaphragms. Besides the process optimization of less stress silicon nitride layer and the introduction of corrugated diaphragm, an investigation is carried out to determine the effects of sputtering parameters of $\mathrm{Cr} / \mathrm{Au}$ metal electrode film (thickness, sputtering process pressure and process power) on the residual stress of silicon nitride/metal diaphragm. Details of modeling, fabrication and experimental results will be presented.

\section{Contributed Papers}

\section{3:00}

2pEAa4. Piezoelectric Cantilevers for Low-Noise Silicon Microphones. Robert Littrell (University of Michigan, 2250 G G Brown Bldg, 2350 Hayward St., Ann Arbor, MI 48109, USA, rlittrel@umich.edu), Karl Grosh (University of Michigan, 2250 G G Brown Bldg, 2350 Hayward St., Ann Arbor, MI 48109, USA, grosh@umich.edu)

Microphones fabricated using microelectromechanical systems (MEMS) technology are one of the fastest growing applications of MEMS. Capacitive sensing has been the dominant detection principle used in MEMS microphones. Piezoelectric sensing, however, offers advantages including simpler accompanying circuitry and the possibility for simpler fabrication. Piezoelectric microphones have been limited primarily by a high noise floor, typically at least an order of magnitude higher than, otherwise similar, capacitive microphones. We present a low noise piezoelectric cantilever microphone to overcome the main limitation of previously constructed piezoelectric microphones. Aluminum Nitride (AlN) has been selected as the piezoelectric material because its piezoelectric coupling coefficient, in combination with its electric permittivity, and its piezoelectric loss coefficient enable low-noise devices. Through both mechanical and electrical optimization, models indicate that by combining several short, thin cantilevers made exclusively of Molybdenum and AlN, microphones with a die size of $1 \mathrm{~mm}$ $\mathrm{x} 1 \mathrm{~mm}, 10 \mathrm{kHz}$ bandwidth, $2 \mathrm{mV} / \mathrm{Pa}$ sensitivity, and noise floor below 40 $\mathrm{dBA}$ can be constructed using a simple 4 mask process. Analytical and numerical models and experimental results will be presented.

\section{3:20}

2pEAa5. A low-noise biomimetic differential microphone. Ronald $\mathrm{N}$. Miles (State University of New York, PO 6000, Vestal Parkway East, Binghamton, NY 13902-6000, USA, miles@binghamton.edu), Quang T.
Su(State University of New York, PO 6000, Vestal Parkway East, Binghamton, NY 13902-6000, USA, be83190@binghamton.edu), Weili Cui (State University of New York, PO 6000, Vestal Parkway East, Binghamton, NY 13902-6000, USA, weilicui@yahoo.com), Stephen A. Jones (State University of New York, PO 6000, Vestal Parkway East, Binghamton, NY 139026000, USA, saj0716@gmail.com), F. Levent Degertekin (Georgia Institute of Technology, G. W. Woodruff School of Mechanical Engineering, 801 Ferst Dr. NW, Atlanta, GA 30332-0405, USA, levent.degertekin@me.gatech.edu), Baris Bicen (Georgia Institute of Technology, G. W. Woodruff School of Mechanical Engineering, 801 Ferst Dr. NW, Atlanta, GA 30332-0405, USA, baris@gatech.edu), Caesar Garcia (Georgia Institute of Technology, G. W. Woodruff School of Mechanical Engineering, 801 Ferst Dr. NW, Atlanta, GA 30332-0405, USA, caesar@gatech.edu), Neal A. Hall (Georgia Institute of Technology, G. W. Woodruff School of Mechanical Engineering, 801 Ferst Dr. NW, Atlanta, GA 30332-0405, USA, nahall@alumni.utexas.net)

A miniature differential microphone is described that has a noise floor that is substantially lower than that of existing devices of comparable size. The sensitivity of a differential microphone suffers as the distance between the two pressure sensing locations decreases, resulting in an increase in the input sound pressure-referred noise floor. In the microphone described here, the two sources of microphone internal noise, the diaphragm thermal noise and the electronic noise, are minimized by a combination of novel diaphragm design and the use of low-noise optical sensing. The differential microphone diaphragm measures $1 \mathrm{~mm}$ by $2 \mathrm{~mm}$ and is fabricated out of polycrystalline silicon. The diaphragm design is based on the coupled ears of the fly Ormia ochracea. The sound pressure input-referred noise floor of this miniature differential microphone has been measured to be less than 36 dBA. 


\section{Contributed Paper}

$5: 20$

2pEAa6. Thin film thermo-viscous damping in miniature condenser microphones. Thierry Le Van Suu (Laboratoire d'Acoustique de l'Université du Maine, Avenue Olivier Messiaen, 72085 Le Mans, France, thierry.levansuu@univ-lemans.fr), Petr Honzik (CVUT v Praze, Fakulta Elektrotechnická, K13137 Katedra Radioelektroniky - Technická 2, 16627 Praha 6, Czech Republic, Petr.Honzik@lf1.cuni.cz), Stephane Durand (Laboratoire d'Acoustique de l'Université du Maine, Avenue Olivier Messiaen, 72085 Le Mans, France, stephane.durand@univ-lemans.fr), Nicolas Joly (Laboratoire d'Acoustique de l'Université du Maine, Avenue Olivier Messiaen, 72085 Le Mans, France, nicolas.joly@univ-lemans.fr), Zdeněk Škvor (CVUT v Praze, Fakulta Elektrotechnická, K13137 Katedra Radioelektroniky - Technická 2, 16627 Praha 6, Czech Republic, skvorzdn@feld.cvut.cz), Michel Bruneau (Laboratoire d'Acoustique de l'Université du Maine, Avenue Olivier Messiaen, 72085 Le Mans, France, michel.bruneau@univ-lemans.fr)
The thermo-viscous damping due to the thin fluid film between the membrane and the backing electrode strongly influences both the sensitivity of the condenser microphones in the lower frequency range and the upper limit of the frequency bandwidth. Nowadays, most of the MEMs microphones use a perforated backing electrode while some authors suggest that a continuously curved backing electrode could enhance their performances (among avantages in the design when etching). The present paper provides two kinds of modeling for such microphones with a tapered fluid film: the first one lies on Kirchhoff-network analysis (neglecting cross-coupling between elements) whereas the second one is based upon the direct resolution of the set of basic equations (including heat transfer phenomena). The results are presented and discussed for both models in the cases of flat, parabolic, and stepped shapes backing electrode. Finally, the pressure field in the fluid film, computed (for an axisymmetrical configuration) using the abovementioned models, is compared to the one computed with a new FEM formulation taking into account both viscous and thermal phenomena in the boundary layers.

\section{Invited Paper}

$$
5: 40
$$

2pEAa7. Miniature diffraction-based optical MEMS microphones with integrated optoelectronics. Levent Degertekin (Georgia Institute of Technology, G. W. Woodruff School of Mechanical Engineering, 801 Ferst Dr. NW, Atlanta, GA 30332-0405, USA, levent@gatech.edu), Kamran Jeelani (Georgia Institute of Technology, G. W. Woodruff School of Mechanical Engineering, 801 Ferst Dr. NW, Atlanta, GA 30332-0405, USA, gtg804t@mail.gatech.edu), Shakeel Qureshi (Georgia Institute of Technology, G. W. Woodruff School of Mechanical Engineering, 801 Ferst Dr. NW, Atlanta, GA 30332-0405, USA, shakeel@ece.gatech.edu), Paul Hasler (Georgia Institute of Technology, School of Electrical and Cumputer Engineering, 777 Atlantic Drive NW, Atlanta, GA 30332-0250, USA, phasler@ece.gatech.edu), Baris Bicen (Georgia Institute of Technology, G. W. Woodruff School of Mechanical Engineering, 801 Ferst Dr. NW, Atlanta, GA 30332-0405, USA, baris@gatech.edu), Weili Cui (State University of New York, PO 6000, Vestal Parkway East, Binghamton, NY 13902-6000, USA, weilicui@yahoo.com), Quang T. Su (State University of New York, PO 6000, Vestal Parkway East, Binghamton, NY 13902-6000, USA, be83190@binghamton.edu), Ronald N. Miles (State University of New York, PO 6000, Vestal Parkway East, Binghamton, NY 13902-6000, USA, miles@ binghamton.edu)

Diffraction-based optical interferometric sensing has been shown to be a low-noise displacement detection method for MEMS microphones. For this method to be useful in many important applications such as hearing aids, it needs to be packaged in a small volume and should have power consumption levels suitable for operation with a battery. In this talk, we describe miniature, packaged optical microphones that use solid state vertical cavity surface emitting lasers (VCSELs) as light sources and custom designed photodetectors. The package carries silicon chips with two micromachined biomimetic differential microphones as well as an omnidirectional microphone. It is made by 3-D laser stereo lithography process and has dimensions suitable for behind-the-ear hearing aids. With this configuration, the input referred noise floor for the differential microphone is measured as 42.6dBA, limited by the VCSEL intensity noise in this particular case. In addition to miniature packaging, an optoelectronic chip including VCSEL pulser, photodetectors, transimpedance amplifiers and 1 bit Sigma-Delta ADC has been implemented in 1.5V, 0.35u CMOS technology. The second order ADC structure providing 14-bits of theoretical resolution with 64 over sampling ratio and $20 \mathrm{kHz}$ input signal is described and initial characterization results are presented.

\section{Contributed Papers}

\section{6:00}

2pEAa8. On determination of microphone response and other parameters by a hybrid experimental and numerical method. Salvador Barrera-Figueroa (Danish Fundamental Metrology, Matematiktorvet 307, 2800 Kgs. Lyngby, Denmark, sbf@dfm.dtu.dk), Finn Jacobsen (Acoustic Technology Department, Technical University of Denmark, Ørsted Plads, B352, DK-2800 Lyngby, Denmark, fja@oersted.dtu.dk), Knud Rasmussen (Danish Fundamental Metrology, Matematiktorvet 307, 2800 Kgs. Lyngby, Denmark, kra@dfm.dtu.dk)

Typically, numerical calculations of the pressure, free-field and randomincidence response of a condenser microphone are carried out on the basis of an assumed displacement distribution of the diaphragm of the microphone; the conventional assumption is that the displacement follows a Bessel function. This assumption is probably valid at frequencies below the resonance frequency. However, at higher frequencies the movement of the membrane is heavily coupled with the damping of the air film between membrane and back plate, and with resonances in the back chamber of the microphone. A solution to this problem is to measure the velocity distribution of the membrane by means of a non-contact method, such as laser vibrometry. The measured velocity distributions can be used together with a numerical formulation such as the Boundary Element Method for estimating the microphone response and other parameters such as the acoustic centres. In this work, a hybrid method is presented. The velocity distributions of condenser Laboratory Standard microphones were measured using a laser vibrometer. This measured velocity distribution was used for estimating the microphone responses and parameters. The agreement with experimental data is good. This method can be used as an alternative for validating the parameters of the microphones determined by classical calibration techniques. 


\section{6:20}

2pEAa9. Diffraction based optical MEMS microphones and accelerometers with active electrostatic force feedback. Baris Bicen (Georgia Institute of Technology, G. W. Woodruff School of Mechanical Engineering, 801 Ferst Dr. NW, Atlanta, GA 30332-0405, USA, baris@gatech.edu), Caesar Garcia (Georgia Institute of Technology, G. W. Woodruff School of Mechanical Engineering, 801 Ferst Dr. NW, Atlanta, GA 30332-0405, USA, caesar@gatech.edu), Neal A. Hall (Georgia Institute of Technology, G. W. Woodruff School of Mechanical Engineering, 801 Ferst Dr. NW, Atlanta, GA 30332-0405, USA, nahall@alumni .utexas.net), Murat Okandan (Sandia National Laboratories, New Mexico, PO Box 5800, Albuquerque, NM 87185-1080, USA, mokanda@sandia .gov), Weili Cui (State University of New York, PO 6000, Vestal Parkway East, Binghamton, NY 13902-6000, USA, weilicui@yahoo.com), Quang T. Su (State University of New York, PO 6000, Vestal Parkway East, Binghamton, NY 13902-6000, USA, be83190@binghamton.edu), Ronald N. Miles (State University of New York, PO 6000, Vestal Parkway East, Binghamton, NY 13902-6000, USA, miles@binghamton.edu), Levent Degertekin (Georgia Institute of Technology, G. W. Woodruff School of
Mechanical Engineering, 801 Ferst Dr. NW, Atlanta, GA 30332-0405, USA, levent@gatech.edu)

Diffraction-based optical displacement detection method and its use in low noise micromachined microphones have been shown earlier. [Hall et al., J. Acoust. Soc. Am. 118, 3000-3009 (2005), Garcia et al., J. Acoust. Soc. Am. 121, 3155 (2007)]. In these devices, the integrated electrostatic port of the sensor is uncoupled from the integrated optical sensing. This structure enables one to use this port for sensitivity tuning, self characterization, and active control to adjust the device dynamics. Given that the displacement noise of integrated optical sensor is below the thermal-mechanical noise of the mechanical structure, one can implement force feedback methods such as active Q-control, or adjust device stiffness without adding substantial noise to the system. We implemented micromachined optical microphones and accelerometers with integrated optoelectronics integrated in a $1.5 \mathrm{~mm} 3$ volume. We present experimental results on force feedback Q-control of low noise omnidirectional, and biomimetic directional optical microphones, as well as adjusting the stiffness of accelerometers to improve their frequency response. [Work supported by NIH Grant 5R01DC005762-03 and the Catalyst Foundation.]

\section{Invited Paper}

\section{6:40}

2pEAa10. Piezoelectric silicon microphones for aeroacoustics applications. Mark Sheplak (University of Florida, 231 MAE-A Building, PO Box 116250, Gainesville, FL 32611-6250, USA, sheplak@ufl.edu)

This talk presents the development of several microelectromechanical systems (MEMS)-based piezoelectric measurement microphone technologies for aeroacoustic applications. Piezoelectric MEMS microphones offer the promise of reducing cost, improving performance, and increasing mounting flexibility over existing conventional microphone technologies. Specifically, a microphone with no external power requirement has a key advantage for a large-channel count, widespread deployment. The modeling and design aspects of these devices are reviewed. First, the electroacoustic transduction is predicted via piezoelectric composite plate theory. Lumped element models are then synthesized to describe the dynamic characteristics of the microphone diaphragm and the cavity/vent structure. Constrained nonlinear design optimization using a sequential quadratic programming scheme is then performed to determine the microphone design parameters. From a fabrication perspective, the main drawback to silicon-micromachined piezoelectric devices is material integration and compatibility with standard micromachining. Several different device structures and fabrication approaches will be discussed in this talk. Representative results from a lead zirconate-titanate-based device will then be presented. Finally, unresolved technical issues are summarized for future sensor development. 


\title{
Session 2pEAb
}

\section{Engineering Acoustics and Signal Processing in Acoustics: Microphone Array Signal Processing II (Poster Session)}

\author{
Gary Elko, Cochair \\ mh acoustics LLC \\ Walter Kellermann, Cochair \\ Univ. of Erlangen-Nuremburg
}

\begin{abstract}
All posters will be on display from 3:40 p.m. to 5:20 p.m. To allow contributors an opportunity to see other posters, contributors of odd-numbered papers will be at their posters from 3:40 p.m. to 4:30 p.m. and contributors of even-numbered papers will be at their posters from 4:30 p.m. to 5:20 p.m.
\end{abstract}

\section{Contributed Papers}

2pEAb1. Advanced Processing of Microphone Array Data for Engineering Applications. Sandro Guidati (HEAD acoustics $\mathrm{GmbH}$, Ebertstrasse 30a, 52134 Herzogenrath, Germany, claudia.erens@headacousitcs.de)

Microphone arrays are tools for the localisation and quantification of sound sources. The use of the technology is strongly related to the progresses in computer technology. For a long time the application was limited to military or scientific use. The focus of the systems designed for industrial applications lies on fast setup and basic evaluation (e.g. delay-andsum beamforming). The resulting boundaries are mainly a limited dynamic range and the limitation to free field environments. Although a wide range of algorithms for advanced evaluation has been developed, only few of them are integrated in industrial systems. This is mainly due to the fact that these algorithms require considerably longer computation time, expert knowledge and the integration of additional hardware. This paper presents techniques for almost real-time processing of microphone array data including multiband beamforming, coherence/incoherence filtering and the integration of measured source characteristics for the application in strongly reverberant environments. By combining the signals of multiple cameras it is possible to detect the distance between the array and a three dimensional source distribution increasing the accuracy of the localisation and quantification.

2pEAb2. Instrumentation Synchonization Techniques for Large Microphone Arrays. Kurt Veggeberg (National Instruments, $11500 \mathrm{~N}$. Mopac C, Austin, TX 78759, USA, kurt.veggeberg@ni.com)

In many acoustic measurement applications, there is a need to correlate data acquired from different systems or synchronize systems together with precise timing. Signal Based and Time Based are the two basic methods of synchronizing instrumentation. In Signal Based synchronization, clocks and triggers are physically connected between systems. Typically this provides the highest precision synchronization. In many acoustic applications size and distance constrains physically connecting the systems needed for making measurements though the inter-channel phase information of simultaneously sampled signals is crucial. In Time Based synchronization, system components have a common reference of what time it is. Events, triggers and clocks can be generated based on this time. This is an overview of how you can use a variety of time references including GPS, IEEE-1588, and IRIG-B to correlate and synchronize measurements anywhere in the world with absolute timing with and without a direct connection between the measurement systems. The level of precision of the variety of methods that can be used for timestamping, generating a trigger at a user specified time as well as synchronizing multiple instrumentation types is covered. Specific applications where this has been applied to large microphone arrays will be described.

2pEAb3. Differential microphone array for speech recognition. Philippe Martin (Ecole Polytechnique Fédérale de Lausanne, EPFL STI LEMA, Station 11, CH 1015 Lausanne, Switzerland, philippe.martin@epfl.ch), Hervé Lissek (Ecole Polytechnique Fédérale de Lausanne, EPFL STI LEMA, Station 11, CH 1015 Lausanne, Switzerland, herve.lissek@epfl.ch), John Dines (IDIAP, Rue du Simplon 4, 1920 Martigny, Switzerland, john.dines@idiap.ch)

Speech recognition applications embedded on a PDA are already available on the market. The usual hardware for this kind of system is a single microphone mounted on the PDA, giving good results within quiet environments. Though, the recognition rate falls drastically as the signal to noise ratio decreases. Arrays of microphones are then particularly interesting, allowing the discrimination of useful sounds sources within parasitic ones, thanks to an improved directivity pattern. In speech recognition applications on PDA, a trade-off is to be found on an array small enough to guarantee the ergonomics of the whole system, while operating on a bandwidth covering the frequency range of human voice, from $300 \mathrm{~Hz}$ up to $6 \mathrm{kHz}$ and low distortion ratio. Differential arrays are well adapted to fulfill these specifications, since they are known to be robust and allow small dimensions for a high directivity index. The performances could be improved by the addition of adaptive post-filtering and noise reduction algorithms. This work describes the design and the implementation of a recording device dedicated to speech recognition applications on PDA, based on overlapping differential arrays. The assessment of its performances in a noisy environment is carried out and show the system efficiencies.

2pEAb4. Speech separation based on law of causality. Kensaku Fujii (University of Hyogo, 2167 Shosha, 671-2280 Himeji, Japan, fujiken@eng.u-hyogo.ac.jp), Hirofumi Nakano (University of Hyogo, 2167 Shosha, 671-2280 Himeji, Japan, er06j025@steng.u-hyogo.ac.jp), Mitsuji Muneyasu (Kansai University, 3-3-35 Yamate-cho, 564-8680 Suita, Japan, muneyasu@ipcku.kansai-u.ac.jp)

This paper proposes a microphone array system separating speech signals, based on a different principle from independent component analysis (ICA). This system applies linear prediction error filters to microphone outputs, and using the prediction errors, adjusts the coefficients of adaptive filters. In this case, only the prediction errors satisfying the low of causality become available for the adjustment; consequently, this system can steer a null toward a direction satisfying it. The permutation problem discussed in 
ICA can be thereby avoided. This system also can compensate the separated speech signals by using adaptive filters, and finally, can provide high quality speech signals. This paper moreover verifies the performance of the proposed system by using speech signal data measured in an ordinary room. This result shows that the proposed system works well even in reverberation environment.

2pEAb5. Time-Shift Sensor Noise Suppression Algorithm. Alain De Cheveigne (CNRS, Universite Paris 5, Ecole Normale Superieure, 29 rue d’Ulm, 75230 Paris, France, alain.de.cheveigne@ens.fr)

We propose a new method to remove sensor noise from multichannel data recordings. Each channel is projected on the span of the time-shifted neighboring channels, and replaced by its projection. Noise specific to any sensor is attenuated, whereas any signal component that loads more than one sensor is preserved. The inclusion of a range of time shifts allows the method to compensate for any delay or convolutional mismatch in the signal paths between sensors. The method is expected to be of use for microphone arrays to reduce sensor-specific noise, e.g. thermal noise, wind noise, or overloading. The new method extends a previous method for sensor noise suppression in physiological recordings (EEG, MEG), that performed the same operation without the delays.
2pEAb6. Acoustic payload on an aerostat detects and locates transient sources. Michael V. Scanlon (US Army Research Laboratory, AMSRDARL-SE-SA, 2800 Powder Mill Road, Adelphi, MD 20783-1197, USA, mscanlon@arl.army.mil), Christian Reiff (US Army Research Laboratory, AMSRD-ARL-SE-SA, 2800 Powder Mill Road, Adelphi, MD 20783-1197, USA, creiff@arl.army.mil), Latasha Solomon (US Army Research Laboratory, AMSRD-ARL-SE-SA, 2800 Powder Mill Road, Adelphi, MD 207831197, USA, 1solomon@ arl.army.mil)

The US Army Research Laboratory (ARL) has conducted experiments using acoustic sensor arrays suspended below tethered aerostats to detect and localize transient signals from mortars, artillery, and small arms fire. The airborne acoustic sensor array calculates an azimuth and elevation to the originating transient, and immediately cues a collocated imager to capture the remaining activity at the site of the acoustic transient. This single array's vector solution defines a ground-intersect region or grid coordinate for threat reporting. Unattended ground sensor (UGS) systems can augment aerostat arrays by providing additional solution vectors from several ground-based acoustic arrays to perform a 3D triangulation on a source location. The aerostat array's advantage over ground systems is that it is not as affected by diffraction and reflection from man-made structures, trees, or terrain, and has direct line-of-sight to most events. Helicopter detection and tracking algorithms can also be implemented.

TUESDAY AFTERNOON, 1 JULY 2008

P3-C, LEVEL 3, 3:40 TO 5:20 P.M.

\title{
Session 2pEAc
}

\section{Engineering Acoustics: Acoustic Evaluation II (Poster Session)}

\begin{abstract}
All posters will be on display from 3:40 p.m. to 5:20 p.m. To allow contributors an opportunity to see other posters, contributors of odd-numbered papers will be at their posters from 3:40 p.m. to 4:30 p.m. and contributors of even-numbered papers will be at their posters from 4:30 p.m. to 5:20 p.m.
\end{abstract}

\section{Contributed Papers}

2pEAc1. Mechanism detection of stress corrosion cracking by acoustic emission and effect of manufacturing process on AE signals. Hossein Heidary (Polytechnic of Tehran, AmirKabir University of Technology, Department of Mechanical Engineering, NDT Lab., 424 Hafez Ave, 158754413 Tehran, Iran, hosseinheidary@gmail.com), Amir Refahi Oskouei (Polytechnic of Tehran, AmirKabir University of Technology, Department of Mechanical Engineering, NDT Lab., 424 Hafez Ave, 15875-4413 Tehran, Iran, amir.refahi@cic.aut.ac.ir), Mehdi Ahmadi (Polytechnic of Tehran, AmirKabir University of Technology, Department of Mechanical Engineering, NDT Lab., 424 Hafez Ave, 15875-4413 Tehran, Iran, ahmadin@cic.aut ac.ir)

Stress corrosion cracking could occur wherever a specific corrodent and sufficient tensile stresses coexist. In the objective to monitor online the SCC on real structures, it seems reasonable to characterize and recognize acoustic emission during static U-bend tests. The present study is concerned with static tests on 304 stainless steels in two different media $(5 \% \mathrm{HCl}$ and $5 \%$ $\mathrm{H} 2 \mathrm{SO} 4)$ in order to find a criterion to distinguish, the two different mechanism of SCC (anodic dissolution and hydrogen embrittlement) by characteristic parameters and waveform analysis of AE signals. In the next stage, effect of residual stress on stress corrosion cracking in chloride solution has been studied. Three samples have been prepared: one of them was stress relieved; the second one was made sensitive to SCC by heat treatment and the last one was cold worked by rolling. Cumulative count, amplitude distribution and waveform analysis were selected as AE-parameters. AE with amplitudes ranging from 39 to $65 \mathrm{~dB}$ with different counts and energy occurred during SCC of SS-304 at room temperature. According to the results, acoustic emission is able to be used as robust technique for mechanism detection of SCC. In addition it can be used to measure the effect of residual stress in manufacturing process on SCC.

2pEAc2. Underground Sonar Using Shear Waves -Resolution improvement Using Pulse Compression and Dynamic Focusing-. Hiraku Kawasaki (Toin University of Yokohama, 1614 Kurogane-cho, Aoba-ku, 225-8502 Yokohama, Japan, hot.hu2.feel.good@gmail .com), Tsuneyoshi Sugimoto (Toin University of Yokohama, 1614 Kurogane-cho, Aoba-ku, 225-8502 Yokohama, Japan, tsugimot@cc.toin.ac .jp)

In order to detect the buried relics and ruins at shallow depth, a method by using shear waves has proposed. The effect of the pulse compression processing is examined for improving the underground imaging resolution. Using a super-magnetostriction vibrator which can change waveforms and use high frequency waves, we generate both chirp waves and high frequency waves. To confirm the resolution of underground images, pulse compression is simulated. And, to confirm the lateral resolution, dynamic focusing is simulated in our laboratory. Then, its exploration experiment is carried out where the buried position has already known. The axial resolutions are smaller than $0.2 \mathrm{~m}$ when the range of start and stop frequencies are more 
than $700 \mathrm{~Hz}$ in the simulation. And the underground images obtained from exploration experiment confirm that the images by the pulse compression have almost the same axial resolution as by the result of the simulation.

2pEAc3. PVB mechanical constants characterization in laminated glasses using low frequency ultrasound. Jaime Ramis Soriano (DFISTS Univ. de Alicante, Carretera de Sant Vicent del Raspeig s/n, 03690 San Vicente del Raspeig, Spain, jramis@ua.es), Jorge Frances Monllor (DFISTS. Univ. de Alicante, Carretera de Sant Vicent del Raspeig s/n, 03690 San Vicente del Raspeig, Spain, jfmonllor@ua.es), Jesús Alba Fernandez (Escola Politècnica Superior de Gandia, Universitat Politècnica de València, Crtra Natzaret-Oliva s/n, 46730 Gandia, Spain, jesalba@fis.upv.es), Enrique E. Segovia Eulogio (Depto Ing. Construcción. Univ. de Alicante, Carretera de Sant Vicent del Raspeig s/n, 03690 San Vicente del Raspeig, Spain, Enrique.Gonzalo@ua.es), Jenaro Vera Guarinos (DFISTS. Univ. de Alicante, Carretera de Sant Vicent del Raspeig s/n, 03690 San Vicente del Raspeig, Spain, jenaro@dfists.ua.es)

To predict the behaviour of a partition made of laminated glasses, it's necessary to know the parameters of the layers composing it. These types of devices are composed by monolithic glass and an intermediate muffling layer usually made of PVB. In this study, we present a method to find the mechanical constants of PVB in laminated glasses. Mechanical constants characterization in these materials is studied using a model-based inverse problem and the data obtained from an ultrasonic transmission setup: a numerical simulation of the system is proposed using a lineal finite elements model of the ultrasonic propagation on the multilayered solid. Parameters are obtained by minimizing divergences between experimental and numerically predicted waveform.

2pEAc4. Ultrasonic Technique for the Quality Control of Water Containing Clay. Adil Hamine (Ibn Zohr University, FS Agadir, 80000 Agadir, Morocco, adil.hamine@Gmail.com), Bouazza Faiz (Ibn Zohr University, FS Agadir, 80000 Agadir, Morocco, faizbou@hotmail.com), Driss Izbaim (Ibn Zohr University, FS Agadir, 80000 Agadir, Morocco,
driss_izbaim@yahoo.fr),Ali Moudden(Ibn Zohr University, FS Agadir, 80000 Agadir, Morocco, ali_moudden@yahoo.fr)

In this work a new method is developed to control the stopping water using an ultrasonic technique. This technique allows controlling the presence of clay grains by measuring the attenuation of the ultrasonic waves. The dimension of clay grains used in this study is about $10 \mu \mathrm{m}$. For slurries with different weight percent of clay $1 \%$ or less, high sensitivity is gained by analyzing attenuation measurements. At high clay concentrations, sufficient sensitivity is obtained by analyzing data from a simple transmission. The experimental results show that the sound attenuation due to particles varies linearly with mass fraction.

2pEAc5. Acoustic Computerized Tomography for Temperature Distribution Measurement in Rectangular Space. Ayumu Minamide (Grad. Sch. of Sys. Inf. Eng., Univ. of Tsukuba, 1-1-1, Tennodai, 305-8573 Tsukuba, Japan, minamide@aclab.esys.tsukuba.ac.jp), Naoto Wakatsuki (Tsukuba Univ., Tsukuba Science City, 305-8573 Ibaraki, Japan, wakatuki@iit.tsukuba.ac.jp), Koichi Mizutani (Tsukuba Univ., Tsukuba Science City, 305-8573 Ibaraki, Japan, mizutani@esys.tsukuba.ac.jp)

In this research, we propose an improved algorithm of Acoustic Computerized Tomography to measure temperature distribution in rectangular space. Temperature distribution measurement is used in various fields, such as, air-conditioning control in offices, temperature control in greenhouse. If acoustic transducers are fixed, they are ordinarily arranged at equal intervals on a circle. However, it is difficult to take circular arrangements of transducers in the rectangular spaces. Thus, we devise to arrange the transducers on a rectangle. Therefore, a problem is occurs that projection data could not be obtained at equal angular intervals. To solve this problem, we propose non-uniform angular interval Back-Projection to weight the projection data with respect to its angular intervals. We confirm usefulness of the proposed method by numerical simulations. In the simulations, 24-acoustic transducers are arranged at equal intervals on a rectangle whose aspect ratio is two. By using the proposed method, the shape approaches to the given temperature distribution, and RMS error of the reconstructed distribution to the given distribution decreases about $25 \%$ compared to the error of conventional CT. 


\title{
Session 2pEAe
}

\section{Engineering Acoustics, Underwater Acoustics, Signal Processing in Acoustics, and ECUA: Sensor Technologies for Autonomous Acoustic Sensing Systems II (Poster Session)}

\author{
Henrik Schmidt, Cochair \\ MIT \\ Andrea Caiti, Cochair \\ University of Genova
}

\begin{abstract}
All posters will be on display from 3:40 p.m. to 5:20 p.m. To allow contributors an opportunity to see other posters, contributors of odd-numbered papers will be at their posters from 3:40 p.m. to 4:30 p.m. and contributors of even-numbered papers will be at their posters from 4:30 p.m. to 5:20 p.m.
\end{abstract}

\section{Contributed Papers}

2pEAe1. High Accuracy Multisensor Hydroacoustic System for Models of Trawl Geometry Measurements. Jacek Marszal (Gdansk University of Technology, Narutowicza 11/12, 80-952 Gdansk, Poland, marszal@eti.pg .gda.pl)

The new high accuracy multisensor hydroacoustic system for models of trawl geometry measurements was designed for developing the construction of cod trawl net for the Baltic fishery. The system consist of 20 miniature hydrophones attached by the light-gauge cables to the measuring microprocessor device and notebook computer. The pulse excitation at high frequency and correlation digital signal processing make it possible to obtain high resolution of measurements. Additionally each of hydrophones is equipped with the thermistor which is used to calculate the local sound velocity and enable precision calibration of obtained measururing data. This method of calibration is particularly important for measurements investigated in the surface water layer with the high temperature gradient. The construction details, the measuring signal processing algorithms as well as examples of obtained data and the accuracy verification will be presented in the paper.

2pEAe2. Ultra-deep low-frequency sub-bottom profiler for AUV and ROV. Yves Le Gall (IFREMER, BP 70, 29280 Plouzané, France, Yves.Le.Gall@ifremer.fr), Anne Pacault (IFREMER, BP 70, 29280
Plouzané, France, Anne.Pacault@ifremer.fr),Frédéric Mosca(Ixsea, 46 Quai François Mitterrand, 13600 La Ciotat, France, fmo@ixsea.com), Gilles Greninguey (Ixsea, 46 Quai François Mitterrand, 13600 La Ciotat, France, ggr@ixsea.com), Marcel Vial (Ixsea, 46 Quai François Mitterrand, 13600 La Ciotat, France, mvi@ixsea.com), Jean-Claude Aissa (Ixsea, 46 Quai François Mitterrand, 13600 La Ciotat, France, jca@ixsea.com)

Acoustical techniques for ultra-deep sub-bottom exploration are still little investigated. IXSEA and IFREMER have launched in 2006 the development of a new deep-sea low-frequency sub-bottom profiler, working up to 6000 meters depth. Based on the Janus-Helmholtz broadband technology, two acoustic sources have been modelled, achieved and in-tank and at-sea measured : a single transducer working in the $[1.8,6.2 \mathrm{kHz}]$ frequency band, and a three-transducer array operating in the $[2,8 \mathrm{kHz}]$ frequency band. In both cases, vertical resolution is better that $20 \mathrm{~cm}$. Comparison between finite element modelling and in-tank measurements is presented, together with the specific constraints linked to very-deep water and AUV mounting. Power electronics and impedance matching unit have been specifically optimised to deliver a sound level of $190 \mathrm{~dB}$ (ref. $1 \mu \mathrm{Pa} @ 1 \mathrm{~m}$ ), with $48 \mathrm{VDC}$ supply voltage and $250 \mathrm{~W}$ available electric power. The receiving part is made up of a three-hydrophone array with a loss of sensitivity lower than 1 $\mathrm{dB}$ between 0 and 600 bar. This ultra-deep light system has been mounted on an IFREMER's AUV and the first results at sea are presented and discussed. 


\title{
Session 2pEAf
}

\section{Engineering Acoustics and Psychological and Physiological Acoustics: Hearing Aid Engineering II (Poster Session)}

\author{
Daniel Warren, Cochair \\ Knowles Electronics \\ Stefan Launer, Cochair \\ Phonak $A G$
}

\begin{abstract}
All posters will be on display from 3:40 p.m. to 5:20 p.m. To allow contributors an opportunity to see other posters, contributors of odd-numbered papers will be at their posters from 3:40 p.m. to 4:30 p.m. and contributors of even-numbered papers will be at their posters from 4:30 p.m. to 5:20 p.m.
\end{abstract}

\section{Contributed Papers}

2pEAf1. Two-input two-output speech enhancement with binaural spatial information using a soft decision mask filter. Satoshi Hongo (Faculty of Design and Computer Applications, Miyagi National College of Technology, 48, Nodayama, Medeshima Shiote, 981-1239 Natori, Japan, hongo@miyagi-ct.ac.jp), Ai Sasaki (R.I.E.C., Tohoku University, 2-1, Katahira, Aoba-ku, 980-8577 Sendai, Japan, asasaki@ais.riec.tohoku.ac.jp), Shuichi Sakamoto (R.I.E.C., Tohoku University, 2-1, Katahira, Aoba-ku, 980-8577 Sendai, Japan, saka@ais.riec.tohoku.ac.jp), Junfeng Li (Japan Advanced Institute of Science and Technology, 1-1, Asahidai, Nomi, 923-1292 Ishikawa, Japan, junfeng@ jaist.ac.jp), Yôiti Suzuki (R.I.E.C., Tohoku University, 2-1, Katahira, Aoba-ku, 980-8577 Sendai, Japan, yoh@ais.riec.tohoku.ac.jp)

A two-input two-output speech enhancement method that preserves binaural spatial information in the output is effective for realizing a comfortable auditory communication system. Such a system benefits from the noise reduction capability provided by the signal processing technology, in addition to the binaural processing of the human auditory system. We investigated a two-input two-output speech enhancement method that calculates soft decision mask filters to attenuate a noisy time-frequency bin. The soft decision mask filter is estimated for each direction of arrival (DOA) based on a target noise ratio calculated using an adaptive filter that cancels the target signal. Results of computer simulations show that the proposed method has superior capabilities for maintaining spatial information in the two-output signals and for segregating the target signal in arbitrary azimuth and elevation DOA.

2pEAf2. Improvement of speech intelligibility by audio hearing systems. Hannes Seidler (Clinic of ORL, Dept. of Medicine, TU Dresden, Fetscherstrasse 74, 01307 Dresden, Germany, Hannes.Seidler@tu-dresden .de)

All hearing aids and cochlea implants have algorithm to improve the speech intelligibility. The idea is to share human speech and noise to find different ratings. The result should be easier to understand by impaired people. On the market there are big efforts to recognize speech and to separate it from noise. In this field we can notice a remarkable progress in the last years. But the reverberant sound in rooms or noise from same direction like the signal need a lot of work to suppress them effective. The simplest way seems to be to gets the original speech signal direct from the source and to processes it individual in hearing devices. Induction loop systems as well as wireless infrared or radio frequency systems are used in churches, cinemas, theatres and conference rooms. But the available systems are very different in costs and benefit for management and clients. If these systems are used there is a considerable improvement of speech intelligibility. The presentation will show the level of most used external audio hearing systems, their possibilities to improve the signal to noise ratio, the speech transmission index (STI) and the benefit for hearing aid or cochlear implant users. 


\title{
Session 2pEAg
}

\section{Engineering Acoustics, Underwater Acoustics, and ECUA: Sonar Transducer Design and Modeling III (Poster Session)}

\author{
John Blottman, Cochair \\ Naval Undersea Warfare Center, Division Newport \\ Pascal Mosbah, Cochair \\ IEMN dpt ISEN
}

\begin{abstract}
All posters will be on display from 3:40 p.m. to 5:20 p.m. To allow contributors an opportunity to see other posters, contributors of odd-numbered papers will be at their posters from 3:40 p.m. to 4:30 p.m. and contributors of even-numbered papers will be at their posters from 4:30 p.m. to 5:20 p.m.
\end{abstract}

\section{Contributed Papers}

2pEAg1. Finite element and boundary element modeling of multimodal arrays. Julien Bernard (Thales Underwater Systems, 525 route des Dolines BP 157 Valbonne Parc d'Activités de Sophia Antipolis, 06903 Sophia Antipolis Cedex, France, julien.bernard@fr.thalesgroup.com)

Modern active sonar arrays exhibit the following characteristics: low operating frequency, large number of transducers, small transducer spacing compared to wavelength, and in some cases, multimodal transducers. This results in strong, complex acoustic interaction phenomena which can involve both a large number of array elements and multiple transducer modes. To predict the performance of such arrays, this paper presents a modeling approach which is both accurate and computationally economical. First, the generic equivalent circuit for an array of multimodal transducers is described. The field of application of such a model, the definition of the parameters, and the characteristics which can be calculated from it are provided. Second, the computation of the equivalent circuit parameters is performed using commercial finite element and boundary element codes. Specifically, a method is described to compute the mechanical, electrical, self and mutual radiation parameters of the various transducer modes. Finally, the methodology is applied to a 12 element volumetric array of flextensional transducers, with both $\lambda / 2$ and $\lambda / 4$ spacings.

2pEAg2. The design of a wideband and widebeam piston transducer in a finite closed circular baffle. Zekeriyya Sahin (Aselsan A.S., Mehmet Akif Ersoy Mah. 16.Cad. no:16, Macunkoy, 06370 Ankara, Turkey, zsahin@aselsan.com.tr), Hayrettin Koymen (Bilkent Univ., Dept. of Electrical and Electronics Engineering, 06800 Ankara, Turkey, koymen@ee .bilkent.edu.tr)

The design of a high power piezoelectric underwater transducer operating at frequency range $45 \mathrm{kHz}-85 \mathrm{kHz}$ with acoustic power capability in excess of $150 \mathrm{~W}$ is described. The transducer consists of two back-to-back elements. Each element is formed by stacked PZT-4 ceramic rings, a matching and a steel backing layer, and placed in a finite rigid circular baffle. We investigate the dependence of bandwidth and beamwidth to the combination of piston and baffle radii, a and b, respectively. With a ka of 2.45 (k:wave number) and b/a ratio of 2 , the transducer resonates at $65 \mathrm{kHz}$ with $70 \%$ bandwidth and has a beamwidth of $60^{\circ}$ at each half space. We show that when two transducers are placed at right angles spatially and driven in parallel, we can obtain an omnidirectional beam pattern horizontally in the lower frequency band. The beam pattern exhibits two dips in each quadrant at the higher end of the frequency band, which are within $7 \mathrm{~dB}$. We also investigated power handling capability of the transducer from thermal point of view using finite element analysis. The input impedance measurements agree well with the numerical results within the pass band.

2pEAg3. A lossy, one-dimensional, linear systems model for piezoelectric transducers containing opposing zones of polarization. Sivaram Nishal Ramadas (Institute of Sound and Vibration Research, University of Southampton, University Road, Highfield, S017 1BJ Southampton, UK,nishal@ieee.org)

Inversion layer transducers (ILT), consisting of two or more active, piezoelectrically opposed regions along their thickness direction, have attracted considerable interest in the recent past, due to the potential for wide bandwidth and even harmonic sensitivity. However, design and manufacture of such ILT devices are not straightforward due to the large number of degree of freedom involved. This paper presents the development of a lossy, computationally efficient analytical model to design ILT configuration. The mathematical formulation for a generalized linear systems model, applicable to arbitrary ILT configurations is presented. The model describes multiple active and passive layers, including zones of variable piezoelectric polarization, matching layers and bondlines. The model is extended further to include mechanical wave absorption in both the active transducer element and the propagating channel, and the effect of such frequency dependant loss on the operational characteristics of an ILT device is reported. Several simulation results are shown to demonstrate the feasibility of the approach together with a comparisonal analysis to conventional finite modeling techniques and experimental evaluations. The results indicate clearly that such approximate analytical techniques can be used effectively to design optimal ILT configurations.

2pEAg4. An analytical model for piezoelectric/elastic axisymmetric bender disks radiating in water. Raphaël Lardat (Thales Underwater Systems, 525 Route des Dolines, BP 157, 06930 Sophia Antipolis cedex, France, raphael.lardat@fr.thalesgroup.com)

Flextensional transducers have been widely studied for low frequency SONAR application due to the low celerity of the flexion wave. In this work, we will describe in detail a complete analytical model for an axisymmetric disk made of one elastic plate on which two piezoelectric disks of smaller radius have been reported on each side. The model, starting from flexural axisymmetric theory and from the piezoelectric equations, finally comprises local radiation impedance and mutual coupling with neighboring disks. In particular, we will explain the continuity conditions that are to be imposed at the external radius of the piezoelectric disk. We also show how to include a radiation force opposed and proportional to the normal velocity 
inside the analytical solution given by a fourth order differential equation. The described model is then able to compute a large number of parameters such as Eigen modes, harmonic admittance, radiated pressure, coupling coefficient and static and dynamic stresses. The validity of the model is as- sessed by comparison to solutions of the coupled problem in water using classical FEM/BEM. We will show an extremely good agreement enabling us to use this simple model for geometry optimization and design improvement.

TUESDAY AFTERNOON, 1 JULY 2008

P2-D, LEVEL 2, 3:40 TO 5:20 P.M.

\title{
Session 2pMUa
}

\section{Musical Acoustics and Physical Acoustics: Brass Instrument Acoustics II (Poster Session)}

\author{
Thomas Moore, Cochair \\ Rollins College \\ Joël Gilbert, Cochair \\ Laboratoire d'Acoustique de l'Université du Maine
}

\begin{abstract}
All posters will be on display from 3:40 p.m. to 5:20 p.m. To allow contributors an opportunity to see other posters, contributors of odd-numbered papers will be at their posters from 3:40 p.m. to 4:30 p.m. and contributors of even-numbered papers will be at their posters from 4:30 p.m. to 5:20 p.m.
\end{abstract}

\section{Contributed Papers}

2pMUa1. Pedal notes of brass instruments, a mysterious regime of oscillation. Joël Gilbert (Laboratoire d'Acoustique de l'Université du Maine, Avenue Olivier Messiaen, 72085 Le Mans, France, joel.gilbert@univ-lemans.fr), Pierre Aumond (Lab. d'Acoustique Université du Maine, UMR CNRS 6613, 72085 Le Mans Cedex 9, France, pierre .aumond.etu@univ-lemans.fr)

In reed and brass instruments, sound is produced by self-sustained mechanical oscillations driven by an airflow coming from a pressure supply such as the player's lungs. Most of the time, the fundamental frequency of a periodic oscillation is close to, and mainly controlled by one given acoustic mode. Even though, these oscillations are the result of a complex non-linear coupling between all the acoustic modes and the mechanical valve. The pedal note, which is the lowest note that can be sounded on a brass instrument, is well-known as a counter-example. Eighty years ago, Bouasse did an experiment by replacing the brass mouthpiece by a reed mouthpiece on a brass instrument, and obtained a pedal note a fifth below! An elementary model dedicated to both cane-reed and lip-reed instruments can be used. In order to investigate the sound production of pedal notes, a simulation method based on modal decomposition of bore and reed dynamics has been carried out. Then, the periodic solutions of this model are obtained, and the mysterious oscillation regimes of Bouasse experiment are discussed.

2pMUa2. Nonlinear effects in the propagation of outgoing and reflected pulses inside a trombone. Pablo L Rendón (CCADET, Universidad Nacional Autónoma de México, Ciudad Universitaria, 04510 Mexico, Mexico, pablo.rendon@ccadet.unam.mx), Felipe Orduña-Bustamante (CCADET, Universidad Nacional Autónoma de México, Ciudad Universitaria, 04510 Mexico, Mexico, felipe.orduna@ccadet.unam.mx), Jacques Sorrentini (Institut Fresnel, UMR 6133 CNRS, Campus de Saint Jérôme, Av. Escadrille Normandie 13397, Cedex 20 Marseille, France, jacques.sorrentini@ec-marseille.fr), Antonio Pérez-López (CCADET, Universidad Nacional Autónoma de México, Ciudad Universitaria, 04510 Mexico, Mexico, antonio.perez@ccadet.unam.mx)

While sound propagation in a trombone can be generally quite properly described by linear models, it is commonly acknowledged that the "brassiness" of sound produced at high amplitudes is associated to nonlinear effects. Whether these effects are due principally to propagation inside a long, narrow pipe, or to the production of sound at the mouthpiece is still unclear. We present experimental measurements taken both from a long, narrow tube, and an actual slide trombone from which it is observed that the scale of nonlinear effects seems to be greater for outgoing pulses than for the reflected pulses at the open end of the trombone or tube. A theoretical model is also proposed, based on weakly nonlinear perturbations of linear theory, and is validated both by experimental results and by numerical simulations. The results indicate that both the amplitude and the shape of the initial perturbation produced at the trombone mouthpiece have an effect on the scale of propagation nonlinearity present inside the trombone resonator, and thus also on the "brassy" quality of sound.

2pMUa3. External sound radiation of vibrating trombone bells. Guillaume Nief (Laboratoire d'Acoustique de l'Université du Maine, Avenue Olivier Messiaen, 72085 Le Mans, France, guillaume .nief.etu@univ-lemans.fr), François Gautier (Laboratoire d'Acoustique de l'Université du Maine, Avenue Olivier Messiaen, 72085 Le Mans, France, francois.gautier@univ-lemans.fr), Jean-Pierre_Dalmont (Laboratoire d'Acoustique de l'Université du Maine, Avenue Olivier Messiaen, 72085 Le Mans, France, Jean-Pierre.Dalmont@univ-lemans.fr), Joël Gilbert (Laboratoire d'Acoustique de l'Université du Maine, Avenue Olivier Messiaen, 72085 Le Mans, France, joel.gilbert@univ-lemans.fr)

The issue of the influence of bell vibrations on the sound of brass instruments is still debated. For such instruments, external sound field is the superposition of the sound field resulting from acoustic oscillations of the air column and the sound field resulting from direct radiation of the bell and walls. The aim of this paper is to quantify the bell contribution with respect to the air column one, and to examinate the conditions for which the former may become audible. For this purpose, the structural modes of a trombone bell are identified using an experimental modal analysis. For each mode shape, the radiation efficiency is computed using a model of the radiated sound based on a distribution of equivalent monopoles. A critical frequency is defined for the bell and allows us to determine at which condition a given structural mode is radiating. Measurements of the sound power radiated by a trombone excited by an harmonic acoustic source are carried out when the bell is free to vibrate and when bell vibrations are damped. Comparison between the two configurations is analyzed using the developed model and is used to quantify the sound power due to bell vibrations. 
2pMUa4. Acoustic radiation of wind instruments resonators. Bastien Mallaroni (Laboratoire de Mécanique et d'Acoustique - CNRS, 31 chemin Joseph Aiguier, 13402 Marseille, France, mallaroni@1ma.cnrs-mrs .fr), Pierre-Olivier Mattei (Laboratoire de Mécanique et d'Acoustique CNRS, 31 chemin Joseph Aiguier, 13402 Marseille, France, mattei@1ma.cnrs-mrs.fr), Philippe Herzog (Laboratoire de Mécanique et d'Acoustique - CNRS, 31 chemin Joseph Aiguier, 13402 Marseille, France, herzog@1ma.cnrs-mrs.fr)

Optimization of the acoustic radiation of wind instruments resonators may be based on the study of finite length circular ducts radiating in unbounded medium, as this is a model realistic enough, but which allows advanced analytics developments. Our approach is based on the calculation of resonance frequencies of such a simplified wind instrument. These are the complex frequency singularities of the operator describing the global resonator/external fluid system. A Newton's method is used to search for the singularities of the multimodal Green's function of the duct. This is obtained by the product of impedance matrices which relate pressure and velocity between two abscissae inside the duct. A variable coupling factor is introduced with external acoustic radiation, described by a multimodal radiation impedance based on the Zorumski formulation. Examples of variation of the resonance frequencies with this coupling factor will be given. Surprisingly, regular variations of the coupling lead to non regular evolution of the resonances. Physical interpretation of this behaviour will be discussed.

2pMUa5. Transient behaviour of human and artificial brass players' lips. Samuel Stevenson (Edinburgh University, 4201 JCMB, Kings Buildings, Mayfield Road, EH9 3JZ Edinburgh, UK, samuel.stevenson @ed.ac.uk), Donald M. Campbell (Edinburgh University, 4201 JCMB, Kings Buildings, Mayfield Road, EH9 3JZ Edinburgh, UK, d.m.campbell@ed.ac.uk), John Chick (Edinburgh University, 4201 JCMB, Kings Buildings, Mayfield Road, EH9 3JZ Edinburgh, UK, john.chick@ed.ac.uk), Joël Gilbert (Laboratoire d'Acoustique de l'Université du Maine, Avenue Olivier Messiaen, 72085 Le Mans, France, joel.gilbert@univ-lemans.fr), Stefan Bilbao (University of Edinburgh, Room 7306B, JCMB, King's Bldgs., Mayfield Rd., EH9 3JZ Edinburgh, UK, sbilbao@ staffmail.ed.ac.uk)

It is widely accepted that the starting transient is an extremely significant perceptual feature of musical sound. For players, the ease with which a note can be started on a particular instrument is also of great importance. However, until recently research has concentrated on the analysis of steady state sounds rather than the study of the starting behaviour of the instrument. The work reported here uses a high speed camera to capture the motion of lip reeds during the starting transient. Simultaneous recordings are made of the pressure signals generated in the mouthpiece of the instrument and radiated from the bell. Preliminary results on human players, already presented, have been extended and supplemented by measurements using an artificial mouth in order to separate effects due to the instrument from those dependent on the expertise of a particular human player. Computational simulations have been compared to these experimental findings.

2pMUa6. Tristan trumpet or English horn? Robert W. Pyle (S. E. Shires Co., 11 Holworthy Place, Cambridge, MA 02138, USA, rpyle@post.harvard.edu), Sabine K. Klaus (National Music Museum, The
University of South Dakota, PO Box 190, Landrum, SC 29356, USA, sabineklaus@alltel.net)

In Act III of his opera "Tristan und Isolde", Richard Wagner wrote an instrumental tune to be (apparently) played by a shepherd. This was originally scored for, and is customarily played by, an off-stage cor anglais. Wagner indicated that he would have preferred some form of wooden trumpet, similar to a small-scale alphorn. A few such instruments have been built. These are pitched in 4-foot $\mathrm{C}$, with a pear-shaped bell similar to that of the cor anglais, and a single metal whole-tone valve. The Utley Collection at the National Music Museum, The University of South Dakota, has two such instruments: one made by Gebrüder Alexander (Mainz), formerly used in the National Opera in Prague (Volkstheater), the other a copy of a trumpet by Martin Lehner, Munich, in the possession of the Staatsoper Dresden, made by Andreas Schöni and Rainer Egger (Bern and Basel). We will compare sound spectra of the Tristan trumpet with that of the cor anglais. We will also compare the acoustic input impedance of the Tristan trumpet with that of an ordinary trumpet of the same pitch.

2pMUa7. Harmonicity of trumpet modes. Peter L. Hoekje (Baldwin-Wallace College, 275 Eastland Rd., Berea, OH 44017, USA, phoekje@bw.edu), Michael Attaway (Richardson ISD, 400 S. Greenville Ave., Richardson, TX 75081, USA, michael_attaway@hotmail .com)

The mouthpiece on a brass instrument is generally removable but constitutes an important part of the whole instrument. In the frequency domain, it can be considered to provide a frequency dependent equivalent length that increases with frequency up to the Helmholtz resonance frequency $f_{\text {pop }}$ of the mouthpiece itself. Geometrical changes that affect the total volume or the $f_{\text {pop }}$ of the mouthpiece will affect the harmonicity of the nearly harmonic normal modes of the instrument. Using heterodyne filter analysis, the development of the frequency components and their amplitudes were measured for low trumpet notes, both for repeated attacks of short notes and for sustained note crescendos from pianissimo to fortissimo. The measurements were repeated after the mouthpiece was modified to improve the harmonicity of the normal modes. During both attack and crescendo tests, the modified mouthpiece was associated generally with more stable frequencies of the partials and more even development of the amplitudes of the partials.

2pMUa8. The influence of braces on the playability of trumpets. Alexander Mayer (Inst. f. Wiener Klangstil, Univ. f. Music, Anton von Webernplatz 1, A-1030 Vienna, Austria, mayer@mdw.ac.at), Rainer Egger (Blechblas-Instrumentenbau Egger, Turnerstrasse 32, 4058 Basel, Switzerland, info@eggerinstruments.ch), Gregor Widholm (Inst. f. Wiener Klangstil, Univ. f. Music, Anton von Webernplatz 1, A-1030 Vienna, Austria,Widholm@mdw.ac.at)

The influence of braces on the playability, response and sound properties of the trumpet is an intense discussed subject for players and instrument makers. In addition to the problem of finding the proper position of the braces, inserted braces can produce stress between the instruments tubes. In this paper the influence of the tube stress is investigated. To ensure an adjustable and reproducible stress between the trumpet tubes special adjustable braces in conjunction with a force monitor are used. The results of blind playing tests are compared with spectral analysis of the played sounds. In addition, the acoustic input impedance, the wall vibrations and the transfer function of the instrument are investigated. 


\title{
Session 2pMUb
}

\section{Musical Acoustics: Edge Tone and Flue Pipes II (Poster Session)}

\author{
Shigeru Yoshikawa, Cochair \\ Kyushu University \\ Judit Angster, Cochair \\ Fraunhofer Institute for Building Physics
}

\begin{abstract}
All posters will be on display from 3:40 p.m. to 5:20 p.m. To allow contributors an opportunity to see other posters, contributors of odd-numbered papers will be at their posters from 3:40 p.m. to 4:30 p.m. and contributors of even-numbered papers will be at their posters from 4:30 p.m. to 5:20 p.m.
\end{abstract}

\section{Contributed Papers}

2pMUb1. Vortex sound of the flute and its interpretation. Andreas Bamberger (Physics Institute University Freiburg, Hermann-Herder-Str. 3, 79104 Freiburg, Germany, bamberger@physik.uni-freiburg.de)

The flute as an example of flue instruments is investigated for its properties of the jet-labium interaction at $1 \mathrm{kHz}$. The jet and the externally excited acoustic flow in the embouchure especially near the labium is recorded with endoscopic Particle-Image-Velocimetry. Extracting the vorticity the data allows us to calculate the vortex sound according to M. How (1975) The far field acoustic power of the flute is measured as well and compared with the vortex sound power, which matches in sign and magnitude up to a factor 2 for different sound levels. The non-trivial interpretation of the sound production is based on the near cancellation of the two vortex layers of the jet along its path except near the labium. In order to gain insight of the interplay of the different ingredients of the vortex sound power the coriolis force, the acoustic field and their phase relation are determined. Scaling of laws for these quantities as a function of the jet velocity/pressure of the standing wave are deduced and compared with the acoustic power dependence. A comparison with recently available numerical simulations (ISMA 2007) is discussed.

2pMUb2. Experimental investigation of the aeroacoustic coupling of self-sustained tones to non-planar modes of a resonator. Martin Glesser (CNRS - LMA, 31 Chemin Joseph Aiguier, 13009 Marseille, France, glesser@1ma.cnrs-mrs.fr), Vincent Valeau (Laboratoire d'Etudes Aérodynamiques (LEA), Université de Poitiers - ENSMA - CNRS, Bâtiment K, 40 Avenue du Recteur Pineau, F-86022 Poitiers, France, vincent.valeau@lea.univ-poitiers.fr), Anas Sakout (LEPTIAB Université de La Rochelle, Avenue Michel Crépeau, 17042 La Rochelle Cedex 01, France, asakout@univ-lr.fr)

Recent studies have shown that a jet-slot oscillator (ie, an aeroacoustic self-sustained source consisting of the impingement of the vortical structures of a plane jet on a slotted plate) can be coupled to non-planar modes of the flow-supply duct. This phenomenon occurs in the particular case where the jet-exit and the obstacle are misaligned in the spanwise direction. This paper presents an experimental investigation of the excited modes as a function of the plate inclination angle, and of the distance between the jet-exit and the slot. It is shown that: (i) the vortex-tubes spanwise morphology is governed by the nature of the transverse excited modes, and (ii) the vortices tend to impinge on the inclined obstacle almost in phase in the spanwise direction, in order to enhance the coherence of the interaction along the slot. Moreover, an elementary analytical model of the resonant acoustic field shows that the ratio between the transverse and planar modes amplitudes is tuned by the system in order to keep this coherence maximum. The existence of a so-called "opposed transverse mode" is also shown, generating vortices with an inclination angle opposed to the plate inclination angle.

2pMUb3. Open and closed loop characterization of an air-jet reflection amplifier. John C. Price (University of Colorado, Department of Physics, 390 UCB, Boulder, CO 80309-0390, USA, john.price@ colorado.edu)

Our laboratory has embarked on an effort to understand the steady-state behavior of a Yamaha YRT-304B tenor recorder. The instrument is split at the head-joint, and both sides are studied with a multi-microphone reflectometer using sinusoidal excitation. For the head joint, data is obtained versus frequency, blowing pressure, and excitation amplitude. We have two goals: First, to use our open-loop reflectometry data to understand the internal acoustic spectrum of the assembled instrument, as a function of blowing pressure and fingering; and second, to provide a well-defined set of openloop observables for comparison with hydrodynamic calculations of the airjet dynamics. This particular instrument is attractive for several reasons: it disassembles at a straight cylindrical section of the bore, and so is easily coupled to straight waveguide; it is large enough for accurate reflection coefficient and internal pressure measurements; it can be studied closed-loop as a complete instrument; and it represents a fiducial geometry that is readily available to any laboratory. 


\title{
Session 2pMUc
}

\section{Musical Acoustics: Acoustic Measurements on Wind Instruments II (Poster Session)}

\author{
Noam Amir, Cochair \\ Tel Aviv University \\ David B. Sharp, Cochair \\ Open Univeristy
}

\begin{abstract}
All posters will be on display from 3:40 p.m. to 5:20 p.m. To allow contributors an opportunity to see other posters, contributors of odd-numbered papers will be at their posters from 3:40 p.m. to 4:30 p.m. and contributors of even-numbered papers will be at their posters from 4:30 p.m. to 5:20 p.m.
\end{abstract}

\section{Contributed Papers}

2pMUc1. Sound power level measurement of Sheng, a Chinese wind instrument. Yue Zhe Zhao (State Key Laboratory of Subtropical Building Science, South China University of Technology, 381 Wushan Street, 510640 Guangzhou, China, arzhyzh@scut.edu.cn), Shuo Xian Wu (State Key Laboratory of Subtropical Building Science, South China University of Technology, 381 Wushan Street, 510640 Guangzhou, China, arshxwu@ scut .edu.cn), Jian Zhen Qiu (State Key Laboratory of Subtropical Building Science, South China University of Technology, 381 Wushan Street, 510640 Guangzhou, China, qjianzhen@gmail.com), Li Ling Wu (Dept. of Musicology, Xinghai Conservatoire of Music, 510500 Guangzhou, China, wushijun@scut.edu.cn), Hong Huang (Dept. of Musicology, Xinghai Conservatoire of Music, 510500 Guangzhou, China, yyxyhuanghong@163.com)

Sheng is one of the Chinese traditional wind instruments. But its sound power level has never been carefully measured. In this paper, the sound power measurements of Sheng were performed for the first time in a reverberation chamber according to ISO standard and Chinese national standard. Two qualified musicians performed on their own instruments in the center of the reverberation chamber. The radiated sound energy and the dynamic ranges of the Sheng were investigated by four channel acoustic measuring equipments. Typical sound power values were obtained through averaging and the results were reported in this paper. It was showed that the mean forte sound power level can reach up to $98 \mathrm{~dB}$ with a dynamic range of $22.5 \mathrm{~dB}$ when music scale was performed. The method discussed here is valuable for the sound power measurements of other musical instruments. The measurement of the sound power radiated by national musical instruments lays foundations for the investigation into the acoustics of national music halls.

2pMUc2. Measurements of woodwind tone-hole parameters using a double impedance head method. Paul Dickens (University of New South Wales, Music Acoustics, School of Physics, NSW 2052 Sydney, Australia, PDickens@ resmed.com.au), Yakov Kulik (University of New South Wales, Music Acoustics, School of Physics, NSW 2052 Sydney, Australia, ykulik@phys.unsw.edu.au), Joe Wolfe (University of New South Wales, Music Acoustics, School of Physics, NSW 2052 Sydney, Australia, J.Wolfe @unsw.edu.au)

A woodwind tone hole is often represented as a T-junction with a shunt and series impedance. We measured the frequency dependence of the series and shunt impedances of open and closed tone holes using a pair of impedance heads, one on either side of a symmetric section of short bore pipe with a finger hole, and each calibrated on resonance-free loads. The shunt impedance is most accurately measured when the hole is located at a pressure antinode (speakers in phase) and the series impedance at a pressure node (speakers in anti-phase). We use both conditions, in this way, to measure series and shunt impedances for all frequencies studied. Pipes with the same length and diameter, but having wall thicknesses $1.5-5.0 \mathrm{~mm}$ and tone hole diameters 1.5-15.0 mm were used. For open holes, results are compared with calculations and results measured using other methods. The results for holes closed with fingers are also used to calculate the effective length of finger intrusion. Examples of the inclusion of the results into woodwind models are given.

2pMUc3. Investigation of clarinet reed auto-oscillations with digital Fresnel holography. Denis Mounier (LPEC/UMR 6087/CNRS/Université du Maine, Avenue Olivier Messiaen, 72085 Le Mans Cedex 09, France, denis.mounier@univ-lemans.fr), Pascal Picart (Laboratoire d'Acoustique de l'Université du Maine, Avenue Olivier Messiaen, 72085 Le Mans, France, pascal.picart@univ-lemans.fr), Julien Leval (Laboratoire d'Acoustique de l'Université du Maine, Avenue Olivier Messiaen, 72085 Le Mans, France, julien.leval.etu@univ-lemans.fr), Francis Piquet (Laboratoire d'Acoustique de l'Université du Maine, Avenue Olivier Messiaen, 72085 Le Mans, France, francis.piquet@univ-lemans .fr), Jean-Pierre Boileau (Laboratoire d'Acoustique de l'Université du Maine, Avenue Olivier Messiaen, 72085 Le Mans, France, jean-pierre.boileau@univ-lemans.fr), Thomas Guimezanes (Laboratoire d'Acoustique de l'Université du Maine, Avenue Olivier Messiaen, 72085 Le Mans, France, thomas.guimezanes@univ-lemans.fr), Jean-Pierre Dalmont (Laboratoire d'Acoustique de l'Université du Maine, Avenue Olivier Messiaen, 72085 Le Mans, France, Jean-Pierre.Dalmont@univlemans.fr)

This paper describes a full field digital holographic method for studying the cinematic of the vibration of the clarinet reed in playing conditions in an artificial mouth. Since many years, LAUM is interested in studying musical instruments and particularly the clarinet. The behaviour of such an instrument is particularly complex because it depends on aerodynamics, contact between reed and beak, and interaction with the lip of the musician. Thus, visualisation of the full movement of the reed in playing conditions is necessary to better understand physics of the clarinet. However, free oscillations have high amplitude of several hundreds of micrometers, thus corresponding to several thousand times the laser wavelength. The analysis of the movement is performed by a laser tracking of the vibration. It is demonstrated that it is possible to reconstruct a synthetic high amplitude deformation of auto-oscillations encoded with digital Fresnel holograms. The setup is applied to the auto-oscillation of the clarinet reed in a synthetic mouth. Tracking of the vibration is performed by using the pressure signal delivered by the mouth. Experimental results show the four steps of the reed movement and especially emphasize the shocks of the reed on the mouthpiece. 


\title{
Session 2pMUd
}

\section{Musical Acoustics: Interaction Between Instrument and Instrumentalist II (Poster Session)}

\author{
Gary Scavone, Cochair \\ Schulich School of Music, McGill University \\ Xavier Boutillon, Cochair \\ Laboratoire de Mécanique des Solides
}

\begin{abstract}
All posters will be on display from 3:40 p.m. to 5:20 p.m. To allow contributors an opportunity to see other posters, contributors of odd-numbered papers will be at their posters from 3:40 p.m. to 4:30 p.m. and contributors of even-numbered papers will be at their posters from 4:30 p.m. to 5:20 p.m.
\end{abstract}

\section{Contributed Papers}

2pMUd1. Chest wall dynamics and respiratory muscle recruitment during flute playing. Isabelle Cossette (McGill University, 555, Sherbrooke St, Montréal, QC H3A 1E3, Canada, isabelle.cossette1@mcgill.ca), Pierpaolo Monaco (McGill University, 555, Sherbrooke St, Montréal, QC H3A 1E3, Canada, pierpal@yahoo.it), Andrea Aliverti (Politecnico di Milano, Dipartimento di Bioingegneria, P.zza Leonardo da Vinci, 32, 20133 Milano, Italy, andrea.aliverti@polimi.it), Peter Macklem (PO Box 250, Lansdowne, ON K0E 1L0, Canada, peter.macklem @ gmail.com)

Respiratory parameters and sound were recorded during professional flute playing in order to assess what physiological processes were associated with the control of sound production that results in 'breath support' which in turn is associated with high quality playing. Four standing young professional flautists played flute excerpts with different technical requirements with and without breath support. Recordings included optoelectronic plethysmographic measurements of chest wall volume $(\mathrm{Vcw})$ and its compartments, surface electromyography of the scalene, lateral abdominal, rectus abdominus, parasternal and sternocleidomastoid muscles, mouth pressure, and sound. Flow was estimated from differentiating Vcw during playing. Comparison in between various types of playing were performed. Results showed that flute support entails antagonistic contraction of nondiaphragmatic inspiratory muscles that tends to hold the rib cage at higher lung volume during long legato phrase playing. This relieves the expiratory muscles from the task of producing the right mouth pressure, especially at the end of the phrases, so they can contribute more to the finer control of mouth pressure modulations required for high quality playing.

2pMUd2. A concise overview of present knowledge of bowed-string gestures and their acoustical consequences. Knut Guettler (Norwegian Academy of Music, P.O.Box 5190 Majorstuen, 0302 Oslo, Norway, knut .guettler@nmh.no)

During the few years of our young millennium the picture of what happens acoustically when the bow is drawn across the string has become considerably more complete. A few surprises emerged along the way: the bow's position on the string does not seem to influence the spectral envelope-and different from what Schelleng predicted-there seems to be no clear correlation between minimum bow force and the bow speed. The intention of this presentation is to give a brief survey of related studies and their results, as well as pointing out some of the blank spots still remaining on the canvas. 


\title{
Session 2pMUe
}

\section{Musical Acoustics and Speech Communication: Singing Voice and Source-Filter Interaction}

\author{
Brad Story, Cochair \\ University of Arizona, Dept. of Speech and Hearing Sciences, 1131 E. 2nd Street, Tucson, AZ 85721-0071, USA \\ Nathalie Henrich, Cochair \\ Département Parole \& Cognition, GIPSA-lab, 46, avenue Félix Viallet, Grenoble Cedex, 38031, France
}

\section{Contributed Paper}

\begin{abstract}
$5: 20$
2pMUe1. How whisper and croak phonation affect vocal tract resonances. Yoni Swerdlin (University of New South Wales, Music Acoustics, School of Physics, NSW 2052 Sydney, Australia, yoniswerdlin@hotmail.com), John Smith (University of New South Wales, Music Acoustics, School of Physics, NSW 2052 Sydney, Australia, john.smith@unsw.edu.au), Joe Wolfe (University of New South Wales, Music Acoustics, School of Physics, NSW 2052 Sydney, Australia, J.Wolfe @unsw.edu.au)
\end{abstract}

The whisper and croak modes of phonation provide convenient broad band excitation of the vocal tract and thus give relatively precise information about tract resonances. How closely do the measured resonances for these mechanisms approximate those of normal speech? We measured the frequencies of the first four resonances (R1-R4) in normal, whisper and croak phonation. Subjects produced pairs of these phonations in the same vocal gesture. Formants were used to measure the frequencies (R1-R4) for the non-periodic phonations and broad band excitation at the mouth was used to measure them with similar precision in normal speech. For R1 to R4 respectively, whispering raised the resonant frequencies by $255 \pm 90 \mathrm{~Hz}$ $(92)^{*}, 115 \pm 105 \mathrm{~Hz}(119)^{*}, 125 \pm 125 \mathrm{~Hz}(109)^{*}$ and $75 \pm 120 \mathrm{~Hz}(118)^{*}$, (mean \pm standard deviation (n), asterisks show significance at the $5 \%$ level). These values, and their decrease with increasing frequency, are consistent with the effect of the increased glottal opening in whispering only if the increased opening is large. A supra-glottal narrowing in the tract could also help raise the frequencies of resonance. Croak phonation raises the resonant frequencies respectively by $45 \pm 50 \mathrm{~Hz}(121)^{*}, 10 \pm 60 \mathrm{~Hz}(124), 65 \pm 120$ $\mathrm{Hz}(109)^{*}$ and $15 \pm 110 \mathrm{~Hz}(108)$.

\section{Invited Papers}

2pMUe2. Vocal folds and ventricular bands in interaction: comparison between 'in vivo' measurements and theoretical predictions. Lucie Bailly (Département Parole \& Cognition, GIPSA-lab, 46, avenue Félix Viallet, 38031 Grenoble Cedex, France, Lucie.Bailly@gipsa-lab.inpg.fr), Nathalie Henrich (Département Parole \& Cognition, GIPSA-lab, 46, avenue Félix Viallet, 38031 Grenoble Cedex, France, Nathalie.Henrich@gipsa-lab.inpg.fr), Xavier Pelorson (Département Parole \& Cognition, GIPSA-lab, 46, avenue Félix Viallet, 38031 Grenoble Cedex, France, pelorson@ @icp.inpg.fr), Joël Gilbert (Laboratoire d'Acoustique de l'Université du Maine, Avenue Olivier Messiaen, 72085 Le Mans, France, joel.gilbert@univ-lemans.fr)

Period-doubling occurrences have been found during singing phonations such as Mongolian Kargyraa throat singing or Sardinian $A$ Tenore Bassu singing. The combined vibrations of vocal folds and ventricular bands have been observed during the production of such low-pitch bass-type sounds. The present study aims at better understanding the physical interaction between the ventricular-bands vibration and the vocal-folds self-sustained oscillations. In this paper, the vibratory properties of both vocal folds and ventricular bands in interaction are analysed on a professional singer by means of acoustical, electroglottographic signals and synchronized glottal images obtained by high-speed cinematography. Using the detected glottal and ventricular areas, the aerodynamic behavior of the laryngeal system is simulated using a simplified aerodynamic modelling previously validated "in vitro" on vocal-folds and ventricular-bands replica. An estimate of the subglottal pressure along with the ventricular aperture extracted from the "in vivo" data allows a theoretical prediction of the glottal aperture. The "in vivo" measurements of the glottal aperture are then compared to the simulated estimations. The influence of the subglottal pressure is also discussed.

\section{6:00}

2pMUe3. Longitudinal voice source and acoustic analysis of English girl cathedral choristers. David M. Howard (University of York, Department of Electronics, Heslington, YO10 5DD York, UK, dh@ohm.york.ac.uk)

Since 1998, the authors have been recording girl choristers who sing at Wells Cathedral in England to enable a longitudinal quantitative exploration of voice development for girls aged between 8 and 16 who are involved in professional choral singing on a daily basis. The recordings are made of their acoustic output and the output from an electrolaryngograph for a series of vocal tasks including speaking and singing. Changes have been observed on a longitudinal basis for larynx closed quotient (the percentage of each cycle for which the vocal folds remain closed), long-term average spectra as well as in the overall spectral coverage in terms of frequency and dynamic ranges. Evidence of observations will be presented and their implications will be discussed with reference to source-filter interaction as appropriate. 


\section{Contributed Papers}

6:20

2pMUe4. Phonetograms of laryngeal source parameters for different vowels and laryngeal mechanisms. Sylvain Lamesch (IJLRA-LAM, 11, rue de Lourmel, 75015 Paris, France, lamesch@lam.jussieu.fr), Boris Doval (LIMSI-CNRS, B.P. 133, 91403 Orsay Cedex, France, boris.doval@limsi.fr), Michèle Castellengo (IJLRA-LAM, 11, rue de Lourmel, 75015 Paris, France, castel@ccr.jussieu.fr)

This paper explores how the laryngeal source is adjusted by the singer when the sung vowel changes. As the source parameter adjustments may depend on intensity and pitch, comparisons are done through phonetograms computed for each vowel. Distinction is made in the phonetograms between the two main laryngeal mechanisms (M1 and M2). Male and female subjects were recorded producing crescendos and decrescendos from $\mathrm{C} 3$ to $\mathrm{C} 5$ on /a/, /i/ and /o/ in order to obtain a reduced phonetogram for each vowel and each laryngeal mechanism. Sound, electroglottographic signals and vertical larynx position (VLP) were recorded. Investigations were done on the open quotient and the VLP. The results show a smaller vocal dynamic on /i/ (and in a smaller extent on $/ \mathrm{o} /$ ) in each laryngeal mechanism, and differences on the intensity low limit of the highest part of the M1-phonetogram. Clear tendencies about the larynx position were measured. They are similar for both laryngeal mechanisms, but differ among singers. The Oq seems to be lower on /i/ than on /a/ and /o/ for M1 productions.

\section{6:40}

2pMUe5. Resonance strategies and glottal behaviour in the two main laryngeal mechanisms for professional operatic singers. Nathalie
Henrich(Département Parole \& Cognition, GIPSA-lab, 46, avenue Félix Viallet, 38031 Grenoble Cedex, France, Nathalie.Henrich@gipsa-lab .inpg.fr), John Smith (University of New South Wales, Music Acoustics, School of Physics, NSW 2052 Sydney, Australia, john.smith@unsw .edu.au), Joe Wolfe (University of New South Wales, Music Acoustics, School of Physics, NSW 2052 Sydney, Australia, J.Wolfe@unsw.edu.au)

In lyrical singing, two principal laryngeal mechanisms are used to produce all the notes across the singer's tessitura. Laryngeal mechanisms M1 and M2 are characterised by a greater and lesser vibrating mass respectively. Different timbres between the two are at least partly explained by their different glottal behaviours. However, different adjustments of the vocal-tract resonances, which can also contribute to different timbres, may also be involved, but have been neglected. We studied seven professional operatic singers, who produced sustained vowels at the same pitch using both laryngeal mechanisms. Vocal-tract resonances were directly measured during phonation, by broadband acoustic excitation at the mouth. Glottal behaviour and laryngeal movement were measured indirectly with an electroglottograph. As expected, we measured lower values of open quotient and higher sound pressure levels in M1 than in M2. In most cases, the first two vocal-tract resonances were characterised by lower frequencies in M2 than in M1. In the singer's formant region, similar resonance-frequency values were found for both laryngeal mechanisms. The effect on the sound spectrum of an open-quotient increase together with a first-formant decrease may be modelled by source-filter theory. The theoretical and measured amplitudes of the first two harmonics will be compared.

\section{Invited Paper}

\section{7:00}

2pMUe6. Chest, head and whistle registers in an untrained female singer analyzed by videokymography, strobolaryngoscopy and sound spectrography. Jan G. Svec (Palacky University Olomouc, Faculty of Science, Dept. Experimental Physics, Laboratory of Biophysics, Tr. Svobody 26, CZ-771 46 Olomouc, Czech Republic, svecjan@vol.cz), Johan Sundberg (KTH, Department of Speech, Music and Hearing, Lindstedtsvägen 24, SE-100 44 Stockholm, Sweden, jsu@csc.kth.se), Stellan Hertegard (Karolinska University Hospital Huddinge, Dept. Logopedics and Phoniatrics, SE-141 86 Stockholm, Sweden, Stellan.Hertegard@ki.se)

There has been a lack of objective data on the singing voice registers, particularly on the so called "whistle" register, occurring in the top part of the female pitch range, which is accessible only to some singers. This study offers unique strobolaryngoscopic and high-speed (7812.5 images/s) videokymographic data on the vocal fold behavior of an untrained female singer capable of producing three distinct voice qualities, i.e., the chest, head and whistle registers. The sound was documented spectrographically. The transition from chest to head register, accompanied by pitch jumps, occurred around tones B4-C\#5 $(500-550 \mathrm{~Hz})$ and was found to be associated with a slight decrease in arytenoids adduction, resulting in decrease of the closed quotient. The register shifts from head to whistle, also accompanied by pitch jumps, occurred around tones E5-B5 $(670-1000 \mathrm{~Hz})$ without any noticeable changes in arytenoids adduction. Some evidence was found for the vocal tract influence on this transition. The mechanism of the vocal fold vibration in whistle register was found principally similar to that at lower registers: vibrations along the whole glottal length and vertical phase differences (indicated by sharp lateral peaks in videokymography) were seen on the vocal folds up to the highest tone G6 (1590 Hz).

\section{Contributed Paper}

$7: 20$

2pMUe7. Sopranos' secondo passagio: a resonance adjustment and/or a laryngeal transition to the whistle voice? Maeva Garnier (University of New South Wales, Music Acoustics, School of Physics, NSW 2052 Sydney, Australia, maeva.garnier@gmail.com), Nathalie Henrich (Département Parole \& Cognition, GIPSA-lab, 46, avenue Félix Viallet, 38031 Grenoble Cedex, France, Nathalie.Henrich @ gipsa-lab.inpg.fr), John Smith (University of New South Wales, Music Acoustics, School of Physics, NSW 2052 Sydney, Australia, john.smith@unsw.edu.au), Joe Wolfe (University of New South Wales, Music Acoustics, School of Physics, NSW 2052 Sydney, Australia, J.Wolfe @unsw.edu.au)

How are glottal source and vocal tract involved in the sopranos' passagio around E5? This pilot study investigates a legit soprano singing C5 to C6 with a lyrical technique and C5 to D7 in a "light" voice. On (de)crescendi and glissandi over the passagio, we observed clear bifurcations from "light" to lyrical mode, with discontinuous changes in f0, larynx vertical position, amplitude and shape of the electroglottographic signal. No such discontinuities occurred from C5 to D7 within the "light" mode. We established phonetograms of both modes and characterised differences in voice quality (Spectrum, Harmonicity, Open quotient). For [oe] in both modes, this singer tunes the first vocal tract resonance (R1) to f0 over the passagio. For a free vowel, a single and strong resonance is observed in the "light" mode, which may correspond to the merging of R1 and R2 and which is tuned to $2 \mathrm{f} 0$ below C6, then to f0 until F6. Above F\#6, two resonances are observed again and R2 is tuned to f0. All these observations suggest that, for this singer, the secondo passagio may correspond to both a resonance adjustment and a transition from the second laryngeal mechanism to the third, also called whistle voice. 


\title{
Session 2pNSa
}

\section{Noise, ASA Committee on Standards and EURONOISE: Sound Quality Tools and Applications I}

\author{
Wade R. Bray, Cochair \\ HEAD acoustics, Inc., 6964 Kensington Road, Brighton, MI 48116, USA \\ Klaus Genuit, Cochair \\ HEAD acoustics GmbH, Ebertstrasse 30a, Herzogenrath, 52134, Germany \\ Etienne Parizet, Cochair \\ Laboratoire Vibrations Acoustique, Insa Lyon, 25 bis, av. J. Capelle, Villeurbanne Cedex, 69621, France
}

\section{Invited Papers}

\section{2:00}

2pNSa1. How loud is low frequency noise? Norm Broner (Sincliar Knight Merz, 590 Orrong Road, Armadale, 3143 Melbourne, Australia, nbroner@skm.com.au)

When it comes to assessing noise, the conventional wisdom is that the loudness of a sound will correlate well with its perceived annoyance. On this basis, the A-weighted sound pressure level is also assumed to correlate with the potential percieved annoyance of a sound. This might well be a reasonable assumption for mid range frequency sounds. But for low frequency sounds, particularly below $63 \mathrm{~Hz}$, the assumption that loudness is correlated highly with perceived annoyance breaks down. Indeed, while loudness adaptation may occur for low frequency sound, the annoyance can increase with time. Thus, with time, the annoyance may keep increasing while the apparent loudness decreases!

2pNSa2. Metric assessment of subjective annoyance due to low frequency aircraft noise. Kathleen Hodgdon (Applied Research Laboratory, The Pennsylvania State University, P.O. Box 30, State College, PA 16804, USA, kkh2@psu.edu), Matthew L. Nickerson (IBM, 3039 Corwallis Road, Durham, NC 27709, USA, mlnicker@us.ibm.com), Anthony A. Atchley (The Graduate Program in Acoustics, The Pennsylvania State University, PO Box 30, State College, PA 16804, USA, atchley@engr.psu.edu), Thomas Gabrielson (The Graduate Program in Acoustics, The Pennsylvania State University, PO Box 30, State College, PA 16804, USA, tbg3@psu.edu)

A low frequency noise study was conducted at the Pennsylvania State University to investigate human response to the low frequency content of aviation noise. Metric assessment included level based and loudness metrics, including time-varying loudness. Current predictive noise models and metrics may underestimate the impact of low-frequency noise produced by aviation noise. Assessing the role of low frequency noise on human response will facilitate understanding how this type of noise can impact communities. Indoor recordings of aircraft arrivals and departures at Washington Dulles International Airport made in 2004 were used to assess subjective noise annoyance. These recordings and low-frequency variants of these recordings were reproduced for subjects through the Gulfstream Supersonic Acoustic Signature Simulator II (SASS II); the signatures were then rated for annoyance. Subjective judgments were statistically analyzed and compared against objective metrics that were calculated for each signature. Results are shown that all things being equal higher levels of low-frequency content in aircraft noise can result in increased annoyance in subjects. The C-weighted sound exposure level was found to correlate well with the subjective annoyance response.

\section{2:40}

2pNSa3. Sound character ratings by experienced and naive listeners. Reinhard Weber (Oldenburg University, Institute of Physics - Acoustics, Carl-von-Ossietzky Str. 9-11, 26111 Oldenburg, Germany, Reinhard.Weber@uni-oldenburg.de), Hans Hansen (IRCAM - Sound Perception \& Design, 1 place Igor Strawinsky, 75004 Paris, France, Hans_Hansen@gmx.de)

In the frame of a cross-cultural study on pitch perception of tones in noise, two different groups of participants took part in the listening tests in France. Trained in psycho-acoustic evaluation tasks, members of the laboratory belonged to the first group, whereas the second one consisted of naïve students. 25 stimuli with different tone-to-noise ratios were presented via headphones and, using a semantic differential of 14 adjective pairs, the participants made their judgements on common seven step scales. For both listening groups a PCA of the results led to the same three orthogonal dimensions of the perceptual spaces. They were identified in accordance to Namba's findings as 'pleasant', 'metallic' and 'powerful' factors with an interesting difference: For the trained, the 'metallic' factor, preferably describing the sound character, explained the largest amount of total variance as first factor. The 'pleasant' factor as 'evaluation' dimension took the second place and the 'powerful' factor ranked behind. However, the order of the first two factors was reversed for the untrained listeners. For them the variance of 'pleasant' factor was a bit higher compared to the 'metallic' factor. So the variance in sound character judgements was higher for the trained compared to the untrained listeners. 


\section{Contributed Papers}

3:00

2pNSa4. Optimising the value of sound quality evaluations by observing assessors' driving strategies. Sebastiano S. Giudice (WMG, University of Warwick, Gibbet Hill Road, CV47AL Coventry, UK, S.D.Giudice@warwick.ac.uk), Paul Jennings (WMG, The University of Warwick, International Manufacturing Centre, CV4 7AL Coventry, UK, paul.jennings@warwick.ac.uk), Rebecca Cain (WMG, The University of Warwick, International Manufacturing Centre, CV4 7AL Coventry, UK, R.Cain.1@warwick.ac.uk), Garry Dunne (Jaguar \& LandRover, Jaguar Engineering Center Abbey Road, Whitley, CV3 4LF Coventry, UK, gdunne@jaguar.com), Mark Allman-Ward (Sound Evaluations Ltd, 18 Walsworth Road, Hitchin, Hertfordshire, SG49SP Hitchin, UK, mallman@soundevaluations.com), Roger Williams (Sound Evaluations Ltd, 18 Walsworth Road, Hitchin, Hertfordshire, SG49SP Hitchin, UK, rwilliams@soundevaluations.com)

Traditionally vehicle sound evaluations have been conducted either whilst driving a car or by auditioning fixed test conditions in a listening room. On-road testing provides the right context but the results are often inconsistent and unrepeatable. Furthermore it is not possible to evaluate prototype sounds or carry out back-to-back comparisons. In-room evaluations improve the statistical confidence, but the context of the assessments is unrepresentative. Interactive $\mathrm{NVH}$ vehicle simulators enable assessments to be performed in a setting representative of real appraisals. Accurate sounds can now be generated in real-time, and assessors can adopt a driving strategy that allows their own interpretation of the attributes. The benefits include the opportunity to understand how preferences are formed by assessors, albeit with added complexities. Different assessors may be associating their preferences on different operating conditions which have different acoustical properties. Therefore for engineers to identify the key features that influence perception they need to be able to relate the driving strategies with the subjective preferences. This paper reports on new observational methods which capture assessors' decision making strategies. It demonstrates how these help in relating subjective preferences to vehicle operating conditions and how to design a structured evaluation to reduce sources of experimental error.

3:20

2pNSa5. Perception of simple sounds from an emotional point of view. Anders Sköld (Chalmers University of Technology, Division of Applied Acoustics, Sven Hultinsgata 8a, 41296 Gothenburg, Sweden, anders.skold@chalmers.se), Penny Bergman (Chalmers University of Technology, Division of Applied Acoustics - Chalmers Room Acoustics Group, Sven Hultins gata 8a, 41296 Gothenburg, Sweden, penny@ chalmers.se), Daniel Vastfjall (Chalmers University of Technology, Division of Applied Acoustics - Chalmers Room Acoustics Group, Sven Hultins gata 8a, 41296 Gothenburg, Sweden, daniel.vastfjall@psy.gu.se), Andreas Colebring (Chalmers University of Technology, Division of Applied Acoustics, Sven Hultinsgata 8a, 41296 Gothenburg, Sweden, andreas.colebring@gmail.com)

In sound perception the focus often lies in the cognitive aspect of the sound. We argue that the emotional aspect has to be added to get a fuller picture of sound perception. By using emotions as parameter in design of auditory alerts, one can reach a more accurate reaction to the alert. In this paper we studied the emotional connection to some attributes, common in music psychology, that are possible to describe by simple parameters. Short stimuli were created from these parameters in a factorial test design. The sounds were presented over headphones, with same signal fed to both ears, to 30 participants. The participants were asked to rate level of valence and activation, using a pictorial scale (SAM). Statistical differences was mostly found in ratings of activation, but differences were also shown in valence ratings. Results will be discussed in relation to theories of sound perception as well as music psychology.

3:40-5:00 Posters

Lecture sessions will recess for presentation of poster papers on various topics in acoustics. See poster sessions for topics and abstracts.

\section{Contributed Paper}

\section{5:00}

2pNSa6. Emotional bias for the perception of rising tones. Ana Tajadura-Jiménez (Chalmers University of Technology, Division of Applied Acoustics, Sven Hultinsgata 8a, 41296 Gothenburg, Sweden, ana.tajadura@chalmers.se), Aleksander Väljamäe (Laboratory for Synthetic Perceptive, Emotive and Cognitive Systems (SPECS) - Universitat Pompeu Fabra, Ocata 1, 08003 Barcelona, Spain, aleksander.valjamae@iua.upf.edu), Daniel Vastfjall (Chalmers University of Technology, Division of Applied Acoustics - Chalmers Room Acoustics Group, Sven Hultins gata 8a, 41296 Gothenburg, Sweden, daniel.vastfjall @psy.gu.se)

Sounds with rising and falling intensity are often perceived, respectively, as approaching or receding sound sources. Research has shown the existence of biases both at perceptual and neural levels towards approaching versus receding sounds. It has been suggested that these effects might be accounted to a greater biological salience of approaching sounds. In the present study we investigated whether this asymmetry could be also explained by emotional theories. Approaching and receding tones, followed by neutral, negative or positive photographs, were presented. Participants were required to make a speeded three-alternative forced choice (3AFC) task regarding their feelings towards the photographs. Reaction times (RTs) to this task, together with self-reported valence (pleasantness) and arousal (activation) ratings for the photographs were collected. In addition, participants' electrodermal activity (EDA) and facial electromyography (EMG) when listening to the sounds were measured. Participants responded faster to photographs preceded by approaching tones, especially for photographs with a negative emotional content. Both the intensity range and the period of intensity change of the sounds had a significant effect in RTs. Taken together, these results suggest that approaching sounds induce a greater emotional response on listeners, which might modulate subsequent attentional and perceptual processes.

\section{Invited Papers}

2pNSa7. Survey of sound quality research and applications in the information technology industry. Terry Baird (HewlettPackard Co., 11311 Chinden Blvd, Boise, ID 83714, USA, terry.baird@hp.com)

Traditionally, emission sound power level has been the key attribute measured and reported for describing IT equipment acoustic performance. More recently, designers and manufacturers of IT equipment have come to realize that customer satisfaction or annoyance is not dictated by sound power alone, but is dependent on many sound quality attributes as well. Additionally, sound power level tends 
to be a poor indicator of equipment condition or component degradation where other tools are required for diagnosis and characterization. This paper reviews key research and trends over the last several years using psychoacoustic principles and analyses, as well as case studies of specific applications in the IT sector.

\section{$5: 40$}

2pNSa8. Identification and Classification of Noise Patterns. Roland Sottek (HEAD acoustics GmbH, Ebertstrasse 30a, 52134 Herzogenrath, Germany, claudia.erens@head-acoustics.de)

Sound quality evaluation is a challenge due to spectral and temporal structures of noise. Tonal components, howling sounds and modulated signals are often the cause of customer complaints. Thus, besides frequency-weighted level like $\mathrm{dB}(\mathrm{A})$ or loudness, additional parameters are required. Besides time-varying loudness, other psychoacoustic parameters like sharpness and roughness can be used for sound quality evaluation. Sharpness considers the amount of high frequency components of a noise, and roughness evaluates modulation characteristics. In addition, a metric combining modulation spectral analysis with loudness calculation has been introduced. Some years ago, a "Hearing Model" was developed with the intention of explaining and describing psychoacoustic effects. Applying the Hearing Model to sound quality tasks allows evaluating the spectral and temporal patterns of a sound ("Relative Approach" analysis) where absolute level or loudness is often without significance. The Relative Approach analysis emphasizes all relevant signal components concerning human auditory perception: tonal and transient signals. For extracting and evaluating individual patterns further signalprocessing steps are necessary. The paper presents different methods for effective sound quality evaluation of noise and their application to several examples.

\section{6:00}

2pNSa9. The Calibration and Validation of a Binaural Room Scanning System Used for Subjective Evaluation of Automotive Audio Systems. Sean E. Olive (Harman International Industries, Inc., R\&D Group, 8500 Balboa Blvd, Northridge, CA 91329, USA, solive@ harman.com), Todd Welti (Harman International Industries, Inc., R\&D Group, 8500 Balboa Blvd, Northridge, CA 91329, USA, twelti@harman.com)

Binaural room scanning (BRS) is a method of capturing the acoustical response of audio systems in rooms and automotive cabins as a set of binaural impulse responses that are stored and later reproduced through head-tracking headphones. All BRS systems have errors that without careful calibration limit their usefulness for subjective measurements of sound quality. This paper discusses a method for calibrating and testing a proprietary BRS system based on how well it reproduces listeners' preference ratings of different automotive audio systems made in situ. A group of trained listeners gave preference ratings for 5 different equalizations of a high quality automotive audio system made both in situ and through the BRS capture/playback system. The tests were repeated in mono and stereo using 6 individualized listener calibrations. The results can be summarized as follows: there were no significant differences in preferences between the in situ and BRS-based evaluations for either the mono or stereo tests. A significant interaction was found between the different equalizations and individualized calibrations that were largely confined to 1 particular listener calibration. The results suggest that a generalized BRS calibration may provide sufficiently accurate preference measurements of audio system sound quality.

\section{Contributed Papers}

\section{6:20}

2pNSa10. Head and torso simulators for acoustic measurements Gunnar Rasmussen (G.R.A.S. Sound \& Vibration A/S, Skovlytoften 33, 2840 Holte, Denmark, gr@gras.dk)

Head and torso simulators which reproduce the acoustical effect of a median human adult, including diffraction and direction-dependent transformation from free field to the ear drum as well as allow for objective testing of sound systems involving hearing aids, headphones, earphones, telephones, mobile phones, headsets and sound quality type of measurements may be realized based on different philosophies. It may be based on geometrically simplified shape or mean values of individuals. The geometrically simplified shape will appear like no human being. The mean values may result in non human like features, while average anthropometric dimensions of an adult human may offer data closer to a subjective test. Especially the shape of the pinna may often be critical to test of earphones and headphones as well as mobile phones. Subjective tests on groups of people may be supplemented with objective tests on a manikin for reference and quality control purposes. Variations in pinna size may have significant influence on the measurement of earphones as well as leakage in telephone testing. The influence of the dress worn may also be of significance especially for the head related transfer function and on the directional response in the middle frequency range.

\section{6:40}

2pNSa11. Capturing blocked-entrance binaural signals from open-entrance recordings. Dorte Hammershøi (Acoustics, Aalborg University, Fredrik Bajers Vej 7 B5, 9220 Aalborg Ø, Denmark, dh@es.aau.dk), Pablo F. Hoffmann (Acoustics, Aalborg University, Fredrik Bajers Vej 7 B5, 9220 Aalborg Ø, Denmark, pfh@es.aau.dk), Søren K. Olesen (Acoustics, Aalborg University, Fredrik Bajers Vej 7 B5, 9220 Aalborg Ø, Denmark, sko@es.aau.dk), Per Rubak (Acoustics, Aalborg University, Fredrik Bajers Vej 7 B5, 9220 Aalborg Ø, Denmark, pr@es.aau.dk)

Binaural recordings enable us to capture all sound attributes including spatial information, room effect, and source characteristics in a given environment. It has been shown that blocked-entrance binaural recordings provide advantages over open-entrance recordings, primarily because the blocked-entrance recordings is not influenced by the ear canal acoustics of the individual for which it is recorded. However, blocking the ear canal for recoding imposes an obvious disruption to normal hearing conditions, which may be unacceptable for applications in which binaural audio capturing is desired but without interfering the individual's hearing and doing. In this work we propose a strategy for the recording of binaural audio with minimal hearing interference, and for transforming these recordings to blockedentrance versions that are more suitable for analysis and reproduction of binaural audio in a more general context. To this purpose, equalization filters are derived from the ratio between blocked and open ear canal transfer functions. Different transfer-function measuring techniques and inverse filtering methods are evaluated. 


\section{Invited Paper}

7:00

2pNSa12. PsySound3: a program for the analysis of sound recordings. Densil Cabrera (University of Sydney, Faculty of Architecture, Design and Planning, NSW 2006 Sydney, Australia, densil@usyd.edu.au), Sam Ferguson (University of Sydney, Faculty of Architecture, Design and Planning, NSW 2006 Sydney, Australia, samferguson@ihug.com.au), Farhan Rizwi (Empirical Musicology Group, University of New South Wales, NSW 2052 Sydney, Australia, farhan.rizwi@unswalumni.com), Emery Schubert (Empirical Musicology Group, University of New South Wales, NSW 2052 Sydney, Australia, E.Schubert@unsw.edu.au)

This paper demonstrates the sound analysis software PsySound3, which was written by the authors. The software currently includes a range of DSP-based analysis techniques (e.g., spectrum, cepstrum, autocorrelation, Hilbert transform, sound level meter emulator), as well as implementations of psychoacoustical algorithms often associated with sound quality (e.g., loudness, sharpness, loudness fluctuation, roughness, pitch, binaural attributes). In some cases, PsySound3 makes available multiple models of the one auditory attribute - for example it implements dynamic and static loudness models using Erb- and Bark-based auditory filters. The program is extensible, and so has the potential to allow researchers to share their analysis models using a common interface. PsySound3 is written in Matlab, and is also available as a stand-alone program. The software is freely available from www.psysound.org.

\section{Contributed Papers}

\section{$7: 20$}

2pNSa13. Hartis, a re-synthesis tool for vehicles sound design. Gaylord Desoeuvre (GIST, 62 bis avenue André Morizet, 92100 Boulogne Billancourt, France, gaylord.desoeuvre@ext.mpsa.com), Florent Richard (PSA Peugeot Citroën, Centre Technique de Vélizy, Route de Gisy, 78943 VélizyVillacoublay, France, florent.richard@mpsa.com), Vincent Roussarie (PSA Peugeot Citroën, Centre Technique de Vélizy, Route de Gisy, 78943 VélizyVillacoublay, France, vincent.roussarie@mpsa.com), Marie Céline Bezat (PSA Peugeot Citroën, Centre Technique de Vélizy, Route de Gisy, 78943 Vélizy-Villacoublay, France, marieceline.bezat@mpsa.com)

For more than thirty years, the main goal for the automotive acoustic engineer was the car's noise level reduction. Within new customers' behaviours, the car manufacturers have now to put products sound quality at the same level as its aesthetic qualities or ergonomics. To improve customer's satisfaction, one solution is to increase sensory coherence and perceived quality by controlling the sound identity and character of a vehicle. To reach this new objective, the Research Department of PSA Peugeot Citroën is developing his own sound design software HARTIS [1] (HArmonics Real TIme Synthesis). HARTIS is a complete re-synthesis tool useful in many stages of vehicle sound design process. In this paper we first present the last version of HARTIS fully interfaced for an easy use in project teams. The second part of the paper presents new integrated module that deals with impulsional noise for diesel application and other sources models. Each type of source can be controlled and filtered to recreate different driving situation.
References [1] Sound design in car passenger compartment Process and tool for the control of engine sound character. V. Roussarie, F. Richard, Journées du Design Sonore 2004.

\section{$7: 40$}

2pNSa14. Ride quality and noise in high speed elevators. Mauro Pierucci (San Diego State University, 5500 Campanile drive, San Diego, CA 92182-3020, USA, mpierucci@mail.sdsu.edu), Michael Frederick (San Diego State University, 5500 Campanile drive, San Diego, CA 92182-3020, USA, mike.frederick-1@nasa.gov)

Ride quality in an elevator is the latest "hot topic" in elevator design and construction. In skyscrapers being built in Asia, Dubai and the US, the new elevators travel close to and beyond $10 \mathrm{~m} / \mathrm{s}$. The rides to the highest floors will last well over one minute. From a comfort point of view, it is important that the ride be as smooth and quiet as possible. There are many factors that influence the vibration of the elevator and the concomitant noise. In this paper we will examine the sudden forces that the elevator experiences as it passes each floor. The side forces on the elevator are caused by the asymmetric flow field about the elevator cab. The pressure and streamline flow field was evaluated by using the CFD code FLUENT. Comparison between the numerical and the measured results are given and the pressure disturbances caused by the passage of the elevator and its appendages are discussed together with some solutions to alleviate the pressure disturbances. 


\title{
Session 2pNSb
}

\section{Noise, Physical Acoustics, and EURONOISE: Aeroacoustics III (Poster Session)}

\author{
Philip Morris, Cochair \\ Penn State University \\ Christophe Bailly, Cochair \\ Ecole Centrale de Lyon
}

\begin{abstract}
All posters will be on display from 3:40 p.m. to 5:20 p.m. To allow contributors an opportunity to see other posters, contributors of odd-numbered papers will be at their posters from 3:40 p.m. to 4:30 p.m. and contributors of even-numbered papers will be at their posters from 4:30 p.m. to 5:20 p.m.
\end{abstract}

\section{Contributed Papers}

2pNSb1. Nonlinear propagation of spark-generated $\mathrm{N}$-waves in atmosphere: theoretical and experimental assessment of the shock front structure. Petr V. Yuldashev (Center for Industrial and Medical Ultrasound, Applied Physics Lab., University of Washington, 1013 NE 40th St., Seattle, WA 98105, USA, petr@acs366.phys.msu.ru), Mikhail V. Averiyanov (Center for Industrial and Medical Ultrasound, Applied Physics Lab., University of Washington, 1013 NE 40th St., Seattle, WA 98105, USA, misha@acs366.phys.msu.ru), Vera A. Khokhlova (Center for Industrial and Medical Ultrasound, Applied Physics Lab., University of Washington, 1013 NE 40th St., Seattle, WA 98105, USA, vera@ acs366.phys.msu.ru), Oleg A. Sapozhnikov (Center for Industrial and Medical Ultrasound, Applied Physics Lab., University of Washington, 1013 NE 40th St., Seattle, WA 98105 , USA, oleg@acs366.phys.msu.ru), Ollivier Sebastien (Ecole Centrale de Lyon, LMFA, UMR CNRS 5509, Ecully, 69134 Lyon, France, Sebastien.Ollivier@ec-lyon.fr), Philippe Blanc-Benon (Ecole Centrale de Lyon, LMFA, UMR CNRS 5509, Ecully, 69134 Lyon, France, Philippe .Blanc-Benon@ec-lyon.fr)

Extensive outdoor and laboratory-scale experiments on sonic boom propagation in turbulent atmosphere have shown that shock wave amplitude and rise time are important parameters responsible for sonic boom annoyance. However, accurate measurement of the shock front structure with standard microphone remains a challenge due to the broadband spectrum of the $\mathrm{N}$-wave shock front. In this work the experimental setup utilizing a spark source has been designed and built to investigate nonlinear

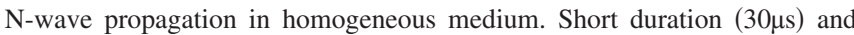
high amplitude $(1 \mathrm{kPa})$ spherically divergent $\mathrm{N}$-waves were generated. In addition to acoustic measurements with $1 / 8^{\prime \prime} \mathrm{B} \& \mathrm{~K}$ microphones, the shadowgraphy method using short duration flash lamp (20 ns) and CCD camera was employed to assess the shock front structure at different distances from the spark. It is shown that the shock rise time measured by the shadowgraphy method was in a good agreement with the theoretical predictions and it was 10 times shorter than in microphone measurements. The widening of the shock in acoustic measurements was therefore due to the limited bandwidth of the microphone. The combination of modeling, acoustic and optical measurements provided an accurate calibration of the shock wave measuring system. [Work supported by RFBR and INTAS.]

2pNSb2. Doppler effect in aeroacoustics. Jean Varnier (ONERA, 29 avenue de la Division Leclerc, 92320 Châtillon, France, jean.varnier@onera .fr)

Doppler effect in aeroacoustics The prediction of acoustic fallout caused at the ground level by aircraft in flight or by launchers at take-off sets the problem of the distortion of the emitted sound spectrum by the Doppler effect. Former studies applied to this problem use a geometrical formalism based on simplifying hypotheses such as homogeneous atmosphere, rectilin- ear trajectory and constant velocity of the sound source. Other studies consider the supersonic case using a model of spherical waves inside the Mach cone. These approaches are in fact not well adapted to the real cases, and the literature does not give many practical examples concerning this topic. In this study, we propose a new time approach of the Doppler effect that allows to avoid the complexity of the geometrical models and to take into account real atmosphere, curvilinear trajectories or accelerations of moving sound sources. This model allows to easily calculate the frequency shift and the change of acoustic level of the signal recorded at a given hearing point, in both cases of subsonic and supersonic sound sources. We present a first validation of this method applied to signals recorded in Kourou during the atmospheric phase of Flight 521 of Ariane 5.

2pNSb3. Noise computation of an axisymmetric free jet using general purpose CFD code. Péter Tóth (Dep. of Fluid Mechanics, Budapest Univ. of Technology and Economics, Bertalan Lajos street 4-6, 1111 Budapest, Hungary, toth@ara.bme.hu), Máté Márton Lohász (Dep. of Fluid Mechanics, Budapest Univ. of Technology and Economics, Bertalan Lajos street 4-6, 1111 Budapest, Hungary, lohasz@ara.bme.hu)

The increasing interest in the noise prediction of the turbulent flows is leading the general purpose Computational Fluid Dynamics (CFD) software developers to integrate noise predicting methods into their code. However the aeroacoustic simulation of free shear flows, where the mean source of noise is the turbulent fluctuations in the free space is challenging with a general purpose CFD application. The aim of this work is to investigate the capabilities of commercial CFD software in the field of noise prediction of free shear flows. The important acoustical properties of a low Reynolds number, low Mach number axisymmetric (2D) jet is computed by means of hybrid Computational Aeroacoustic method using Fluent. The noise source is computed without modeling instantaneously using the compressible solver of the code, and the far field acoustical data is evaluated by the Ffowcs-Williams-Hawkings acoustical analogy. The results is compared to the Direct Numerical Simulation of [Jiang2004 J. Sound Vib.] in terms of frequency spectra in the far field and directivity pattern of the jet.

2pNSb4. Application of RANS modelling and LES to the prediction of noise from a simple cold propane jet. Tanya Stanko (The University of Leeds, School of Process, Environmental and Materials Engineering, LS2 9JT Leeds, UK, pmtss@leeds.ac.uk), Michael Fairweather (The University of Leeds, School of Process, Environmental and Materials Engineering, LS2 9JT Leeds, UK, M.Fairweather@leeds.ac.uk), Mohamed Pourkashanian (The University of Leeds, School of Process, Environmental 
and Materials Engineering, LS2 9JT Leeds, UK, m.pourkashanian @ leeds.ac.uk), Derek Ingham (The University of Leeds, School of Process, Environmental and Materials Engineering, LS2 9JT Leeds, UK, D.B.Ingham @leeds.ac.uk)

Numerical simulation, combining computational fluid dynamic and acoustic models, is useful as an aeroacoustic tool that is effective in identifying components or surfaces that generate significant amounts of noise, and can thereby provide opportunities for early design changes. In this work, numerical solutions of a cold turbulent jet of propane issuing from a circular nozzle are used to provide velocity information at a Reynolds number of 68,000 . Predictions provided by large eddy simulation are compared with Reynolds-averaged Navier-Stokes solutions derived using a second-moment turbulence closure, as well as with experimental data available in the literature. Reasonable agreement with data is found, and these solutions are used subsequently as the basis for noise predictions. The first acoustic model employed is based on Lighthill's theory using the Goldstein broadband noise source formalisation that postulates axisymmetric turbulence superposed on the mean flow. The second approach is based on Ffowcs-Williams and Hawkings theory which implies that the far field sound is computed from an integral formulation with all quantities evaluated on a control surface, with the control surface itself assumed to enclose all nonlinear flow effects and noise sources. The simulation results reported in this paper confirm previous experimental works presented in the literature demonstrating that jet noise is modified by inlet conditions.

2pNSb5. Uncertainties of Measured and Calculated Aircraft Noise and Consequences in Relation to Noise Limits. Georg Thomann (Empa, Ueberlandstrasse 128, 8600 Duebendorf, Switzerland, georg.thomann@empa .ch)

The uncertainties of measuring and calculating aircraft noise have been analysed in a thesis at the ETH Zurich. The thesis provides information and methods for estimating uncertainties. It shows possibilities for handling these uncertainties and provides guidance to legal and administrative bodies on how to deal with such uncertainties when applying the legal noise limits. To estimate the uncertainties of calculations and measurements the Swiss aircraft noise calculation program FLULA2 and a great deal of measured data have been analysed. Using radar data it is possible to achieve a standard uncertainty of the calculated Leq between $0.5 \mathrm{~dB}$ for day-time and $1.0 \mathrm{~dB}$ for night-time. The analyses also show that the uncertainty of measurements at automated stations is in the same order of magnitude as the calculations. Therefore the annual calculations and measurements of aircraft noise show no significant deviations. Furthermore with FLULA2 it is possible to present the uncertainty at a confidence level of $90 \%$. It is now up to administrative and legal bodies to set up rules on how to account for uncertainties of calculations in evaluating noise situations close to the legal noise limits.

2pNSb6. Acoustic test facility for aero engine fans. Yuri Khaletskiy (Central Institute of Aviation Motors, 2, Aviamotornaya Str., 111116 Moscow, Russian Federation, noise@ciam.ru), Victor Mileshin (Central Institute of Aviation Motors, 2, Aviamotornaya Str. 111116 Moscow, Russian Federation, mileshin@ciam.ru), Rollan Shipov (Central Institute of Aviation Motors, 2, Aviamotornaya Str., 111116 Moscow, Russian Federation, noise@ ciam.ru)

The facility for acoustic tests of aero engine fans in conditions of anechoic chamber is created in Central Institute of Aviation Motors (Moscow). The facility is designed for testing models of single rotating fans and counter rotating fans. The measurements of the sound are carried out in forward and rearward semi-spheres simultaneously. Design features of the anechoic chamber and its acoustic performance are presented. Also a fan drive system and systems of acoustic and aerodynamic measurements are described. In particular fan rotor max rotation frequency makes up 13,000 rpm at each shaft max power $2.5 \mathrm{MW}$. The rig acquisition system allows checking in real time results of complete three-octave analysis of machine noise on all 24 channels in a measurement frequency range of $0 œ 80 \mathrm{kHz}$.
2pNSb7. Nonlinear Propagation of Screech Noise. Jothi Punekar(School of Engineering and Materials, Queen Mary, University of London, 327 Mile End Road, E1 4NS London, UK, j.punekar@qmul.ac.uk), Eldad Avital (School of Engineering and Materials, Queen Mary, University of London, 327 Mile End Road, E1 4NS London, UK, e.avital@qmul.ac.uk)

This study investigates the non-linear propagation of screech noise emitted by supersonic jets. Supersonic jet noise consists of three components: mixing, broadband and screech noise. In order to generate broadband and screech noise the jet has to expand imperfectly, meaning shock waves are formed inside the potential core and interact with the surrounding shear layer. The feedback between the generated noise and the nozzle cause the screech noise pattern. It is associated with high frequency (annoying to the human ear). Near field screech noise obtained from Large Eddy Simulation (LES) of circular supersonics jets is investigated using the Burgers equation, taking into account thermo-viscous effects. The Burgers equation is commonly used to investigate non-linear propagation effects. The initial screech signal is found to consist of two main frequency modes. Significant nonlinear effects appear during propagation although the pressure amplitude is no more than a few percentages of the ambient pressure. Saw tooth waves are generated and the amplitude is found to decrease as $1 / \mathrm{sqrt}(\mathrm{t})$ for low viscous medium according to Lighthill' 'bunching' theory. Further high frequencies are also generated. Finally, geometrical attenuation is discussed by transforming the plane wave propagation to cylindrical form.

2pNSb8. Instability wave control in a subsonic round jet. Georgy Faranosov (TsAGI, Acoustic Division, 17, Radio str, 105005 Moscow, Russian Federation, georgefalt@ rambler.ru)

It is generally accepted that one of the main sources of acoustic radiation from a turbulent jet is due to spatial instability wave packets propagating downstream within the jet. This approach has enabled to explain and predict the principal features of sound radiated by a supersonic jet. Therefore the problem of noise control for the jet could be considered as a problem of instability wave control. Instability wave developed in the tone-excited jet issued from axisymmetric, semi-infinite duct is considered. The flow field in the vicinity of the nozzle edge is investigated for the purpose of identifying instability wave parameters. Instability wave turned out to be damped completely on the condition that the amplitude and the phase of the external forcing are chosen in accordance with those of the instability wave. The robust strategy of instability wave experimental suppression based on the near field measurements is formulated. Similar problem in two-dimensional case was recently analyzed in Kopiev \& Faranosov (Acoust. Phys. 2008, V.54, N.2).

2pNSb9. Decomposition of the Lighthill source term and analysis of acoustic radiation from mixing layers. Florent Margnat (Arts et Métiers Paris Tech - Sinumef Lab, 151 bd de l'Hopital, 75013 Paris, France, florent.margnat@paris.ensam.fr), Véronique Fortuné (Univ. Poitiers, CNRS Laboratoire d'Etudes Aérodynamiques, Bat. K - 22 avenue du recteur Pineau, $86022 \quad$ Poitiers, France, veronique.fortune@lea.univ-poitiers.fr), Peter Jordan (Univ. Poitiers, CNRS Laboratoire d'Etudes Aérodynamiques, 43, rue de l'Aérodrome, 86036 Poitiers, France, peter.jordan@lea.univ-poitiers.fr), Yves Gervais (Laboratoire d'Etudes Aérodynamiques (LEA), Université de Poitiers - ENSMA - CNRS, Bâtiment K, 40 Avenue du Recteur Pineau, 86022 Poitiers, France, yves.gervais@lea.univ-poitiers.fr)

Acoustic radiation from jet flow has been studied extensively by means of theoretical, experimental and numerical approaches over the past decades. Unfortunately, the mechanisms responsible for the production of sound by unbounded turbulence in subsonic flows remain unclear. For advancing our fundamental understanding of these mechanisms, the development of specific analysis tools is needed. Our study is based on a decomposition of the Lighthill source term, which is known to contain all the existing links between the fluid flow and the acoustic field. Ten subterms are written with the help of physically meaningful quantities such as velocity, density, dilatation and vorticity. The methodology is tested through the two-dimensional compressible mixing layer flow in spatial development, at a Reynolds number of 400 and a Mach number of 0.25 . A direct numerical simulation of the flow and its acoustic radiation is performed and used as a reference solution. 
Acoustic field generated by each source terms is predicted computing the integral solution of Lighthill's equation. Effect of the compressibility on the balance between the contribution of major subterms is of key importance. Directivity and convective effects are identified as well.

2pNSb10. Acoustic and aerodynamic dissipations induced by a sound wave-impacted Helmholtz resonator. Jean-Michel Roche (ONERA, BP 72 - 29 avenue de la Division Leclerc, 92322 Châtillon, France, jean-michel.roche@onera.fr), Laurent Leylekian (ONERA, BP 72 - 29 avenue de la Division Leclerc, 92322 Châtillon, France, laurent.leylekian@onera.fr), François Vuillot (ONERA, BP 72 - 29 avenue de 1a Division Leclerc, 92322 Châtillon, France, francois.vuillot@onera.fr)

The purpose of the work introduced herein is to study the dissipation of the acoustic energy carried by sound waves, by a Helmholtz resonator. Twodimensional direct numerical simulations (DNS) are performed with the CEDRE code, a Navier-Stokes equations solver developed by Onera, for a resonator with a $0.8 \times 0.8 \mathrm{~mm}$ square opening. The incident sound pressure level (SPL) varies between $100 \mathrm{~dB}$ and $150 \mathrm{~dB}$ : it is checked that when the SPL is low, the acoustic energy is dissipated in the resonator opening by viscous wall friction, whereas when the SPL is high, the acoustic energy is converted in kinetic energy carried by micro-vortices induced at the opening mouths. However, when the SPL takes intermediate values, both mechanisms coexist. Thus, for each case, we aim at splitting the aeraoacoustic field into respective acoustic and aerodynamic contributions, and at evaluating the ratio between these two distinct mechanisms in order to quantify their relative weight on the global dissipation. Our results are compared with those obtained by Tam et al. for the same geometry. A three-dimensional study for a Helmholtz resonator with a cubic opening is eventually considered.

2pNSb11. The multiple side-branch system as a model for a corrugated pipe. Devis Tonon (Technische Universiteit Eindhoven, CC.2.25, N-LAAG, Postbus 513, 5600 MB Eindhoven, Netherlands, d.tonon@tue.nl), Stefan Belfroid (TNO Science and Industry, Stieltjesweg 1 PO Box 155, 2600 AD Delft, Netherlands, stefan.belfroid@tno.nl), Jan F. Willems (Technische Universiteit Eindhoven, CC.2.25, N-LAAG, Postbus 513, 5600 MB Eindhoven, Netherlands, J.F.H.Willems@tue.nl), Avraham Hirschberg (Technische Universiteit Eindhoven, CC.2.25, N-LAAG, Postbus 513, 5600 MB Eindhoven, Netherlands, a.hirschberg@tue.nl)

Corrugated pipes are used as flexible risers in offshore natural gas production and for vacuum cleaners. Such pipes can display whistling. As theoretical models are available to predict the aero-acoustical behaviour of closed side branches [1], we consider a row of equally spaced closed side branches along a pipe, as a model for a corrugated pipe. We consider side branches with a diameter $\mathrm{D}$ and depth $\mathrm{L}$ equal to the main pipe diameter, placed at a distance of three diameters from each other. For systems of 11 or 12 side branches the lowest resonance modes are reasonably well predicted by assuming $n(\lambda / 2)$ standing wave modes with an effective speed of sound $c_{\text {eff }}$ [2]. Whistling is observed for the $\mathrm{n}=2$ and $\mathrm{n}=3$ modes with a pressure fluctuation amplitude $p^{\prime} /\left(\rho_{0} \mathrm{c}_{0} \mathrm{U}_{0}\right)=\mathrm{O}\left(2 \times 10^{-2}\right)$ similar to that observed for sharp edges corrugations in a corrugated pipe (with $\rho_{0}$ the fluid density, $c_{0}$ the speed of sound and $U_{0}$ the main flow velocity). The Strouhal number of these oscillations is $S r_{D}=(f \cdot D) / U_{0} \simeq 0,70$. This is higher than typical values observed for corrugated tubes [3]. References: [1] J.C. Bruggeman et al., J. Sound and Vibration 1991 150(4) 371-393. [2] J.W. Elliot, in "Lecture Notes on the Mathematics of Acoustics" M.C.M. Wrigth, Imperial College Press, London (2005). [3] S. Belfroid et al., ASME paper PVP2007-26503.

2pNSb12. The sound wave reflection from the corrugated plane streamlined by the low Mach number shear flow (generalized Miles mechanism). Mikhail Mironov (Andreev Acoustics Institute, Shvernik, 4, Moscow, 117036 Moscow, Russian Federation, mironov@akin.ru), Ivan Belyaev (Central Aerohydrodynamics Institute (TsAGI), Acoustic Division, 17 Radio str., 105005 Moscow, Russian Federation, belyaev_iv@bk.ru), Vasilisa Solntseva (Andreev Acoustics Institute, Shvernik, 4, Moscow, 117036 Moscow, Russian Federation, mironov@akin.ru)

The plane sound wave reflection from the periodically corrugated plane streamed by the parallel shear flow is studied. The incident wave scatters on corrugations and gives both the mirror reflected sound wave and two inhomogeneous nonpropagating in the normal to the plane direction waves. One of the inhomogeneous waves goes in the same direction along the plane of the flow and the second one in the opposite direction. The first inhomogeneous wave interacts with critical layer of flow according to Miles mechanism (which was originally applied to the generation of surface waves by the wind (JFM: 1957, 1959)) and reflects from this layer back to the plane. After that this inhomogeneous wave scatters from the corrugated plane and gives some additional part to the mirror reflected sound wave. The total reflection coefficient of sound wave appeared to be more than 1 . The angle dependence of the reflection coefficient is analyzed. The equivalent impedance is calculated. The obtained amplification is compared with thermal and viscous surface losses

2pNSb13. On a stability of the shear flow with a tangential discontinuity in the presence of a small scatterer. Nikolay Kanev (Andreev Acoustics Institute, Shvernik, 4, Moscow, 117036 Moscow, Russian Federation, nikolay.kanev@mail.ru), Mikhail Mironov (Andreev Acoustics Institute, Shvernik, 4, Moscow, 117036 Moscow, Russian Federation, mironov@akin.ru)

It is well known a tangential discontinuity of velocity in the shear flow is unstable. That instability is convective i.e. all disturbances of the tangential discontinuity grow exponentially but they are carried away by the flow downstream so that after all the amplitude of sound field generated by the tangential discontinuity will decrease at any fixed point. In the presented paper we consider the problem when a small scatterer is placed near the tangential discontinuity. It appears this system can be absolutely unstable under the definite position of the scatterer. It means that the appearance of any disturbance of the tangential discontinuity will result in rising of the amplitude of sound field at every point of space. The cause of absolute instability is a feedback provided by the scatterer and the type of instability does not depend on the size of the scatterer. An absolutely unstable system behaves as an autogenerator and radiates tone or multitone sound. So the obtained result explains the possible mechanism of noise generation in systems with tangential discontinuities and gives some hints about noise control in such systems.

2pNSb14. Spectral attenuation of sound in dilute suspensions with nonlinear particle relaxation. Max Kandula (ASRC, Mailstop ASRC5211, NASA Kennedy Space Center, Kennedy Space Center, FL 32899, USA, max.kandula-1@ksc.nasa.gov), Michael Lonergan (NASA, Kennedy Space Center, Mailstop NE-M1, Kennedy Space Center, FL 32899, USA, michael.lonergan-1@ksc.nasa.gov)

Theoretical studies on the dissipation and dispersion of sound in twophase suspensions have been briefly reviewed. Previous studies (theory of Temkin \& Dobbins, JASA, Vol. 40, No. 2, pp. 314-324, 1966,) on the sound attenuation in particle-laden flows under Stokesian drag and conductioncontrolled heat transfer have been extended to accommodate the nonlinear drag and heat transfer. It has been shown that for large particle-to-fluid density ratio, the particle Reynolds number bears a cubic relationship with $\{$ lomega $\}\{t$ tau, where $\{$ lomega $\}$ is the circular frequency and $\{\mid$ tau $\}$ the Stokesian particle relaxation time. This dependence leads to the existence of a peak value in the linear absorption coefficient occurring at a finite value of \{lomega\} $\{\backslash t a u\}$. Comparison of the predictions with the test data of Norum (AIAA-2004-2976) for the spectral attenuation of sound with water injection in a perfectly expanded supersonic jet (Mach number of 1.45) shows a satisfactory trend of the proposed theory. It is found that the nonlinear particle relaxation processes (particle drag and heat transfer) are primarily responsible for reduction in the linear absorption coefficient of sound at high frequencies.

2pNSb15. Aeroacoustic simulation of automotive ventilation outlets. Jean-Luc Adam (Renault SAS, 1 avenue du golf, 78288 Guyancourt, France, jean-luc.adam@renault.com), Denis Ricot (Renault SAS, 1 avenue 
du golf, 78288 Guyancourt, France, denis.ricot@renault.com),Flavien Dubief (Renault SAS, 1 avenue du golf, 78288 Guyancourt, France, flavien.dubief@renault.com), Christine Guy (Ligeron, Les Algorithmes Bâtiment Euclide, 91194 Saint Aubin, France, christine.guy@ligeron.com)

In this work we have numerically studied aeroacoustics of automotive ventilation outlets. Simulations are performed with the CFD software PowerFLOW based on Lattice Boltzmann method (LBM). Low dissipative LBM scheme enables to compute aeroacoustic sources generated by turbulence fluctuations and to propagate them in the same simulation. In a first step we validate the ability of LBM for propagating acoustic waves in ducts and radiating them at open end terminations. In a second step, aeroacoustic simulations on automotive vents will be presented and compared with experimental data obtained from a DoE (Design of Experiment). This DoE is based on an idealized outlet with variing parameters (number and lenght of grid blades, grids spacing ...) which gives 18 distinct geometrical configurations. All these configurations have been simulated with PowerFLOW and measured with a new test facility (built in the Renault Research Department). The large number of tested geometries and the statistical analysis of the DoE give an accurate idea of the capability of PowerFLOW, and more generally LBM, to correctly simulate the generation and propagation of aeroacoustic sources for a complex geometry. Results will be presented and discussed.

2pNSb16. Increase of sound proofing of air duct. Igor Shubin (Research Institute of Building Physics (NIISF RAABS), 21, Lokomotivny pr., 127238 Moscow, Russian Federation, marina_niisf@mail.ru), Vladimir Gusev (Research Institute of Building Physics (NIISF RAABS), 21, Lokomotivny pr., 127238 Moscow, Russian Federation, marina_niisf@mail.ru), Marina Pestereva (Research Institute of Building Physics (NIISF RAABS), 21, Lokomotivny pr., 127238 Moscow, Russian Federation, marina_niisf@mail.ru)

One of ways of noise protection from transit air duct is increase of sound insulation of air duct by different acoustical materials. The main interest of this work is in comparative analysis of acoustic efficiency different thermal insulation materials for use as sound insulation coverings. The report con- tains analysis of the distribution of noise through round and rectangular air ducts. There was discovered different tipes of thermal insulation materials such as easy made foam materials and fibrous materials. Tests are executed in the reverberation chamber. The received results have shown advantage of a fibrous material.

2pNSb17. On the correlation of the acoustic signal of microphones mounted on a flat plate to the turbulence of an impinging jet. Christoph Reichl (Arsenal Research - Austrian Research Centers, Giefinggasse 2, 1210 Wien, Austria, Christoph.Reichl@arsenal.ac.at), Michelle Boeck (Arsenal Research - Austrian Research Centers, Giefinggasse 2, 1210 Wien, Austria, michelle.boeck@arsenal.ac.at), Wolfgang Tilser (Arsenal Research - Austrian Research Centers, Giefinggasse 2, 1210 Wien, Austria, wolfgang.tilser@arsenal.ac.at), Hermann Lang (Arsenal Research - Austrian Research Centers, Giefinggasse 2, 1210 Wien, Austria, hermann.lang@arsenal.ac.at), Klaus Haindl (AKG Acoustics GmbH, Lemboeckgasse 21-25, 1230 Wien, Austria, HaindlK@akg.com), Friedrich Reining (AKG Acoustics GmbH, Lemboeckgasse 21-25, 1230 Wien, Austria, ReiningF@akg.com), Martin Opitz (AKG Acoustics GmbH, Lemboeckgasse 21-25, 1230 Wien, Austria, OpitzM@akg.com)

An axial symmetric fan driven by a brushless actuator with variable frequency is used to generate a turbulent circular jet leading to typical corevelocities of around $10 \mathrm{~m} / \mathrm{s}(\operatorname{Re}=99000)$. The jet impinges on a flat plate. Turbulence is generated using different sets of turbulence generators in the pipe consisting of meshes, rods, cubes and spikes. The turbulent flow field downstream of the pipe outlet is measured using two-axis hot wire anemometry with a temporal resolution of a minimum of $48 \mathrm{kHz}$. The CTA probe is positioned using an automated three-axis traversing system. 12 Microphones are mounted on a flat plate and are used to capture the wall pressure fluctuations. CFD calculations are performed for the different turbulence generators and are compared to the acoustic and CTA signals. An important aspect of the work is the simultaneous recording of both the CTA- and the acoustic signals in the experimental and numerical approach. This allows for the calculation of correlation patterns between turbulence and the acoustic signals of the plate mounted microphones. 


\title{
Session 2pNSc
}

\section{Noise, ASA Committee on Standards, and EURONOISE: Comparing Noise Regulations and Codes in USA and Europe (Poster Session)}

\author{
Robert Bruce, Cochair \\ CSTI, 15835 Park Ten Place, Suite 105, Houston, TX 77084-5131, USA \\ Wolfgang Probst, Cochair \\ DataKustik GmbH, Gewerbering 5, 86926 Greifenberg, Germany
}

\begin{abstract}
All posters will be on display from 3:40 p.m. to 5:20 p.m. To allow contributors an opportunity to see other posters, contributors of odd-numbered papers will be at their posters from 3:40 p.m. to 4:30 p.m. and contributors of even-numbered papers will be at their posters from 4:30 p.m. to 5:20 p.m.
\end{abstract}

\section{Contributed Papers}

2pNSc1. Noise limits and noise levels along motorways in Europe Wiebe Alberts (Rijkswaterstaat: Centre for Transport and Navigation, Postbus 5044, 2600 GA Delft, Netherlands, wiebe.alberts@ rws.nl)

Environmental noise caused by traffic is one of the main local environmental problems in Europe. The National Road Authorities (NRA's), combined in the Conference of European Directors of Roads (CEDR), are aware of the environmental noise problem along their motorways. One of the main objectives of CEDR is to facilitate knowledge sharing on noise abatement issues among European NRA's. The CEDR noise group has made a comprehensive questionnaire survey on how the CEDR member states handle noise issues. The paper focuses on the part of the survey on noise limits and noise levels along motorways throughout Europe. It will deal with the: - status of noise limits; - noise indicators to express noise limits; - noise limits (outdoor and indoor) for motorways; - noise models to calculate noise levels. Due to the use of different noise indicators and different noise calculation models, the comparison of noise levels and noise limits along motorways in Europe is complicated to say the least. The only way to solve this problem is to have and to use a European noise model for road traffic based on the outcome of the HARMONOISE/IMAGINE projects.

2pNSc2. Origins of the modern noise ordinance in the County of San Diego, California. John C. Bennett (6110 Loukelton Circle, San Diego, CA92120, USA, jc.bennett@cox.net)

An unofficial history of the origins of San Diego County's noise regulations has been compiled as an investigative tool to review current and future zoning schemes in this jurisdiction. These regulations date back to the 1970's and overlap with the development of city regulations. The focus of the history is the acoustical aspect of their development. A general discussion of performance standards is included in a comparison with similar regu- lations in two other California jurisdictions developed within the last five years. Some practical similarities and differences with European regulations in local jurisdictions are identified to encourage an open discussion in this session.

2pNSc3. Implementation of the noise directive in music and entertainment sector in Finland. Esko Toppila (Finnish Institute of Occupational Health, P.O:Box 486, 33101 Tampere, Finland, esko.toppila@ttl.fi), Pekka Olkinuora (FIOH, Topeliuksenkatu 41, 00250 Helsinki, Finland, pekka.olkinuora@ttl.fi), Pekka Airre (FIOH, P.O.Box 486, 33101 Tampere, Finland, erkko.airo@ttl.fi)

In the noise directive (2003/10/EC) the member countries were obliged to develop codes of conduct for the music and entertainment sector. In Finland the code of conduct was adopted into use in February 2007. According to this code of conduct the workers in the music and entertainment sector consist of performers (musicians, singers, conductors and actors), staff exposed to music (waiters,...) and teacher who teach music in schools. Guidelines were designed based on the worker group and the type of employment. In this approach the performers, who actually produce the "noise", must be able to monitor the sound levels in real time. Restaurants and music halls design the protective actions for other staff based on the assumption that predefined sound level is not exceeded the premises. The same principle is applied to schools. For temporary staff there must be hearing protectors available. Also the need for damping and use of quieter instruments is recognised. The code of conduct give the principles for the hearing conservation, but for the music and entertainment sector direct application of the principles is too complicated. Implementation examples are needed for all exposure patterns. This work is going on now in Finland. 


\title{
Session 2pNSd
}

\section{Noise, ASA Committee on Standards, and EURONOISE: Sound Quality Tools and Applications II (Poster Session)}

\author{
Wade Bray, Cochair \\ HEAD acoustics, Inc. \\ Klaus Genuit, Cochair \\ HEAD acoustics $\mathrm{GmbH}$ \\ Etienne Parizet, Cochair \\ Laboratoire Vibrations Acoustique, Insa Lyon
}

\begin{abstract}
All posters will be on display from 3:40 p.m. to 5:20 p.m. To allow contributors an opportunity to see other posters, contributors of odd-numbered papers will be at their posters from 3:40 p.m. to 4:30 p.m. and contributors of even-numbered papers will be at their posters from 4:30 p.m. to 5:20 p.m.
\end{abstract}

\section{Contributed Papers}

2pNSd1. Two-level description of environmental sounds: comparison and generalization of $\mathbf{4}$ timbre studies. Antoine Minard (IRCAM - UMR CNRS 9912, Equipe Perception et Design Sonores, 1, place Igor Stravinsky, 75004 Paris, France, antoine.minard@ ircam.fr), Patrick Susini (IRCAM UMR CNRS 9912, Equipe Perception et Design Sonores, 1, place Igor Stravinsky, $75004 \quad$ Paris, France, susini@ircam.fr), Nicolas Misdariis (IRCAM - UMR CNRS 9912, Equipe Perception et Design Sonores, 1, place Igor Stravinsky, 75004 Paris, France, misdarii@ircam .fr), Guillaume Lemaitre (IRCAM - UMR CNRS 9912, Equipe Perception et Design Sonores, 1, place Igor Stravinsky, 75004 Paris, France, lemaitre@ircam.fr), Stephen McAdams (Centre for Interdisciplinary Research in Music Media \& Technology (CIRMMT) - Schulich School of Music - McGill Univ., 555 Sherbrooke Street West, Montreal, QC H3A1E3, Canada, smc@music.mcgill.ca), Etienne Parizet (Laboratoire Vibrations Acoustique, Insa Lyon, 25 bis, av. J. Capelle, 69621 Villeurbanne Cedex, France, etienne.parizet@insa-lyon.fr)

The purpose of this study is to propose a scheme of environmental sound description based on the comparison and generalization of 4 timbre studies of diverse environmental sounds. In the medium term, the aim is to make the indexing and classification processes of this kind of sound automatic, which is essential for sound content-based searching and browsing methods using perceptual models of environmental sounds. First, we inventoried the stimuli, experiments and perceptual results of these 4 studies. Then, we experimentally identified 3 main environmental sound categories that constitute this overall sound dataset: impact sounds, motor sounds and instrumentlike sounds. We developed an efficient tool that predicts these categories according to only two acoustic features calculated on the signal. However, because of the specificities of the sound production of these three categories, each of them has its own timbre space. Therefore, we finally used perceptually relevant acoustic features to explain these timbre spaces, according to the main acoustic characteristics that define each sound class. We found that timbral brightness is a common feature used to discriminate sounds in all classes, while other particular features are specifically used within each class. [Work partially supported by the SamplerOrchestrator project of the ANR program]

2pNSd2. Relations between acoustic parameters and perceptual properties: an approach by regressions tree applied to car door closure sounds. Marie Céline Bezat (PSA Peugeot Citroën, Centre Technique de
Vélizy, Route de Gisy, 78943 Vélizy-Villacoublay, France, marieceline.bezat@mpsa.com), Vincent Roussarie (PSA Peugeot Citroën, Centre Technique de Vélizy, Route de Gisy, 78943 VélizyVillacoublay, France, vincent.roussarie@mpsa.com), Richard Kronland-Martinet (CNRS-LMA, 31, chemin Joseph Aiguier, 13402 Marseille, France, kronland@lma.cnrs-mrs.fr), Solvi Ystad (CNRS-LMA, 31, chemin Joseph Aiguier, 13402 Marseille, France, ystad@1ma.cnrs-mrs .fr)

The aim of this study is to identify perceptually pertinent parameters for the evaluation of car door closure sounds. For this purpose, door closing sounds are finely decomposed into perceptual properties: analytical properties (which are obtained thanks to sensory analysis), natural properties (linked to perception of sources and events) and evocations. The acoustic characterisation of the sound is then processed by means of an analysissynthesis model which aims, not at reproducing the exact replica of the door closing sounds, but to synthesize sounds that preserve perceptual properties with a reduced number of signal parameters. The model consists in decomposing the sound in several independent impact sources, each impact being modelled by a set of gains and damping factors in frequency bands. Listening tests on controlled sounds are then carried out to observe the effects of acoustic parameters on the perceptual properties. The complex relations between acoustic parameters and perceptual properties are finally established via regressions tree analyses.

2pNSd3. Acoustic feedback of control elements in cars. Alexander S. Treiber (Heilbronn University, Max-Planck-Str. 39, 74081 Heilbronn, Germany, treiber@hs-heilbronn.de), Gerhard Gruhler (Heilbronn University, Max-Planck-Str. 39, 74081 Heilbronn, Germany, gruhler@hs-heilbronn.de)

Due to the complexity of modern car's on-board systems, car manufacturers tend to use menu based user interfaces. These interfaces offer a relatively low number of control elements for a large number of functions. Optimization of both haptical and acoustical feedback of the remaining control elements can improve both acceptance and security of operation. Since cars nowadays are no longer sold simply as technical but as lifestyle products it is crucial for the success that the potential customer perceives every single aspect of the car to be valuable. Obvious examples are exterior design and material quality. Since the user interface of the car can be judged even before a test drive the feeling of buttons and switches is a key aspect. This work presents the results of a jury test which is part of an ongoing research project focused on acoustical feedback. Subjects were asked to judge both 
recordings of real control elements as well as synthesized signals which were presented on purpose-built hardware. Based on the subjective results the recorded signals were analyzed in order to identify spectral features which correlate with the subject's responses.

2pNSd4. Influence of time-varying booming noise on automotive comfort. Florent Richard (PSA Peugeot Citroën, Centre Technique de Vélizy, Route de Gisy, 78943 Vélizy-Villacoublay, France, florent.richard@mpsa.com), Francois Costes (PSA Peugeot Citroën, Centre Technique de Vélizy, Route de Gisy, 78943 Vélizy-Villacoublay, France, francois.costes@free.fr), Vincent Roussarie (PSA Peugeot Citroën, Centre Technique de Vélizy, Route de Gisy, 78943 Vélizy-Villacoublay, France, vincent.roussarie@mpsa.com)
Noise level reduction is the main preoccupation for acoustic engineers in the automotive industry. Huge progresses were made during the past decades thanks to the use of acoustic materials and specific intake or exhaust silencers. In the future, vehicles will have to be lighter and engines optimized to ensure very low energy consumption. This sets new compromises between mass, performances and acoustics, in particular for booming noise that can be very affected by mass reduction or exhaust modifications. Acoustic specifications must therefore be optimized to guarantee customer's comfort in spite of these new constraints. In this paper, we study the impact of time-varying booming noise on customer's comfort on acceleration sound samples. We performed several customer tests to evaluate the impact of different time-varying booming profiles on comfort and to find out an acceptability threshold for booming.

TUESDAY AFTERNOON, 1 JULY 2008

P2-A, LEVEL 2, 3:40 TO 5:20 P.M.

\title{
Session 2pNSe
}

\section{Noise and EURONOISE: General Topics in Noise II (Poster Session)}

\begin{abstract}
All posters will be on display from 3:40 p.m. to 5:20 p.m. To allow contributors an opportunity to see other posters, contributors of odd-numbered papers will be at their posters from 3:40 p.m. to 4:30 p.m. and contributors of even-numbered papers will be at their posters from 4:30 p.m. to 5:20 p.m.
\end{abstract}

\section{Contributed Papers}

2pNSe1. Measurement of the acoustic pressure distribution occurring around an aerial substation adjacent to apartment buildings. Sebastian Borucki (Technical University of Opole, ul. Miko ajczyka 5, 45-271 Opole, Poland, s.borucki@po.opole.pl), Tomasz Boczar (Technical University of Opole, ul. Miko ${ }^{3}$ ajczyka 5, 45-271 Opole, Poland, t.boczar@po.opole.pl), Andrzej Cichon (Technical University of Opole, ul. Miko ${ }^{3}$ ajczyka 5, 45-271 Opole, Poland, a.cichon@ po.opole.pl)

The subject matter of the research work presented in this paper refers to the measurements of the values of the acoustic pressure levels (noise) occurring around a main feeding-point aerial substation adjacent to apartment buildings. The paper presents the values of the noise accompanying the particular power devices, mainly transformers, during their regular operation. The main aim of this research work was the comparison and assessment of the acoustic pressure values measured with the permissible values defined by environmental standards binding in Poland. The research analysis carried out proved that during a long-term operation of power appliances installed in substations the acoustic pressure level that they emit into the environment is not constant but is subject to changes. Thus the increase of the noise level above the permissible values can be the cause of violation of environmental standards. Due to a significant increase of people's awareness and readiness to claim their rights, the main consequence of violating the standards is a growing number of claims. Therefore it is imperative that the level of the acoustic pressure be monitored during routine tests, especially around highpower transformers.

2pNSe2. Investigation and application of theoretical acoustic field model evaluating the change of environmental conditions. Robertas Mikalauskas (Kaunas University of Technology, K.Donelaicio str. 73, LT44029 Kaunas, Lithuania, robertas.mikalauskas@ktu.lt), Vitalijus Volkovas (Kaunas University of Technology, K.Donelaicio str. 73, LT44029 Kaunas, Lithuania, tsdi@ktu.lt)

The effectiveness of the means used to reduce the noise depends on many factors. One of the main factors is the sources which generates acoustic field. In industrial premises there are many sources, their impact to acoustic field differs, and the source may also change due to environmental conditions. Therefore the task of the creation of the mobile noise reduction means, their rational arrangement and control becomes more and more relevant. In this case theoretical modeling of interaction of acoustic noise control systems with environment has significant importance for creating such systems. This paper provides acoustic field model using FEM, which imitates changing conditions of real industrial premise, evaluates the effectiveness and application possibilities of the mobile noise reduction system. It also provides the comparison of the results of theoretical experiment with experimental results. The results showed that with the help of the model mobile noise reduction system can be modeled, their effectives might be evaluated considering the change of sources and reflection planes, and the structural model of the investigative room can be supplemented with acoustic noise reduction means - noise reduction screens, selecting their geometric dimensions, arrangement in the space and selecting the materials and predicting the values of acoustic field parameters in the real object point under consideration.

2pNSe3. Effects of personal stereo use: pilot results from 20 university students. Eric De Santis (Acoustics, Aalborg University, Fredrik Bajers Vej 7 B5, 9220 Aalborg Ø, Denmark, ems@es.aau.dk), Rodrigo Ordoñez (Acoustics, Aalborg University, Fredrik Bajers Vej 7 B5, 9220 Aalborg Ø, Denmark, rop@es.aau.dk), Karen Reuter (Acoustics, Aalborg University, Fredrik Bajers Vej 7 B5, 9220 Aalborg $\varnothing$, Denmark, kr@es.aau.dk), Dorte Hammershøi (Acoustics, Aalborg University, Fredrik Bajers Vej 7 B5, 9220 Aalborg Ø, Denmark, dh@es.aau.dk)

Modern personal stereo systems have the ability to store thousands of music files in small, relatively low-cost, compact devices. The popularity and widespread presence of the MP3 player cannot go unnoticed in contemporary society. Given a sufficient noise level and listening duration, consumers are at risk of developing a noise-induced hearing loss. A study into the effects of personal stereo use will be carried out with the intention of investigating common noise exposure levels in realistic settings, user listening habits and the hearing status in a group of listeners. Noise exposure levels are to be obtained by using the manikin technique described in the ISO 
11904-2 standard. The questionnaire to be presented to interested participants is designed to report personal stereo listening habits and to screen subjects for the hearing status evaluation. The hearing status of selected subjects will be evaluated through the use of conventional and possibly extended high-frequency audiometry, transient-evoked otoacoustic emissions and distortion product otoacoustic emission fine structures.

2pNSe4. Classroom noise in schools containing children learning English as a second language. Gina Ramirez (5924 N. Campbell Avenue, Chicago, IL 60659, USA, gina.ramirez@loop.colum.edu)

This study analyzes the acoustics of two elementary schools containing both native English speaking children and large English learning populations, to determine if facilities are best being utilized acoustically. As stated by the ASA, children learning English require a more favorable signal-to- noise ratio and should thus be instructed in quieter learning environments. The study examined at least 20 classrooms in each facility by measuring ambient noise level and reverberation time by methodologies outlined in the ANSI S12.60 (2002) standard. Creating a noise mapping allowed for the evaluation of each school based on utilization of pre-existing facilities. Based on preliminary results all classrooms tested failed to meet the maximum A-weighted steady background noise level specified in the standard furthermore schools placed English learning students in environments that were up to $10 \mathrm{~dB}$ louder than classrooms used for native English speaking students, demonstrating that more attention to ambient noise level and its effect on speech intelligibility is needed when planning the layout of learning facilities containing large English learning populations. The ongoing analysis of the data aims at assessing the impact of the measured acoustical environments in terms of perceived intelligibility by using a survey tool among the student population.

TUESDAY AFTERNOON, 1 JULY 2008

P2-A, LEVEL 2, 3:40 TO 5:20 P.M.

\title{
Session 2pNSf
}

\section{Noise, Computational Acoustics, and EURONOISE: Time-Domain Modeling Methods in Acoustics I (Poster Session)}

\author{
Paul Calamia, Cochair \\ Rensselaer Polytechnic Institute \\ Maarten Hornikx, Cochair \\ Applied Acoustics, Chalmers University of Technology
}

\begin{abstract}
All posters will be on display from 3:40 p.m. to 5:20 p.m. To allow contributors an opportunity to see other posters, contributors of odd-numbered papers will be at their posters from 3:40 p.m. to 4:30 p.m. and contributors of even-numbered papers will be at their posters from 4:30 p.m. to 5:20 p.m.
\end{abstract}

\section{Contributed Papers}

2pNSf1. Optimization of compact finite difference method for wave acoustic simulation. Hideo Tsuru (Nittobo Acoustic Engineering, 1-21-10 Midori Sumida-ku, 130-0021 Tokyo, Japan, tsuru@noe.co.jp), Reima Iwatsu (Tokyo Denki University, Kanda-Nishikicho 2-2, Chiyoda-ku, 1018457 Tokyo, Japan, iwatsu@cck.dendai.ac.jp)

Recently, the finite difference method, a tool for wave acoustic simulations, can be applied to practical analysis. However, the numerical dispersion which makes propagation speed in simulations change according to the wave length, is a severe problem to maintain high accuracy in the analysis. The numerical error accompanied by the time integration hinders to carry out an accurate simulation. An examination of a compact finite difference scheme which can approximate differentiation precisely on a comparatively large grid spacing was made. It was shown that the adjusting the coefficients in the compact finite difference scheme can simulate a wave propagation accurately with a small amount of computation. The improvement method of the time integration was also discussed. The possibility of improvement of accuracy through a multi-step integration is shown.

2pNSf2. Time-domain modeling of porous media acoustics. David K. Wilson (U.S. Army Engineer Research and Development Center, 72 Lyme Rd., Hanover, NH 03755-1290, USA, D.Keith.Wilson@usace .army.mil), Sandra L. Collier (U.S. Army Research Lab, Attn: AMSRDARL-CI-EE, 2800 Powder Mill Rd., Adelphi, MD 20783, USA, sandra.collier@us.army.mil), Vladimir E. Ostashev (NOAA/Earth System Research Laboratory, 325 Broadway, Boulder, CO 80305, USA,
vladimir.ostashev@noaa.gov),David H. Marlin(U.S. Army Research Lab, Attn: AMSRD-ARL-CI-EE, 2800 Powder Mill Rd., Adelphi, MD 20783, USA, dmarlin@arl.army.mil), David F. Aldridge (Sandia National Laboratories, PO 5800, MS 0750, Albuquerque, NM 87185-0750, USA, dfaldri@sandia.gov), Neill P. Symons (Sandia National Laboratories, PO 5800, MS 0750, Albuquerque, NM 87185-0750, USA, npsymon@sandia .gov)

Sound waves propagating in porous media are subject to strong dissipation and dispersion. This paper elaborates upon several recent publications by the authors regarding the time-domain description of these effects. The foundation is a relaxational description of the viscous and thermal dissipation in a rigid porous medium, which is shown to possess an exact, analytical conversion from the frequency to the time domain. The complex density and bulk modulus operators transform to temporal convolutions between a causal response function and the acoustic field variables. When the convolutions are neglected, the equations reduce to the well known ZwikkerKosten phenomenological model. The relaxation function can be inverted to provide an equivalent time-domain formulation from the complex volume and compressibility operators. Although a direct time-domain transformation of the specific impedance from the relaxation model has not been found, an accurate broadband approximation thereof can be transformed. The resulting time-domain boundary condition (TDBC) describes the absorptive and reactive response of the material. The response of the boundary decays slowly, as the inverse square root of time, but efficient numerical procedures are formulated that allow the TDBC to be approximated with a small number of recursive filters. 


\title{
Session 2pNSg
}

\section{Noise and EURONOISE: EU Projects for Aircraft Noise Reduction I (Poster Session)}

\author{
Dominique Collin, Chair \\ Snecma - Safran Group
}

\begin{abstract}
All posters will be on display from 3:40 p.m. to 5:20 p.m. To allow contributors an opportunity to see other posters, contributors of odd-numbered papers will be at their posters from 3:40 p.m. to 4:30 p.m. and contributors of even-numbered papers will be at their posters from 4:30 p.m. to 5:20 p.m.
\end{abstract}

\section{Contributed Papers}

2pNSg1. Effect of curvature on the scattering coefficients of Herschel-Quincke tubes. Benjamin Poirier (Université de Technologie de Compiègne, Centre de Recherche Royallieu, BP20529, 60205 Compiègne, France, bpoirier@utc.fr), Jean-Michel Ville (Université de Technologie de Compiègne, Centre de Recherche Royallieu, BP20529, 60205 Compiègne, France, jean-michel.ville@utc.fr), Cédric Maury (Université de Technologie de Compiègne, Centre de Recherche Royallieu, BP20529, 60205 Compiègne, France, cedric.maury@utc.fr), Félix Foucart (Université de Technologie de Compiègne, Centre de Recherche Royallieu, BP20529, 60205 Compiègne, France, felix.foucart@utc.fr)

The concept of Herschel-Quincke (HQ) tubes has been known since the beginning of the 20th century and was shown to be of interest to reduce tonal and broadband noise from turbofan engine. To help to understand the physical phenomena underlying the HQ concept, Hallez \& Burdisso have developed a 3D theoretical model. The 3D modelling technique considers the tubes-inlet interfaces as finite piston sources that couple the acoustic field inside a hard-walled duct with the acoustic field within the HQ tubes. This model makes a geometrical approximation by considering the tube as a straight duct whereas in reality it corresponds to a curved duct. In this paper, a model is presented which takes into account the curvature of the bend by integrating two differential equations for the pressure and velocity in the bend, projected on the local transverse modes. Results of some typical coefficients of the scattering matrix [S] deduced from the curved HQ model are compared with the 3D straight tube model, the experimental results and the numerical results. The curvature is shown to modify the HQ resonances and then have to be taken into account in the analytical formulation.

2pNSg2. Assessment of noise impacts of the air traffic. Katrin Ohlau (Universität Stuttgart / IER, Heßbrühlstraße 49a, 70565 Stuttgart, Germany, katrin.ohlau@ier.uni-stuttgart.de), Peter Bickel (Universität Stuttgart/IER, Heßbrühlstraße 49a, 70565 Stuttgart, Germany, peter.bickel@ier .uni-stuttgart.de), Rainer Friedrich (Universität Stuttgart/IER, Heßbrühlstraße 49a, 70565 Stuttgart, Germany, rainer.friedrich@ier.uni-stuttgart.de)
Against the background of the generally increasing traffic and especially the expansion plans in doubling the air traffic until 2020, this paper presents an assessment of noise effects of air traffic and underlines its future meaning. In several projects including the EC funded projects HEATCO (Developing Harmonised European Approaches for Transport COsting and Project Assessment), GRACE (Generalisation of Research on Accounts and Cost Estimation) and ASSET (ASsessing SEnsitiveness to Transport), methodologies for assessing noise including aircraft noise have been developed, improved and applied. The methodologies focus on the impact pathway approach, i.e. first noise levels are estimated, and then level- response functions are applied to calculate health impact and annoyance level distributions. Afterwards it is evaluated by transforming the impacts into monetary levels. Our studies for assessing annoyance have been carried out with the contingent valuation method. Results of the monetary valuation will be shown for the airports Frankfurt, Hamburg, Berlin-Tegel, Düsseldorf, Köln/Bonn, München and Stuttgart. The total air traffic noise costs for Germany range from EUR 150 million to EUR 1200 million per year.

2pNSg3. Efficiency of shielding effect on installed double stream nozzles. Sébastien Aeberli (SNECMA - groupe SAFRAN, Villaroche Rond-point R. Ravaud, 77550 Moissy-Cramayel, France, sebastien.aeberli @ snecma.fr)

In spite of acoustic improvements on jet noise brought by innovative technologies such as chevrons, a way to ensure important acoustic gains is to optimize the power plant integration on aircrafts in order to benefit of the shielding effect due to wing, empennage and fuselage surfaces. In this presentation, these effects are illustrated thanks to a jet noise test campaign at $1 / 10$ th scale in an anechoic chamber as part of a European project. This campaign gives a better understanding of shielding effect by a wing and also of the acoustic impact of pylon azimutal direction on two representative BPR 9 nozzles (Baseline - smooth - and Low Noise - with chevrons). The efficiency of shielding effect on jet noise sources in these configurations is assessed. 


\title{
Session 2pNSh
}

\section{Noise and EURONOISE: Action Planning and Global Solutions for Urban Noise II (Poster Session)}

\author{
Klaus Genuit, Cochair \\ HEAD acoustics $\mathrm{GmbH}$ \\ Itziar Aspuru Soloaga, Cochair \\ Labein-Tecnalia
}

\begin{abstract}
All posters will be on display from 3:40 p.m. to 5:20 p.m. To allow contributors an opportunity to see other posters, contributors of odd-numbered papers will be at their posters from 3:40 p.m. to 4:30 p.m. and contributors of even-numbered papers will be at their posters from 4:30 p.m. to 5:20 p.m.
\end{abstract}

\section{Contributed Papers}

2pNSh1. A proposal for doing a touch of anova with noise levels. Michel Maurin (INRETS-LTE, case 24, 69675 Bron cedex, 69500 Bron, France, maurin@inrets.fr)

Because noise levels are logarithm of additive variables, the usual numerical processings are not suitable for them (mind $60+60 \neq 120$ ). Then one has to deal with them differently, following some other syntaxic rules which may be called in a general way the "logic of levels". Of course this question is correctly resolved with the "energetic mean" but it fails as soon as one has to deal with variances of levels or covariances with other variables (data analysis, linear regressions, œ). Some (necessarily) approximate compromises may be developped between the logic of levels and the arithmetical logic, (transforming variables in statistics for instance). Here we introduce a new statistic, the h-dispersion, which is not a variance but has many properties of variance, and very fortunatly takes account of the logarithmic status of data. This new compromise may be employed for levels in some cases of design of experiments, such as one-way cases and comparisons "beforeafter" in relation to acoustics operations for instance, and also more or less for two-ways cases. It remains approximate (a syntaxic compromise), but in any time this is an improvement in comparison with a crude ANOVA on levels.

2pNSh2. Study of the surface traffic inside the Cluj-Napoca urban agglomeration. Geamilia Solea (Technical University of Cluj-Napoca, Faculty of Machines Design; Department of Mechanics and Computer Programming, B-dul Muncii; Nr. 103-105, 400641 Cluj-Napoca, Romania,
marylandprod@gmail.com),Mariana Arghir(Technical University of ClujNapoca, Faculty of Machines Design; Department of Mechanics and Computer Programming, B-dul Muncii; Nr. 103-105, 400641 Cluj-Napoca, Romania, marianaarghir@yahoo.com), Dan Borza (National Institute of Applied Sciences of Rouen, INSA Rouen, LMR, Ave l'Universite, BP8 76800 Rouen, France, dan.borza@insa-rouen.fr), Mariana Runcan (National Institute of Applied Sciences of Rouen, INSA Rouen, LMR, Ave 1'Universite, BP8 76800 Rouen, France, mariruncan@yahoo .com)

The environment's protection is one of the most actual problems, because of the fact that pollution is a major danger for planet life. The environment's pollution is caused because some pollution agents are in too large a quantity for a long time and they become dangerous for human health, for the flora, for the fauna and for the constructions too. The pollution sources can be classified as natural and artificial sources. The quality of the environment from a specific area at a certain time can be determined by: a) the air quality; b) the water quality; c) the soil quality; d) the health level of the population; e) the decrease of animals and plants species that are accounted for. In the paper there are given the urban traffic on the principal routes inside the Cluj-Napoca municipality. The traffic is the constituent part of the research activity of the national project having the destination the monitoring of the noise and vibrations into an urban agglomeration, applied to the Cluj - Napoca town. This study is the first step for the "noise map" asked for by the Romania integration in the European Union for the team researchers of TUCN under the the direction of Dr. Mariana Arghir. 


\title{
Session 2pNSi
}

\section{Noise and EURONOISE: Railway Noise and Vibration I (Poster Session)}

\author{
Richard Carman, Cochair \\ Wilson, Ihrig \& Associates \\ Franck Poisson, Cochair \\ SNCF DIR \\ David Thompson, Cochair \\ Inst. of Sound and Vibration
}

\begin{abstract}
All posters will be on display from 3:40 p.m. to 5:20 p.m. To allow contributors an opportunity to see other posters, contributors of odd-numbered papers will be at their posters from 3:40 p.m. to 4:30 p.m. and contributors of even-numbered papers will be at their posters from 4:30 p.m. to 5:20 p.m.
\end{abstract}

\section{Contributed Papers}

2pNSi1. On effectiveness of vibration isolation using super-elastic rail support combined with booted sleeper or floating slab. Tianxing X. Wu (Shanghai Jiao Tong University, School of Mechanical Engineering, 800 Dong Chuan Road, 200240 Shanghai, China, txwu@sjtu.edu.cn)

Ground vibration or structure vibration of viaduct due to railway traffic can be reduced by use of super-elastic rail support, booted sleeper and floating slab to isolate vibration transmission from the track to the infrastructure. In this study modeling in the frequency domain of track structure dynamics and wheel/rail interaction is carried out. Wheel/rail interaction and transmission to the infrastructure of the interaction force due to a relative displacement excitation are simulated. The effectiveness of vibration isolation is analyzed for booted sleeper or floating slab combined with super-elastic rail fastener. It is found that the combination of super-elastic rail fastener with booted sleeper or floating slab can effectively reduce the wheel/rail interaction force in the medium frequencies, compared with the booted sleeper or floating slab track using usual rail pad. The combination also shows better ability to block vibration transmission from the track to the infrastructure in the medium and high frequencies.

2pNSi2. Overview of Railway Noise Control in Europe. Jakob Oertli (Swiss Federal Railways, Schanzenstrasse 5, 3000 Bern, Switzerland, jakob.oertli@sbb.ch)

European and national legislation, increasing freight traffic, political pressure and many more factors contribute to make noise an issue of increasing importance for the railways. This paper provides an overview of railway noise abatement efforts throughout Europe. Different countries in Europe have adopted varying noise abatement strategies, ranging from an emphasis on infrastructure based measures to a combination of rolling stock measures with noise barriers. Important in the current European discussion is the development and retrofitting of freight wagons with K- and LL-brake blocks. To support this retrofitting a wide variety of incentives, such as differential track access charges, operational restrictions or direct subsidies are being discussed. The current efforts by the European Commission, the International Union of Railways (UIC), the Community of European Railways (CER) and other international organisation to promote silent railways are presented.

2pNSi3. A guideline for prediction and control of noise in railroads maintenance activities. Sergio Luzzi (Vie En.Ro.Se. Ingegneria, Via Stibbert, 1, 50134 Florence, Italy, sergio.luzzi@ vienrose.it)

In railroads routine maintenance as well as emergency maintenance can be represented by the acoustic point of view as a system of classified yard activities, including catalogued single and grouped noise sources, that is moved along the tracks areas. RFI (Rete Ferroviaria Italiana), the Italian National Railway Company, has provided a guideline for the correct evaluation of noise impact on receivers surrounding the yards, which can be adapted to many situations fitting the specific national law and local regulations disposals about noise assessment, prediction and reduction in temporary activities. Starting from their long term experience, the authors have developed a method for the production of local reports on maintenance railroad yard activities based on the modelling of standard scenarios. Each machinery employed in yards (for superstructure laying or repairing, electric traction, etc,) has been measured and characterized as noise source or as part of a noise sources system (emission box). The noise propagation scenarios are then build using an MS Excel based adaptation software. This methodology represents the technical and procedural architecture of the guideline which provides materials for the management of all the different typologies of maintenance yards operating on the RFI railroad network.

2pNSi4. Railway vibration mitigation - An innovative concept. Frederic Le Corre (Alstom Transport, TGS Engineering - 33, rue des Bateliers, 93400 Saint-Ouen, France, frederic.le-corre@transport.alstom .com), Christine Charles (Alstom Transport, TGS Engineering - 33, rue des Bateliers, 93400 Saint-Ouen, France, christine.charles@transport.alstom .com), Marcel Girardi (Alstom Transport, TGS Engineering - 33, rue des Bateliers, 93400 Saint-Ouen, France, marcel.girardi@transport.alstom.com)

These last decades, rail transportation has known an amazing development. Hence, the resulting pollution on people living alongside lines or above underground lines has become a crucial subject of consideration. The disturbance has two origins: the direct noise or vibration from the railway traffic and the noise produced by vibration of the building walls, the so-called re-radiated noise. Noise and vibration excitation is essentially due to wheel and rail roughness at the contact point. From this excitation point, the track acts as a filter of vibrations. Hence, spectrum of emitted vibrations can be controlled with the design of railway track. Providing vibration mitigation with the track is now commonly encountered, especially on urban projects (LRT MRT). This is usually achieved by introducing an adequate resilient layer to provide the required attenuation. Alstom Transport and Sateba are developing a system to mitigate vibrations. This system called AFST is based on the booted sleeper system. After a complete analysis of critical parameters, a special design has been drawn. The resulting antivibratile solution is a high-performance system suiting areas where vibration mitigation and/or attenuation of re-radiated noise are required. 
2pNSi5. Noise and vibrations at tram track intersection. Stjepan Lakusic (University of Zagreb, Faculty of Civil Engineering, Kaciceva 26, 10000 Zagreb, Croatia, laki@grad.hr), Marijan Bogut (KONCAR - Electrical Engineering Institute, Fallerovo setaliste 22, 10000 Zagreb, Croatia, mbogut@koncar-institut.hr), Visnja Tkalcevic Lakusic (University of Zagreb, Faculty of Civil Engineering, Kaciceva 26, 10000 Zagreb, Croatia, visnjat@grad.hr)

In urban areas noise and vibration due to public traffic diminish the quality of life. In Zagreb, tram traffic is the most important form of public transport. Tram network has $120 \mathrm{~km}$ of the tracks and 190 trams are used for passengers transport every day. In the movement of the vehicle over track, the interaction between the wheel and track plays the most important role in the field of noise and vibrations. The increasing of noise and vibrations are particularly emphasized at track intersection where a given number of track switches and crossings are installed. This paper presents an experimental study of noise and ground vibrations through the passage of three types of tram vehicles. The measurements were done on the tram intersection with the highest traffic volume in Zagreb's tram network. Two types of track construction were analyzed, discrete and continuous rail fastening system. During the track reconstruction at this intersection, the existing discrete rail fastening system was replaced with a continuous. Because of that, the measurements were carried before and after reconstruction at the same test points. Test results have showed significant reductions of noise and vibrations at track intersection in the case of continuous rail installation.

2pNSi6. Numerical and analytical models for high speed train pantograph radiated noise prediction. Massimo Viscardi (Dept. of
Aerospace Engineering - University of Naples, Via Claudio, 21, 80125 Naples, Italy, massimo.viscardi@unina.it), Michele Iadevaia (Dept. of Aerospace Engineering - University of Naples, Via Claudio, 21, 80125 Naples, Italy, miadevaia@inwind.it), Salvatore Melchionna (Dept. of Aerospace Engineering - University of Naples, Via Claudio, 21, 80125 Naples, Italy, s.melchionna@active-group.it), Leonardo Lecce (Dept. of Aerospace Engineering - University of Naples, Via Claudio, 21, 80125 Naples, Italy, leonardo@unina.it)

The present work deals with the development and comparison of an analytical and numerical models for the evaluation of the noise radiated from a pantograph of an high speed train. Under the numerical point of view, a simplified model has been developed for the pantograph; the model that substructures the problem approaching a complex structure as a combination of simple components, has been derived from a similar aeronautical problem (landing gear noise) but has been self-modified to adapt the formulation to the specific problem. The output of the model is the radiated noise level and spectra as a function of the pantograph speed. The CFD numerical model has been developed for the pantograph based upon a commercial code; only the upper part of the system has been herein simulated because it was addressed as the main noise source during previous laboratory acoustic tests. As an output of the model, the radiated noise has been derived with special reference to two main speed to which experimental data could be referred. Analytical and numerical results will be within the paper discussed and compared to highlight the single approach's advantage and drawbacks. At the and of the paper, some line experimental results will be also introduced and discussed.

TUESDAY AFTERNOON, 1 JULY 2008

P2-A, LEVEL 2, 3:40 TO 5:20 P.M.

\title{
Session 2pNSj
}

\section{Noise and EURONOISE: Noise, Structure Borne Noise from Building Technical Equipment, and Ground Borne Noise from Railways I (Poster Session)}

\author{
James Nelson, Cochair \\ Wilson, Ihrig and Associates, Inc. \\ Michel Villot, Cochair \\ CSTB
}

\begin{abstract}
All posters will be on display from 3:40 p.m. to 5:20 p.m. To allow contributors an opportunity to see other posters, contributors of odd-numbered papers will be at their posters from 3:40 p.m. to 4:30 p.m. and contributors of even-numbered papers will be at their posters from 4:30 p.m. to 5:20 p.m.
\end{abstract}

\section{Contributed Papers}

2pNSj1. Low frequency noise reduction from building technical equipment: A case study. Kathleen Kondylas (NEVA Associates Noise Control, 15 Beck Street, Newburyport, MA 01950, USA, kondylas@earthlink.net), Chris R. Fuller (Virginia Tech, 131 Durham Hall, Blacksburg, VA 24061, USA, christopher.r.fuller@nasa.gov), Joseph A. King (DuPont, 5401 Jefferson Davis Highway, Richmond, VA 23234, USA, joseph.a.king@usa.dupont.com), Natalia Levit (DuPont, 5401 Jefferson Davis Highway, Richmond, VA 23234, USA, natalia.v.levit@usa.dupont .com)

Mechanical equipment (fans, chillers, motors) produces airborne and structure-borne noise with a significant low frequency component. However, in many applications, mitigation of the low frequency noise requires imple- mentation of significant mass barrier in combination with an air space. This work presents case studies on the use of a novel lightweight and thin acoustical material to mitigate low frequency noise from mechanical equipment. This advanced material, based on patented distributed absorber technology, was used to treat the metal housing of the mechanical equipment. The novel material effectively increased transmission loss of the metal housing below $300 \mathrm{~Hz}$. The total noise reduction was 10-14 dB (A) with $5 \mathrm{~dB}$ improvement over typical flexible acoustical blankets below $300 \mathrm{~Hz}$.

2pNSj2. Experimental and numerical study of the ground transmission of structure-borne sound generated by trams. Guillaume Coquel (RATP - CSTB, 13, Rue Jules Vallès, 75547 Paris Cedex 11, France, 
guillaume.coquel@cstb.fr),Philippe A.Jean(CSTB, 24 rue JosephFourier, 38400 Saint-Martin-d'Hères, France, philippe.jean@cstb.fr), Michel Villot (CSTB, 24 rue Joseph Fourier, 38400 Saint-Martin-d'Hères, France, michel.villot@cstb.fr)

The increase of railway traffic in urban areas leads to developments in modelling and understanding the propagation of structure borne sound through soil, foundation and structure. Vibration propagation through the ground, is still not well known. The present paper focuses on the propagation of trams vibrations $(20-250 \mathrm{~Hz})$ in the ground and the energy transmission to the structure. In the first part, calculations performed using a $2 \mathrm{D}$ code based on a FEM/BEM approach are compared to measurements performed on a test site. The railway excitation is produced with a vibrator in order to recreate a line of uncorrelated forces. Soil properties are evaluated by an analysis of surface waves. Both bending and in-plane waves are measured on the structure. The ratio of the soil velocity over the structure velocity is computed in order to evaluate the accuracy of the computation. A 2,5D modelisation is also performed in order to evaluate the effect of a point source on the velocity fields on the soil and on the structure. In the second part, a parametric study using the 2D code is performed on typical cases in order to evaluate the modification of vibration transmission from the ground to the foundation.

2pNSj3. A parametric study on the isolation of ground-borne noise and vibrations in a building using a coupled numerical model. Péter Fiala (Budapest University of Technology and Economics, BME Dept. of
Telecommunications, Magyar tudósok körútja 2, H-1117 Budapest, Hungary, fiala@hit.bme.hu), Shashank Gupta (KU Leuven, Department of Civil Engineering, Kasteelpark Arenberg 40, B-3001 Leuven, Belgium, shashank.gupta@bwk.kuleuven.be), Geert Degrande (KU Leuven, Department of Civil Engineering, Kasteelpark Arenberg 40, B-3001 Leuven, Belgium, geert.degrande@bwk.kuleuven.be), Fülöp Augusztinovicz (Budapest University of Technology and Economics, BME Dept. of Telecommunications, Magyar tudósok körútja 2, H-1117 Budapest, Hungary, fulop@ hit.bme .hu)

Underground traffic induced vibrations and noise in buildings are a major environmental concern in urban areas. To quantify these vibrations a numerical prediction model has been developed and validated. A coupled FE-BE model is used to compute the incident ground vibrations due to the passage of a train in the tunnel. A dynamic soil-structure interaction model is used to determine the vibration levels of the building. The soil-structure interaction problem is solved by means of a 3D boundary element method for the soil coupled to a 3D finite element method for the structural part. An acoustic 3D spectral finite element method is used to predict the acoustic response. The coupled numerical model is used to quantify the efficiency of vibration and noise mitigation measures at different stages of the vibration propagation chain. Vibration isolation with a floating slab track is modeled on the source side, base isolation is incorporated in the structure model, and a box-within-box arrangement is considered for the isolation of re-radiated noise in the building's rooms. The insertion gain of the three methods is compared using the model of a multi-story portal frame office building subjected to ground-borne vibrations from an underground railway line.

TUESDAY AFTERNOON, 1 JULY 2008

P2-A, LEVEL 2, 3:40 TO 5:20 P.M.

\title{
Session 2pNSk
}

\section{Noise and EURONOISE: Physical and Psychophysical Evaluation of Vehicle Exterior Noise II (Poster Session)}

\author{
Paul Donavan, Cochair \\ Illingworth \& Rodkin, Inc. \\ Hugo Fastl, Cochair \\ AG Technische Akustik, MMK, TU München
}

\begin{abstract}
All posters will be on display from 3:40 p.m. to 5:20 p.m. To allow contributors an opportunity to see other posters, contributors of odd-numbered papers will be at their posters from 3:40 p.m. to 4:30 p.m. and contributors of even-numbered papers will be at their posters from 4:30 p.m. to 5:20 p.m.
\end{abstract}

\section{Contributed Papers}

2pNSk1. Questionnaire survey on vehicle horn use. Masahiko Takada (Omihachiman Community Medical Center, 1379 Tsuchida-cho, 523*0082 Omihachiman, Japan, takada@design.kyushu-u.ac.jp), Yoko Fukuda (Foster Electric Company, Limited, 512 Miyazawa-cho, Akishima City, 196-8550 Tokyo, Japan, yoko_19831228@yahoo.co.jp), Shin-Ichiro Iwamiya (Kyushu University, 4-9-1 Shiobaru, Minami-ku, 815-8540 Fukuoka, Japan, iwamiya@design.kyushu-u.ac.jp)

The Japanese Road Traffic Law provides that a vehicle horn should be utilized in the dangerous situation or the place where the horn sign was installed. However, drivers are frequently seen to use their vehicle's horn as a signal in the situations when they pass each other. Given such situations, the mentioned above law is likely to become a law in name only. Therefore, a questionnaire survey was carried out to ascertain the current circumstance of a vehicle horn use. The respondents were asked the latest or last remem- bered case of horn use in various situations of both their driving or as pedestrians. With regards to an experience of being tooted at by another driver, the questionnaire included questions concerning place, traffic volume, the aim of the horn use, time pattern of horn tooting, respondent's mental reaction when tooted at by another driver, etc. As a result, drivers mostly had negative mental reactions (i.e., startled, noisy and irritating) by long toots. Pedestrians' negative mental reactions were mainly aroused by horn use calling another's attention to something or to inform of a danger. Furthermore, nearly 40 percent of the respondents were discontented with the volume and the sound quality of horn.

2pNSk2. Sound Quality Evaluation for the Axle Gear Noise in the vehicle. Hyun Ho Lee (Inha University, Mechanical Engineering, 253 Yonghyun Dong, 402-751 Inchon, Republic of Korea, hyunho0424@naver 
.com),Ho Wuk Kim(Inha University, Mechanical Engineering, 253 Yonghyun Dong, 402-751 Inchon, Republic of Korea, mowugi@gmail.com) , Sang Kwon Lee (Inha University, Mechanical Engineering, 253 Yonghyun Dong, 402-751 Inchon, Republic of Korea, sangkwon@inha.ac.kr)

A gear whine sound due to the axle system is one of the most important sound qualities in a sport utility vehicle (SUV). In the previous works about the gear whine sound, it was known that it is difficult to evaluate the gear whine sound objectively by using the only A-weighted sound pressure level because of the masking effect. In this paper, for the objective evaluation of the axle-gear whine sound, the characteristics of the axle-gear whine sound is at the first investigated based on the synthetic sound technology and the new objective evaluation method for the axle-gear whine sound is developed by using the sound metrics, which is the psychoacoustic parameters, and the artificial neural network (ANN) used for the modeling of the correlation between objective evaluation and subjective evaluation. This model is successfully applied the objective evaluation of the axle-gear whine sound for real sport utility vehicles and the output of the model are compared with subjective evaluation. The results have a good correlation over 90 per cent.

2pNSk3. Effect of coolant temperature on combustion and combustion noise fluctuations in a Diesel engine at idle conditions. Matthieu Gazon (Coria CNRS UMR6614, Site Universitaire du Madrillet, Avenue de l’Université BP12, 76801 St Etienne du Rouvray, France, gazon@coria.fr) , Jean Bernard Blaisot (Coria CNRS UMR6614, Site Universitaire du Madrillet, Avenue de l'Université BP12, 76801 St Etienne du Rouvray, France, Jean-Bernard.Blaisot@coria.fr), Nicolas Duclaux-De-L'Estoille (Renault SAS, 1 Allée Cornuel, 91510 Lardy, France, nicolas.duclaux-de-lestoile @ renault.com), Shanjin Wang (Renault SAS, 67, rue des Bons Raisins, 92508 Rueil Malmaison, France, shanjin.wang@renault.com)

At idle conditions, the cycle-to-cycle fluctuations in Diesel engines lead to combustion noise variations that could be annoying for people, even if the mean level is low, and the cold start condition is one of the worst cases. This study focuses on the effect of injection parameters (duration and timings) and the effect of coolant temperature on combustion characteristics and on the combustion noise. A Direct Injection Diesel engine (four cylinders, 16 valves) equipped with a common-rail injection system and piezoelectric injectors was used. The coolant temperature is changed at idle from the ambient temperature to the temperature at normal working conditions. The combustion is analysed in terms of rate of heat release and combustion noise through the measurement of the cylinder pressure. The results show the dependency of the combustion behavior on the increase of coolant temperature at constant engine adjustment parameters. It is also shown that acting on adjustment parameters can modify the combustion behavior and, consequently, the cycle-to-cycle fluctuations. A trade-off between combustion noise and engine emissions can thus be reached.

2pNSk4. Sound perception of urban delivery trucks. Florence Manca-Demirdjian (Laboratoire Vibrations Acoustique - INSA Lyon, 25 bis avenue Jean Capelle, Bâtiment Saint-Exupéry, F-69621 Villeurbanne cedex, France, florence.manca@gmail.com), Etienne Parizet (Laboratoire Vibrations Acoustique, Insa Lyon, 25 bis, av. J. Capelle, 69621 Villeurbanne Cedex, France, etienne.parizet@insa-lyon.fr)

The study deals with the annoyance of urban delivery trucks noises. Sound sequences of two lorries were recorded during a typical delivery : a commercial one and a prototype specially designed to reduce sound emissions. Various sources could be heard during these sequences (engine, doors, hydraulic rear doors). These recordings (the duration of which being more than 5 minutes) were evaluated by subjects in a sound proofed room by two methods. The first one was a continuous assessment of sequences : the listeners had to continuously assess their annoyance by moving a cursor sliding along a five levels graduated scale (from "not at all annoying" to "extremely annoying"). The second one was an overall evaluation (using the same scale) of each event of the sequences (for example : the arrival of the truck, or the closing of the driver's door, etc...). The comparison of the results obtained from these two methods for both lorries will be presented, as well as the reduction of the annoyance could be realized on the prototype. 


\title{
Session 2pNSI
}

\section{Noise and EURONOISE: Environmental Noise Mapping III (Poster Session)}

\author{
Kenneth Cunefare, Cochair \\ The Georgia Inst. of Technol. \\ Gaetano Licitra, Cochair \\ ARPAT - Dept. Firenze
}

\begin{abstract}
All posters will be on display from 3:40 p.m. to 5:20 p.m. To allow contributors an opportunity to see other posters, contributors of odd-numbered papers will be at their posters from 3:40 p.m. to 4:30 p.m. and contributors of even-numbered papers will be at their posters from 4:30 p.m. to 5:20 p.m.
\end{abstract}

\section{Contributed Papers}

2pNSI1. Low frequency noise impact of road traffic in the Netherlands. J. Jabben (RIVM, A. van Leeuwenhoeklaan 9, 3720 BA Bilthoven, Netherlands, jan.jabben@rivm.nl), Eric Schreurs (RIVM, A. van Leeuwenhoeklaan 9, 3720 BA Bilthoven, Netherlands, eric.schreurs@rivm.nl)

Current traffic noise impact assessments are usually based on broadband A-weighted noise indicators. A more complete picture and better correlation with annoyance and health effects may result from indicators that include temporal aspects and frequency character. These aspects are currently under investigation at RIVM. This paper gives an evaluation of low frequency noise impact from road traffic in the Netherlands. The results are based on low frequency noise maps covering the entire road network. In particular the effectiveness of various types of noise control measures, such as noise barriers or walls, porous asphalt layers and reduction of noise emission, with regards to low frequency impact were studied. The results indicate for low frequency noise levels, powertrain noise and freight traffic in general, are of major importance for environmental quality.

2pNS12. In Situ Characterization and Noise Mapping of Ships Moored in the Port of Venice. Antonino Di Bella (Dept. Technical Physics - University of Padova, Via Venezia 1, 35131 Padova, Italy, antonino.dibella@unipd.it), Andrea Tombolato (Studio di Acustica, Via Tripoli 16, 35141 Padova, Italy, tombolato@acusticapd.191.it), Stefano Cordeddu (Studio di Acustica, Via Tripoli 16, 35141 Padova, Italy, cordeddu@acusticapd.191.it), Emanuele Zanotto (Autorità Portuale di Venezia, Porto Commerciale - Molo A - Fabbricato 448, 30175 Marghera, Italy, emanuele.zanotto@port.venice.it), Marco Barbieri (Autorità Portuale di Venezia, Porto Commerciale - Molo A - Fabbricato 448, 30175 Marghera, Italy, marco.barbieri@port.venice.it)

The acoustical characterization and the mapping of noise emitted from ships were carried out on behalf of the Venice Port Authorithy in the frame of the European Project NoMEPorts (Noise Management in European Ports). According to the Directive 2002/49/CE (END), different noise sources (like roads, railways, aircraft, industrial sites, ports) have to be considered and mapped separately; so, in the first step, the acoustical characterization of ships is required. Annex IV of the Directive, in particular, establishes that the strategic noise maps for agglomerations shall put a special emphasis on the noise emitted by, inter alia, industrial activities, including ports. Three kinds of ships were investigated, using the Italian technical standards UNI 10855 and UNI 11143: 1. inland navigation ships (along wharves); 2. ferries (along wharves and when maneuvering); 3. cruises (along wharves and when maneuvering). As for the maneuvering, it could be said that a supplementary noise indicator is needed, as the END, Annex I, forsees in the case the average number of noise events in one or more of the day periods is very low (for example, less than one noise event an hour).

2pNSI3. Mapping urban noise quota generated by a transport service. Sergio Luzzi (Vie En.Ro.Se. Ingegneria, Via Stibbert, 1, 50134 Florence, Italy, sergio.luzzi@vienrose.it)

The paper refers to a recent noise mapping experience, regarding the determination of quotas generated by urban transport services in complex areas. The first results of this study have been presented at Euronoise 2006 in Tampere and at Transport Noise 2006 congress in Saint Petersburg. Now the study is completed. Italian Law established that noise levels produced by buses, coaches and trams, must respect noise limits of area and road buffers classification. Public and Private transport companies are directly requested to point out their specific contributions levels and action plans for the reduction of those levels. At the same time, data for 2002/49/EC Directive have to be collected by them and given to authorities in agreement with formats and standards. The original methodology of source analysis, noise mapping and action planning studied and experienced by the authors in the Florentine area, applies a multi-variable couple-checking algorithm for the optimal choice of critical sites, to the whole road network of each company, reducing the number of calculations and conducting measurement campaigns only for the chosen critical "pivot" scenarios. Noise contribution quotas, generated by bus and coach traffic on linear sources and road systems, have been investigated and considered in models.

2pNSI4. Uncertainty of noise mapping software. Jacek Wierzbicki (AGH University of Science and Technology, Al. Mickiewicza 30, 30-059 Kraków, Poland, wierzbic@agh.edu.pl), Wojciech Batko (AGH University of Science and Technology, Al. Mickiewicza 30, 30-059 Kraków, Poland, batko@agh.edu.pl)

Noise map is a tool to come to noise policy and especially to an action plan. The most important question connected with noise mapping realized by computational methods is about reliability of results. It is closely connected with quality of input data and calculation algorithms and their parameters. An influence of elements location, buildings height and number of vehicles accuracy on calculated results was considered in first stage. Before implementation of Harmonoise/Imagine P2P algorithms there are differences between various software. The paper presents first results from investigations with two commercial prediction programs. The absolute accuracy (uncertainty) of predicted by software sound levels is very difficult to quantify because an uncertainty in the measurements. The main result of 
investigations is a table of differences in sound level calculations related to variation of input data (geometry of objects and acoustic parameters) and calculation algorithm

2pNS15. Noise mapping of major roads in Milan District. Alessandro Bisceglie (Department of Environmental Sciences of the University of Milano - Bicocca, Piazza della Scienza, 1, 20126 Milan, Italy, alessandro.bisceglie@unimib.it), Giovanni Zambon (Department of Environmental Sciences of the University of Milano - Bicocca, Piazza della Scienza, 1, 20126 Milan, Italy, giovanni.zambon@unimib.it), Fabrizio Artom (Phoneco S.r.l., Via San Cristoforo 84, 20090 Trezzano Sul Naviglio, Italy, artom@phoneco.it), Mattia Viganò (Phoneco S.r.l., Via San Cristoforo 84, 20090 Trezzano Sul Naviglio, Italy, vigano@phoneco.it), Franco Bertellino (Microbel s.r.l., via Livorno 60, 10144 Torino, Italy, franco.bertellino@microbel.it), Marco Longoni (Provincia di Milano, Corso di Porta Vittoria 27, 20122 Milano, Italy, m_longoni@provincia milano.it)

The paper deals with noise map calculation, according to the first deadline of the END (Environmental Noise Directive, 2002/49/EC), for Milan district major roads. The roads with more than six million vehicle passages per year have been estimated to cover $405 \mathrm{~km}$ out of $1090 \mathrm{~km}$ total. Noise emission has been calculated from peak hour flow data for each segment of the whole road network and from some local traffic direct measurements. A statistical method to assign mean flow values to each road segment has been used, according to Italian reference periods. These data enable to use an acoustic model on a wide area with several line sources in order to obtain noise propagation maps of Lden and Lnight indicators. A procedure to estimate people exposed to different noise levels, as required by the END, has been also studied. Through a geographic information system it has been possible to intersect population input data (demographic density for different areas, buildings use type and buildings volumetric data) and noise mapping data. Every step of the procedure has been carried out complying with WGAEN Good Practice Guide recommendations, on the basis of the best available data in Milan district.

2pNSI6. 3D noise map of an hospital and noise sources evaluation Paulo J. Sequeira Goncalves (EST / Instituto Politecnico de Castelo Branco, Av Empresario, 6000-767 Castelo Branco, Portugal, pgoncalves@est.ipcb .pt)

This paper presents the tri-dimensional acoustical characterization of Castelo Branco Hospital. Traffic and air conditioning machines produce noise that influences the Hospital and the buildings nearby. The results are presented in two dimensions at a height of four meters above the ground and in the vertical direction to access the noise levels in the building floors. For the construction of the model were used and compared to types of measurements of vehicles counting, for characterization of the traffic noise. The first approach was performed at every hour during the 24 hours of the day, by counting periods of 10 minutes. In the second approach counting was performed for 30 minutes on six time intervals, identified by inquiries to the nearby population and by a 24 hour noise measurement. The air conditioning machines were modelled using the Portuguese norm, NP EN ISO 3744. Correlation results taken from the two previous approaches and the noise measurements used to validate the Cadna/A model showed values of 0.87 and 0.85 . Since they are very similar, one can conclude that both approaches can be used in practice. Taking the economical factor, the second approach is preferable because it takes less time to be performed.

2pNS17. Management of the Tourist \& Entertainment Noise Using Noise Mapping Technique. Alan Štimac (DARH2 Acoustics \& Civil Eng. Ltd., Ljubicin prolaz 3, HR-10430 Samobor, Croatia, alan@darh2.hr), Aleš Globevnik (A-PROJEKT Natasa Kepe-Globevnik s.p., Vinarje 110b, SI-2000 Maribor, Slovenia, aprojekt@siol.net), Lilijana Kuhelj (Environmental Agency of the Republic of Slovenia, Vojkova 1B, SI-1000 Ljubljana, Slovenia, lilijana.kuhelj@gov.si)

In countries with strong orientation in tourism and entertainment, especially during summer months, noise problems are always present. This paper represents some applications of noise mapping techniques for the management of tourist $\&$ entertainment noise that were conducted in Croatia and
Slovenia. The noise mapping techniques were applied for the prediction of noise levels on several places inside the cities of Varaždin and Pola (Croatia) including famous Roman amphitheatre, and in the city of Nova Gorica (Slovenia) especially during entertainment live-shows (concerts, movie festival etc). Such approach enables the city authorities to prepare the base for the implementation of noise policy with introducing such a system that would represent background documents for providing criteria of setting out areas on one side intended to entertainment and quiet areas on the other. For the daily activities in areas that are the most attractive for tourists, noisemodelling techniques have been used for predicting noise emitted from pubs and bars including terraces and loudspeakers. Such approaches have been used by city authorities to establish technique for "fixed quotas" that will be able to designate maximum area of terraces concerning number of seating places in relation with the type of bar/pub and noise emission.

2pNS18. Managing Input Data Uncertainty within Strategic Noise Mapping by Utilising Data Schema Specifications. Simon J. Shilton (Acustica Ltd, Trident One, Styal Road, M22 5XB Manchester, UK, simon.shilton@acustica.co.uk), Nigel Jones (Extrium Ltd, Calverley House, 55 Calverley Road, TN1 2TU Tunbridge Wells, UK, nigel.jones@extrium.co.uk), James Trow (Hepworth Acoustics Ltd, 5 Bankside, Crosfield Street, WA1 1UP Warrington, UK, james.trow @hepworth-acoustics.co.uk)

With the proclamation of the Directive on Environmental Noise (2002/49), the process of noise mapping and action planning has begun around Europe. Large scale wide area noise modelling requires an extensive range of data inputs. The requirements for these inputs are driven by several factors, including the noise calculation methodologies, noise mapping software system, GIS and the deliverables set out within the Directive and recommended Reporting Mechanism. Utilising a staged approach to project design led to the development of detailed noise mapping data schema specifications prior to the collation of input datasets for the calculation process. The data schema may be used throughout the mapping project as a framework to manage dataset concatenation, data capture or the use of defaults and GPGv2 Toolkits to infill data gaps. They also enable data preprocessing guidelines to be passed to non-acoustic GIS specialists, and help to guide and catalogue data processing steps. This paper presents experience gained whilst developing a number of noise mapping data schema specifications and their implementation within successful projects during the first round of mapping. Railway noise mapping is used as an example of how a data schema may be used to develop solutions to technical problems as well as coordinate data capture.

2pNS19. On railway noise modelling - an approach to the european interim method. J. Luis Bento Coelho (CAPS, Instituto Superior Técnico, TU Lisbon, Av. Rovisco Pais, P-1049-001 Lisbon, Portugal, bcoelho@ist.utl.pt), Diogo Alarcão (CAPS, Instituto Superior Técnico, TU Lisbon, Av. Rovisco Pais, P-1049-001 Lisbon, Portugal, diogo.alarcao@ist .utl.pt)

Railway noise results from a number of sources, with airborne and structure borne transmission mechanisms being responsible for the noise radiation and propagation. The European Directive 2002/49/EC requires the drawing of noise maps for areas near major transport infrastructures. The Portuguese Noise Act of 2007 further requires the drawing of noise maps for all transport infrastructures. This paper reports on our experience on noise mapping of the major Portuguese railway lines. The interim calculation model RMR96/SRMII, recommended in Directive 2002/49/EC, was adopted for the prediction of the railway noise, where a $1 / 1$ octave band noise spectrum curve fitting approach was followed. Details on the implementation, adaptation and validation of the calculation procedure are reported. Emphasis on the required data such as train categories, superstructure, and speed profiles will be given. Examples and results for a number of major railway lines will be presented. 
2pNS110. Is DNL appropriate for airport noise zoning? Jules G. Slama (UFRJ, Av. Horacio Macedo, 2030, Centro de Tecnologia, Bl. G, Sala 204, Cidade Universitária, 21941-914 Rio de Janeiro, Brazil, julesslama@yahoo.com.br), Félix A. Mora-Camino (Ecole Nationale de l'Aviation Civile, 7 avenue Edouard-Belin BP 54005, 31055 Toulouse, France, felix.mora@enac.fr), Teo C. Revoredo (UFRJ, Av. Horacio Macedo, 2030, Centro de Tecnologia, Bl. G, Sala 204, Cidade Universitária, 21941-914 Rio de Janeiro, Brazil, teorevoredo@yahoo.com.br)

The use of the DNL metric in airport noise zoning is based in the works of Schultz, that were later improved by Fidell. The basic idea is to use the percentage of highly annoyed people as a parameter to organize the area around airports. In urban zoning it is recommended to use the equivalent sound level LAEQ, which uses different criteria to day and night periods to define the land use. With regards to airport noise one may use LAD and LAN metrics which are the equivalent sound levels determined for day and night period, respectively. In this case, the maximum levels chosen to the establishment of the zoning are based on the land use. It is possible, through a simple equation, to determine the DNL level associated to a LAD, LAN pair. The inverse problem, however, has not a unique solution, once this relation depends on the number of daily and night flights of the airport. Thus, the use of DNL in airport noise zoning may lead to completely different results for different airports. Exemples are presented and discussed for two main brazilian airports: Recife and Congonhas.

TUESDAY AFTERNOON, 1 JULY 2008

P2-A, LEVEL 2, 3:40 TO 5:20 P.M.

\title{
Session 2pNSm
}

\section{Noise and EURONOISE: Noise Mapping Techniques and Uncertainties II (Poster Session)}

\author{
Wolfgang Probst, Chair \\ DataKustik GmbH, Gewerbering 5, Griefenberg, 86926, Germany
}

\begin{abstract}
All posters will be on display from 3:40 p.m. to 5:20 p.m. To allow contributors an opportunity to see other posters, contributors of odd-numbered papers will be at their posters from 3:40 p.m. to 4:30 p.m. and contributors of even-numbered papers will be at their posters from 4:30 p.m. to 5:20 p.m.
\end{abstract}

\section{Contributed Papers}

2pNSm1. Strategic noise mapping in slovak republic. Milan Kamenicky (Norsonic Slovensko s.r.o., Letisko M.R. Stefanika 63, 82001 Bratislava, Slovakia, kamenicky@norsonic.sk), Peter Petrak (AkusTech s.r .o., Lubovnianska 14, 85107 Bratislava, Slovakia, akustech@akustech.sk)

In the EU there is an ongoing process of strategic noise mapping and action planning. The goal is to determine the noise produced by the main noise sources - road, rail and air transport and industrial facilities and the exposure caused by them in densely populated agglomerations. Subsequently it is necessary to consider possibilities, how to reduce additional increase of noise levels in the environment. Authors participated in the strategic noise map development process and this paper comprises the information about the measurement and calculation results of traffic and industrial noise in Bratislava and in the slovak republic. Presentation of strategic noise maps and actions plans in Slovakia.

2pNSm2. Mapping of noise caused by military airplane operation. Milan Kamenicky (Norsonic Slovensko s.r.o., Letisko M.R. Stefanika 63, $82001 \quad$ Bratislava, Slovakia, kamenicky@norsonic.sk), Peter Petrak (AkusTech s.r.o., Lubovnianska 14, 85107 Bratislava, Slovakia, akustech@akustech.sk)

Noise from military airplane operations is a serious problem in affected surroundings of military airports. Usually, military airplane noise emission data are not available in databases of common programs, which causes problems in noise load modeling. In such cases it is necessary to obtain emission data by measurement. Measurement must be performed in modes which are not used in ordinary airplane operation. Based on these measurements, acoustic emission data will be processed in the required form according to a used mathematical model. This paper will include examples of acoustic data acquisition and their utilization in mapping of noise load caused by military airplane operations in the surroundings of military airports. Also, verification of data acquired by prediction and measurement in the airport surrounding ordinary airplane operations will be present.

2pNSm3. Effect of limitations of ray-tracing software on predicting community from petrochemical plants. Frank Brittain (Bechtel, Corp., 2255 Peavine Valley Road, Reno, NV 89523, USA, fhbritta@bechtel.com), Marlund Hale (Advanced Engineering Acoustics, 663 Bristol Ave., Simi Valley, CA 93065, USA, noisedoc@aol.com)

Ray-tracing software has proven to be a valuable and powerful tool to predict community noise from outdoor petrochemical and power plants. Accurate predictions are necessary to determine noise reductions needed to meet regulations and/or project noise limits, and then to determine individual equipment noise limits, select add-on noise controls, and confirm the plant will comply with its noise limits. Modeling using ray-tracing software is much more powerful and versatile than a spreadsheet model, which had often been used. Ray tracing and similar image source software have many limitations, particularly for outdoor petrochemical and power plants. To effectively use ray-tracing software, the user needs to understand those limitations. For example, ray tracing does not adequately predict for configurations where distances are significant compared to the wave length of sound, reflections from dense pipe racks, or screening by pipelines and equipment. This paper identifies and discusses some of the limitations of ray-tracing software for predicting community noise. Examples are given. This paper emphasizes limitations of ray-tracing methodology, and not those arising from atmospheric effects. 


\title{
Session 2pNSn
}

\section{Noise and EURONOISE: Railway Noise and Vibration II}

\author{
Richard A. Carman, Cochair \\ Wilson, Ihrig \& Associates, 5776 Broadway, Oakland, CA 94618, USA \\ Franck Poisson, Cochair \\ SNCF DIR, 45, rue de Londres, Paris, 75379, France \\ David Thompson, Cochair \\ Institute of Sound and Vibration Research, University Road, Highfield, Southampton S017 1BJ, UK
}

\section{Invited Papers}

$5: 20$

2pNSn1. The railway noise reductions achieved in the Silence project. Franck Poisson (SNCF DIR, 45, rue de Londres, 75379 Paris, France, franck.poisson@sncf.fr), Pierre Etienne Gautier (SNCF DIR, 45, rue de Londres, 75379 Paris, France, pierre-etienne .gautier@sncf.fr)

The European project Silence is dedicated to the reduction of railway and roadway noise in urban areas. Within the framework of the subproject $\mathrm{E}$ and $\mathrm{G}$, the reduction of the railway noise is addressed. Source ranking of state of the art rolling stocks has been carried out. Then, the Diesel engine, the exhaust, the cooling unit, the electrical engine, the wheels and the track have been optimised by the manufacturers and prototypes have been constructed. The most promising have been selected through laboratory measurement to be installed on the trains and trams. Last measurement campaigns have been carried out to characterise the combination of optimised rolling stocks running on optimised track. Then, relevant reductions of the pass-by noise have been achieved for electrical and Diesel multiple unit trains, freight trains and trams. In the same time, a software called VAMPPASS has been developed in subproject B to simulate the time signal of a train pass-by. The paper focuses on the results of the last measurement campaigns which validate the noise reduction achieved on the global pass-by noise of several rolling stocks and tracks. A comparison with the reduction predicted by VAMPPASS will be also presented. Some improvement of the solutions will be also addressed.

\section{$5: 40$}

2pNSn2. Principles for selection of rail fastening systems for control of railway noise and vibration. Stephen J. Cox (Pandrol Rail Fastenings Ltd, 63 Station Road, KT15 2AR Addlestone, UK, s.cox@pandrol.com)

Railways generate noise and vibration as trains pass along the track. It is usually ultimately noise that causes annoyance to those above or alongside the track, but this noise is often generated as a result of vibrations transmitted along and away from the track. Examples of ground-borne vibration at the surface above railway tunnels; airborne noise from trains passing at grade; and noise from trains passing over structures such as viaducts and bridges are each considered in turn. The selection of suitable rail fastening systems, and in particular their stiffness, to control each of these problems is discussed. This often requires compromise - for example, reduction of the noise emitted from the rail may suggest a high stiffness, while reduction of noise from the supporting structure may require a low stiffness. These considerations lead to different solutions to different problems. Some general conclusions are drawn as to how, and to what extent, each of the different types of railway noise and vibration problem may be addressed by modifications to the track fastening system.

\section{6:00}

2pNSn3. Cost-effectiveness of railway noise measures in practice. Chiel Roovers (ProRail, P.O. Box 2038, Department VMJB, Railway Noise Knowledge Centre, NL-3500 GA Utrecht, Netherlands, Chiel.Roovers@prorail.nl)

The Dutch noise nuisance law enforces railinframanager ProRail to take noise measures when railway transport is growing, new lines are built or changes are made to the existing lines. Also, measures are taken by the Dutch government at existing "hotspots". At the moment the toolbox of measures includes noise barriers, sleeper renewal, raildampers, acoustic grinding and façade insulation. The life-cycle costs of these measures have been derived from recent projects. This paper focuses on the evaluation of the cost-effectiveness of these measures in typical situations where the choice of the application of noise mitigation measures was made recently. In what circumstances is it considered to be appropriate to take measures, and how much money will be available? Are source measures like raildampers more cost-effective compared to noise barriers? The introduction of a track-access-charge system which differentiates on the noise emission of trains concludes this paper. 


\section{Contributed Papers}

\section{6:20}

2pNSn4. Comparing railway noise prediction results for passenger trains using various models. Malgorzata Reiter (Gdansk University of Technology, Multimedia Systems Department, 11/12 Gabriela Narutowicza Street, 80-952 Gdansk, Poland, reiter.m@sound.eti.pg.gda.pl), Bozena Kostek (Gdansk University of Technology, Multimedia Systems Department, 11/12 Gabriela Narutowicza Street, 80-952 Gdansk, Poland, bozenka @ sound.eti.pg.gda.pl)

Railway noise modeling is a requirement for all European countries. Under the European Directive on the Assessment and Management of Environmental Noise, 2002/49/EC all member states were obliged to create strategic noise maps of the major railways by June 30th, 2007. Some of the participating states have their own national schemes dedicated to noise prediction. For those not having their own noise prediction model, the recommendation is to use the Dutch SRM II Model. The most important assessment criterion is an inaccuracy value, which is defined as a difference between the result calculated based on a model and the actual measurements under the same atmospheric conditions simulated. Therefore, it is essential to check out which of the main calculation schemes produces most accurate results. Therefore, all above models were implemented numerically and calculations of the noise maps were performed with the use of: Schall 03 developed in Germany, Dutch SRM II and the Nordic model engineered in Norway. Those models were tested employing sample passenger trains data exploited in Gdansk, Poland. Noise maps were drawn in CadnaA software and then were compared to the field noise measurement test results.

\section{6:40}

2pNSn5. Dynamic wheel/rail forces induced by trams at low frequencies. Olivier Chiello (INRETS, Transport and Environment Lab., 25 avenue François Mitterrand, 69675 Bron cedex, France, olivier.chiello@inrets.fr), Marc Maldonado (Research Inst. of Civil Eng. and Mech., UMR CNRS 6183, 1 rue de la Noë, 44321 Nantes Cedex 3, France, marc.maldonado@ec-nantes.fr)

This work is part of various experimental and theoretical investigations into the noise and the vibrations caused by trams in Nantes, France. It focuses on the problem of the low frequency vibrations induced in the track and the ground and particularly on the excitation mechanism at the wheel/rail interface. In order to estimate the corresponding dynamic wheel/rail forces, axle-box vibrations have been measured on a carrying bogie in various vehicle and track configurations. Additionally, a specific instrument has been used to measure the rail unevenness at large wavelengths likely to excite the vehicle/track system in the low frequency range. Finally, the vertical receptance of the different tracks has been measured by using an impact hammer. In the paper, all these experimental results are presented and the validity of a simple vehicle/track interaction model is discussed.

\section{7:00}

2pNSn6. A fast time-domain model for wheel/rail interaction demonstrated for the case of impact forces caused by wheel flats. Astrid Pieringer (Chalmers University of Technology, Division of Applied Acoustics, SE-41296 Gothenburg, Sweden, astrid.pieringer@chalmers .se), Wolfgang Kropp (Chalmers University of Technology, Division of Applied Acoustics, SE-41296 Gothenburg, Sweden, wolfgang.kropp @ chalmers.se)

The prediction of impact forces caused by wheel flats requires the application of time-domain models that are generally more computationally demanding than are frequency-domain models. In this paper, a fast timedomain model is presented to simulate the dynamic interaction between wheel and rail, taking into account the non-linear processes in the contact zone. Track and wheel are described as linear systems using impulse- response functions that can be precalculated. The contact zone is modelled by non-linear contact springs, allowing for loss of contact. This general model enables the calculation of the vertical contact forces generated by any kind of roughness excitation between wheel and rail. Here, the model is adapted to the excitation caused by wheel flats by introducing the irregular wheel shape as a form of extreme roughness. A brief parameter study is presented to demonstrate the functioning of the model. The results from the model are discussed and compared with results from literature.

\section{$7: 20$}

2pNSn7. Testing a new rail roughness measurement standard. Chris J. Jones (Institute of Sound and Vibration Research, Univeristy of Southampton, Highfield, SO17 1BJ Southampton, UK, cjcj@isvr.soton.ac.uk), Fabien Létourneaux (SNCF - Agence d'Essai Ferroviaire, 21 avenue Salvador Allende, F-94407 Vitry-Sur-Seine, France, fabien.letourneaux@ sncf .fr), Pascal Fodiman (SNCF - Direction Générale Déléguée Infrastructure, 34 rue du Commandant Mouchotte, 75699 Paris Cedex 14, France, pascal .fodiman@sncf.fr)

Railway rolling noise arises from the combined roughness of the wheel and rail surfaces. The rail roughness is therefore an important parameter in the assessment of train pass-by noise. The Technical Specifications for Interoperability (TSI's) in Europe require noise to be measured on a 'reference track' the conditions of which are controlled. A spectral limit for the rail roughness is a major part of that control. The TSIs needed a new standard for rail roughness measurement so the CEN commissioned TC 256 Working Group 3 to draft one. The final draft of the new standard is now complete and will soon be published. This paper describes a 'road test' that was part of the standard development process. This test involved asking eight teams from all over Europe with seven different instruments to measure roughness at the same site, independently making their own interpretation of the standard. The paper outlines the aims of the new standard and describes the road test. It presents results and conclusions of the test that have been used already to improve the standard. Consideration had to be made of how railhead defects are treated and how different measurement technologies are used.

\section{$7: 40$}

2pNSn8. Brake noise measurements on mixed freight trains with composite brake blocks. Erwin H. Jansen (TNO Science and Industry, PO Box 155, 2600 AD Delft, Netherlands, erwin.jansen@tno.nl), Michael G. Dittrich (TNO Science and Industry, PO Box 155, 2600 AD Delft, Netherlands, michael.dittrich@tno.nl), Erik L. Sikma (Lloyd's Register Rail Europe B.V., PO Box 2016, 3500 GA Utrecht, Netherlands, erik.sikma @ lrrail.com)

In the Netherlands, the noise emission of shunting yards, which are often located in urban areas, are a source of disturbance. The noise emission of shunting yards is legislated in terms of maximum allowable equivalent sound pressure levels and peak noise levels of the façade of residences. Brake noise is known to be a major contributor to the total sound emission of shunting yards, in particular from trains with cast-iron block brakes. Within the scope of the Dutch Innovation Programme on Noise Reduction, the rolling noise reducing effect of alternative composite block brakes, such as LL and K blocks, was successfully demonstrated. Additionally, the effect of composite brake blocks on braking noise was studied. For this purpose, noise measurements were conducted on mixed freight trains according to the applicable protocols. Measurements were conducted on trains slowing down from a speed of $30 \mathrm{~km} / \mathrm{h}$ to a standstill and trains passing-by with brake systems applied. Some composite brake blocks show a considerable reduction of both braking pass-by levels and peak noise levels. Results are presented. If squeal noise is absent, braking noise of most considered brake blocks show a speed dependency similar to that for rolling noise. 


\title{
Session 2pNSo
}

\section{Noise and EURONOISE: Noise, Structure Borne Noise from Building Technical Equipment, and Ground Borne Noise from Railways II}

\author{
James Nelson, Cochair \\ Wilson, Ihrig \& Associates, Inc., 5776 Broadway, Oakland, CA 94618, USA \\ Michel Villot, Cochair \\ CSTB, 24 rue Joseph Fourier, Saint-Martin-d'Hères, 38400, France
}

Invited Papers

2pNSo1. Prediction method for structure borne noise generated in buildings by tools such as drills. Michel Villot (CSTB, 24 rue Joseph Fourier, 38400 Saint-Martin-d'Hères, France, michel.villot@cstb.fr), Philippe A. Jean (CSTB, 24 rue Joseph Fourier, 38400 Saint-Martin-d'Hères, France, philippe.jean@cstb.fr)

Tools such as drills generate structure borne noise which propagate in the building structure and radiate loud noise even in rooms far from the source. This paper presents a method for predicting this type of noise, frequent in buildings under construction or renovation. First the structural power injected by the tool to the receiving structure (floors or walls) is estimated from field measurements of the vibrational (bending) energy stored in the receiving plate. Then the vibration propagation through the building structures and the noise radiated in the receiving room is estimated using Statistical Energy Analysis (SEA), where both bending and in plane waves are taken into account. Comparisons between predictions and results measured in an apartment building under construction show that SEA gives quite acceptable results down to $1 / 3$ octave $50 \mathrm{~Hz}$ for structure borne sound sources such as drills.

\section{$5: 40$}

2pNSo2. Structure borne sound source data of building service equipments for EN 12354 part 5 - Round Robin measurements. Christian Simmons (Simmons Akustik \& Utveckling, Krokslätts Fabriker 1, 43137 Mölndal, Sweden, info@simmons.se)

At the conference, results will be presented of a round robin test where sound pressure levels and vibration data have been measured in 7 sites using two laundry machines and one heating compressor as sources. The vibrations are determined as transversal vibration levels of the concrete slabs (with the structural loss factors) according to a draft standard prEN 15657-1, as well as relative levels compared to the standardized tapping machine (ISO 140-8). The purpose is to study whether these data may be applied to predict structure borne sound in nearby spaces, according to the draft standard prEN 12354 parts 5 and 2, with a reasonable accuracy as compared to the measured sound pressure levels. The variations in space and time will be monitored carefully to observe any modal coincidence and whether the time and spatial average is the best descriptor of these types of source.

\section{6:00}

2pNSo3. A combined approach for base isolation design. Albano Neves E Sousa (IST, Department of Civil Engineering and Architecture, Av. Rovisco Pais, 1, 1049-001 Lisbon, Portugal, albano.nsousa@civil.ist.utl.pt), Luís Guerreiro (IST, Department of Civil Engineering and Architecture, Av. Rovisco Pais, 1, 1049-001 Lisbon, Portugal, luisg@civil.ist.utl.pt), Rui Gomes (IST, Department of Civil Engineering and Architecture, Av. Rovisco Pais, 1, 1049-001 Lisbon, Portugal, ruigomes@civil.ist.utl.pt)

New buildings in the vicinity of underground railway lines should be protected against noise and vibrations induced by railway traffic. In the present paper a case study is presented. A 2D FEM model of the tunnel, ground and building was built in order to identify the transfer function of vibration from the tunnel to building foundations. A 3D model of the building was built, including not only structural elements, but also other non structural walls, in order to identify the transfer function of vibration from foundations to walls and floors. As the impedance of heavy walls and floors is much higher than the acoustic impedance of air, the numerically assessed vibration fields of walls and floors were used to calculate sound fields in rooms by means of natural mode analysis. The vibration and sound fields were then compared with comfort criteria in order to design a base isolation system. With this combined approach, the analysis was performed up to $170 \mathrm{~Hz}$, which is more than one can usually do with integrated 3D models.

$$
\text { 6:20 }
$$

2pNSo4. Variance in predicted structure borne sound power due to simplified characterisation of the source. Tomos A. Evans (University of Salford, Acoustic Research Centre, Newton Building, M5 4WT Salford, UK, t.a.evans@pgr.salford.ac.uk), Andy T. Moorhouse (University of Salford, Acoustic Research Centre, Newton Building, M5 4WT Salford, UK, a.t.moorhouse@ salford.ac.uk)

Theoretically the structure borne sound power transmission from a vibration source to a receiving structure can be predicted using the mobility method. In order for all the power transmission mechanisms to be accounted for the source and receiver mobility matrices must contain the mobilities for all degrees of freedom (three translational and three rotational) for each contact point and the transfer mobilities between; each contact point, each degree of freedom and each degree of freedom at each contact point. In practice reliable 
measured data for some degrees of freedom is difficult to obtain, so simplified source characterisation methods are required, for example using only certain degrees of freedom. The novel contribution of this paper is to investigate the variance in the predicted structure borne sound power transmission calculated when some degrees of freedom are missing, and when the source is approximated as a single equivalent excitation. The investigation was carried out using numerical simulations of simplified source and receiver structures.

\section{6:40}

2pNSo5. Uncertainties and repeatability of the Reception Plate Method. Lieven De Geetere (Belgian Building Research Institute, Lombardstraat 42, B-1000 Brussel, Belgium, lieven.de.geetere@bbri.be), Bart Ingelaere (BBRI, rue du Lombard, 42, B-1000 Brussel, Belgium, bart.ingelaere@bbri.be), Pieter Schevenels (Lab. ATF, Katholieke Universiteit Leuven, Celestijnenlaan 200D, B-3001 Leuven, Belgium, pieter.schevenels@bwk.kuleuven.be)

The Reception Plate Method (RPM) as proposed by CEN/TC126/WG7 in prEN 15657-1 estimates the structure-borne sound power injected from a (high mobility) vibrating source into a (low mobility) building structure. In the near future, a round-robin test is planned to investigate repeatability and reproducibility issues of the RPM. As a preparation of this round-robin, uncertainties of the RPM are studied using the standard ISO tapping machine, placed on a 4 feet table on a reception plate. The injected power into the reception plate is calculated using different methods. The influence of the source position, accelerometer positions and airborne excitation are investigated, together with repeatability tests. Also, differences in injected power between two different brands of the ISO tapping machine will be studied. Finally, in-situ installed structure-borne sound power level will be predicted using prEN 12354-5 and compared to measurements on a simple building structure.

\section{7:00}

2pNSo6. Investigation of a vibro-acoustic reciprocal method to derive the contact forces of building equipment. Pieter Schevenels (Lab. ATF, Katholieke Universiteit Leuven, Celestijnenlaan 200D, B-3001 Leuven, Belgium, pieter.schevenels@bwk.kuleuven.be), Arne Dijckmans (Lab. ATF, Katholieke Universiteit Leuven, Celestijnenlaan 200D, B-3001 Leuven, Belgium, arne.dijckmans@bwk.kuleuven.be), Peter J. Van Der Linden (Qsources BVBA, Ketelwinning 38, 3293 Diest, Belgium, info@qsources.be), Gerrit Vermeir (Lab. ATF, Katholieke Universiteit Leuven, Celestijnenlaan 200D, B-3001 Leuven, Belgium, Gerrit.Vermeir@bwk.kuleuven.be)

Structure-borne sound caused by installations can be derived from the injected structure-borne sound power into the connected building element. Theoretically, this is as simple as summing the products of force and velocity at each contact point of the installation. The velocity can be well approximated by the velocity measured close to the contact point. But to measure the force, installation and building element would have to be separated to insert a force sensor. This is generally impractical or even impossible. In this paper, a method is analyzed to derive the force between an installation and a building element in a vibro-acoustic reciprocal way. First, a dedicated volume sound source is placed in an adjacent room and the transfer function of its volume acceleration with the acceleration of the building element is determined. Next, the sound pressure is measured at the source's position while the installation is operating. Since most installations have an important lower frequency contribution, below $50 \mathrm{~Hz}$, the sound source must cover this frequency range with sufficient level. Moreover, an accurate measure of the in-situ volume acceleration of the used source is required. Finally, the method is compared to other methods that approximate the injected structure-borne sound power.

\section{Contributed Papers}

$$
7: 20
$$

2pNSo7. Building Isolation Design for Noise Critical Applications George P. Wilson (Wilson, Ihrig \& Associates, Inc., 5776 Broadway, Oakland, CA 94618, USA, gwilson@wiai.com)

Because it is a complex, multi-degree-of-freedom system, the success of a building isolation installation depends on careful selection of a number of design factors. The new Toronto Opera House, the Four Seasons Centre for the Performing Arts, opened in June 2006, is located directly adjacent to a surface light rail trackway and to a subway rail transit system. The results characterized as "the quietest Opera House in the world" demonstrate that groundborne noise and vibration is not a factor precluding the use of a noisy site for a noise-sensitive facility. Measurement of the existing groundborne noise provided the basis to determine the required noise reduction. Very stiff and massive foundation elements with stiff and massive structure directly above the isolation bearings allows the isolation design to be simplified to a single-degree-of-freedom system for deriving the performance of the isolation at low frequencies. Natural rubber bearings with thickness adjusted to achieve a specific amount of insertion loss for the structure-borne noise can be used to determine the expected performance at frequencies above 50-80 Hz. Isolation system design parameters for the new 2000-seat Opera House Performance Hall and graphical results from vibration and noise projections and tests are presented.

\section{$7: 40$}

2pNSo8. Ground vibration requirements in vehicle projects. Janne Färm (Bombardier Transportation Mainline and Metros Division, Östra
Ringvägen 2 MLN/TSSV, 72173 Västerås, Sweden, janne.farm@se .transport.bombardier.com), Siv Leth (Bombardier Transportation Centre of Competence Acoustics and Vibration, Östra Ringvägen $2 \mathrm{CoC} \mathrm{AcV}$, 72173 Västerås, Sweden, siv.leth@se.transport.bombardier.com), Philipp Rüst (Bombardier Transportation Bogie Division, Zürcherstrasse 39 Postfach 414, 8401 Winterthur, Switzerland, philipp.ruest@ch.transport .bombardier.com)

The climate is right for trains and the environmental targets regarding noise and vibration have to be met to enhance the competitiveness of rail over other forms of transport. Ground vibration is one part that needs to be further addressed. Customer requirements on ground vibration are often included in vehicle contracts even if the vehicle is only one part of the system determining the ground vibration at the receiver point. For the design of new lines there is a need for a better optimization of overall generation of noise and vibration where both the infrastructure and vehicle parameters are included. An overview of the diversity of ground vibration requirements in different vehicle projects is given and the relation to a number of vehicle and track parameters. The potential conflict between ground vibration reduction and increased airborne noise generation is analyzed for particular examples. The background analysis clearly points out the need for a standardization regarding both setting and evaluation of requirements. There is also a need for developing one commonly used, widely accepted, validated prediction tool for ground vibration. It should facilitate engineering level decision making and allow assessment of mitigation measures. 


\title{
Session 2pPAa
}

\section{Physical Acoustics: Nonlinear Acoustics in Earthquake Processes and Other Earth Processes I}

\author{
Paul A. Johnson, Cochair \\ EES-11 (Geophysics) - Los Alamos National Laboratory, MS D443, Los Alamos, NM 87545, USA \\ Fabrice Cotton, Cochair \\ Université Joseph Fourier, Laboratoire de Géophysique Interne et Tectonophysique, CNRS, BP53, France
}

\section{Invited Papers}

\begin{abstract}
2:00
2pPAa1. Stick-slip dynamics of a granular medium. Alberto Petri (Institute of Complex Systems - CNR, via del Fosso del Cavaliere 100, 00133 Rome, Italy, alberto.petri@isc.cnr.it), Andrea Baldassarri (Dipartimento di Fisica -Università di Roma La Sapienza, P.le Aldo Moro 5, 00186 Rome, Italy, andrea.baldassarri@ roma1.infn.it), Fergal Dalton (Institute of Complex Systems - CNR, via del Fosso del Cavaliere 100, 00133 Rome, Italy, fergal.dalton@isc.cnr.it), Giorgio Pontuale (Institute of Complex Systems - CNR, via del Fosso del Cavaliere 100, 00133 Rome, Italy, giorgio.pontuale@isc.cnr.it), Stefano Zapperi (CNR-INFM, S3, Dipartimento di Fisica Universita’ di Modena e Reggio Emilia, via Campi 213/A, 41100 Modena, Italy, stefano.zapperi@roma1.infn.it)

Under certain limits, granular materials share many features with fluids and solids. Most of the time, however, they exhibit peculiar features which originate in the non-equilibrium and dissipative character of the grain dynamics. We have investigated the dynamics of a granular medium in a channel, when subject to continuous shear stress from a plate connected to a motor by an elastic spring. We have shown [1] that the statistical features of granular stick-slip dynamics are not simply Gaussian and that [2] can be described in a quantitative way by a simple stochastic equation. Extant work, such as the statistical properties of friction in solid-on-solid systems, further suggests that a large class of driven instabilities can be described in terms of similar general mechanisms. The intensity of the acoustic signals emitted by the granular medium during the slip events displays a non trivial correlation with the plate velocity. [1] F. Dalton et al., Phys. Rev. Lett. 95, 138001 (2005) [2] A. Baldassarri et al., Phys. Rev. Lett. 96, 118002 (2006)
\end{abstract}

$$
\text { 2:20 }
$$

2pPAa2. Monitoring changes in crustal properties with seismic noise. Michel Campillo (LGIT - CNRS - Université Joseph Fourier, Maison des Géosciences, 1381 rue de la Piscine, BP 53, 38041 Grenoble, France, Michel.Campillo@obs.ujf-grenoble.fr), Florent Brenguier (Laboratoire de Sismologie IPGP, 4 place Jussieu, 75252 Paris, France, fbrengui@ipgp.jussieu.fr), Celine Hadziioannou (LGIT Université Joseph Fourier/CNRS, Maison des Géosciences Domaine Universitaire, 38041 Grenoble, France, eline.hadziioannou@ujf-grenoble.fr), Nikolai Shapiro (Laboratoire de Sismologie IPGP, 4 place Jussieu, 75252 Paris, France, nshapiro@ipgp.jussieu.fr), Eric Larose (LGIT - CNRS - Université Joseph Fourier, Maison des Géosciences, 1381 rue de la Piscine, BP 53, 38041 Grenoble, France, Eric.Larose@obs.ujf-grenoble.fr)

The seismic noise is a continuous source of waves due to the interaction between the solid Earth and its fluid envelopes. Theoretically, the time correlation of the field measured at two distant points allows retrieving the Green function between the points for a random distribution of sources or in presence of a diffuse field. We use this property and study the stability of the correlation functions. We show that the velocity measurements made with correlations are very stable. Using doublet analysis, we found that that we can monitor relative velocity changes smaller than $10^{-4}$. We present applications to the forecast of volcanic eruptions and to the monitoring of an active fault.

\section{Contributed Paper}

\section{2:40}

2pPAa3. Force fluctuations in granular disks near the jamming threshold. Robert E. Ecke (Los Alamos National Laboratory, Center for Nonlinear Studies, MS-B258, Los Alamos, NM 87545, USA, ecke@lanl.gov), Mahesh Bandi (Los Alamos National Laboratory, Condensed Matter and Thermal Physics, MS-K764, Los Alamos, NM 87545, USA, mbandi@lanl.gov), Michael K. Rivera (Los Alamos National Laboratory, Condensed Matter and Thermal Physics, MS-K764, Los Alamos, NM 87545, USA, mkrivera@lanl.gov)

We study experimentally the force fluctuations felt by a probe disk as it is dragged at a nominally constant rate through a two-dimensional bi- disperse system of randomly packed photo-elastic disks. As the packing fraction increases from loose packing to a value where the disks are jammed, lines of connected force - stress chains - develop that couple the entire domain. We consider the transition from an unjammed state with no long range force distribution to a jammed state with stress chains spanning the system size. We characterize this transition using the measured force fluctuations and the determination of stress chains as visualized by the photo-elastic disks. Length and time scales obtained from these measurements are used to describe both the transition to jamming and the nature of the jammed state. The reorganization of disks and the force fluctuations have a stick-slip character in the jammed state. 


\section{Invited Paper}

3:00

2pPAa4. Rheology and mobility in a sono-fluidized granular packing. Eric Clement (Laboratoire PMMH, ESPCI, 10 rue Vauquelin, 75231 Paris Cedex 5, France, erc@ccr.jussieu.fr), Gabriel Caballero (Laboratoire PMMH, ESPCI, 10 rue Vauquelin, 75231 Paris Cedex 5, France, g.a.caballerorobledo@tnw.utwente.nl), Chay Goldenberg (Laboratoire PMMH, ESPCI, 10 rue Vauquelin, 75231 Paris Cedex 5, France, chayg@pmmh.espci.fr)

Dynamics near jamming (glassy phase, aging, memory effects and intermittency) shows amazing analogies among a variety of very different systems (colloids, dense suspensions, foams, granular materials). Recently, several proposals have emerged with the aim of describing in a general and unified way this behavior. With the purpose of testing experimentally some of these ideas, we performed several experimental studies on dry granular materials under vibration. We found that even with a weak level of vibration, such as sound injection, the behavior of granular matter is changed dramatically. We directly observe that the grains undergo a sub diffusive collective dynamics on a very long time scale which results on global compaction effects. In this regime, we have studied the rheology of an intruder moving in the bulk as a function of the level of energy injected, the driving velocity and the shape of the moving object. We also present a simple model to account for the observed behavior.

\section{Contributed Paper}

\section{$3: 20$}

2pPAa5. Irreversible interaction of sound waves with dense granular matter. Jérôme Laurent (Université Paris-Est Marne la Vallée, 5 Bd. Descartes, 77454 Marne la Vallée, France, jerome.laurent @univ-mlv.fr), Thomas Brunet (Université Paris-Est Marne la Vallée, 5 Bd. Descartes, 77454 Marne la Vallée, France, Thomas.Brunet @insp.jussieu.fr), Xiaoping Jia (Université Paris-Est Marne la Vallée, 5 Bd. Descartes, 77454 Marne la Vallée, France, jia@univ-mlv.fr)

In this talk, we will describe quantitatively the irreversible interaction between sound and the granular medium under low confining pressure $(<$ $0.1 \mathrm{MPa}$ ). Both resonance measurement and pulsed wave propagation show that as the acoustic pressure goes up to a few percent of the confining pressure, the dynamic bulk modulus and shear one can be reduced to $10 \%$ and
$20 \%$, respectively. This observation of modulus softening is qualitatively in agreement with the prediction by a model based on the Mindlin hysteretic nonlinearity at the grain contact level. Beyond certain thresholds of acoustic amplitude, the irreversible sound-granular matter interaction is accompanied by continue or intermittent sample compactions. No visible grain motion is observed at this stage; however the multiply scatted shear waves [1] allow probing a significant evolution of the contact networks, which coincide with slow recovery of elastic moduli. This work may have the implication for better understanding the physical origin of dynamic triggering of the fault core [2]. [1] X. Jia, "Coda-like multiple scattering of elastic waves in dense granular media," Phys. Rev. Lett. 93, 154303 (2004) [2] P.A. Johnson \& X. Jia, "Nonlinear dynamics, granular media and dynamic earthquake triggering," Nature 437, 871-874 (2005)

Lecture sessions will recess for presentation of poster papers on various topics in acoustics. See poster sessions for topics and abstracts.

\section{Contributed Papers}

\section{5:20}

2pPAa6. Acoustic emissions for the prediction of avalanches. Pierre De Guibert (Université Paul Sabatier, PHASE, 118, route de Narbonne, 31062 Toulouse cedex 9, France, dguibert@cict.fr), Vincent Gibiat (Université Paul Sabatier, PHASE, 118, route de Narbonne, 31062 Toulouse cedex 9, France, gibiat@cict.fr), Eric Plazza (Université Paul Sabatier, PHASE, 118, route de Narbonne, 31062 Toulouse cedex 9, France, ericvpp@ hotmail .com)

Although we know well enough the main zones where occur avalanches, the main difficulty results in forecasting avalanches because of the constant evolution of the snow coat. The coat, composed of various slopes have different properties according to the weather conditions not only during falls but also after. Weather modifications after snow falls generate instability of slopes which create stress, fissure and failure states. These different states generate acoustic emissions whose parameters can lead to forecast avalanches. The aim is to detect in situ these precursors, that initiate a low frequency signal. We have settled an experiment to monitor and register this signal. It includes two geophones to detect the acoustic signals, an acquisition system, one aerial of temperature sensor and a wireless connection to a deported PC. The system is controlled by ADSL in our offices. It will be shown on examples that any movement of the snow cap provides a signal. The rate of repetition of these elementary signals is the indication of an increasing instability of the snow precursor to an avalanche. By comparing the acoustic signals to temperature variation evolution of the snow pack we could define an alarm degree allowing to secure the area.

\section{5:40}

2pPAa7. The effect of acoustic waves on stick-slip behaviour in sheared granular media, with implications to earthquake processes. Paul A.
Johnson(EES-11 (Geophysics) - Los Alamos National Laboratory, MS D443, Los Alamos, NM 87545, USA, paj@lanl.gov), Heather Savage (University of California, Department of Earth and Planetary Science, Santa Cruz, CA 95064, USA, hsavage@ucsc.edu), Matt Knuth (University of Wisconsin, Department of Geology and Geophysics, Madison, WI 53707, USA, mwknuth@wisc.edu), Joan Gomberg (US Geological Survey, University of Washington, Department of Earth and Space Sciences, Box 351310, Seattle, WA 98195-1310, USA, gomberg@usgs.gov), Robert Behringer (Duke University, Department of Physics, Durham, NC 27708, USA, bob@phy.duke.edu), Chris Marone (Pennsylvania State University, Department of Earth and Planetary Science, University Park, PA 16802, USA, cjm@geosc.psu.edu)

We are studying the effects of acoustic waves on sheared granular material, with two goals in mind: one is to understand the intriguing physics that arises in this experimental system, and the other is to see if such experiments offer insight into earthquake processes, in particular the phenomenon where one earthquake triggers another nearby, or distant, earthquake ('dynamic earthquake triggering'). We conducted laboratory experiments of stick-slip in granular media using a double-direct, shear apparatus, while applying low amplitude vibration as well as pulsed waves. We find that vibration and pulses significantly perturb the shearing behaviour of the granular material, and that the manifestation of vibration is extremely complex, including strong material memory of the acoustic perturbation, that persists. We note that the wave disturbance must take place near the critical point, where the granular material is near failure, otherwise no effect is observed. Also, horizontal loads on the system can eliminate the effect if they arelarge $(\geqslant 4-5 \mathrm{MPa})$. 


\title{
Invited Paper
}

6:00

2pPAa8. Observations and models of dynamic earthquake triggering. Joan Gomberg (US Geological Survey, University of Washington, Department of Earth and Space Sciences, Box 351310, Seattle, WA 98195-1310, USA, gomberg@usgs.gov), Paul A. Johnson (EES-11 (Geophysics) - Los Alamos National Laboratory, MS D443, Los Alamos, NM 87545, USA, paj@lanl.gov)

Seismologists have long accepted the idea that step-function perturbations to the deformation field acting on a fault can change its likelihood of, or 'trigger', failure as fault slip. We review the observations that have lead to the very recent recognition that transient perturbations (e.g., associated with seismic waves) also affect failure probabilities, and more broadly, observations of the spatial and temporal variations in both triggering deformations and triggered responses. Many of these cannot be explained by conventional models of earthquake nucleation, requiring consideration of ideas developed in other disciplines, such as those describing and explaining nonlinear dynamic elasticity from rock-mechanics. In addition to the scientific challenges, these observations and models significantly impact earthquake forecasts and hazard assessments. We focus on observations of natural earthquakes and from the rock-mechanics laboratory, and some of the explanatory models that we and others have proposed. While many of these observations and models are just being vetted now, even newer ones related to slow aseismic fault slip and non-volcanic tremor (seismic radiation that scales very differently from that from earthquakes) may lead to substantive modifications and advances. We conclude with a few tantalizing examples as a prelude to a companion presentation.

\section{Contributed Paper}

6:20

2pPAa9. Dynamic asperity failure triggering during the rupture process? Carene Larmat (EES-11 (Geophysics) - Los Alamos National Laboratory, MS D443, Los Alamos, NM 87545, USA, carene@lanl.gov)

Dynamic triggering by the transient deformations associated with seismic waves has been proposed as an alternative mechanism for inducing aftershocks in contrast to the Coulomb stress. This idea is supported by observations of triggered seismicity like for the $\mathrm{Mw}=7.3$ Landers earthquake (1992), the $\mathrm{Mw}=7.1$ Hector Mine (1999), and after the $\mathrm{Mw}=7.4$ Izmit earthquake for which small events began in Greece immediately after the passage of the mainshock surface waves (Brodsky et al., 2000). Gomberg et al. (2003) conducted a rigorous analysis of aftershock distribution for 15 mainshocks and showed that most of the triggered events are located in the rupture direction which corresponds to a maximum of the radiated seismic energy. Another argument is the fact that the correctly scaled amplitude of the dynamic deformations are consistent with laboratory observations of failure processes (Gomberg \& Johnson, 2005). Finally, work by Felzer and Brodsky (2006) indicates that aftershocks are due to dynamic triggering rather than quasistatic stress changes. Thus instabilities can be triggered as aftershocks as well as at remote distances by seismic waves, suggesting asperities located on the mainshock fault may fail due to transient strains. In this study, we propose to explore that hypothesis.

TUESDAY AFTERNOON, 1 JULY 2008

ROOM 362/363, 2:20 TO 7:20 P.M.

\section{Session 2pPAb}

\section{Physical Acoustics: Nonlinear Acoustics of Unconsolidated Granular Media I}

\author{
Paul A. Johnson, Cochair \\ EES-11 (Geophysics) - Los Alamos National Laboratory, MS D443, Los Alamos, NM 87545, USA \\ Vitali Gusev, Cochair \\ LPEC/UMR 6087/CNRS/Université du Maine, Avenue Olivier Messiaen, Le Mans Cedex 09, 72085, France
}

\section{Invited Papers}

$$
\text { 2:20 }
$$

2pPAb1. Strongly nonlinear behavior of granular chains and granular composites. Vitali F. Nesterenko (University of California, San Diego, 9500 Gilman Drive, Department of Mechanical and Aerospace Engineering, La Jolla, CA 92093-0411, USA, vnesterenko@ucsd.edu), Eric B. Herbold (University of California, San Diego, 9500 Gilman Drive, Department of Mechanical and Aerospace Engineering, La Jolla, CA 92093-0411, USA, eherbold@gmail.com), David J. Benson (University of California, San Diego, 9500 Gilman Drive, Department of Mechanical and Aerospace Engineering, La Jolla, CA 92093-0411, USA, dbenson@ucsd.edu), Jeonghoon Kim (University of California, San Diego, 9500 Gilman Drive, Materials Science and Engineering Program, La Jolla, CA 92093, USA, jek008@ucsd.edu), Chiara Daraio (California Institute of Technology, Aeronautics (GALCIT) and Applied Physics, Pasadena, CA 91125, USA, daraio@caltech.edu)

Strongly nonlinear behavior of granular phononic crystals composed of stainless steel cylinders and PTFE spheres is considered experimentally and numerically. The tenability of phononic band gap in this two-mass prestressed chain was investigated. The results show that the phononic band gap exists within audible frequency regime $(20-20,000 \mathrm{~Hz})$ and can be tuned by changing the value of prestress. Influence of the weak and strong nonlinearity on the propagation of signals with frequencies inside band gap was investigated. The mechanical properties of reactive materials (e.g., high density mixtures of polytetraflouroethylene (PTFE), aluminum (Al) and 
tungsten (W) powders) where unconsolidated granular packing of metal particles plays significant role in the dynamic behavior was investigated. It was found that dynamic strength can be tailored by changing the morphology of the particles and porosity. Cold isostatically pressed PTFE-Al-W powder composites with fine metallic particles and a higher porosity exhibited higher ultimate compressive strength than less porous composites having equivalent mass ratios with coarse $\mathrm{W}$ particles. Based on the numerical analysis it was concluded that a mesoscale force chains between the fine metallic particles are responsible for this unusual phenomenon. This work was supported by the NSF DCMS03013220 and by MURI ONR N00014-07-1-0740.

\section{2:40}

2pPAb2. Elastic waves in one-dimensional unconsolidated medium with Hertz nonlinearity. Alexandr I. Korobov (Dept. of Acoustics, Physics Faculty, M.V. Lomonosov Moscow State University, Leninskie gory 1, 119991 Moscow, Russian Federation, akor@acs465a-1.phys.msu.ru), Maria Y. Izosimova (Center for Industrial and Medical Ultrasound, Applied Physics Lab., University of Washington, 1013 NE 40th St., Seattle, WA 98105, USA, maria.izossimova@mail.ru), Yurii A. Brazhkin (Dept. of Acoustics, Physics Faculty, M.V. Lomonosov Moscow State University, Leninskie gory 1, 119991 Moscow, Russian Federation, akor@acs465a.phys.msu.su), Elizaveta Sovetskaya (Dept. of Acoustics, Physics Faculty, M.V. Lomonosov Moscow State University, Leninskie gory 1, 119991 Moscow, Russian Federation, akor@acs465a.phys.msu.su)

Results of experimental study of elastic longitudinal harmonic wave's propagation in one-dimensional unconsolidated medium are presented. A 1-D chain of identical pre-pressed beads is used as an experimental model. To describe elastic properties of such medium the Hertz model is used. Presence of periodical structure causes elastic waves to be dispersive in such medium and to have a cutoff frequency. A cutoff frequency depends not only on material's elastic properties and beads' geometrical sizes but also on pre-press value. Velocity dependence on frequency of longitudinal harmonic wave of small magnitude is measured experimentally, and dispersion curves are built under different pre-press value. Pre-press value is regulated with applied external static force along chain axis. Measured dispersion dependences are in good agreement with theoretically calculated ones. Dispersion impedes observation of higher harmonics generation in such chain. Therefore to study nonlinear properties of 1-D structure, velocity dependence on longitudinal wave amplitude were investigated. The wave velocity depends not only on its magnitude but also on pre-press value on beads. It was experimentally shown the one-dimensional chain of elastic beads is a medium with dispersion, linear and nonlinear properties to be controlled with the applied external static force. Work supported by RFBR.

\section{Contributed Papers}

\section{3:00}

2pPAb3. Breathing in Granular Chains and Conversion of Wave Energy to Useful Forms. Surajit Sen (State University of New York at Buffalo, Dept of Physics, SUNY-Buffalo, Buffalo, NY, Buffalo, NY 142601500, USA, sen@nsm.buffalo.edu)

Estimates suggest that a meter of a wave front carries $~ 100 \mathrm{~kW}$ of power. A significant amount of renewable energy is hence dissipated across the time scale of minutes as surface gravity waves dissipate on any beach. Given that the majority of the world population lives near the ocean, it makes sense to explore the conversion of wave energy into electrical energy. Here we consider a new concept - that of a potentially scalable system that converts wave energy into energy in mechanical pulses and then to voltage drops. The envisioned system would exploit the well studied physics of nonlinear repulsion between elastic grains encased in cylinders to continuously convert the incident wave energy into dispersionless energy bundles, which can be subsequently converted to stress pulses in a piezo-electric slab. The key points involved in the concept will be presented.

\section{3:20}

2pPAb4. Wave propagation in wetted Hertzian granular chains: analysis of a single contact dynamics. Stéphane Job (Supméca Paris, 3 rue Fernand Hainaut, 93407 Saint-Ouen Cedex, France, stephane.job @ supmeca.fr), Francisco Santibañez (Univ. de Santiago de Chile - Dept. de Fisica, Avenida Ecuador 3493, Casilla 307, Correo 2, Santiago de Chile, Chile, fsantibanez@fisica.usach.cl), Francisco Melo (Univ. de Santiago de Chile - Dept. de Fisica, Avenida Ecuador 3493, Casilla 307, Correo 2, Santiago de Chile, Chile, fmelo@usach.cl)

A one-dimensional dry granular medium, a chain of beads which interact via the nonlinear Hertz potential, exhibits strongly nonlinear behaviors. When such an alignment contains some viscous fluid between grains, it exhibits new interesting features. We report some recent experiments, analysis and numerical simulations concerning nonlinear wave propagation in dry and wet chains of spheres. We consider first a monodisperse chain as a reference case. We then analyze how the pulse characteristics are modified in the presence of an interstitial viscous fluid. The fluid not only induces dissipation but also strongly affects the intergrain stiffness: in a wet chain, wave speed is enhanced and characteristic pulse duration is shorter. Our observations are in a satisfactory agreement with ultrasound propagation measurements in 3D wetted granular beds reported in the literature. Recent experiments performed with a single sphere colliding a wall covered by a thin film of fluid allowed us to determine the rheology of the contact dynamics, and confirmed that a complex elastohydrodynamic interaction takes place close to solids contact. 


\section{Invited Paper}

5:00

2pPAb5. Dynamic effective mass of granular media. David L. Johnson (Schlumberger-Doll Research, One Hampshire St., Cambridge, MA 02139, USA, johnson10@slb.com)

We report an experimental and theoretical investigation of the frequency-dependent effective mass, $M(\omega)$, of loose granular particles which occupy a rigid cavity to a filling fraction of $48 \%$. We demonstrate that this is a sensitive and direct way to measure those properties of the granular medium that are the cause of the changes in acoustic properties of structures containing grain-filled cavities. The dominant features of $\mathrm{M}(\omega)$ are a sharp resonance and a broad background, which we analyze within the context of simple models as well as with molecular dynamic simulations. We find that: a) These systems may be understood in terms of a height-dependent and diameter-dependent effective sound speed $(\sim 130 \mathrm{~m} / \mathrm{s})$ and an effective viscosity $\left(\sim 2 \times 10^{4}\right.$ Poise). b) There is a dynamic Janssen effect in the sense that, at any frequency, and depending on the method of sample preparation, approximately one-half of the effective mass is borne by the side walls of the cavity and one-half by the bottom. c) On a fundamental level, dissipation is dominated by adsorbed films of water at grain-grain contacts in our experiments, not by global viscous damping.

\section{Contributed Paper}

\section{$5: 20$}

2pPAb6. Mechanisms of acoustic absorption in weakly wet granular media. Thomas Brunet (Université Paris-Est Marne la Vallée, 5 Bd. Descartes, 77454 Marne la Vallée, France, Thomas.Brunet@insp.jussieu .fr), Xiaoping Jia (Université Paris-Est Marne la Vallée, 5 Bd. Descartes, 77454 Marne la Vallée, France, jia@univ-mlv.fr), Pierre Mills (Université Paris-Est Marne la Vallée, 5 Bd. Descartes, 77454 Marne la Vallée, France, mills@univ-mlv.fr)

Multiply scattered elastic waves provide a sensitive probe for studying the viscoelastic properties of a granular medium at the length scale of grains.
Based on a diffusion model, we can infer the structural and viscoelastic properties of the material from the fitting parameters such as the diffusion constant D and quality factor Q. In this work, we characterize quantitatively the acoustic dissipation occurring at the grain contacts by measuring the $\mathrm{Q}$ factor in different granular samples. We focus a particular attention on the effects of on the internal loss by adding a small amount of liquid in the granular medium $(\sim 0.05 \%)$. Using the Hertz-Mindlin contact theory we have identified two different mechanisms of acoustic absorption: frictional and viscous dissipations.

\section{Invited Papers}

\section{$5: 40$}

2pPAb7. Static and dynamic nonlinear behaviors of unconsolidated granular media. Zhiqu Lu (University of Mississippi, NCPA, 1 Coliseum Drive, University, MS 38677, USA, zhiqulu@olemiss.edu), James M. Sabatier (University of Mississippi, NCPA, 1 Coliseum Drive, University, MS 38677, USA, sabatier@olemiss.edu)

Unconsolidated granular media such as sands, soils, and glass beads, exhibit astonishing static and dynamic nonlinear behaviors that are quite different from those of elastic materials such as liquids and crystalline solids. They can generally classified as mesoscopic hysteretic nonlinear materials since hysteresis is a universal phenomenon for these materials. In order to understand the mechanism and to quantify the nonlinearity of unconsolidated granular materials, a series of studies have been conducted on re-molded unconsolidated soils. The systematic research consists of three parts: (1) a triaxial cell test with pre-defined stress-paths and sound speed measurement, focusing on the exploration of the role of hysteresis in both linear and nonlinear acoustics; (2) the phase shift method, using nonclassical nonlinear acoustic theory to measure the hysteretic nonlinearity parameter; (3) the study of fluid effects on the hysteretic nonlinearity parameter. The study reveals many static and dynamic nonlinear behaviors of soils, including: nonlinear stress/strain relationship, hysteresis loop, end-point memory, much greater dynamic elastic modulus than static elastic modulus, strain-amplitude dependence of elastic modulus, linear resonant frequency shift and phase shift relationships against strain, extremely high values of the hysteretic nonlinearity parameter, harmonics generation, and moisture effects.

\section{6:00}

2pPAb8. Acoustic waves in granular materials. Stefan Luding (UTwente, Multi Scale Mechanics, P.O.Box 217, 7500 AE Enschede, Netherlands, s.luding@utwente.nl), Orion Mouraille (UTwente, Multi Scale Mechanics, P.O.Box 217, 7500 AE Enschede, Netherlands, o.j.p.mouraille@utwente.nl)

The numerous sound propagation mechanisms in granular materials are rather complex and strongly depend on the material microscale parameters, such as inter-particle friction or damping. The use of DEM (Discrete Element Simulations) allows us to tune these parameters and hence to understand their influence. In both regular (crystal-like) and polydisperse (sand-like) systems of spheres, a small perturbation is created on one side of the simulation box and examined during its propagation and on arrival at the opposite side. Starting from a regular packing, a tiny size variation comparable to the typical contact deformation already changes sound propagation considerably [1]. The transmission spectrum becomes discontinuous, i.e., a lower frequency band is transmitted well, while higher frequencies are not, mainly due to scattering effects. Furthermore, the wave propagation and transmission properties are studied on fully polydisperse, frictional and cohesive packings under increasing load until breakage of the material [2]. [1] O. Mouraille and S. Luding, Sound wave propagation in weakly polydisperse granular materials, Ultrasonics, submitted. [2] O. Mouraille, O. Herbst and S. Luding, Testing cohesive and frictional granular materials with the discrete element model, in preparation. 
2pPAb9. Acoustic wave propagation in granular systems. Ellak Somfai (University of Warwick, Department of Physics and Centre for Complexity Science, CV4 7AL Coventry, UK, E.Somfai@warwick.ac.uk), Jean-Noel Roux (Laboratoire des Materiaux et des Structures du Genie Civil, Institut Navier, 2 allee Kepler, Cite Descartes, 77420 Champs-sur-Marne, France, jean-noel.roux@lcpc.fr), Jacco H. Snoeijer (University of Bristol, Department of Mathematics, University Walk, BS8 1TW Bristol, UK, jacco.snoeijer@bristol.ac.uk), Martin Van Hecke (Universiteit Leiden, Kamerlingh Onnes Laboratory, P.O. Box 9504, 2300RA Leiden, Netherlands, mvhecke@lorentz.leidenuniv.nl), Wim Van Saarloos (Universiteit Leiden, Kamerlingh Onnes Laboratory, P.O. Box 9504, 2300RA Leiden, Netherlands, saarloos@ lorentz.leidenuniv.nl)

We present numerical simulations of acoustic wave propagation in granular systems consisting of particles interacting with the three-dimensional Hertz-Mindlin force law. The response to a short mechanical excitation on one side of the system is found to be a propagating coherent wavefront followed by random oscillations made of multiply scattered waves. We find that the coherent wavefront is insensitive to details of the packing: force chains do not play an important role in determining this wavefront. The coherent wave has a well defined velocity, which is roughly compatible with the predictions of macroscopic elasticity, and its amplitude and width depend as a power law on distance. As there is at present no theory for the broadening and decay of the coherent wave, we numerically and analytically study pulse-propagation in a one-dimensional chain of identical elastic balls. The results for the broadening and decay exponents of this system differ significantly from those of the disordered packings. We briefly discuss the eigenmodes of the system and effects of damping are investigated as well.

\section{Contributed Papers}

\section{6:40}

2pPAb10. Localization of sound in a three-dimensional elastic network. John H. Page (Dept. of Physics and Astronomy, Univ. of Manitoba, Winnipeg, MB R3T 2N2, Canada, jhpage@cc.umanitoba.ca), Hefei Hu (Dept. of Physics and Astronomy, Univ. of Manitoba, Winnipeg, MB R3T 2N2, Canada, hefeihu2@uiuc.edu), Anatoliy Strybulevych (Dept. of Physics and Astronomy, Univ. of Manitoba, Winnipeg, MB R3T 2N2, Canada, anatoliy@physics.umanitoba.ca), Sergey Skipetrov (Université Joseph Fourier, Laboratoire de Physique et Modélisation des Milieux Condensées/CNRS, Maison des Magistères, 38042 Grenoble, France, Sergey.Skipetrov@grenoble.cnrs.fr), Bart Van Tiggelen (Université Joseph Fourier, Department of Applied Physics, Hokkaido University, 060-8628 Sapporo, Japan, Bart.Van-Tiggelen@grenoble.cnrs.fr)

A long standing question in physical acoustics has been whether or not the Anderson localization of sound can be demonstrated unambiguously in three dimensions. Here we address this question by reporting evidence for the localization of ultrasonic waves in a three-dimensional granular network of weakly sintered aluminum beads. In the upper part of the intermediate frequency regime, where the ultrasonic wavelength is comparable with the sizes of the pores and beads, the intensity time-of-flight profile of the multiply scattered waves exhibits non-exponential decay, which may be construed as a slowing down of the diffusion coefficient with propagation time and is consistent with predictions for localized waves. We use a quasi point source and detector to demonstrate how localization cuts off the transverse spreading of the multiply scattered waves, an effect, which we call 3D transverse localization, that has not been observed previously for any type of wave. These results are interpreted using recent theoretical predictions based on the self-consistent theory of the dynamics of localization, allowing the localization length to be determined. Further evidence is obtained from intensity statistics, which reveal a Thouless conductance g less than unity. These results unambiguously demonstrate the localization of sound in this system.

\section{7:00}

2pPAb11. Surface elastic waves in granular media under gravity and their relation to booming avalanches. Lénaïc Bonneau (Laboratoire PMMH, ESPCI, 10 rue Vauquelin, 75231 Paris Cedex 5, France, bonneau@pmmh.espci.fr), Bruno Andreotti (Laboratoire PMMH, ESPCI, 10 rue Vauquelin, 75231 Paris Cedex 5, France, Andreotti@ pmmh.espci.fr), Eric Clement (Laboratoire PMMH, ESPCI, 10 rue Vauquelin, 75231 Paris Cedex 5, France, erc@ccr.jussieu.fr)

Due to the nonlinearity of Hertzian contacts, the speed of sound $\mathrm{c}$ in granular matter is expected to increase with pressure as P1/6. A static layer of grains under gravity is thus stratified so that the bulk waves are refracted toward the surface. The reflection at the surface being total, there is a discrete number of modes (both sagittal plane and transverse ones) localized close to the free surface. The shape of these modes and the corresponding dispersion relation are investigated in the framework of an elastic description taking into account the main features of granular matter: the nonlinearity between stress and strain and the existence of a yield transition. We show in this context that the surface modes localized at the free surface exhibit a waveguide effect related to the nonlinear Hertz contact. Sound propagation measurements in a laboratory scale channel as well as in the field (slip face of a singing dune), will be presented and compared to the theoretical predictions. Finally, these results will be used to constrain the possible dynamical mechanisms at the origin of booming avalanches. 


\title{
Session 2pPAc
}

\section{Physical Acoustics: Diffraction of Waves on Periodical Structures: Acoustic, Ultrasonic and Acousto-Optical Diffraction Phenomena II (Poster Session)}

\author{
Nico Declercq, Cochair \\ Georgia Tech Lorraine - G.W. Woodruff School of ME \\ Nataliya Polikarpova, Cochair \\ M.V. Lomonosov Moscow State University, Department of Physics
}

\begin{abstract}
All posters will be on display from 3:40 p.m. to 5:20 p.m. To allow contributors an opportunity to see other posters, contributors of odd-numbered papers will be at their posters from 3:40 p.m. to 4:30 p.m. and contributors of even-numbered papers will be at their posters from 4:30 p.m. to 5:20 p.m.
\end{abstract}

\section{Contributed Papers}

2pPAc1. Information losses minimization in spectral devices based on acousto-optic Bragg cells. Boris S. Gurevich (Scientific Instruments, Rizhsky prospekt 26, 190103 St. Petersburg, Russian Federation, bgurevich@mail.ru), Simon B. Gurevich (A.F. Ioffe Physical-Technilcal Institute RAS, Politekhnicheskaya, 26, 194021 St. Petersburg, Russian Federation, sbgurevich@mail.ru), Sergey V. Andreyev (Scientific Instruments, Rizhsky prospekt 26, 190103 St. Petersburg, Russian Federation, svan51@mail.ru), Andrey V. Belyaev (Institute for analytical instrumentation RAS, Rizhsky pr. 26, 190103 St. Petersburg, Russian Federation, bgurevich@mail.ru)

Spectral devices such as spectrophotometers are intended, as many other devices, for information transmission and processing. Hence, they can be characterized by specific information characteristics such as information transmission capability and information capacity, which can be connected with some physical and technical parameters of the devices. The use of acousto-optic components in these devices introduce some peculiarities in information transmission and processing in spectrophotometers. We have considered the information transmission limitations based on signal and noise in acousto-optic Bragg cells. The spectral resolving power is also considered as one of decisive characteristics influencing on acousto-optic spectral device transmission capability.

2pPAc2. Theoretical study of the diffraction fields generated by a fluid-loaded plate with two-surface corrugation. Sarah W. Herbison (Georgia Tech Lorraine - G.W. Woodruff School of ME, UMI Georgia Tech - CNRS 2958, 2 rue Marconi, 57070 Metz, France, sherbison@gatech.edu), Nico F. Declercq (Georgia Tech Lorraine - G.W. Woodruff School of ME, UMI Georgia Tech - CNRS 2958, 2 rue Marconi, 57070 Metz, France, nico.declercq@me.gatech.edu)

A renewed interest has recently emerged in the study of the interaction of elastic waves with periodic structures, including periodically corrugated surfaces. From prior theoretical and experimental studies of the diffracted fields generated by such surfaces, it is well known that periodically corrugated surfaces can be used to generate ultrasonic surface waves, which are especially important in the field of ultrasonic non-destructive evaluation. In general, anomalies appear in the reflection and transmission spectra due to the presence of the corrugation, and these anomalies can be correlated with the surface waves generated. The present work has extended the theory of diffraction on periodically corrugated surfaces to the case of a fluid-loaded plate with both sides corrugated. The unique filtering effects and diffraction phenomena that can be achieved with such a structure are examined and related to the creation of surface and plate waves.

2pPAc3. 2D-ultrasonic tomography using first-order Born and canonical approximations. Philippe Lasaygues (Laboratory for Mechanics and Acoustics CNRS, 31 chemin Joseph Aiguier, 13009 Marseille,
France, lasaygues@1ma.cnrs-mrs.fr),Loic Le Marrec(Institute of Mathematical Research of Rennes IRMAR, Campus de Beaulieu, 35042 Rennes, France, loic.lemarrec@univ-rennes1.fr), Thierry Scotti (CNRS - LMA, 31 Chemin Joseph Aiguier, 13009 Marseille, France, scotti@lma.cnrs-mrs.fr)

This paper deals with the two-dimensional image reconstruction of an elastic tubes using ultrasonic tomographic method based on first-Born approximation and a canonical approximations. The latter improvement makes it possible to extend the scope of tomography from lower impedance contrast media to higher impedance contrast situations, even when the ultrasonic propagation is greatly perturbed by the difference in acoustic impedance between the scatterer and the surrounding medium. The strategy used to solve this problem was based on comparisons between the experimental diffracted field and the canonical solution approached as a forward problem. The algorithm adopted, using the analytical solution to the local forward problem and an iterative process to recover the unknowns, is fast enough to yield real-time information about the shape. A single frequency does not suffice to determine the real solution as the global minimum of the cost function, and the inversion is improved by using all the frequencies present in the broadband of the transducers. The method presented is robust and is not perturbed by the experimental measurements or small errors in the material properties. The results are most promising. This method gives an image with an error, which is lower than the wavelength.

2pPAc4. Light interaction with leaky acoustic wave radiation in YX-LiTaO3. Paulius Kazdailis (Vilnius Univ., Dept. of Radiophysics, Saulëtekio al. 9, LT-10222 Vilnius, Lithuania, paulius.kazdailis@ff .vu.lt), Romualdas Rimeika (Vilnius Univ., Dept. of Radiophysics, Saulëtekio al. 9, LT-10222 Vilnius, Lithuania, romualdas.rimeika@ff .vu.lt), Daumantas Ciplys (Vilnius Univ., Dept. of Radiophysics, Saulëtekio al. 9, LT-10222 Vilnius, Lithuania, daumantas.ciplys@ff.vu.lt)

The theoretical model and experimental studies of $633 \mathrm{~nm}$ laser light interaction with radiation from leaky surface acoustic waves (LSAW) at various frequencies in YX-lithium tantalate $(\mathrm{LiTaO})$ are presented. The acousto-optic diffraction with and without polarization rotation has been observed. The values of BAW propagation angle and of the corresponding velocity have been calculated and compared to the ones extracted from the experiment. The differences of the light diffraction process in YX-lithium tantalate from the one in ZX-lithium niobate have been pointed. The potential of the described acousto-optic interaction for applications in light control devices and acoustic wave probing has been demonstrated.

2pPAc5. Scattering of ultrasonic waves in randomly layered materials. Michal Pakula (Université Paris 6, Laboratoire d'Imagerie Paramétrique, 15, rue de l'Ecole de Médecine, 75006 Paris, France, michalp@ukw.edu.pl), Mieczyslaw Cieszko (Institute of Environmental 
Mechanics and Applied Computer Science, Kazimierz Wielki University in Bydgoszcz, ul. Chodkiewicza 30, 85-064 Bydgoszcz, Poland, cieszko@ukw.edu.pl), Jozef Kubik (Institute of Environmental Mechanics and Applied Computer Science, Kazimierz Wielki University in Bydgoszcz, ul. Chodkiewicza 30,85-064 Bydgoszcz, Poland, kubik@ukw.edu.pl)

The goal of the paper is the proposal of a new macroscopic description of scattering of elastic waves at internal inhomogeneities of the material. Within the studies the microscopic inhomogeneity of the medium is modeled as the alternately arranged two kinds of elastic layers of random thicknesses and different mechanical properties. Calculations of the acoustical characteristics of such medium (reflection and transmission coefficients) are performed in two stages: (i) first the problem of interaction of plane harmonic wave with the half space of the randomly layered medium for the case of normal wave incidence is analyzed; (ii) then the interaction of the harmonic wave with the slice composed of randomly layered structure is considered. Such approach allowed to derive analytical relations for the phase velocity of wave propagation and attenuation as the explicit functions of frequency, stochastic structural parameters of the medium and material properties of layers. The obtained formulas are helpful for interpretation of experimental ultrasonic data and may serve for identification of the characteristic size of material inhomogeneity based on the measured wave parameters like frequency dependent phase velocity, attenuation as well as backscattering coefficient.

2pPAc6. Design of III-Nitride multi-layer structures for optical and surface acoustic wave interaction. Samuel Dupont (IEMN CNRS 8520 DOAE, Université de Valenciennes et du Hainaut Cambrésis Le Mont Houy, 59313 Valenciennes, France, samuel.dupont@univ-valenciennes .fr), Jean-Claude Kastelik (IEMN CNRS 8520 DOAE, Université de Valenciennes et du Hainaut Cambrésis Le Mont Houy, 59313 Valenciennes, France, jean-claude.kastelik@univ-valenciennes.fr), Victor Y. Zhang (IEMN-CNRS, Av. Poincare, Cite Scientifique, B.P. 60069, 59652 Villeneuve d'Ascq Cedex, France, victor.zhang@iemn.univ-lille1 .fr), Federica Causa (Dept. of Electronic and Electrical Engineering, Univ. of Bath, BA2 7AY Bath, UK, f.causa@bath.ac.uk), Qingbin Meng (Dept. of Electronic and Electrical Engineering, Univ. of Bath, BA2 7AY Bath, UK, Q.Meng@bath.ac.uk), Jayanta Sarma (Dept. of Electronic and Electrical Engineering, Univ. of Bath, BA2 7AY Bath, UK, J.Sarma@bath.ac.uk)

Research efforts into Surface Acoustic Waves (SAW) devices built on III-Nitrides has recently significantly intensified because of the increasing availability of good-quality epitaxial material. Owing to their strong piezoelectricity and high acoustic velocity, high-Al-content materials are of particular interest in high-speed applications ( $\mathrm{GHz}$ electronics). For optoelectronics applications III-Nitrides cover a wide range of wavelengths from ultra-violet $(\mathrm{AlN})$ to blue $(\mathrm{GaN})$ to infra-red $(\mathrm{InN})$; thus providing great flexibility in the choice of operating wavelength. III-Nitrides, therefore, become particularly attractive for the development of acousto-optic modulators where the SAW is used to create a controllable (moving) optical grating, causing the diffraction of the Optical Guided Wave (OGW). The interaction is governed by the momentum and energy conservation and importantly by the field overlap of the two waves. We present here design guidelines and modelling results for the development of III-Nitride-based multi-layer structures to support both OGW and SAW. The purpose of this study is to attempt to optimise the device efficiency compared to single GaN-layer structures typically used in the literature where only the SAW characteristics are optimised.

2pPAc7. Short access time acousto-optic deflector based on two cascaded Paratellurite devices. Nabil Ouail (IEMN CNRS 8520 DOAE, Université de Valenciennes et du Hainaut Cambrésis Le Mont Houy, 59313 Valenciennes, France, nabil.ouail@univ-valenciennes.fr), Jean-Claude Kastelik (IEMN CNRS 8520 DOAE, Université de Valenciennes et du Hainaut Cambrésis Le Mont Houy, 59313 Valenciennes, France,
jean-claude.kastelik@univ-valenciennes.fr),Michel_Pommeray(IEMN CNRS 8520 DOAE, Université de Valenciennes et du Hainaut Cambrésis Le Mont Houy, 59313 Valenciennes, France, michel.pommeray @univ-valenciennes.fr), Samuel Dupont (IEMN CNRS 8520 DOAE, Université de Valenciennes et du Hainaut Cambrésis Le Mont Houy, 59313 Valenciennes, France, samuel.dupont@univ-valenciennes.fr)

Acousto-optic deflectors (AODs) are used in many applications requiring optical scanning devices with various specifications regarding access time, resolution and wavelength. High time-bandwidth products can be achieved with AODs made in a tellurium dioxide ( $\mathrm{TeO} 2)$ crystal. As paratellurite crystals are available with relatively large dimensions, the timebandwidth product can be increased by enlarging the optical aperture. However, the access time is increased which is not suitable for random access scanning mode. A new laser scanning system is then presented based on two wide-band paratellurite acousto-optic deflectors. Anisotropic interactions take place under two different tangential phase matching (TPM) configurations in such a way that the acousto-optic bandwidths add up. The optical arrangement of the two cascaded AODs is detailed. The feasibility of such a cascade deflection system has been demonstrated for the green wavelength $\lambda=514 \mathrm{~nm}$ of an argon laser. The total frequency bandwidth is $\Delta \mathrm{ft}=100$ $\mathrm{MHz}$, equally distributed between the two acousto-optic deflectors. The total angular scan at the output is $\Delta \theta t=4.5^{\circ}$ leading to more than 120 resolvable spots for a $1 \mathrm{~mm}$ truncated Gaussian beam and a short access time (as low as $1.5 \mu \mathrm{s})$.

2pPAc8. Peculierities of acoousto-optic interaction in nanodimensional laser heterostructures. Liudmila Kulakova (Ioffe Physico-Technical Institute RAS, Polytekhnicheskaya 26, 194021 St. Petersburg, Russian Federation, L.Kulakova@mail.ioffe.ru)

In the report, we present results of study of acousto-optic and acoustoelectron interactions in active and optical resonator regions of nanodimensional laser heterostructures. It was established that an ultrasonic wave introduced into a laser heterostructure produces modulation of the laser radiation frequency with a period equal to that of the acoustic wave. The static and dynamic analysis of the spectral parameters change under the ultrasonic strain has been fulfilled. The appreciable contribution of the acousto-optic interaction (comparable with the acousto-electron), resulting in the modulation in time of the positions of the heterolaser optical resonator lines was found out. The assumption about basic influence of quasi-two dimensional configuration of the laser optical resonator on the photoelastic properties causing unexpectedly large acousto-optic efficiency is stated.

2pPAc9. 3D Simulation of periodic surface wave inter-digital transducer using finite element/boundary element analysis. Sylvain Ballandras (CNRS FEMTO-ST, 32 Avenue de l'Observatoire, 25044 Besançon, France, sylvain.ballandras@femto-st.fr), William Daniau (CNRS FEMTO-ST, 32 Avenue de l'Observatoire, 25044 Besançon, France, william.daniau@femto-st.fr), Mikaël Wilm (Imasonic, ZA rue des Savourots, $\quad 70190 \quad$ Voray/Ognon, $\quad$ France, mikael.wilm@imasonic.com), Alexandre Reinhardt (CEA-LETI, 17 rue des Martyrs, 38054 Grenoble, France, alexandre.reinhardt@cea.fr), Raphaël Lardat (Thales Underwater Systems, 525 Route des Dolines, BP 157, 06930 Sophia Antipolis cedex, France, raphael.lardat@fr.thalesgroup.com), William Steichen (THALES Safare, 525 Route des Dolines, 06560 Sophia Antipolis, France, william.steichen @ fr.thalesgroup.com)

Spectacular advances have been made during the past years in the development of surface acoustic wave thanks to technology improvements but also to a strong effort in the modelling and design of such devices, taking into account their actual structure to benefit from second order effects (for instance the influence of the metal strip shape used in inter-digital transducers on the wave propagating under periodic gratings). In this paper, we present a $2 \mathrm{D}$-periodic 3D-simulation approach to compute the spectral response of one period of a surface wave transducer, accounting for the actual shape of the electrodes as well as realistic guiding conditions and acoustic properties of the propagation surface. The idea consists in meshing the inhomogeneous part of the transducer, i.e. the electrodes and the bus bar, as well as a thin layer of the substrate to match the finite element section with 
surface boundary elements simulating the acoustic contribution of the substrate (propagation and radiation). Periodic boundary conditions are applied in both surface direction, allowing for the exploitation of spectral Green's function-based boundary elements, but additional absorbing conditions are added in the transverse direction (from both part of the bus bars) to suppress any unwanted modes generated by the mesh.

2pPAc10. Acoustic wave scattering from infinite cylinders made from functionally graded materials. Jalil Jamali (Faculty of Mechanical Engineering, K. N. Toosi University of Technology, Pardis St., Molla Sadra Ave., Vanak Sq., Postal code 1999143344, 16579 Tehran, Iran, ja_ja032@yahoo.com), Farhang Honarvar (Faculty of Mechanical Engineering, K. N. Toosi University of Technology, Pardis St., Molla Sadra Ave., Vanak Sq., Postal code 1999143344, 16579 Tehran, Iran, honarvar@mie .utoronto.ca), Mohammad Hasan Naei (Faculty of Mechanical Engineering, K. N. Toosi University of Technology, Pardis St., Molla Sadra Ave., Vanak Sq., Postal code 1999143344, 16579 Tehran, Iran, mhnaei@ut.ac.ir)

Functionally graded materials (FGM's) are superheat-resistive materials which show attractive properties in many applications including furnace liners. FGM's consist of two distinct materials, for example a ceramic and a metal alloy. These two materials are mixed such that the composition of each material changes continuously along a specific direction resulting in a continuous change of microstructure along that direction. The change in microstructure induces chemical, material, and microstructural gradients, and makes functionally graded materials different in behavior from homogeneous materials and traditional composites. In this paper, the scattering of an incident plane acoustic wave from an infinite solid cylinder made from functionally graded materials is studied. Expressions are derived for the far-field scattered pressure generated by illumination of the infinite FGM cylinder by an infinite plane acoustic wave. The propagation direction of the incident wave can make an arbitrary angle with the normal to the cylinder axis. The mathematical equations are derived and numerical results for cylinders made from specific functionally graded materials are presented at different incidence angles.
2pPAc11. Pendellösung phenomenon in two-dimensional sonic crystals. Mitko Angelov (Politechnic University of Valencia, Wave Phenomena Group. Department of Electronic Engineering, C/ Camino Vera s.n, ES46022 Valencia, Spain, mitan@doctor.upv.es), Daniel Torrent (Politechnic University of Valencia, Wave Phenomena Group. Department of Electronic Engineering, C/ Camino Vera s.n, ES-46022 Valencia, Spain, datorma1@upvnet.upv.es), Francisco Cervera (Politechnic University of Valencia, Wave Phenomena Group. Department of Electronic Engineering, C/ Camino Vera s.n, ES-46022 Valencia, Spain, fcervera@fis.upv.es), José Sanchez-Dehesa (Polytechnic University of Valencia, Cami de Vera $\mathrm{s} / \mathrm{n}$, 46022 Valencia, Spain, jsdehesa@upvnet.upv.es)

The phenomenon of "Pendelösung" oscillations, which is well known in X-ray diffraction [P.P. Ewald, Phys. Z. 14, 465 (1913); Ann. Phys. 54, 519 (1917); Rev. Mod. Phys. 37, 189 (1965)] is predicted here and experimentally verified for the case of two-dimensional sonic crystals made of hexagonal arrays of fluidlike cylindrical bars embedded in water. The dependence of diffraction intensity is studied as a function of layer thickness; i.e., of the layer number N. Nearly periodic oscillations have been observed at specific frequencies and the oscillation period is also a function of the frequency. The periodic behaviour is evidence of the exchange of energy between two modes in the sonic crystal. [Work supported by MEC of Spain.]

2pPAc12. Sound diffraction in periodic surfaces in ancient architectural structures. Jorge Antonio Cruz Calleja (Instituto Pólitecnico Nacional, Av Santa Ana No. 1000 col. San Francisco Culhuacan, Del Coyoacan, 04430 México, Mexico, jorgeacruzc@hotmail.com)

In this work is analyzed the sound diffraction in architectural structures caused by periodic surfaces. Is studied the sound interaction with architectural structures using different types of sources, materials and sizes. The apparently scattering effect of the staircases in ancient constructions (like the staircase of the Kukulcan pyramid in Chichen Itza), that allows the speech communication to large distances and the spatial filter effect using the glide repetition pitch theory are also analyzed (Is the Chirp echo of the ancient prehispanic pyramids an intentional effect?). Computational models of the phenomenon and real measurements were used to establish the phenomena physics principles. 


\title{
Session 2pPAd
}

\section{Physical Acoustics: Outdoor Sound Propagation and Uncertainties II (Poster Session)}

\author{
Keith Wilson, Cochair \\ U.S. Army Cold Regions Research and Engineering Laboratory \\ Michel Berengier, Cochair \\ Laboratoire Central des Ponts et Chaussées
}

\begin{abstract}
All posters will be on display from 3:40 p.m. to 5:20 p.m. To allow contributors an opportunity to see other posters, contributors of odd-numbered papers will be at their posters from 3:40 p.m. to 4:30 p.m. and contributors of even-numbered papers will be at their posters from 4:30 p.m. to 5:20 p.m.
\end{abstract}

\section{Contributed Papers}

2pPAd1. Lossy propagation of the Gulfstream Quiet Spike. Joe Salamone (Gulfstream Aerospace, 500 Gulfstream Rd., M/S R-01, Savannah, GA 31402, USA, joe.salamone@gulfstream.com)

The Gulfstream Quiet Spike is a telescoping nose extension with multiple segments of decreasing diameter intended to reduce the outdoor loudness of a sonic boom. This work examines the influence of the atmosphere on a propagated sonic boom signature for this device. First, atmospheric data, averaged by season, has been collected as a function of latitude to reasonably bound the range of meteorological conditions. These atmospheric profiles are subsequently input to nonlinear, lossy propagation code that computes the primary sonic boom carpet for a supersonic aircraft in steady, level flight. This code is an augmented implementation of the KZK model equation for one-dimensional propagation. The confluence of shocks generated by the Quiet Spike remains distinct and do not coalesce, as intended per the design. The amplitudes, rise times and spacing of the shocks from the individual spike segments are strongly governed by dispersion and attenuation. Loudness levels calculated from the resulting ground signatures show an observable change of over $4 \mathrm{~dB}$ at certain azimuths due to the various atmospheric conditions. Based on these results, minor design changes are recommended to improve the acoustic performance of the Gulfstream Quiet Spike.
2pPAd2. Practical analysis of the influence of basic meteorological phenomena on the long-term noise level. Barbara Joanna Lebiedowska (Warsaw University of Technology, ul. Lukasiewicza 17, 09400 Plock, Poland, barbara_lebiedowska@yahoo.fr)

Current regulations on the prognosis of the acoustic climate outdoors require that meteorological conditions should be examined as part of longterm noise level calculations. The impact of meteorological conditions on the noise level was analysed using simulation calculations performed with NMPB-96 method and Mithra software. Their aim was to determine the relationship between the value of these influences and the distance between the receiver point and the noise source and its height above the ground.The distance between a source and the built-up area varied. A number of its locations in relation to the built-up area ranging between $200 \mathrm{~m}$ and $4000 \mathrm{~m}$ were considered. The built-up area consisted of a row of 11-storey buildings ca. $600 \mathrm{~m}$ long. The ground floor height and the floor height were accepted to be $3.5 \mathrm{~m}$ and $3.0 \mathrm{~m}$, respectively. Calculations were performed for various meteorological conditions: from $\mathrm{P}=0$ (homogenous conditions) to $\mathrm{P}=1.0$, increasing by every 0.1 . The receiver point was located $2 \mathrm{~m}$ away from the façade at varying heights: from the lowest point positioned at $5 \mathrm{~m}$ to the highest point at $27.5 \mathrm{~m}$, increasing by $2.5 \mathrm{~m}$, which corresponds to the median position of each successive storey, from first floor to tenth floor. 


\title{
Session 2pPAe
}

\section{Physical Acoustics: Phononic Crystals II (Poster Session)}

\author{
Pierre Deymier, Cochair \\ University of Arizona \\ Jérôme Vasseur, Cochair \\ IEMN, UMR CNRS 8520
}

\begin{abstract}
All posters will be on display from 3:40 p.m. to 5:20 p.m. To allow contributors an opportunity to see other posters, contributors of odd-numbered papers will be at their posters from 3:40 p.m. to 4:30 p.m. and contributors of even-numbered papers will be at their posters from 4:30 p.m. to 5:20 p.m.
\end{abstract}

\section{Contributed Papers}

2pPAe1. Advances in acoustics of liquid crystals. Olga Kapustina (Institute of Acoustics, 4 Shvernik Street, 117036 Moscow, Russian Federation, oakapustina@yandex.ru)

Advances in the fundamental problem of the orientation transition in liquid crystals produced by acoustical action are considered. The theoretical models to describe a variety of liquid crystals orientation transitions in acoustic fields for the homeotropically and planar oriented layers which were developed in the framework of the Leslie-Ericksen hydrodynamics and the new methodology based on the ideas of non-equilibrium thermodynamics and nonlinear hydrodynamics are discussed and are substantiated experimentally. The orientation phenomena in liquid crystals were classified and the physical factors defining a kind of transition (threshold or nonthreshold type) and a choice of approach correct to describe adequately the experimental data on a new molecular arrangement induced by acoustical action were established. Among them a macrostructure type of liquid crystals layer, a layer thickness-viscous wavelength in liquid crystal ratio, an angle between the medium optical axe (the liquid crystal director) and the line of acoustical wave propagation, a sound intensity, a type of acoustical boundary conditions at the layer edges. The modern ideas on the distortion peculiarities of liquid crystals above a certain threshold of the structure instability under non-stationary dynamic action on the liquid crystal layer of sound-induced oscillating flows for a wide frequency band were considered.

2pPAe2. SH-wave propagation and localization anomalies in semi-infinite 1D magnetic phononic crystal. Tetyana V. Laptyeva (Donetsk Institute for Physics and Technology, 72, R. Luxemburg str., 83114 Donetsk, Ukraine, lapteva@mail.fti.ac.donetsk.ua), Sergey V. Tarasenko (Donetsk Institute for Physics and Technology, 72, R. Luxemburg str., 83114 Donetsk, Ukraine, tarasen@mail.fti.ac.donetsk.ua), Olga S. Tarasenko (Donetsk Institute for Physics and Technology, 72, R. Luxemburg str., 83114 Donetsk, Ukraine, tarasen@mail.fti.ac.donetsk .ua), Vladimir M. Yurchenko (Donetsk Institute for Physics and Technology, 72, R. Luxemburg str., 83114 Donetsk, Ukraine, yurch@mail.fti.ac .donetsk.ua)

At the present, the composite magnetic materials with a superstructure (magnetic photonic crystals) are actively investigated in view of conditions of electromagnetic wave transmission. Some of these materials are acoustically continuous media and, then, can be considered as magnetic phononic crystals (MPC). However, the dynamic acoustic peculiarities of such structures have been disregarded. Theoretical investigation of SH-wave propagation in the gyrotropic and nongyrotropic semi-infinite one dimensional magnetic phononic crystals (1D MPC) is presented here. We have taken into account magnetoelastic interaction and the commensurability a wavelength and 1D MPC period. The transfer matrix method allowed us to determine the specific features of volume SH-wave localization and propagation near the rigid interface of 1D MPC and nonmagnetic medium. In particular, the necessary conditions of existence and dispersion relations for three types of shear surface acoustic waves localized near the external surface of 1D MPC have been found. This type of elastic excitation are exists also for a case when elastic properties of MPC components are identical. The conditions of reflectionless transmission of volume SH-waves through a finite 1D MPC have been determined.

2pPAe3. Zone center phonons in thalium fluoroborate. Hem Chandra Gupta (Indian Institute of Technology, Physics Department, 110016 New Delhi, India, hcgupta@physics.iitd.ac.in), Jaya Singh (Indian Institute of Technology, Physics Department, 110016 New Delhi, India, hcgupta @ physics.iitd.ac.in)

The study of phonons in thalium fluoroborate is very important in order to understand the phenomenon of phase transition in perchlorates and fluoroborates.Few studies of structural phase transition have been made of perchlorates and fluoroborates with a higher thalium ion, but their zone center phonons have not been investigated thoroughly. In order to understand the above, the Raman and the infrared frequencies of thalium fluoroborate have been investigated for the first time theoretically in its orthorhombic phase of space group Pnma. A short range force constant model (SRFCM) as well as rigid ion model (RIM) with stretching and bending force constants has been applied to obtain the zone center Raman and infrared phonons. The calculated values of Raman and infrared phonons are in very good agreement with the available observed values. The effect of these calculated interatomic force constants on the phase transition of thalium fluoroborate is discussed.

2pPAe4. Study of full band gaps and propagation of acoustic waves in two-dimensional piezoelectric phononic plates. Jin-Chen Hsu (Institute of Applied Mechanics, National Taiwan University, No. 1, Sec. 4, Roosevelt Road, 106 Taipei, Taiwan, hsu@ndt.iam.ntu.edu.tw), Tsung-Tsong Wu (Institute of Applied Mechanics, National Taiwan University, No. 1, Sec. 4, Roosevelt Road, 106 Taipei, Taiwan,wutt@ndt.iam.ntu.edu.tw)

Acoustic wave propagation in periodic structures composed of multiconstituents, namely the phononic crystals (PCs), has received much attention in the past decade. Recently, PCs constructed in the form of plate structures with two-dimensional lattices have been investigated, and some important phenomena, such as the full band-gap and waveguiding effects, induced by the periodicities of the phononic plates were reported. One of the virtues using Lamb waves is to perfectly confine the acoustic energy within the plate thickness and guided in structures with only 2D lattices rather than 3D lattices; however, the additional free surfaces derive much more complicated characteristics which are still awaiting study further. In this study, we 
investigate the band-gap and propagation properties of waves in twodimensional phononic plates that consist of either elastic or piezoelectric materials by employing a revised full 3D plane wave expansion (PWE) method and so on. To apply the PWE method efficiently, Fourier expansions are performed in the thickness direction for an imaginary 3D periodic structure by stacking the phononic plates and vacuum layers alternatively. Moreover, effects of piezoelectricity, lattices, and filling ratios on band gaps are discussed.

2pPAe5. Shannon Entropy as a Characterization Tool in Acoustics Helios Sanchis-Alepuz (Polytechnic University of Valencia, Cami de Vera s/n, 46022 Valencia, Spain, hesana@alumni.uv.es), José SanchezDehesa (Polytechnic University of Valencia, Cami de Vera s/n, 46022 Valencia, Spain, jsdehesa@upvnet.upv.es)

Shannon entropy [1] was introduced as a measure of the uncertainty associated with a random variable and initially it was used to quantify the information contained in a binary message. In this work, a definition of Shannon's information entropy is introduced in acoustics, defined in terms of the displacement field, as a measure of the localization of resonant modes. As an example, it is applied to study the avoided crossing appearing in the resonant Zener-like phenomenon in ultrasonic superlattices made of two different fluidlike metamaterials. It is shown that acoustic Shannon entropy gives a correct physical insight of the localization effects taking place and manifest the informational exchange of the involved acoustic states in the narrow region of parameters where the avoided crossing occurs. Results for ultrasonic structures consisting of alternating layers of polymethylmethacrylate (Plexiglas) and water cavities, in which the acoustic Zener effect were recently demonstrated [2], are also reported. It is concluded that Shannon entropy is a useful tool to characterize localization effects in acoustical systems. [1] C.E. Shannon, Bell Sys. Tech. J. 27, 623 (1948). [2] H. Sanchis-Alepuz, Yu.A. Kosevich, and J. Sanchez-Dehesa, Phys. Rev. Lett. 98134301 (2007).

2pPAe6. Acoustic wave band gaps in triangular and honeycomb two-dimensional phononic crystals. Fu-Li Hsiao (Institute of Optical Sciences, National Central University, 320 Jung-Li, Taiwan, flhsiao@ios.ncu.edu.tw), Abdelkrim Khelif (Institut FEMTO-ST/CNRS, 32 avenue de l'Observatoire, 25044 Besançon cedex, France, abdelkrim.khelif@femto-st.fr), Boujemaa Aoubiza (Institut FEMTOST/CNRS, 32 avenue de l'Observatoire, 25044 Besançon cedex, France, boujemaa.aoubiza@univ-fcomte.fr), Abdelkrim Choujaa (Institut FEMTOST/CNRS, 32 avenue de l'Observatoire, 25044 Besançon cedex, France, abdelkrim.choujaa@femto-st.fr), Hanane Moubchir (Institut FEMTOST/CNRS, 32 avenue de l'Observatoire, 25044 Besançon cedex, France, hanane.moubchir@femto-st.fr), Chii-Chang Chen (Institute of Optical Sciences, National Central University, 320 Jung-Li, Taiwan, trich@ios.ncu.edu.tw), Vincent Laude (Institut FEMTO-ST/CNRS, 32 avenue de l'Observatoire, 25044 Besançon cedex, France, vincent.laude @ femto-st.fr)

The ultrasonic band gap properties of phononic crystals composed of arrays of steel cylinders immersed in water and arranged according to square, triangular or honeycomb lattices are compared theoretically and experimentally. In all three cases, complete band gaps are obtained, and the conditions of existence are identified by observing the complete band gap width as a function of the size of the inclusions. However, the measured transmission spectra reveal in the hexagonal symmetry cases (triangular and honeycomb lattices) the existence of deaf bands that cause strong attenuation in the transmission, with no band gap being involved. Band gaps and deaf bands are identified by comparing band structure computations for the infinite phononic crystal, obtained by a periodic-boundary finite element method (FEM), with transmission simulations for the finite phononic crystal, obtained using the finite difference time domain (FDTD) method. The possibility of managing phononic wave guides within a defect line channel inside an otherwise perfect triangular lattice phononic crystal is further demonstrated experimentally.
2pPAe7. Shock waves in nanomechanical resonators. Robert $\mathrm{H}$. Blick (University of Wisconsin-Madison, Electrical and Computer Engineering, 1415 Engineering Drive, Madison, WI 53705, USA, blick@engr.wisc.edu), Florian W. Beil (University of Munich, Center for NanoScience, Geschwister Scholl Platz 1, 80539 Munich, Germany, beil_florian@bah.com), Achim Wixforth (University of Augsburg, Universitaetstrasse 1, 86135 Augsburg, Germany, achim.wixforth@physik .uni-augsburg.de), Werner Wegscheider (University of Regensburg, Fakultaet fuer Physik, 93040 Regensburg, Germany, werner.wegscheider @ physik.uni-regensburg.de), Dieter Schuh (University of Regensburg, Fakultaet fuer Physik, 93040 Regensburg, Germany, dieter.schuh @ physik.uni-regensburge.de), Max Bichler (Technical University of Munich, Coulombweg 1, 86748 Garching, Germany, max.bichler@wsi.tum.de)

The dream of every surfer is an extremely steep wave propagating at the highest speed possible. The best waves for this are would be shock waves, but are very hard to surf. In the nanoscopic world the same is true: the surfers in this case are electrons riding through nanomechanical devices on acoustic waves. Naturally, this has a broad range of applications in sensor technology and for communication electronics for which the combination of an electronic and a mechanical degree of freedom is essential. But this is also of interest for fundamental aspects of nano-electromechanical systems (NEMS), when it comes to quantum limited displacement detection and the control of phonon number states. Here, we study the formation of shock waves in a NEMS resonator with an embedded two-dimensional electron gas using surface acoustic waves. The mechanical displacement of the nanoresonator is read out via the induced acoustoelectric current. Applying acoustical standing waves we are able to determine the anomalous acoustocurrent. This current is only found in the regime of shock wave formation. We ontain very good agreement with model calculations.

2pPAe8. Experimental measurements of the band structure of Lamb waves in phononic lattices. Bernard Bonello (CNRS and Paris VI University, INSP - 140 rue de Lourmel, 75015 Paris, France, bernard.bonello@insp.jussieu.fr), Thomas Brunet (CNRS and Paris VI University, INSP - 140 rue de Lourmel, 75015 Paris, France, thomas.brunet@insp.jussieu.fr), Jiu-Jiu Chen (CNRS and Paris VI University, INSP - 140 rue de Lourmel, 75015 Paris, France, chen99nju@gmail .com)

We have measured the band structure of Lamb waves propagating in 2D phononic crystals. Different cases were investigated. First we have studied the phononic film deposited on a homogeneous substrate. The phononic film is made of cylindrical iron scatterers embedded into a copper background; the substrate is a $700 \mu \mathrm{m}$ silicon plate. The propagation is along the crystallographic direction [100] of Si. At low filling fraction, a frequency band gap for the antisymmetric mode arises at reduced Brillouin edge. At high filling fraction, a band gap opens also on the symmetric branch. We have also studied the case of phononic slabs with very high contrast between the scatterers and the matrix. The samples are made of $200 \mu \mathrm{m}$ silicon plates with air holes lattices drilled throughout. Whatever the symmetry of the lattice, square of centered rectangular, broad band gaps open at first and second reduced Brillouin zone edges. Experimental data are then compared to theoretical predictions obtained using a plane wave expansion method.

2pPAe9. Order/disorder transition for ultrasonic propagation in 2D scatterer arrays. Alice Bretagne (Laboratoire Ondes et Acoustique, ESPCI, Université Paris 7, CNRS, 10 rue Vauquelin, 75005 Paris, France, alice.bretagne@espci.fr), Arnaud Tourin (Laboratoire Ondes et Acoustique, ESPCI, Université Paris 7, CNRS, 10 rue Vauquelin, 75005 Paris, France, arnaud.tourin@espci.fr), Mathias Fink (Laboratoire Ondes et Acoustique, ESPCI, Université Paris 7, CNRS, 10 rue Vauquelin, 75005 Paris, France, mathias.fink@espci.fr)

Recently, intriguing phenomena in quantum mechanics, such as the Aharonov-Bohm effect [P. Roux et al., Phys. Rev. Lett. 79, 3170 (1997)], weak localization in disordered media [A. Tourin et al., Phys. Rev. Lett. 79, 3637 (1997)] and maybe one of the most striking, tunnelling [S. Yang et al., Phys. Rev. Lett. 88, 104301 (2002)], have been revisited using acoustic waves. The advantages of using ultrasound for such studies lie in the macroscopic character of samples and the possibility of directly measuring the 
phase of the wavefield. Here we explore propagation of $\mathrm{MHz}$-ultrasound in a medium which is either ordered or disordered in the two transverse dimensions $(\mathrm{x}, \mathrm{y})$ but invariant in the propagation direction $(\mathrm{z})$. An equivalent scheme has been recently used in optics to demonstrate transverse localization of light [T. Schwartz et al., Nature 446, 52 (2007)]. Our samples are made of a parallel arrangement of cylindrical scatterers $(0.8 \mathrm{~mm}$ in diameter $)$ embedded in a PVA matrix. In the ordered case, the probe beam undergoes ballistic transport. In the disordered case transport becomes diffusive and as the sample length increases the wave tends to be laterally confined.

2pPAe10. Experimental and theoretical investigations of focusing of ultrasonic waves by two-dimensional flat phononic crystals. Alexey Sukhovich (Universidad de Murcia, Dept. of Physics and Astronomy, Univ. of Manitoba, Winnipeg, MB R3T 2N2, Canada, alexei@physics.umanitoba.ca), John H. Page (Dept. of Physics and Astronomy, Univ. of Manitoba, Winnipeg, MB R3T 2N2, Canada, jhpage@cc.umanitoba.ca), Bassam Merheb (University of Arizona, Materials Science and Engineering Department, Mines Bldg., P.O. Box 210012 , Tucson, AZ 85721, USA, bassam@merheb.net), Jérôme Vasseur (IEMN, UMR CNRS 8520, avenue Poincaré, BP 60069, 59652 Villeneuve d'Ascq, France, jerome.vasseur@univ-lille1.fr), Pierre Deymier (University of Arizona, Materials Science and Engineering Department, Mines Bldg., P.O. Box 210012, Tucson, AZ 85721, USA, deymier@u.arizona.edu)

We compare experimental and theoretical results demonstrating focusing of sound fields emitted by subwavelength sources using two-dimensional flat phononic crystals. Our phononic crystals are made of stainless steel rods assembled in a triangular crystal lattice and immersed in a liquid. Focusing is achieved due to negative refraction of ultrasonic waves propagating through the crystal, which is realized at the frequencies corresponding to the 2nd band, where the group velocity and wave vector are antiparallel. High quality images are obtained experimentally with a methanol-filled phononic crystal surrounded by water, in which the source and detecting transducers are located. Excellent resolution approaching the diffraction limit is achieved for the frequency at which the equifrequency contours inside the crystal match those in water. Our experimental results are compared with theoretical predictions by the Finite Difference Time Domain (FDTD) method. Good agreement is observed for the width of the focal spot in the direction parallel to the crystal surface, while along the perpendicular direction, we find experimentally that the focal spot is narrower and closer to the crystal surface than in the theoretical predictions.

2pPAe11. Complete band gap in the phonon spectra of compact ceramics. Evgenii I. Salamatov (Physical-Technical Institute UrB RAS, 132 Kirov str., 426000 Izhevsk, Russian Federation, salam@otf.pti.udm.ru)

The processes of sound propagation in inhomogeneous systems have long the focus of researchers attention. The new interest in this problem $i$ last years has been inspired by the appearance of artificial elastic periodic structures - phononic crystals. Since the lattice parameter artificial phononic crystals is about several millimetres the complete bandgap is assumed to lie in the megahertz range. It is of fundamental importance for modern radio equipment to obtain phononic crystals with forbidden bandgap in the gigahertz range, which requires generation of phononic lattices with the period of the order of tens and hundreds nanometers. The author believes nanoceramics compacted from a superfine powder in a special way to be very promising systems for the generatio such phononic lattices. In this work the phonon spectra of the model two-dimensional ceramics numerically calculated using the finite-difference time-domain (FDTD) method. To interpret the results, the analytical expressions obtained for an elastic limit are used, which enables formalization of the conditions necessary for the appearance of a gap in a phonon spectrum. The possibility of creation of bulk acoustic waves resonators is discussed.

2pPAe12. A Transient Grating approach to elastic wave and thermal propagation in a 2D free-standing micrometric phononic crystal Iacopo Malfanti (European Lab. for Non-Linear Spectroscopy (LENS), Univ. di Firenze, via Nello Carrara 1, 50019 Sesto Fiorentino (Fi), Italy,
malfanti@lens.unifi.it),Renato Torre(European Lab. for Non-Linear Spectroscopy (LENS), Univ. di Firenze, via Nello Carrara 1, 50019 Sesto Fiorentino (Fi), Italy, torre@lens.unifi.it), Paolo Bartolini (European Lab. for Non-Linear Spectroscopy (LENS), Univ. di Firenze, via Nello Carrara 1, 50019 Sesto Fiorentino (Fi), Italy, bart@lens.unifi.it), Andrea Taschin (European Lab. for Non-Linear Spectroscopy (LENS), Univ. di Firenze, via Nello Carrara 1, 50019 Sesto Fiorentino (Fi), Italy, taschin@lens.unifi.it), Francesco Vita (via Brecce Bianche, 1, 60131 Ancona, Italy, f.vita@univpm.it), Francesco Simoni (via Brecce Bianche, 1, 60131 Ancona, Italy, f.simoni@univpm.it)

We investigate the phonon propagation in a $2 \mathrm{D}$ phononic crystal (with typical dimensions on the micrometric scale) by means of a transient grating (TG) heterodyne detected experiment. In a TG experiment both a temperature and a density grating are induced by means of optical techniques. The relaxation dynamics of the induced grating are then monitored live-time over 6 temporal decades with a probe beam. Our sample is a freestanding 100 micrometer thick polymer matrix with empty rods (filled with gas) arranged in a triangular lattice. Evidence of the presence of two different bulk wave acoustic modes are experimentally found. The excited acoustic modes show a correlation with the orientation of the sample with respect to the induced grating wave vector, while the thermal properties show a significant dependence on the magnitude of the induced grating wave vector.

2pPAe13. Thermal shifting of phononic bandgaps in barium strontium titanate-based structures. Kwok Lung Jim (Department of Applied Physics and Materials Research Centre, The Hong Kong Polytechnic University, Hunghom, Kowloon Hong Kong, China, jim.kl@polyu.edu.hk), Chi Wah Leung (Department of Applied Physics and Materials Research Centre, The Hong Kong Polytechnic University, Hunghom, Kowloon Hong Kong, China, apleung@inet.polyu.edu.hk), Chung Loong Choy (Department of Applied Physics and Materials Research Centre, The Hong Kong Polytechnic University, Hunghom, Kowloon Hong Kong, China, scclchoy@inet .polyu.edu.hk), Helen L. W. Chan (Department of Applied Physics and Materials Research Centre, The Hong Kong Polytechnic University, Hunghom, Kowloon Hong Kong, China, apahlcha@inet.polyu.edu.hk)

Phononic crystals, which are structures with periodic variations of density and/or sound velocities, can exhibit phononic bandgaps where propagation of acoustic waves is forbidden. The ability of phononic crystals to manipulate sound, in a similar manner which photonic crystal control light, makes them particularly useful for applications such as acoustic filters or very efficient waveguides. Since the positions of the phononic bandgaps depend solely on the densities and the sound velocities of the constituent materials, it is expected that a modulation in the densities and/or sound velocities of the constituent materials would result in a phononic bandgap shift. It is known that ferroelectric ceramic, such as barium strontium titanate (BST), will undergo a ferroelectric-to-paraelectric phase transition across the Curie temperature. During the phase transition, there is a large variation in the sound velocities of BST which result in a shift of the phononic bandgaps. In this work, we calculated the phononic bandstructures of BST-based structures by the plane-wave expansion method and showed that the phononic bandgaps can be shifted thermally.

2pPAe14. Nondiffractive propagation of sound in sonic crystals. Isabel Perez-Arjona (IGIC - Universitat Politècnica de València, Cra. NazaretOliva S/N, E-46730 Gandia, Spain, iparjona@upvnet.upv.es), Victor Sánchez-Morcillo (IGIC - Universitat Politècnica de València, Cra. Nazaret-Oliva S/N, E-46730 Gandia, Spain, victorsm@fis.upv.es), Víctor Espinosa (IGIC - Universitat Politècnica de València, Cra. Nazaret-Oliva S/N, E-46730 Gandia, Spain, vespinos@ fis.upv.es), Kestutis Staliunas (Departament de Fisica i Enginyeria Nuclear, Colom 11, 08222 Terrassa (Barcelona), Spain, Kestutis.Staliunas@icrea.es), Javier Redondo (IGIC - Universitat Politècnica de València, Cra. Nazaret-Oliva S/N, E-46730 Gandia, Spain, fredondo@fis.upv.es)

In this work we present the study of the nondiffractive propagation regime of ultrasonic waves in a two-dimensional periodic acoustic media (sonic crystal) both from the theoretical and the experimental point of view. An analytical treatment of this regime sonic wave propagation is presented, which allows evaluating the parameters of the nondiffractive regime, just as 
the minimum size of the nondiffractively propagating acoustic beam. Experimentally, we report on the first experimental demonstration of the subdiffractive propagation (self-collimation) of an ultrasound beam in a sonic crystal formed by steel cylinders in water. The experimental measurements show a good agreement with the theoretical predictions, where the effect of the finite size of the crystal was also considered. The preliminary results on self-collimation of sound in three-dimensional sonic crystal, in progress at the present moment, will be also presented.

TUESDAY AFTERNOON, 1 JULY 2008

P3-A, LEVEL 3, 3:40 TO 5:20 P.M.

\title{
Session 2pPAf
}

\section{Physical Acoustics: Acoustics of Porous Media III (Poster Session)}

\author{
Walter Lauriks, Cochair \\ Lab. ATF, Katholieke Universiteit Leuven \\ Keith Attenborough, Cochair \\ Open University
}

\begin{abstract}
All posters will be on display from 3:40 p.m. to 5:20 p.m. To allow contributors an opportunity to see other posters, contributors of odd-numbered papers will be at their posters from 3:40 p.m. to 4:30 p.m. and contributors of even-numbered papers will be at their posters from 4:30 p.m. to 5:20 p.m.
\end{abstract}

\section{Contributed Papers}

2pPAf1. In-situ measurement of interface permeability. Lin Lin (Universisty Of Maine, 5711 Boardman Hall, Orono, ME 04469, USA, lin.lin@umit.maine.edu), Michael Peterson (Universisty Of Maine, 5711 Boardman Hall, Orono, ME 04469, USA, michael.peterson @ maine.edu), Alan Greenberg (Univesity of Colorado, 427 UCB Dept. of Mechanical engineering, Boulder, CO 80309, USA, Alan.Greenberg @colorado.edu), Benjamin McCool (ExxonMobil Research and Engineering Company, 1545 Route 22 East, Annandale, NJ 08801, USA, Benjamin .a.mccool@exxonmobil.com)

Permeability of a porous interface is important for many areas of research and technology ranging from transport of nutrients in marine sediments to produce separations in bio-pharmaceuticals. Biot theory has shown that porous materials are able to support the propagation of two longitudinal waves: fast and slow wave. By measuring the propagation threshold of the slow longitudinal wave when the wave number is higher than the critical wave number kcr, we can obtain the intrinsic permeability of a porous interface. The ability to sense permeability change due to differences in pore size and fluid viscosity is demonstrated. The measurement is compared to an exact solution for kcr as well as previously published analytical solutions. This technique for measuring permeability has been shown in a model material and can now be extended to a wide range of materials of scientific and technical importance.

2pPAf2. Sound absorption of advanced textile materials. Ivan Bolkovac (Faculty of EE and Computing, Unska 3, Department of Electroacoustics, HR-10000 Zagreb, Croatia, ibolkovac@gmail.com), Hrvoje Domitrovic (Faculty of EE and Computing, Unska 3, Department of Electroacoustics, HR-10000 Zagreb, Croatia, hrvoje.domitrovic@fer .hr), Martina Bozic (Faculty of textile technology, University of Zagreb, Ljubijska 51a, 10000 Zagreb, Croatia, martina.bozic.ttf@gmail.com)

Today's acoustic applications demand an ever greater array of materials used for acoustic treatment of spaces for both professional or nonprofessional use. Elements like absorbers, diffusers and silencers are met with increasingly strict demands, both aesthetic and functional. That is the reason why we were interested in acoustic qualities (absorption coef.) of different kinds of textile materials that could be used in building or finishing such elements. Textile materials range from conventional to high- performance textiles. The latter, with their advanced qualities (both acoustic and mechanical) promise a much wider area of application and further specialized use, both as stand-alone elements or their parts.

2pPAf3. Influence of sound absorption on close proximity noise of porous pavement. S. E. Paje (Laboratory of Acoustics Applied to Civil Engineering (LA2IC), Universidad de Castilla-La Mancha, Department of Applied Physics, 13071 Ciudad Real, Spain, santiago.exposito@uclm .es), Moises Bueno (Laboratory of Acoustics Applied to Civil Engineering (LA2IC), Universidad de Castilla-La Mancha, Department of Applied Physics, 13071 Ciudad Real, Spain, moises.bueno@uclm.es), Urbano Viñuela (Laboratory of Acoustics Applied to Civil Engineering (LA2IC), Universidad de Castilla-La Mancha, Department of Applied Physics, 13071 Ciudad Real, Spain, urbano.vinuela@uclm.es), Fernando Terán (Laboratory of Acoustics Applied to Civil Engineering (LA2IC), Universidad de Castilla-La Mancha, Department of Applied Physics, 13071 Ciudad Real, Spain, fernando.teran@uclm.es)

The main objective of this research is to correlate sound absorption of a new bituminous porous pavement (air void content 21\%) with the close proximity noise. Different core samples were taken from a porous road surface. The normal incidence sound absorption spectra were measured for these samples using the two-microphone impedance tube. Noise levels close to the tire/pavement contact patch were measured continuously in a georeferenced way, when the test vehicle was rolling. The emission properties of the porous surface as a function of vehicles speed are analyzed in the acoustic frequency range showing different behaviors for the relationships between noise levels and speed. An abrupt variation in the relationship between the coefficient $\mathrm{B}$ and frequencies below $1 \mathrm{kHz}$ was observed $(\mathrm{Lcp}=\mathrm{A}+\mathrm{B} \cdot \log (\mathrm{V}))$. This feature could be highly influenced by sound absorption mechanism, particularly noticeable for a non-clogged porous surface with a high content of air voids.

2pPAf4. Physico-chemical properties and ultrasonic characterization of calcium phosphate ceramics. Daniela Predoi (National Institute of Materials Physics, Atomistilor 105 bis, 77123 Magurele, Romania, 
dpredoi_68@yahoo.com),Serge Derible(LOMC FRE CNRS 3102, Université du Havre, Place Robert Schuman, 76610 le Havre, France, serge.derible@univ-lehavre.fr), Hugues Duflo (LOMC FRE CNRS 3102, Université du Havre, Place Robert Schuman, 76610 le Havre, France, hugues.duflo@univ-lehavre.fr), Mihai Valentin M. Predoi (University Politechnica of Bucharest, Department of Mechanics, 060032 Bucharest, Romania, predoi@cat.mec.pub.ro), Cristian Catalin Petre (University Politechnica of Bucharest, Department of Mechanics, 060032 Bucharest, Romania, cristian.petre@promteh.ro)

Calcium phosphate compounds have been studied for biomedical applications due to their chemical and structural similarity to the mineral phase of bone and tooth. The composition, physical and chemical properties, crystal size and morphology of synthetic apatite are extremely sensitive to preparation conditions and sometimes it resulted into non- stoichiometric calcium deficient hydroxyapathite (Hap) powders. The present paper refers to calcinations of calcium phosphate ceramics at 800 and $1000^{\circ} \mathrm{C}$. The effect of heat treatment were investigated by X-ray diffraction (XRD), Fourier transform infrared (FTIR), differential thermal analysis (DTA) and thermal gravimetric analysis (TGA). FTIR spectra showed the presence of various $\mathrm{PO}_{4} 3-$ and $\mathrm{OH}$-groups present in the powders. Powders compacted and sintered at 800 and $1000^{\circ} \mathrm{C}$ showed an increase in density. The main objective of the paper is a comparison of the results obtained by the previous methods to those obtained using the ultrasonic air-coupling technique. Modulated ultrasonic signals of $500 \mathrm{kHz}$ central frequency have been transmitted through the calcium phosphate ceramics specimens. Correlation between signals allowed some conclusions concerning density, porosity and preparation temperature influence on these specimens. These comparisons and correlation of methods, allow a better characterisation of such important materials

2pPAf5. Ultrasonic monitoring of hardening concrete. Julien Bue (Institut Jean le Rond D'Alembert / UMR 7190, 2 Place de la Gare de Ceinture, 78210 Saint Cyr L'Ecole, France, julien.bue@wanadoo .fr), Frederic Cohen Tenoudji (Institut Jean le Rond D'Alembert / UMR 7190, 2 Place de la Gare de Ceinture, 78210 Saint Cyr L'Ecole, France, fcohentenoudji@yahoo.fr)

Monitoring results of velocities and attenuations of compressional and transverse ultrasonic waves in hardening concrete using short pulse throughtransmission are presented. A fluid concrete is a highly attenuating heterogeneous medium, composed of several phases and granular classes. Different attenuation mechanisms are considered in order to account for experimental results: high impedance mismatch between air layers and solid-fluid clusters at early ages, Energy loss by viscous dissipation and wave scattering by heterogeneities (Rayleigh scattering at low frequencies, single or multi diffusion scatterings at intermediate and high frequencies) during the beginning of hardening. At early ages, the medium may be described as composed of a succession of large slabs of fresh concrete separated by very thin slabs of air. Attenuation is then computed from the effective transmission coefficient of this medium. This model is in good agreement with experiments. During the beginning of hardening, the medium may be seen as composed of big particles immersed in an effective medium formed by the ensemble of the particles of lesser granular sizes and water. Then, the Rayleigh scattering model at low frequencies and the multiple diffusion model predictions at intermediate frequencies are in good agreement with experiments.

2pPAf6. Flow resistivity profile inversion for a porous medium. Claude Depollier (Laboratoire d'Acoustique de l'Université du Maine, Avenue Olivier Messiaen, 72085 Le Mans, France, claude.depollier@univ-lemans .fr), Naima Sebaa (Laboratoire d'Acoustique de l'Université du Maine, Avenue Olivier Messiaen, 72085 Le Mans, France, naima.sebaa@univ-lemans .fr), Mouna Naas (Laboratoire d'Acoustique de l'Université du Maine, Avenue Olivier Messiaen, 72085 Le Mans, France, mouna.naas.etu @ univ-lemans.fr), Bernard R. Castagnede (Laboratoire d'Acoustique de
l'Université du Maine, Avenue Olivier Messiaen, 72085 Le Mans, France, bernard.castagnede@univ-lemans.fr), Zine Fellah (CNRS-Laboratoire de Mécanique et d'Acoustique, 31 Chemin Joseph Aiguier, 13402 Marseille, France, fellah@lma.cnrs-mrs.fr), Walter Lauriks (Lab. ATF, Katholieke Universiteit Leuven, Celestijnenlaan 200D, B-3001 Leuven, Belgium, Walter.Lauriks@fys.kuleuven.be)

Predictions for the low frequency sound waves propagation in a porous medium needs the knowledge of the flow specific resistance of the medium. We present a scheme for the flow resistivity profile inversion for a layered medium. In the framework of the fluid equivalent model a closed-form relation of the resistivity profile with the Fourier transform of the reflection is derived. It provide a straightforward way to perform the reconstruction of the profile. The result show a strong correlation between the flow resistivity profile and the reflection coefficient in this model. Some numerical simulations are given as examples of the applicability of this scheme.

2pPAf7. Wave equation in non-integer-dimensional porous media. Claude Depollier (Laboratoire d'Acoustique de l'Université du Maine, Avenue Olivier Messiaen, 72085 Le Mans, France, claude.depollier @univ-lemans.fr), Jean-Baptiste Legland (Laboratoire d'Acoustique de l'Université du Maine, Avenue Olivier Messiaen, 72085 Le Mans, France, jean-baptiste.legland.etu@univ-lemans.fr)

Over some range of scales, porous media display properties of fractals. It particular both pore sizes and pore-interfaces exhibit the fractal characteristics. One of the fascinating interests of fractals is their capability to model objects with complicate structure. This is due to an important property of fractal objects that their structure is characterized by a small number of parameters. One of them is the fractal dimension which tells how the fractal fills the space in which it lies. Very early, fractal concept has been incorporated in the study of porous media to investigate various phenomena as flow in porous media, pores and fractures of geological media. We present generalized Biot's wave equations for a fractional dimensional system of 3-spatial coordinates used as an effective description of porous media. Some specific examples are given as an application of this model.

2pPAf8. Investigation of the limp model validity for porous materials. Olivier Doutres (Laboratoire d'Acoustique de l'Université du Maine, Avenue Olivier Messiaen, 72085 Le Mans, France, olivier.doutres.etu @univ-lemans.fr), Nicolas Dauchez (Laboratoire d'Acoustique de l'Université du Maine, Avenue Olivier Messiaen, 72085 Le Mans, France, nicolas.dauchez@univ-lemans.fr), Jean Michel Genevaux (Laboratoire d'Acoustique de l'Université du Maine, Avenue Olivier Messiaen, 72085 Le Mans, France, jean-michel.genevaux@univ-lemans.fr), Olivier Dazel (Laboratoire d'Acoustique de l'Université du Maine, Avenue Olivier Messiaen, 72085 Le Mans, France, olivier.dazel@univ-lemans.fr)

A new criterion to test the validity of using the limp model for porous materials is proposed. The limp model is derived from the poroelastic Biot model assuming that the frame has no bulk stiffness. Being an equivalent fluid model accounting for the motion of the frame, it has fewer limitations than the usual equivalent fluid model assuming a rigid frame. A criterion is derived to identify the porous materials for which the limp model can be used. This criterion relies on a new parameter, the Frame Stiffness Influence (FSI) based on porous material properties. The critical values of FSI under which the limp model can be used, are determined using a 1D analytical modeling for three boundary sets: absorption of a porous layer backed by a rigid wall, radiation of a vibrating plate covered by a porous layer and transmission loss of a double leaf panel filled in by a porous layer. Compared with other criteria, the criterion associated with FSI provides information in a wider frequency range and can be used for configurations which include vibrating plates.

2pPAf9. An index to quantify the through-thickness symmetry of sound absorbing materials. Yacoubou Salissou (Groupe d'Acoustique de l'Université de Sherbrooke, 2500, Boul. de l'Université, Département de génie mécanique, Sherbrooke, QC J1K-2R1, Canada, Yacoubou.Salissou 
@USherbrooke.ca),Raymond Panneton(Groupe d'Acoustique de l'Université de Sherbrooke, 2500, Boul. de l'Université, Département de génie mécanique, Sherbrooke, QC J1K-2R1, Canada, raymond.panneton @usherbrooke.ca)

In this work, an extension of the theoretical formulation of the transfer matrix to non symmetrical sound absorbing porous materials is carried out. From this extension, an index of asymmetry is proposed and discussed. This index allows one to quantify the through-thickness asymmetry of a sound absorbing porous material. This index may be used for quality control or to assess the symmetry of the material in terms of its acoustic properties (absorption, reflection, impedance, propagation constant). To validate the application of the index of asymmetry, samples made up from two layers of porous materials are studied. Each so-constructed two-layered sample is seen as an equivalent asymmetrical single porous layer with a sudden change in its physical properties. The acoustic properties of each sample are then measured in the direct and inverted configurations (i.e., when both sides of the sample are facing successively the incident wave). Their values are compared in terms of the asymmetry index of the equivalent single layer. From these tests, an index value is suggested. Above this value, the acoustic properties of a sound absorbing material should be considered as being not symmetrical.

2pPAf10. Ultrasonic critical angle reflectometry applied to porous nuclear fuel mechanical characterization. Vivian Cereser Camara (Université Montpellier II, Place Eugène Bataillon, 34095 Montpellier, France, cereser@lain.univ-montp2.fr), Didier Laux (Université Montpellier II, Place Eugène Bataillon, 34095 Montpellier, France, laux@lain .univ-montp2.fr), Gilles Despaux (Université Montpellier II, Place Eugène Bataillon, 34095 Montpellier, France, despaux@lain.univ-montp2 .fr), Daniel Baron (EDF, CEA Cadarache, 13108 St Paul Lez Durance, France, daniel.baron@edf.fr)

An ultrasonic reflectometer working from 2 to $10 \mathrm{MHz}$ on zones around $4 \mathrm{~mm}$ has been built in order to assess in a non destructive way the reflection coefficient $\mathrm{R}(\theta, \mathrm{f})$ as a function of incident angle and frequency. Many experiments were performed on various materials (glass, aluminium,...) showing that with this goniometer and the related signal processing it is possible to assess very accurately $\mathrm{R}(\theta, \mathrm{f})$ for bulk or thin samples (Lamb modes). Longitudinal and shear velocities (VL, Vs) deduced from $\mathrm{R}(\theta, \mathrm{f})$ have been used to determine the elastic moduli $\mathrm{E}$ and $\mathrm{G}$ which values match very favourably with literature. Hence the Ultrasonic Critical angle Reflectometry is a powerful tool for materials characterization. In a second step, this device has been used on civil power plants fuel (UO2) with various volume fraction of porosity. The elastic moduli obtained are in good agreement with the literature data. The relations between VL, VS and the porosity (p) on the one hand, and $\mathrm{E}$ and $\mathrm{G}$ with $\mathrm{p}$ on the other hand will be presented and discussed. In particular, a link between these relations and the pores morphology (deduced with numerical simulations) will be pointed out.

2pPAf11. Hybrid inversion technique for predicting geometrical parameters of porous materials. Paresh Shravage (Dipartimento di Ingegneria - University of Ferrara, Via Saragat 1, 44100 Ferrara, Italy, paresh.shravage@unife.it), Paolo Bonfiglio (Dipartimento di Ingegneria University of Ferrara, Via Saragat 1, 44100 Ferrara, Italy, paolo.bonfiglio @ unife.it), Francesco Pompoli (Dipartimento di Ingegneria - University of Ferrara, Via Saragat 1, 44100 Ferrara, Italy, francesco.pompoli@unife.it)

In prediction of acoustical behavior of porous materials, five geometrical parameters play a very important role, but some of these geometrical properties are very difficult to measure directly. So many authors have suggested different inversion strategies for getting these properties from directly measured both characteristic and surface properties of the material using standing wave tube. These approaches can be divided in two different categories: analytical (based on the limit behaviour of the bulk properties) and minimization based methods (which make use of searching algorithms to determine the best solution that minimizes a cost function calculated by means of a prediction model). Recent studies have shown the reliability of the analytical methods for the determination of the airflow resistivity and the minimization based approach by using genetic algorithms for getting the other physical parameters. Consequently, a hybrid inversion technique can be proposed for the complete calculation of the geometrical quantities and here it is presented in detail. Moreover the paper compares the results from the hybrid approach with the experimentally measured parameters and the values of the five parameters obtained by using genetic algorithms. Finally, the paper presents the effect of both inversion techniques on acoustical properties using Johnson-Allard-Champoux model.

2pPAf12. Absorption and dispersion of acoustical waves in synthetic and natural compressed fibrous materials. Bernard $R$. Castagnede (Laboratoire d'Acoustique de l'Université du Maine, Avenue Olivier Messiaen, 72085 Le Mans, France, bernard.castagnede @ univ-lemans.fr), Bruno Brouard (Laboratoire d'Acoustique de l’Université du Maine, Avenue Olivier Messiaen, 72085 Le Mans, France, bruno.brouard@univ-lemans.fr), Claude_Depollier (Laboratoire d'Acoustique de l'Université du Maine, Avenue Olivier Messiaen, 72085 Le Mans, France, claude.depollier@univ-lemans.fr), Olivier Dazel (Laboratoire d'Acoustique de l'Université du Maine, Avenue Olivier Messiaen, 72085 Le Mans, France, olivier.dazel@univ-lemans.fr), Denis Lafarge (Laboratoire d'Acoustique de l'Université du Maine, Avenue Olivier Messiaen, 72085 Le Mans, France, denis.lafarge@univ-lemans.fr)

When a fibrous slab is compressed along its thickness, there are some changes in the basic physical parameters which describe the propagation of sound waves. For instance during compression, the acoustical resistivity and the tortuosity increase, while at the same time the porosity as well as the viscous and thermal characteristic lengths diminish. For a 1-D compression along the thickness of the porous layer, these changes are decribed by straightforward linear equations, as long as the compression ratio is kept small (e.g. between 1 and 5). Such simple expressions are derived theoretically on the base of conservation principles relying on some fundamental metrology indicators. Next, the influence of these changes on the 5 fundamental physical properties of any porous networks are studied numerically on the dispersion and attenuation curves versus frequency, in the frame of the "equivalent-fluid" model [4]. Finally, experiments are performed in order to check some of these predictions on various felt fibrous materials having different compression ratio.

2pPAf13. Acoustic properties of partly saturated porous soils. Kirill V. Horoshenkov (University of Bradford, School of Engineering, Design and Technology, BD7 1DP Bradford, UK, k.horoshenkov@Bradford .ac.uk), Mostafa H. Mohamed (University of Bradford, School of Engineering, Design and Technology, BD7 1DP Bradford, UK, m.h.a.mohamed@Bradford.ac.uk), Siow N. Ting (University of Bradford, School of Engineering, Design and Technology, BD7 1DP Bradford, UK, s.n.ting@bradford.ac.uk)

Controlled experiments are performed on soil samples saturated with different types of liquid such as water and oil to determine the relations between the degree of saturation, type of saturated liquid and the acoustic surface admittance. These experiments are conducted using a Buchner funnel attached directly to an acoustic impedance tube. The results show a very sensitive dependence of the acoustic admittance upon the degree of saturation irrespective of the liquid present. It is found that the relationship between the volumetric water content and the real part of the surface admittance in the frequency range of $500-1000 \mathrm{~Hz}$ can be expressed using a logarithmic equation. The coefficients of the proposed equation can be determined using a soil property called "uniformity coefficient" and the acoustic admittance of dry soil. Results of volumetric water content obtained using the proposed equation are validated using those obtained from an independent test. The results of the validation exercise demonstrate that the proposed relations can be used to determine very accurately the volumetric water content within soil from the acoustical data. The accuracy of the acoustically measured degree saturation is found to be within $2.0 \%$. 


\title{
Session 2pPAg
}

\section{Physical Acoustics: Photoacoustics II (Poster Session)}

\author{
Gerald Diebold, Cochair \\ Brown Univ. \\ Christ Glorieux, Cochair \\ Lab. ATF, Katholieke Universiteit Leuven
}

\begin{abstract}
All posters will be on display from 3:40 p.m. to 5:20 p.m. To allow contributors an opportunity to see other posters, contributors of odd-numbered papers will be at their posters from 3:40 p.m. to 4:30 p.m. and contributors of even-numbered papers will be at their posters from 4:30 p.m. to 5:20 p.m.
\end{abstract}

\section{Contributed Papers}

2pPAg1. Fiber Bragg grating applied pulsed photoacoustic detection technique for online monitoring concentration of liquid. Atsushi Yarai (Osaka Sangyo University, 3-1-1 Nakagaito, 574-8530 Daito, Osaka, Japan,yarai@osaka-sandai.ac.jp), Takuji Nakanishi (Osaka Sangyo University, 3-1-1 Nakagaito, 574-8530 Daito, Osaka, Japan, a.yarai@ieee.org)

The need for real-time monitoring of the concentration of raw liquids and wastewater flowing in ducts is one of the significant process controls in chemical plants. A concentration sensor applicable to acidic fluids, however, has not been practically developed because of the maintenance required to sustain performance due to the metal erosion of the electrode from the acidity. To overcome this problem, we propose the fiber Bragg grating applied pulsed photoacoustic (PA) detection technique for online monitoring concentration of liquid. In our technique, optical fiber made of quartz is used for the PA signal generation and detection, for which a 0.6-mm-diameter step index fiber and Bragg grating composed of single mode fiber are used as an object, respectively, so that our metal-free sensor head is not only of small size but is also maintenance-free. The concentration of dye (Rhodamine 6G) dissolved into distilled water was measured to estimate the capability. A Q-switched frequency doubled Nd:YAG laser was used as the PA exiting beam, which is fed to the PA sensor through the fiber. It was experimentally confirmed that the detection linearity was significantly obtained in a concentration range from a few ppm to approximately $200 \mathrm{ppm}$.

2pPAg2. Film thickness determination by laser ultrasonics. Michaël Lemaire (Institut d'Electronique de Microélectronique et de Nanotechnologie, Département Opto-Acousto-Electronique (UMR CNRS 8520), Université de Valenciennes et du Hainaut-Cambrésis, Le Mont-Houy, 59313 Valenciennes cedex 9, France, Michael.Lemaire@meletu.univ-valenciennes .fr), Frédéric Jenot (Institut d'Electronique de Microélectronique et de Nanotechnologie, Département Opto-Acousto-Electronique (UMR CNRS 8520), Université de Valenciennes et du Hainaut-Cambrésis, Le Mont-Houy, 59313 Valenciennes cedex 9, France, frederic.jenot@univ-valenciennes .fr), Mohammadi Ouaftouh (Institut d'Electronique de Microélectronique et de Nanotechnologie, Département Opto-Acousto-Electronique (UMR CNRS 8520), Université de Valenciennes et du Hainaut-Cambrésis, Le Mont-Houy, 59313 Valenciennes cedex 9, France, mohammadi.ouaftouh @univ-valenciennes.fr), Wei Jiang Xu (Institut d'Electronique de Microélectronique et de Nanotechnologie, Département Opto-AcoustoElectronique (UMR CNRS 8520), Université de Valenciennes et du Hainaut-Cambrésis, Le Mont-Houy, 59313 Valenciennes cedex 9, France, weijiang.xu@univ-valenciennes.fr), Marc Duquennoy (Institut d'Electronique de Microélectronique et de Nanotechnologie, Département
Opto-Acousto-Electronique (UMR CNRS 8520), Université de Valenciennes et du Hainaut-Cambrésis, Le Mont-Houy, 59313 Valenciennes cedex 9, France, Marc.Duquennoy@univ-valenciennes.fr), Mohamed Ourak (Institut d'Electronique de Microélectronique et de Nanotechnologie, Département Opto-Acousto-Electronique (UMR CNRS 8520), Université de Valenciennes et du Hainaut-Cambrésis, Le Mont-Houy, 59313 Valenciennes cedex 9, France, Mohamed.Ourak@univ-valenciennes.fr), Renaud Côte (Lab. ATF, Katholieke Universiteit Leuven, Celestijnenlaan 200D, B-3001 Leuven, Belgium, renaud.cote@fys.kuleuven.be), Robbe Salenbien (Lab. ATF, Katholieke Universiteit Leuven, Celestijnenlaan 200D, B-3001 Leuven, Belgium, robbe.salenbien@fys.kuleuven.be), Bart Sarens (Laboratorium voor Akoestiek en Thermische Fysica - Departement Natuurkunde en Sterrenkunde - Katholieke Universiteit Leuven, Celestijnenlaan 200D, B-3001 Leuven, Belgium, bart.sarens@fys .kuleuven.be), Walter Lauriks (Lab. ATF, Katholieke Universiteit Leuven, Celestijnenlaan 200D, B-3001 Leuven, Belgium, Walter.Lauriks@fys .kuleuven.be), Christ Glorieux (Lab. ATF, Katholieke Universiteit Leuven, Celestijnenlaan 200D, B-3001 Leuven, Belgium, christ.glorieux@ fys .kuleuven.be)

The thickness of films deposited on substrates is crucial for their thermal, electrical, optical behaviour. These properties are essential in thin film applications, especially in the field of microelectronics. In this study, we are interested in thickness determination of silver and gold films deposited by evaporation on a silicon substrate by using an ultrasonic non-destructive technique. In particular, the well-known laser ultrasonic technique is used to generate and detect the surface acoustic waves. Results obtained by two complementary methods allowing a non-contact measurement in a large bandwidth (from $5 \mathrm{MHz}$ to $200 \mathrm{MHz}$ ) are presented, and the dispersion of the Rayleigh wave propagation velocity is analyzed to determine the film thickness.

2pPAg3. Photoacoustic metrology of nanoimprint polymers. Timothy Kehoe (Tyndall National Institute, Lee Maltings, University College Cork, Prospect Row, Cork, Ireland, tim.kehoe@tyndall.ie), Juerg Bryner (ETH Zurich, Institute of Mechanical Systems, Dept. of Mechanical and Process Engineering, CH 8092 Zurich, Switzerland, bryner@imes.mavt .ethz.ch), Jacqueline Vollmann (ETH Zurich, Institute of Mechanical Systems, Dept. of Mechanical and Process Engineering, CH 8092 Zurich, Switzerland, vollmann@imes.mavt.ethz.ch), Clivia Sotomayor Torres (Tyndall National Institute, Lee Maltings, University College Cork, Prospect Row, Cork, Ireland, clivia.sotomayor@tyndall.ie), Laurent Aebi (ETH Zurich, Institute of Mechanical Systems, Dept. of Mechanical and Process Engineering, CH 8092 Zurich, Switzerland, aebi@imes.mavt.ethz.ch), Juerg 
Dual(ETH Zurich, Institute of Mechanical Systems, Dept. of Mechanical and Process Engineering, CH 8092 Zurich, Switzerland, juerg.dual@imes .mavt.ethz.ch)

Nanoimprint lithography (NIL) is an alternative lithography method for patterning of thin polymer films using a rigid stamp, which is being developed as desired minimum feature sizes reduce to the scale of tens of nanometres. To characterise nanoimprinted structures, there is a need for more convenient and non-destructive wafer-scale metrologies to complement scanning electron microscopy and atomic force microscopy. The photoacoustic method, with a resolution in the range of $10 \mathrm{~nm}$, and normally used to measure metal and dielectric layer thicknesses and physical properties, has been used for the first time to study nanoimprinting polymer layers. A good signal was obtained from the top and the bottom interfaces of two polymers, mr-I PMMA 75k300 and mr-NIL 6000.3, with thicknesses ranging from 100 to $500 \mathrm{~nm}$. From the measured time of flight of the acoustic wave, and modelling physical parameters of the polymers, thicknesses calculated agree well with those measured by profilometry. The measurements are performed on a short pulse laser pump-probe setup, where bulk wave packets are excited and detected using near infrared laser pulses of less than $100 \mathrm{fs}$ duration. The entire experimental setup is also simulated numerically.

2pPAg4. Line-focus beam photoacoustic imaging of surface and undersurface defect simulated on a planar specimen. Tsutomu Hoshimiya (Tohoku Gakuin University, 13-1, Chuo 1, 985-8537 Tagajyo, Japan, tpth@tjcc.tohoku-gakuin.ac.jp), Mika Hatake-Yama (Tohoku Gakuin University, 13-1, Chuo 1, 985-8537 Tagajyo, Japan, hatakemail @ yahoo.co.jp)

A theoretical formulation of photoacoustic (PA) imaging with a linefocus beam (LFB) for surface and undersurface simulated defect was performed. Equivalence between 2D surface defect photoacoustic tomography (PAT) and X-ray CT was derived. PAT imaging experiment was carried out A second harmonics of a LD-pumped YAG laser was used as a LFB. The laser power was $45 \mathrm{~mW}$. The length and width of a laser beam on a specimen was $25 \mathrm{~mm}$ times $0.65 \mathrm{~mm}$ The measured area was $27 \mathrm{~mm} \times 27 \mathrm{~mm}$, while the reconstructed area was $18 \mathrm{~mm} \times 18 \mathrm{~mm}$. 64 times 64 resolution image was reconstructed from the rotation and translation scanning. Reconstructed PA image agreed with the PA image obtained with a point-focus PA imaging. The frequency dependence of thermally diffused image agreed well with the theoretical prediction (thermal diffusion length is inversely proportional to the square root of modulation frequency) Under surface PAT image was obtained by a thermal wave diffraction formula, and the simulated image agreed well with the experimental data.

2pPAg5. Generation of Acoustic Wavelength Shorter Than 10nm By Means Of Nonlinear Acoustic. Shuo Zhang (INSP - UMR 7588 CNRS \& Université Pierre et Marie Curie, 140 Rue de Lourmel, 75015 Paris, France, Shuo.Zhang@insp.jussieu.fr), Emmanuel Péronne (INSP - UMR 7588 CNRS \& Université Pierre et Marie Curie, 140 Rue de Lourmel, 75015 Paris, France, emmanuel.peronne @insp.jussieu.fr), Laurent Belliard (INSP - UMR 7588 CNRS \& Université Pierre et Marie Curie, 140 Rue de Lourmel, 75015 Paris, France, lbelliar@ccr.jussieu.fr), Bernard Perrin (INSP UMR 7588 CNRS \& Université Pierre et Marie Curie, 140 Rue de Lourmel, 75015 Paris, France, bernard.perrin@insp.jussieu.fr)

As the size of the structure decrease toward the nanoscale, their acoustic properties get closer to the $\mathrm{THz}$ range. Traditional techniques based on piezoelectric transducer or the Brillouin diffusion can hardly reach the adequate frequency range. However, if we tightly focus short optical pulses, we can expect to generate high frequencies due to nonlinear effect during the propagation of the acoustic pulses. Moreover, such small source could be used to image buried structures. We have developed an pump-probe experiment based on a Ti:Sa oscillator using microscope objectives and 2 axis displacement stage to study nonlinear propagation and diffraction of acoustic wave in 3 dimension. We have studied $356-\mu \mathrm{m}$ thick GaAs substrate and have demonstrated the generation of acoustic longitudinal wavelength as short as $10 \mathrm{~nm}$. Due to the acoustic dispersion during the propagation, acoustic solitons are observed for high excitation power. Thanks to the use of interferometric detector, the acoustic pulses are characterized in great details. Such experimental development can help us to study the properties of transmission and reflection of buried nanosystem such as single quantum well or phonon nanocavities.

2pPAg6. The theoretical analyse of the surface acoustic waves propagated on the surface of the bulk materials with subsurface defect. Xiaodong Xu (Modern Acoustics, Institute of Acoustics, Nanjing University, 210093 Nanjing, China, xdxu@nju.edu.cn), Ran Ding (Nanjing University, P.O.Box44\#, Pukou Campus, 210089 Nanjing, China, snail_dr@hotmail.com), Christ Glorieux (Lab. ATF, Katholieke Universiteit Leuven, Celestijnenlaan 200D, B-3001 Leuven, Belgium, christ.glorieux@fys.kuleuven.be), Xiaojun Liu (Modern Acoustics, Institute of Acoustics, Nanjing University, 210093 Nanjing, China, xjliu@nju .edu.cn)

A theoretical model based on finite element method is used to analyse the properties of surface acoustic wave (SAW) propagated on the surface of the bulk materials with subsurface defect. The simulation results reveal the modes conversion between SAW and Lamb wave at the border of the defect and explain the diffusion properties of SAW propagated on the bulk material with subsurface defect. The modes conversion and wave reflection at the border of the defect are theoretically explained why there are a lot of riffles after the SAW passed through Al sample with circle subsurface defect, which detected by laser ultrasonic method. The relationships between the wavelength of wave and the physical state of the defect in the bulk material are also fully described in theoretical simulation.

2pPAg7. LED-based Stroboscopic Schlieren System. Kalle Hanhijärvi (Electronics Research Unit, University of Helsinki, P.O.Box 64 (Gustaf Hällströmin katu 2), FIN-00014 Helsinki, Finland, kalle.hanhijarvi@helsinki.fi), Ivan Kassamakov (Electronics Research Unit, University of Helsinki, P.O.Box 64 (Gustaf Hällströmin katu 2), FIN00014 Helsinki, Finland, ivan.kassamakov@helsinki.fi), Juha Aaltonen (Electronics Research Unit, University of Helsinki, P.O.Box 64 (Gustaf Hällströmin katu 2), FIN-00014 Helsinki, Finland, juha.aaltonen@helsinki.fi), Edward Hæggström (Electronics Research Unit, University of Helsinki, P.O.Box 64 (Gustaf Hällströmin katu 2), FIN00014 Helsinki, Finland, edward.haeggstrom @ helsinki.fi)

A stroboscopic Schlieren system is proposed to characterize acoustic fields in transparent media. Schlieren imaging has been used to visualize beam profiles of ultrasonic transducers mostly in liquids. The method is sensitive to gradients in refractive index, and can thus be used with solids and fluids. A stroboscopic illumination synchronized with the ultrasonic vibration (controllable phase delay between the illumination and the ultrasonic drive signal), allows obtaining an image of a propagating tone burst. An LED source provides relatively fast response time for high measurement bandwidth. Incoherent light doesn't suffer from unwanted interference, as with laser sources. Our setup employs two $90^{\circ}$ off-axis parabolic mirrors. Non-reflected light is blocked with spatial filtering which is achieved with a knife-edge, placed at the focal point of the focusing mirror. We first present a time-averaged Schlieren image of the acoustic field of a $7.5 \mathrm{MHz}$ focusing transducer, as a proof-of-principle result. Then a custom-built LED-based stroboscopic illumination system is used to visualize wave propagation in water emitted by a high power $20 \mathrm{kHz}$ transducer. Schlieren imaging is useful in applications, where non-contact characterization of acoustic fields is necessary.

\section{2pPAg8. Stroboscopic White Light Interferometry for Dynamic} Characterization of Capacitive Pressure Sensors. Ivan Kassamakov (Electronics Research Unit, University of Helsinki, P.O.Box 64 (Gustaf Hällströmin katu 2), FIN-00014 Helsinki, Finland, ivan.kassamakov@helsinki.fi), Kalle Hanhijärvi (Electronics Research Unit, University of Helsinki, P.O.Box 64 (Gustaf Hällströmin katu 2), FIN$00014 \quad H e l s i n k i, \quad$ Finland, kalle.hanhijarvi@helsinki.fi), Juha Aaltonen (Electronics Research Unit, University of Helsinki, P.O.Box 64 
(Gustaf Hällströmin katu 2), FIN-00014 Helsinki, Finland, juha.aaltonen@helsinki.fi), Lauri Sainiemi (Micro and Nanosciences Laboratory, Helsinki University of Technology, P.O.Box 3500, FI-02015 TKK, Finland, lauri.sainiemi@tkk.fi), Kestutis Grigoras (Micro and Nanosciences Laboratory, Helsinki University of Technology, P.O.Box 3500, FI02015 TKK, Finland, kestas.grigoras@tkk.fi), Sami Franssila (Micro and Nanosciences Laboratory, Helsinki University of Technology, P.O.Box 3500, FI-02015 TKK, Finland, sami.franssila@tkk.fi), Anu Kärkkäinen (VTT Technical Research Centre of Finland, P.O.Box 1000, FIN-02044 VTT, Finland, anu.karkkainen@vtt.fi), Edward Hæggström (Electronics Research Unit, University of Helsinki, P.O.Box 64 (Gustaf Hällströmin katu 2), FIN-00014 Helsinki, Finland, edward .haeggstrom@helsinki.fi)

Scanning white light interferometry (SWLI) is a well-established method for accurate static out-of-plane 3-D profiling of micromechanical devices. Periodic displacement can be measured using stroboscopic illumination synchronized to an arbitrary phase angle of the sample oscillation. We modified an existing SWLI setup for dynamic MEMS characterization. A two-channel function generator drives the sample and the stroboscopic illumination, and controls the inter-channel phase. A phosphorous white light LED or single-color LED is used as light source. Currently our shortest stroboscopic pulse is $50 \mathrm{~ns}$. We measured the out-of-plane displacement of a thermal microbridge fabricated on SOI wafer with $20 \mathrm{~nm}$ accuracy. The microbridge was driven with sinusoidal and square wave signals $(1 \mathrm{~Hz}-960$ $\mathrm{Hz}$ ). The stroboscopic duty cycles were $0.1 \%-5 \%$. We also characterize the vibration modes of the membrane of a capacitive pressure sensor. Stroboscopic SWLI is useful for nanoscale profile measurements of periodic oscillations.

2pPAg9. Laser-generated narrow-band ultrasonic wave for detection of subsurface defect. Ran Ding (Nanjing University, P.O.Box44\#, Pukou Campus, 210089 Nanjing, China, snail_dr@hotmail.com), Xiaodong Xu (Modern Acoustics, Institute of Acoustics, Nanjing University, 210093 Nanjing, China, xdxu@nju.edu.cn)

Laser ultrasonics employs laser beams to excite ultrasound. Spatial an$\mathrm{d}$ /or temporal modulated intensity of laser on the surface of the specimen (aluminum) can serve to narrow the band of the generated ultrasound around the desired central frequency and meanwhile obtains higher signal-noise ratio. In the present work, we use Computer Generated Holograms (CGHs) to spatial-modulate the laser beam. CGHs provide flexibility of adjustment of the intensity profile of the laser on the specimen and the online reconstruction of CGHs by digital and electronic devices helps to reduce the complexity of the experimental system and is promising in detection of subsurface defect and depth profiling as the surface acoustic waves penetrate into the solids a depth proportional to the wavelength. Discussion was carried out between experimental results and a theoretical model which based on finite element analysis.

2pPAg10. Laser-Ultrasonic Measurement of Stress in Metal. Aleksei Podolsky (Moscow State University, MSU, 1, building 2, GSP-2, Leninskiye Gory, 119992 Moscow, Russian Federation, uzh@yandex .ru), Vladimir Koshkin (Moscow State University, MSU, 1, building 2, GSP-2, Leninskiye Gory, 119992 Moscow, Russian Federation, uzh@ya.ru), Viktor Bel'Ko (Moscow State University, MSU, 1, building 2, GSP-2, Leninskiye Gory, 119992 Moscow, Russian Federation, uzh@ya.ru), Andrei Fetisov (Moscow State University, MSU, 1, building 2, GSP-2, Leninskiye Gory, 119992 Moscow, Russian Federation, uzh@ya.ru), Alexander Karabutov (Moscow State University, MSU, 1, building 2, GSP-2, Leninskiye Gory, 119992 Moscow, Russian Federation, akarabutov@gmail.com)

Stress in metal was investigated by acousto-elastic effect. The dependence of longitudinal ultrasonic wave velocity on transverse stress was considered. A laser-ultrasonic defectoscope was used for precise ultrasonic velocity measurement. Optoacoustic transducer provides laser excitation and piezoelectric wide-band detection of ultrasonic pulse. The accuracy of the measurement exceeded $0.1 \%$ for $2 \mathrm{~mm}$ thick metal sample. Samples in the form of plates with the thickness of 2 to $4 \mathrm{~mm}$ made of aluminum and titanium alloys and stainless steel were tested. The samples were loaded quasistatically, the load was changing from zero to yield point stress. Load, deformation and velocity of ultrasound were measured. It is shown that the velocity changes significantly with the load. Some features of the velocity change under tensile stress are presented. The possibility of the local residual stress measurement with the laser-ultrasonic technology is discussed.

2pPAg11. Application of nonlinear laser photoacoustic technique to crack detection. Jacek Zakrzewski (LPEC/UMR 6087/CNRS/Université du Maine, Avenue Olivier Messiaen, 72085 Le Mans Cedex 09, France, jzakrzew@fizyka.umk.pl), Nikolay Chigarev (LPEC/UMR 6087/CNRS/Université du Maine, Avenue Olivier Messiaen, 72085 Le Mans Cedex 09, France, Nikolay.Chigarev@univ-lemans.fr), Vincent Tournat (LPEC/UMR 6087/CNRS/Université du Maine, Avenue Olivier Messiaen, 72085 Le Mans Cedex 09, France, vincent.tournat@univ-lemans .fr), Denis Mounier (LPEC/UMR 6087/CNRS/Université du Maine, Avenue Olivier Messiaen, 72085 Le Mans Cedex 09, France, denis.mounier@univ-lemans.fr), Vitali Gusev (LPEC/UMR 6087/CNRS/Université du Maine, Avenue Olivier Messiaen, 72085 Le Mans Cedex 09, France, vitali.goussev@univ-lemans.fr)

Nonlinear acoustics is a promising method for non-destructive testing (NDT) as it allows to improve the sensitivity and contrast of defects detection. The application of contactless laser photoacoustic technique for the tasks of nonlinear acoustics looks very promising. Meanwhile, real examples of industrial systems using nonlinear photoacoustics are still absent. In this work nonlinear photoacoustic response of artificially prepared cracks has been studied. Acoustic vibrations have been excited through the loASAHGRcal heating caused by the absorption of a focused beam of visible or near-infrared laser radiation. Several methods have been applied for the detection of surface displacement including piezo-transducers, aircoupled transducers and optical laser interferometry. It has been shown, that nonlinear acoustic response increases drastically when both pump and probe points are localized near the crack. For this reason, the last two methods allowing local probing look very promising. Two dimensional scans of generation and detection points on the sample surface could be used to obtain the images of crack at new spectral components induced by crack nonlinearity. The goal of this work is to find optimal methods of the excitation and detection of nonlinear photoacoustic response of the crack. It should make the technique attractive for the industrial applications.

2pPAg12. Looking for shear waves in glass forming liquids. Thomas Pezeril (Massachusetts Institute of Technology, 77 Mass. Avenue, room 6-026, Cambridge, MA 02215, USA, pezeril@mit.edu), Christoph Klieber (Massachusetts Institute of Technology, 77 Mass. Avenue, room 6-026, Cambridge, MA 02215, USA, cklieber@mit.edu), Stephane Andrieu (Laboratoire de Physique des Matériaux UMR7556, Université H. Poincaré/Nancy I, 54506 Vandoeuvre, France, stephane.andrieu@lpm .u-nancy.fr), Keith Nelson (Massachusetts Institute of Technology, 77 Mass. Avenue, room 6-026, Cambridge, MA 02215, USA, kanelson@mit .edu)

The ability of the generation of picosecond shear acoustic pulses in solids using ultrashort optical pulses have been employed to look for high frequency shear waves in glass forming liquids. As a transducer we used a canted iron thin film deposited on a glass substrate. The strong shear efficiency of generation provided by this kind of samples allowed the study of several glass forming liquids. As a first try, we have detected shear brillouin scattering in m-toludine at $100 \mathrm{~K}$, that is below the glass transition temperature, at a frequency of $9 \mathrm{GHz}$. Then, we observed shear waves propagating at room temperature in glycerol at frequencies below $50 \mathrm{GHz}$. 
Session 2pPAh

\title{
Physical Acoustics and Signal Processing in Acoustics: Acoustic Landmine Detection II (Poster Session)
}

\author{
James Sabatier, Cochair \\ University of Mississippi \\ Keith Attenborough, Cochair \\ Open University
}

\begin{abstract}
All posters will be on display from 3:40 p.m. to 5:20 p.m. To allow contributors an opportunity to see other posters, contributors of odd-numbered papers will be at their posters from 3:40 p.m. to 4:30 p.m. and contributors of even-numbered papers will be at their posters from 4:30 p.m. to 5:20 p.m.
\end{abstract}

\section{Contributed Papers}

2pPAh1. Vector surface velocity measurement using contour scanning laser vibrometers for the detection of landmines. Patrick $F$. O’Malley (Catholic University, 620 Michigan Ave, Washington, DC 20064, USA, 68omalley@cua.edu), Woods J. Teresa (Catholic University, 620 Michigan Ave, Washington, DC 20064, USA, 59woods@cua.edu), Joseph F. Vignola (Catholic University, 620 Michigan Ave, Washington, DC 20064, USA, vignola@cua.edu), John A. Judge (Catholic University, 620 Michigan Ave, Washington, DC 20064, USA, judge@cua.edu), Jacek Jarzynski (Catholic University, 620 Michigan Ave, Washington, DC 20064, USA, jjarzynski@comcast.net)

Much work has been done studying the detection of buried landmines using acoustic excitation and measuring the vibration response of the ground using Laser Doppler Vibrometry (LDV). We examine the benefits of measuring the full three-dimensional velocity response of the ground surface when a landmine is present. Three-dimensional velocity measurements provide a richer data set for identification of buried targets and avoid potential errors associated with projecting the surface velocity vector onto a single laser line of sight direction. We present results of a study conducted using landmines buried in sand in a laboratory environment. The data were obtained using a novel five-axis laser vibrometry system, which uses a single point LDV system to measure the surface of the sand from multiple angles at each measurement location. Data will be presented showing the consequences of a buried landmine on the three-dimensional velocity response of a contoured ground surface.

2pPAh2. Scattering of a buried circular membrane imbedded in a rigid substrate in a layered fluid waveguide. Michelle B. Mattingly (Physics Dept., United States Naval Academy, Chauvenet Hall Room 295, 572 C Holloway Road, Annapolis, MD 21402, USA, mini_matisick@ hotmail.com) , James L. Buchanan (Mathematics Dept., United States Naval Academy, 572 C Holloway Road, Annapolis, MD 21402, USA, jlb@usna.edu), Reza Malek-Madani (Mathematics Dept., United States Naval Academy, 572 C Holloway Road, Annapolis, MD 21402, USA, mm@usna.edu), Murray S. Korman (Physics Dept., United States Naval Academy, Chauvenet Hall Room 295, 572 C Holloway Road, Annapolis, MD 21402, USA, korman @ usna.edu)

A study of mathematical modeling of the buried landmine detection problem involves wave propagation in a layered waveguide in the presence of a flush mount buried circular target. In this study, emphasis is placed on acoustic to seismic coupling of an airborne $\mathrm{CW}$ point source located over an iso-velocity fluid layer. The top plate of the buried landmine is modeled as a circular elastic membrane stretched flush over a cylindrical cavity in a rigid substrate beneath the fluid layer. The finite fluid layer affords a manageable study of the modal resonances in the waveguide system with and without the target. The Helmholtz equation is solved in the atmospheric layer using cylindrical coordinates and a point source. The homogeneous Helmholtz equation is used in the fluid layer. Green's function techniques involving vibrations of the membrane are incorporated into the boundary conditions. Results of the closed form normal mode solution will be presented in a MATLAB ${ }^{\mathrm{TM}}$ user interface; describing the effects of frequency, depth, density, sound, absorption, including radius and elastic parameters of the membrane. Comparison of the results (involving the fluid surface particle velocity profiles across the target) will be made with experiments reported in the literature to evaluate its usefulness.

2pPAh3. Nonlinear scattering of a surface wave by an object buried in soil. Evgenia A. Zabolotskaya (Applied Research Laboratories, The University of Texas, P.O. Box 8029, Austin, TX 78713-8029, USA, zhenia@arlut.utexas.edu), Yurii A. Ilinskii (Applied Research Laboratories, The University of Texas, P.O. Box 8029, Austin, TX 78713-8029, USA, yura@arlut.utexas.edu), Mark F. Hamilton (Applied Research Laboratories, The University of Texas, P.O. Box 8029, Austin, TX 78713-8029, USA, hamilton@mail.utexas.edu)

Nonlinear scattering of a surface wave by an object buried in soil is investigated theoretically. The object is supposed to be excited by a monochromatic seismic wave and its pulsations are considered to be nonlinear. Specific features of soil are taken into account, namely, shear modulus less than compressibility modulus and anomalously high nonlinearity. Two analytical approaches are applied. One is based on theory of elasticity and the other is based on fluid mechanics. The two approaches yield identical results for the second harmonic in the scattered field. The second harmonic is evaluated in the near field where the medium can be considered as incompressible. The analysis is performed with perturbation theory for small displacement amplitudes in the soil. The second harmonic is also investigated with an asymptotic expression obtained from the general equation for the second harmonic generated by a pulsating object in a compressible medium. The equation is expanded for a small object at distances that are small compared with a wavelength. The influence of a stress-free boundary on the scattered field is evaluated using Green's functions. [Work supported by ONR.]

2pPAh4. Numerical simulations of resonant granular layers. Joseph A. Turner (University of Nebraska, Dept. of Engineering Mechanics, W317.4 NH, Lincoln, NE 68588, USA, jaturner@unl.edu), Florin Bobaru (University of Nebraska, Dept. of Engineering Mechanics, W317.4 
NH, Lincoln, NE 68588, USA, fbobaru2@unl.edu),Kitti Rattanadit (University of Nebraska, Dept. of Engineering Mechanics, W317.4 NH, Lincoln, NE 68588, USA, kittir@ bigred.unl.edu)

A coupled Discrete Element Method-Finite Element Method (DEMFEM) model is developed and implemented for simulating the dynamic response of a dry granular layer on top of an elastic beam. The implementation is first validated against the quasi-static results for spherical particles which are compared with well-bonded effective-medium models. In the dynamic regime however, the effective-medium analytical result differs from experi- mental values in terms of the dependence of particle size. The simulations developed are used to understand the discrepancy between the theory and the experiments. The first resonance of the system is examined with respect to particle size using a variety of particle sizes and a frequency sweep input. The particle size dependence observed experimentally is reproduced in the simulations only through inclusion of cohesive forces between the particles. Rolling resistance and friction between particle is shown to be of minor importance. This new modeling tool offers promise for understanding the dynamic interactions of granular materials. [Work supported by ARL]

TUESDAY AFTERNOON, 1 JULY 2008

P3-A, LEVEL 3, 3:40 TO 5:20 P.M.

\title{
Session 2pPAi
}

\section{Physical Acoustics: Nonlinear Acoustics of Unconsolidated Granular Media II (Poster Session)}

\author{
Paul Johnson, Cochair \\ EES-11 (Geophysics) - Los Alamos National Laboratory \\ Vitali Gusev, Cochair \\ LPEC/UMR 6087/CNRS/Université du Maine
}

\begin{abstract}
All posters will be on display from 3:40 p.m. to 5:20 p.m. To allow contributors an opportunity to see other posters, contributors of odd-numbered papers will be at their posters from 3:40 p.m. to 4:30 p.m. and contributors of even-numbered papers will be at their posters from 4:30 p.m. to 5:20 p.m.
\end{abstract}

\section{Contributed Papers}

2pPAi1. Low-frequency acoustoelasticity in glass beads saturated with water using a nonlinear wave-coupling technique. Guillaume Renaud (LUSSI, 10 Bd Tonellé, 37032 Tours, France, guillaume.renaud28 @etu.univ-tours.fr), Samuel Callé (LUSSI, 10 Bd Tonellé, 37032 Tours, France, calle_s@med.univ-tours.fr), Jean-Pierre Remenieras (LUSSI, 10 Bd Tonellé, 37032 Tours, France, remenier@med.univ-tours.fr), Marielle Defontaine (LUSSI, 10 Bd Tonellé, 37032 Tours, France, defontai@med .univ-tours.fr)

To study "non-classical" acoustical nonlinearities in cracked materials, we developed a nonlinear (NL) wave coupling technique. Propagation velocity and amplitude of short high-frequency (HF, $600 \mathrm{kHz}$ ) bursts are modulated as result of nonlinear interaction with a low-frequency (LF, 3 $\mathrm{kHz}$ ) wave. Time Of Flight Modulation (TOFM) is indeed related to both elasticity and density variations. Because high acoustic nonlinearities in glass beads have been reported, we applied our technique to glass beads with different diameters (hundreds micrometers). A small container is filled up with glass beads saturated with water and placed below the LF source. The large HF to LF ratio (200) and the small sample size $(6 \mathrm{~cm})$ compared to the LF wavelength in water $(50 \mathrm{~cm})$ allow: 1) to measure instantaneous Time Of Flight Modulation (TOFM) and attenuation as functions of the instantaneous LF pressure, 2) to consider the LF pressure field a quasi-static hydrostatic variation of the ambient pressure in the medium. The LF pressure amplitude in water is approximately $10 \mathrm{kPa}$. Different TOFM and NL attenuation behaviors in tension and in compression, as well as different hysteresis patterns, are observed. The influence of the LF pressure amplitude is discussed.

2pPAi2. Sound in a linear array of magnetic spheres. J. Carlos Ruiz-Suarez (CINVESTAV-IPN, Antigua Carretera a Progreso $\mathrm{Km} 6$, $97310 \quad$ Merida, Mexico, cruiz@mda.cinvestav.mx), Florent Malloggi (PMMH-ESPCI Paris, 10 Rue Vaquelin, 75005 Paris, France,
F.G.J.Malloggi@tnw.utwente.nl),Osvaldo Carvente(CINVESTAV-IPN, Antigua Carretera a Progreso $\mathrm{Km}$ 6, 97310 Merida, Mexico, ocarven@yahoo.com.mx), Jose Cruz-Damas (CINVESTAV-IPN, Antigua Carretera a Progreso Km 6, 97310 Merida, Mexico, josia.damas @ gmail.com), Felipe Pacheco (CINVESTAV-IPN, Antigua Carretera a Progreso Km 6,97310 Merida, Mexico, fpacheco@mda.cinvestav.mx)

We study the propagation of sound in a chain of strongly magnetized spheres. The strong dipole-dipole magnetic force allows us to hold the chain in different positions to evaluate the effect gravity produces on sound transmission. This strong cohesion permits us also to insert in the chain a periodical array of impurities and study the effect this array has in the transmission. A well defined gap of frequencies, where no sound propagation exists, is observed. We investigate as well square lattices, and look into the effects dipole anisotropy causes to sound propagation. Finally, we study the effects produced by vacancies.

2pPAi3. Linear and non linear acoustic waves in macroscopically inhomogeneous unconsolidated granular crystals. Aurélien Merkel (LPEC/UMR 6087/CNRS/Université du Maine, Avenue Olivier Messiaen, 72085 Le Mans Cedex 09, France, aurelien.merkel.etu @ univ-lemans.fr), Xavier Jacob (LPEC/UMR 6087/CNRS/Université du Maine, Avenue Olivier Messiaen, 72085 Le Mans Cedex 09, France, xavier.jacob@univ-lemans.fr), Vincent Tournat (LPEC/UMR 6087/CNRS/Université du Maine, Avenue Olivier Messiaen, 72085 Le Mans Cedex 09, France, vincent.tournat@univ-lemans.fr), Vitali Gusev (LPEC/UMR 6087/CNRS/Université du Maine, Avenue Olivier Messiaen, 72085 Le Mans Cedex 09, France, vitali.goussev@univ-lemans.fr)

Ordered unconsolidated structure of spherical beads in the absence of external loading except the gravity field constitutes a macroscopically inhomogeneous and strongly nonlinear phononic crystal. We report the experimental and theoretical investigation of linear and nonlinear acoustic phenomena in these granular crystals of finite thickness along the gravity 
direction. In particular the dependence of the resonance frequencies on the thickness of the crystal (the number of layers) is evaluated. The linear transmission of acoustic waves from the bottom to the free surface of the granular crystal exhibits complicated features. It is compared to the linear transmission through the same structure but with a macroscopically homogeneous static stress. Some frequency regions of the acoustic response function are shown to be insensitive to the transition from an inhomogeneous static stress (gravity induced) to a homogeneous one (additional ex- ternal load). The nonlinear acoustic phenomena of resonance frequency shift and resonance curve broadening with increasing amplitude of acoustic oscillations are observed. It allows to measure the nonlinear parameters of the crystal for different amounts of layers, accounting for the absolute particle velocity amplitude detected at the free surface with a laser vibrometer. Corresponding theoretical models describing the acoustic eigenmodes in macroscopically homogeneous and inhomogeneous granular crystals are developed.

TUESDAY AFTERNOON, 1 JULY 2008

P3-A, LEVEL 3, 3:40 TO 5:20 P.M.

\title{
Session 2 pPAj
}

\section{Physical Acoustics: Nonlinear Acoustics in Earthquake Processes and Other Earth Processes II (Poster Session)}

\author{
Paul Johnson, Cochair \\ EES-11 (Geophysics) - Los Alamos National Laboratory \\ Fabrice Cotton, Cochair \\ Université Joseph Fourier
}

\begin{abstract}
All posters will be on display from 3:40 p.m. to 5:20 p.m. To allow contributors an opportunity to see other posters, contributors of odd-numbered papers will be at their posters from 3:40 p.m. to 4:30 p.m. and contributors of even-numbered papers will be at their posters from 4:30 p.m. to 5:20 p.m.
\end{abstract}

\section{Contributed Papers}

2pPAj1. Elastic-anisotropic properties of rocks along the Finnish Drill Hole (OKU) section in the depth range down to $1 \mathrm{~km}$. Mikhail Kovalevskiy (Geological Institute of Kola Science Centre of Russian Academy of Sciences, 14, Fersman St., 184209 Apatity, Russian Federation, koval@geoksc.apatity.ru)

The Finnish drill hole OKU was drilled in the south-eastern Baltic Shield (Finland). The drill hole limiting depth is $2516 \mathrm{~m}$. The goal of investigations was determination of elastic and non-elastic characteristics of 14 rock samples taken within $1.0 \mathrm{~km}$ depth. The determinations were done on the basis of the latest improvement of the acoustopolarization method with devices for determining elastic properties [1]. The study of the rock sample properties from the drill hole section showed that they are all elastic anisotropic and pertain to the transverse-isotropic and orthorhombic symmetry types. A slight change in the compression and shear wave velocities with depth can be observed. The effect of linear acoustic anisotropic absorption has been registered in the samples. The nature of this effect manifestation is related to the presence of microcracks of the natural character. The effect of depolarization of shear waves was registered in some samples which suggests the presence of the angular unconformity between the directions of the LAAA elements and elastic symmetry elements. The pattern of change in the anisotropy factors for compression and shear waves with depth is manifested in a similar way. REFERENCES 1. Gorbatsevich F.F. Acoustopolariscopy of rocks. Apatity, Acad. Sciences, 1995, 204 p.

2pPAj2. Generation and Evolution of Cavitation in Magma under Decompression Waves. Valeriy K. Kedrinskiy (Lavrentyev Institute of Hydrodynamics, Siberian Division of the Russian Academy of Sciences, Lavrentyev prospect 15, 630090 Novosibirsk, Russian Federation, kedr@hydro.nsc.ru), Maxim N. Davydov (Lavrentyev Institute of Hydrodynamics, Siberian Division of the Russian Academy of Sciences,
Lavrentyev prospect 15, 630090 Novosibirsk, Russian Federation, davydov @ hydro.nsc.ru)

It's considered that presence of the dissolved water (with concentration up to 5-7 \% (wt.)) and viscosity of magma play one of the key roles in volcanic processes. According to the geophysical data, both a character and a structure of explosive volcano eruptions are defined by dynamics of cavitation development and the viscosity increasing orders during the diffusion of the dissolved gas from magma in bubbles. This paper represents a full system of the equations, including the kinetics of phase transitions, which allows us to describe dynamics of a magma melt state in a gravity field behind the front of a decompression wave. The problem is solved numerically, using a known expression for the nucleation frequency as a function of current concentration of gas dissolved in magmatic melt. The notion of diffusion zones which arise around cavitation nuclei is introduced. The latter allow us to define real density of cavitation kernels generated in an unit of magmatic melt volume as a result of phase transitions behind a rarefaction-wave front. The dynamics of the size distribution of cavitation bubbles along a magmamelt column ( $1 \mathrm{~km}$ height) and also of the magma viscosity changing dynamically vs. a concentration of dissolved water are investigated. (RFBR 06-01-00317a financial support).

2pPAj3. Features of acoustic emission at various influences on rock samples. Alexander S. Voznesenskiy (Moscow State Mining University, Leninskiy Prospect 6, 119991 Moscow, Russian Federation, a148@mail.ru), Sergey V. Viljamov (Moscow State Mining University, Leninskiy Prospect 6, 119991 Moscow, Russian Federation, serjo@mail.ru)

In the report special features of acoustic emission (AE) in the rock samples during its deforming, loading, dissolving and heating are discussed. The AE-activity and spectral characteristics of $\mathrm{AE}$ at the frequency band from on $30 \mathrm{kHz}$ up to $500 \mathrm{kHz}$ versus time at various influence stages and forms are analyzed. For example the amplitudes at the frequencies 30-90 $\mathrm{kHz}$ during deforming of carnallite are higher than these ones during its 
dissolving. It means the cracks at the deforming are about 4-10 $\mathrm{mm}$ and these ones at the dissolution are about 1-3 mm. These results can be used for the recognition of influence forms and stages.

2pPAj4. Formation of ripples on sand surface as result of nonlinear interaction of sound waves and wind drift particles. Nora A. Vilchinska (LAA-Latvian Acoustics Association, 3 Kurzemes pr, LV-1064 Riga, Latvia, vilcinska@hotmail.com)

The phenomenon speaks within the limits of nonlinear acoustics, considering it as amplification of surface acoustic waves (SAWs) by drift of carriers. Interaction and amplification of SAWs, borne from falling sand particles on dry sand surface and drift sand particles under wind action, is responsible for the first ripple forming on a dry sand flat surface. SAWs interact with drift sand particles in the moment, when the velocity of drift is equal to velocity of a sound propagation. The sound wave acts on drifting particles so that sand grains, a little bit advancing wave, will be braked, and lagging behind be accelerated. Particles will gather in a phase of a wave take place grouping of particles and ripple forming. The distance between groups is a ripples wave length $\lambda$. Experimental in-situ are measured weak sound field on frequencies $25 \mathrm{~Hz}$ in just forming sand massif, and near surface velocity of sand drug under wind action. Measured ripples wave lengths $\lambda$ are in good agreement with measured diapason of wind velocitys. Measurements were carried out on the sand beach of the gulf of Riga, Latvia. Theoretically the phenomenon speaks within the limits of nonlinear acoustics or within the limits of nonlinear mechanic.

2pPAj5. A numerical study of the onset of granular avalanches. Lydie Staron (Institut Jean le Rond d'Alembert, 4 place Jussieu, case 161-162, 75252 Paris Cedex 05, France, staron@1mm.jussieu.fr)

Granular media can either exist in a jammed state, in which their behaviour can be seen as solid-like, or they can exist in a dilute state and flow as a fluid would. In this contribution, we are interested in the mechanisms prevailing in the transition between solid-like and fluid-like behaviour, specifically in the case of the onset of granular avalanches. Therefore, discrete numerical simulations of granular flows are carried out. The focus is set on the analysis of the stick-slip dynamics preceeding the trigger of the avalanche; the existence of precursors to the flow, and their distribution in space and time will be investigated.

TUESDAY AFTERNOON, 1 JULY 2008

P3-A, LEVEL 3, 3:40 TO 5:20 P.M.

Session 2pPAk

Physical Acoustics: Sonic, Ultrasonic, and Megasonic Cleaning II (Poster Session)

\author{
Ralph Muehleisen, Cochair \\ Illinois Institute of Technology \\ Claus-Dieter Ohl, Cochair \\ University of Twente
}

\begin{abstract}
All posters will be on display from 3:40 p.m. to 5:20 p.m. To allow contributors an opportunity to see other posters, contributors of odd-numbered papers will be at their posters from 3:40 p.m. to 4:30 p.m. and contributors of even-numbered papers will be at their posters from 4:30 p.m. to 5:20 p.m.
\end{abstract}

\title{
Contributed Paper
}

2pPAk1. Ultrasonic cleaning of submerged membranes for drinking water applications. Sonja Lauterborn (TU Darmstadt, Institut WAR, Petersenstraße 13, 64287 Darmstadt, Germany, s.lauterborn@iwar .tu-darmstadt.de), Wilhelm Urban (TU Darmstadt, Institut WAR, Petersenstraße 13, 64287 Darmstadt, Germany, w.urban@iwar.tu-darmstadt.de)

Ultrasonic cleaning of membranes used in water purification and waste water treatment is under investigation for avoiding fouling and scaling on the membranes. So far chemicals are used, but their use is under scrutinity for safety, waste removal and health issues. Chemicals often even do not solve the cleaning problem durably. For applications in the part of drinking water treatment it is necessary to ensure the filtered water is really clean.
Therefore in the presented experiment the outflow is constantly controlled by turbidity measurements and by using a particle counter in an online system. A pilot plant for sonication of submerged membranes to produce drinking water from surface water was constructed and placed at the Rhine water works in Biebesheim. It could be shown that sonication with $130 \mathrm{kHz}$ when backflushing the membranes only works with following air overflow. Then the permeability keeps high. No damage of the membranes occurs like often has been found in former studies. Only 30 seconds of sonication after 30 min of filtration are enough to keep high performance of the membranes. Thus energy demand is low, which is a pre-condition for an economical use in technical applications. 


\title{
Session 2pPAl
}

\section{Physical Acoustics: Mathematical and Numerical Methods II (Poster Session)}

\begin{abstract}
All posters will be on display from 3:40 p.m. to 5:20 p.m. To allow contributors an opportunity to see other posters, contributors of odd-numbered papers will be at their posters from 3:40 p.m. to 4:30 p.m. and contributors of even-numbered papers will be at their posters from 4:30 p.m. to 5:20 p.m.
\end{abstract}

\section{Contributed Papers}

2pPAl1. Finite element modelling of thermoviscous acoustics in closed cavities. Nicolas Joly (Laboratoire d'Acoustique de l'Université du Maine, Avenue Olivier Messiaen, 72085 Le Mans, France, nicolas.joly @univ-lemans.fr)

A numerical methodology is presented to compute the acoustic field in a closed domain filled by a thermoviscous fluid, using the finite element method. The formulation based upon temperature variation and particle velocity is well suited for both (i) modelling the acoustic, thermal and viscous effects in the fluid bulk and (ii) accounting for the non-slip and thermal boundary conditions on the solid. The set of coupled equations presents usual scalar- and vector- operators for finite element modelling. Due to the development of thin thermal- and viscous- boundary layers compared to the acoustic wavelength, very different scales are present in the computed fields: the finite-element mesh needs to be dramatically refined along the direction normal to the boundary, while a coarse mesh is often sufficient along the directions parallel to the boundary, and far from the boundaries. The optimised mesh is obtained iteratively, using a loop procedure computing (a) the thermoviscous solution, (b) the metric map suited for this field, and (c) the unstructured anisotropic adapted mesh based on this metric, until convergence. Results are presented for two-dimensional and axisymmetric three-dimensional applications, illustrating thermoviscous effects for various geometries of cavities.

2pPA12. Wave propagation in stratified fluid flows: Application of the Stroh formalism to Lagrangian acoustic perturbations. Olivier Poncelet (LMP, UMR CNRS 5469, Université Bordeaux I, 351, cours de la Libération, 33405 Talence, France, o.poncelet@1mp.u-bordeaux1 .fr), Mélanie Ottenio (INRETS - Univ. Claude Bernard Lyon 1, UMR-T 9406, Laboratoire de Biomecanique et Mecanique des Chocs, 25, Avenue Francois Mitterand, 69675 Bron, France, melanie.ottenio@inrets.fr)

We consider the problem of harmonic waves propagating in non uniform fluid flows in presence of either solid interfaces (elastic walls), or jumps of mechanical properties or jumps of fluid convection-speed. The acoustic field is described by taking into account Lagrangian perturbations referred to an Eulerian frame related to the moving fluid. In contrast to the case of eulerian perturbations, the lagrangian description enables us to introduce the acoustical displacement in the problem and therefore to write the continuity conditions in a simple and unambiguous way: normal acoustical displacement is always continuous through an interface between either solids or (moving) fluids; the normal acoustical stress is also continuous in most of the practical cases (boundary layers, vibrating walls). We express the wave equation as an ordinary differential system by making use of the Stroh formalism from which analytical and semi-analytical results can be obtained for any arbitrary profile of the flow properties. This formalism, coupled with PeanoNeumann series expansion and Frobenius power series, enable us to compute modes dispersion in elastic ducts, and reflective properties of a mixing layer in between two fluids in relative motion. Some analytics on those problems is provided.

2pPAl3. Treatment of the asymptotic behaviour of the piezolectric Green's function for finite element/boundary element analysis of surface waveguides. Sylvain Ballandras (CNRS FEMTO-ST, 32 Avenue de
l'Observatoire, 25044 Besançon, France, sylvain.ballandras@femto-st .fr), Michel Lenczner (CNRS FEMTO-ST, 32 Avenue de l'Observatoire, 25044 Besançon, France, michel.lenczner@femto-st.fr), Thierry Larroche (CNRS FEMTO-ST, 32 Avenue de l'Observatoire, 25044 Besançon, France, thierry.larroche@femto-st.fr)

The simulation of surface waveguides has been dramatically improved by the combination of analytic description of piezoelectric materials using surface Green's function and numerical approaches sur as plane wave expansion, finite diffrence, finite element, etc. A lot of work has been dedicated to treat the singularities of such Green's function generally derived in the spectral domain. An interesting approach consists in using the Green's function which relates the surface stresses to the displacements which is particularly well-suited for mixed finite element/boundary element formulations. This Green's function does not exhibit any pole but presents an asymptotic behavior which tends to infinity along increasing wavenumber values, which prevents the computation of its Fourier transform. In this work, we show how this difficulty can be overcome and we propose a formulation in which the Green's function is factorized in order to change its asymptotic behaviour to a form allowing for Fourier transform computation for non periodic problems and an analytic treatment of its asymptotic behaviour for the simulation of periodic structures. Examples are provided to show the interest of the proposed approach in terms of computation delays and precision.

2pPAl4. 'Maxwellian' macroscopic acoustics and acoustic metamaterials. Denis Lafarge (Laboratoire d'Acoustique de l'Université du Maine, Avenue Olivier Messiaen, 72085 Le Mans, France, denis.lafarge@univ-lemans.fr), Aroune Duclos (Laboratoire d'Acoustique de l'Université du Maine, Avenue Olivier Messiaen, 72085 Le Mans, France, aroune.duclos@univ-lemans.fr)

For electromagnetic wave propagation it was found that there exist composite media exhibiting strong spatial dispersion. This has raised the question of the relevance of the spatial dispersion in the characterization of the new metamaterials. The present communication intends to show that exactly the same problematic occurs in acoustics when considering long-wavelength acoustic wave propagation in a fluid in presence of solid obstacles of any arbitrary shape. When these obstacles resemble "Helmholtz" resonators the macroscopic equivalent medium may exhibit strong spatial dispersion. It is demonstrated that the corresponding Macroscopic Acoustics, which may be worked out in detail, is very similar to the general nonlocal Maxwellian Macroscopic Electrodynamics. This raises the question of the relevance of the spatial dispersion in the characterisation of porous materials, and specifically the question of the possible design of acoustic metamaterials exhibiting enhanced absorption due to spatial dispersion.

2pPAl5. Theoretical proof of acoustic source property single definition in liquid and solid. Leo Zagorskiy (Moscow Mining Institute, Lenin Street, 6, 16-14, Settl. Vugi, 140004 Luybertsy, Russian Federation, ZAGORSKIY_LEV@MAIL.RU)

The problem of acoustic waves property sourse single definition consists in unknown density and elastic modules of the medium. The Green's function definition is difficult as well as the real sourse isn't point and has the 
time dependence. In the early author's papers Green's function had been defined by using Levitan's polynomials. In the liquid or solid medium it is necessary for that to know only the first eigen values and shear or P-wave velocity at the first step from the free surface. It provides to calculate amplitudes of first mode of SH waves, $\alpha$ and $\beta$ - the limits of lacunas in the spectrum. The time function of not moving sourse $F$ is defined from the equation $\mathrm{F}^{*} \mathrm{G} \equiv \mathrm{U}$, where $\mathrm{U}$ is displacement, G- Green's function, * denominates rolling up. That equation is solved by direct and inverse fast Fourie transform. The further definition of source's location from the direct task for P or S body waves with defined velocity isn't hard by using modern finite element method. The rounding of the fronts gives the location of the sourse.

TUESDAY AFTERNOON, 1 JULY 2008

P2-C, LEVEL 2, 3:40 TO 5:20 P.M.

\title{
Session 2pPPa
}

\section{Psychological and Physiological Acoustics: General Topics in Psychological and Physiological Acoustics I (Poster Session)}

\author{
Elizabeth Strickland, Cochair \\ Purdue University \\ Armin Kohlrausch, Cochair \\ Philips Research Europe \\ Alain De Cheveigne, Cochair \\ CNRS, Universite Paris 5, Ecole Normale Superieure
}

\begin{abstract}
All posters will be on display from 3:40 p.m. to 5:20 p.m. To allow contributors an opportunity to see other posters, contributors of odd-numbered papers will be at their posters from 3:40 p.m. to 4:30 p.m. and contributors of even-numbered papers will be at their posters from 4:30 p.m. to 5:20 p.m.
\end{abstract}

\section{Contributed Papers}

2pPPa1. Detection and Cortical Representations of the Break in Interaural Correlation of Narrowband Noises Are Affected by Center Frequency and Interaural Delay. Ying Huang (Dept. of Psychology, Peking Univ., 5 Yeheyuan Road, Haidian District, 100871 Beijing, China, innhuang@gmail.com), Lingzhi Z. Kong (Dept. of Psychology, Peking Univ., 5 Yeheyuan Road, Haidian District, 100871 Beijing, China, konglingzhi@gmail.com), Silu Fan (Graduate School of the Chinese Academy of Sciences, 100049 Beijing, China, slfan@gucas.ac.cn), Xihong H. Wu (Dept. of Machine Intelligence, Speech and Hearing Research Center, 2 Science Building, Peking Univ., 5 Yeheyuan Road, Haidian District, 100871 Beijing, China, wxh@cis.pku.edu.cn), Liang Li (Dept. of Psychology, Peking Univ., 5 Yeheyuan Road, Haidian District, 100871 Beijing, China, liangli@pku.edu.cn)

When either broadband or narrowband arbitrary noises presented at the two ears are correlated, a fused noise image is perceived inside the head if the interaural interval (interaural time difference, ITD) is sufficiently short, indicating that acoustic-waveform information can be binaurally integrated At both the perceptual level and neurophysiological level, this study investigated whether the binaural integration of correlated noises is affected by center frequency (for narrowband noise) and ITD. Results of the psychophysical experiment show that the duration threshold for detecting a break in correlation (BIC) in the correlated noises at the two ears was higher for high-frequency noises than for low-frequency noises, and dramatically elevated with the increase of the ITD from 0 to $4 \mathrm{~ms}$. Moreover, the ITDinduced threshold elevation was much larger for high-frequency narrowband noises than for low-frequency narrowband noises. Results of the neurophysiological experiments show that the cortical components of scalp eventrelated potentials to the BIC were markedly modulated by both the center frequency (for narrowband noise) and ITD. Thus, temporal integration of acoustic waveform details of correlated noises at the two ears is both frequency and ITD dependent. Supported by the National Natural Science Foundation of China.

2pPPa2. Object-related negativity indexes perception of sounds beyond the echo threshold. Lisa D. Sanders (Univ. of Massachusetts, Department of Psychology, Tobin Hall, Amherst, MA 01003, USA, lsanders@psych.umass.edu), Benjamin Zobel (Univ. of Massachusetts, Department of Psychology, Tobin Hall, Amherst, MA 01003, USA, benjaminzobel@yahoo.com), Rachel Keen (University of Virginia, Department of Psychology, P. O. Box 400400, Charlottesville, VA 22904-4400, USA, rachelkeen@ virginia.edu), Richard L. Freyman (Univ. of Massachusetts, Dept. of Communication Disorders, 358 N. Pleasant St., Amherst, MA 01003, USA,rlf@comdis.umass.edu)

The ability to isolate a single sound source among concurrent sources and reverberant energy is crucial for understanding the auditory world. The precedence effect describes an experimental finding that when listeners are presented with identical sounds from two locations with a short onset asynchrony, they report hearing a single source with a location dominated by the lead sound. We recently showed that event-related potentials (ERPs) elicited by click pairs differ for trials on which listeners do and do not report hearing the lag sound as a separate source. Specifically, when participants report hearing two sounds, we observe a negativity between 100 and $250 \mathrm{~ms}$, previously termed the object-related negativity (ORN). These results indicate that the precedence effect reflects top-down cognitive influence over early neurosensory processing. Additionally, the study provides support for the claim that the ORN, initially reported for sounds perceived as two concurrent pitches, indexes auditory object perception. The effects on the ORN of repeating identical click pairs, a condition that has previously been shown to 
increase echo thresholds, will also be discussed in the context of listeners forming complex models of room acoustics based on what they hear in a specific setting.

2pPPa3. Improving source localisation in multi-source, reverberant conditions: exploiting local spectro-temporal location cues. Heidi Christensen (University of Sheffield, Regent Court, Dept. of Computer Science, 211 Portobello Road, s1 4dp Sheffield, UK, h.christensen@dcs .shef.ac.uk), Ning Ma (University of Sheffield, Dept. of Computer Science, Regent Court, 211 Portobello Street, S1 4DP Sheffield, UK, n.ma@dcs.shef.ac.uk), Stuart N. Wrigley (University of Sheffield, Regent Court, Dept. of Computer Science, 211 Portobello Road, s1 4dp Sheffield, UK, s.wrigley@dcs.shef.ac.uk), Jon Barker (University of Sheffield, Dept. of Computer Science, Regent Court, 211 Portobello Street, S1 4DP Sheffield, UK, j.barker@dcs.shef.ac.uk)

This paper examines the use of binaural cues for estimating the location of individual sources in multi-source acoustic mixtures. In most environments, location cues such as interaural time difference (ITD) and interaural intensity difference (IID) are rendered unreliable by noise due to competing sources and reverberation. We propose a method that examines spectrotemporal regions where such effects are minimised. Source location is estimated by combining two processing stages. First, following [Christensen et al, Interspeech (2007)], as pitch cues are generally less affected by noise, a multi-pitch estimation and tracking algorithm is used to identify local spectro-temporal 'fragments' in which the SNR is high. Second, the confidence of ITD and IID estimates arising from each time-frequency 'pixel' is estimated based on interaural coherence [Faller and Merimaa, J. Acoust. Soc. Am. 116, 3075--3089 (2004)] and reverberation estimation [Heckmann et al, Int. Conf. Int. Robots and Systems (2006)]. A location estimate is then constructed by integrating suitably weighted cues from each pixel within the fragment. Experiments are carried out on a large corpus of multi-speaker data, mixed from binaural recordings in a real, reverberant environment. The fragment based processing is shown to provide significant improvements when compared to competitive baseline approaches.

2pPPa4. The effect of spectral overlap on sensitivity to interaural time differences. Christopher A. Brown (Arizona State University, Dept. of Speech \& Hearing Science, P.O. Box 870102, Tempe, AZ 85287-0102, USA, c-b@asu.edu), William A. Yost (Arizona State University, Dept. of Speech \& Hearing Science, P.O. Box 870102, Tempe, AZ 85287-0102, USA, William.Yost@asu.edu)

Cochlear implant users who have some residual low-frequency hearing in both their implanted and unimplanted ears may have access to interaural time differences (ITDs), which could provide a cue for sound source segregation. Speech intelligibility has been shown in simulation to improve under these conditions. However, implant patients who stand to benefit from ITDs may have a significant asymmetry in the configuration of loss in the low-frequency region. It is unknown how a difference in corner frequency of loss across ears will affect ITD sensitivity. As a first step toward characterizing this relationship, the present study measured ITD just-noticeable differences (JNDs) of bands of noise that varied in the amount of spectral overlap across ears in five normal-hearing listeners. In one ear, the center frequency was $250 \mathrm{~Hz}$, and the bandwidth was either $1 / 3$ or $2 / 3$ octaves. In the other ear, the center frequency was $250,281,315,354,397$, or $794 \mathrm{~Hz}$, and the bandwidth was $1 / 3$ or $2 / 3$ octaves. Results showed that although ITD JNDs increased as spectral overlap decreased, ITDs were only detectable so long as some spectral overlap was present.

2pPPa5. Perception of speaker's facing angle. Hiroaki Kato (ATR Cognitive Information Science Laboratories, 2-2-2 Hikaridai, Seika-cho Soraku-gun, 619-0288 Kyoto, Japan, kato@atr.jp), Hironori Takemoto (ATR Cognitive Information Science Laboratories, 2-2-2 Hikaridai, Seika-cho Soraku-gun, 619-0288 Kyoto, Japan, takemoto@atr.jp), Ryouichi Nishimura (National Institute of Information and Communications Technology/ATR Cognitive Information Science Laboratories, 2-2-2 Hikaridai, Seika-cho, 619-0288 Kyoto, Japan, ryou@ atr .jp)

In pursuit of an ultimately realistic human-to-human telecommunication technology, the ability to auditorily perceive the facing direction of a human speaker was explored. A male speaker sat on a pivot chair in an anechoic chamber and spoke a short sentence (about 5 seconds) while facing either of eight azimuth angles $(0=$ listener's direction, $45,90,135,180,225,270$, or 315 degrees) and either of three elevation angles $(0=$ horizontal direction, -45 , or 45 degrees). The azimuth angles were set solely by turning the pivot chair. Twelve blindfolded listeners heard the spoken sentence at a distance of either 1.2 or 2.4 meters from the speaker and were asked to indicate the speaker's facing angle. In separate sessions, the speaker changed facing angles while speaking and the listeners indicated the perceived direction of horizontal movement (clockwise or counter-clockwise) or vertical movement (up or down). Overall results showed that the listeners were more accurate in indicating the movement of the speaker than in simply indicating the facing angles. Effective acoustic cues were then discussed on the basis of the transfer characteristics from the speaker's mouth to the listener's ears measured by the cross-spectral method using the speaker's own voice.

2pPPa6. Auditory capture in a spatial cueing task. Marieke Van Der Hoeven (TNO, Kampweg 5, 3769ZG Soesterberg, Netherlands, marieke.vanderhoeven@tno.nl), Adelbert W. Bronkhorst (TNO, Kampweg 5, 3769ZG Soesterberg, Netherlands, adelbert.bronkhorst@tno.nl)

This study examined how an auditory non-informative spatial cue influences detection and localization of auditory targets. In a go-no go task subjects attended a centrally placed loudspeaker surrounded by 6 other loudspeakers and were asked to respond only to centre targets. The cue came from one of the loudspeakers and preceded the target tone with an SOA of 100 or $400 \mathrm{~ms}$. A baseline condition with a spatially diffuse cue was included, using out-of-phase presentation through two loudspeakers. Results show that, compared to the baseline, cues shortened reaction times at small cue-target angles (up to $7^{\circ}$ ) and increased them at larger angles. At the shortest SOA, the reaction time difference was up to $80 \mathrm{~ms}$. Furthermore, cueing seems to have no effect on sound localization: false alarms were normally distributed around the hits. These findings demonstrate that strong auditory capture effects occur that depend on cue-target distance.

2pPPa7. Spatiotemporal interactions between audition and vision. Adelbert W. Bronkhorst (TNO, Kampweg 5, 3769ZG Soesterberg, Netherlands, adelbert.bronkhorst@tno.nl), Thomas Koelewijn (Vrije Universiteit, van der Boechorststraat 1, 1081 BT Amsterdam, Netherlands, T.Koelewijn $@$ @ psy.vu.nl), Erik Van Der Burg (Vrije Universiteit, van der Boechorststraat 1, 1081 BT Amsterdam, Netherlands, E.van.der.Burg@psy.vu.nl)

It is well known that spatial auditory cues can affect performance on spatial visual tasks. One example is crossmodal cueing: response times for a visual spatial discrimination task are shortened when the location of the visual stimuli is cued auditorily, and prolonged when an invalid location is cued. Another example is auditory support of visual search: we normally find lower search times for visual items when the location of the target item is indicated by an auditory cue. In our research we have explored two questions: one related to crossmodal cueing, and the other to audiovisual search. In our crossmodal cueing paradigm we have looked at the interaction between (exogenous) cueing and endogenous information given before each trial. In this way we wanted to determine whether auditory cues are able to capture visual attention even when this attention has already been focused endogenously. In our audiovisual search paradigm we have studied whether nonspatial auditory cues, presented simultaneously with the visual target item, are able to improve visual search. Because of the temporal synchrony, the auditory stimuli do not act as actual cues but they are probably fused with the visual stimuli into an integrated percept.

2pPPa8. The acoustical bright spot and the erroneous localization of tones by human listeners. Brad Rakerd (Michigan State University, Dept. of Communicative Sciences and Disorders, East Lansing, MI 48824, USA, 
rakerd@msu.edu),Zach Ryan(Michigan State University, Dept. of Physics and Astronomy, East Lansing, MI 48824, USA, ryanzach@msu.edu), Eric Macaulay (Michigan State University, Dept. of Physics and Astronomy, East Lansing, MI 48824, USA, macaula5@msu.edu), William M. Hartmann (Michigan State University, Dept. of Physics and Astronomy, East Lansing, MI 48824, USA, hartmann@pa.msu.edu)

In free-field sound localization experiments, human listeners estimated the locations of sine tones originating at 13 loudspeakers that spanned a range of azimuths from 0 degrees (straight ahead) to 90 degrees (extreme right). During the experiments the tone amplitudes and phases were measured in the listener's ear canals. Because of the acoustical bright spot, measured interaural level differences (ILD) were found to be a nonmonotonic function of azimuth at frequencies above about $1000 \mathrm{~Hz}$. In source identification experiments at $1500 \mathrm{~Hz}$, there was no correlation between estimated location and the interaural phase difference. There was high correlation with ILD, causing listeners to make large localization errors for azimuths greater than about 55 degrees. In a second experiment, listeners judged the direction of movement of sounds from one source to another. Errors in the second experiment could be well predicted from the localization responses in the source identification experiment. The ear canal measurements showed that, in principle, listeners could localize successfully by using the levels in the two ears independently. However, localization performance remained poor even after extensive training. Work supported by the NIDCD, grant DC00181.

2pPPa9. Human cortical responses to change in sound lateralization suggest non-topographical coding of interaural time differences. David A. Magezi (MRC Institute of Hearing Research, University Park, NG7 2RD Nottingham, UK, david.magezi@ihr.mrc.ac.uk), Katrin Krumbholz (MRC Institute of Hearing Research, University Park, NG7 2RD Nottingham, UK, katrin@ihr.mrc.ac.uk)

It is generally assumed that interaural time differences (ITDs) are coded by a parametric array of finely tuned ITD channels (topographic code). An alternative theory is that ITD is coded by a rate code, which involves only one channel in each hemisphere, broadly tuned to the contralateral hemispace. The aim of the current study was to investigate ITD coding in humans by using electroencephalography (EEG) with a continuous stimulation paradigm (CSP). In this paradigm, a test sound, with a given ITD, is immediately preceded by an appropriately matched control sound with a different ITD. At the transition from the test to the control sound, only neural elements that respond to the test but not the control ITD would be expected to be activated. Thus, the size of the transition response would be assumed to be determined by the degree of overlap between the neural responses to the control and test ITDs. The pattern of results was found to be inconsistent with the topographic model, and thus lend support to the hemispheric channel model.

2pPPa10. Both prime length and prime position affect the spatial release of nonsense speech from informational masking. Zhigang Yang (Dept. of Psychology, Peking Univ., 5 Yeheyuan Road, Haidian District, 100871 Beijing, China, synrhine@163.com), Jing Chen (Dept. of Machine Intelligence, Speech and Hearing Research Center, 2 Science Building, Peking Univ., 5 Yeheyuan Road, Haidian District, 100871 Beijing, China, chenj@cis.pku.edu.cn), Qiang Huang (Dept. of Machine Intelligence, Speech and Hearing Research Center, 2 Science Building, Peking Univ., 5 Yeheyuan Road, Haidian District, 100871 Beijing, China, QIANG.HUANG@SPREADTRUM.COM), Ying Huang (Dept. of Psychology, Peking Univ., 5 Yeheyuan Road, Haidian District, 100871 Beijing, China, innhuang@gmail.com), Xihong H. Wu (Dept. of Machine Intelligence, Speech and Hearing Research Center, 2 Science Building, Peking Univ., 5 Yeheyuan Road, Haidian District, 100871 Beijing, China, wxh@cis.pku.edu.cn), Yanhong Wu (Dept. of Psychology, Peking Univ., 5 Yeheyuan Road, Haidian District, 100871 Beijing, China, wuyh@pku.edu.cn), Liang Li (Dept. of Psychology, Peking Univ., 5 Yeheyuan Road, Haidian District, 100871 Beijing, China, liangli@pku.edu.cn)

When masking speech is present, pre-presentation of early part of nonsense target speech improves recognition of the rest of target speech, indicating a content and/or voice priming effect (Freyman et al., 2004; Yang et al., 2007). Here, we examined both the prime-length effect and the primeposition effect on recognition of nonsense target speech with twelve syllables and three keywords. Target speech started $1 \mathrm{sec}$. after the onset of two-talker masking speech. The results show that a longer prime with 10 syllables (including the 1st and 2nd keywords) significantly improved recognition of the last (3rd) keyword in target speech. However, when the 1st four syllables (including the 1st keyword) were pre-presented, recognition of either the 2nd or 3rd keyword was not improved. Interestingly, when the 2nd four syllables (including the 2nd keyword) were pre-presented, recognition of the 1st but not the 3rd keyword was significantly improved. Thus under speech-on-speech masking conditions, both the prime length and the prime position in the sentence influence the priming effect on recognition of target speech, and listeners contribute more attentional resource to the initial part of target speech. Supported by the National Natural Science Foundation of China.

2pPPa11. Masking release based on fine-grain temporal pitch cues in speech-like stimuli - mechanisms and implications for the cocktail-party phenomenon. Nicholas R. Clark (MRC Institute of Hearing Research, University Park, NG7 2RD Nottingham, UK, n.clark@ihr.mrc .ac.uk), Katrin Krumbholz (MRC Institute of Hearing Research, University Park, NG7 2RD Nottingham, UK, katrin@ihr.mrc.ac.uk)

One of the most important cues for segregating wanted from unwanted sounds when listening in noisy environments is temporal regularity (or harmonicity), which gives rise to the perception of pitch. This study measured masked signal detection thresholds when both the signal and the masker are complex tonal stimuli similar to speech. Detection was considerably easier when the pitch of the masker differed from that of the signal. A control experiment involving non-simultaneous masking revealed that this pitch-based release from masking was not due to spectral differences between the signal and the masker. A second experiment investigated the effect of a pitch difference between the signal and the masker on the audibility of certain perceptual features of the signal when the signal is presented at a supra-threshold level. Two features were used that are known to be particularly important for speech perception: amplitude modulation and spectral profile related to formant frequencies. The supra-threshold tasks enabled to quantify the unmasking effect of pitch on the processing of these features over and above the effect on the signal detection threshold. The results provide new insights into the low-level processes involved in the cocktail-party phenomenon. Acknowledgement: Work supported by the Medical Research Council.

2pPPa12. Perceptual sensitivity to high-frequency interaural time differences created by rustling sounds. Lutz Wiegrebe (Biocenter, University of Munich, Großhaderner Str. 2, 82152 Munich, Germany, lutzw @1mu.de)

ITDs are recruited to localize sounds in azimuth. ITDs can be extracted from low-frequency sounds or from the envelopes of high-frequency, complex sounds. Studies of the latter have included amplitude-modulated or transposed tones. A recent study showed that not the degree of envelope fluctuation, as quantified by the envelope 4th moment, determines sensitivity to envelope ITDs but the envelope spectrum and with it the interaural crosscorrelation function of the auditory envelopes provide a good description of envelope ITD sensitivity. Here we use a different class of high-frequency stimuli, namely noise stimuli generated with different degrees of roughness which resemble natural rustling sounds. Stimuli are generated with a Gaussian-noise carrier and an aperiodic, rectangular modulator of statistically different duty cycle. The results show that ITD sensitivity increases both with increasing roughness and increasing bandwidth of the rustling sounds. While the effect of bandwidth on ITD sensitivity is in line with previous conclusions, the effect of roughness is not. ITD sensitivity for rustling sounds elicited ITD thresholds as low as $32 \mu$ s, i.e., considerably lower than reported in previous studies with transposed stimuli. The data show that high-frequency rustling sounds provide strong temporal localization cues which the auditory system can effectively exploit. 
2pPPa13. Lateralization of binaural stimuli with independent fine-structure and envelope-based temporal disparities. Mathias Dietz (Carl von Ossietzky Universität Oldenburg, Ammerländer Heerstraße 114-118, 26111 Oldenburg, Germany, mathias.dietz@uni-oldenburg .de), Stephan D. Ewert (Carl von Ossietzky Universität Oldenburg, Ammerländer Heerstraße 114-118, 26111 Oldenburg, Germany, stephan.ewert@uni-oldenburg.de), Volker Hohmann (Carl von Ossietzky Universität Oldenburg, Ammerländer Heerstraße 114-118, 26111 Oldenburg, Germany, volker.hohmann@uni-oldenburg.de)

A computational model for the lateralization of binaural stimuli, motivated by recent physiological findings in the literature and psychoacoustic data is presented. The model is based on the evaluation of the interaural phase difference (IPD). In the model, IPDs are separately assessed for the stimulus' fine-structure and envelope. Psychoacoustic measurements were conducted and compared to model predictions. Sinusoidally amplitude modulated $1-\mathrm{kHz}$ tones with a modulation frequency of 25,50 , and $100 \mathrm{~Hz}$ were employed. The IPD of the fine-structure and the envelope IPD were independently matched with an interaural level difference or were traded against each other. Lateralization increased for increasing IPDs up to $135^{\circ}$ of either the fine-structure or envelope independent of the modulation frequency. However, trading a fine-structure IPD with an opposing envelope IPD revealed a most persistent fine-structure IPD at $45^{\circ}$. The data could be modeled assuming a physiological distribution of the best IPDs of binaural neurons clustered around $45^{\circ}$. The model was also utilized to correctly predict the perceived lateralization of critical stimuli from literature. Individual differences in the perceptual salience of envelope and fine-structure cues, also known from the literature, could be modeled by a personal weighting coefficient for the fine-structure cue.

2pPPa14. A binaural advantage in the subjective modulation transfer function with simple impulse responses. Eric R. Thompson (Centre for applied hearing research, Technical University of Denmark, DTU, Bygn. 352, 2800 Lyngby, Denmark, et@oersted.dtu.dk), Torsten Dau (Centre for applied hearing research, Technical University of Denmark, DTU, Bygn. 352, 2800 Lyngby, Denmark, tda@ oersted.dtu.dk)

The speech transmission index (STI) has been a popular method for predicting speech intelligibility in rooms. It is based on the magnitude of the modulation transfer function, which can be derived from the impulse response of the room and the background noise levels. However, it does not take into account that humans listen with two ears. There can be large interaural phase differences in the modulation transfer functions, which can create detectable interaural level difference fluctuations. Measurements were made to determine whether these interaural modulation phase differences can be used to enhance the detectability of sinusoidal intensity modulations imposed on a broadband noise carrier and then convolved with simple, dichotic impulse responses. The results show that there can be a significant advantage to listening with two ears over listening with just one. Some further investigations were made to determine whether this advantage in signal detection can also be used as an advantage for speech intelligibility.

2pPPa15. Temporal weighting of interaural level differences in high-rate click trains. Christopher Stecker (Dept. of Speech and Hearing Sciences, University of Washington, 1417 NE 42nd St, Seattle, WA 98105, USA, cstecker@u.washington.edu)

When high-carrier-frequency stimuli are amplitude modulated at rates above $\sim 200 \mathrm{~Hz}$, lateralization based on envelope interaural time differences (ITD) becomes dominated by onset ITD. The role of onset cues in interaural-level-difference (ILD) processing, however, is less well understood. Although theoretical predictions suggest that onset dominance should have similar effects upon ILD as upon ITD, some experimental data suggest that ILD processing retains greater sensitivity during post-onset segments of a high-rate stimulus. In this study, subjects lateralized ILDs carried by trains of 16 Gaussian-filtered clicks (carrier frequency $4000 \mathrm{~Hz}$ ) that varied in interclick interval (ICI) from 2 to $10 \mathrm{~ms}$ (modulation rate 100-500 $\mathrm{Hz}$ ). ILDs of individual clicks in each train were randomly perturbed. Multiple linear regression of listener responses onto the perturbed ILD values was used to generate temporal weighting functions (TWFs) that describe how a listener's sensitivity to ILD changes over the stimulus duration. The resulting TWFs were compared to predictions from models of onset dominance as well as to TWFs measured for ITD and in free-field conditions. The results have implications for binaural processing of high-rate pulsatile stimuli experience by users of bilateral cochlear implants.

2pPPa16. Modeling the precedence effect in inferior colliculus neurons using converging excitatory and inhibitory inputs. Jing Xia (Boston University Hearing Research Center, 677 Beacon Street, Boston, MA 02215, USA, xiajing0928@hotmail.com), Andrew Brughera (44 Cummington St., Boston, MA 02215, USA, brughera@bu.edu), H Steven Colburn (44 Cummington St., Boston, MA 02215, USA, colburn@bu.edu), Barbara Shinn-Cunningham (Boston University Hearing Research Center, 677 Beacon Street, Boston, MA 02215, USA, shinn@cns.bu.edu)

Responses of a population model of low-frequency inferior colliculus (IC) neurons to a pair of broadband clicks were evaluated. IC inputs came from Hodgkin-Huxley models of medial superior olive (MSO) and cochlear nucleus bushy cells driven by a stochastic auditory nerve model. Each IC cell received converging inputs that were excitatory from ipsilateral MSO and inhibitory from contralateral MSO. Convergence of multiple excitatory, ipsilateral MSO inputs with similar interaural time difference (ITD) tuning improved ITD sensitivity and reduced the influence of MSO onset responses caused by monaural, rather than binaural, coincidences. Inhibition that lasted over several milliseconds suppressed IC responses to the lagging click. The effect of the inhibition depended on the ITD tuning of the excitation and inhibition converging on a given IC neuron. With appropriate convergence, the IC population showed no response to the lagging click at short delays, and responded to the lagging click but did not encode its ITD at intermediate delays. Only at longer inter-click delays did IC responses show sensitivity to the lagging click ITD, consistent with the perceptual phenomenon of the precedence effect. [Work supported by NSF and NIH].

2pPPa17. Relationship between reading and language ability and auditory temporal processing measured with the precedence effect. Cynthia M. Zettler (University of Wisconsin, Waisman Center, 1500 Highland Ave, Madison, WI 53705, USA, zettler@waisman.wisc.edu), Rose A. Sevcik (Georgia State University, Dept of Psychology, University Plaza, Atlanta, GA 30303, USA, psyras@langate.gsu.edu), Robin D. Morris (Georgia State University, Dept of Psychology, University Plaza, Atlanta, GA 30303, USA, psyrdm@langate.gsu.edu), Mary Ann Romski (Georgia State University, Dept of Psychology, University Plaza, Atlanta, GA 30303, USA, mromski@gsu.edu), Colleen O'Rourke (Georgia State University, Dept of Psychology, University Plaza, Atlanta, GA 30303, USA, corourke@gsu.edu), Marsha G. Clarkson (Georgia State University, Dept of Psychology, University Plaza, Atlanta, GA 30303, USA, mclarkson @ gsu.edu)

Processing of timing information by the auditory system contributes to numerous abilities, including spatial hearing and higher-order language and reading skills. However, relationships among these abilities are poorly understood. The present study was aimed at investigating potential common mechanisms that involve timing. Auditory timing was measured by click stimuli presented via two loudspeakers at a rate of $1.5 / \mathrm{sec}$. Fourteen-ms clicks were presented from one (single-source condition) or two (dualsource condition) loudspeakers. In the latter condition, the onset of one loudspeaker was delayed relative to the other by $5,10,20,50,100,200$, or $400 \mathrm{~ms}$. Seventy-five naïve adults (mean age 21.23 years) listened to the auditory stimuli and were asked to identify the source of the leading sound. Additionally, all participants completed a battery of language and reading measures. Results on the auditory task revealed that performance was significantly above chance on single-source trials, dropped at short delays, and improved with increasing delay. The mean threshold for correctly identifying the source of the sound was $43.48 \mathrm{~ms}$. A significant correlation $(\mathrm{p}<.05)$ found between this threshold and a measure of word identification suggests a relationship between timing required for auditory localization and timing required for reading ability.

2pPPa18. Lateralization of unidirectional frequency sweeps at high frequencies. I-Hui Hsieh (University of California, Department of Cognitive Sciences, Irvine, CA 92697, USA, ihsieh@uci.edu), Kourosh 
Saberi(University of California, Department of Cognitive Sciences, Irvine, CA 92697, USA, saberi@uci.edu)

The ability to lateralize FM sweeps at high frequencies was investigated in a 2IFC 2-down 1-up adaptive design. Four conditions were examined in which the unidirectional FM stimulus swept either up or down in frequency, and either linearly or logarithmically between 3 and $8 \mathrm{kHz}$ with the onset frequency roved by $15 \%$ on each presentation. The signal was an interaural delay in the waveform's fine-structure and hence its instantaneous frequency. The waveforms to the left and right ears had simultaneous envelopes and were filtered with the inverse of the headphone transfer functions to eliminate frequency-dependent interaural level differences. Results showed that sweep durations exceeding $25 \mathrm{~ms}$ produce near-chance performance. Thresholds monotonically improved with increasing sweep rate to a lower asymptote of approximately 100 microseconds. Counterintuitive reversals of lateral position percepts were observed for down-sweeps which were inconsistent with lateralization based on envelope outputs of frequency-matched filters.

2pPPa19. The localization of multiple simultaneous sounds is mediated by attention. Brian D. Simpson (AFRL, 2610 Seventh Street, WPAFB, OH 45433, USA, Brian.Simpson@wpafb.af.mil), Douglas S. Brungart (AFRL, 2610 Seventh Street, WPAFB, OH 45433, USA, douglas.brungart@wpafb.af.mil), Robert H. Gilkey (Wright State University, Department of Psychology, 335 Fawcett Hall, Dayton, 45435, USA, gilk@wright.edu), Nandini Iyer (AFRL, 2610 Seventh Street, WPAFB, OH 45433, USA, nandini.iyer@wpafb.af.mil), James T. Hamil (Ohio State University, Department of Electrical Engineering, Columbus, 43210, USA, hamil.3@osu.edu)

Real-world listening experiences suggest that listeners possess a much greater ability to recover spatial information from multiple simultaneous sounds than would be predicted from the results of most multisource listening experiments. Recent data from our laboratory are consistent with these real-world experiences, and indicate that listeners can reliably report the location of a sound that has been deleted from a multisource scene. However, performance degrades as the number of competing sounds increases, and it is unclear if this 'set size effect' arises from changes in signal-to-noise ratio (SNR) related to the number of competing sources, or from attentional constraints that limit the number of simultaneous sources that can be monitored. In this study, SNR was held roughly constant by fixing the number of competing sources at 8 , and the 'relevant set size' was varied by displaying on a visual monitor, prior to an observation interval, a list of $1,2,3,4,6$, or 8 environmental sounds, one of which was the target sound (the sound that would be deleted). The ability to localize this target sound decreased as the relevant set size increased, suggesting that the ability to monitor complex auditory scenes is mediated by attentional constraints.

2pPPa20. Better-ear listening strategies for normal-hearing and hearing-impaired individuals. William M. Whitmer (GN Auditory Research Laboratory, 2601 Patriot Blvd, Chicago, IL 60026, USA, whitmerb@beltone.com), Maureen Coughlin (GN Auditory Research Laboratory, 2601 Patriot Blvd, Chicago, IL 60026, USA, mcoughlin@gnresound.com), Jeff Bondy (GN Auditory Research Laboratory, 2601 Patriot Blvd, Chicago, IL 60026, USA, jbondy@gnresound.com), Andrew Dittberner (GN Auditory Research Laboratory, 2601 Patriot Blvd, Chicago, IL 60026, USA, adittberner @ gnresound.com)

The current study was designed to examine how hearing-impaired (HI) listeners use better-ear listening. Better-ear performance was initially assessed for normal-hearing (NH) and $\mathrm{HI}$ participants using a connectedspeech recognition test at two signal-to-noise-ratio (SNR) levels presented over insert earphones. Test conditions consisted of monaural, symmetric and asymmetric combinations of better and worse SNR conditions. SNRs were different for NH and HI groups. Stimuli were presented at each participant's most comfortable level; to account for audibility with HI participants, stimuli were spectrally shaped based on audiometric data. A second experiment investigated the role of spatial information using recordings of the same stimuli presented in a sound-dampened chamber at the same SNR differences, with the speech signal at $0^{\circ}$ azimuth/elevation, and uncorrelated babble presented from eight speakers at the corners of the chamber. Listening strategies between $\mathrm{NH}$ and $\mathrm{HI}$ groups were similar across experiments; neither group consistently used an optimal listening strategy. Results will be discussed in terms of hearing-impaired pathologies, rehabilitation and amplification strategies.

2pPPa21. Numerical Analysis of the Effects of Pinna Shape and Position on the Characteristics of Head-Related Transfer Functions. Yukio Iwaya (R.I.E.C., Tohoku University, 2-1, Katahira, Aoba-ku, 9808577 Sendai, Japan, iwaya@riec.tohoku.ac.jp), Yôiti Suzuki (R.I.E.C., Tohoku University, 2-1, Katahira, Aoba-ku, 980-8577 Sendai, Japan, yoh@ais .riec.tohoku.ac.jp)

There are distinctive notches and peaks in Head-Related Transfer Functions (HRTFs). Some of them are considered as important cues in the perception of the elevation angle and thus the roles of these peaks and notches should be clarified. It is known that the characteristics of HRTFs are deeply related to listener's anthropometry. It is thus naturally expected that frequencies of the peaks and notches also change according to the individuality of listeners' anthropometry. Therefore, in this study, effects of ear shapes and positions on frequency positions of the peaks and notches are examined by numerical analyses. The analysis was performed with boundary element method (BEM). A three-dimensional model of a dummy-head was constructed with a three-dimensional laser scanner and HRTFs of the model were numerically computed with a BEM solver. The model was modified on some features as follows: 1) pinna position, 2) pinna size, 3) angle of pinna toward listener's head, and 4) existence of wrinkles of pinna. HRTFs of the modified models were also computed. From the comparison among the results of calculation, several systematic changes were found. For example, the frequency position of notches around $8 \mathrm{kHz}$ is shifted toward lower frequency as the size of pinna increases.

2pPPa22. Are interaural time and level cues processed by separate or integrated networks in the human auditory cortex? Barrie A. Edmonds (MRC Institute of Hearing Research, University Park, NG7 2RD Nottingham, UK, barrie@ihr.mrc.ac.uk), Katrin Krumbholz (MRC Institute of Hearing Research, University Park, NG7 2RD Nottingham, UK, katrin @ihr.mrc.ac.uk)

Listeners use two cues to localise the position of a sound source in the horizontal plane: interaural time differences (ITDs) and interaural level differences (ILDs). It is thought that these two cues are encoded separately at the level of the brainstem, but are likely to be combined at higher levels in order to produce a representation of sound azimuth. In the current study, we used electro-encephalography (EEG) to investigate whether the cortical representation of a stimulus containing both ITDs and ILDs reflects separate processing of ITDs and ILDs or a combined code for perceived sound location. Stimuli were presented over headphones using a continuous stimulation paradigm; a diotic control sound was presented for 1.5 seconds followed immediately by a dichotic test sound for 250 milliseconds. Evoked responses were recorded for test stimuli containing an ITD only, an ILD only, or both an ITD and an ILD indicating either the same or opposite source azimuths. When the two cues were in opposition, the stimuli were perceived as central and were indistinguishable from the diotic control sound. However, they still elicited a strong cortical response. This suggests that ITDs and ILDs are encoded separately in the auditory cortex. 


\title{
Session 2pPPb
}

\section{Psychological and Physiological Acoustics and ASA Committee on Standards: Applications of Psychoacoustics II (Poster Session)}

\author{
Patricia Davies, Cochair \\ Ray W. Herrick Lab., School of Mechanical Engineering, Purdue Univ. \\ Hugo Fastl, Cochair \\ AG Technische Akustik, MMK, TU München
}

\begin{abstract}
All posters will be on display from 3:40 p.m. to 5:20 p.m. To allow contributors an opportunity to see other posters, contributors of odd-numbered papers will be at their posters from 3:40 p.m. to 4:30 p.m. and contributors of even-numbered papers will be at their posters from 4:30 p.m. to 5:20 p.m.
\end{abstract}

\section{Contributed Papers}

2pPPb1. Loudspeaker sound quality: comparison of assessment procedures. Vincent Koehl (LISyC EA 3883, 6 avenue Victor Le Gorgeu, CS 93837, 29238 Brest Cedex 3, France, vincent.koehl @ univ-brest.fr), Mathieu Paquier (LISyC EA 3883, 6 avenue Victor Le Gorgeu, CS 93837, 29238 Brest Cedex 3, France, mathieu.paquier@univbrest.fr)

In listening tests involving different loudspeakers and aimed at assessing the sound quality of these sound-reproducing systems, the level is generally adjusted to compensate for differences in sensitivity. The loudness sensation must be alike for each system under test. Because of the non-stationary nature of the musical signals used as test material in loudspeaker ratings, loudness assessment by using the current models (Zwicker, Mooreœ) remains slightly inaccurate. In practice, loudness is often equalized by ear by the experimenter. This study deals with the comparison of various test procedures. The first experiment was a paired comparison of loudspeakers where short-duration stimuli were presented to listeners for preference ratings. In the second experiment, the same listeners were allowed to switch, at any time, from one loudspeaker to another one so that the proposed stimuli were longer. In both experiments the loudness was equalized by the experimenter. However, under normal listening conditions, the listener is usually free to adjust by himself the reproduction level. At last, in a third experiment, the listeners had the opportunity, at any time, to not only to switch from one system to another one, but also to adjust the loudness of the stimuli.

2pPPb2. Study of the perceptive space linked to dashboard tapping sounds. Francois Montignies (Renault Technocentre, FR TCR LAB 252, 1 avenue du Golf, 78288 Guyancourt Cedex, France, francois.montignies @insa-lyon.fr), Etienne Parizet (Laboratoire Vibrations Acoustique, Insa Lyon, 25 bis, av. J. Capelle, 69621 Villeurbanne Cedex, France, etienne .parizet@insa-lyon.fr)

It was observed that dashboard tapping sounds can be produced by customers exploring static vehicles. To master the perceived quality of the dashboard, a global sound design process has thus been engaged. The specific aim of the present study was to identify the acoustical and technological characteristics being major determinants in the discrimination of the sounds and in the evaluation of the sound sources. 47 sounds were used for a perceptive test in laboratory. They were recorded in 22 vehicles by handtapping on different dashboard parts. In a same session, each subject performed 3 different tasks: categorisation (free sorting of sounds), description (free verbalisations) and evaluation (the score of a freely selected prototype was attributed to the whole group). Data analysis took advantage of the combination of various multidimensional analysis techniques, already used in psychoacoustics or sensory analysis. This low-cost methodology allowed us to describe a wide range of product samples with complementary psychological data. Results will help us to propose a sound metric which represent the evaluation of dashboard quality evoked by the sound. Furthermore, they give first hypotheses for the technological variables that could be decisive in the design process.

2pPPb3. Perceptual pitch compensation for low frequency bandwidth extension. Kim Seung (So. EECS/EE at Seoul National University, INMC, 132-302 Seoul National University, 599 Gwanangno, Gwanak-gu, 151-742 Seoul, Republic of Korea, bandwalk@acoustics.snu .ac.kr), Koeng-Mo Sung (Applied Acoustics Lab., INMC, 132-302, Seoul National University, San 56-1, Sillim-dong, Kwanak-gu, 151-742 Seoul, Republic of Korea, kmsung@snu.ac.kr)

As the size of speakers decreases with miniaturization of audio devices and thickness of TV, there is increased demand to ensure low-frequency sound quality. Psychoacoustics bandwidth extension for low-frequency has been applied to recent audio devices. A perceptual pitch does not correspond with fundamental frequency of missing fundamental, because the perceptual pitch is heard highly than desirable fundamental frequency. Consequently, the algorithm compensating perceptual pitch with difference is needed. This paper describes the algorithm compensating the difference using mel scale curve, and the listening test result of sound source applying the algorithm in comparison with sound source applying conventional algorithm.

2pPPb4. Towards an hearing threshold prediction model in car noise. Jean-François Sciabica (PSA Peugeot Citroën, Centre Technique de Vélizy, Route de Gisy, 78943 Vélizy-Villacoublay, France, jean-francois.sciabica @mpsa.com), Florent Richard (PSA Peugeot Citroën, Centre Technique de Vélizy, Route de Gisy, 78943 Vélizy-Villacoublay, France, florent.richard@mpsa.com), Vincent Roussarie (PSA Peugeot Citroën, Centre Technique de Vélizy, Route de Gisy, 78943 Vélizy-Villacoublay, France, vincent.roussarie@mpsa.com)

Sound design is an important challenge at PSA Research Center. Engine noise is rich in harmonics and interacts with aerodynamic and road noise. In this context, comprehension of masking phenomenon is indispensable to obtain a good description of sound attributes like roughness and booming. In this paper, we present a study about interaction between engine harmonics and road and aerodynamic noise. Thresholds for the detection of one harmonic in noise are firstly measured as a function of noise level. We propose also a prediction model for thresholds as a linear function of noise level in critical band. Results were compared to data available in the literature. Secondly, masking of harmonics by a harmonic and noise is measured. The 
masker frequency is $100 \mathrm{~Hz}$ and its level varies from 65 to $85 \mathrm{~dB}$ SPL in 5 $\mathrm{dB}$ steps. Noise level is $80 \mathrm{~dB}$ SPL. Masking patterns are traced for 8 frequencies (50 to $225 \mathrm{~Hz}$ ).

2pPPb5. The noise induced harmful effects assessment using psychoacoustical noise dosimeter. Jozef Kotus (Gdansk University of Technology, Multimedia Systems Department, 11/12 Gabriela Narutowicza Street, 80-952 Gdansk, Poland, joseph@ sound.eti.pg.gda.pl), Bozena Kostek (Gdansk University of Technology, Multimedia Systems Department, 11/12 Gabriela Narutowicza Street, 80-952 Gdansk, Poland, bozenka@sound.eti.pg.gda.pl), Andrzej Czyzewski (Gdansk University of Technology, Multimedia Systems Department, 11/12 Gabriela Narutowicza Street, 80-952 Gdansk, Poland, andcz@sound.eti.pg.gda.pl), Krzysztof Kochanek (The Institute of Physiology and Pathology of Hearing, Pstrowskiego 1, 01-943 Warsaw, Poland, k.kochanek@ifps.org.pl), Henryk Skarzynski (The Institute of Physiology and Pathology of Hearing, Pstrowskiego 1, 01-943 Warsaw, Poland, h.skarzynski@ifps.org.pl)

A new way of assessment of noise-induced harmful effects on human hearing system was presented in the paper. Employing the developed psychoacoustical noise dosimeter the new indicators of noise harmfulness were verified on the basis of hearing examinations and noise measurement results. The indicators were based on some psychoacoustical properties of the human hearing system and, at the same time, on evaluation of the time and frequency characteristics of noise. Additionally, time properties of the Temporary Threshold Shift are calculated during the noise exposure. The evaluation of the proposed indicators were conducted on the basis of hearing examinations in the real noise exposure situations and also on the basis of simulation results employing standard test signals (such as: white, pink and brown noise). The standard noise dose analysis results were also presented for the purpose of comparison. The performed analysis and obtained results confirmed correctness and practical usefulness of the proposed indicators.

2pPPb6. Subjective and objective evaluation of localisation accuracy in wave field sysnthesis. Joseph Sanson (IRCAM, 1 Place Igor Stravinsky, 75004 Paris, France, joseph.sanson@ircam.fr), Etienne Corteel (Sonic Emotion, Eichweg, 6, CH-8154 Oberglatt, Switzerland, etienne.corteel@sonicemotion.com), Olivier Warusfel (IRCAM, 1 Place Igor Stravinsky, 75004 Paris, France, Olivier.Warusfel@ircam.fr)

Wave Field Synthesis (WFS) is a holophonic technique that relies on the reproduction of physical properties of sound fields in an extended listening area. Limitations of this technique are due to approximations regarding the mathematical basis and have been studied in [Ber88] [dB04]. The synthesis remains correct up to a corner frequency referred to as the spatial aliasing frequency and inside an area limited by the length of the WFS array and the position of the virtual sources. A Multichannel Equalization filter (MEQ) is applied to the driving limentation signals of the loudspeakers, as described in [Cor06]. The aim of the present study is to evaluate localisation in WFS depending on both listening and source positions. An objective analysis of the binaural signals is compared to the results of a localisation task for which spatialised high-pass filtered white noises served as stimuli. The results of the psycho-acoustic test are compared to the binaural localisation cues, namely ITD (interaural time differences) and ILD (interaural leve differences). Above the aliasing frequency, ITD and ILD are conflicting and ILD errors account in most cases for the perceptual errors.

2pPPb7. Analysis of everyday sounds which are extremely annoying for children with autism. Örjan Johansson (Luleå University of Technology, Dept Human Work Sciences, Div of Sound and Vibration, SE-97187 Luleå, Sweden, orjan.johansson@ltu.se), David Lindegren (Luleå University of Technology, Dept Human Work Sciences, Div of Sound and Vibration, SE-97187 Luleå, Sweden, david.lindegren@gmail.com)

Extreme sensitivity to noise is a problem that almost all autistic children suffer from. A sound that is extremely annoying does not need to be loud. However, the characteristics and temporal variations of these sounds are sparsely investigated. The aim of this study is to increase the knowledge about these extremely annoying sounds so they can be avoided by better design criteria for classrooms and venues like that. By interviewing teachers and parents a number of everyday sounds were identified and binaurally recorded. Examples are vacuum cleaners, ventilation noise, washing machines and pouring water. Detailed psychoacoustic analyses of this type of sounds were achieved by a listening test procedure in three parts. First 16 children composed different types of vacuum cleaner sounds trying to minimize annoyance in two different tests, a) keeping original sound pressure level. b) adjusting to acceptable loudness. In the second part, teachers working with autistic children performed a listening test to evaluate some of the composed sounds from part 1 and modified versions of them. The third part was performed by children to validate the results. The results showed that Roughness, Loudness and an index defined as High frequency tonality were the most important characteristics.

2pPPb8. Application of psychoacoustic tests for the evaluation of acoustical characteristics of Bartók Béla Concert Hall in Palace of Arts, Budapest. Zsuzsanna Alabardos (St Stephan King Technical School of Music Arts, Columbus u.11, H-1143 Budapest, Hungary, alabardos.zsuzsanna@aratokft.hu), Eva Arato-Borsi (Arato Acoustics Ltd, Mezo u.7, H-1038 Budapest, Hungary, arato.eva@ aratokft.hu)

The objective characterisation of the subjective listening effect of single number quantities are well known in concert halls such: reverberation time, clarity, centre time, lateral energy fraction and so on. The Bartók Béla Concert Hall in the Palace of Arts in Budapest has been inaugurated at 15th of March 2005. The acoustic consultant office of the hall was ARTEC Ltd. A set of objective measurements has been carried out in the Concert Hall and a series of listening tests were accomplished. Results of the experiment show clear correlations in the relationship of architectural and room acoustical parameters. Apart from the important conclusions, there are some questions revealed.

2pPPb9. Parametric quality prediction for IP-based audio. Alexander Raake (Deutsche Telekom Laboratories, Berlin Institute of Technology, Ernst-Reuter-Platz 7, 10587 Berlin, Germany, alexander.raake @ telekom.de), Marieneige Garcia (Deutsche Telekom Laboratories, Berlin Institute of Technology, Ernst-Reuter-Platz 7, 10587 Berlin, Germany, marie-neige.garcia@telekom.de), Bernhard Feiten (T-Systems Enterprise Services GmbH, Goslarer Ufer 35, 13437 Berlin, Germany, bernhard.feiten@telekom.de), Sebastian Möller (Deutsche Telekom Laboratories, Berlin Institute of Technology, Ernst-Reuter-Platz 7, 10587 Berlin, Germany, sebastian.moeller@telekom.de)

Different multimedia services are more and more transmitted over a common network infrastructure, e.g. using the Internet Protocol (IP). Examples are the widespread voice over Internet Protocol (VoIP), and Internet Protocol Television (IPTV). The streaming of pure audio over IP even has a longer tradition, with applications such as internet radio. For an efficient development, planning and monitoring of such services, models can be used that predict user-perceived quality based on technical service characteristics. Speech quality models for telephony are among the most advanced ones in this context, with different model types like the signal-based PESQ (ITU-T Rec. P.862, 2001) or the parametric E-model (ITU-T Rec. G.107, 2005). In this paper, we describe a parametric approach for predicting the quality of IP-based audio. The main parameters are the audio codec, codec bitrate, packet loss characteristics and the audio content. We base our considerations on own listening tests conducted in the framework of IPTV quality assessment, on approaches and test data described in the literature and on complementary knowledge from the fields of speech and video quality models. In this context, we identify similarities and discrepancies between different types of services in the light of a common model framework. 


\title{
Session 2pPPc
}

\section{Psychological and Physiological Acoustics: Loudness, from Controlled Stimuli to Environmental Sounds I (Poster Session)}

\author{
Jeremy Marozeau, Cochair \\ Northeastern University \\ Sabine Meunier, Cochair \\ CNRS-LMA
}

\begin{abstract}
All posters will be on display from 3:40 p.m. to 5:20 p.m. To allow contributors an opportunity to see other posters, contributors of odd-numbered papers will be at their posters from 3:40 p.m. to 4:30 p.m. and contributors of even-numbered papers will be at their posters from 4:30 p.m. to 5:20 p.m.
\end{abstract}

\section{Contributed Paper}

2pPPc1. Effect of reverberation on loudness perception. Andrew Raimond (Reading University, Department of Psychology, RG6 6AL Reading, UK, a.raimond@ reading.ac.uk), Anthony J. Watkins (Reading University, Department of Psychology, RG6 6AL Reading, UK, syswatkn@rdg.ac .uk)

Stimuli with a slow-attack/fast-decay (S-F) envelope are judged to be louder than stimuli with a temporally-reversed, equal-energy version of the same envelope (fast-attack/slow-decay or F-S). It may be that the energy in the tails of F-S stimuli is discounted from loudness judgments because it is perceptually attributed to room reverberation. Here we examine the characteristics of real-room reverberation that affect loudness perception, particularly the way that reverberant tails tend to be de-correlated at the two ears. Our experiments used reverberation from real-room measurements of binaural room impulse responses (BRIRs). We ask if the perceived reduction in loudness of F-S stimuli relative to S-F stimuli is still apparent with dichotic sounds processed by the BRIRs. 


\title{
Session 2pPPd
}

\section{Psychological and Physiological Acoustics: Integrated Approaches to Auditory Scene Analysis II (Poster Session)}

\author{
Mounya Elhilali, Cochair \\ Johns Hopkins University \\ Daniel Pressnitzer, Cochair \\ CNRS UMR 8158; Univ. Paris Descartes; Ecole Normale Supérieure
}

\begin{abstract}
All posters will be on display from 3:40 p.m. to 5:20 p.m. To allow contributors an opportunity to see other posters, contributors of odd-numbered papers will be at their posters from 3:40 p.m. to 4:30 p.m. and contributors of even-numbered papers will be at their posters from 4:30 p.m. to 5:20 p.m.
\end{abstract}

\section{Contributed Papers}

2pPPd1. The ability of temporally integrating acoustic waveforms is associated with release of speech from informational masking under reverberant conditions. Liang Li (Dept. of Psychology, Peking Univ., 5 Yeheyuan Road, Haidian District, 100871 Beijing, China, liangli@pku.edu.cn), Ying Huang (Dept. of Psychology, Peking Univ., 5 Yeheyuan Road, Haidian District, 100871 Beijing, China, innhuang@gmail.com), Qiang Huang (Dept. of Machine Intelligence, Speech and Hearing Research Center, 2 Science Building, Peking Univ., 5 Yeheyuan Road, Haidian District, 100871 Beijing, China, QIANG.HUANG@SPREADTRUM.COM), Xun Chen (National Key Lab. on Machine Perception, Peking Univ., 5 Yiheyuan Road, 100871 Beijing, China, Xun.Chen@sonyericsson.com), Xihong H. Wu (Dept. of Machine Intelligence, Speech and Hearing Research Center, 2 Science Building, Peking Univ., 5 Yeheyuan Road, Haidian District, 100871 Beijing, China, wxh @ cis.pku.edu.cn)

Perceptual integration of the sound wave directly emanating from the source with reflections of the source needs both bridging temporal gaps and calculating correlations between sound waves. In this study, we examined whether the temporal integration of source/reflection signals is frequency dependent and associated with speech unmasking under simulated reverberant conditions. In Experiment 1, a break in correlation (BIC) between correlated wideband or narrowband noises at the two ears was detectable even when an interaural interval (IAI) was introduced. The longest IAI varied markedly across listeners. In wideband noise, it could be up to $21 \mathrm{~ms}$; in narrowband noise, it decreased as the center frequency was increased. In Experiment 2 , when the interval between target speech and its single-reflection simulation (inter-target interval, ITI) was reduced from 64 to $0 \mathrm{~ms}$, intelligibility of target speech was markedly improved under the speech-masking condition but not the noise-masking condition. The longest effective ITI under the speech-masking condition significantly correlated with the longest IAI for detecting the BIC in low-frequency $(<$ or $=400 \mathrm{~Hz})$ narrowband noises. Thus the frequency-dependent temporal integration of soundwaveform signals is critical for releasing speech from informational masking in reverberant environments. Supported by the National Natural Science Foundation of China.

2pPPd2. A computational model for auditory scene analysis. Maria E. Niessen (University of Groningen, Dept. Artificial Intelligence, P.O. Box 407, 9700 AK Groningen, Netherlands, m.niessen@ai.rug.nl), Ronald A. Van Elburg (University of Groningen, Dept. Artificial Intelligence, P.O. Box 407, 9700 AK Groningen, Netherlands, r.van.elburg@ai.rug.nl), Dirkjan J. Krijnders (University of Groningen, Dept. Artificial Intelligence, P.O. Box
407, 9700 AK Groningen, Netherlands, j.d.krijnders@ai.rug.nl),Tjeerd C. Andringa (University of Groningen, Dept. Artificial Intelligence, P.O. Box 407, 9700 AK Groningen, Netherlands, t.andringa@ai.rug.nl)

Primitive auditory scene analysis (ASA) is based on intrinsic properties of the auditory environment. Acoustic features such as continuity and proximity in time or frequency cause perceptual grouping of acoustic elements. Various grouping attributes have been translated into successful signal processing techniques that may be used in source separation. A next step beyond primitive ASA is source identification through schema-based ASA. We present a computational model for ASA that is inspired by models from cognitive research. It dynamically builds a hierarchical network of hypotheses, which is based on (learned) knowledge of the sources. Each hypothesis in the network, initiated by bottom-up evidence, represents a possible sound event. The network is updated for each new input event, which may be any sound in an unconstrained environment. The analysis of new input events is guided by knowledge of the environment and previous events. As a result of this adaptive behavior, information about the environment increases and the set of possible hypotheses decreases. With this method of continuously improving sound event identification we make a promising advance in computational ASA of complex real-world environments.

2pPPd3. Pitch-based streaming of vowel sequences, speech-in-speech segregation, and frequency selectivity. Etienne Gaudrain (Laboratoire Neurosciences Sensorielles, Comportement, Cognition, UMR 5020, Université Lyon 1 - CNRS, 50 av. Tony Garnier, 69366 Lyon Cedex 07, France, egaudrain@gmail.com), Nicolas Grimault (Laboratoire Neurosciences Sensorielles, Comportement, Cognition, UMR 5020, Université Lyon 1 CNRS, 50 av. Tony Garnier, 69366 Lyon Cedex 07, France, ngrimault@olfac.univ-lyon1.fr), Eric W. Healy (University of South Carolina, Speech Psychoacoustics Laboratory, Department of Communication Sciences and Disorders, William Brice Bldg., 1621 Greene St., Columbia, SC 29208, USA, ewh@sc.edu), Jean-Christophe Béra (INSERM U556, 151 cours Albert Thomas, 69424 Lyon Cedex 03, France, jean-christophe .bera@ec-lyon.fr)

Simultaneous and sequential segregation are described as the base mechanisms for auditory scene analysis and are likely to be involved in concurrent speech segregation. However, speech-in-noise perception has been found to be uncorrelated to simultaneous segregation, but related to puretone fusion threshold. This study aimed to clarify the relationship between pitch-based speech-in-speech segregation, pitch-based streaming and frequency selectivity. Twenty-five listeners with close to normal hearing were involved. Auditory filter widths were derived from a notched-noise method. Speech-in-speech perception was measured using words presented in a time reversed single talker background, with various pitch differences between 
target and masker. The streaming performance was measured using an objective order-naming task on vowel sequences. The results showed a correlation between frequency selectivity and performance in speech-in-speech perception suggesting that intelligibility relies on simultaneous masking. A correlation was also found between the effect of pitch on speech-in-speech perception and the effect of pitch on streaming performance. However, no correlation was found between streaming and frequency selectivity. These latter results suggest that pitch-based segregation probably relies on pitch discrimination which is only weakly correlated to frequency selectivity.

2pPPd4. Speech- synchronized visual cues release speech from informational masking. Mengyuan Y. Wang (Dept. of Psychology, Peking Univ., 5 Yeheyuan Road, Haidian District, 100871 Beijing, China, motoluto@163.com), Jingyu Y. Li (Dept. of Machine Intelligence, Speech and Hearing Research Center, 2 Science Building, Peking Univ., 5 Yeheyuan Road, Haidian District, 100871 Beijing, China, lijy@cis.pku.edu.cn), Ying Huang (Dept. of Psychology, Peking Univ., 5 Yeheyuan Road, Haidian District, 100871 Beijing, China, innhuang@gmail.com), Yanhong Wu (Dept. of Psychology, Peking Univ., 5 Yeheyuan Road, Haidian District, 100871 Beijing, China, wuyh@pku.edu.cn), Xihong H. Wu (Dept. of Machine Intelligence, Speech and Hearing Research Center, 2 Science Building, Peking Univ., 5 Yeheyuan Road, Haidian District, 100871 Beijing, China, wxh@cis.pku.edu.cn), Liang Li (Dept. of Psychology, Peking Univ., 5 Yeheyuan Road, Haidian District, 100871 Beijing, China, liangli@pku.edu.cn)

Visual speech information, such as lipreading cues, can assist listeners to segregate a target voice from competing voices (Helfer and Freyman, 2005). However, because signals contained in lipreading are multidimensional, it is not clear whether a simple visual cue, such as the light flash that is synchronous to the onset of each syllable in target speech, is sufficient to release target speech from noise or speech masking. In this study, when target speech was of a constant rate, the speech-synchronized light flash had no effects on speech recognition under either speech or noise masking condition. However, when the rate of target speech was artificially manipulated unstable or an intense noise burst occurred in the middle of the target sentence, the speech-synchronized light flash improved speech recognition when the two-talker speech masker but not the speech-spectrum noise masker was co-presented. These data suggest that only when the rate of target speech cannot be predicted and the masker is speech, speechsynchronized visual cues play a role in helping listeners attend to the target voice and follow the stream of target speech, leading to a release of target speech from informational masking. Supported by the National Natural Science Foundation of China.

2pPPd5. Phonemic restoration with hearing-impaired listeners. Deniz Baskent (Starkey Hearing Research Center, 2150 Shattuck Ave, Suite 408, Berkeley, CA 94704, USA, deniz_baskent@starkey.com), Cheryl Eiler (Starkey Hearing Research Center, 2150 Shattuck Ave, Suite 408, Berkeley, CA 94704, USA, cheryl_eiler@starkey.com), Brent Edwards (Starkey Hearing Research Center, 2150 Shattuck Ave, Suite 408, Berkeley, CA 94704, USA, brent_edwards@starkey.com)

Baskent et al. (ISAAR 2007) showed that simulated undershoot that may happen due to release from hearing-aid compression may reduce phonemic restoration by normal-hearing $(\mathrm{NH})$ listeners. Phonemic restoration was measured as the increase in recognition of interrupted speech when the silent intervals were filled with loud noise bursts. These results would be more crucial for hearing-impaired (HI) listeners who might encounter such problems in real life as hearing-aid users. However, it has not been previously shown if HI listeners benefit from phonemic restoration similar to $\mathrm{NH}$ listeners. Factors such as adverse effects of background noise on speech perception and increased forward masking observed with HI listeners might affect phonemic restoration. The present study explored phonemic restoration with mildly and moderately HI listeners with a method similar to the previous study. NH listeners participated as the control group. Perception of speech interrupted at rates of 1.5 and $2.2 \mathrm{~Hz}$ was measured with silent intervals and with noise bursts filling the silent intervals. The preliminary re- sults showed that many HI listeners, especially with mild hearing loss, were able to benefit from phonemic restoration. However, in each subject group, there were a small number of listeners who did not show any benefit.

2pPPd6. Effect of lip movement cues on auditory streaming of concurrent speech. Aymeric Devergie (Université Lyon 1, UMR 5020 CNRS, 50 av. T. Garnier, 69366 Lyon cedex 07, France, aymeric.devergie@olfac.univ-lyon1.fr), Nicolas_Grimault (Laboratoire Neurosciences Sensorielles, Comportement, Cognition, UMR 5020, Université Lyon 1 - CNRS, 50 av. Tony Garnier, 69366 Lyon Cedex 07, France, ngrimault@olfac.univ-lyon1.fr), Frédéric Berthommier (Gipsa-lab UMR 5216, 46 avenue Félix Viallet - INPG, F - 38031 Grenoble cedex, France, frederic.berthommier@gipsa-lab.inpg.fr), Etienne Gaudrain (Laboratoire Neurosciences Sensorielles, Comportement, Cognition, UMR 5020, Université Lyon 1 - CNRS, 50 av. Tony Garnier, 69366 Lyon Cedex 07, France, egaudrain@gmail.com), Eric W. Healy (University of South Carolina, Speech Psychoacoustics Laboratory, Department of Communication Sciences and Disorders, William Brice Bldg., 1621 Greene St., Columbia, SC 29208, USA, ewh@sc.edu)

Lip reading is known to induce audio-visual integration processes that enhance speech-in-speech intelligibility. Recent work in auditory scene analysis [see Gaudrain et al., Acoustic'08] found correlations between sequential segregation performance and intelligibility of speech-in-speech. Our working hypothesis is that an audiovisual binding process is involved in speech understanding in multi-talker situations. However, the potential effect of audio-visual integration on auditory stream segregation remains unclear. A single previous study [Gaudrain et al., J. Acoust. Soc. Am. 122, 3065 (2007)] found that lip movements congruent with the presentation of vowels may enhance segregation. In this paradigm, audio-visual sequences of vowels with alternating low and high fundamental frequencies have been generated, in which the lip movements are paired with the second ones. The current experiment is built to test further the potential effect of visual cues on segregation. As in the previous study, lips movements were either steady (control condition), open-closed (rhythm condition) or congruent with each particular vowel. Moreover, a congruent but asynchronous audio-visual condition has been introduced as a new control. This is expected to disrupt the audiovisual binding process. Results are interpreted as an evaluation of the contribution of visual cues in Cocktail Party situations.

2pPPd7. Modelling the temporal response of the auditory nerve to the pitch of complex tones in reverberation. Lowel P. O'Mard (Centre for the Neural Basis of Hearing, The Physiological Laboratory, Downing Street, CB2 3EG Cambridge, UK, lpo21@cam.ac.uk), Mark Sayles (Centre for the Neural Basis of Hearing, The Physiological Laboratory, Downing Street, CB2 3EG Cambridge, UK, ms417@ cam.ac.uk), Ian M. Winter (Centre for the Neural Basis of Hearing, The Physiological Laboratory, Downing Street, CB2 3EG Cambridge, UK, imw1001@cam.ac.uk)

A computational model of the auditory nerve has been used to investigate the effect of reverberation on the response to the fundamental frequency (F0) of frequency-swept harmonic complexes. The model used a computationally efficient dual resonance non-linear (DRNL) model of basilar membrane frequency selectivity. The DRNL architecture comprises two parallel filter paths providing linear and non-linear responses. The model successfully reproduced the results of a physiological investigation using the same stimuli in a population of single units from the ventral cochlear nucleus (VCN). In particular the effect of reverberation was dependent upon unit best frequency, F0 and source-to-receiver distance. The model results suggest that the effects of reverberation observed at the level of the VCN are already present in the auditory periphery. The use of the model has also enabled us to examine the effect of hearing impairment by the removal of the non-linear path response. This simulation of a sensorineural hearing loss accentuated the loss of the temporal representation of F0 in the presence of reverberation 
2pPPd8. Blind source separation and sound source localization on time-frequency domain considering time lag information. Shogo Ueda (Department of Architecture, Tokyo University of Science, 1-3 Kagurazaka, Shinjuku-ku, 162-8601 Tokyo, Japan, shogo_0604@yahoo .co.jp), Fumio Sasaki (Department of Architecture, Tokyo University of Science, 1-3 Kagurazaka, Shinjuku-ku, 162-8601 Tokyo, Japan, fsasaki@rs.kagu.tus.ac.jp), Osamu Tanaka (Department of Architecture, Tokyo University of Science, 1-3 Kagurazaka, Shinjuku-ku, 162-8601 Tokyo, Japan, otanaka@rs.kagu.tus.ac.jp), Masahito Yasuoka (Department of Architecture, Tokyo University of Science, 1-3 Kagurazaka, Shinjuku-ku, 162-8601 Tokyo, Japan, shogoarc@ybb.ne.jp)

The blind source separation and sound source localization based on independent component analysis on time-frequency domain considering time lag information between source signals and observation signals are conducted. The formulation based on the independency of time-frequency domain and the linearity of source signal is presented. The method which can be conducted not only the separation of source signals but also the specification of location of source signals is proposed through the consideration of time lags. Using this method, it can be analyzed even if observation signals include an intermittent noise, under the assumption of some independency of source signals. First of all, the number of source signals is specified through the quotient of complex valued time-frequency information of two observation signals. Next, the locations of source signals are specified using the relationship of relative time lags between source signal and observation signal. Then, the source signals are obtained by use of the Fourier information. The numerical test is conducted to confirm our method, and then the locations of source signals and source signals are obtained by high accuracy.

TUESDAY AFTERNOON, 1 JULY 2008

P2-C, LEVEL 2, 3:40 TO 5:20 P.M.

\title{
Session 2pPPe
}

\section{Psychological and Physiological Acoustics: Cochlear Implants: Going Beyond the Envelope II (Poster Session)}

\author{
Bernhard Seeber, Cochair \\ MRC Institute of Hearing Research \\ H Steven Colburn, Cochair \\ Boston University
}

\begin{abstract}
All posters will be on display from 3:40 p.m. to 5:20 p.m. To allow contributors an opportunity to see other posters, contributors of odd-numbered papers will be at their posters from 3:40 p.m. to 4:30 p.m. and contributors of even-numbered papers will be at their posters from 4:30 p.m. to 5:20 p.m.
\end{abstract}

\section{Contributed Papers}

2pPPe1. Perception of speech and environmental sounds in cochlear implant patients. Valeriy Shafiro (Rush University Medical Center, Dept. Communication Disorders, 600 S. Paulina Str., 1015 AAC, Chicago, IL 60612, USA, valeriy_shafiro@ rush.edu), Brian Gygi (East Bay Institute for Research and Education, 150 Muir Road 151-I, Martinez, CA 94552, USA, bgygi@ebire.org), Min-Yu Cheng (Rush University Medical Center, Dept. Communication Disorders, 600 S. Paulina Str., 1015 AAC, Chicago, IL 60612, USA, min-yu_cheng@rush.edu), Megan Mulvey (Rush University Medical Center, Dept. Communication Disorders, 600 S. Paulina Str., 1015 AAC, Chicago, IL 60612, USA, megan_mulvey@rush.edu), Blythe Holmes (Rush University Medical Center, Dept. Communication Disorders, 600 S. Paulina Str., 1015 AAC, Chicago, IL 60612, USA, blythe_holmes @ rush.edu)

Limited research has investigated cochlear implant (CI) patients' ability to perceive environmental sounds. In this study, environmental sound perception was assessed with a large-item test composed of 40 sound sources, each represented by four different tokens. The relationship between speech and environmental sound perception and the role of working memory and basic auditory abilities was examined based on patient performance on a battery of speech tests (HINT, CNC, and individual consonant and vowel tests), tests of basic auditory abilities (audiometric thresholds, gap detection, temporal pattern and temporal order tests), and a backward digit recall test. Twelve postlingually deaf adult CI patients, having 1-5 years of implant experience participated. The results indicate substantially reduced ability to identify common environmental sounds in CI patients. Speech test scores correlated strongly with the scores on the environmental sound test. Both speech and environmental sound tests moderately correlated with gap detection, temporal order test and backward digit recall test. However, the correlation between speech and environmental sounds changed little after partial- ling out the variance due to other variables. These results suggest that speech and environmental sounds may overlap considerably in their perceptual processing, being largely independent of peripheral limitations that may affect both sound classes.

2pPPe2. Intelligibility of interrupted speech in normal-hearing listeners and cochlear implantees. Dan Gnansia (Univ Paris Descartes, CNRS, Ecole Normale Superieure, DEC, 29 rue d'Ulm, 75005 Paris, France, dan.gnansia@ens.fr), Vincent Pean (MXM / Neurelec, 2729 Chemin St Bernard, 06224 Vallauris Cedex, France, vpean@neurelec .com), Christian Lorenzi (Univ Paris Descartes, CNRS, Ecole Normale Superieure, DEC, 29 rue d'Ulm, 75005 Paris, France, lorenzi@ens.fr)

The intelligibility of a target speech signal is substantially degraded in the presence of a competing talker in cochlear implantees. The current study aimed to test whether this degradation is caused by an inability to i) make use of the partial target speech information glimpsed into the competing talker "valleys", and ii) fuse perceptually those successive glimpses into coherent speech streams. This hypothesis was tested by assessing the intelligibility of periodically interrupted vowel-consonant-vowel (VCV) signals pronounced by a male $(\mathrm{F} 0=113 \mathrm{~Hz})$ and female $(\mathrm{F} 0=216 \mathrm{~Hz})$ speaker in normal-hearing listeners and implantees. A 4-Hz square-wave modulator with random phase was used to interrupt periodically each signal. The interrupted VCVs from each speaker were either presented alone (Experiment 1) or interleaved (Experiment 2: the two speakers were alternated). In experiment 1 , the mean identification score calculated for each voice was about $90 \%$ in normal-hearing listeners, and $40 \%$ in implantees. In experiment 2, the mean identification score corresponding to correct identification of both voices was about $50 \%$ in normal-hearing listeners, but at chance 
level in implantees. Taken together, these data suggest that implantees can make use of partial speech information, but cannot organize this partial speech information into coherent streams.

2pPPe3. Electrode Discrimination and the effect on Speech Perception for Adults with Cochlear Implants. Debi A. Vickers (UCL Ear Institute, 332-336 Gray's Inn Road, WC1X 8EE London, UK, d.vickers@ucl.ac.uk), Leah Meerton (UCL Cochlear Implant Team, 332336 Gray's Inn Road, WC1X 8EE London, UK, leah.meerton@royalfree .nhs.uk)

Zwolan et al (1997) showed that electrode discrimination can have an effect on speech perception for adult cochlear implant users with the Nucleus Mini 22 multi-channel device. The purpose of this study was to evaluate electrode discrimination in subjects using different implant systems and speech processing strategies to observe the effect that switching off undiscriminable electrodes has on speech perception. 17 postlingually deafened adults were tested; they used either a Nucleus 24 device (either using SPEAK or ACE processing strategies) or an Advanced Bionics HiRes cochlear implant (either using HiRes or HiRes120 strategies). Only electrodes that were activated in the subjects' clinical maps were tested in the electrode discrimination task. Electrode discrimination was performed using standard clinical software at comfortable listening level and pairs of electrodes that scored less than $60 \%$ were deemed undiscriminable. One of the undiscriminable pair was switched off and the electrode selected was determined from the results of the other electrode pairs. Performance was evaluated prior to and immediately after switch off and at a follow up appointment 1 month later. Zwolan T, Collins L and Wakefield G (1997). J. Acoust. Soc. Am. 102 (6), 3673-3685

TUESDAY AFTERNOON, 1 JULY 2008

P2-C, LEVEL 2, 3:40 TO 5:20 P.M.

\title{
Session 2pPPf
}

\section{Psychological and Physiological Acoustics: Auditory Perception and Signal Processing by Prostheses II (Poster Session)}

\author{
Huanping Dai, Cochair \\ University of Arizona \\ Birger Kollmeier, Cochair \\ Universität Oldenburg, Medizinische Physik
}

\begin{abstract}
All posters will be on display from 3:40 p.m. to 5:20 p.m. To allow contributors an opportunity to see other posters, contributors of odd-numbered papers will be at their posters from 3:40 p.m. to 4:30 p.m. and contributors of even-numbered papers will be at their posters from 4:30 p.m. to 5:20 p.m.
\end{abstract}

\section{Contributed Papers}

2pPPf1. A model for predicting hearing aid benefit. Michael Nilsson (Sonic Innovations, 2795 East Cottonwood Parkway, Suite 660, Salt Lake City, UT 84121, USA, mnilsson@sonici.com), Victor Bray (Sonic Innovations, 2795 East Cottonwood Parkway, Suite 660, Salt Lake City, UT 84121, USA, vbray@ sonici.com)

A 'large n' database was created incorporating demographic information as well as speech-recognition-in-noise measurements from several hundred subjects who participated in field trials with wearable DSP hearing aids. Performance measures were obtained across multiple hearing aid styles (CIC to BTE) with multiple signal processing technologies (multi-channel compression, digital noise reduction, directionality). The database was previously analyzed using various regression techniques (results presented at AAS2005, IHCON-2006, AAS-2007). The current analysis includes additional parameters that were not in the earlier models and uses a more robust statistical analysis. Aided speech-understanding-in-noise can be predicted based on factors of (a) unaided thresholds for tones (b) unaided thresholds for speech in quiet, (c) unaided thresholds for speech in noise, (d) hearing aid technology, and (e) subject age. We conclude that a model for hearing aid benefit can be used as a new tool for evidence-based management of hearing aid clients. Patient parameters may be entered into the model to serve as a basis for prediction of benefit in speech-understanding-in-noise using various forms of amplification treatment.
2pPPf2. Temporal manipulations in multi-channel compression effecting sound quality and performance. Michael Nilsson (Sonic Innovations, 2795 East Cottonwood Parkway, Suite 660, Salt Lake City, UT 84121, USA, mnilsson@sonici.com), Michelle Hicks (Sonic Innovations, 2795 East Cottonwood Parkway, Suite 660, Salt Lake City, UT 84121, USA, mhicks@sonici.com), Robert Ghent (Sonic Innovations, 2795 East Cottonwood Parkway, Suite 660, Salt Lake City, UT 84121, USA, bghent @ sonici.com), Victor Bray (Sonic Innovations, 2795 East Cottonwood Parkway, Suite 660, Salt Lake City, UT 84121, USA, vbray@sonici.com)

Multi-channel compression (in four or more compression channels) is expected in modern hearing aids, but compression temporal characteristics vary without an understanding of what is optimal and why. This study takes a 16 channel system using linearly spaced compressors with minimal overlap and evaluates sound quality ratings and speech recognition performance with the HINT in quiet and noise with various attack and release settings in a $2 \times 2 \times 2$ design. The first variable is the relationship between attack and release times, with asymmetric time constants (attack faster than release) or symmetric time constants (attack and release set the same). The second variable is the relationship of time constants across frequency channels, with uniform settings across frequencies, or frequency dependent time constants (faster settings for higher frequencies with shorter wavelengths). The third variable is absolute speed, with fast or slow time constants. No characteristics other than the temporal settings of the compressor were changed between conditions. Results will be presented showing optimal settings for 
sound quality as well as speech recognition. The interaction between compression characteristics and additional signal processing, such as singlemicrophone noise reduction, will be discussed.

2pPPf3. Speech Intelligibility in Virtual Environments Simulating an Asymmetric Directional Microphone Configuration. Lorenzo Picinali (De Montfort University, Music, Technology and Innovation Research Centre, LE1 9BH Leicester, UK, lorenzo@limsi.fr), Silvano Prosser (Dipartimento di Audiologia, Università degli Studi di Ferrara, Corso Giovecca 203, 44100 Ferrara, Italy, prssvn@unife.it), Antonio Mancuso (DICO-LIM, Università degli Studi di Milano, Via Comelico, 39, 20135 Milano, Italy, knmbm@tin.it), Giancarlo Vercellesi (DICO-LIM, Università degli Studi di Milano, Via Comelico, 39, 20135 Milano, Italy, giancarlo.vercellesi@dico.unimi.it)

The benefit of directional processing and binaural listening in terms of speech intelligibility for frontal sound sources has been well documented in recent and past studies. Nevertheless, only the $20 \%$ of the situations in real life present a speaker located exactly in the frontal position. Different attempt have been done so far in order to allow, manually or automatically, a change in the directional pattern of the hearing aid, but the results of these studies are far from being satisfying. The purpose of this study is to explore the advantages brought by the asymmetric directional microphone configuration of hearing aids in terms of speech intelligibility for frontal, lateral and rear sound sources in noisy environments, and in terms of the quality of the "spatial perception" of the surrounding sound-scape. Through a 3D Ambisonic virtual environment manipulation, the presence of two microphones (the two hearing aids) is simulated in a noisy environment with a speech sound source. The listeners are presented with the signal recorded from the two simulated microphones, calibrated with symmetrical and asymmetrical directional patterns, and played through a pair of headphones. The speech intelligibility is measured for all the directional microphones configurations and for all the speech sound source positions.

2pPPf4. Simulation of an electro-acoustic implant (EAS) with a hybrid vocoder. Fabien Seldran (Univ. Lyon 1 - Lab. Neurosciences, Service Pr Collet, Pavillon U, Hôpital Edouard Herriot, F-69003 Lyon, France, fseldran@yahoo.fr), Eric Truy (Hôpital Edouard Herriot, Service ORL, Place d'Arsonval, 69003 Lyon, France, eric.truy@chu-lyon.fr), Stéphane Gallégo (Univ. Lyon 1 - Lab. Neurosciences, Service Pr Collet, Pavillon U, Hôpital Edouard Herriot, F-69003 Lyon, France, iakhoun@olfac.univ-lyon1.fr), Christian Berger-Vachon (Univ. Lyon 1 Lab. Neurosciences, Service Pr Collet, Pavillon U, Hôpital Edouard Herriot, F-69003 Lyon, France, iakhoun@olfac.univ-lyon1.fr), Lionel Collet (Univ. Lyon 1 - Lab. Neurosciences, Service Pr Collet, Pavillon U, Hôpital Edouard Herriot, F-69003 Lyon, France, iakhoun@olfac.univ-lyon1.fr), Hung Thai-Van (Univ. Lyon 1 - Lab. Neurosciences, Service Pr Collet, Pavillon U, Hôpital Edouard Herriot, F-69003 Lyon, France, iakhoun@olfac.univlyon1.fr)

Electroacoustic stimulation (EAS) is indicated for hearing impaired patients with enough residual hearing in low frequencies and severe hearing loss in high frequencies. We aimed at simulating the speech intelligibility provided by EAS with a hybrid vocoder model. The French Fournier word set was used in this study. We therefore tested several parameters on 24 normal hearing adults. First, the boundary between acoustic and electric stimulation frequency areas $(\mathrm{Fc})$ was taken at 500, 707, 1000 and $1414 \mathrm{~Hz}$. Second, we assessed the effect of electrical stimulation channel numbers (1 to 4). Third, we tested the effect of background noise with a cocktail party noise at $-6,0$ and $+6 \mathrm{~dB}$ SNR. It appeared that the 3 electrical channels \& $707 \mathrm{~Hz} \mathrm{Fc}$ condition produced normal-hearing-like results (at least in quiet). In noisy auditory scene, 4 electrical channels \& $500 \mathrm{~Hz} \mathrm{Fc}$ could produce fair speech intelligibility. [Work supported by CNRS, Lyon1 University and Medel].

2pPPf5. Cartilage conduction hearing aid for the patient with atresia auris. Takefumi Sakaguchi (Nara Medical University, 840 Shijo-cho, 634$8522 \quad$ Kashihara, Japan, t-saka@naramed-u.ac.jp), Osamu Saito (Department of Otorhinolaryngology, Nara Medical University, 840 Shijo-cho, 634-8522 Kashihara, Japan, osam0502@yahoo.co.jp), Hiroshi Hosoi (Nara Medical University, 840 Shijo-cho, 634-8522 Kashihara, Japan, hosoi@naramed-u.ac.jp)

Although bone conduction hearing aid is considered to be better to compensate the hearing loss of the patients who have atresia auris, some of these patients tend to wear air conduction hearing aid because of the feeling of tightness when wearing bone conduction haring aid. We expected cartilage conduction would reduce feeling of tightness compared to bone conduction, and improve compensation compared to air conduction. In this study, we report the result of the basic studies performed to evaluate the usefulness of cartilage conduction hearing aid. Patient who has atresia auris participated in the hearing test. Stimuli were presented to the patient by means of two types of transmitter. One was piezoelectric transducer placed on antilobium, another one was insertion earphone. We found that the hearing threshold of the patient improved about $25 \mathrm{~dB}$ or greater when the transducer was located on antilobium compared to the result when insertion earphone was used. These results suggest that the cartilage conduction hearing aid can be an option of the hearing aid for the patient who has atresia auris which is better than air conduction hearing aid in compensating their hearing, and more comfortable to wear compared to the bone conduction hearing aid.

2pPPf6. Verifying the attenuation of earplugs in situ: variability of transfer functions among human subjects. Annelies Bockstael (Ghent University, De Pintelaan 185 2P1, 9000 Gent, Belgium, annelies.bockstael@ugent.be), Dick Botteldooren (University Ghent - Department Information Technology, Sint-Pietersnieuwstraat 41, 9000 Gent, Belgium, dick.botteldooren@intec.ugent.be), Bart Vinck (Ghent University, De Pintelaan 185 2P1, 9000 Gent, Belgium, bart.vinck@ugent.be)

The use of in situ measurements of hearing protectors' (HPD's) attenuation following the MIRE-protocol (Microphone In Real Ear) is increasing. The attenuation is hereby calculated from the difference in sound levels outside the ear and inside the ear canal behind the HPD. Custom-made earplugs have been designed with an inner bore that allows inserting a miniature microphone. A thorough understanding of the difference, henceforth called 'transfer function', between the sound pressure of interest at the eardrum and the one measured at the inner bore of the HPD is indispensable for optimizing this technique and extending its field of application. Of particular interest is the variation of these transfer functions among humans. This was checked experimentally on 19 subjects. Differences in sound pressure were measured at the HPD's inner bore, by the MIRE-microphone, and at the eardrum by inserting an extra tube microphone in the ear canal. All transfer functions showed a comparable shape, however variability was substantial for the exact frequency and amplitude of the resonance peaks. The link between this variability and the morphology of the individual's HPD and ear canal was addressed using FDTD-simulations (Finite-Difference TimeDomain) of the outer ear canal occluded by an earplug with inner bore. 


\title{
Session 2pPPg
}

\section{Psychological and Physiological Acoustics: Loudness, from Controlled Stimuli to Environmental Sounds II}

\author{
Jeremy Marozeau, Cochair \\ Northeastern University, 360 Huntington Ave - 106A Forsyth Building - SLPA, Institute for Hearing, Speech \& Language, \\ Boston, MA 02115, USA \\ Sabine Meunier, Cochair \\ CNRS-LMA, 31, chemin Joseph Aiguier, Marseille, 13402, France
}

\section{Invited Papers}

\author{
$5: 20$
}

2pPPg1. Does induced loudness reduction explain contextual effects in loudness judgment? Lawrence E. Marks (John B. Pierce Laboratory, 290 Congress Avenue, New Haven, CT 06519, USA, MARKS@JBPIERCE.ORG)

Briefly presenting an inducing tone of 70-80 dB can substantially reduce the loudness of a subsequent test tone at or near the inducer's frequency, a phenomenon called Induced Loudness Reduction (ILR). The study of ILR emerged from earlier observations on differential contextual effects in loudness judgment: Tones of a given SPL and frequency were judged softer when presented as part of an ensemble of high rather than low SPL tones at the same frequency, relative to judgments of loudness of tones at a different sound frequency. At first, these effects of stimulus context on loudness judgment were assumed to reflect decisional processes, that is, to reflect biases in loudness judgment. On the other hand, ILR is often assumed to reflect a depression in the intensity response of the auditory system. While it is tempting to explain differential contextual effects in loudness judgment wholly in terms of ILR, the properties of ILR and the properties of contextual effects may not be identical, leaving open a possible role for decisional processes as well as sensory processes in contextual effects, and perhaps also in ILR, in both laboratory and 'real-world' settings.

\section{$5: 40$}

2pPPg2. The effects of induced loudness reduction on tone-burst otoacoustic emissions. Michael Epstein (Northeastern University, 360 Huntington Ave - 106A Forsyth Building - SLPA, Institute for Hearing, Speech \& Language, Boston, MA 02115, USA, m.epstein@neu.edu), Jeremy Marozeau (Northeastern University, 360 Huntington Ave - 106A Forsyth Building - SLPA, Institute for Hearing, Speech \& Language, Boston, MA 02115, USA, marozeau@gmail.com)

Induced loudness reduction (ILR) is a phenomenon by which the loudness of a sound is reduced when it is preceded by a higherlevel sound (inducer). Because the effects of ILR can last for at least several minutes after exposure to an inducer, many clinical and laboratory test procedures may be unintentionally confounded. However, little is known about the physiologic manifestation of ILR or how it might affect clinical tests. The present study examined whether ILR could be observed in otoacoustic emission measurements, which have been shown to correlate well with loudness. Otoacoustic emissions were measured monaurally in 12 normal-hearing listeners in response to a $1 \mathrm{kHz} 70 \mathrm{~dB}$ SPL tone-burst stimulus before and after a series of $1 \mathrm{kHz} 90 \mathrm{~dB}$ SPL tone-burst inducers. Additionally, otoacoustic emissions were measured in two control conditions in which ILR was not expected to occur. For most listeners, the level of otoacoustic emissions decreased after inducer exposure and did not decrease in the control conditions. The results indicate that at least some component of ILR is likely to arise as a cochlear process and that although the observed effects are small, ILR may affect measures beyond just loudness judgments.

6:00

2pPPg3. Modelling categorical loudness perception for arbitrary listeners and sounds. Birger Kollmeier (Universität Oldenburg, Medizinische Physik, Carl-von-Ossietzky Str. 9-11, 26111 Oldenburg, Germany, birger.kollmeier@uni-oldenburg.de), Jens E. Appell (Universität Oldenburg, Medizinische Physik, Carl-von-Ossietzky Str. 9-11, 26111 Oldenburg, Germany, jens.appell@offis.de), Jesko Verhey (Universität Oldenburg, Medizinische Physik, Carl-von-Ossietzky Str. 9-11, 26111 Oldenburg, Germany, jesko.verhey@uni-oldenburg.de), Volker Hohmann (Carl von Ossietzky Universität Oldenburg, Ammerländer Heerstraße 114-118, 26111 Oldenburg, Germany, volker.hohmann@uni-oldenburg.de)

While "classical" loudness models predict loudness in sone using the concepts of Stevens' compressive power law, (subdivided) categorical loudness perception after Heller follows the compressive logarithmic Weber-Fechner law. To bridge the gap between both approaches, this contribution reviews various steps towards a loudness model that predicts categorical loudness (in categorical units, $\mathrm{CU}$ ) for normal and hearing-impaired listeners for arbitrary sounds. It uses a (modified) classical loudness model for stationary signals to derive the loudness in sone and a nonlinear transformation from sone to CU. This transformation is approximated by a cubic polynomial equation wich is derived from categorical loudness data of 84 normal-hearing subjects. The model parameters are further set to predict the standard isophones that are in good agreement with the equal loudness level contours derived from categorical loudness data. Also, the model predicts the loudness functions near threshold both for normal and hearing-impaired listeners and can be extended to predict duration-dependent loudness perception. Since categorical loudness can be measured more easily and directly than loudness in sone, the current modelling approach can be experimentally tested and can be used in various applications, such as, e.g. hearing aid processing and fitting procedures. 
2pPPg4. Binaural loudness summation in and out of the laboratory. Mary Florentine (Northeastern University, 360 Huntington Ave - 106A Forsyth Building - SLPA, Institute for Hearing, Speech \& Language, Boston, MA 02115, USA, florentin@ neu.edu), Michael Epstein (Northeastern University, 360 Huntington Ave - 106A Forsyth Building - SLPA, Institute for Hearing, Speech \& Language, Boston, MA 02115, USA, m.epstein@neu.edu)

Textbooks state that a tone presented binaurally is louder than the same tone presented monaurally. This is called Binaural Loudness Summation, BLS. Recent data and classroom demonstrations of BLS using speech stimuli from a visually present talker yield much less BLS than previously reported. Are conclusions about BLS drawn from tones presented via earphones in the laboratory applicable to live- voice speech in a room? To answer this question, eight normal listeners were presented three types of stimuli [monitored live voice (MLV) spondees, recorded spondees, and tones] monaurally and binaurally across a wide range of levels. The same stimuli were presented via earphones and loudspeakers. Loudness was measured using magnitude estimation. Results show that the amount of BLS was significantly less for MLV spondees than for tones or recorded spondees. It was also significantly less for loudspeaker presentation than for earphone presentation. The amount of BLS was least for MLV spondees presented via loudspeakers. Results indicate that BLS in loudspeaker conditions is significantly less than BLS in typical laboratory test conditions using earphones. A new phenomena-called "Binaural Loudness Constancy"-will be described that may result from expectations about loudness of a visually present talker. [Work supported by NIH-NIDCD grant R01DC02241]

6:40

2pPPg5. Loudness asymmetry ratings between accelerating and decelerating car sounds. Patrick Susini (IRCAM - UMR CNRS 9912, Equipe Perception et Design Sonores, 1, place Igor Stravinsky, 75004 Paris, France, susini@ircam.fr), Stephen McAdams (Centre for Interdisciplinary Research in Music Media \& Technology (CIRMMT) - Schulich School of Music - McGill Univ., 555 Sherbrooke Street West, Montreal, QC H3A1E3, Canada, smc@music.mcgill.ca)

Loudness change has been studied for tones with linearly varying levels revealing an asymmetry depending on the direction of change (increasing vs. decreasing) and the range of levels (high vs. low). Different assumptions were proposed to explain this asymmetry in favour of linearly increasing sounds. Teghtsoonian et al. (2005) and, more recently, Susini et al. (2006) explain that loudness of an increasing sound is influenced by the end level. Neuhoff (1999) describes this result by a survival advantage for detecting an approaching sound source. Whatever the assumption is, the results show that loudness judgments for abstract sounds (1000-Hz tones) and synthetic vowel sounds were significantly higher for increasing ramps. Those results are compared here with continuous and global ratings obtained on everyday sounds such as accelerating and decelerating car sounds with a same duration (43 s), but with different temporal functions and range of levels. Global loudness judgments made after the end of the sound are significantly higher for accelerating than decelerating car sounds, but are judged on average similar using continuous judgments. In addition, an increase in the speed (acceleration) is evaluated primarily in terms of its level at the end. These results confirm the previous studies.

\section{Contributed Papers}

7:00

2pPPg6. Temporal weighting in loudness judgments of time-varying sounds containing a gradual change in level. Daniel Oberfeld (Dept. of Psychology, Johannes Gutenberg-Universitaet Mainz, Staudingerweg 9, 55128 Mainz, Germany, oberfeld@uni-mainz.de)

Two experiments studied how listeners weight the level information from different portions of a noise consisting of ten temporal segments, the levels of which were sampled independently from a normal distribution. If a gradual increase in level ("fade-in") is imposed on the first few segments in such a sound, temporal weights in global loudness judgments in a oneinterval paradigm show a delayed primacy effect [Oberfeld and Plank, Fortschritte der Akustik - DAGA2005, 227-228 (2005)]. The maximum weight is assigned to the first segment presented at the full level, while the weights assigned to the fade-in segments are close to zero. In Experiment 1, a similar pattern of weights was observed in a two-interval paradigm. Thus, listeners do not use intensity information from the fade-in segments even when making within-trial comparisons. In Experiment 2, the first three segments gradually decreased rather than increased in level (i.e., the first segment had the highest level). Listeners gave near exclusive weight to the first segment. The results are compatible with a tendency to assign greater weight to loud elements [Lutfi and Jesteadt, J. Acoust. Soc. Am. 120, 3853-3860 (2006)] rather than with segmentation of the noise into a "gradually changing" and a "stable" part.

\section{$7: 20$}

2pPPg7. Magnitude judgments of loudness change for discrete, dynamic, and hybrid stimuli. Jesse D. Flint (Binghamton University (SUNY), Department of Psychology, Binghamton, NY 13902-6000, USA, jdflint@binghamton.edu), Richard E. Pastore (Binghamton University (SUNY), Department of Psychology, Binghamton, NY 13902-6000, USA,
pastore@binghamton.edu),Jeremy R. Gaston(Binghamton University (SUNY), Department of Psychology, Binghamton, NY 13902-6000, USA, jgaston1@binghamton.edu)

Recent investigations of loudness change across dynamically varying stimuli have identified differences between increasing versus decreasing power that depends upon stimulus complexity and intensity range [e.g., Canévet, Acustica, 62, 2136-2142 (1986); Neuhoff, Nature, 395(6698), 123124, (Sep 1998)]. Some explanations of these findings are based upon known psychophysical effects [Teghtsoonian, Teghtsoonian \& Canévet, Perception \& Psychophysics, 67(4), 699-712 (2005)], whereas others posit qualitative differences in the perception of discrete laboratory and dynamic natural sound structures (Neuhoff, 1998). For discrete, static stimuli, the relationship between loudness and power is dependant upon a number of factors, including frequency, intensity range, perceptual anchoring, temporal order, and integration of power across time. The current investigation examines listener perception of loudness for sound structures that vary systematically from discrete to dynamic changes in power, allowing evaluation of the contributions of static versus dynamic change, as well as other factors in the perception of loudness change within complex stimuli. Relevant methodology, as well as commonalities and differences with predictions of traditional psychophysics, are discussed.

\section{$7: 40$}

2pPPg8. Loudness of impulsive sounds as a function of duration: a parametric study of the effect of level and frequency. Sabine Meunier (CNRS-LMA, 31, chemin Joseph Aiguier, 13402 Marseille, France, meunier@1ma.cnrs-mrs.fr), Guy Rabau (CNRS-LMA, 31, chemin Joseph Aiguier, 13402 Marseille, France, rabau@lma.cnrs-mrs.fr)

Loudness of brief signals increases when signal duration increases up to about $100 \mathrm{~ms}$, this increase is called temporal summation. The question examined in this study is to determine how loudness change as a function of 
duration. Literature shows different results that lead to different conclusions: when duration increases (i) the loudness increases in direct proportion to energy (intensity $\mathrm{x}$ duration); (ii) the loudness increases faster than energy; (iii) the loudness increases slower than energy. We have shown, in previous experiments, that loudness change with duration can follow either (i) or (ii) depending on signal level. The present study provides a parametric exami- nation of this question. Loudness was measured for signals whose energy was kept constant while the duration was varied. The parameters of the experiment were the signal frequency and level. The results allow us to explain part of the discrepancies found in literature. Moreover, the results of this study are used into a model of impulsive sounds that calculate with a good precision the loudness of environnemental short-duration sounds.

\title{
Session 2pSAa
}

\section{Structural Acoustics and Vibration and EURONOISE: Acoustic Imaging in Confined Space I}

\author{
Earl G. Williams, Cochair \\ Naval Research Laboratory, 4555 Overlook Ave, Washington, DC 20375, USA \\ Alexandre Garcia, Cochair \\ CNAM, 292 rue Saint Martin, Paris, 75003, France
}

Invited Papers

\begin{abstract}
2:00
2pSAa1. Analysis of enclosed sound fields using spherical microphone array processing. Boaz Rafaely (Ben-Gurion University of the Negev, Department of Electrical and Computer Engineering, 84105 Beer-Sheva, Israel, br@ee.bgu.ac.il)

Enclosed sound fields in rooms and auditoria are characterized by multiple reflections in addition to the direct sound. The spatial and temporal manner in which sound propagates from the sound source to a listener determines the acoustics of the enclosed space. Recent studies showed that spherical microphone arrays and associated array processing can be used effectively in the experimental study of the acoustics of auditoria. The main advantages presented are the true three-dimensional analysis and the effectiveness of array processing in the spherical harmonics domain. Spherical microphone array design issues will first be reviewed in this work, in particular concerning the design of open-sphere arrays useful in room acoustics analysis with high spatial resolution. Then, beam-forming methods will be presented which can facilitate identification of individual room reflections, and computation of directional room impulse responses. Finally, the use of optimal array processing methods will be presented which facilitates improved sound field analysis.
\end{abstract}

$$
2: 20
$$

2pSAa2. Resolution improvement of beamformers using spherical microphone array. Jean-Claude Pascal (ENSIM - LAUM, Université du Maine, rue Aristote, 72085 Le Mans, France, Jean-Claude.Pascal@univ-lemans.fr), Jing-Fang Li (Visual VibroAcoustics, 51 rue d'Alger, 72000 Le Mans, France, jingfang.li@ visualvibroacoustics.com)

Spherical microphone array has been studied for various applications. In enclosed space, spherical array eliminates forwardbackward grating lobes occurring in two-dimensional arrays. Beamforming and spherical harmonic decomposition are both used to map the distribution of source strength. The two approaches show similar performance at frequencies where the upper spherical harmonic order equals the product of the wave number and sphere radius. However, at lower frequencies, processing using spherical harmonics maintains the same directivity while the spatial resolution for delay-and-sum beamformer deteriorates. It has been shown that the scattering of sound by a rigid sphere improves the directivity of the same open sphere microphone beamformer, by increasing the path length travelled by incident sound. This paper concerns focused beamformer using rigid and open sphere. The concept of statistically optimized array processing (SOAP) is applied to adapt to a particular interior geometry, considering the scattered field in case of rigid sphere. The regularization process makes it possible to use a model of partially coherent sound field in the enclosed space. A deconvolution post-processing based on the knowledge of the actual steering vector leads to an efficient and robust solution to improve considerably the resolution of the source strength distribution.

$$
\text { 2:40 }
$$

2pSAa3. Measurement of low-frequency sources in non-anechoic room using near-field acoustic holography. Manuel Melon (CNAM, 292 rue Saint Martin, 75003 Paris, France, melon@cnam.fr), Christophe Langrenne (CNAM, 292 rue Saint Martin, 75003 Paris, France, christophe.langrenne@cnam.fr), Alexandre Garcia (CNAM, 292 rue Saint Martin, 75003 Paris, France, garcia@cnam.fr), Philippe Herzog (Laboratoire de Mécanique et d'Acoustique - CNRS, 31 chemin Joseph Aiguier, 13402 Marseille, France, herzog@lma.cnrs-mrs.fr)

Low frequency $(20 \mathrm{~Hz}-200 \mathrm{~Hz})$ measurements of sound sources are rather difficult to perform because free field conditions can not be easily achieved properly. Moreover, some industrial sources have to be measured in situ. In such a case, a Field Separation Method (FSM) can be used to subtract the pressure field reflected by walls of the testing room from the measured data. This approach required the knowledge of both acoustic pressure and velocity on a closed surface surrounding the source. In this paper, a spherical harmonic expansion of measured data is used to solve the problem. The proposed method is applied to the measurement of the frequency response 
of a closed box subwoofer tested under various conditions: in a room with variable reverberation time ( $6.4 \mathrm{~s}$ to $0.6 \mathrm{~s}$ ). Theoretical frequency response of the subwoofer is also calculated using the Thiele and Small model. Results show a good agreement between separated data and simulations. The influences of the measurement distance and of the measurement point number required on the separation process are discussed.

\title{
3:00
}

2pSAa4. Vector intensity reconstructions in a volume surrounding a rigid spherical measurement array. Earl G. Williams (Naval Research Laboratory, 4555 Overlook Ave, Washington, DC 20375, USA, earl.williams@nrl.navy.mil), Kazuhiro Takashima (Nittobo Acoustic Engineering Co., Ltd., 1-21-10, Midori, Sumida-Ku, 130-0021 Tokyo, Japan, taka@noe.co.jp)

An approach is presented that provides a prediction of the vector intensity field throughout a volume exterior to a rigid spherical measurement array consisting of 31 flush mounted microphones. The theory is based on spherical harmonic expansions of the measured field with the radial variation of the near-field pressure obtained using the Greens function with vanishing normal derivative at the rigid sphere surface. Experimental results with rigid spherical arrays of differing radii are presented using multiple incoherent sources. Successful intensity reconstructions are obtained over a volume three times the sphere radius up to a frequency of $1.5 \mathrm{kHz}$ that clearly reveal the locations and levels of the two sources. This volumetric intensity probe is very similar mathematically to one described recently by the author (EGW) that used 50 microphones in an open array. The latter was used successfully inside an aircraft cabin in flight to uncover sources of noise. This work was supported by the US Office of Naval Research and Nittobo Acoustic Engineering Co. Ltd.

$$
\text { 3:20 }
$$

2pSAa5. A boundary element method for near-field acoustical holography in bounded noisy environment. Christophe Langrenne (CNAM, 292 rue Saint Martin, 75003 Paris, France, christophe.langrenne@cnam.fr), Manuel Melon (CNAM, 292 rue Saint Martin, 75003 Paris, France, melon@cnam.fr), Alexandre Garcia (CNAM, 292 rue Saint Martin, 75003 Paris, France, garcia @cnam.fr)

This paper presents a boundary element method to recover free field conditions from noisy bounded space situations. The proposed approach is based on the Helmholtz integral formulation and requires the knowledge of double layer pressure fields on two parallel closed surfaces surrounding the source. First, the outgoing and ingoing pressure fields are separated. Then, the incident field scattered by the tested source is subtracted from the outgoing field to estimate the pressure field which would have been radiated in free field. The method had been numerically tested and an experimental example is given here. The source is a rectangular box with seven loudspeakers mounted on it driven by bandwith limited white noise. The source is put at $0.4 \mathrm{~m}$ from the ground of a semi-anechoïc room. The ground plays a disturbant role because it produces secondary sources. The results show the effectiveness of the method particularly at frequencies where stationary waves between the ground and the underside of the box exist.

3:40-5:20 Posters

Lecture sessions will recess for presentation of poster papers on various topics in acoustics. See poster sessions for topics and abstracts.

\section{Invited Papers}

\author{
$5: 20$
}

2pSAa6. Acoustically invisible cylinder. Yuri Bobrovnitskii (Mechanical Engineering Research Institute, 4, M. Kharitonievky Str., 101990 Moscow, Russian Federation, yuri@imash.ac.ru)

Coatings of new type recently proposed by the author (Acoustical Physics, 2007, vol. 53, N5, pp. 535-545) are applied to bodies of cylindrical geometry to reduce reflection or scattering of sound and thus to make them undetectable by imaging systems. Such a coating, called as a coating with extended reaction, represents a periodic set of small elements with coupling between the neighboring elements. Appropriate choice of the coupling parameters makes its efficiency much higher than that of commonly used coatings. In the present paper, it is shown by computer simulation that a rather simple coating of this type can reduce the back-scattered pressure amplitude more than $40 \mathrm{~dB}$ (with respect to the rigid cylinder) practically at all frequencies. Considerable reduction of the scattered power can also be achieved in a low frequency range. The width of this range and the reduction index depend on the number of couplings introduced into the coating.

\section{$5: 40$}

2pSAa7. Acoustic source identification in an enclosed space using the inverse phased beam tracing at medium frequencies. Jeong-Guon Ih (KAIST, Dept. of Mechanical Engineering, Science Town, 305-701 Daejeon, Republic of Korea, J.G.Ih@kaist.ac.kr), Cheol-Ho Jeong (Acoustic Technology, DTU Elektro, Technical University of Denmark, Building 352, Ørsted plads, DK-2800 Kgs. Lyngby, Denmark, chj@oersted.dtu.dk)

When the source is enclosed by room surfaces, it is not easy, though not totally impossible, to apply conventional modal methods for source identification. This is because there are too many complicated wave interferences and effects of wall impedance, in particular at medium frequencies. The phased beam tracing method was suggested as a fast and efficient acoustic simulation tool at the medium frequencies in an enclosure, which overcomes the defects of geometrical acoustics techniques. In this study, the phased beam tracing method, implemented in its inverse form, was applied to the identification of the acoustic sources inside a chamber. In the simulation 
tests, spherical and rectangular shaped sources operating in a room were taken as examples. When the source pressure distribution was reconstructed by this numerical inverse technique, the sound power spectrum radiated from the source could be estimated by eliminating the contribution from surface reflections. Reconstruction error was investigated by the Monte Carlo simulation in terms of the signalto-noise ratio.

\section{Contributed Papers}

\section{6:00}

2pSAa8. Noise source characterisation using patch impedance technique. G Pavić (INSA de Lyon - LVA, Bâtiment St. Exupéry, 25 bis avenue Jean Capelle, F-69621 Villeurbanne Cedex, France, goran.pavic @insa-lyon.fr), Nicolas Totaro (INSA de Lyon - LVA, Bâtiment St. Exupéry, 25 bis avenue Jean Capelle, F-69621 Villeurbanne Cedex, France, nicolas.totaro@insa-lyon.fr)

A novel approach of an environment-independent sound source characterisation is discussed. The source is defined via a suitable enveloping interface surface by its blocked sound pressure and its surface impedance. Both the blocked pressure and the impedance are discretised using the averaging patch concept. Such a definition avoids singularity of point acoustic impedance and is suitable for numerical as well as experimental implementation. The characterisation of a source by the patch concept allows for the acoustical sub-structuring, which in turn enables the prediction of the sound field created by the source coupled to an arbitrary environment. Numerical simulations are presented which demonstrate the feasibility of the approach. It is hoped that the proposed approach can serve as a universal tool for noise synthesis of complex equipment incorporating noise sources.

\section{6:20}

2pSAa9. Local patch acoustic holography methods in enclosed spaces. Zdenek Havranek (Brno University of Technology, UAMT-FEEC, Kolejni
4, 61200 Brno, Czech Republic, havranek@feec.vutbr.cz),Ludvik Bejcek (Brno University of Technology, UAMT-FEEC, Kolejni 4, 61200 Brno, Czech Republic, bejcek@feec.vutbr.cz)

The paper compares reconstruction accuracy and computational cost of three patch acoustic holography algorithms applicable in the enclosed spaces for prediction of sound field near sources. Algorithms under investigation were DL-SONAH, IBEM and classical NAH with spatial transformations and hologram aperture enlargement. All of the selected algorithms take an advantage of using double layer microphone array for measurement of sound pressure field. The reconstruction accuracy of all algorithms is determined by using of simple 3D model of curved radiating surface on the basis of calculation of difference between true acoustic quantity (pressure, velocity or intensity) values very close to the surface and predicted sound field at same positions. All these methods use different calculation procedure to obtain predicted sound field near source surface, thus comparison of effectiveness of these algorithms including prediction accuracy-to-computational cost ratio is useful to determination their applicability in practice. Results of prediction error and calculation time including regularization with different parameter choice methods (L-curve, GCV, etc., are presented and compared. Initial comparison of the selected methods confirms shorter calculation time in non-element based methods, especially in NAH with transformations, while reconstruction error is in the same order.

\section{Invited Papers}

\section{6:40}

2pSAa10. Identification of source velocities with Inverse Patch Transfer Functions method. Mathieu Aucejo (INSA de Lyon LVA, Bâtiment St. Exupéry, 25 bis avenue Jean Capelle, F-69621 Villeurbanne Cedex, France, mathieu.aucejo@insa-lyon.fr), Nicolas Totaro (INSA de Lyon - LVA, Bâtiment St. Exupéry, 25 bis avenue Jean Capelle, F-69621 Villeurbanne Cedex, France, nicolas.totaro@insa-lyon.fr), Jean-Louis Guyader (INSA de Lyon - LVA, Bâtiment St. Exupéry, 25 bis avenue Jean Capelle, F-69621 Villeurbanne Cedex, France, jean-louis.guyader@insa-lyon.fr)

The identification of source velocities remains an important problem in noise control. For this purpose, several methods were developed such as Near-field Acoustic Holography (NAH) or inverse Boundary Elements Method (iBEM). An alternative method, based on the double measurement of pressure and particle velocity fields surrounding the source is presented. This method has been developed in the SILENCE European project framework. In this method, called inverse Patch Transfer Functions method (iPTF), measurement and identification surfaces are divided into elementary areas called patches. Then, source velocities are computed from acoustic field and inversion of impedances matrices obtained by FEM. Theoretically, this method presents two main advantages: it can be applied to sources with complex 3D geometries and measurements can be carried out in a non-anechoic environment, even in the presence of other sources. In the present paper, theoretical background of iPTF is exposed and results are presented on a source with simple geometry (an L-shaped plate) and discussed.

2pSAa11. Detection of acoustic radiating areas of a generic helicopter cabin by beamforming. Gérard Martin (ONERA, 2 avenue Edouard Belin, BP 4025, 31055 Toulouse, France, Gerard.Martin@onecert.fr), Frank Simon (ONERA, 2 avenue Edouard Belin, BP 4025, 31055 Toulouse, France, Frank.Simon@onecert.fr), Daniel Biron (ONERA, 2 avenue Edouard Belin, BP 4025, 31055 Toulouse, France, Daniel.Biron@onecert.fr)

Improvement of the helicopter internal noise is essential to decrease the fatigue effects of passengers. This requires having a metrological tool, able to give information on acoustic radiating areas in cabin in order to target appropriate acoustic passive or active solutions. In this context, the authors have shown, in previous papers, capability of modified beamforming using a cross-shaped array of microphones, associated with an acoustic mask, to localize isolated sources in a generic composite helicopter cabin (VASCo), thus, in spite of free field's hypothesis. The present paper deals with the feasibility of this measurement method to identify main acoustic pressure areas radiated by an helicopter mechanical deck whose vibration is generated by gear box beams between 800 and $5000 \mathrm{~Hz}$. This configuration is performed, thanks to simulations and experiments on a sandwich composite panel of VASCo, excited by 4 cor- 
related shakers. It appears that beamforming can be used to identify main acoustic area coming from the radiating of many coupled vibration modes. Nevertheless, the dynamic range decreases with the number of pressure anti-nodes and it is necessary to introduce an inverse method to reject the ghost images and to reconstruct amplitude and phase of synthesized sources.

\title{
Contributed Papers
}

\section{$7: 20$}

2pSAa12. 3D localization of acoustic sources with a spherical array. Karim Haddad (Brüel \& Kjær Sound \& Vibration Measurement A/S, Skodsborgvej 307, DK-2850 Nærum, Denmark, khaddad@bksv .com), Jørgen Hald (Brüel \& Kjær Sound \& Vibration Measurement A/S, Skodsborgvej 307, DK-2850 Nærum, Denmark, JHALD@bksv.com)

This paper describes a technique dedicated for the localization of acoustic sources in all directions and in the far-field. Classical beamforming techniques based on planar arrays provides an acoustic map restricted to a limited aperture, but a spherical array does not have such a limitation since there is no preferential direction. In this processing called Spherical Harmonics Beamforming, the sound field on the sphere is decomposed with spherical harmonics functions, and then a corrected summation gives the acoustic contribution from a given direction. We have used a rigid spherical array, which has the advantage that cabling of microphones and integrated cameras can be hidden inside the sphere. A rigid surface also provides better numerical stability in connection with Spherical Harmonics Beamforming. In this study, this technique is evaluated in connection with the resolution and the dynamic range. Simulated and experimental results are presented.

\section{$7: 40$}

2pSAa13. Near-field Acoustic Holography for partial measurements inside complex structures. Nicolas Valdivia (Naval Research Laboratory,
4555 Overlook Ave, Washington, DC 20375, USA, valdivia@pa .nrl.navy.mil), Earl G. Williams (Naval Research Laboratory, 4555 Overlook Ave, Washington, DC 20375, USA, earl.williams@nrl.navy.mil)

Near-field acoustical holography (NAH) requires the measurement of the near-field pressure field over a closed surface in order to recover the acoustic field on a nearby conformal surface. Very often we encounter applications where pressure measurements are available only over a patch of the measurement surface. In these cases the strict NAH theory does not hold, but still there are techniques that have been developed to overcome this difficulty. The best-known technique for planar surfaces is patch NAH and, recently proposed, for arbitrarily shaped surfaces patch IBEM and patch ESM. It was found in a recent study by the authors that these techniques will be affected by the problem of back-source contamination for interior NAH, but it was showed that this problem could be overcome by the use of "Cauchy" measurements. In this work we will compare the reconstruction of the acoustic field from patch based techniques with the technique that uses Cauchy measurements, and discuss the problem of back-source contamination in more detail. We use a cylindrical surface excited by a point force as an example to validate our results. This work was supported by the US Office of Naval Research.

TUESDAY AFTERNOON, 1 JULY 2008

P2-B, LEVEL 2, 3:40 TO 5:20 P.M.

\section{Session 2pSAb}

\section{Structural Acoustics and Vibration and EURONOISE: Source Characterization in Structure Borne Noise Problems II (Poster Session)}

\author{
Evan Davis, Cochair \\ The Boeing Company \\ Charles Pezerat, Cochair \\ Laboratoire Vibrations Acoustique - INSA Lyon
}

\begin{abstract}
All posters will be on display from 3:40 p.m. to 5:20 p.m. To allow contributors an opportunity to see other posters, contributors of odd-numbered papers will be at their posters from 3:40 p.m. to 4:30 p.m. and contributors of even-numbered papers will be at their posters from 4:30 p.m. to 5:20 p.m.
\end{abstract}

\section{Contributed Papers}

2pSAb1. The significance of cross-order terms in interface mobilities for structure-borne sound source characterization. Hannes A. Bonhoff (Berlin Institute of Technology, Einsteinufer 25, TA7, 10587 Berlin, Germany, hannes.bonhoff@tu-berlin.de), Björn A. Petersson (Berlin Institute of Technology, Einsteinufer 25, TA7, 10587 Berlin, Germany, b.a.t .petersson@tu-berlin.de)

For the characterization of structure-borne sound sources and the description of the associated transmission process, the source descriptor and coupling function were introduced. The concept of source descriptor and coupling function can be reformulated by incorporating the interface mobilities. The applicability for source-receiver assemblies with a multipoint or continuous connection is thereby granted. The accuracy of the results and consequently the validity of this approach, however, depends on the significance of the so-called cross-order terms. Such cross-order terms consist of force orders and cross-order interface mobilities. In recent theoretical and experimental work, the influence of cross-order interface mobilities as well as the distribution of force-orders have been investigated. Based on this knowledge, the significance of the cross-order terms is assessed in the present contribution. 
2pSAb2. Measurement of force and moment mobilities using a finite difference technique. Andrew Elliott (University of Salford, Acoustic Research Centre, Newton Building, M5 4WT Salford, UK, a.s.elliott @ pgr.salford.ac.uk), G Pavić (INSA de Lyon - LVA, Bâtiment St. Exupéry, 25 bis avenue Jean Capelle, F-69621 Villeurbanne Cedex, France, goran.pavic@insa-lyon.fr), Andy T. Moorhouse (University of Salford, Acoustic Research Centre, Newton Building, M5 4WT Salford, UK, a.t .moorhouse@salford.ac.uk)

Measuring only force mobilities it is possible to derive a moment mobility without the need for an externally applied moment. The method uses finite differences of both forces and velocities about a central point. In this way pure forces and moments can be extracted as can pure velocities and angular velocities. There are, however, errors associated with finite difference techniques. In this case the purity of the extracted quantities depends on the area over which they are measured and its significance in terms of wavelength. Meanwhile experimental errors such as noise dictate that the area is sufficiently large to provide differences which are incorruptible. The paper aims to quantify the finite difference error in a general way free of situation specifics.

TUESDAY AFTERNOON, 1 JULY 2008

P2-B, LEVEL 2, 3:40 TO 5:20 P.M.

\title{
Session 2pSAc
}

\section{Structural Acoustics and Vibration and EURONOISE: Acoustic Imaging in Confined Space II (Poster Session)}

\author{
Earl Williams, Cochair \\ Naval Research Laboratory \\ Alexandre Garcia, Cochair \\ CNAM
}

\begin{abstract}
All posters will be on display from 3:40 p.m. to 5:20 p.m. To allow contributors an opportunity to see other posters, contributors of odd-numbered papers will be at their posters from 3:40 p.m. to 4:30 p.m. and contributors of even-numbered papers will be at their posters from 4:30 p.m. to 5:20 p.m.
\end{abstract}

\section{Contributed Papers}

2pSAc1. Experimental feasibility of in-duct sound source reconstruction. Teresa Bravo (Université de Technologie de Compiègne, Centre de Recherche Royallieu, BP20529, 60205 Compiègne, France, teresa.bravo-maria@utc.fr), Cédric Maury (Université de Technologie de Compiègne, Centre de Recherche Royallieu, BP20529, 60205 Compiègne, France, cedric.maury@utc.fr)

Due to the expected air transport growth and stringent environmental regulations, there is a strong need to develop noise reduction techniques at acceptable cost in the aeronautical sector. A sound characterisation of the aero-acoustic sources in the nacelle acoustic duct problem plays a crucial role. In this study, it is shown how the liners optimized impedances strongly depend on the noise source characteristics under both tonal and broadband excitation conditions, and in the latter case, for varying degree of correlations between the random source strengths. The limitations for source identification methods, such as pointwise model-based inverse techniques, have been studied. These methods are able to provide reliable models of equivalent sound sources from a limited number of in-duct measurements, but require a priori knowledge of the source location. The performance of such techniques are compared with two different approaches, namely the use of focussed in-duct beamforming techniques to locate the unknown sources, and a decomposition of the assumed source strengths into angular Fourier series for both the location and the determination of the source amplitudes. Experimental results are presented for the location and the reconstruction of the particle velocity spectrum of wall-mounted compression drivers from induct measurements.

2pSAc2. Vector intensity measurement with a rigid spherical microphone array in a vehicle cabin. Kazuhiro Takashima (Nittobo Acoustic Engineering Co., Ltd., 1-21-10, Midori, Sumida-Ku, 130-0021 Tokyo, Japan, taka@noe.co.jp), Hiroshi Nakagawa (Nittobo Acoustic
Engineering Co., Ltd., 1-21-10, Midori, Sumida-Ku, 130-0021 Tokyo, Japan, nakagawa@noe.co.jp), Earl G. Williams (Naval Research Laboratory, 4555 Overlook Ave, Washington, DC 20375, USA, earl.williams@nrl.navy .mil)

Measurement results showing intensity vector reconstructions using a rigid spherical microphone array in a vehicle cabin are presented. The measurement equipment developed by Nittobo was used in previous demonstrations to visualize the acoustic field using a beamforming technique superimposed on a picture from an on-board camera. In the present study intensity reconstructions are shown and are successful in locating and quantifying sources, demonstrating the usefulness of this technique in an enclosed space like a vehicle cabin, aircraft cabin, small room, etc. This work was supported by the US Office of Naval Research and Nittobo Acoustic Engineering Co. Ltd.

2pSAc3. An improved method of visualizing the energy flow: the Rayleigh wave. Cleon E. Dean (Physics Department, Georgia Southern University, P. O. Box 8031, Statesboro, GA 30460-8031, USA, cdean $@$ GeorgiaSouthern.edu), James P. Braselton (Department of Mathematical Sciences, Georgia Southern University, P.O.B. 8093, Statesboro, GA 304608093, USA, jbraselton@GeorgiaSouthern.edu)

A variety of ways of visualizing the energy flux or elastodynamic Poynting vector field are demonstrated including gridded vector field, color coding, and other methods for the simple example of a Rayleigh wave. In particular an improved version of a technique shown at the 8th International Conference on Theoretical and Computational Acoustics, ICTCA 2007 is demonstrated [David M. F. Chapman, "Visualizing acoustic energy flow into the seabed using energy streamlines," Eighth International Conference on Theoretical and Computational Acoustics, ICTCA 2007, Heraklion, Crete, GREECE, 2-5 July 2007.]. The improved streamline method shows both the direction and the intensity of the energy flow. 


\title{
Session 2pSAd
}

\section{Structural Acoustics and Vibration and EURONOISE: Vibration and Radiation from Complex Structural Systems III (Poster Session)}

\author{
David Feit, Cochair \\ Applied Physical Sciences Corp. \\ Jean-Louis Guyader, Cochair \\ INSA de Lyon - LVA
}

\begin{abstract}
All posters will be on display from 3:40 p.m. to 5:20 p.m. To allow contributors an opportunity to see other posters, contributors of odd-numbered papers will be at their posters from 3:40 p.m. to 4:30 p.m. and contributors of even-numbered papers will be at their posters from 4:30 p.m. to 5:20 p.m.
\end{abstract}

\section{Contributed Papers}

2pSAd1. Implementation of the high order asymptotic models of linings and coatings to the SAW spectra evaluation. Dmitry Zakharov (LMP, UMR CNRS 5469, Université Bordeaux I, 351, cours de la Libération, 33405 Talence, France, dmitrii.zakharov@gmail.com), Alexander Shuvalov (LMP, UMR CNRS 5469, Université Bordeaux I, 351, cours de la Libération, 33405 Talence, France, a.shuvalov@1mp.u-bordeaux1 .fr), Olivier Poncelet (LMP, UMR CNRS 5469, Université Bordeaux I, 351, cours de la Libération, 33405 Talence, France, o.poncelet@1mp.ubordeaux1.fr)

High order asymptotic models of the relatively thin linings and coatings are used to reduce the problem dimension when dealing with a reasonable frequency range. The essential point consists in the use of asymptotic expansion for the internal stress and strain state of a laminate, subjected to the tight conditions at least on one of its faces, i.e., the conditions formulated for the displacements. The respective algorithm, based on it, is shorter than for the direct evaluation of the SAW spectra. It can be implemented to the calculation of Rayleigh waves or Lamb waves in layered media. Numerical examples are presented with both asymptotical and numerical evaluation of error.

2pSAd2. Plate Sound Radiation Prediction in Machines Using Multiple Input Techniques. Armand Nejade (Institut National de Recherche et de Sécurité (INRS), Ave. de Bourgogne, B.P. 27, F-54501 Vandoeuvre Cedex, France, armand.nejade@inrs.fr)

In systems with various types of noise sources, the characterization and ranking of each source may require a different approach. Also, one of the main challenges in such investigations, consists of isolating each source from the influence of the others. Often in the machines, the constituting plates are efficient radiators whose specific contributions need to be properly determined. In this presentation, we describe a method of plate radiation characterization using multiple input techniques. These techniques have frequently been used in case of discrete sources such as, for instance, vehicle engine cylinders where the pressure variations are modelled as the inputs. This application differs from the traditional ones by the fact that the inputs are now, the elements of a continuous medium. Here the surface of a plate is meshed, each element is taken as a sub-source and consequently, the plate is modelled as an assembly of sub-sources. The plate contributions at the operational modes, are then obtained through vibration measurements and therefore, are not affected by the noise from the other sources. Investigations on structures of various geometries and materials have so far, demonstrated the efficiency of this type of application.
2pSAd3. Forced vibrations of a cylindric panel with regular orthogonal system of stiffeners. Leonid A. Lazarev (Central Aerohydrodynamic Institute (TsAGI), 17, Radio Str., 105005 Moscow, Russian Federation, leonid174@mail.ru), Boris M. Efimtsov (Central Aerohydrodynamic Institute (TsAGI), 17, Radio Str., 105005 Moscow, Russian Federation, efimtsov @ prob-lab.ru)

Forced vibrations of a cylindrical panel freely supported in contour, with regular orthogonal system of stiffeners (typical of aircraft fuselage structure) are investigated. The connection of all three components of displacement of the skin and of discrete stiffeners as well as of the respective elastic and inertial forces and moments are taken into account in the case of excitation by normal and tangential forces. This connection can be described correctly at joint application of the recreptance method and the method of spaceharmonics. The task of forced vibrations is solved directly except the procedure of solving the task in terms of eigen-values. Some illustrative examples of the aircraft fuselage panel by a concentric harmonic force are presented. They demonstrate a high efficiency of applying this combined method. Potential possibilities of using this combined method for solving the tasks related to vibrations of a framed skin modeling the aircraft fuselage section and to the sound field in the volume bounded by it are considered.

2pSAd4. Optimisation of building vibration isolation by means of numerical methods. Fülöp Augusztinovicz (Budapest University of Technology and Economics, BME Dept. of Telecommunications, Magyar tudósok körútja 2, H-1117 Budapest, Hungary, fulop@ hit.bme.hu)

Ground-borne vibrations pose a steadily increasing environmental problem in densely populated areas of large cities. Although not the cheapest, the application of resilient elements in the construction of new buildings is an effective and viable method. Obviously, experimentation with parameters and placement of the isolators is not possible in this case. Therefore, a careful and watchful optimization of the static and dynamic characteristics of the supporting elements is a must. The proposed paper gives an overview of the vibration isolation design and reports on the expected effect of some basic design versions of a typical multi-storey building. Standard structural finite element method is used as a basic design tool, but a number of vibroacoustic aspects are also considered and treated. Experimental results as well as comparisons of measurements and predictions will be reported too. 
2pSAd5. Refracto-vibrometry - a novel method for visualizing sound waves in transparent media. Lothar Zipser (HTW University of Applied Sciences, Friedrich-List-Pl. 1, D-01069 Dresden, Germany, zipser @et.htw-dresden.de), Heinz H. Franke (HTW University of Applied Sciences, Friedrich-List-Pl. 1, D-01069 Dresden, Germany, zipser@et.htwdresde.de)

For optimization of new sonic techniques, for development of acoustic sensors or for understanding of complex sonic phenomena it is often desirable to visualize the generation and distribution of the invisible sound waves. A novel method for measuring and visualizing the sound waves in transparent media was developed and tested by the authors. This non-contact method is based on a scanning laser-Doppler vibrometer. It can be used for measuring and visualizing sound fields in gases, fluids and even pellucid solid objects. The variation of the optical refractive index $\mathrm{n}$ of transparent media, caused by the sound waves is used as measuring effect. This justifies the denomination "refracto-vibrometry". The paper describes the theoretical fundamentals and the technical equipment required for the novel method. As examples, the generation and radiation of sound in air, water and transparent solid objects are presented. The animation of the results as videos is very impressive and instructive. In research, education and system design refracto-vibrometry has a large potential to make acoustics clearer and more feasible for application.

2pSAd6. Influence of the pre-stress state on the wave propagation in a shearable Timoshenko beam. Nicolas Bideau (Institute of Mathematical Research of Rennes IRMAR, Campus de Beaulieu, 35042 Rennes, France, nicolas.bideau@univ-rennes1.fr), Polynice Eyi-Assoumou (Institut de Recherche Mathématique de Rennes, Université de Rennes 1, Campus de Beaulieu, 35042 Rennes, France, polynice.eyi-assoumou @ univ-rennes1.fr), Loic Le Marrec (Institute of Mathematical Research of Rennes IRMAR, Campus de Beaulieu, 35042 Rennes, France, loic.lemarrec@univ-rennes1.fr), Lalaonirina Rakotomanana (Institut de Recherche Mathématique de Rennes, Université de Rennes 1, Campus de Beaulieu, 35042 Rennes, France, lalaonirina.rakotomanana-ravelonarivo @ univ-rennes1.fr)

It is very common to use pre-stressed beam models in the structure design. However in the nonlinear domain, the modal analysis remains difficult especially when shearability is taken into account. This work aims to study the natural vibrations of pre-stretched nonlinear shearable Timoshenko beam using Cosserat continuum mechanics. In this paper, a three dimensional nonlinear beam model is first developed. Then we analyse waves which are superimposed on a finite pre-stretch state. Namely, analytical dispersion relations are given for different values of pre-strech. The complete spectrum, including propagating and evanescent traction/compression, shear and bending modes, of a pre- stretched shearable beam is calculated through dispersion pattern. Spectrum strongly depends on initial pre-stress value. A significant qualitative and quantitative difference with the classical Timoshenko beam theory is pointed out even for small pre-stretch values.

2pSAd7. Temporal prediction of the acoustic radiation of vibrating structures: subjective evaluation of a simplified approach. Nacer Hamzaoui (LVA, INSA de Lyon, Bat. Saint-Exupéry, 25 bis avenue Jean Capelle, 69621 Villeurbanne, France, nacerhamzaoui @insa-lyon.fr), Juliette Pierangelo (LVA, INSA de Lyon, Bat. SaintExupéry, 25 bis avenue Jean Capelle, 69621 Villeurbanne, France, juliette.pierangelo@insa-lyon.fr), Antoine Levy (LVA, INSA de Lyon, Bat. Saint-Exupéry, 25 bis avenue Jean Capelle, 69621 Villeurbanne, France, antoine.levy@insa-lyon.fr)

The goal of the approach presented in this paper is to predict the noise radiated by vibrating structures with acquired vibratory measurements, in the temporal field, on their surface. In the frequential field this prediction is based on the resolution of the Kirchhoff integral equation, which requires a lot of computing time and is thus limited to low and intermediate frequencies; the boundary element method (BEM) is used for the resolution. Simplifications were already brought to low $(<$ Flim1) and to high $(>$ Flim2) frequencies, leading to a simple calculation of a fast integral. The limiting frequencies (Flim1 and Flim2) depend on the structure dimensions and its position towards the observation point. In this paper we will present an approach developed in the temporal field in which a complete calculation $(\mathrm{BEM})$ is avoided by choosing a minimal distance in order to have Flim $1=$ Flim2 and use only the simplified calculations. The vibratory data is measured in the temporal field and the studied structure is a parallelepipedic box excited by two mechanical excitation pots which excite one or two faces. The acoustic pressures calculated in the temporal field will be confronted with measurements, by using similarity subjective tests and integrating a variation of the principal parameters intervening in this approach.

2pSAd8. Radiation efficiency of natural modes of plates with beam stiffeners. Andreas Rousounelos (Loughborough University, Department of Aeronautical and Automotive Engineering, Ashby Road, LE11 3TU Loughborough, UK, a.rousounelos@lboro.ac.uk), Stephen J. Walsh (Loughborough University, Department of Aeronautical and Automotive Engineering, Ashby Road, LE11 3TU Loughborough, UK, S.J.Walsh@lboro.ac.uk), Victor V. Krylov (Loughborough University, Department of Aeronautical and Automotive Engineering, Ashby Road, LE11 3TU Loughborough, UK, V.V.Krylov@lboro.ac.uk)

In this paper, the radiation efficiency of the natural modes of finite plates with attached beams is considered. Firstly, the scattering of flexural waves, by the beams and the boundaries, in the plate is considered. The reflection and transmission coefficients along with the near-field coefficients of the beam are used to calculate the vibration field of the plate, by taking into account an infinite number of reflections from the boundaries. A wavenumber transform of the vibration velocity field is used to calculate the sound power and the radiation efficiency. Numerical results are presented, for a number of attached beams, and show that the beams introduce ripples in the radiation efficiency curve, below a certain frequency. The amplitude of the ripples is controlled by the mass and the torsional stiffness of the attached beams whereas the stiffness of the beam has little influence. The results are compared with measurements of the radiation efficiency of plates with attached beams.

2pSAd9. Virtual statistical energy analysis for vibroacoustic industrial prediction. Gérard Borello (InterAC, 10 impasse Borde-Basse, ZA. La Violette, 31240 L’Union, France, gerard.borello@interac.fr), Laurent Gagliardini (PSA Peugeot Citroën, Route de Gisy, 78943 VélizyVillacoublay Cedex, France, laurent.gagliardini@mpsa.com), Denis Thenail (PSA Peugeot Citroën, Route de Gisy, 78943 Vélizy-Villacoublay Cedex, France, denis.thenail@mpsa.com)

In the mid-frequency range $(200-2000 \mathrm{~Hz})$, difficulty is encountered when modeling car body vibroacoustic interactions, mainly due to the complexity of automotive design. Analytical Statistical Energy Analysis (ASEA) is efficient to bring to the fore regions of interest regarding NVH design but was proven to be accurate only above $2000 \mathrm{~Hz}$. To overcome ASEA limitations at lower frequencies, Virtual SEA (VSEA) technique was introduced to translate the dynamic information contained in a finite element (FE) model into an SEA model. Any FE model, whatever its complexity, can thus be processed thanks to an automatic sub-structuring algorithm and a built-in VSEA modal synthesis solver, fast leading to robust numerical SEA model. VSEA also addresses structure-borne noise problems by coupling structural VSEA subsystems to analytical acoustic subsystems through a virtual wave number. Investigation of damping and trim treatment effects on mixed acoustic and structural subsystems are thus possible. While reviewing VSEA theory, industrial application cases will be presented.

2pSAd10. Noise Prediction for a Pumping Loop using the Noise Synthesis Technology Approach. Henri Pepin (CETIM, 52 Avenue Felix Louat, 60300 Senlis, France, henri.pepin@ cetim.fr), Axel Hass (52, avenue Félix Louat, 60304 Senlis, France, Axel.Haas@cetim.fr)

A collective project involving the french manufacturers of industrial pumps and valves is dedicated to the noise prediction in pumping plants. This paper presents a methodology based on virtual acoustic prototyping, and specifically on the noise synthesis technology (NST). Prediction of radiated noise in particular locations, such as operator stations or contractual specifications, allows us to control from the conception stage if the industrial plant will meet the prescribed noise criteria (regulations or user specifications). In addition, related noise control recommendations can be 
achieved by comparison of data obtained from the individual response of the components and their interaction. This methodology does not work as an absolute predictive process, and thus requires experimental validation. Components are taken into account by establishing their detailed vibroacoustic characteristics (technical data or measurements); in the present work on a simplified pumping loop, selected components are a centrifugal pumping unit, an open pipe circuit and several type of valves. Vibroacoustic and hydroacoustic measurements have to be adjusted according to the NST requirements. Comparison of the NST results with experimental data show a wide validity domain, covering the $100-10 \mathrm{kHz}$ third-octave range.

2pSAd11. Design of air springs for improved frequency response characteristics using computational fluid dynamics. Grace $R$. Kessenich (IPTRADE, Inc, 1 Gateway Center Suite 601, Newton, MA 02458, USA, grace.kessenich@iptrade.com), Allan D. Pierce (College of Enginering, Boston University, 110 Cummington St, Boston, MA 02215, USA, adp@bu.edu), Baruch Pletner (IPTRADE, Inc, 1 Gateway Center Suite 601, Newton, MA 02458, USA, baruch.pletner@iptrade.com)
High performance air springs can be limited as vibration isolation devices by aeroacoustic cavity resonances. Such systems require detailed knowledge of their frequency response over all frequencies of interest. Transient computational fluid dynamics (CFD) simulations can derive quantitative magnitude and phase data early in the design cycle, before manufacturing. CFD models are unaffected by ambient noise, resonances from other sources, and sensor sensitivity, major experimental concerns. Unlike analytical methods, CFD modeling is applicable to complex as well as simple geometries. The modeling methodology is illustrated in the present study with 2D and 3D simulations of three different prototypical chambers. A chamber wall section, representing a piston, is moved with a sinusoidal displacement at constant frequency. Measurement of the magnitude and phase from the resulting sinusoidal force on this surface provides one point of a frequency response. By running multiple simulations over a wide frequency range, the null and resonant frequencies are readily apparent. Comparison with analytical predictions for simple geometries validates this technique. Insertion of porous membranes in the air spring cavity is modeled and is shown to be an effective method for mitigating aeroacoustic effects on air spring performance. Simulations provide crucial information for membrane parameter and placement optimization.

TUESDAY AFTERNOON, 1 JULY 2008

P2-B, LEVEL 2, 3:40 TO 5:20 P.M.

Session 2pSAe

\title{
Structural Acoustics and Vibration and EURONOISE: General Topics in Structural Acoustics and Vibration III (Poster Session)
}

\author{
Wolfgang Kropp, Cochair \\ Chalmers University of Technology \\ Sean Wu, Cochair \\ Wayne State University
}

\begin{abstract}
All posters will be on display from 3:40 p.m. to 5:20 p.m. To allow contributors an opportunity to see other posters, contributors of odd-numbered papers will be at their posters from 3:40 p.m. to 4:30 p.m. and contributors of even-numbered papers will be at their posters from 4:30 p.m. to 5:20 p.m.
\end{abstract}

\section{Contributed Papers}

2pSAe1. The investigation on sound souce indentification in semi-space by NAH. Dejiang Shang (Harbin Engineering University, College of Underwater Acoustic Engineering, 150001 Harbin, China, chizhafengyun1979@126.com), Yongwei Liu (Harbin Engineering University, College of Underwater Acoustic Engineering, 150001 Harbin, China, chizhafengyun1979@126.com)

The near field acoustic holography (NAH) theory model in semi-space has been constructed on the foundation of NAH theory in full-space. The technique of semi-space acoustic field reconstruction by NAH has been developed on Fast Fourier Transform Algorithm. This method has considered the water surface as soft boundary condition. The equivalent free sound field can be acquired if the measured sound field has been handled dissymmetrically by the principle of the mirror. This method can overcome the disadvantages of the boundary element method which can be used to resolve the problems of sound source identification in semi-space, such as the using of the semi-space Green function, complex transformation matrix calculation, more time consummation and singularity solution existence. This method has been verified by numerical calculation and by experiment respectively. The experimental frequency is between 3 and $10 \mathrm{kHz}$. The distance between the hologram plane and source plane is 3 centimeter. The results show that it's an efficiency way to deal with the problem of sound source identification in semi-space.

2pSAe2. An analysis of the modes of wave propagation in cylindrical shells. Yu-Cheng Liu (Graduate Institute of the Mechanical \& Aeronautical Engineering, Feng Chia University, No. 100 Wenhwa Rd., Seatwen, 40724 Taichung, Taiwan, p9245215@fcu.edu.tw), Yun-Fun Hwang (Electroacoustic Graduate Program, No. 100 Wenhwa Rd., Seatwen, $40724 \quad$ Taichung, Taiwan, yfhwang@fcu.edu.tw), Jin-Huang Huang (Department of Mechanical and Computer-Aided Engineering, No. 100 Wenhwa Rd., Seatwen, 40724 Taichung, Taiwan, jhhuang@fcu.edu.tw)

Similar to a recent paper by Karczub [J. Acoust. Soc. Am., Vol. 119(6), 3553-3557, 2006], this work utilizes a symbolic math solution to obtain analytical solutions for the dispersion relation in a thin circular cylindrical shell. Analyses on the propagating wave mode shapes are particularly emphasized. The analytical solutions provide a straightforward calculation for all four branches of the dispersion curves, and the cumbersome numerical root searching technique is avoided. The dispersion relations for the breathing and torsional modes $(\mathrm{n}=0$ circular harmonic), which were neglected by 
Karczub, are included. Mode shapes that characterize the types of wave that are predominantly transversal, longitudinal, circumferential, or in any combination of the three are analyzed in considerable detail. Effects by thickness and Poisson ratio variations are considered and examined. The cutoff frequencies for the higher order propagating modes are compared among the various orders of circular harmonics, from $\mathrm{n}=0$ to 10. Finally, comparison among several shell theories in the dispersion relation prospective, which is directly related to their accuracy in predicting the vibratory resonant frequencies of a finite shell, is also included in the discussion.

2pSAe3. Influence of Constructional Parameters of Smal Reciprocating Compressors on Sound Power Emissions. Peter J. Kral (Vienna University of Technology, Institute for Engineering Design and Logistics Engineering, Getreidemarkt 9/E307, 1230 Vienna, Austria, peter.kral@tuwien.ac.at)

Noise in the environment is an increasing problem in industrial countries and is often strongly restricted by governmental laws. Therefore reducing sound levels must be one target in every design process. This paper describes in detail the influence of constructional parameters on the total sound power emitted by a small reciprocating compressor $\left(V_{H}=159 \mathrm{~m}^{3}\right)$ with reed valves. Measurements (sound power levels, sound pressure levels, FFTanalysis, time-signal analysis) on a test bed situated in an anechoic room by varying the operating conditions - for example - rotational speed, maximum pressure, and suction terms, were done. Additionally sound power measurements with regard to reed valve parameters (thickness of the reed, area of the inlet and outlet port of the valve plate, material of the valve plate) were achieved. Theoretically researches with the aid of computational methods (FEM-Analysis with Ansys, Calculations with MatLab) are presented and show very good accordance compared to the sound level measurements. These results should lead to a better understanding of the causal connection between constructional (valve) parameters/operating conditions and the resulting sound power emissions. Finally the differentiation of airborne and structure born noise may be helpful to minimize the noise emissions of compressors already within the design process.

2pSAe4. Modelling of the hydro and aero acoustic sources in piping. Véronique Villouvier (EDF/R\&D, 1 avenue du Général de Gaulle, 92141 Clamart, France, veronique.villouvier@edf.fr)

In the industrial piping networks, various components are crossed by flows, generally in air or in water. The pressure fluctuations generated locally close to these singularities are propagated then in all the network and can lead to important vibrations of the structures. A research program based on many laboratory tests made it possible to highlight a hydroacoustic modelling of the butterfly valves in the low frequencies domain. The model, which describes the acoustic sources, generated by the turbulence, has a general applicability. In this paper, one presents the application of this model to orifices (openings and slits) working in air and in water. These various orifices were studied from an acoustic point of view on different test loops and for several hydraulic conditions (flow rate and head loss). These experimental cases were then simulated using the source model of turbulence noise, associated to the transfer matrix of each singularity, which is determined by another way. The comparison between these calculations and the corresponding measurements makes it possible to consolidate the general character of the model of turbulence noise for singularities in piping.

2pSAe5. Hydroacoustic modeling of the butterfly valves - some industrial applications. Véronique Villouvier $(\mathrm{EDF} / \mathrm{R} \& \mathrm{D}, 1$ avenue du Général de Gaulle, 92141 Clamart, France, veronique.villouvier@edf.fr)

The French PWR units include a large number of control valves which are sometimes at cavitation working for certain operating conditions. The very high excitations which are then created may lead to the cracking of some small lines located nearby. These devices have been tested on laboratory test loops. An hydroacoustic behaviour model has been set up on the basis of very precise identifications obtained from numerous experimental tests. The model obtained describes the acoustic transfers through the valve and the sources generated by turbulence and cavitation in the low frequency domain, according to the main operating parameters: aperture, flow rate, cavitation number, upstream pressure and diameter. Hydraulic and vibratory measurements were carried out on several Residual Heat Removal circuits for many control configurations. When this circuit is functioning under certain conditions, the two valves which regulate the hot and cold flow rates operate according to a cavitation system. The hydroacoustic valve model is used to simulate of the valves under the hydraulic conditions of measurements. The results are compared at the vibratory levels recorded on site. The model also makes it possible to determine the optimal operating conditions in order to limit the vibrations of the circuit.

2pSAe6. Use of noise and vibration signal for detection and monitoring of cavitation in kinetic pumps. Jan Černetič (University of Ljubljana, Faculty of Mechanical Engineering, Aškerčeva 6, 1000 Ljubljana, Slovenia, jan.cernetic@fs.uni-lj.si), Jurij Prezelj (University of Ljubljana, Faculty of Mechanical Engineering, Aškerčeva 6, 1000 Ljubljana, Slovenia, jurij.prezelj@fs.uni-lj.si), Mirko Čudina (University of Ljubljana, Faculty of Mechanical Engineering, Aškerčeva 6, 1000 Ljubljana, Slovenia, mirko .cudina@fs.uni-lj.si)

Cavitation in kinetic pumps causes lowering performance, mechanical damage and increase of vibrations and noise. Therefore, it is important to detect inception and development of cavitation phenomenon in the pump. This paper deals with signals of vibrations and noise, which will be used for detection and monitoring of cavitation in kinetic pumps, and also to prevent the effect of cavitation in the pump and pumping system. When the cavitation is increasing, the flowing conditions are changing, which leads to an increase of vibrations of the pump and emitted noise in the surroundings. Because vibrations and noise are transferred from the pump through its casing, the signal is non-uniformly distorted due to transfer losses and structure of the casing surfaces. Even so, it is possible to determine development of cavitation phenomena from the measured signal. Noise and vibrations are increasing steadily, but in some specific frequency ranges the signal is more pronounced than in other part of the spectrum. Experimental results have shown that when the cavitation is fully developed, the measured signals at a characteristic frequency or range of frequencies start to decrease. This characteristic frequency or band of frequency is discussed in this paper, and also comparison between theoretical expectations and measurement results was performed.

2pSAe7. The wavelet analysis the acoustic emission signals generated by multi-source partial discharges. Andrzej Cichon (Technical University of Opole, ul. Miko ${ }^{3}$ ajczyka 5, 45-271 Opole, Poland, a.cichon@po.opole.pl), Sebastian Borucki (Technical University of Opole, ul. Miko ${ }^{3}$ ajczyka 5, 45-271 Opole, Poland, s.borucki@po.opole.pl), Tomasz Boczar (Technical University of Opole, ul. Miko ${ }^{3}$ ajczyka 5, 45-271 Opole, Poland, t.boczar@po.opole.pl)

Within the research work, the results of which are presented in this paper, a comparative analysis of the acoustic emission (AE) signals generated by multi-source partial discharges (PDs) was carried out. The research investigations were carried out in a model system in which PDs were generated with two spark-gaps. A spark-gap in the surface system and a pointplane spark-gap were used due to the fact that these are the most commonly occurring PD forms in power transformers. The AE signals were registered with a contact transducer placed on the external part of the tub. The analysis in the time-frequency domain using a short-time Fourier transform (STFT) was carried out for the $\mathrm{AE}$ signals generated by PDs. The results of the analysis are shown in the form of two- and three-dimensional spectrograms of the power spectral density and three-dimensional spectrograms of the amplitude spectrum

2pSAe8. Using wavelet transform to locate acoustic emission source in composite plate with one sensor. Amir Refahi Oskouei (Polytechnic of Tehran, AmirKabir University of Technology, Department of Mechanical Engineering, NDT Lab., 424 Hafez Ave, 15875-4413 Tehran, Iran, amir.refahi@cic.aut.ac.ir), Mehdi Ahmadi (Polytechnic of Tehran, 
AmirKabir University of Technology, Department of Mechanical Engineering, NDT Lab., 424 Hafez Ave, 15875-4413 Tehran, Iran, ahmadin@cic.aut .ac.ir)

In this paper a new method for locating the AE source with one sensor is presented. When acoustic emission signals propagate in wave-guided, they have multi-mode and dispersion characteristics. The separation of the modes at the sensors could make it possible to extract the exact information about the source that produced the waves. Based on the modal nature of AE, it can be understood that a good location would have two methods to determine the arrival times. One is determined on the same part of the waves (not only the same mode, but also the same frequency) at all sensors. The other is determined on the different modes at one sensor, which makes it possible to reduce the number of sensors needed. First, through modal analysis of the propagation of elastic waves in a thin plate, the dispersion characteristics of the modes are predicted. Second, the wavelet transform theory is briefly outlined and its application in elastic waves is explained. It is shown that by using the peak of the magnitude of the wavelet transform, the arrival times of the different modes can be determined. Additionally, experiments were undertaken using a lead break on the edge of the plate. These demonstrated the feasibility of the one sensor linear location scheme.

2pSAe9. Effect of boundary restraints on sound radiation. Wen Li (Wayne State University, Department of Mechanical Engineering, 5050 Anthony Wayne Dr., Detroit, MI 48202, USA, wli@wayne.edu)

This study is focused on the effect of boundary restraints on the sound radiation from a rectangular plate. The modal and acoustic characteristics of square plates are studied for different restraining stiffnesses and configurations. It is shown that the modes of the restrained plates can be considerably different from those for the familar simply supported boundary condition, so are the corresponding modal radiation efficiencies. The proposed method generally applies to the plates elastically restrained along any edge(s) and for any stiffness values. The acoustic calculations are also valid for any acoustic wavenumber, instead of only for some extreme acoustic/structural wavenumbers as in other investigations.

2pSAe10. Progress in Primary Calibration of Acoustic Emission Sensors. Jiri Keprt (Brno University of Technology, UAMT-FEEC, Kolejni 2906/4, 61200 Brno, Czech Republic, keprt@feec.vutbr.cz), Petr Benes (Brno University of Technology, UAMT-FEEC, Kolejni 2906/4, 61200 Brno, Czech Republic, benesp@feec.vutbr.cz)

The paper reviews the background of the primary calibration of acoustic emission sensors and the determination of uncertainty by this calibration. There are discussed the aims and the purpose of the primary calibration and main sources of uncertainty in practical usage of calibration results. The comparison of the results of the both method is presented. The shape of calibration characteristics corresponds well up to $500 \mathrm{kHz}$. The calculation of the uncertainty in the reciprocity and step function calibration is closely described. The problem of propagation of uncertainty in the fast fourier algorithm was solved. Uncertainty of measurement by primary calibration is determined and presented in the paper. The uncertainty in reciprocity calibration of UT 1000 in range from $60 \mathrm{kHz}$ to $285 \mathrm{kHz}$ was $\pm 3 \mathrm{~dB}$ and from $285 \mathrm{kHz}$ to $1 \mathrm{MHz}$ up to $\pm 8 \mathrm{~dB}$ and the same results in the calibration by step function. The calculation of the uncertainty includes all the presumable sources. Such as variation of input coefficient of reciprocity parameter, impact of thickness of glass capillary Also the main problems, that were solved and have to be solved, are presented.

2pSAe11. High-speed electronic speckle pattern interferometry of a struck flat plate. Thomas Moore (Rollins College, Department of Physics, Winter Park, FL 32789, USA, tmoore@ rollins.edu), Daniel Zietlow (Rollins College, Department of Physics, Winter Park, FL 32789, USA, dzietlow@rollins.edu), Christopher Gorman (Rollins College, Department of Physics, Winter Park, FL 32789, USA, cgorman@ rollins.edu)

An electronic speckle pattern interferometer capable of imaging 33,000 frames per second has been used to study the transient motion of a flat plate which was struck at a point near the edge. Multiple interferograms, each with an exposure time of 25 microseconds, were combined to construct a video of the plate motion that can be used for both qualitative and quantitative analysis. The interferograms reveal the expected evolution of the bending waves created when a thin circular plate is struck near the edge; however, anomalous displacement of the surface of the plate is also observed. This unusual displacement of the surface takes the form of a transverse wave that precedes the motion directly attributable to the normal propagation of the initial bending wave.

2pSAe12. The Investigation on Measuring the Reflection Coefficient of Materials in Semi-space by Spatial Fourier Transforms. Yongwei Liu (Harbin Engineering University, College of Underwater Acoustic Engineering, 150001 Harbin, China, chizhafengyun1979@126.com), Dejiang Shang (Harbin Engineering University, College of Underwater Acoustic Engineering, 150001 Harbin, China, chizhafengyun1979@126.com), Qi Li (Harbin Engineering University, College of Underwater Acoustic Engineering, 150001 Harbin, China, chizhafengyun1979@126.com)

A new method using SFT (Spatial Fourier Transforms) has been investigated to measure reflection coefficient at arbitrary angles of incidence by NAH(Near-field Acoustical holography ) in semi-space. The paper gives an experimental verification of this method. The experimental frequency is from $3 \mathrm{kHz}$ to $10 \mathrm{kHz}$. The size of test steel plate is $1.6 \mathrm{~m} \times 1.5 \mathrm{~m}$, the size of hologram surfaces is $1.32 \mathrm{~m} \times 1.14 \mathrm{~m}$, the distance between two hologram surface and the test material is about $2 \mathrm{~cm}$, the distance between sound source and water surface is about $4 \mathrm{~cm}$. The measured results are generally in good agreement with the theoretical value; the mean-square error of data is below 0.06 . The experimental results show that this method eliminates the effect of the scattered sound field of the water surface completely. Because the sound source and the test material are set near the water surface during the experiment, it is very easy to set the measuring system, and this is very useful in practice.

2pSAe13. Optimizing the Dynamic Behaviour of a Large Portal Robot. Christian R. Koenig (University of Bremen, Badgasteiner Str. 1, FZB Room 2140, 28359 Bremen, Germany, chkoenig@uni-bremen.de), Markus Kochmann (University of Bremen, Badgasteiner Str. 1, FZB - Room 2140, 28359 Bremen, Germany, kochmann@uni-bremen.de), Jan Ohlendorf (University of Bremen, Badgasteiner Str. 1, FZB - Room 2140, 28359 Bremen, Germany, johlendorf@uni-bremen.de), Dieter H. Müller (University of Bremen, Badgasteiner Str. 1, FZB - Room 2140, 28359 Bremen, Germany, ml@biba.uni-bremen.de), K.-D. Thoben (University of Bremen, Badgasteiner Str. 1, FZB - Room 2140, 28359 Bremen, Germany, tho@biba.uni-bremen.de)

The object under investigation in this work is a portal robot used for the production of large fibre reinforced structures. The achieved product quality and the production speed of the device are influenced by the vibration and the damping behaviour of the system. The required process time is determined by kinematic parameters, i.e. speed and acceleration of the system and the dead time required for the vibration level to decay to a threshold level given by the required precision of process. In the first step, a Design of Experiments is used in order to identify the ideal combination of acceleration and speed. At this optimum, the sum of the drive time and the dead time reaches a minimum for a given precision. In the second step, an experimental Modal Analysis is performed in order to identify the potential for an optimization of the structure.

2pSAe14. Evaluation of hand-transmitted vibration exposure on basis of a questionnaire. Rauno Pääkkönen (Finnish Institute of Occupational Health, P.O:Box 486, 33101 Tampere, Finland, rauno.paakkonen@ttl.fi), Riitta Sauni (Finnish Institute of Occupational Health, P.O:Box 486, 33101 Tampere, Finland, riittta.sauni@ttl.fi), Jukka Uitti (Finnish Institute of Occupational Health, P.O:Box 486, 33101 Tampere, Finland, jukka.uitti@ttl.fi), Esko Toppila (Finnish Institute of Occupational Health, P.O:Box 486, 33101 Tampere, Finland, esko.toppila@ttl.fi)

We made a survey to see if metal workers had symptoms caused by vibration. The vibration exposure over lifetime was also asked. The cumulative HAV dose was calculated as a HAV index determined on the basis of the questionnaire, in which jobs, tools and active work time (hours/day, days/week, moths/year) had been requested. An index, I, describing the total 
HAV exposure was determined by $\mathrm{I}=\mathrm{a} 8 \mathrm{~h} 2 \times \mathrm{y}$ x d, where $\mathrm{I}=$ cumulative exposure index $(\mathrm{m} 2 \mathrm{ad} / \mathrm{s} 4), \mathrm{y}=$ exposure time in years $(\mathrm{a})$, and $\mathrm{d}=$ annual exposure time in days. The exposure of metal workers varied 1-5 m/s2, and the average was $2.4 \mathrm{~m} / \mathrm{s} 2$. The limit value of European directive $5 \mathrm{~m} / \mathrm{s} 2$ is not usually exceeded. However, there is a wide unaccuracy area that needs to be cleared one way or another. We also connected symptoms and exposure on statistical level. The total exposure times did not differ significantly in these groups, but, when the daily HAV exposure was taken into account in the calculation of the cumulative index, the difference was statistically significant.

2pSAe15. Experimental investigation of the acoustic black hole effect for vibration damping in elliptical plates. François Gautier (Laboratoire d'Acoustique de l'Université du Maine, Avenue Olivier Messiaen, 72085 Le Mans, France, francois.gautier@univ-lemans.fr), Jacques Cuenca (LAUM, CNRS, Université du Maine, Lab. d'Acoustique Université du Maine, UMR CNRS 6613, 72085 Le Mans Cedex 9, France, Jacques.Cuenca.etu@univ -lemans.fr), Victor V. Krylov (Loughborough University, Department of Aeronautical and Automotive Engineering, Ashby Road, LE11 3TU Loughborough, UK, V.V.Krylov@lboro.ac.uk), Laurent Simon (LAUM, CNRS, Université du Maine, Lab. d'Acoustique Université du Maine, UMR CNRS 6613, 72085 Le Mans Cedex 9, France, Laurent.Simon@univ-lemans.fr)

It is well known that flexural waves in beams or plates slow down if their thickness decreases. A beam or a plate with a decreasing thickness in the vicinity of one of their boundaries can be designed so that the travel time needed for a wave to reach the edge becomes infinite. Thus, the reflection coefficient associated with the corresponding region is zero. This effect is referred to as the acoustic black hole effect. The use of 'acoustic black holes' in combination with thin damping layers for wedges of power-law profile has been theoretically and experimentally studied by V.V. Krylov and R.E.T.B. Winward (JSV 300 (2007) 43-49). The aim of this paper is to show experimentally the acoustic black hole effect in a two-dimensional configuration comprising an elliptical plate with a pit of power-law profile placed in one of its focuses. The elliptical shape of the plate induces a focalization of waves towards the black hole, which is observed by means of scanning laser vibrometry. Comparison of input mobility measurements for plates with and without black hole shows the reduction in vibrational level induced by the black hole effect.
2pSAe16. Characterising elastic layers as non-lightly damped SDOF systems for the reduction of impact transmission noise. Francisco Simon (Instituto de Acústica (CSIC), C/ Serrano 144, 28006 Madrid, Spain, psimon@ia.cetef.csic.es), David K. Anthony (Instituto de Acústica (CSIC), C/ Serrano 144, 28006 Madrid, Spain, iaca344@ia.cetef.csic.es)

There is a wealth of literature dealing with modal determination of lightly damped viscous systems, however there is relatively little reported for medium and heavily damped systems or with non-viscous damping. The most commonly reported and applied methods have significant errors in the determination of both modal frequency and damping ratio for systems with high damping. For the lightly damped case simplification can be often conveniently made to allow the performance of each method to be studied theoretically, however for the non-lightly damped case this is not possible and the analysis must proceed on a heuristic basis. For the application modal parameter determination of elastic floor layers designed to reduce building transmission noise by impact testing, traditional and new methods of modal parameter determination for non-lightly are presented and heuristically evaluated for both viscous and non-viscous SDOF systems. It is also shown the performance often depends on the type of damping. This either must be known a priori or can be gleaned by comparing results from different methods.

2pSAe17. Eigenvalue imaging method for subsurface defects detection. Kenbu Teramoto (Saga University, 1-Honjo, 8408502 Saga, Japan, tera @ me.saga-u.ac.jp)

This paper proposes the eigenvalue imaging method to be independent of frequency and phase velocity and to characterize the Lamb-wave field. Lamb-waves play important roles in NDE fields. Being placed on the surface of a plate, a sound source excites several kinds of waves: (a) pressure and shear waves, (b) a Rayleigh wave, and (c) symmetric and antisymmetric Lamb-waves traveling along the plate. The first two waves are utilized for traditional ultrasonic techniques such as pulse-echo methods. In contrast to these classical techniques, in which the wavelength is shorter than the plate thickness, the Lamb-wave has the advantage of propagating over large area. The variation of the Lamb-wave velocity, however, causes difficulties for interpretation of observed signals. It is important, therefore, to establish the crack detection criterion independent of local wave numbers. This paper proposes a novel imaging method which utilizes the 3 rd and 4th eigenvalues of the covariance matrix, which is defined over the 4- dimensional vector space which is spanned by following components: (1) a vertical displacement, (2) its particle velocity, (3) the orthogonal pair of out-ofplane strains. This study shows that the 3rd eigenvalue detects a wave scattered by defects and the 4 th one has an ability to localize defects. 


\title{
Session 2pSCa
}

\section{Speech Communication: Speech Perception II}

\author{
Souhila Messaoud-Galusi, Chair \\ University College London, Wolfson House, Department of Phonetics and Linguistics, 4 Stephenson Way, London, NW1 2HE, \\ $U K$
}

\section{Contributed Papers}

2:00

2pSCa1. Cue switching in the perception of approximants: Evidence from two English dialects. Rachael-Anne Knight (City University, Department of Language and Communication Science, Northampton Square, EC1V 0HB London, UK, knight@city.ac.uk), Christina Villafana Dalcher (City University, Department of Language and Communication Science, Northampton Square, EC1V 0HB London, UK, cvdalcher@mac.com), Mark J. Jones (University of Cambridge, Phonetics Lab, Department of Linguistics, Raised Faculty Building, Sidgwick Avenue, CB3 9DA Cambridge, UK, markjjones@ hotmail.com)

A surprising dissimilarity is attested in the perception of approximants by speakers of American and Standard Southern British English. Eighteen subjects (6 AE and $12 \mathrm{SSBE}$ ) performed an identification task in which they judged whether stimuli were more like $/ \mathrm{r} /$ or $/ \mathrm{w} /$. The stimuli comprised five sounds, copy-synthesised from a source $/ \mathrm{r} /$, where the values of $\mathrm{F} 2$ and F3 we adjusted to fall between the frequencies typical for ["turned $\left.r^{\prime \prime}\right]$ and $[w]$. The only significant difference between the two dialect groups' performance occurred with a token in which F3 was at a frequency typical for $/ \mathrm{r} /$ and F2 was lowered to that of /w/. AE and SSBE speakers identified this token as $/ \mathrm{r} / 90 \%$ and $59 \%$ of the time, respectively. This is unexpected as $/ \mathrm{r} /$ in both dialects is characterised by a low F3. However, the difference may be due to the existence of the 'labiodental' variant of $/ \mathrm{r} /$ in SSBE. As this variant does not have a low F3, SSBE speakers must tolerate a wider diversity of /r/-types than AE speakers. Therefore, the /r/ category in SSBE may be becoming increasingly defined by F2, rather than F3, which also has implications for the future production of $/ \mathrm{r} /$ in this accent

\section{2:20}

$2 \mathrm{pSCa}$. Dialectal effects in the perception of vowels produced by first and second language speakers: North Carolinian versus Southern Welsh listeners. Ricardo Augusto Hoffmann Bion (SISSA - Cognitive Neuroscience, VL Stock 2/2, 34135 Trieste, Italy, ricardobion@ gmail.com), Paola Escudero (University of Amsterdam, Institute of Phonetic Sciences, Spuistraat 210, 1012 VT Amsterdam, Netherlands, P.R.EscuderoNeyra@uva.nl) , Geoffrey Stewart Morrison (Australian National University, School of Language Studies, Building 110, ACT 0200 Canberra, Australia, geoff .morrison@anu.edu.au)

This paper investigated the effect of listeners' regional origin on the perception of vowels. Listeners from North Carolina and South Wales were presented with natural tokens of the four English vowels /i/, /I/, /E/ and / $/$ / which were produced by L1 and L2 speakers. Randomization tests revealed a significant difference between the listener groups' confusion matrices. Examination of the confusion matrixes suggested that the dialectal differences were primarily in the vowel contrasts /I/-/E/ and /E/-/\{/. In order to explore these findings, logistic regression models were fitted to each listener group's responses (predictor variables in the models were initial and final formant values and duration). Territorial maps based on the logistic regression models indicated that there was a substantial difference in the locations of the $/ \mathrm{E} /-/\{/$, and a smaller difference in the location of the /I/-/E/ boundary.
2:40

2pSCa3. Is there evidence of an allophonic mode of speech perception in dyslexic children. Souhila Messaoud-Galusi (University College London, Wolfson House, Department of Phonetics and Linguistics, 4 Stephenson Way, NW12HE London, UK, souhila@phon.ucl.ac.uk), Valerie Hazan (University College London, Wolfson House, Department of Phonetics and Linguistics, 4 Stephenson Way, NW1 2HE London, UK, val@ phon.ucl.ac.uk), Stuart Rosen (UCL, Wolfson House, 4, Stephenson Way, NW12HE London, UK, stuart@phon.ucl.ac.uk)

Recent studies have suggested that dyslexics perceive phonemic categories in a less categorical fashion than individuals of average reading ability due to increased within-category discrimination ('allophonic' mode of perception). In the present study, 62 dyslexic and 51 typically-developing (TD) children aged between 6;6 and 13;7 years old were tested on the perception of a /pi-bi/ continuum using an adaptive identification task presented in clear and in background noise, and a fixed-level discrimination task presented in clear. They also undertook a battery of tests of reading, IQ, phonological awareness, phonological short-term memory and word perception in noise. The gradient of the identification function was significantly weaker for the dyslexic than TD group in clear, but the two groups did not differ in the noisy condition. In the discrimination test, there was no evidence of increased within-category discrimination in the dyslexic group but the TD group showed better cross-category discrimination. These results do not support the existence of an allophonic model of speech perception in dyslexic children. Also, they do not support the view that dyslexic children are more affected by noise as a result of more poorly specified phonemic categories. [Work supported by Wellcome Trust].

\section{3:00}

2pSCa4. fMRI evidence for central auditory processing of speech in deaf infants under sedation. Scott K. Holland (Cincinnati Children's Hospital Medical Center, Imaging Research Center, 3333 Burnet Ave, Cincinnati, OH 45229, USA, scott.holland@cchmc.org), Prasanna Karunanayaka (Cincinnati Children's Hospital Medical Center, Imaging Research Center, 3333 Burnet Ave, Cincinnati, OH 45229, USA, KAR4RP@cchmc.org), Akila Rajagopal (Cincinnati Children's Hospital Medical Center, Imaging Research Center, 3333 Burnet Ave, Cincinnati, OH 45229, USA, RAJA7Y@ cchmc.org), Kristen Smith (Cincinnati Children's Hospital Medical Center, Imaging Research Center, 3333 Burnet Ave, Cincinnati, OH 45229, USA, SMIJ6D@cchmc.org)

Functional MRI (fMRI) performed in infants with congenital hearing loss provides evidence that auditory language stimulation produces activation of the central auditory system in the brain. Babies are normally sedated for clinical MRI scans. With IRB approval we have added a 10 minute fMRI scanning procedure to clinically indicated MRI scans in infants with severe to profound hearing impairment $(n=18)$ or normal hearing $(n=22)$. Sedation is performed according to clinical guidelines at our institution using either Propofol ((200-250 mcg/kg/min i.v.) or pentobarbital $(5 \mathrm{mg} / \mathrm{kg}$ orally). fMRI scanning is performed using Echo-planar gradient echo acquisitions on a 3 Tesla clinical MRI system while a mothers voice reads stories to the babies at a sound level $10 \mathrm{~dB}$ above the measured hearing threshold. Brain activation is measured in primary auditory cortex and planum temporale speech recognition areas. Results in the normal hearing group of infants demonstrates that the central auditory system is stimulated by speech in in- 
fants, although sedation does attenuate brain activation in a dose dependent manner. Central auditory and language activation is also detected in hearing impaired infants with levels of activation correlating to measured hearing thresholds.

\section{3:20}

2pSCa5. Left parietal activation during the production of pointing in several modalities: prosodic focus, syntactic extraction, digital- and ocular- pointing. Hélène Loevenbruck (Speech and Cognition Department, GIPSA-lab, 46 avenue Félix Viallet, 38031 Grenoble, France, Helene.Loevenbruck@gipsa-lab.inpg.fr), Coriandre Vilain (Speech and Cognition Department, GIPSA-lab, 46 avenue Félix Viallet, 38031 Grenoble, France, coriandre.vilain@gipsa-lab.inpg.fr), Francesca Carota (Centre de Neuroscience Cognitive, 67, Boulevard Pinel, 69675 Bron, France, Francesca.Carota@isc.cnrs.fr), Monica Baciu (Laboratoire de Psychologie et NeuroCognition, UFR Sciences de l'Homme et de la Société, BP47, 38040 Grenoble, France, monica.baciu@upmf-grenoble .fr), Christian Abry (Centre de Recherche sur 1'Imaginaire (CRI), Université Stendhal, Grenoble 3, BP 25, 38040 Grenoble cedex 9, France, christian.abru@u-grenoble3.fr), Laurent Lamalle (Plateforme régionale
IRM 3Tesla, IFR n 1, RMN Biomédicale : de la cellule à l'homme, CHU de Grenoble, BP 217, 38043 Grenoble Cedex 9, France, Laurent.Lamalle @ujf-grenoble.fr), Cédric Pichat (Laboratoire de Psychologie et NeuroCognition, UFR Sciences de l'Homme et de la Société, BP47, 38040 Grenoble, France, cedric.pichat@upmf-grenoble.fr), Christoph Segebarth (Grenoble Institut de Neurosciences, CHU de Grenoble - B.P. 217, 38043 Grenoble, France, csegebar@ujf-grenoble.fr)

Deixis, or pointing, is the ability to draw the viewer/listener's attention to an object, a person, a direction or an event. Pointing is gradually acquired by children, first with the eyes, then with the finger, then with intonation and finally with syntax. The crucial role of digital pointing in language acquisition suggests that all modalities of pointing may share a common cerebral network. An fMRI study of the production of multimodal pointing was carried out on 15 subjects. Subjects were scanned during the execution of index finger pointing gestures, eye pointing gestures, prosodic pointing (focus) and syntactic pointing (extraction). The results of a random effect group analysis show that the left superior parietal lobule (BA 7) was activated in all three digital, ocular and prosodic pointing but not in syntactic pointing. These results indicate that pointing in different modalities may recruit the left superior parietal lobule, with ocular pointing more anterior than prosodic pointing, itself more anterior than digital pointing. A grammaticalisation process is suggested to explain the lack of parietal activation in syntactic pointing.

\title{
Session 2pSCb
}

\section{Speech Communication: Speech Articulation I}

\author{
Helen M. Hanson, Chair \\ Union College, 807 Union St., Schenectady, NY 12308, USA
}

\section{Contributed Papers}

\section{2:00}

2 pSCb1. Acoustic cues to the voicing contrast in coda stops in the speech of 2-year-olds learning American English. Helen M. Hanson (Union College, 807 Union St., Schenectady, NY 12308, USA, hansonh@union.edu), Stefanie Shattuck-Hufnagel (Massachusetts Institute of Technology, Research Laboratory of Electronics, Speech Communication Group, 77 Massachusetts Ave., Cambridge, MA 02139, USA, stef@speech mit.edu)

Developing a model of how children acquire the phonological feature contrasts and phonetic cues of their adult speech community requires a detailed understanding of how production changes during development. Earlier studies based on listening provided an initial estimate of these patterns; recent instrumental studies of the acoustic and articulatory details of production during development have revealed some surprises, including covert contrasts between distinctive feature categories (e.g., Scobbie et al., 1996) and of incomplete acquisition of adult feature-cue patterns (Imbrie, 2005), which can be difficult for adults to hear. Few instrumental studies, however, have focused on the child's cue patterns for coda consonants (although see Song and Demuth, in press). Our study of cues to the feature [voice] for coda stops used quantitative acoustic analyses of tokens from the Imbrie corpus of 2-year-old speech to compare child productions with those of their adult caretakers. Results show that a) many children produce a noisy region at the end of the vowel for voiceless codas (in duck, cup) but a long strong voice bar during closure for voiced codas (in bug, tub), and b) these cues may be exaggerated versions of the feature cues of their adult caretakers.

\section{2:20}

$2 \mathrm{pSCb2}$. Are acoustic and articulatory changes of speech produced in noise only related to the increase in vocal intensity? Maeva Garnier (University of New South Wales, Music Acoustics, School of Physics, NSW 2052 Sydney, Australia, maeva.garnier@gmail.com), Nathalie Henrich (Département Parole \& Cognition, GIPSA-lab, 46, avenue Félix Viallet, 38031 Grenoble Cedex, France, Nathalie.Henrich@gipsa-lab .inpg.fr), Danièle S. Dubois (CNRS, LCPE/LAM 11 rue de Lourmel, 75015 Paris, France, ddubois@ccr.jussieu.fr)

Speech acoustics and articulation are modified in speech produced in noisy environments. Is this simply a result of the increase in vocal intensity or may these modifications be some communicative strategies to increase audiovisual intelligibility? We recorded a first acoustic database with ten speakers and a second acoustic and articulatory database with three speakers, for quiet and for two kinds of background noise. We first verified previous observations about speech modifications in noise and observed that some are not strongly correlated to the increase in vocal intensity and cannot be entirely explained by previous models of vocal effort. We also made additional observations. Pitch does not only rise in noise but also extends its dynamic. For female speakers, pitch not only rises but also fits spectrum "holes" of cocktail-party noise. Speech modifications are not only global 
over the whole utterance but also specific to some of its units. Articulatory modifications consist not only of an amplification of lip movements, but also correspond to an enhancement of the potentially visible contrast between vowels. Lastly, some articulatory changes may be compensation for formant modifications induced by the increase in vocal intensity, rather than a consequence of it.

\section{2:40}

2pSCb3. Muscle saturation effect in / $\mathbf{i} /$ production: Counterevidence from a 3D biomechanical model of the tongue. Stéphanie Buchaillard (ICP/GIPSA-lab, INPG, 46 Avenue Félix Viallet, 38031 Grenoble Cedex 01, France, stephanie.buchaillard@gipsa-lab .inpg.fr), Pascal Perrier (ICP/GIPSA-lab, INPG, 46 Avenue Félix Viallet, 38031 Grenoble Cedex 01, France, Pascal.Perrier@gipsa-lab .inpg.fr), Yohan Payan (TIMC-IMAG, Pavillon Taillefer, Faculté de Médecine, 38706 La Tronche cedex, France, yohan.payan@imag.fr)

Numerous works suppose the existence of a saturation effect to facilitate the control of the constriction area when producing vowel \il. This study exploits a 3D biomechanical Finite Element model of the vocal tract to evaluate the effectiveness of this assumption. The model includes the tongue and its major muscles, represented as a hyperelastic body, inserted in the oral cavity including jaw, palate, pharyngeal walls and hyoid bone. Muscles activations are controlled with the lambda model (Equilibrium-Point Hypothesis). After determining a set of possible motor commands to generate vowel $\backslash \mathrm{i} \backslash$, the impact of local variations of these commands on tongue shape was studied. The main tongue muscles motor commands were independently modified (by $\pm 2 \%, 5 \%, 8 \%$ around their values at target) and the effect on the tongue shape and on the acoustic signal was studied. The simulations showed a global stabilization of the tongue body, thanks to the palatal contacts, but also variability in the alveolar groove due to the anterior genioglossus activation. Though extremely localized, this variation has an important impact on the constriction size and, then, on the acoustic signal. Consequently, a specific control of the articulatory variability could be necessary to explain experimental data for vowel li〉.

\section{3:00}

2pSCb4. Compensatory tongue patterns in glossectomy patients. Emi Murano (University of Maryland Dental School, $650 \mathrm{~W}$ Baltimore St. \#8402, Department of Biomedical Sciences, Baltimore, MD 21201, USA, emurano@umaryland.edu), Maureen Stone (Vocal Tract Visualization Lab, Depts of Biomedical Sciences and Orthodontics, University of Maryland Dental School, 650 W. Baltimore St., Baltimore, MD 21201, USA, mstone@umaryland.edu), Xiaofeng Liu (The Johns Hopkins University, 3400 N Charles Street, 105 Barton Hall, Baltimore, MD 21218, USA, xiaofeng@jhu.edu), Jiachen Zhuo (University of Maryland Medical
System, 22 South Greene Street, Department of Diagnostic Radiology, Baltimore, MD 21201, USA, JZHUO@umm.edu), Andrew Salama (University of Maryland Dental School, $650 \mathrm{~W}$ Baltimore St. \#8402, Department of Biomedical Sciences, Baltimore, MD 21201, USA, asalama@umm.edu), Rao Gullapalli (University of Maryland Medical System, 22 South Greene Street, Department of Diagnostic Radiology, Baltimore, MD 21201, USA, RGULLAPALLI@umm.edu), Robert Ord (University of Maryland Dental School, 650 W Baltimore St. \#8402, Department of Biomedical Sciences, Baltimore, MD 21201, USA, rord@umm.edu), Jerry Prince (The Johns Hopkins University, 3400 N Charles Street, 105 Barton Hall, Baltimore, MD 21218, USA, prince@jhu .edu)

To examine the effects of glossectomy surgery on tongue deformation strategies, a unique combination of data was used, including internal tongue strain patterns from tagged-cine-MRI and detailed muscle architecture from high-resolution MR images. Two glossectomy patient groups were studied and compared to normal controls. They differed in the type of reconstruction after cancer excision: primary closure or reconstruction with forearm flap. All patients included in this study had the same tumor type (Squamous Cell Carcinoma), size (T1 and $\mathrm{T} 2$ ) and location (unilateral mid-third of oral tongue). The tongue developed distinct deformation strategies that move the remaining tongue tissue, the flap and the scar tissue. Compensatory patterns such as pivot points and areas of rigidity will be shown and discussed. These appear to be compensatory strategies to optimize speech output after surgery. This presentation is based on viewing the tongue as a muscular hydrostat, which provides the basis for hypotheses about the formation and release of areas of rigidity within the tongue. The rigidity might be a way to simplify sound-specific strategies for moving the tongue and controlling changes in its shape in normal and patient subjects. Supported by NIH K99DC009279, and R01DC01758 from the NIDCD.

\section{3:20}

2pSCb5. Statistical identification of critical, dependent and redundant articulators. Philip J. Jackson (University of Surrey, Centre for Vision, Speech and Signal Processing, GU2 7XH Guildford, UK, p.jackson@surrey.ac.uk), Veena D. Singampalli (University of Surrey, Centre for Vision, Speech and Signal Processing, GU2 7XH Guildford, UK, v.singampalli@surrey.ac.uk)

An automatic method for identifying critical, dependent and redundant roles in speech articulation is presented. Critical articulators are identified using the Kullback-Leibler divergence between phone-specific and model pdfs, which are initialised to the grand pdfs for each articulator. Model pdfs of critical and dependent articulators, those significantly correlated with the critical ones, are updated accordingly for both 1D and 2D cases, as long as the divergence exceeds the threshold. Those unaffected are termed redundant. Algorithm performance is evaluated on the MOCHA-TIMIT database by comparison with phonetic features. Results are also given for an exhaustive search, and principal component analysis of articulatory fleshpoints. Implications of being able to extract phonetic constraints automatically from articulatory recordings are discussed. 


\title{
Session 2pSCc
}

\section{Speech Communication: General Topics in Speech Communication II (Poster Session)}

\author{
Yukari Hirata, Cochair \\ Colgate University, Dept. of East Asian Lang. and Lit., 13 Oak Dr., Hamilton, NY 13346, USA \\ James Hillenbrand, Cochair \\ Western Michigan University, Dept. Speech Path. and Aud., 1903 W. Michigan Ave., Kalamazoo, MI 49008, USA \\ Natasha Warner, Cochair \\ University of Arizona, MPI NIjmegen, Dept. of Linguistics, Box 210028, Tucson, AZ 85721, USA
}

\begin{abstract}
All posters will be on display from 3:40 p.m. to 5:20 p.m. To allow contributors an opportunity to see other posters, contributors of odd-numbered papers will be at their posters from 3:40 p.m. to 4:30 p.m. and contributors of even-numbered papers will be at their posters from 4:30 p.m. to 5:20 p.m.
\end{abstract}

\section{Contributed Papers}

2pSCc1. A grouping approach to harmonic complexes. Dirkjan J. Krijnders (University of Groningen, Dept. Artificial Intelligence, P.O. Box 407, 9700 AK Groningen, Netherlands, j.d.krijnders@ai.rug.nl), Maria E. Niessen (University of Groningen, Dept. Artificial Intelligence, P.O. Box 407, 9700 AK Groningen, Netherlands, m.niessen@ai.rug.nl), Tjeerd C. Andringa (University of Groningen, Dept. Artificial Intelligence, P.O. Box 407, 9700 AK Groningen, Netherlands, t.andringa@ai.rug.nl)

Humans seem to perform sound-source separation for quasi-periodic sounds, such as speech, mostly on harmonicity cues. To model this function, most machine algorithms use a pitch-based approach to group the speech parts of the spectrum. In these methods the pitch is obtained either explicitly, in autocorrelation methods, or implicitly, as in harmonic sieves. If the estimation of pitch is wrong, the grouping will fail as well. In this paper we show a method that performs harmonic grouping without first calculating the pitch. Instead a pitch estimate is associated with each grouping hypothesis. Making the grouping independent of the pitch estimate makes it more robust in noisy settings. The algorithm obtains possible harmonics by tracking energy peaks in a cochleogram. Co-occuring harmonics are compared in terms of frequency difference. Grouping hypotheses are formed by combining harmonics with similar frequency differences. Consistency checks are performed on these hypotheses and hypotheses with compatible properties are combined into harmonic complexes. Every harmonic complex is evaluated on the number of the harmonics, the number of subsequent harmonics and the presence of a harmonic at the pitch position. By using the number of subsequent harmonics octave errors are prevented. Multiple concurrent harmonic complexes can be found as long as the spectral overlap is small.

2pSCc2. The role of generalization in learning perceptual contrasts. Meghan Sumner (Stanford University, Department of Linguistics, Margaret Jacks Hall, Stanford, CA 94305-2150, USA, sumner@ stanford.edu)

Understanding spoken language is a difficult task. Variation caused by accents make this task even more daunting. This project examines the generalization of novel phonetic cues by listeners of non-native speech. Native speakers of English were trained on Korean-English pronunciations of minimal pairs with word-final contrasts (e.g., bed - bet). While native English speakers rely on vowel duration to cue a final voicing contrast, native Korean speakers of English do not produce a vowel duration contrast, but use a different distinguishing phonetic cue (e.g., voicing into closure duration). Listeners were trained on a subset of minimal pairs ending in final stops (bet - bed). Following successful training, listeners participated in an immediate or long-term form priming task. Four conditions included: Trained Identity (bet - bet), Trained Non-identity (bed - bet), Novel Non-identity, Trained Rime (wet - wed), and Novel Non-identity, Novel Rime (beat - bead). Preliminary results show episodic treatment of words in the immediate priming task, but generalization to novel forms in the long-term priming task. These data suggest that early learning is mediated by episodic representations learning proceeds episodically until sufficient information is available to support a more abstract generalization.

2pSCc3. Vocalizations of deaf infants before and after cochlear implantation. Mary K. Fagan (Indiana University School of Medicine, Riley Research Wing 044, 699 West Drive, Indianapolis, IN 46202, USA, mkfagan@indiana.edu)

Critical aspects of spoken language depend on perceiving and understanding speech sounds. For deaf infants, however, neither sound perception nor awareness of the sound-making consequences of their actions is available. The goal of this study was to uncover and document early, measurable effects of hearing loss on infant vocalization and changes in these behaviors following cochlear implantation. Participants were 8 deaf infants, 7-11 months old, and 8 infants with cochlear-implants, 12-20 months old and 1-6 months post-implantation. Dependent variables include number of vocalizations, mean vocalization duration, and mean frequency before and after cochlear implantation and in relation to reduplicated babble onset and word production. Post-implant changes in vocal behaviors may have clinical implications for decisions regarding age of implantation and auditory habilitation as well as for understanding post-implant variability.

2pSCc4. Contrastive Focus in Taiwan Mandarin. Ho-Hsien Pan (Dept. of Foreign Languages and Literatures, National Chiao Tung University, 1001 TA Hsueh Road, 300 Hsinchu, Taiwan, hhpan@faculty.nctu .edu.tw), Hui-Ying Tsou (Dept. of Foreign Languages and Literatures, National Chiao Tung University, 1001 TA Hsueh Road, 300 Hsinchu, Taiwan, hytsou@ms11.hinet.net), Wan-Ting Huang (Dept. of Foreign Languages and Literatures, National Chiao Tung University, 1001 TA Hsueh Road, 300 Hsinchu, Taiwan, caissybelle@yahoo.com.tw)

This study investigated the production and perception of given, new, and contrastive information in Taiwan Mandarin. Spontaneous dialogues elicited through a game were analyzed to access the acoustical parameters marking different information structures. In perceptual studies, two perceptual experiments, (1) a dialogue history experiment, of which listeners identified the questions preceding the sentences, and (2) a pair comparison experi- 
ment, of which subject compared sentence with same wording but different information structures were used. The stimuli were either natural utterances form elicited spontaneous dialogues or resynthesized utterances with conflicting duration and $\mathrm{f} 0$ cues swapped from utterances of different information structures. Preliminary results of production experiments showed that duration elongation is a more consistent cue for marking narrow and contrastive foci than $\mathrm{f} 0$ range expansion. Listeners performed better in the pair comparison experiments than in the dialogue history experiment. For nature utterances, listeners were able to identify sentences with given information, given versus new information, and given versus contrastive information, but were not able to identify utterances with more than two contrastive foci. Listeners' performances declined when resynthesized utterances with conflicting duration and fo cues were presented. When presented with conflicting cues, listeners relied more on durational than f0 cues.

2pSCc5. Comparison of Japanese expressive speech perception by Japanese and Taiwanese listeners. Chun-Fang Huang (Japan Advanced Institute of Science and Technology, 1-1, Asahidai, Nomi, 923-1292 Sendai, Japan, chuang@jaist.ac.jp), Donna Erickson (Showa University of Music, 808 Sekiguchi, Atsugi, 243-8521 Kanagawa, Japan, EricksonDonna2000@gmail.com), Masato Akagi (Japan Advanced Institute of Science and Technology, 1-1, Asahidai, Nomi, 923-1292 Sendai, Japan, akagi@jaist.ac.jp)

Language is an important tool in speech communication. Even without the understanding of one language, we can still judge the expressive content of a voice, such as happiness or sadness. However, sometimes misunderstanding of emotional communication occurs. It is not clear what the common/different features are that help or hinder people with different culture/native-languages background in making judgments about the expressivity of speech. In order to explore this question, we focus on Japanese and Taiwanese listeners perception of Japanese expressive speech utterances. We used the perceptual model proposed by [Huang and Akagi, InterSpeech 2005; 2007], which involves a concept called "semantic primitives"-- adjectives for describing voice perception. This concept simplifies and clarifies the discussion of common/different features in terms of acoustic cues and expressive speech perception categories. Using this model, we found some common/different features in the perception of expressive speech. Specifically, our results suggest that there may be primary and secondary semantic primitives associated with acoustic speech characteristics which are involved in the perception of expressive speech, and that people from different cultures/native-language background tend to use the same primary semantic primitives in perceiving expressive speech but different secondary ones.

2 pSCc6. Acoustic analysis of the vocal tract during vowel production by finite-difference time-domain method. Hironori Takemoto (ATR Cognitive Information Science Laboratories, 2-2-2 Hikaridai, Seika-cho Soraku-gun, 619-0288 Kyoto, Japan, takemoto@atr.jp), Parham Mokhtari (ATR Cognitive Information Science Laboratories, 2-2-2 Hikaridai, Seika-cho Soraku-gun, 619-0288 Kyoto, Japan, parham@atr.jp), Tatsuya Kitamura (Konan University, Okamoto 8-9-1, Higashinada, 658-8501 Kobe, Japan, t-kitamu@konan-u.ac.jp)

An acoustic simulator based on the finite-difference time-domain (FDTD) method was evaluated by acoustic measurements on solid models of the vocal tract. Three-dimensional vocal tract (3D VT) shapes for a male subject during production of the five Japanese vowels were measured by magnetic resonance imaging. Transfer functions of the 3D VT shapes were computed by the acoustic simulator. The accuracy of the finite-difference algorithm was second-order in time and fourth-order in space. From the same 3D VT shapes, solid models were made of epoxide resin by a stereolithographic technique, and their acoustic transfer functions were measured using a time-stretched pulse signal. The calculated and measured spectra were compared up to $8 \mathrm{kHz}$. Although locations of major poles and zeros were common between calculated and measured spectra, the calculated spectra showed a larger number of small zeros, possibly caused by underestimation of VT acoustic losses in the simulation. However, the lower four formants were simulated remarkably accurately, with a mean absolute error of only $2.2 \%$ compared with the acoustic measurements. These facts indicated that the acoustic simulator can reasonably account for acoustic phenomena within the VT.

2pSCc7. Speech intelligibility determined with various tests presented against noise. Edward Ozimek (Umultowska 85, 61-614 Poznan, Poland, ozimaku@amu.edu.pl), Dariusz Kutzner (Umultowska 85, 61-614 Poznan, Poland, konsbol@wp.pl), Anna Warzybok (Umultowska 85, 61-614 Poznan, Poland, a_warzybok@ poczta.onet.pl)

The paper describes three Polish tests for assessment of speech intelligibility under noisy conditions: the matrix test, the sentence test and the digit triplet test. The matrix test contains semantically unpredictable sentences of fixed syntactical structures. This test is very useful for long-term intelligibility measurements and is applicable for subjects with profound hearing loss or cochlear implants users. The sentence test comprises utterances taken from everyday speech and aims at clinical speech intelligibility measurements. The digit triplet test contains sequences of three digits that are spoken separately and is aimed at screening measurements, especially via telephone. The statistical and phonemic properties of the tests are analyzed and compared. The correlation between results obtained in the respective tests and the tests applicability are also discussed.

2pSCc8. Consonants, but not vowels, prime lexical decision following masked priming. Boris New (CNRS - Université Paris Descartes, 71 Avenue Edouard Vaillant, 92100 Boulogne-Billancourt, France, boris.new@univ-paris5.fr), Veronica Araujo (CNRS - Université Paris Descartes, 71 Avenue Edouard Vaillant, 92100 Boulogne-Billancourt, France, veronica.cruzaraujo@gmail.com), Nathalie Bour (CNRS - Université Paris Descartes, 71 Avenue Edouard Vaillant, 92100 BoulogneBillancourt, France, psynat93@hotmail.com), Thierry Nazzi (CNRS - Université Paris Descartes, 45 rue des Saints-Pères, 75006 Paris, France, thierry .nazzi@univ-paris5.fr)

Recently, Nespor et al (2003) have argued that consonants play a more important role at the lexical level than vowels. This proposal has received direct support from studies showing that 16/20/30-month-old infants are better at processing specific consonantal than vocalic information while learning new words (Nazzi, 2005; Nazzi \& New, 2007). This proposal is also supported, although indirectly, by studies on adults. Adults have been found to rely more on consonants than on vowels when asked to transform a nonword into a real word (Cutler et al., 2000). Moreover, adults presented with a continuous stream of syllables are able to segment it into "lexical units" by relying on consonantal but not vocalic information (Bonatti et al., 2005). In our study, we directly investigate this issue using a visual masked priming lexical decision task (an oral version being currently implemented). The test items are bisyllabic (CVCV or VCVC) and trisyllabic (CVCVCV or VCVCVC) words, which are presented following four different primes: identity (e.g., joli-joli), unrelated (vabu-joli), same consonants (jalu-joli) and same vowels (vobi-joli). Priming was found for the identity and same consonants conditions, not for the same vowels condition, supporting the privileged role of consonants at the lexical level.

2pSCc9. Frequency importance function of Mandarin Chinese speech. Jing Chen (Dept. of Machine Intelligence, Speech and Hearing Research Center, 2 Science Building, Peking Univ., 5 Yeheyuan Road, Haidian District, 100871 Beijing, China, chenj@cis.pku.edu.cn), Tianshu S. Qu (Dept. of Machine Intelligence, Speech and Hearing Research Center, 2 Science Building, Peking Univ., 5 Yeheyuan Road, Haidian District, 100871 Beijing, China, qutianshu@cis.pku.edu.cn), Xihong H. Wu (Dept. of Machine Intelligence, Speech and Hearing Research Center, 2 Science Building, Peking Univ., 5 Yeheyuan Road, Haidian District, 100871 Beijing, China, wxh@cis.pku.edu.cn), Qiang Huang (Dept. of Machine Intelligence, Speech and Hearing Research Center, 2 Science Building, Peking Univ., 5 Yeheyuan Road, Haidian District, 100871 Beijing, China, QIANG 
.HUANG@SPREADTRUM.COM),Ying Huang(Dept. of Psychology, Peking Univ., 5 Yeheyuan Road, Haidian District, 100871 Beijing, China, innhuang@ gmail.com), Liang Li (Dept. of Psychology, Peking Univ., 5 Yeheyuan Road, Haidian District, 100871 Beijing, China, liangli@pku.edu.cn), Huisheng S. Chi (Dept. of Machine Intelligence, Speech and Hearing Research Center, 2 Science Building, Peking Univ., 5 Yeheyuan Road, Haidian District, 100871 Beijing, China, chi@pku.edu.cn)

The speech intelligibility index (SII) theory objectively assesses speech intelligibility, and the frequency-importance function (FIF), which reflects the relative importance of various frequency bands to speech intelligibility for various languages, occupies the central part of the theory. However, the FIF has not been examined for tonal Chinese Mandarin speech. In this study, considering the characteristics of Mandarin speech, 50 phonemicallybalanced one-syllable words were selected as the speech stimuli from the speech corpus "A Method for Calculating Articulation Index (GB/T 155081995)", which meets the National Standards of China, and the 1/3 octave FIF was measured and computed with the Fletcher's method. The results show that spectrum regions with frequencies $2000-4000 \mathrm{~Hz}$ are more important in Chinese speech than those in English speech. The results predicted by SII model with the new FIF fit the result of human psychophysical studies well, which indicate that the new FIF model is effective and appropriate for assessing the intelligibility of Mandarin speech even when a steady-state noise masker is co-presented. [Supported by the NSFC 60435010; 60535030; 60605016; 30670704]

2pSCc10. Arabic Diagnostic Rhyme Test using minimal pairs. Bachir Boudraa (USTHB Faculté d'électronique et d'informatique, BP 32, El-Alia, 16031 Alger, Algeria, b.boudraa@yahoo.fr), Malika Boudraa (USTHB Faculté d'électronique et d'informatique, BP 32, El-Alia, 16031 Alger, Algeria, mk.boudraa@yahoo.fr), Bernard Guerin (INPG, Avenue Félix Viallet, 38031 Grenoble, France, Bernard.Guerin@enserg.fr)

In this paper, we try to adapt to Arabic the diagnostic rhyme test (DRT) developed by Voiers for English and adapted to French by Peckels \& Rossi. DRT is specific to each language and is essential to subjective assessments of coders and synthesizers. Six Arabic pertinent dimensions are used which are acuteness, compactness, tenseness, stridence, nasality and flatness. 72 monosyllabic meaningful pairs of words called minimal pairs have been developed. The apprehensibility of every attribute is tested in each of six vowel contexts. Every feature has been repeated six times and has been paired twice with every vowel.

2pSCc11. A fascinating personage in the history of phonetics: The phonetics and the politics of Elise Richter. Natasha Warner (University of Arizona, MPI NIjmegen, Box 210028, Dept. of Linguistics, Tucson, AZ 85721-0028,USA, nwarner@u.arizona.edu)

Elise Richter (1865-1943) was the first woman lecturer or professor in Austria or Germany, and she was also an experimental phonetician and even an experimental phonologist. Richter's career was delayed by the roles allowed for women at the time, and the end of her career, and her life, came about through the Nazis' policies toward Jews. Richter began her scientific work in Romance philology, but decided to seek explanations for historical sound change in phonetics. This led her to a productive career in experimental phonetics, and she even applied phonetic methods to study questions of phonological structure. Richter was a leader in exploring new methods and bringing together questions across widely differing fields. Although her specific experimental findings were superseded with time, Richter's refusal to be constrained by the limits of a field led her to ideas that have become current in phonetics just recently. In addition to her scientific career, Richter was politically active, both in working to expand women's opportunities and in general politics. This poster presents a new perspective on Richter's contributions to phonetics and on her view of feminism. Furthermore, it makes new observations of a parallel between Richter's political activities and her scientific work.
$2 \mathrm{pSCc12}$. The role of tongue articulation for $/ \mathrm{s} /$ and $/ \mathrm{z} /$ production in whispered speech. Hirohide Yoshioka (University of Tsukuba, 1-4-5 Shinmei, Urawa, 336-0023 Saitama, Japan, hirohide-y@ mtc.biglobe.ne.jp)

Although the timing of the initiation and cessation of vocal fold vibrations is crucial to characterize the voiced and voiceless cognates, other cues, such as the duration of preceding vowels, the patterns of the formant transitions in the following vowels, and the period of stop closure, may also play important roles in performing these distinctions. The present study is to further investigate the role of tongue articulatory movements during voiced and unvoiced consonant production, specifically when the vocal folds do not vibrate during the production of fricative consonants, /s/ and /z/ in whispered speech. A normal Japanese speaker served as the subject. The palato-lingual contact patterns during the intervocalic consonant $/ \mathrm{s} /$ and $/ \mathrm{z} /$ in whispered speech were recoded using dynamic electro-palatography. The results show that the area of palato-lingual contact is clearly wider during /z/ production than /s/ production; the opening for fricative turbulent noise production is narrower and longer for $/ \mathrm{z} /$ than for $/ \mathrm{s} /$. In addition, the contact pattern is unstable for $/ \mathrm{s} /$ production.

2pSCc13. Production and perception of V1V2 described in terms of formant transition rates. René Carré (Laboratoire Dynamique du Langage, UMR 5596, CNRS, Université Lyon 2, 14 Avenue Marcelin Berthelot, 69363 Lyon cedex 07, France, recarre@wanadoo.fr)

Vowels can be produced with static articulatory configurations leading to stable formant frequencies (targets). Here, an algorithm computes area functions according to the criterion of minimal deformation leading to maximal acoustic variations. Within this evolutionary dynamics, the deformations of the tube are not performed to reach targets, unknown during the process, but to move in the acoustic space in order to increase acoustic contrast. The corresponding formant trajectories in the acoustic space can be described in terms of formant transition rates. For example, following this dynamic approach, to produce [ae] from [a], the transition rates of $\mathrm{F} 1$ and $\mathrm{F} 2$ are necessary and sufficient to represent $[\mathrm{e}]$ and at the very beginning of the transition and throughout the transition there is sufficient information to detect [e]. This assertion means that the transition duration is more or less constant. Studies of V1V2 production and perception characterized by their formant transition rates are presented. Such a representation leads to new interpretations of vowel reduction, coarticulation, perceptual overshoot, hyper and hypo speech, normalization.

2pSCc14. The effect of facal dynamics on infant perception of adult-directed speech in noise. Linda Polka (McGill University, School of Communication Sciences and Disorders, Beatty Hall, 1266 Pine Avenue West, Montreal, QC H3G 1A8, Canada, linda.polka@mcgill.ca), Robin Panneton (Virginia Polytechnic Instit. \& State Univer., Williams Hall, Blacksburg, VA 24061-0436, USA, Panneton@vt.edu), Jessica Versele (Virginia Polytechnic Instit. \& State Univer., Williams Hall, Blacksburg, VA 24061-0436, USA, Versele@ vt.edu)

Recently Polka and Rvachew (in press) found that 6- to 8-month-old infants discriminated a native contrast("bu" - "gu") when familiarized and tested with syllables presented in quiet, but not when familiarized with syllables mixed with a competing noise, even when testing was conducted in quiet. Because the competing noise (segments of cricket and bird song) did not create energetic masking, infant failure to encode the familiarization syllable was due to a disruption in attention to the speech stream. Importantly, in this study, infants watched a checkerboard while listening to syllables spoken in an adult-directed (AD) style. The current study investigated if the addition of a dynamic face facilitates infants' speech processing in the same task. Six-month-olds were habituated to repetitions of the native syllable "ba" mixed with the same noise but these infants saw and heard syllables produced in AD style. Following habituation, infants were presented familiar (repetitions of "ba") and novel test trials (repetitions of "ga"). Infants failed to show syllable discrimination as indexed by a reliable preference for 
the novel test trials. These findings show that a dynamic face producing AD speech does not engage infant attention to speech enough to support syllable discrimination in noise.

2pSCc15. Voicing offsets and onsets in relation to intraoral pressure values in lingual obstruents of German. Laura L. Koenig (Haskins Labs. and Long Island Univ., Brooklyn, New York, NY 11201-8423, USA, koenig@ @askins.yale.edu), Susanne Fuchs (ZAS, Schuetzenstr. 18, 10117 Berlin, Germany, fuchs@zas.gwz-berlin.de)

Phonation requires that tracheal pressure remain higher than intraoral pressure (Pio). In obstruent consonants, a major constriction in the upper vocal tract yields an increase in Pio, inhibiting phonation. The degree of Pio increase in consonants varies as a function of laryngeal and supraglottal apertures. Voiceless stops involve a rapid buildup and discharge of Pio, whereas fricatives involve more gradual changes in Pio. This work quantifies phonation offsets and onsets in German obstruents in relation to the Pio at these times. Pio signals were recorded via a pressure transducer affixed to the posterior end of an EPG palate while 9 speakers of standard German produced intervocalic voiceless consonant sequences (stops, fricatives, affricates, and clusters). Past theoretical work suggests that phonation offsets and onsets will show a hysteresis effect, with onsets requiring higher driving pressures than offsets. Of particular interest here is whether the extent of hysteresis differs among stops, fricatives, and obstruent sequences. Data on intraoral pressure change will also be compared with EPG data to explore how supraglottal constrictions affect Pio, and thus, phonation.

2pSCc16. Magnetic Resonance investigation of palatalized stop consonants and spirants in Russian. Galina Y. Kedrova (Moscow State Lomonosov University, GSP-2, Leninskije Gory, MGU, 1st Humanities, R. 983, Centre for New Technologies in Humanities, 119992 Moscow, Russian Federation, kedr@philol.msu.ru), Nikolay V. Anisimov (Moscow State Lomonosov University, GSP-2, Leninskije Gory, MGU, 1st Humanities, R. 983, Centre for New Technologies in Humanities, 119992 Moscow, Russian Federation, kedr@philol.msu.ru), Leonid M. Zaharov (Moscow State Lomonosov University, GSP-2, Leninskije Gory, MGU, 1st Humanities, R. 983, Centre for New Technologies in Humanities, 119992 Moscow, Russian Federation, kedr@philol.msu.ru), Yurij A. Pirogov (Moscow State Lomonosov University, GSP-2, Leninskije Gory, MGU, 1st Humanities, R. 983, Centre for New Technologies in Humanities, 119992 Moscow, Russian Federation, kedr@philol.msu.ru)

The research in question is aimed at the experimental MRI-investigation of articulatory gestures corresponding to the palatalized Russian stop con-

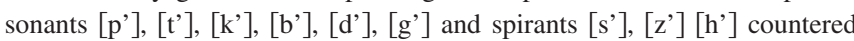
with their non-palatalized counterparts $[\mathrm{p}],[\mathrm{t}],[\mathrm{k}],[\mathrm{b}],[\mathrm{d}],[\mathrm{g}],[\mathrm{s}],[\mathrm{z}][\mathrm{h}]$ in Russian speech production. An experimental dataset was based upon 2D MR-images, audio- and video recordings taken from 4 native speakers of Russian (3 males and 1 female) producing VCCV sequencies (Russian pseudo-words with the second vowel stressed) containing Russian consonants under investigation in the vocalic contexts [a]_[a] and [a]_[i]. All speaking subjects had standard pronunciation and were without any perceptible articulation disease. MRI investigation of the Russian consonantal phonemes was based upon admitted procedures and techniques though expanded with several new original methods elaborated by the Russian team of experimentalists. Experimental data was collected through several MRI sessions (done in a month and in a year with participation of the same speaking subjects), ensuring thereby credibility and robustness of the experimental results. A detailed analysis of the whole dataset of MR-images of palatalised Russian consonants countered with their non-palatalized counterparts revealed special articulatory pattern of palatalisation in Russian.

2pSCc17. Duration of Japanese singleton and geminate obstruents in two- to four-mora words. Yukari Hirata (Colgate University, 13 Oak Drive, Department of East Asian Languages and Literatures, Hamilton, NY 13346, USA, yhirata@mail.colgate.edu), Hiroaki Kato (ATR Cognitive
Information Science Laboratories, 2-2-2 Hikaridai, Seika-cho Soraku-gun, 619-0288 Kyoto, Japan, kato@atr.jp), Keiichi Tajima (Hosei University, 2-17-1 Fujimi, Chiyoda-ku, 102-8160 Tokyo, Japan, tajima@hosei.ac.jp)

Hirata and Whiton [J. Acoust. Soc. Am. 118, 1647-1660 (2005)] found an invariant durational structure for Japanese stop quantity distinction in two- and three-mora words across different speaking rates. The present study examined whether their finding extends to include fricative and affricate quantity distinctions and three- and four-mora words. Stimuli were stop, fricative, and affricate contrasts in four types of words, including (1) a long vowel (e.g., ka.so.o vs. ka.s.so.o), (2) a moraic nasal (e.g., ji.se.n vs. ji.s.se .n), (3) CV sequence (e.g., ho.so.ku vs. ho.s.so.ku), and (4) shorter words (e.g., i.shi vs. i.s.shi), spoken in isolation at three speaking rates by four native Japanese speakers. Duration of contrasting obstruents, words, and the interval between the onsets of the first and the second vowels (VOI) was measured. Results indicated durational patterns similar to those found previously for stop contrasts. In addition, duration of words, regardless of their segmental composition, reflected well the number of moras they contained. Finally, the ratio of the contrasting obstruent to the word and the ratio of the VOI to the mean mora duration were useful in classifying the singleton and geminate categories across rates. [Supported by Grant-in-Aid for Scientific Research, JSPS]

2pSCc18. Effects of hand gesture and lip movements on auditory learning of second language speech sounds. Spencer Kelly (Colgate University, 13 Oak Drive, Department of Psychology, Hamilton, NY 13346, USA, skelly@mail.colgate.edu), Yukari Hirata (Colgate University, 13 Oak Drive, Department of East Asian Languages and Literatures, Hamilton, NY 13346, USA, yhirata@mail.colgate.edu), Jen Simester (Colgate University, 13 Oak Drive, Department of Psychology, Hamilton, NY 13346, USA, jsimester@gmail.com), Jackie Burch (University of Rochester, 585 Elmwood Ave., Box 645, Rochester, NY 14642, USA, Jaclyn_Burch @ mail.urmc.rochester.edu), Emily Cullings (Colgate University, 13 Oak Drive, Department of East Asian Languages and Literatures, Hamilton, NY 13346, USA, ecullings@mail.colgate.edu), Jason Demakakos (Colgate University, 13 Oak Drive, Department of Psychology, Hamilton, NY 13346, USA, jdemakakos@mail.colgate.edu)

Previous research found that auditory training helps native English speakers to perceive phonemic vowel length distinction in Japanese, but that their performance has never reached native levels (Hirata et al., 2007). Given that multimodal information, such as hand gesture and lip movements, influences semantic aspects of language processing and development (Kelly et al., 2002), we examined whether this multimodal information helps to improve native English speakers' ability to perceive Japanese vowel length distinction. Forty-five native English speakers participated in one of three types of training: (1) audio alone; (2) audio with hand gestures; and (3) audio with lip movements and hand gestures. Before and after training, participants were given phoneme perception tests that measured their ability to distinguish between short and long vowels in Japanese, e.g., /kato/ versus /kato:/. Our original prediction was that more modalities in training would result in greater learning. Although all three groups improved from pre- to post-test, there were no significant differences among the three training groups. Unlike the original prediction, hand gestures and lip movements did not seem to augment learning of difficult sound distinctions. We will discuss possible benefits and limitations of using multimodal information in second language speech learning.

2pSCc19. MRI-based 3-D vocal tract acoustic analysis of an American English lateral sound. Xinhui Zhou (Speech communication lab, Institute of systems research and department of electrical and computer engineering, University of Maryland, College Park, MD 20742, USA, zxinhui @ umd.edu), Carol Y. Espy-Wilson (Speech communication lab, Institute of systems research and department of electrical and computer engineering, University of Maryland, College Park, MD 20742, USA, espy 
@ glue.umd.edu),Mark Tiede(Haskins lab and MIT Research lab of electronics, 300 George street suite 900, New Haven, CT 06511, USA, tiede@haskins.yale.edu), Suzanne Boyce (Department of communication sciences and disorders, University of Cincinnati, Mail location 0394, Cincinnati, OH 45267, USA, boycese@email.uc.edu)

The lateral sound /1/ in American English involves a complex articulatory configuration that includes one or two lateral channels along the sides of the tongue, and sometimes a lingual-alveolar contact. The acoustic characteristics of /1/ consist of a third formant (F3) that is close in frequency to the F3 of the adjacent vowel(s), but is often considerably weaker in amplitude. Generally, there is relatively weak energy in the F3-F5 region. The articulatory-acoustic relationship is not well understood. Previous studies did not consider the 3-D vocal tract model and the area function extraction was based on some assumed model. In this study, we constructed a 3-D vocal tract geometry based on magnetic resonance images from one subject during sustained production of syllabic dark /1/ (as in "pole"), and performed finite-element analysis. The harmonic analysis showed that the frequency response of the 3-D vocal tract is comparable in many ways to the spectrum of the acoustic signal, suggesting good agreement between the actual and reconstructed vocal tracts. A pole-zero pair is found in F3-F4 region, which may explain the weak energy level in this frequency region. Application of the 3-D wave propagation property to vocal tract area function extraction will also be discussed. [Research supported by NIH.]

2 pSCc20. The acoustics of yer and non-yer vowels [e] and [o] in Slovak Stefan Benus (Constantine the Philosopher University, Stefanikova 67, 94901 Nitra, Slovakia, sbenus@ukf.sk)

Yers of Slavic languages are vowels that alternate with zero and historically developed from high short lax vowels. In Slovak, both front and back yers were preserved and surface as [e] and [o] respectively. For example, the second [o] in kotol 'cauldron-Nom-Sg' is a yer because it disappears with adding a suffix vowel: kotla 'cauldron-Gen-Sg'. Compare with a non-yer [o] in kostol 'church-Nom-Sg', kostola 'church-Gen-Sg'. Traditional phonological accounts of this difference (e.g. Rubach 1993) assume that yer vowels are underlyingly different from non-yer vowels and merge with [e] and [o] through a phonological process. Therefore, these accounts predict that yer and non-yer vowels should be phonetically identical since they enter the phonetic component already merged as [e] or [o]. The results of our acoustic experiments show that yer vowels are phonetically different from non-yer vowels. The most salient differences were observed in the first formant and duration: yers have lower F1 than non-yers, and for some subjects they are also shorter. This finding supports the view that the phonetic component has access to deep phonological alternations and that phonetics-phonology is a single cognitive system in which the components have different granularities and interact bidirectionally.

2pSCc21. Behavioral and physiological correlates of language preference. Megha Sundara (UCLA Department of Linguistics, 3125 Campbell Hall, Los Angeles, CA 90095-1543, USA, megha.sundara@humnet.ucla.edu), Christine Kitamura (MARCS Auditory Laboratories, Bankstown Campus, Building 1, University of Western Sydney, Locked Bag 1797, 1797 Penrith South DC NSW, Australia, c.kitamura@uws.edu.au), Thierry Nazzi (CNRS - Université Paris Descartes, 45 rue des Saints-Pères, 75006 Paris, France, thierry.nazzi@univparis5.fr)

With increasing experience listening to language, infants' sensitivity to the patterns of their native language becomes more specialized. In monolingual, hearing infants, this tuning-in into the native language has been demonstrated in an elegant experiment. Hayashi et al. (2001) tested Japaneselearning infants between 4 and 14 months on their preference for listening to Japanese when compared to English. Not surprisingly, Japanese-learning infants preferred to listen to Japanese over English. Critically, the magnitude of this native language preference increased linearly from 4- to 14-months. In this study, we replicated and extended Hayashi et al.'s results. We tested 65 Australian-English learning infants aged 3 - 16 months on their preference for Australian-English compared to Japanese sentences. Our results confirm the findings of Hayashi et al.: Australian-English hearing infants' prefer their native language, and this preference increases linearly with age.
We also tested preference when the same infants heard Australian-English and German sentences. English and German are rhythmically similar, while differing in segmental characteristics. Results indicate that similar to early language discrimination, rhythmic distance affects the emergence of language preference. We are currently analysing concomitant heart rate measured from these infants when they were listening to native and non-native sentences.

2pSCc22. Improvement of speech recognition thresholds by spectral modulation enhancement. Chang Liu (University of Texas, Dept. of Comm. Sci. \& Dis., 1 University Station A1100, Austin, TX 78712, USA, changliu@mail.utexas.edu), David A. Eddins (University of Rochester, Department of Otolaryngology, Rochester, NY 14618, USA, David_Eddins @URMC.Rochester.edu)

Our previous studies have shown that vowel identification in noise was significantly improved by spectral enhancement resulting from modifications in the spectral modulation domain. The present study investigates whether spectral enhancement results in improved speech recognition in background competition. Speech recognition thresholds (SRT) were measured in a long-term speech shaped (LTSS) noise and in multi-talker babble using NU-6 words with and without spectral enhancement for young normal-hearing listeners. Results indicated that SRTs were significantly reduced, by $2.2 \mathrm{~dB}$, in LTSS noise and in babble when speech sounds were spectrally enhanced between 2.0 and 2.5 cycles/octave, while no significant changes in SRTs occurred for enhancement between 1.5 and 2 cycles/octave or between 1.5 and 2.5 cycles/octave. Spectral modulation transfer functions were also measured for each listener. Linear regression analyses showed that SRT improvement was moderately correlated with modulation detection thresholds at a spectral modulation frequency of 0.5 cycles/octave. Thus, it appears that better modulation detection at low spectral modulation frequencies is associated with greater improvement in word recognition in background competition.

2pSCc23. Perception of sinewave vowels. James M. Hillenbrand (Western Michigan University, Dept of Speech Path \& Aud., 1903 W. Michigan Ave., Kalamazoo, MI 49008, USA, james.hillenbrand@wmich.edu), Michael J. Clark (Western Michigan University, Dept of Speech Path \& Aud., 1903 W. Michigan Ave., Kalamazoo, MI 49008, USA, michael.clark@wmich.edu)

There is a significant body of research examining the intelligibility of sinusoidal replicas of naturally spoken utterances. Discussion has followed about what the sinewave speech phenomenon might imply about the auditory and perceptual mechanisms that are involved in phonetic recognition. However, this work has typically been conducted using meaningful and syntactically well formed sentences, making it unclear what the relative contributions are of higher level, top-down processes as opposed to lower level auditory and phonetic mechanisms. The simple purpose of this study was to measure vowel intelligibility using sinusoidal replicas of naturally spoken $/ \mathrm{hVd} /$ syllables. The sinusoidal signals were modeled after 300 utterances selected from a database of 1,668 syllables spoken by men, women, and children [Hillenbrand, Getty, Clark, and Wheeler, J. Acoust. Soc. Am., 97, 3099-3111 (1995)]. Listeners consisted of 74 students enrolled in an introductory phonetics course. Recognition rates for the sinusoidal vowels averaged $55 \%$, with considerable variability across listeners. This figure is substantially lower than the $\sim 95 \%$ intelligibility (measured in several earlier studies) of the naturally spoken signals upon which the sinusoidal replicas were modeled. Attempts to improve performance using three different training methods met with modest success, with post-training recognition rates rising by $\sim 5-12$ percentage points, depending on the training method.

2pSCc24. Complexity of acoustic-production-based models of speech perception. Geoffrey Stewart Morrison (Australian National University, School of Language Studies, Building 110, ACT 0200 Canberra, Australia, geoff.morrison@anu.edu.au)

Discriminant analysis models trained on acoustic vowel production data have been found to have significant correlation with listeners' perception. Two regularised discriminant analysis models were trained on monolingual speakers' vowels. One model was trained on North Central Peninsular Span- 
ish vowel tokens, and the other on Western Canadian English vowel tokens. For each language the model which resulted in the lowest cross-validated classification error rate was close to the least complex model possible, i.e., close to linear discriminant analysis using the variances of each acoustic variable but not the covariances between variables. In order to make crosslanguage vowel perception predictions the Spanish model was used to classify English vowel tokens and the English model was used to classify Spanish vowel tokens. Results suggest that monolingual North Central Peninsular Spanish listeners would assimilate most tokens of Western Canadian English /i/ and /I/ to Spanish /i/ and /e/ respectively, and thus for this combination of dialects, Spanish-speaking learners of English would not be expected to have difficulty with the English /i/-/I/ contrast.

2pSCc25. Letter sound and letter name recognition for automated literacy assessment of young children. Shrikanth Narayanan (University of Southern California, 3740 McClintock Ave, EEB430, Los Angeles, CA 90089-2561, USA, shri@sipi.usc.edu), A Kazemzadeh (University of Southern California, 3740 McClintock Ave, EEB430, Los Angeles, CA 90089-2561, USA, kazemzad@usc.edu), Matthew Black (University of Southern California, 3740 McClintock Ave, EEB430, Los Angeles, CA 90089-2561, USA, Matthew.Black@usc.edu), Joseph Tepperman (University of Southern California, 3740 McClintock Ave, EEB430, Los Angeles, CA 90089-2561, USA, tepperma@usc .edu), Sungbok Lee (University of Southern California, 3740 McClintock Ave, EEB430, Los Angeles, CA 90089-2561, USA, sungbok1@usc.edu), Abeer Alwan (University of California, 405 Hilgard Ave, Los Angeles, CA 90095, USA, alwan@ee.ucla.edu)

Evaluations of letter naming and letter sounding are commonly used to measure a young child's growing reading ability, since performance in them is well-correlated with future reading development. Assessing a child's oral reading skills requires teachers, as well as technologies that attempt to automate such assessment, to form an item-level accept/reject decision based on speech cues and prior knowledge of the child's literacy level and linguistic background. With data collected from $171 \mathrm{~K}-2$ children, both learners and native speakers of American English, we designed and evaluated an automated letter naming assessment method using a simple word-loop HMM decoding for the word-level letter names. The automated accept/reject evaluation performance, $81.9 \%$, approached the agreement of human raters, $83.2 \%$ (0.62 kappa). However, the task where children must produce the sound that the letter represents was more difficult: English orthography allows one-to many letter-to-sound mapping, teachers showed less agreement in their assessment $(80.9 \%, 0.55 \mathrm{kappa})$, and the brief durations of some of the letter sounds made it difficult to distinguish them from each other and from background noises. Phone-level HMM based evaluation accuracy was $58.2 \%$. Preprocessing the recordings into speech, silence, and noise improved these results, especially for plosive sounds. [Supported by NSF]

2pSCc26. Neighbors as competitors: Phonological analysis of spoken word recognition errors. Adam Buchwald (NYU, 665 Broadway, Suite 910, New York, NY 10012, USA, buchwald@nyu.edu), Robert A Felty (Indiana University - Speech Research Laboratory, 1101 E 10th St., Dept. of Psychology, Bloomington, IN 47401, USA, robfelty@indiana.edu), David B. Pisoni (Indiana University - Speech Research Laboratory, 1101 E 10th St., Dept. of Psychology, Bloomington, IN 47401, USA, pisoni@indiana.edu)

Theories of speech perception and spoken word recognition widely assume that phonetically similar words compete for selection during lexical access. These competitors - called lexical neighbors - are classically defined as differing by a single segment, with little attention paid to other levels of phonological structure such as featural content or syllabic structure. This paper reports on analyses of over 15,000 word recognition errors made on a representative sample of the lexicon $(\mathrm{N}=1428)$ that includes the same variety of syllable structures, lengths, and lexical frequencies that exists in the English lexicon. The analyses reveal that competitors share segmental information as has been previously suggested, but that the classic definition of neighbor cannot capture the level at which featural and syllabic properties of the target constrain the error responses. We will discuss several analyses on these issues and discuss the implications for our understanding of the organization of the lexicon. [Work supported by NIH-NIDCD R01 00111 and T32 00012]

2pSCc27. The prosody of second position clitic placement and focus in Croatian. Kristine Yu (Dept. of Linguistics, University of California, 3125 Campbell Hall, Los Angeles, CA 90095, USA, krisyu@humnet.ucla .edu)

Since Browne 1974, the placement of second position clitics in Bosnian/Croatian/Serbian has inspired debate about interaction at the syntaxphonology interface. The placement of these clitics can alternate quite freely: either after the first phonological word or after the first syntactic constituent. While it's generally agreed that prosodic phonology, in addition to morphosyntax, plays a role in clitic placement in Bosnian/Croatian/Serbian, the prosodic patterns for the different placements have not been studied acoustically. In addition, it has been suggested that clitic placement and pragmatic focus may interact (Bošković 2001), but this has not been systematically studied. We recorded adult Zagreb Croatian speakers producing subject noun phrases with initially stressed trisyllabic adjectives and nouns in transitive sentences. We varied clitic placement (after the first word or first constituent) and focus domain (broad focus, and narrow focus on adjective, noun, both the adjective and the noun, or the entire noun phrase) and controlled for dialect and pitch accents. Preliminary results from three speakers indicate that gross differences in prosodic patterns occur only as a function of focus domain but not clitic placement. Further work will confirm if these patterns hold for more speakers and if clitic placement is correlated with finer-grained prosodic differences.

2pSCc28. English /r/-/l/ pronunciation training for Japanese speakers. Kota Hattori (University College London, Department of Phonetics and Linguistics, 4, Stephenson Way, NW1 2HE London, UK, k.hattori@ucl.ac.uk), Paul Iverson (University College London, Department of Phonetics and Linguistics, 4, Stephenson Way, NW1 2HE London, UK, p.iverson@ucl.ac.uk)

Previous studies have demonstrated that perceptual training improves both perception and production by adult second-language (L2) learners. The present study examined whether production training likewise improves both perception and production. Japanese speakers underwent ten sessions of production training for English $/ \mathrm{r} /$ and $/ 1 /$. The training combined three methods: (1) subjects received audio-visual examples and pronunciation instructions for $/ \mathrm{r} /$ and $/ 1 /$; (2) they produced mono syllables (i.e., /ra/ and /la/) and minimal-pair words, with online feedback about their formant frequencies using a real-time spectrogram as well as pronunciation feedback from an instructor; and (3) they made recordings of minimal-pair words and compared them to "enhanced" versions of these recordings (i.e., signalprocessed versions with the correct F3 frequencies and transition durations). All participants completed a battery of pre/post training tests (i.e., English $/ \mathrm{r} /$-/1/ identification, discrimination, perceptual mapping of best exemplars, and production). The preliminary results indicate that this training approach improves production; the results will be further discussed in terms of its effect on perception and the underlying representation of these categories.

2pSCc29. Production of American English [eI] and $[\varepsilon]$ by Mandarin speakers: mono- vs. disyllabic words. Chung-Lin Yang (Dept. of Linguistics, Indiana Univ.-Bloomington, Memorial Hall 322, Bloomington, IN 47405, USA, cy1@indiana.edu)

Mandarin productions of English tense vs. lax vowels are difficult for English speakers to differentiate (Chen et al. 2006). In this study the production of American English [eI] and $[\varepsilon]$ by Mandarin and American participants was investigated. The target vowels were embedded in monosyllabic and disyllabic words with a stop-V-voiceless stop context in carrier sentences of variable length. There were two formant measurements for each vowel (20\% after the onset and before the end of the vowel). In the monosyllabic condition, Mandarin speakers' performance was similar to American speakers in duration and formant values except that $[\mathrm{eI}]$ started too low. However, in the disyllabic condition, Mandarin speakers' formants for $[\mathrm{eI}]$ and $[\varepsilon]$ were overlapped and there was no significant durational distinction between $[\mathrm{eI}]$ and $[\varepsilon]$. One possible account for this difference is that 
when producing a disyllabic word, Mandarin speakers tend to shorten the vowel duration of the first syllable to compensate for the production of the following syllable. The difference in syllable conditions may reflect a tendency that Chinese regularizes word durations regardless of the number of syllables. Reference: Chen, Y. et al. (2006). Vowel production by Mandarin speakers of English. Clinical linguistics and phonetics, 15, 427-440

$2 \mathrm{pSCc} 30$. A numerical analysis of fluctuations in pressure wave within the larynx using two-dimensional asymmetrical vocal folds model. Hideyuki Nomura (Dept. of Electronic Eng., Univ. of ElectroCommunications, 1-5-1, Chofugaoka, 182-8585 Chofu-shi, Japan, nomu@ee.uec.ac.jp), Tomoo Kamakura (Dept. of Electronic Eng., Univ. of Electro-Communications, 1-5-1, Chofugaoka, 182-8585 Chofu-shi, Japan, kamakura@ee.uec.ac.jp), Tetsuo Funada (Div. of Electronic Eng. and Computer Sci., Kanazawa Univ., Kakuma-machi, 920-1192 Kanazawa-shi, Japan, funada@t.kanazawa-u.ac.jp)

Numerical simulations of pathological voice production and estimations of pressure wave fluctuations are performed based on a two-dimensional asymmetrical vocal folds (VFs) model. The asymmetrical VFs model takes into account of geometrical asymmetries (the thickness, effective depth of vibration region, and lateral rest position) and mechanical asymmetries (the Young's modulus, density, and viscosity of VF tissues). Simulation results based on the asymmetrical VFs model show that the left and right VFs vibrate with a phase difference. Obtained pressure waves within the larynx and vocal tract indicate fluctuations of fundamental frequency, amplitude, and waveform. In order to quantitatively evaluate the fluctuations, the coefficient of variation of the fundamental frequency, the coefficient of variation of the amplitude, and the harmonic-to-noise ratio are estimated. With increasing the VF asymmetries, especially on the effective depth and the density of VF elements, remarkable fluctuations are observed not only at the glottis but also in the regions downstream of the glottis and faraway from the glottis. This suggests that the estimation of fluctuation in pressure wave is useful for the diagnosis of pathological VFs.

2pSCc31. Differential effects of the phonemes on identification of previously unknown speakers. Kanae Amino (Dept. of Electrical and Electronics Engineering, Sophia University, 7-1 Kiyoi-cho, Chiyoda-ku, 102-8554 Tokyo, Japan, amino-k@sophia.ac.jp), Takayuki Arai (Dept. of Electrical and Electronics Engineering, Sophia University, 7-1 Kiyoi-cho, Chiyoda-ku, 102-8554 Tokyo, Japan, arai@ sophia.ac.jp)

In perceptual speaker identification, it is known that the identification accuracy depends on the contents of the stimuli presented to the listeners. In our previous experiments, we found that the stimuli containing a nasal sound are effective for human speaker identification, and that coronal sounds are more effective than labial ones. This tendency was observed in the identifications of both familiar and previously unknown speakers. In this present study, we investigated the effects of the speech contents again, by focusing on the vowels in $\mathrm{CV}$ monosyllabic stimuli. Through the experiment we obtained several findings: 1) stimuli containing a nasal gained significantly higher accuracy compared to stimuli with only oral sounds; 2) coronal sounds were more effective than labial sounds; 3) palatalisation caused an improvement in performance; and 4) back vowels were more effective than front vowels significantly. These results can be explained by speakerspecific morphologies of the nasal cavity and the paranasal sinuses, and also of the pharyngeal cavity. We will also show analyses and discussions on the acoustical properties of the stimuli and the performance differences among the subjects. [Work supported by Sophia University Open Research Centre from MEXT.]

2pSCc32. Coarticulation in $\mathrm{CV}$ sequences: Locus Equation data. Simone Graetzer (University of Melbourne, Royal Parade, Parkville Victoria, 3010 Melbourne, Australia, n.graetzer@pgrad.unimelb.edu.au)

Background: Speech sounds are known to be influenced by the context in which they occur, and to be produced in an overlapping way. These processes are referred to by the term "coarticulation". Objective: The aim of the study was to compare the magnitude of anticipatory coarticulation of oral voiceless plosives by following vowels in four Australian languages, Arrernte, Burarra, Gupapuyngu, and Warlpiri. Methods: The corpus consists of approximately six hundred isolated real words spoken by eight female adult speakers of the four languages. Locus equations are calculated for intervocalic consonants /p,c,t,tr,k/ (where "/tr/" represents a voiceless oral retroflex plosive) and all following vowels in consonant-vowel sequences. Measurement points are 0.1 and 0.5 into the vowel. The relationship between these two measurement points is linear and is expressed as a locus equation. The slope of the fitted line associated with the equation is a measure of the magnitude of coarticulation of the consonant by the following vowels. Results: There was a general tendency towards relatively highly coarticulated bilabial and velar plosives, weakly coarticulated retroflex plosives, and moderately coarticulated alveolar plosives. Results were variable for palatal plosives. These results are for the most part in accordance with findings for other Australian languages.

2pSCc33. Lexical analysis of spoken word recognition errors. Robert A. Felty (Indiana University - Speech Research Laboratory, 1101 E 10th St., Dept. of Psychology, Bloomington, IN 47401, USA, robfelty@indiana.edu), Adam Buchwald (NYU, 665 Broadway, Suite 910, New York, NY 10012, USA, buchwald@nyu.edu), David B. Pisoni (Indiana University - Speech Research Laboratory, 1101 E 10th St., Dept. of Psychology, Bloomington, IN 47401, USA, pisoni@indiana.edu)

In speech perception and spoken word recognition, a lexical "neighbor" of a target word is a phonetically similar word that competes with the target during lexical access. A commonly-used definition is that neighbors differ from one another by a single segment. While this basic definition accounts for many results of lexical decision and word recognition tasks among monosyllabic words, it also suggests that approximately half of the words in the English lexicon are hermits (words without neighbors). To generate a more inclusive definition of neighbor, we created a representative sample of the lexicon $(\mathrm{N}=1428)$ to examine spoken word recognition errors, and we used the incorrectly selected competitors as a means of indexing "neighbor" status. Analysis of over 15,000 errors reveals several patterns: (1) Errors tend to be of higher frequency than the target word; (2) Less than $1 / 3$ of the errors were neighbors according to the traditional definition of neighborhood density; and (3) Errors differed in phonetic similarity more as the $\mathrm{S} / \mathrm{N}$ ratio decreased. We use these data to test several alternative definitions of "neighbor" and their ability to predict actual word recognition errors. [Work supported by NIH-NIDCD R01 00111 and T32 00012]

2pSCc34. Articulatory constaints on coarticulation in fricatives as a function of tongue involvement. Carole E. Gelfer (William Paterson University, 300 Pompton Road, Wayne, NJ 07470, USA, gelferc@wpunj.edu), Fredericka Bell-Berti (St. John's University, 8000 Utopia Parkway, Queens, NY 11439, USA, bellf@stjohns.edu)

Numerous studies have shown coarticulatory effects of vowel rounding on the spectra of sibilants. However, the extent to which the tongue contributes to coarticulation is not clear because most studies have used only fricatives whose constriction is formed with the tongue. This study examines the effects of vowel environment on coarticulation in fricatives under two conditions: when the constriction is formed with the tongue (i.e., [s]) and when it is not (i.e., [f]), paired with the English vowels occurring at the extremes of the vowel quadrilateral. Preliminary results for adult native speakers of American English suggest that in the absence of tongue constraint (i .e., [f]), there is a greater effect of vowel context on the fricative spectrum. This result reinforces the importance of considering the freedom of an articulator to anticipate an upcoming gesture when examining the extent of coarticulation.

2pSCc35. Acquisition of rhythm: evidence from spontaneous L2 speech. Irina Shport (University of Oregon, Department of Linguistics, 1290, Eugene, OR 97403, USA, ishport@uoregon.edu)

This study examined the acquisition of Japanese rhythm by comparing the rhythmic characteristics of elicited spontaneous speech of English learners in the setting of an oral proficiency test. Two metrics for the quantification of speech rhythm that have been shown to reliably discriminate rhythmic classes in both L1 and L2 speech (White and Mattys, 2007) were utilized for the analysis - 1) the proportion of vowel intervals within the sentence $(\% \mathrm{~V})$, and 2$)$ the standard deviation of vowel intervals divided by the 
mean vowel interval duration and multiplied by 100 (VarcoV). A difference in the rhythm scores of novice-mid and intermediate-high learners of Japanese was found. Furthermore, the learners' scores were significantly different from Japanese native speakers' scores, suggesting that speakers start acquiring speech rhythm relatively early, but they are still short of the target at the intermediate level of proficiency. By comparing the obtained scores with scores of stress-timed languages, such as English, the difference in the scores may be attributed to an L1 transfer into the rhythmic organization of the L2 speech.

2pSCc36. Effects of training, style, and rate of speaking on speech perception of young people in reverberation. Nao Hodoshima (Dept. of Electrical and Electronics Engineering, Sophia University, 7-1 Kiyoi-cho, Chiyoda-ku, 102-8554 Tokyo, Japan, n-hodosh@sophia.ac.jp), Takayuki Arai (Dept. of Electrical and Electronics Engineering, Sophia University, 7-1 Kiyoi-cho, Chiyoda-ku, 102-8554 Tokyo, Japan, arai@sophia.ac.jp), Kiyohiro Kurisu (TOA Corporation, 2-1 Takamatsucho, Takarazuka,665-0043 Hyogo, Japan, kurisu_kiyohiro@toa.co.jp)

Because of the difficulty of listening to speech in reverberation (e.g. train stations or airports), we need to find characteristics of intelligible speech sounds that are appropriate for announcements over loudspeakers in public spaces. This study investigated the effects of training (seven talkers who have received speech training or not), style (conversational/clear) and rate (normal/slow) of speaking on speech perception of young people in simulated reverberant environments. The talkers were instructed to speak nonsense words embedded within a carrier sentence clearly or normally in an anechoic room, and listening tests were carried out with young people in simulated reverberant environments. Results showed that correct rates significantly differed among the talkers, but no difference in correct rates was found between the two speaking rates, and conversational speech had significantly higher correct rates than clear speech. Casual inspections of the stimuli indicate that vowels are enhanced as well as consonants in clear speech so that clear speech had lower correct rates than conversational speech due to increased reverberant masking in clear speech compared to conversational speech. [Work supported by Sophia University Open Research Center from MEXT.]

2pSCc37. Prosody in French theatrical declamation traced backwards in time. George Brock-Nannestad (Patent Tactics, Resedavej 40, DK-2820 Gentofte, Denmark, pattac@image.dk)

Listening to early recordings of a French theatrical personality, such as Sarah Bernhardt, the emphatic emission of words is very noticeable. It is sometimes ascribed to Bernhardt's theatrical mannerisms, but possibly also caused by an erroneous speed of reproduction. But even at correct speed, huge variations in intenseness are very noticeable. Literature does not give a clear indication whether this manner of performance was considered deviating at the time (ca. 1900). New research into the original handwritten patent documents of Édouard-Léon Scott (filed 1857 and 1859) has analyzed his method of recording the performance of the human voice. Scott documented the performance of a complete stanza from a French play, and looking at his graphical output and interpretation it becomes clear that the prosody of Mme. Bernhardt was already in place in 1859. Having identified the French play, even earlier descriptions of the prosodic approach in French declamation were found. The paper also discusses the patents of ÉdouardLéon Scott and his desire for the use of his invention, which was different from that of his manufacturer, Rudolph Koenig. Furthermore, the prosodic documentation will be demonstrated, and a historically informed reproduction of a recording by Sarah Bernhardt will be presented.

2pSCc38. Articulatory coordination in German and English onset and coda clusters. Marianne Pouplier (Institute of Phonetics and Speech Processing, University of Munich, 80799 Munich, Germany, pouplier@phonetik.uni-muenchen.de), Stefania Marin (Institute of Phonetics and Speech Processing, University of Munich, 80799 Munich, Germany, stefania.marin@gmail.com)

Articulatorily, onset consonants differ from coda consonants in their spatial as well as their temporal characteristics in that onsets exhibit greater spatial displacement and are generally less variable than codas. As to their temporal organization, it has been claimed that onsets, but not codas exhibit a so-called c-center organization in which all consonant gestures of a cluster are organized as a single complex structure with respect to the vowel, while consonant clusters in codas have been hypothesized to be organized sequentially [Browman \& Goldstein 1988 Phonetica]. While some studies have confirmed the c-center hypothesis for onsets but not codas, others found a c-center organization for both onset and coda consonants. The current study uses German and English onset and coda clusters to test systematically the c-center hypothesis on the basis of EMA data. Results for both languages indicate that a c-center organization may be observed in both onset and coda position, yet it does not appear consistently for all clusters. Also cross wordboundary clusters may exhibit c-center organization, possibly indicating resyllabification.

2pSCc39. Acoustic consequences of gestural intrusion errors. Stefania Marin (Institute of Phonetics and Speech Processing, University of Munich, 80799 Munich, Germany, stefania.marin@gmail.com), Marianne Pouplier (Institute of Phonetics and Speech Processing, University of Munich, 80799 Munich, Germany, pouplier@phonetik.uni-muenchen .de), Jonathan Harrington (Institute of Phonetics and Speech Processing, University of Munich, 80799 Munich, Germany, jmh@phonetik .uni-muenchen.de), Susanne Waltl (IPS, LMU, Schellingstr. 3/II, 80799 München, Germany, susanne@phonetik.uni-muenchen.de)

An increasing number of studies have elicited speech errors in articulatory experiments, and thus evaluated errors on the basis of their articulatory properties, yet there has been no study that relates the articulatory variability observed in errors to the acoustic consequences. The present study aims to evaluate the acoustic properties of errorful/error-free $[\mathrm{k}]$ and $[\mathrm{t}]$ stimuli with known articulatory configurations (and articulatorily categorized as errorful/error-free), and determine the extent to which articulatory errors have acoustically observable consequences. The most frequent error observed in the articulatory data is an intrusion of a second gesture, resulting in a simultaneous presence of both tongue tip and tongue body gestures during production of $[\mathrm{k}]$ or $[\mathrm{t}]$. Spectral analysis of these stimulis' bursts shows that $[\mathrm{k}]$ and $[\mathrm{t}]$ are differently affected by intrusion gestures: intrusion errors during intended $[\mathrm{k}]$ tokens are more robustly grouped with error-free $[\mathrm{k}]$ across speakers, while intrusion errors during intended $[\mathrm{t}]$ tokens are more variable, some being acoustically like [t], some like [k], and some like neither category, matching previous perceptual results (Pouplier \& Goldstein 2004, Journal of Phonetics). These results will be discussed in the context of different timing patterns between the two co-occurring gestures during intrusion errors.

2pSCc40. Embodiment, not imitation, leads to the replication of timing phenomena. Piers Messum (112 Warner Road, SE5 9HQ London, UK, p.messum@gmail.com)

In many languages, a spoken vowel is shorter before phonologically voiceless consonants than before voiced ones. In West Germanic languages, including English, tense and lax vowels have different lengths and there is a characteristic 'stress-timed' rhythm. For these phenomena and some others, it is generally assumed that time is the controlled variable for production, and that children replicate these speech behaviors through the reproduction of timing patterns abstracted from the adult input (i.e. by imitation). The developmental data does not support these assumptions, and an imitative mechanism would present a young speaker with a highly complex challenge. Instead, these behaviors are more plausibly the result of the embodiment of speech. Embodiment goes beyond laryngeal and articulatory function. A child's speech breathing is not a scaled-down version of the adult model but a distinctly different skill, and one that must be learnt during speech production. Similarly, the aerodynamic setting of child speech differs significantly from that of adults. The constraints that these factors impose become manifest in speech as changes in timing, but these changes are epiphenomenal, not modelled directly. Phenomena particular to West Germanic languages reflect the style of speech breathing these languages require of a child. 
2pSCc41. Investigating the perception of noise-vocoded speech - an individual differences approach. Carolyn McGettigan (University College London, Institute of Cognitive Neuroscience, 17 Queen Square, WC1N 3AR London, UK, c.mcgettigan@ucl.ac.uk), Stuart Rosen (UCL, Wolfson House, 4, Stephenson Way, NW1 2HE London, UK, stuart@phon.ucl.ac.uk), Sophie K. Scott (University College London, Institute of Cognitive Neuroscience, 17 Queen Square, WC1N 3AR London, UK, sophie.scott@ucl.ac.uk)

We used a cochlear implant simulation (noise-vocoded speech) to investigate speech recognition and perceptual learning in normal-hearing adult speakers of English. In two separate sessions (1-2 weeks apart), 28 listeners were tested on recognition of noise-vocoded Sentences, Words, and isolated segments (Consonants and Vowels). There was evidence of significant perceptual learning that survived until Session 2 for all tasks. An individual differences analysis of Session 1 data suggested two independently-varying 'levels' of processing at work in the initial perception of the distorted speech stimuli - a 'top-down' listening mode making use of contextual and lexical information, and a 'bottom-up' mode focussed on acoustic-phonetic discriminations. By Session 2, a more generalised listening mode emerged, reflecting listeners' consolidation of basic sound-to-representation mappings. Further exploration of Consonant and Vowel confusion data (using Information Transfer analyses) suggested that better speech recognition performance may be achieved through more efficient use of the preserved cues to duration and voicing in noise-vocoded stimuli, but that listeners failed to take full advantage of such information. We conclude that training regimes involving directed attention to specific features, such as vowel length, may help to improve performance with noise-vocoded speech.

2pSCc42. Speed-curvature relations in speech production: a multilingual experimental and modeling study. Pascal Perrier (ICP/GIPSA-lab, INPG, 46 Avenue Félix Viallet, 38031 Grenoble Cedex 01, France, Pascal.Perrier@gipsa-lab.inpg.fr), Susanne Fuchs (ZAS, Schuetzenstr. 18, 10117 Berlin, Germany, fuchs@zas.gwz-berlin.de)

Regularities in the relations between tangential velocity and trajectory curvature have been found in a number of human movements. They were formalized in the $1 / 3$ power law [Viviani and Terzuolo, Neuroscience 7: 431-437, 1982]. Viviani and colleagues also found that modifying the speedcurvature relations would influence the perception of synthetic movements in terms of naturalness and classification. Hence, these relations could be at the core of the perception-action link in human movements. In this paper, tongue movement collected from speakers of French, German and Mandarin are analyzed. They are compared with data from simulations using a biomechanical tongue model, which is controlled on a target-to-target basis. Our results for experimental data and simulations are very similar. It is found that the $1 / 3$ power law offers a fair description of the velocity-curvature relations when a large amount of movements are considered together. However, a refined analysis of individual movements reveals that the exceptions to this law are numerous. It is concluded that the velocity-curvature relation is not controlled in speech movements, and that the 1/3 power law only accounts for general characteristics of movements, which could arise from dynamical properties of the articulators.

2pSCc43. Vowel spaces in Swedish children with cochlear implants. Tina Ibertsson (Lund University, Dept. Logopedics, Phoniatrics \& Audiology, University Hospital, SE-221 85 Lund, Sweden, Tina.Ibertssont@med.lu.se), Birgitta Sahlén (Lund University, Dept. Logopedics, Phoniatrics \& Audiology, University Hospital, SE-221 85 Lund, Sweden, Birgitta.Sahlen@med.lu.se), Anders Lofqvist (Lund University, Dept. Logopedics, Phoniatrics \& Audiology, University Hospital, SE-221 85 Lund, Sweden, Anders.Lofqvist@med.lu.se)

This study analyzed vowel productions of Swedish children with cochlear implants, capitalizing on the rich vowel system of Swedish with nine vowels. Minimal word pairs were used as linguistic material. A group of 12 children with CI (mean age 184 months, range 144-229 months), and a group of children with normal hearing (mean age 105 months, range 103118 months) were recruited. The age at implantation of the CI was 79 months (range 31-155 months). The first two formants were measured for each vowel. To estimate the vowel space, the average Euclidean distance in the F1-F2 plane between each vowel and the mean first and second formant frequencies of all the vowels was calculated for each child. The results show a significantly smaller vowel space for the children with a cochlear implant. However, there was no overall significant correlations between the vowel space and measures of speech recognition and vowel production in the CI group. Although the present results show that speech recognition ability affects intelligibility and vowel production, the vowel space may not be a suitable measure for assessing speech production in this population. [Work supported by the Sunnderdahl Disability Foundation.]

2pSCc44. Detection of obstruent consonant landmark for knowledge based speech recgonition system. Jung-In Lee (Yonsei University, 134 Sinchon-dong, Seodaemun-gu, 120-749 Seoul, Republic of Korea, junginida@dsp.yonsei.ac.kr), Jeung-Yoon Choi (Yonsei University, 134 Sinchon-dong, Seodaemun-gu, 120-749 Seoul, Republic of Korea, jychoi @ yonsei.ac.kr)

Obstruent consonant landmarks are detected using spectral energy difference profiles. This study expands upon previous work by Liu. A[J. Acoust. Soc. Am. 100, 3417-3430, 1996]. The proposed algorithm detects four types of landmarks : [stop closure], [stop release], [fricative closure] and [fricative release], where affricates are detected by combining [stop closure], [fricative closure] and [fricative release]. In addition to finding abrupt changes in energy differences, we use energy contours, relative energy and spectral center of gravity differences. This method results in improved performance particularly for $\mathrm{CV}$ obstruents. Overall detection rates for stop closure and release are $76.9 \%$ and $85.7 \%$ for obstruent landmarks in TIMIT, and fricatives yield $82.2 \%$ and $83.6 \%$ respectively. For strident fricatives, the figures are $94.7 \%$ and $93.6 \%$.

2pSCc45. Acoustic-phonetic features for stop consonant place detection in clean and telephone speech. Jung-Won Lee (Yonsei University, 134 Sinchon-dong, Seodaemun-gu, 120-749 Seoul, Republic of Korea, jaesuk2002@dsp.yonsei.ac.kr), Jeung-Yoon Choi (Yonsei University, 134 Sinchon-dong, Seodaemun-gu, 120-749 Seoul, Republic of Korea, jychoi @yonsei.ac.kr)

This work classifies voiceless stop consonant place in CV tokens of English using burst release cues for clean (TIMIT) and telephone speech (NTIMIT). We compared the performance of cepstral coefficients to acoustic phonetics-motivated features such as center of gravity, burst amplitude and relative difference of formant amplitudes. In clean speech, cepstral coefficients resulted in better classification. However, for test data from NTIMIT, acoustic phonetic-based features outperformed cepstral coefficients, particularly if models were trained on clean speech. In addition, augmenting cepstral coefficients with acoustic phonetic-based measurements resulted in the best performance. These findings suggest that cepstral coefficients are able to model speech in a given environment in finer detail, whereas acoustic phonetic-based features are more robust to changes in environment, so that combining both types of measurements leads to the best performance.

2pSCc46. Vowel place detection for a knowledge-based speech recognition system. Sukmyung Lee (Yonsei University, 134 Sinchondong, Seodaemun-gu, 120-749 Seoul, Republic of Korea, pooh390@dsp.yonsei.ac.kr), Jeung-Yoon Choi (Yonsei University, 134 Sinchon-dong, Seodaemun-gu, 120-749 Seoul, Republic of Korea, jychoi @yonsei.ac.kr)

This work aims to detect vowel place as part of a knowledge-based speech recognition system. Vowel place was classified into 6 groups based on tongue advancement [Front/Back] and height [High/Mid/Low]. Experiments were performed using $300 / \mathrm{hVd} /$ utterance data from Hillenbrand [J. Acoust. Soc. Am. 97, 3099-3111] and 6600 TIMIT vowels. Features used include fundamental frequency (F0) and formant value (F1 F3), where formant measurements were classified into separate groups using F0 measurements. The nearest class was found using a simple Mahalanobis distance measure, and yielded a $91.5 \%$ classification rate for the $/ \mathrm{hVd} /$ data. 
The results for the TIMIT data were $64.4 \%$, and error analysis with regard to adjacent segment manner and place was carried out to observe the effects of coarticulation, which was not observed in the $/ \mathrm{hVd} /$ data

2pSCc47. Cross-modal perceptual learning of spectrally degraded speech: Behavioral and neuroimaging studies. Frank Eisner (University College London, Institute of Cognitive Neuroscience, 17 Queen Square, WC1N 3AR London, UK, f.eisner@ucl.ac.uk), Carolyn McGettigan (University College London, Institute of Cognitive Neuroscience, 17 Queen Square, WC1N 3AR London, UK, c.mcgettigan@ucl.ac.uk), Stuart Rosen (UCL, Wolfson House, 4, Stephenson Way, NW12HE London, UK, stuart@phon.ucl.ac.uk), Andrew Faulkner (UCL, Wolfson House, 4, Stephenson Way, NW1 2HE London, UK, andyf@phon.ucl.ac.uk), Sophie K. Scott (University College London, Institute of Cognitive Neuroscience, 17 Queen Square, WC1N 3AR London, UK, sophie.scott@ucl.ac.uk)

We investigated normal-hearing listeners' ability to adapt to a speech signal that simulates aspects of the stimulation received from a cochlear implant. The training materials were spoken sentences which were spectrally degraded by noiseband-vocoding and shifted upwards in frequency. A control condition consisted of spectrally inverted, unintelligible versions of these stimuli. Participants listened passively to these sentences, and on each trial received visual feedback which could be either a written version of the sentence or a video of the talker who originally produced it. Learning under both feedback conditions was relatively fast: Subjects improved on average by $25 \%$ on keyword recognition scores after 100 trials. No learning effects were observed in the control condition. We further used functional magnetic resonance imaging to investigate which cortical areas may be recruited during learning. A comparison of degraded and learnable sentences with the unlearnable control stimuli showed activity in the left superior temporal sulcus both during passive listening and receiving feedback. In contrast, the left inferior frontal gyrus was activated only when subjects were receiving feedback in the learnable condition. These results suggest that the inferior frontal gyrus plays an important role in integrating acoustic-phonetic processing with externally provided feedback.

2pSCc48. Robust harmonic grouping by octave error correction. Hedde Van De Vooren (University of Groningen, Dept. Artificial Intelligence, P.O. Box 407, 9700 AK Groningen, Netherlands, h.van.de.vooren@ai.rug.nl), Renante R. Violanda (University of Groningen, Dept. Artificial Intelligence, P.O. Box 407, 9700 AK Groningen, Netherlands, R.Violanda@ai.rug.nl), Tjeerd C. Andringa (University of Groningen, Dept. Artificial Intelligence, P.O. Box 407, 9700 AK Groningen, Netherlands, t.andringa@ai.rug.nl)

Harmonic grouping is a frequently applied technique in computational auditory scene analysis and automatic speech recognition systems. However, grouping is easily disrupted by noise and reverberation. For instance, a noise induced signal component positioned roughly between two harmonics, might undesirably be assigned to the harmonic complex (HC) as well. This results in an octave error: harmonics in an $\mathrm{HC}$ are assigned to harmonic numbers twice as high as the correct values. We propose a cost function based method to correct these octave errors. This function is designed to, on the one hand, improve the balance between odd and even harmonic numbers, and, on the other hand, minimize the amount of signal components to be rejected. As a preprocessing step we applied short-time Fourier analysis to derive an instantaneous frequency representation from which we obtained the signal components. We used these as input for our harmonic grouping algorithm to obtain the HCs. Then we selected the optimal solution from the cost function and modified the composition of the HCs accordingly. As long as enough harmonics are sufficiently above the local noise level, this octave error correction mechanism works well for various sorts of harmonic sounds including speech.

2pSCc49. A cross-language familiar talker advantage? Susannah Levi (University of Michigan, 440 Lorch Hall, 611 Tappan Street, Ann Arbor, MI 48109, USA, svlevi@umich.edu), Stephen Winters (University of
Calgary, Social Sciences Bldg. Room 820, Department of Linguistics, Calgary, AB T2N 1N4, Canada, swinters@basesproduced.com), David B. Pisoni (Indiana University - Speech Research Laboratory, 1101 E 10th St., Dept. of Psychology, Bloomington, IN 47401, USA, pisoni@indiana.edu)

Previous research has shown that familiar talkers are more intelligible than unfamiliar talkers. In the current study, we tested the source of this familiar talker advantage by manipulating the type of talker information available to listeners. Two groups of native English listeners were familiarized with the voices of five German-English bilingual talkers; one group learned the voices from German stimuli and the other from English stimuli. Thus, English-trained listeners had access to both language-independent and English-specific talker information, while German-trained listeners had access to language-independent and German-specific talker information. After three days of voice learning, all listeners performed a word recognition task in English. Consistent with previous findings, English-trained listeners found the speech of familiar talkers to be more intelligible than unfamiliar talkers, as measured by whole words and phonemes correct. In contrast, German-trained listeners showed no familiar talker advantage, suggesting that listeners must have knowledge of talker-specific, linguistically relevant information to elicit the familiar talker advantage and that knowledge of language-independent talker information - such as size and shape of the vocal tract - does not facilitate speech perception.

2pSCc50. Prosodic effects on articulatory coordination in initial consonant clusters in German. Lasse Bombien (Institut für Phonetik und Sprachverarbeitung, Schellingstr. 3, 80799 München, Germany, lasse@phonetik.uni-muenchen.de), Christine Mooshammer (Haskins lab and MIT Research lab of electronics, 300 George street suite 900, New Haven, CT 06511, USA, tine@ haskins.yale.edu), Phil Hoole (Institut für Phonetik und Sprachverarbeitung, Schellingstr. 3, 80799 München, Germany, hoole@phonetik.uni-muenchen.de), Barbara Kuehnert (Laboratoire de Phonétique et Phonologie - UMR 7018 CNRS - Paris 3, 19, rue des Bernadins, 75005 Paris, France, barbara.kuhnert@univ-paris3.fr)

This study examines how articulatory coordination in heterorganic initial consonant clusters is modulated by prosodic condition. Extending the paradigm of articulatory strengthening at domain edges to clusters, we provide EPG data of 7 speakers as well as EMA data of two speakers including domain-initial clusters $(/ \mathrm{kl} /, / \mathrm{kn} / \mathrm{and} / \mathrm{sk} /)$ in different prosodic positions, varying the strength of the preceding boundary and the position of lexical stress. (e.g. 'Claudia vs. Klau'sur, i.e. stressed vs. unstressed target cluster). The results show that $\mathrm{C} 1$ was consistently lengthened at higher boundaries, but only if the cluster was preceded by a clear pause. C2 was only weakly and inconsistently affected. There were no effects on the spatial domain at all. No consistent effects of lexical stress were found for either $\mathrm{C} 1$ or $\mathrm{C} 2$. Regarding overlap of $\mathrm{C} 1$ and $\mathrm{C} 2$ there was a tendency, albeit quite weak, in the direction of more overlap at the lower boundary levels and in unstressed condition. However, differences in timing between segmentally different clusters were consistent and much larger than boundary and stress effects. Especially for $/ \mathrm{kl} / \mathrm{vs}$. $/ \mathrm{kn} /$ we find that the lateral's timing to $/ \mathrm{k} /$ is much closer than the nasal's.

2pSCc51. Hierarchical approach to voice activity detection in realistic environmental noise. Jörg-Hendrik Bach (Universität Oldenburg, Medizinische Physik, Fakultät V, 26111 Oldenburg, Germany, j.bach@uni-oldenburg.de), Jörn Anemüller (Universität Oldenburg, Medizinische Physik, Fakultät V, 26111 Oldenburg, Germany, joern.anemueller @uni-oldenburg.de)

The automatic identification of acoustic objects, particularly speech, in adverse backgrounds can pose a difficult problem. In this work, the problem of speech detection in different backgrounds is tackled using a hierarchical decision tree: The root node provides a coarse classification of the noise background, while the next nodes are optimised to detect speech in one particular background each. The results of this approach are compared to the ITU G729B standard VAD and to a binary classifier trained on speech vs a mixture of the backgrounds. The features employed are amplitude modulation spectra of the signals (Kollmeier \& Koch, 1993) that represent the modulation content of a signal as a two-dimensional (modulation-frequency vs centre frequency) spectral pattern. The best features for classification at 
each node are selected independently (node-specific) using a sequential floating forward search algorithm. The statistical learner is an open source support vector machine (Chang \& Lin, 2001).

2pSCc52. Visual Influences on Alignment to Voice Onset Time Kauyumari Sanchez (University of California, Riverside, Department of Psychology, 900 University Ave., Riverside, CA 92521, USA, ksanc004@student.ucr.edu), Rachel M. Miller (University of California, Riverside, Department of Psychology, 900 University Ave., Riverside, CA 92521, USA, rmill002@ucr.edu), Lawrence D. Rosenblum (University of California, Riverside, Department of Psychology, 900 University Ave., Riverside, CA 92521, USA, rosenblu@ citrus.ucr.edu)

In speech alignment, interlocutors inadvertently imitate aspects of each others utterances. Alignment also occurs when talkers are asked to shadow (identify out loud) recorded words heard over headphones. For example, the voice onset time (VOT) of consonants produced by subjects will be greater when shadowing heard words whose consonants have been modified to have extended VOTs [K. Shockley, et al., Percept. Psychophys. 66, 422 (2004)]. An experiment was conducted to test whether alignment to VOT has a purely acoustic basis. For this purpose, visual speech tokens composed of a face articulating /ba/ syllables at three different rates, were each dubbed onto a series of seven audio /ba/ syllables varying in VOT. Subjects were asked to simply shadow the audiovisual $/ \mathrm{ba} / \mathrm{s}$. Results revealed that both the visible syllable rate and audio VOT of the stimuli influenced the VOTs of the shadowers' produced syllables. The implications of these results for theories of alignment, audiovisual integration, and the perceptual primitives of speech are discussed.

2pSCc53. The '[+English]' effect in acquisition of English as a second language: Novel- category biases in second language perception and production. Kenneth De Jong (Indiana University, 322 Memorial Hall, 1021 E. 3rd Street, Bloomington, IN 47405, USA, kdejong@indiana .edu), Yen-Chen Hao (Indiana University, 322 Memorial Hall, 1021 E. 3rd Street, Bloomington, IN 47405, USA, yehao@indiana.edu), Hanyong Park (Indiana University, 322 Memorial Hall, 1021 E. 3rd Street, Bloomington, IN 47405, USA, hanypark@indiana.edu), Noah H. Silbert (Indiana University, 322 Memorial Hall, 1021 E. 3rd Street, Bloomington, IN 47405, USA, nosilber@indiana.edu)

Silbert \& de Jong (JASA, 122: 3092) investigated correlations between response bias and segment frequency in a number of previously published segment identification data sets. In general, bias tends to be positively correlated with frequency. When applied to second language (L2) learning, this pattern predicts biases toward identifying novel L2 categories as L1 categories, since the number of instances of L1 categories far outweighs those of novel categories in learners' experience. Nagao, Lim, \& de Jong (2003, $\mathrm{ICPhS}$ ) confirm this: Japanese listeners tend to label ambiguous English coda consonants as onsets. However, younger and less experienced learners of English tend to label ambiguous tokens as codas; they tend toward using the novel category even though it is less frequent in their experience. This paper reports experiments in which Korean EFL learners identified English obstruents and in which they produced them. Cases of novel category response bias are observed in identification, and less so in production. Such novel category effects suggest a model in which novel categories serve, in part, as indicators of the L2 itself and stand out as response options in L2 identification tasks. These effects are likely counteracted by motor difficulties in $\mathrm{L} 2$ production tasks.

2pSCc54. Study on voice quality parameters for anger degree estimation. Yoshiko Arimoto (Tokyo University of Technology, Graduate School of Bionics, Information and Media Science, Faculty of MS, Iida labolatory, 1404-1 Katakura, Hachioji, 192-0982 Tokyo, Japan, ar@mf.teu.ac.jp), Sumio Ohno (Tokyo University of Technology, 1404-1 Katakura, 192-0982 Hachioji, Japan, ohno@cc.teu.ac.jp), Hitoshi Iida (Tokyo University of Technology, 1404-1 Katakura, 192-0982 Hachioji, Japan, iida@media.teu.ac.jp)

With great advance of automatic speech recognition (ASR) systems and a voice command system are demanded to be more sensitive to user's intention or emotion. These systems currently process linguistic information, but not process nonlinguistic information or paralinguistic information which users expressed during dialogs. For that reason, computers can obtain less information about a user through a dialog than human listeners can. If computers will recognize user's emotions conveyed by acoustic information, more appropriate response can be made toward users. For realization of emotion recognition, we have continued our study on anger degree estimation by both prosodic features and segmental features with anger utterances which were recorded during two kinds of pseudo-dialogs. This report focuses on only segmental features related to voice quality and examines them for capabilities to estimate anger degree. The first cepstral coefficient of anger utterances has been analyzed to obtain acoustic parameters related to spectral tilt in our previous works. In addition to the previous voice quality parameters, features related to amplitude of formants and harmonics were extracted from the same anger utterances as that in the previous works. Comparisons were made with the previous parameters to evaluate estimation accuracy of each voice quality parameters.

2 pSCc55. Identification of place of articulation and voicing in white and babble noise. Magnus Alm (Norwegian University of Science and Technology, Psychology Dept, NO 7491 Trondheim, Norway, magnusal@stud.ntnu.no), Dawn Behne (Norwegian University of Science and Technology, Psychology Dept, NO 7491 Trondheim, Norway, dawn .behne@svt.ntnu.no)

Previous research shows that white noise influences the degree auditory and visual modalities are used in audio-visual (AV) speech perception. This study assesses identification of voicing and place of articulation (POA) in the infrequently studied natural babble noise. Incongruent monosyllabic AV stimuli were presented to 15 young adults in white and babble noise at 0 and $-12 \mathrm{~dB}$ SNR where voicing stimuli differed in voicing and voicing structure and POA stimuli differed in POA and POA structure. In white noise POA stimuli received fewer audio responses than in babble whereas voicing stimuli received more audio responses in white noise than in babble. Voiced syllables received more audio responses than voiceless. Findings suggest that differences in noise type for POA and voicing identification are attributable to discrepancies in acoustical attributes of noise and target stimuli. In voicing identification, the spectral transition between aspiration (stabile signal) and voicing (fluctuating signal) is less distinct in the fluctuating babble noise than in the flat power white noise. Voiceless consonants are more spectrally similar to white noise than voiced consonants, making the latter more auditorily accessible. Visual cues aid voicing identification, but only when POA is visually salient and auditorily susceptible to the noise type.

2pSCc56. A comparison of task and non-task related variability in speech production and acoustics, with implications to speech perception. Khalil Iskarous (Haskins Laboratories, 300 George St, New Haven, CT 06511, USA, iskarous@ haskins.yale.edu)

Task Dynamics provides a way of testing whether production goals are acoustic or articulatory. According to this theory, during the production of a segment, the vocal tract can be divided into locations where a task is specified and locations where no task is specified. The goal is for articulators to synergetically achieve the constriction in the task location. Articulatory variability in the non-task locations is permitted due to overlap. However, articulatory activity in both task and non-task locations affects the area function and acoustic output. Therefore it is possible that an articulatory synergy stabilizes an articulatory task variable, without stabilizing the acoustics, evidence that the goal is articulatory. An experiment on the articulation and acoustics of $/ \mathrm{Vd} /$ in Ameican English by 26 subjects, using the Wisconsin Xray Microbeam database, shows that there is a synergy between jaw and tongue tip that explains $79 \%$ of the variability in articulator locations. This synergy stabilizes the position of the tongue tip in the alveolar region. But the formant transitions and bursts are highly variable, since the back of the tongue and jaw assume positions consistent with the preceding vowelevidence that the goal is articulatory (Funded by NIH DC-02717). 
2pSCc57. Evaluation of a simplified phonetic annotation scheme for disordered speech. Kathleen Murray (Head and Neck Surgery, Drexel University College of Medicine, Department of Otolaryngology - Head and Neck Surgery, 1721 Pine Street, Philadelphia, PA 19103, USA, kathleen.murray@drexel.edu), David Graff (Linguistic Data Consortium, 3600 Market St. Suite 810, Philadelphia, PA 19104-2653, USA, graff@ldc.upenn.edu), Yolanda D. Heman-Ackah (Head and Neck Surgery, Drexel University College of Medicine, Department of Otolaryngology Head and Neck Surgery, 1721 Pine Street, Philadelphia, PA 19103, USA, Phillyvoicemd@aol.com), Robert T. Sataloff (Head and Neck Surgery, Drexel University College of Medicine, Department of Otolaryngology Head and Neck Surgery, 1721 Pine Street, Philadelphia, PA 19103, USA, rtsataloff@phillyent.com)

Attempts to train a computer to mimic a vocal pathology expert's perception of perceivable voice problems have had limited success. A recent study successfully used a Cepstrum-based calculation (CPPs) to detect dysphonic speech, but it made significant false negative and false positive errors [1]. Appropriate training data could improve calculation accuracy, but logistical and legal issues from the medical domain render large amounts of detailed, consistent training data difficult to produce. A simple scheme has been developed based on labeling one point in time for each of a few vowels in a read statement. Calculations from [1] and [2], centered on neighborhoods of these vowels will augment the CPPs classifier. The annotation scheme will be evaluated with respect to its ability to assist a classifier to automatically predict perceptual labels [1] from data, and practicalities such as inter/intra-annotator agreement. 1. Heman-Ackah, Y.D., Heuer, R.J., Michael, D.D., Ostrowski, R., Horman, M., Baroody, M., Hillenbrand, J.M., and Sataloff, R.T. (2003). "Cepstral peak prominence: A more reliable measure of dysphonia," Annals of Otology, Rhinology, and Laryngology, 112, 324-333. 2. "Perceived Level of Noise by Mark VII and Decibels (E)" S. S. Stevens, J. Acoustical Soc. Am. v. 51(2, part 2) 1972. pp. 575-602.

2pSCc58. Neural correlates of speech and non-speech processing: Role of language experience in brain activation. Alexis $\mathrm{N}$. Bosseler (University of Washington, Dept. of Speech \& Hearing Sciences, and Institute for Learning \& Brain Sciences, Box 357988, Seattle, WA 98195, USA, bosseler@u.washington.edu), Toshiaki Imada (University of Washington, Dept. of Speech \& Hearing Sciences, and Institute for Learning \& Brain Sciences, Box 357988, Seattle, WA 98195, USA, imada@u.washington.edu), Elina Pihko (BioMag Laboratory, Helsinki University Central Hospital, 00029 Helsinki, Finland, pihko@biomag .hus.fi), Jyrki Mäkelä (BioMag Laboratory, Helsinki University Central Hospital, 00029 Helsinki, Finland, jyrki.makela@hus.fi), Samu Taulu (Elekta-Neuromag Oy, 22 Elimäenkatu, 00510 Helsinki, Finland,
samu@squid.neuromag.fi),Antti Ahonen(Elekta-Neuromag Oy, 22 Elimäenkatu, 00510 Helsinki, Finland, antti.ahonen@elekta.com), Patricia K. Kuhl (University of Washington, Dept. of Speech \& Hearing Sciences, and Institute for Learning \& Brain Sciences, Box 357988, Seattle, WA 98195, USA,pkkuhl@u.washington.edu)

In adults, neural responses to the acoustic properties of native and nonnative speech sounds differ. Recent magnetoencephalography (MEG) studies indicate that two regions of the brain, the superior temporal area and inferior parietal area (Broca's), in both the left and right hemispheres, may contribute to how the adult brain responds to the acoustic properties of speech. The aim of the present study was to investigate neural activation in adults when listening to native and non-native speech, and to non-speech analogs of speech. We used MEG to examine activation of the superior temporal and inferior parietal areas. Native speakers of Finnish were presented with Finnish (/pa/ and /ta/) and Chinese (/t "looped c" "superscript h" i/ and /"looped c" i/) syllables, and sine-wave replicas of /pa/ and /ta/, in a passive oddball paradigm. Our analysis examines the properties of localized neural activities to determine how activation of sensory and motor speech areas differs for speech and non-speech, and the role of language experience in brain activation.

2pSCc59. Why words do not have a psychological alphabet. Robert F. Port (Indiana University, 330 Memorial Hall, Bloomington, IN 47405, USA, port@indiana.edu)

Linguists and psychologists share a powerful intuition that the psychological form of language (in memory) employs letter-like units for spelling words and any other linguistic material. However evidence accumulating over the decades shows that memory for linguistic material actually consists of continuous-time representations containing much more information than we thought, plus there are episodic representations with much redundancy. So Liberman's /di/ and /du/ share nothing in their representation. There need not be an abstract/d/ in the memory representations of language. Our intuition that a letter-like representation is necessary for speech perception and production turns out to be shared only with literate people. Because we cultivate our reading and writing skills, we bias our brains (and consciousness) to model speech using something like letters (e.g, phones) either from an orthographic or a technical alphabet. So our conscious experience of language is shaped by our cultural tradition of training children to hear speech as letters and letters as speech - all to exploit the technology of literacy. When we can overcome this education-based bias toward phones and phonemes, we will discover the phonemic emperor has no supporting evidence. Linguistic material in memory is richly detailed - relative to what an alphabet represents. 


\title{
Session 2pSPa
}

\section{Signal Processing in Acoustics: Beamforming, Localization, and Tracking I}

\author{
Michael Roan, Cochair \\ Virginia Tech, 917 Village Way North, Blacksburg, VA 24060, USA \\ Natalia Sidorovskaia, Cochair \\ Department of Physics, University of Louisiana, UL BOX 44210, Lafayette, LA 70504-4210, USA
}

\section{Contributed Papers}

\begin{abstract}
2:00
2pSPa1. Acoustic vector-sensor array beamforming based on fourth-order cumulants. Tingting Li (College of Underwater Acoustic Engineering, Harbin Engineering University, 150001 Harbin, China, liqiaocheng@hrbeu.edu.cn), Xiukun Li (College of Underwater Acoustic Engineering, Harbin Engineering University, 150001 Harbin, China, xiukun _li@yahoo.com.cn)

Abstract: Some high-resolution direction-of-arrival (DOA) estimation methods, such as the MUSIC method, have been developed based on vectorsensor array. However, these high-resolution methods suffer from serious drawbacks. Indeed, they are not able to estimate coherent signals and sensitive to calibration errors. Mainly to overcome these limitations and in particular to satisfy practical engineering application, vector-sensor array beamforming based on fourth-order cumulants is adopted in this paper. The main interest in using fourth-order cumulants instead of second-order ones in vector-sensor array processing application is the aperture extension property of higher-order cumulants, which makes it possible to increase both the resolution and the number of sources to be processed from a given array, and suppress the Gaussian noise. Based on this method, smaller size of array and higher accuracy of DOA estimation can be realized, besides, this method is less sensitive to the coherence of the signals and calibration errors, which makes it possible to work well in engineering application. The results of computer simulation and analysis of data from outfield trials show that this method excels traditional direct estimation and can improve estimation capability of vector-sensor arrays.
\end{abstract}

\section{2:20}

2pSPa2. Robust array pattern synthesis with uncertain manifold vector. Shefeng Yan (Department of Electronics and Telecommunications, Norwegian University of Science and Technology, O.S. Bragstads plass 2A, 7491 Trondheim, Norway, sfyan@ieee.org)

The knowledge of the array manifold vectors of an acoustic array can be imprecise, which is often the case in practice. This may cause undesirably high sidelobes for a nominal optimal beamformer where the array manifold vectors are assumed to be known exactly. Although the norm constraint on beamformer weights can be imposed to improve the robustness of the optimal beamformer, it is not clear how to choose the optimal constrained parameter based on the known level of uncertainty of the array manifold vectors. A pattern synthesis approach to arbitrary arrays with robustness against array manifold vectors errors is developed. Our technique optimizes the worst-case performance by minimizing the worst-case sidelobe level while maintaining a distortionless response to the worst-case signal steering vector. The parameters can be optimally chosen based on the uncertainty of the array manifold vector. The robust beamformer problem is shown to be convex, which can be efficiently solved using second-order cone programming. A simple lower bound on how much worse the robust optimal beamformer will be compared to the nominal one is also derived. Computer simulations show better performance of the proposed robust beamformer.
2:40

2pSPa3. Analytical prediction of sample eigenvector quality deterioration in large arrays due to SNR or sample size constraints. Raj Nadakuditi (MIT, Department of Mathematics, 77 Massachusetts Avenue, Cambridge, MA 02139, USA, raj@mit.edu), Arthur B. Baggeroer (MIT, Department of Mathematics, 77 Massachusetts Avenue, Cambridge, MA 02139, USA, abb@boreas.mit.edu)

It is well-known that subspace-based estimation methods in adaptive array processing suffer a rapid degradation in performance as either the signalto-noise ratio (SNR) or the number of available snapshots drops below a certain threshold value. In the large system, relative large sample size limit, one can use random matrix theory to analytically predict this threshold and the degradation in the "quality" of the corresponding subspace estimates. In certain settings, one observes a "phase transition" phenonemon so that if the signals are too weak or there are insufficient number of snapshots or both, the subspace estimates are, statistically speaking, noise-like. We discuss the implication of these results for the subspace based detection of signals in white and colored noise using large arrays and illustrate the accuracy of the predictions with numerical simulations.

\section{3:00}

2pSPa4. Efficient Replica Generation for Space Time Adaptive Processing of Acoustic Signals. Neena Imam (Oak Ridge National Laboratory, 1 Bethel Valley Road, Oak Ridge, TN 37831-6015, USA, imamn@ornl.gov), Jacob Barhen (Oak Ridge National Laboratory, 1 Bethel Valley Road, Oak Ridge, TN 37831-6015, USA, barhenj@ornl.gov), Michael Traweek (Office of Naval Research, 875 North Randolph Street, Arlington, VA 22203, USA, Mike.Traweek@navy .mil)

This paper addresses an innovative method for real-time replica generation using generalized Green's function for Space Time Adaptive Processing (STAP) technology for sonar applications. In hypothesis processing for STAP algorithms, the first step is to generate replicas for various target hypothesis such as the infinite-range fixed-direction (IRFD) target hypotheses or the tracking (trajectory) hypotheses. Once generated, these replicas are used in multiple steps of the STAP algorithm for computing system parameters such as the signal to noise ratio. Replicas are traditionally precomputed or hard-wired due to computational load constraints. Such a precomputation precludes direct application of array shape corrections, and limits the ability to process arbitrary target trajectories. W propose to use a generalized Green's function for efficient and ultra-fast real-time generation of replicas on multi-core processors Specifically, for an arbitrary target trajectory $r_{T}\left(t_{i}\right)=\left[x_{T}\left(t_{i}\right), \quad y_{T}\left(t_{i}\right), \quad z_{T}\left(t_{i}\right)\right]$, arbitrary receive array trajectory $r_{R(k)}\left(t_{i}\right)=\left[x_{R(k)}\left(t_{i}\right), y_{R(k)}\left(t_{i}\right), z_{R(k)}\left(t_{i}\right)\right],\left(k=1, \ldots, N_{E L}\right)$, arbitrary vector of frequencies in homogeneous acoustic medium $f=\left[f_{1} f_{2}, \ldots, f_{n}, \ldots\right]$, and sound speed $c_{0}$, we can write $G\left[r_{T}\left(t_{i}\right), r_{R(k)}\left(t_{i}\right), f_{n} \mid c_{0}\right]=\exp \left[j\left(2 \pi f_{n} / c_{0}\right)\left|r_{R(k)}\left(t_{i}\right)-r_{T}\left(t_{i}\right)\right|\right] / \mid$ $r_{R(k)}\left(t_{i}\right)-r_{T}\left(t_{i}\right) \mid$ In terms of rationale, this is the simplest possible replica set for any STAP algorithm. The efficient implementation of the generalized Green's function on a multi-core processor will allow real-time adjustment/modification of acoustical target tracking resulting in improved numerical accuracy and time performance. 


\section{3:20}

2pSPa5. Beam-forming with progressive focalisation. Gaetano Caronna (Università La Sapienza - Dept. Fisica Tecnica, Via Eudossiana 18, $00184 \quad$ Roma, Italy, gaetano.caronna@uniroma1.it), Pierluigi Testa (Università La Sapienza - Dept. Fisica Tecnica, Via Eudossiana 18, 00184 Roma, Italy, pierluigi.testa@uniroma1.it)

This investigation is aiming to detect and track a moving target using its acoustic emissions and applying a new beam-forming approach. An original algorithm of medium computational complexity is reported that is capable of locating, with good accuracy, the direction of an acoustic source with only a few microphones. This algorithm circumvents the known localisation ambiguities due to the unwanted "grating lobes", by using all spectral content of the acoustic source and progressively and narrowly focusing its beam in the direction of the target. The algorithm starts by locating the direction of the target with low angular resolution, using only low frequency components of the acoustic emissions; in this way, no grating lobes occur even with only a few microphones. Then, the angular resolution is increased by processing higher frequencies of the spectrum, but profiting of the information on the angular position achieved in the previous stages. Some algorithmic means are introduced to increase the performance of the algorithmic against environmental noise. This scheme is described in detail. Some experimental tests are included and discussed.

TUESDAY AFTERNOON, 1 JULY 2008

P3-C, LEVEL 3, 3:40 TO 5:20 P.M.

Session $2 \mathrm{pSPb}$

\title{
Signal Processing in Acoustics: Beamforming, Localization, and Tracking II (Poster Session)
}

\author{
Michael Roan, Cochair \\ Virginia Tech \\ Natalia Sidorovskaia, Cochair \\ Department of Physics, University of Louisiana
}

\begin{abstract}
All posters will be on display from 3:40 p.m. to 5:20 p.m. To allow contributors an opportunity to see other posters, contributors of odd-numbered papers will be at their posters from 3:40 p.m. to 4:30 p.m. and contributors of even-numbered papers will be at their posters from 4:30 p.m. to 5:20 p.m.
\end{abstract}

\section{Contributed Papers}

2pSPb1. Particles filter applied in the real-time bearings-only tracking problem of a sonar target. Cleide V. Rodrigues (Ipiru 2 Ilha do Governador, 21931-095 Rio de Janeiro, Brazil, clevital@ hotmail.com), William Soares-Filho (Ipiru 2 Ilha do Governador, 21931-095 Rio de Janeiro, Brazil, wsoaresf@hotmail.com)

The development of a passive sonar tracking system in real-time needs algorithms each time more accurate and that can be able to follow rapid changes in the signal characteristics. In a bearings-only tracking problem, the bearing model is a nonlinear function of the target states. Nowadays, methods that deal with nonlinear and non-Gaussian estimation are receiving great attention. Particles filter is one of these methods. Particles filters are Sequential Monte Carlo methods that represent the required probability density function as a set of random samples. This present paper describes the particles filter application in the bearings-only tracking problem. Real data of targets are displayed in energy versus bearing graphics obtained in an operational naval environment. These data were used with the Sampling Importance Resampling algorithm (SIR). The particles filter is formulated in the Cartesian coordinates and then are transformed to modified polar coordinates. Results are compared with Kalman Extended Filter and the effectiveness and limitations of SIR algorithm for tracking target using a bearing/time record are examined.
2pSPb2. Tracking of underwater acoustic tone sources with linear antenna arrays. Denis Orlov (Institute of Applied Physics of the Russian Academy of Sciences, 46 Ulyanov Street, 603950 Nizhny Novgorod, Russian Federation, denis@hydro.appl.sci-nnov.ru), Alexander Rodionov (Institute of Applied Physics of the Russian Academy of Sciences, 46 Ulyanov Street, 603950 Nizhny Novgorod, Russian Federation, alexr@hydro.appl.sci-nnov.ru)

Track determination is a necessary task for a variety of underwater measurement applications, such as measurement of acoustic fields of ships. The use of linear antenna arrays provides great advantages for measurements and tracking. The investigated ship may be equipped by a special sound source of either pulse or tone signal utilized for tracking. The work considers two scenarios involving the case of a tone source. The scenarios are conditioned by parameters of the array and the source, which may vary depending on task. When a source is of relatively high frequency (several thousands $\mathrm{Hz}$ ), tracking can be based on the dependence of the Doppler frequency shift and bearing on time; in this case, the parameters to be estimated are the ones of the whole track (velocity, traverse instant, etc.). If the source frequency is relatively low (up to $300 \mathrm{~Hz}$ ), the array is large (greater than a typical distance from the source to the array aperture), the array focusing ability allows to perform the determination of "instant" locations of the source at various 
instances; estimating of parameters of the track as a whole is then based on the set of the obtained coordinates. The developed methods have been successfully tested using a large amount of experimental data.

2pSPb3. Robust beamforming and beamspace DOA estimation using measured array manifold of arbitrary geometry acoustic array. Yixin Yang (Institute of Acoustic Engineering, Northwestern Polytechnical University, 710072 Xi'an, China, yxyang@nwpu.edu.cn), Chao Sun (Institute of Acoustic Engineering, Northwestern Polytechnical University, 710072 Xi’an, China, csun@nwpu.edu.cn)

The acoustic diffraction of array supporting structure will result in the large difference of the amplitude and phase response of each hydrophone, which leads to the distortion of beampattern. A method for obtaining the array manifold of arbitrary geometry acoustic array is proposed in this paper.
We measured the array response vector in many directions with a fixed angular step in anechoic water tank. The array response vectors were smoothed to eliminate the measurement system error. Using these smoothed array response vectors, the robust beampattern of arbitrary geometry array is obtained via beam synthesis method. A 16 element volumetric acoustic array was constructed and tested in an anechoic water tank. Robust low sidelobe beampatterns based on the measured manifold were formed, and then the beamouputs were used to apply to beamspace high resolution DOA estimation methods. The experimental results show that our method can suppress noise and interferences outside the beam coverage regions, and get high resolution DOA estimation results. When the array geometry has some distortion, the weighting vectors of beamforming can still work well to form good beampatterns, and then get accurate DOA estimation results. This verifies the effectiveness of our proposed method.

TUESDAY AFTERNOON, 1 JULY 2008

P3-C, LEVEL 3, 3:40 TO 5:20 P.M.

\title{
Session 2pSPc
}

\section{Signal Processing in Acoustics: Acoustical Nondestructive Evaluation, Ultrasonics, and Imaging II (Poster Session)}

\author{
Grace Clark, Cochair \\ Lawrence Livermore National Laboratory \\ Ning Xiang, Cochair \\ Rensselaer Polytechnic Institute
}

\begin{abstract}
All posters will be on display from 3:40 p.m. to 5:20 p.m. To allow contributors an opportunity to see other posters, contributors of odd-numbered papers will be at their posters from 3:40 p.m. to 4:30 p.m. and contributors of even-numbered papers will be at their posters from 4:30 p.m. to 5:20 p.m.
\end{abstract}

\section{Contributed Papers}

2pSPc1. Identification of Noise Emission in a Gear Unit. Ales Belsak (University of Maribor, Faculty of Mechanical Engineering, Smetanova ulica 17, 2000 Maribor, Slovenia, ales.belsak@uni-mb.si), Jurij Prezelj (University of Ljubljana, Faculty of Mechanical Engineering, Aškerčeva 6, 1000 Ljubljana, Slovenia, jurij.prezelj@fs.uni-lj.si)

Today it is very important to ensure a stable production without unscheduled outages. To achieve this objective it is required to use advanced production technologies, to ensure adequate maintenance of mechanical systems and to monitor the condition of a device or machine. Reliable and accurate operation of machines and devices with as few outages as possible is desired. The significance of a life cycle design of machines and devices is growing. Possible damages in gear units can be defined by means of monitoring acoustic emission. A crack in the tooth root is usually indicated by significant changes in tooth stiffness. A difference in dynamic responses of an undamaged gear and of a damaged gear can be noted. The possibility of the use of an acoustic method in the field of condition diagostics is dealt with. The noises produced by a gear unit have been analysed, the noise sources within a gear unit have been determined and the corresponding time-frequency analysis of these sources have been performed, using an acoustic camera.
2pSPc2. Detection of Ultrasonic closer flaws using Nonlinear signal processing. Abdessalem Benammar (Image and signal processing laboratory. Welding and NDT Centre, Route de Dely-Ibrahim, BP 64, Chéraga, 16035 Alger, Algeria, Abs_benammar@yahoo.fr), Redouane Drai (Image and signal processing laboratory. Welding and NDT Centre, Route de Dely-Ibrahim, BP 64, Chéraga, 16035 Alger, Algeria, drai_r@yahoo.fr), Ahmed Kechida (Image and signal processing laboratory. Welding and NDT Centre, Route de Dely-Ibrahim, BP 64, Chéraga, 16035 Alger, Algeria, Abs_benammar@yahoo.fr), Abderrezak Guessoum (Image and signal processing laboratory. Welding and NDT Centre, Route de Dely-Ibrahim, BP 64, Chéraga, 16035 Alger, Algeria, Abs _benammar@yahoo.fr)

The ultrasonic flaw detection is an important problem in the nondestructive evaluation (NDE) of materials. In order to successfully detect and classify flaw echoes from high scattering grain echoes, an efficient and robust method is required. In this paper, a method using split-spectrum processing (SSP) combined with a neural network (NN) has been developed and applied on the ultrasonic signals to perform the detection of closer echoes. SSP can display signal diversity and is therefore able to provide the signal feature vectors for signal classification. The neural network (NN) performs 
highly complex nonlinear mapping by which signals can be classified according to their feature vectors. Therefore, the combination of SSP and NN (SSP-NN) presents a powerful technique for ultrasonic NDE. The SSP is achieved by using Gaussian bandpass filters. Then, an adaptive three layer neural network using a backpropagation learning process is applied to perform the classification processing of frequency diverse data. The SSP-NN method has been tested using both simulated and experimental ultrasonic signals, and the results show that SSP-NN has good sensitivity in the detection of ultrasonic closer flaws echoes drowned in the noise.

2pSPc3. Quantitative Ultrasonics for Inclusion and Pore Characterization of Steel Billets. Ville Kananen (Univ. of Helsinki / Dept. of Physical Sciences, POB 64 (Gustaf Hällströmin katu 2), 00014 Helsinki, Finland, ville.kananen@helsinki.fi), Joona Eskelinen (Univ. of Helsinki / Dept. of Physical Sciences, POB 64 (Gustaf Hällströmin katu 2), $00014 \quad H e l s i n k i, \quad F i n l a n d, \quad j o o n a . e s k e l i n e n @ h e l s i n k i . f i)$, Edward Hæggström (Electronics Research Unit, University of Helsinki, P.O.Box 64 (Gustaf Hällströmin katu 2), FIN-00014 Helsinki, Finland, edward .haeggstrom@helsinki.fi)

We present ultrasonic quantitative inclusion and pore characterization in bearing steel $100 \mathrm{Cr} 6$. A $9.5 \mathrm{MHz}$ focused transducer $(14 \mathrm{~cm}$ focal length, $6.3 \mathrm{MHz}$ bandwidth) scanned across the top surface of immersed $22 * 12 * 6 \mathrm{~cm}^{3}$ production samples (8pcs). Automatic pre-processing was employed to detect inclusions in samples. Recorded RF-data were analyzed. Continuous wavelet transform and cross-correlation were applied to measured and registered RF-signals from known (verified by SEM) inclusions and pores. This allowed characterizing the echoes by shape from wavelet coefficients (WC) and cross-correlation coefficients: the echo is a superposition of the reflection from the inclusion front and back surface, whereas pores exhibit no back surface echo due to their large acoustic impedance mismatch. Fourier transform allows characterizing echo RF-signals by frequency content. Dissimilarities -signal phase and WCs- in echo characteristics between different inclusion and pore classes allows quantitative inclusion and pore characterization. We discriminate three classes: oxideinclusions (aluminium-oxides, calcium-aluminates), sulphides (manganesesulphide), and pores. We also estimate inclusion size, position, and orientation. Rolled (reduction ratio 4 and 10) and unrolled production samples are characterized. The instrument functionality was validated by blind tests using SEM. Inclusion distributions in different classes of steel $100 \mathrm{Cr} 6$ and classification probabilities are presented. Our software saves sample preparation and image analysis work.

2pSPc4. Digital High Frequency Coded Imaging System. Marcin Lewandowski (Institute of Fundamental Technological Research, Swietokrzyska 21, 00-049 Warsaw, Poland, mlew@ippt.gov.pl), Andrzej
Nowicki(Institute of Fundamental Technological Research, Swietokrzyska 21, 00-049 Warsaw, Poland, anowicki@ippt.gov.pl)

Coded transmission is an approach to solve the inherent compromise between penetration and resolution required in ultrasound imaging. It is universally acknowledged that this technique gives major improvement in SNR. A novel high frequency (20-35 MHz) ultrasound real-time imaging system for research and evaluation was developed. The digital programmable coder-digitizer module based on the field programmable gate array (FPGA) supports arbitrary waveform coded transmission and RF echoes sampling up to $200 \mathrm{MSPS}$, as well as real-time streaming of digitized RF data via a high speed USB interface to the PC. All RF and image data processing were implemented in the software. A novel balanced software architecture using CPU and GPU processing supports real-time processing and display at rates up to 30 frames/sec. The system was used to acquire data for sine burst and 16-bit Golay code excitation using a single element scanning head with thick film focused spherical transducer $25 \mathrm{MHz}$ center frequency and $56 \%$ system bandwidth. SNR gain for the Golay codes (referenced to single burst) of $15 \mathrm{~dB}$ for $20 \mathrm{MHz}$ and $16 \mathrm{~dB}$ for $35 \mathrm{MHz}$ were obtained. In water the axial resolution for both single burst and the Golay codes was the same $\mathrm{FWMH}=20 \mathrm{~ns}$ at $20 \mathrm{MHz}$ and $\mathrm{FWMH}=15 \mathrm{~ns}$ at $35 \mathrm{MHz}$.

2pSPc5. Wavelet threshold enhancement by an energetic characterization of ultrasonic signal Ndt analysis. Fairouz Bettayeb (Research Center on Welding and Control, CSC, Route de Delly Brahim, Bp: 64. Chéraga, 16800 Algiers, Algeria, fairouz_bettayeb@email .com)

In ultrasonic NDE of industrial components, the visibility of flaw echoes is corrupted by noise due to multiple scattering. Grain boundaries can reach the size of the same order of magnitude than discontinuities to be detected, becoming scatters of ultrasonic noise and could be confused with defect indications. Many studies have been conducted on the use of the wavelet theory for ultrasonic signal de-noising, but nothing has been done on the structural noise features and its analyzing wavelet function. In the framework of the automation of the ultrasonic signal analysis project, we have followed the exploration of the wavelet theory, from the continuous transforms to the discrete ones, and the experiments give us some ambivalent results. So for a best threshold control, our idea was directed to the investigation of the noise analyzing function. In this work the noise features were extracted by an energetic smoothing algorithm that allows the exploration of the noise analyzing function; by which the random nature of the noise in the spatial domain is bypassed. The energetic characterization of the noise and the defects allows an improved filtering process. The new smoothing algorithm performs an accurate signal reconstruction in an interesting computing time. 


\title{
Session 2pSPd
}

\section{Signal Processing in Acoustics and Physical Acoustics: Biomedical Applications of Time-Reversal II (Poster Session)}

\author{
Oleg Sapozhnikov, Cochair \\ Center for Industrial and Medical Ultrasound, Applied Physics Lab. \\ Mickael Tanter, Cochair \\ Laboratoire Ondes et Acoustique
}

\begin{abstract}
All posters will be on display from 3:40 p.m. to 5:20 p.m. To allow contributors an opportunity to see other posters, contributors of odd-numbered papers will be at their posters from 3:40 p.m. to 4:30 p.m. and contributors of even-numbered papers will be at their posters from 4:30 p.m. to 5:20 p.m.
\end{abstract}

\section{Contributed Papers}

2pSPd1. Time reversal elastography in soft solids. Thomas Gallot(LGIT Université Joseph Fourier/CNRS, Maison des Géosciences Domaine Universitaire, 38041 Grenoble, France, thomas.gallot@obs.ujf-grenoble .fr), Stefan Catheline (LOA, Université Paris 7 Denis Diderot, ESPCI, 10 rue Vauquelin, 75005 Paris, France, stefan.catheline@espci.fr), Nicolas Benech (Science University of Montevideo, 11400 Montevideo, Uruguay, nbenech@fisica.edu.uy), Javier Brum (Science University of Montevideo, $11400 \quad$ Montevideo, Uruguay, jbrum@ fisica.edu.uy), Carlos Negreira (Science University of Montevideo, 11400 Montevideo, Uruguay, carlosn@ fisica.edu.uy), Philippe Roux (LGIT - CNRS - Université Joseph Fourier, Maison des Géosciences, 1381 rue de la Piscine, BP 53, 38041 Grenoble, France, philippe.roux@obs.ujf-grenoble.fr)

One channel time-reversal (TR) experiments allow focalization of waves in reverberant cavities. According to Rayleigh criteria, the focal spot width is directly related to wavelength and therefore depends on the mechanical properties of the medium. Thus the general idea of this work is to extract quantitative estimations of these mechanical properties from TR experiments. They were performed inside 3D soft solid cavities (gelatin based phantoms). An external source creates mechanical waves in the audible frequency range and one component of the vectorial field is measured as function of time by ultrasonic techniques developed in elastography. The wavelength information of shear waves, that dominates the vectorial field, is deduced from TR experiments. The advantage is that the technique works with sources of any shape and with any time dependence. This robustness as regard to shear wave source allows to think about many applications in the medical field, including deep or moving organs. Examples of elasticity image will be shown in inhomogeneous soft tissues.

2pSPd2. Time Reversal Acoustic focusing with random reverberator. Laurent Fillinger (Artann Laboratories/ Stevens Institute of Technology, 711 Hudson St, Hoboken, NJ 07030, USA, laurent.fillinger@artannlabs .com), Alexander Sutin (Stevens Institute of Technology, Castle Point on Hudson, Hoboken, NJ 07030, USA, asutin@stevens.edu), Armen Sarvazyan (Artann Laboratories, 1753 Linvale-Harbourton, Lambertville, NJ 08350, USA, armen@artannlabs.com)

Time Reversal Acoustic (TRA) focusing system based on an external reverberator can provide good focusing with minimum side lobes even with a few transducers. The focusing ability of such system can be increased using reverberator with rough boundaries or by adding internal random scatterers. The experiments were conducted with a reverberator made of a polyethylene bottle filled with water and a single piezoceramic disk attached externally to its wall. Experiments demonstrated that inserting in the bottle random hard scatterers or altering of the bottle surface significantly decreases the size of the focused spot and the level of side lobes. The new pseudo-impulse method of TRA focusing allowed suppressing piezotransducer resonances and provided formation of short wide band signals. The ability of such TRA focusing system based on random reverberator to form simultaneously multiple focuses and produce focal spots of the complex shape has been demonstrated. The application of binary radiation regime led to several fold increasing of the amplitude of the focal spot while the focal structure practically was not affected. Applications of such random focusing system for medical imaging and HIFU treatment is discussed. 


\title{
Session 2pSPe
}

\section{Signal Processing in Acoustics, Acoustical Oceanography, and ECUA: Bayesian Signal Processing I (Poster Session)}

\author{
Zoi-Heleni Michalopoulou, Cochair \\ Department of Mathematical Sciences, New Jersey Institute of Technology \\ Simon Godsill, Cochair \\ Dept. of Engineering, University of Cambridge, Trumpington St., CB2 IPZ Cambridge, UK
}

\begin{abstract}
All posters will be on display from 3:40 p.m. to 5:20 p.m. To allow contributors an opportunity to see other posters, contributors of odd-numbered papers will be at their posters from 3:40 p.m. to 4:30 p.m. and contributors of even-numbered papers will be at their posters from 4:30 p.m. to 5:20 p.m.
\end{abstract}

\section{Contributed Papers}

2pSPe1. Continuous estimation of VTL from vowels using a linearly VTL-covariant speech feature. Christian Feldbauer (Centre for the Neural Basis of Hearing, Department of Physiology, Development and Neuroscience, University of Cambridge, Downing Site, CB23EG Cambridge, UK, feldbauer@tugraz.at), Jessica J. Monaghan (Centre for the Neural Basis of Hearing, Department of Physiology, Development and Neuroscience, University of Cambridge, Downing Site, CB23EG Cambridge, UK, jjmm2 @ cam.ac.uk), Roy D. Patterson (Centre for the Neural Basis of Hearing, Department of Physiology, Development and Neuroscience, University of Cambridge, Downing Site, CB23EG Cambridge, UK, rdp1@cam.ac.uk)

Vowel sounds encode information about the speaker's vocal tract length (VTL) as well as vowel type. This paper shows how VTL can be estimated on a frame-by-frame basis from vowels to facilitate speaker tracking and make automatic speech recognition (ASR) more robust in multi-speaker environments. The algorithm is based on a new, VTL-covariant speech feature that encodes the size information linearly. The paper shows that this new speech feature is better suited for VTL estimation than the more traditional cepstral coefficients. The VTL estimation is based on a Gaussian mixture model that was trained on the speech material of speakers with known body height but unknown VTL. Research supported by the Austrian Science Fund (FWF) [J2541-N15], EOARD [FA8655-05-1-3043], and the UK-MRC [G0500221].

2pSPe2. A sequential Bayesian approach to vertical slice tomography of a shallow water environment. Olivier Carrière (Université libre de Bruxelles (U.L.B.) - Environmental hydroacoustics lab, av. Franklin D. Roosevelt 50, CP 194/5, 1050 Bruxelles, Belgium, ocarrier@ulb.ac .be), Jean-Pierre Hermand (Université libre de Bruxelles (U.L.B.) - Environmental hydroacoustics lab, av. Franklin D. Roosevelt 50, CP 194/5, 1050 Bruxelles, Belgium, jhermand@ulb.ac.be)

A major challenge of acoustic tomography in shallow water environments is to track physical features that are highly variable in time and space and to properly account for sound interaction with the subbottom. In a previous work an extended Kalman filter (EKF) scheme was proposed to track the sound-speed field variations in a vertical slice of the water column for known bottom geoacoustic parameters. Although the results were encouraging, it was shown that some biases were encountered when using the standard EKF, principally because of the high nonlinearity between the observations (the multi-frequency pressure field) and the environmental parameters (here the sound-speed field). In this paper, we show that Ensemble Kalman or sequential Monte Carlo filtering significantly enhance the estimation of the sound-speed field, for both range-independent and rangedependent cases. Reproducible results show that the temporal variations are well tracked, even in presence of measurement noise and model uncertainty. Furthermore the sampling approach allows to increase the time interval between successive measurements, which is an advantage from an operational viewpoint. The performances of the different nonlinear filters are also discussed.

2pSPe3. Source localization and tracking in a waveguide via sequential bayesian estimation. Kevin Cockrell (MIT, 77 Mass Ave, 5-204, Cambridge, MA 02139, USA, cockrell@mit.edu), Henrik Schmidt (MIT, 77 Mass Ave, 5-204, Cambridge, MA 02139, USA, henrik@mit.edu)

This talk addresses the problem of sequentially estimating the location of a moving acoustic source in a waveguide, given a series of acoustic field measurements from an array. The approach taken, in it's most general form, is known as sequential Bayesian estimation. A well known special case of sequential Baysian estimation is the Kalman filter. However, the Kalman filter is only optimal when the relationship between the measured parameters and the ones to be estimated is linear with Gaussian noise. Given the highly non-linear relationship between the acoustic source location and the measured acoustic field, other approaches must be taken. The performance and computational feasibility of various approaches, such as grid-based methods, will be explored using simulated data.

2pSPe4. The role of reproducing densities in a joint likelihood approach to multi-sensor detection and estimation. Michael Roan (Virginia Tech, 917 Village Way North, Blacksburg, VA 24060, USA, mroan@vt.edu), Elizabeth Hoppe (Virginia Polytechnic Institute and State University, 141 Durham Hall, Blacksburg, VA 24060, USA, ehoppe@vt .edu)

In this work, a Bayesian, joint estimation-detection approach is used for computation of sufficient statistics and development of a general multisensor information fusion architecture. An approach borrowed from sequential Bayesian processing is used to compute prior densities for joint Bayesian estimation-detection. In this approach, a posteriori densities calculated at one sensor become priors at the next sensor after a coordinate transformation that transforms the outputs of each sensor to a common reference frame for all sensors. Reproducing prior densities are used to simplify the Bayesian iteration scheme and reduce communications requirements. The framework that is developed is equally applicable to networks where all sensors communicate with a centralized detection and estimation processor as well as those networks where sensors relay information from point to point. 
We anticipate using Bayesian iteration to convert posterior information into prior information on the next data gather cycle, iterate on estimates by exchanging information between sensors, i.e., "turbo likelihood fusion," and having the central decision maker decide when sensors should or should not communicate, depending on a cost criterion which combines information gain versus communication energy expenditure. Simulation are used to show detection and estimation performance under various conditions of sensor position and orientation uncertainty.

TUESDAY AFTERNOON, 1 JULY 2008

P3-C, LEVEL 3, 3:40 TO 5:20 P.M.

\title{
Session 2pSPf
}

\section{Signal Processing in Acoustics, Biomedical Ultrasound/Bioresponse to Vibration, and Underwater Acoustics: Time Reversal Methods for Array Imaging and Signal Processing II (Poster Session)}

\author{
David Chambers, Cochair \\ $L L N L$ \\ Claire Prada, Cochair \\ Laboratoire Ondes et Acoustique
}

\begin{abstract}
All posters will be on display from 3:40 p.m. to 5:20 p.m. To allow contributors an opportunity to see other posters, contributors of odd-numbered papers will be at their posters from 3:40 p.m. to 4:30 p.m. and contributors of even-numbered papers will be at their posters from 4:30 p.m. to 5:20 p.m.
\end{abstract}

\section{Contributed Papers}

2pSPf1. Isolation of backscattering resonances of a thin spherical shell using iterative time reversal. Zachary J. Waters (Boston University, Dept. of Aerosp. and Mech. Eng., 110 Cummington St., Boston, MA 02215, USA, zjwaters@bu.edu), Benjamin R. Dzikowicz (Naval Surface Warfare Center, Panama City Division, Code HS-11, 100 Vernon Ave., Panama City, FL 32407, USA, benjamin.dzikowicz@navy.mil), R. Glynn Holt (Boston University, Dept. of Aerosp. and Mech. Eng., 110 Cummington St., Boston, MA 02215, USA, rgholt@bu.edu), Ronald A. Roy (Boston University, Dept. of Aerosp. and Mech. Eng., 110 Cummington St., Boston, MA 02215, USA, ronroy@bu.edu)

The backscattering spectrum of thin spherical shells show resonance peaks due to several different physical processes including high frequency Lamb wave excitation and low frequency modal ringing [Kaduchak et al., J. Acoust. Soc. Am. 97, 2699-2708 (1995)]. These different processes can be isolated in both time and frequency by using simulated iterative time reversal. This is accomplished by windowing in the time domain and/or filtering in the frequency domain. Iterative time reversal techniques developed for buried target detection [Waters et al., J. Acoust. Soc. Am. 122, 3023 (2007)] are applied to the partial wave series solution for the backscattering of plane acoustic waves by a thin-walled spherical shell. Tank experiments are performed to verify theoretical results. Targets loaded with both water and sediment are considered. Understanding the relationship between the time reversal window's size/position and the dominant target scattering mechanisms is key to the development of time reversal as a detection and identification technique. [Work supported by The Office of Naval Research.]

2pSPf2. The Decomposition of the Time Reversal Operator with virtual transducers. Jean-Luc Robert (Philips Research, 345 Scarborough Road, Briarcliff Manor, NY 10510, USA, jean-luc.robert@philips.com), Mathias Fink (Laboratoire Ondes et Acoustique, ESPCI, Université Paris 7, CNRS, 10 rue Vauquelin, 75005 Paris, France, mathias.fink@espci.fr)

The FDORT method (French acronym for Decomposition Of the Time Reversal Operator using Focused beams) is a variant of the DORT method using focused transmits instead of single element transmits. An intuitive interpretation of the method is presented here. It is shown that the FDORT method is equivalent to a DORT method with two different arrays: the actual array and a virtual array whose transducers are located at the foci of the transmits. By changing the focal depth, it is possible to change the position of the virtual array and thus obtain different information. An application to aberration correction in a far field phase screen model will be presented.

2pSPf3. Reconstruction of temporal invariants of the time-reversal operator for communication in shallow water. Franck D. Philippe (Laboratoire Ondes et Acoustique, ESPCI, Université Paris 7, CNRS, 10 rue Vauquelin, 75005 Paris, France, franck.philippe @ espci.fr), Thomas Folégot (NATO Undersea Research Center, Viale San Bartolomeo 400, 19126 La Spezia, Italy, folegot@nurc.nato.int), Claire Prada (Laboratoire Ondes et Acoustique, ESPCI, Université Paris 7, CNRS, 10 rue Vauquelin, 75005 Paris, France, claire.prada-julia@espci.fr), Mathias Fink (Laboratoire Ondes et Acoustique, ESPCI, Université Paris 7, CNRS, 10 rue Vauquelin, 75005 Paris, France, mathias.fink@espci.fr)

Time-reversal techniques allow focusing in waveguides with application for detection or telecommunication (Kupperman et al., J. Acoust. Soc. Am. 103(1), 25-40, (1998)). It has been shown that the DORT method (French acronym for Decomposition of the Time Reversal Operator) can be used to achieve detection and selective focusing on pointlike scatterers in a waveguide (Mordant et al., J. Acoust. Soc. Am. 105, 2634-2642 (1999)). However, the decomposition is done in frequency domain providing eigenvectors $\mathrm{V}(\omega)$ of undetermined phase. In order to achieve temporal focusing, it is necessary to built 'temporal eigenvectors' $\mathrm{V}(\mathrm{t})$ as an appropriate combination of the eigenvectors $\mathrm{V}(\omega)$. Due to dispersion, the reconstruction of the temporal eigenvectors is difficult in waveguides. One solution was proposed by Mordant et al., using the symmetry of the array response matrix and assuming continuous frequency dependence of the invariants. However this method does not work if eigenvalues crossings occur. Furthermore, it cannot be applied to distinct arrays of transmitters and receivers. We propose a simple method to reconstruct the temporal invariants by using the back propagation of eigenvector in free space. Application to data from a laboratory experiment and from the DOREV 2005 shallow water experiment are presented. 
2pSPf4. Modeling time-reversal focusing in a multiple scattering medium. Kevin J. Haworth (University of Michigan, Department of Radiology, Kresge III, R3320, Ann Arbor, MI 48109-0553, USA, khaworth@umich.edu), Jeffrey B. Fowlkes (University of Michigan, Department of Radiology, Kresge III, R3320, Ann Arbor, MI 48109-0553, USA, fowlkes@umich.edu), Paul L. Carson (University of Michigan, Department of Radiology, Kresge III, R3320, Ann Arbor, MI 48109-0553, USA, pcarson@umich.edu), Oliver D. Kripfgans (University of Michigan, Department of Radiology, Kresge III, R3320, Ann Arbor, MI 48109-0553, USA, greentom@umich.edu)

Time-reversal acoustics has been shown to be effective at focusing sound through high-order multiple-scattering media. In addition to signals being refocused, large amplitudes via pulse compression have also been observed. Previously, a statistical model was developed to simulate a timereversal experiment where an impulse is sent into the scattering medium and the entire response is time-reversed [A. Derode et al., J. App. Phys. 85, 6343-6352 (1999)]. The high-order multiple-scattering process is modeled as a shot noise process. Again postulating a shot noise process the model has been extended to allow for arbitrary input functions and arbitrary windowing before time-reversal. The model has been shown to accurately predict the variance and expectation value of ensembles of simulated data. The analytical model is also tied directly to the governing physical processes, which is useful in attempting to optimize refocused signals. This extension is motivated by a desire to achieve high-amplitude long-pulse focusing, as would be useful in thermal therapies and radiation force experiments. However, the model can find application beyond this specific end-point. The model, it's physical implications, and several time-reversal simulations will be presented.

2pSPf5. Spectral properties of the backscattering matrix in random scattering media. Alexandre Aubry (Laboratoire Ondes et Acoustique, ESPCI, Université Paris 7, CNRS, 10 rue Vauquelin, 75005 Paris, France, alexandre.aubry@espci.fr), Arnaud Derode (Laboratoire Ondes et Acoustique, ESPCI, Université Paris 7, CNRS, 10 rue Vauquelin, 75005 Paris, France, arnaud.derode@ujf-grenoble.fr)

We investigate, both experimentally and theoretically, the spectral properties of the backscattering operator in random media. The experimental set up consists in an array of 64 programmable ultrasonic transducers placed in front of a random scattering medium. The impulse responses between each couple of transducers are measured and form the interelement matrix.The evolution of the singular values with time and frequency is computed by means of a short-time Fourier analysis. Our aim is to compare the mean distribution of the singular values with the predictions of Random Matrix Theory (RMT). Two kinds of random media have been investigated: a highly scattering medium in which multiple scattering predominates and a weakly scattering medium. In both cases, residual correlations that may exist between matrix elements are shown to be a key parameter. The mean distribution of singular values exhibits, surprisingly, very different behaviors in the single and multiple scattering regimes. The agreement between experimental results and RMT predictions is found to be very good in both cases. The results are applied to the detection of a target embedded in a random scattering medium with the DORT method.

TUESDAY AFTERNOON, 1 JULY 2008

P3-C, LEVEL 3, 3:40 TO 5:20 P.M.

\title{
Session 2pSPg
}

\section{Signal Processing in Acoustics, Physical Acoustics, Biomedical Ultrasound/Bioresponse to Vibration, and Underwater Acoutics: Overview of Time Reversal in Acoustics II (Poster Session)}

\author{
David Chambers, Cochair \\ $L L N L$ \\ Claire Prada, Cochair \\ Laboratoire Ondes et Acoustique
}

\begin{abstract}
All posters will be on display from 3:40 p.m. to 5:20 p.m. To allow contributors an opportunity to see other posters, contributors of odd-numbered papers will be at their posters from 3:40 p.m. to 4:30 p.m. and contributors of even-numbered papers will be at their posters from 4:30 p.m. to 5:20 p.m.
\end{abstract}

\section{Contributed Paper}

2pSPg1. Numerical modeling of the effects of finite size transducers for time reversal acoustics in solid media. Michele Griffa (EES-11 (Geophysics) - Los Alamos National Laboratory, MS D443, Los Alamos, NM 87545, USA, mgriffa@lanl.gov), Brian E. Anderson (EES-11 (Geophysics) - Los Alamos National Laboratory, MS D443, Los Alamos, NM 87545, USA, bea@lanl.gov), Paul A. Johnson (EES-11 (Geophysics) Los Alamos National Laboratory, MS D443, Los Alamos, NM 87545, USA, paj@lanl.gov)

Time Reversal Acoustics (TRA) has been shown to be very robust not only in fluids but also in solid bounded media. The most relevant limitations to the Time Reversal Process (TRP) in solid specimens are the co-existence of several propagation modes, mode conversion at each interface (inhomogeneities or boundaries), attenuation mechanisms and the multidimensional nature of the propagating wave fields. Additional limitations arise in practical applications, for example for Non Destructive Evaluation purposes, when the Time Reversal Mirror (TRM) piezoelectric transducers are usually attached to the surface of the solid. We have investigated the role of the finite size of the TRM transducers and their sensitivity to only certain components of the incident wave fields in the TRP when they are attached to the surface of the sample under study. We have developed a theoretical analysis and performed numerical simulations and laboratory experiments in order to examine the robustness of TRA in solid media, including where the TRM is composed of finite size elements attached to the specimen surface. The results lead to useful information about the efficiency of the TRP as well as the optimization of the TRM setup in terms of transducer size. 


\title{
Session 2pSPh
}

\section{Signal Processing in Acoustics: Advances in Acoustic Sensors and Networks for Defense Applications I}

\author{
Brian G. Ferguson, Cochair \\ Defence Science and Technology Organisation, PO Box 44, Pyrmont NSW, Sydney, 2009, Australia \\ Pierre Naz, Cochair \\ ISL, 5 rue du Général Cassagnou, 68300 Saint Louis, France
}

\section{Contributed Papers}

\begin{abstract}
5:00
2pSPh1. Acoustic signal processing methods for detecting intrusions and threats in the battlespace. Brian G. Ferguson (Defence Science and Technology Organisation, PO Box 44, Pyrmont NSW, 2009 Sydney, Australia, Brian.Ferguson@dsto.defence.gov.au), Kam W. Lo (Defence Science and Technology Organisation, PO Box 44, Pyrmont NSW, 2009 Sydney, Australia, Kam.Lo@dsto.defence.gov.au)

The processing of acoustic sensor data enables the detection, classification, localization and tracking of intrusions and threats in the battlespace Initially, passive acoustic signal processing methods that are used in unattended ground sensor networks and onboard uninhabited aerial vehicles are reviewed. These methods process radiated noise (acoustic signature) data generated by sources of military interest and extract tactical information on air and ground vehicles as well as direct (rifle) and indirect (artillery/mortar) fire weapons. Examples of applying various passive acoustic signal processing methods to real sensor data collected during field experiments are presented. The tactical parameter estimates derived from these acoustic methods are then compared with ground truth data. Next, the application of active sonar signal processing to the automatic detection and tracking of a fast inshore watercraft in a cluttered harbor environment is demonstrated using real data. The air bubbles associated with wakes from high-speed surface craft are highly reflective of incident high frequency sonar signal transmissions. Finally, various high frequency active sonar methods that use both real and synthetic apertures to image sea mines are reviewed and demonstrated.
\end{abstract}

\section{$5: 20$}

2pSPh2. Networking and Fusion of Disparate Acoustic Sensors for Battlespace Applications. Tien Pham (US Army Research Laboratory, 2800 Powder Mill Road, Attn: AMSRD-ARL-SE-SA, Adelphi, MD $20783-$ 1197, USA, tien.pham1@us.army.mil), Gene Whipps (US Army Research Laboratory, 2800 Powder Mill Road, Attn: AMSRD-ARL-SE-SA, Adelphi, MD 20783-1197, USA, gene.whipps@us.army.mil)

A network of distributed acoustic sensor systems on the ground and/or in the air can be used effectively for autonomous and remote intelligence, surveillance, and reconnaissance (ISR) applications. However interoperability of disparate sensor systems is a major challenge for current coalition force applications. Specifically, a network of acoustic sensor nodes can exhibit heterogeneity in a variety of dimensions. At the sensor-level, the acoustic sensors can vary in their types (e.g., cardioid vs. omni-direction) and in their responsiveness to transient and continuous sources. At the node-level, the acoustic systems can vary in their array configuration, platform mobility, node reactiveness (e.g., timeliness of response), and information processing and output. At the network-level, the acoustic systems can vary in their communication protocols and access mediums. Current R\&D efforts within the US-UK International Technology Alliance seek to develop a "sensor fabric" technology to seamlessly connect disparate systems for networked sensing applications. Results from a recent field experiment to detect and locate moving ground targets, weapon firings, and explosions via a network of disparate acoustic sensor systems and sensing platforms (e.g., PDA's, workstations, motes and unattended ground sensor systems) are presented.

\section{$5: 40$}

2pSPh3. Performace Bounds for a Multi-Array Network Approach to Gunfire Detection and Localization. Michael Roan (Virginia Tech, 917 Village Way North, Blacksburg, VA 24060, USA, mroan@ vt.edu), Elizabeth Hoppe (Virginia Polytechnic Institute and State University, 141 Durham Hall, Blacksburg, VA 24060, USA, ehoppe@vt.edu), Marty Johnson (Virginia Tech, Mechanical Engineering, 143 Durham 0238, Blacksburg, VA 24061, USA, martyj@vt.edu)

Acoustics-based gunfire detection and sniper localization have become important in both military and civilian sectors. For the case of rifle fire, most current localization algorithms rely on estimating parameters using the projectile's cone-shaped shockwave and the muzzle blast. Previous studies have attempted to quantify shockwave detection performance as a function of range for various approaches such as wavelet matched filtering for the " $\mathrm{N}$ " shaped shockwave. Accurately detecting the shockwave is important because the shockwave shape can be used to determine bullet trajectory if the bullet velocity is known. A second layer of direction of arrival (DOA) estimation can be added by using the time difference of arrival between the shockwave and the muzzle blast. These DOA methods require a two step process of detection and estimation. In this work, a signal model is developed for a network of microphone arrays that receives the acoustic waveform generated by supersonic projectiles. The best-case localization performance of the multi-array network is quantified via the Cramer-Rao lower bound on the error in estimating shooter position. Results are illustrated using simulated data to generate CRLB ellipses on shooter position estimation error and via live fire experiments using several calibers and a multi-array network of microphones.

\section{6:00}

2pSPh4. Automated Identification of Vehicles using Acoustic Signal Processing. Naoko Evans (University of York, Department of Electronics, Heslington, YO10 5DD York, UK, ne502@ohm.york.ac.uk), David Chesmore (University of York, Department of Electronics, Heslington, YO10 5DD York, UK, edc1@ohm.york.ac.uk)

Automated identification of unauthorised intruding vehicles approaching protected infrastructure is becoming increasingly important for security purposes. This three-year project, which is now in its second year, aims to develop a real-time acoustic vehicle type recognition system that will be predominantly composed of three main parts; acoustic signal pre-processing, feature extraction, and decision making (or classification). The main study area covers various signal processing techniques in time, time-frequency and potentially frequency domains with signal classification implemented using a range of artificial intelligence techniques such as artificial neural networks. So far the focus has been on time domain signal processing and neural network classification. Whilst the work is at an early stage, the time domain methods such as Time Domain Signal Coding (TDSC) and CoOccurrence Matrix combined with neural networks have already shown some promise. The presentation will introduce the project, describe methodologies involved as well as the results to date for the novel acoustic identification of categories of vehicles. 
6:20

2pSPh5. Source detection and tracking in 3-D space using efficient subspace methods. Colin Barnhill (Johns Hopkins University, $3400 \mathrm{~N}$. Charles St, Baltimore, MD 21218, USA, shaolin_cb@yahoo.com)

This presentation will demonstrate the versatility and robustness of a MUSIC-based subspace algorithm for acoustic source detection and tracking. The new algorithm (MUSIC3D) is designed for use with spherical arrays and performs three dimensional source detection and tracking. MUSIC3D operates on a spherical decomposition of the received array signals which results in its high efficiency. Spherical decomposition of the array signals allows for data reduction, frequency selection, and direct subspace calculations. The appeal of the MUSIC3D algorithm is that it offers a large variety of implementations which can be tailored for specific environments, signal sources or noise sources. Source tracking is accomplished through a combination of a Kalman-based filter and the MUSIC3D algorithm.
$6: 40$

2pSPh6. Optimal estimation of aircraft noise with four microphones in a spatial configuration. Nico Roosnek (Roosnek Research \& Development, Vlaskamp 82, 2592 AC The Hague, Netherlands, nico@ roosnek.nl)

By using all time shifts between the arrivals of the acoustic signal at the four microphones - in a spatial configuration - the source direction is obtained. The used correlation technique reduces significantly noise from other sources and the error in the estimated level of noise from the tracked aircraft/source. By taking into account a motion model for the source, the history of that motion and the measured acoustic data a very robust sound monitor system is obtained. The sensitivity for other sources, wind and rain noise is greatly reduced - theoretically almost unlimited - in comparison with classical monitor systems. Four aircraft can be tracked simultaneously. Another system capability is the estimation of the true flightpath with a system error depending on the system configuration and the environmental conditions. Using this path information the soundscape can be calculated by inter- and extrapolation. The design goal of the system is truly met!

TUESDAY AFTERNOON, 1 JULY 2008

AMPHI BORDEAUX, 2:00 TO 6:40 P.M.

\title{
Session 2pUWa
}

\section{Underwater Acoustics and ECUA: Auralization of Sonar Signals}

\author{
Paul C. Hines, Cochair \\ Defence R\&D Canada - Atlantic, P.O. Box 1012, Dartmouth, NS B2Y3Z7, Canada \\ Paul R. White, Cochair \\ Institute of Sound and Vibration, Univ. of Southampton, University Road, Highfield, Southampton, SO17 1BJ, UK
}

\section{Invited Papers}

2pUWa1. Active sonar clutter and auralization. Douglas Abraham (CausaSci LLC, PO Box 5892, Arlington, VA 22205, USA, abrahad@ieee.org)

Target-like false alarms generically termed clutter are the primary hindrance to active sonar in shallow water operational areas. Clutter can arise from a myriad of sources with geologic, biologic and anthropogenic origin. The clutter-source echoes are often determined by automatic signal processing to be target-like and therefore potentially of interest. These numerous false alarms consume either the sonar operator's time or flood automated data and information processing algorithms. Aural analysis of clutter echoes (auralization) can increase active sonar performance through improved operator or algorithmic classification. An overview of active sonar clutter is presented with a focus on the role of auralization for clutter reduction. [Supported by the Office of Naval Research Code 321 US under contract number N0001407C0092]

2pUWa2. Insights to dolphin sonar discrimination capabilities with human listening experiments. Whitlow W. Au (Univ. of Hawaii, P.O. Box 1106, Kailua, HI 96734, USA, wau@hawaii.edu), Caroline M. Delong (Brown University, Box 1953, Providence, RI 02912, USA, Caroline_Delong@brown.edu)

Dolphins have a very keen sonar system that is able to make fine discriminations between complex targets such as proud and buried mines. To understand the cues that dolphins use for fine target discrimination, human listening experiments have been conducted with echoes from real targets using a simulated broadband dolphin echo-ranger. Echoes are stretched in time to translate the echoes into the human auditory range. The human performance is usually as accurate as the dolphin, with the additional benefit of being able to get feedback on cues used. Various types of discrimination and detection experiments have been conducted paralleling dolphin sonar experiments. Discrimination of material composition of cylinders and spheres, detection of target in clutter and cylinder wall thickness difference discrimination experiment have been performed. The human listeners performed as well or better than the dolphins at the task of discriminating between a standard target and comparison targets. Click pitch, echo duration, time-separation pitch and timbre are some of the cues used by human listeners to discriminate targets. Dolphins may use some of the same echo features as humans. Human listening studies can quickly identify salient combinations of echo features that permit object discrimination and also help refine dolphin experiments. 
2pUWa3. Similarity-Based Perceptual Feature Identification for Active Sonar Signal Classification. Scott M. Philips (MIT Lincoln Laboratory, 244 Wood Street, Lexington, MA 02420-9108, USA, scott.philips@11.mit.edu), James W. Pitton (Office of Naval Research Global, Blenheim Crescent, West Ruislip, Middlesex, HA4 7HL London, UK, jpitton@onrglobal.navy.mil)

In many acoustic signal processing applications human listeners are able to outperform automated processing techniques, particularly in the identification and classification of acoustic events. This paper develops a framework for employing perceptual information from human listening experiments to improve automatic classification of active sonar signals. We focus on the identification of new signal features that are able to predict the human performance observed in formal listening experiments. Using this framework, our newly identified features have the ability to elevate automatic classification performance closer to the level of human listeners. We develop several new methods for learning a perceptual feature transform from human similarity measures. In addition to providing a more fundamental basis for uncovering perceptual features than previous approaches, these methods also lead to a greater insight into how humans perceive sounds in a dataset. We also develop a new approach for learning a perceptual distance metric. This metric is shown to be applicable to modern kernel-based techniques used in machine learning and provides a connection between the fields of psychoacoustics and machine learning.

3:00

2pUWa4. Auditory-motivated techniques for detection and classification of passive sonar signals. Guy J. Brown (University of Sheffield, Dept. of Computer Science, Regent Court, 211 Portobello Street, S1 4DP Sheffield, UK, g.brown@ dcs.shef.ac.uk), Robert W. Mill (University of Sheffield, Dept. of Computer Science, Regent Court, 211 Portobello Street, S1 4DP Sheffield, UK, r.mill@dcs.shef.ac.uk), Simon Tucker (University of Sheffield, Dept. of Information Studies, Regent Court, 211 Portobello Street, S1 4DP Sheffield, UK, s.tucker@dcs.shef.ac.uk)

Hydrophone arrays used to make passive sonar recordings are becoming increasingly complex, thus placing great demands on sonar operators who are required to identify transient and tonal events. Machine hearing systems can mitigate this problem, by performing initial classification and detection of passive sonar signals based on perceptually relevant principles. We describe two such systems. In the first system, transient sounds are characterised using measures of timbre and acoustic pattern. Acoustic features relating to timbre were identified by a multidimensional scaling study, in which human listeners judged the similarity of transient sonar sounds. The perceptual features give improved classification performance compared to commonly-used statistical measures derived from the power spectrum. The second system is an auditory-motivated approach to narrowband detection, which is based on information in zerocrossing intervals rather than signal power. This is motivated by the observation that the fine time structure of an acoustic stimulus is preserved by the phase locking of auditory nerve fibres. We show that a power detector outperforms the interval detector when the signal is centred on the analysis filter. However, when the signal frequency deviates from the centre of the analysis band, interval-based detection improves and power-based detection worsens.

\section{$3: 20$}

2pUWa5. Auditory models and nonlinear filterbanks in underwater auralization. Stefan Bleeck (National Physical Laboratory, Hampton Road, TW11 OLW Teddington, UK, bleeck@gmail.com), Paul D. Fox (National Physical Laboratory, Hampton Road, TW11 OLW Teddington, UK, pf@isvr.soton.ac.uk), Paul R. White (Institute of Sound and Vibration, Univ. of Southampton, University Road, Highfield, SO17 1BJ Southampton, UK, prw@isvr.soton.ac.uk), Niamh O’Meara (National Physical Laboratory, Hampton Road, TW11 OLW Teddington, UK, niamh17@gmail.com)

Mammals like dolphins and humans have the ability to distinguish between different objects by listening to their scattered signals. We explore this phenomenon by evaluating the "Auditory Image Model" (AIM), a biologically inspired model of the human auditory system from the outer ear up to central processing. AIM aims to simulate the spectral analysis of the basilar membrane, the neural encoding and the temporal integration performed by the auditory system. Simulated scattered signals from the same object objects of different sizes and different objects of same size subjected to an incident pulse were analysed numerically with AIM, simulating the response of the human auditory system. The resulting neuronal activity patterns were analysed in two independent dimensions using the filter centre frequency and the relative timing intervals of the responses. The results are interpreted globally as reflecting the size and the shape/properties of the object respectively. We show for a series of calibration stimuli that the model can distinguish between same objects of different size and different objects of the same size. We also discuss how a nonlinear model of the basilar membrane, performing a Gammachirp/Mellin transformation, may act as a possible feature extraction tool for classification methods

3:40-5:20 Posters

Lecture sessions will recess for presentation of poster papers on various topics in acoustics. See poster sessions for topics and abstracts. 


\section{Contributed Papers}

\section{$5: 20$}

2pUWa6. What it is like to be a bat: A sonar system for humans. Cameron Morland (Boston University, Dept. of Cognitive and Neural Systems, 677 Beacon St., Boston, MA 02215, USA, cjmorlan@bu.edu), David Mountain (Boston University, Biomedical Engineering Dept., 44 Cummington St., Boston, MA 02215, USA, dcm@bu.edu)

Echolocation is a method of perceiving the world by emitting noises then listening to the reflections of these noises off objects in the world. It is used by animals for hunting and navigation. Some visually impaired humans use echolocation as part of their orienting repertoire. This work describes a device designed to enhanced one's existing echolocation ability. The device repeatedly emits an inaudible, ultrasonic "'click", several times per second. Each click reflects off surfaces; this reflection is detected by head-mounted microphones. Computer processing converts the signals into audible signals, which are presented to the user over custom open-ear earphones. ILD, ITD, HRTF, and Doppler shifts in the reflections allow the user to distinguish the location and size/surface properties of objects. The spatial cues presented by our device match those used to localize ordinary audible objects. The auditory "image" generated in this way causes objects in the world to seem to emit sounds, and for objects with different shapes/textures to have subtly different sounds. A prototype has been constructed. Simple detection of objects and open spaces is readily possible, as well as Doppler-based gross surface texture. Further refinement in the areas of emission design, spectral spatialization, externalization, and miniaturization is required.

\section{$5: 40$}

2pUWa7. Modeling the mechanism and neural substrate for aural categorization of sonar echoes. Jason E. Summers (U.S. Naval Res. Lab., Acoust. Div., Code 7142, 4555 Overlook Ave. SW, Washington, DC 20375, USA, jason.summers@nrl.navy.mil), Charles F. Gaumond (U.S. Naval Res. Lab., Acoust. Div., Code 7142, 4555 Overlook Ave. SW, Washington, DC 20375, USA, charlie.gaumond@nrl.navy.mil), Derek Brock (U.S. Naval Res. Lab., Acoust. Div., Code 7142, 4555 Overlook Ave. SW, Washington, DC 20375, USA, derek.brock@ nrl.navy.mil), Ralph N. Baer (U.S. Naval Res. Lab., Acoust. Div., Code 7142, 4555 Overlook Ave. SW, Washington, DC 20375, USA, ralph.baer@nrl.navy.mil)

Previously, human perception of impulsive active-sonar echoes was investigated through paired-comparison ratings and multidimensional scaling (MDS). In the resulting MDS configuration, stimuli formed clusters representing aurally distinct categories. An interpretation is presented which suggests that dissimilarity judgments reflect separate processes for within- and between- category comparisons. The process of categorization is not based on decision boundaries in a low- dimensional space of ordinal-scale perceptual features, while within-category judgments do reflect such features. A class-specific paradigm seems appropriate as a model of human categorization: signals are compared to category archetypes, each described by a set of features that may be unique to that category. Regardless of the specific mechanism, listeners must carry out categorization on a particular signal representation: the neural substrate of the process. The suitability of various temporal, spectral, and time-frequency signal representations are investigated through observing categories formed by hierarchical clustering. A signal-processing model of the spectrotemporal receptive fields of the auditory cortex and the processing of the auditory periphery [T. Chi et al., J. Acoust. Soc. Am. 118, 887-906 (2005)] is shown to yield good agreement with human categorization. Techniques for feature selection from this representation are discussed. [Work supported by ONR.]

\section{6:00}

2pUWa8. Automatic classification of vocalizations with Gaussian Mixture Models and Hidden Markov Models. Judith C. Brown (MIT Media Lab, 37 Indian Ridge Rd, Natick, MA 01760, USA, brown@media.mit.edu), Paris Smaragdis (Adobe Systems Inc., 275 Grove St., Newton, MA 02466, USA, paris@media.mit.edu)

The automatic classification of marine mammal sounds is very attractive as a means of assessing massive quantities of recorded data, freeing humans and offering rigorous and consistent output. Calculations on a set of vocalizations of Northern Resident killer whales using Dynamic Time Warping have been reported recently. [Brown, J. C., and P.J.O. Miller, "Automatic classification of killer whale vocalizations using dynamic time warping," J. Acoust. Soc. Am. 122, 1201-1207, (2007).] Since this method requires the time-consuming pre-processing measurement of the frequency contours, we have explored the use of Gaussian Mixture Models (GMM) and Hidden Markov Models (HMM). These methods can be applied directly to timefrequency decompositions of the recorded signals. Calculations have been made on a set of 75 calls previously classified perceptually into 7 call types. Preliminary results give an agreement of roughly $85 \%$ agreement with the perceptual classification for the GMM and over $90 \%$ for an HMM.

\section{6:20}

2pUWa9. Incorporation of acoustic level analysis with sonar operator workstation workflow using hierarchical hidden Markov models. Justin Nevitt (Naval Research Laboratory, 4555 Overlook AVE SW, Washington, DC 20375, USA, justin.nevitt@nrl.navy.mil), James Ballas (Naval Research Laboratory, 4555 Overlook AVE SW, Washington, DC 20375, USA, james.ballas@nrl.navy.mil)

Future Anti Submarine Warfare (ASW) systems will be highly automated, and utilize multiple sonar signal processing, non-acoustic information, off platform information, and environmental information to maximize the detection, tracking, and classification of undersea threats. Recently, a hidden Markov model (HMM) was developed to describe and characterize the workflow strategies that expert sonar operators used during the IUSW-21 (Integrated UnderSea Warfare for the 21st Century) Sea Test. Abandoning the normative workflow designed into the system, the sonar operators developed and utilized unanticipated strategies to gather evidence to confirm or disconfirm automated classifications or to manually classify contacts. The workflow included detailed visual inspection of and listening to the sonar return. This initial HMM did not include a characterization of the auditory processing. Hidden Markov models are well suited to this purpose. Adding this component to the operator workflow was achieved by a hierarchical HMM which represents the acoustic processing as an embedded HMM in the overall workflow HMM. The resulting model is not just an operator workflow but defines a system processing model that could be used as a framework to develop automated processing of selected components. 


\title{
Session 2pUWb
}

\section{Underwater Acoustics and ECUA: Sonar System and Transducer Calibration Methodology I}

\author{
Kenneth G. Foote, Cochair \\ Woods Hole Oceanographic Institution, Woods Hole, MA 02543, USA \\ Stephen P. Robinson, Cochair \\ National Physical Laboratory, Hampton Road, Teddington, TW11 OLW, UK
}

Invited Papers

2:00

2pUWb1. The sonar equations: definitions, dimensions and units of individual terms. Michael A. Ainslie (TNO Defence, Security and Safety, Oude Waalsdorperweg 63, 2597 AK The Hague, Netherlands, michael.ainslie@tno.nl)

The passive and active sonar equations are reviewed with regard to the definitions of individual terms and their units. It is shown on the one hand that ambiguities exist in conventional definitions that can lead to misinterpretation, and on the other that under special conditions correction terms are needed that are not included in the standard equations. Revised definitions are proposed that remove the need for some of the correction terms and reduce the likelihood of misunderstanding. Particular emphasis is placed on the terms 'propagation loss' and 'source level' for both active and passive sonar. For example, the effect on changes in impedance between the source and receiver of the sound is considered in the case of passive sonar, and between the source, receiver and scatterer of sound in the case of active sonar.

$$
\text { 2:20 }
$$

2pUWb2. Validation of primary hydrophone calibrations by inter-laboratory comparisons and by independent calibration methods. Stephen P. Robinson (National Physical Laboratory, Hampton Road, TW11 OLW Teddington, UK, Stephen.Robinson@npl.co.uk), Peter D. Theobald (National Physical Laboratory, Hampton Road, TW11 OLW Teddington, UK, pdt @npl.co.uk)

A description is presented of two approaches which may be used to validate primary calibration methods for hydrophones and transducers. Firstly, a comparison may be made with another independent absolute calibration method, preferably one based on a different physical principle (and therefore with few common sources of uncertainty). Secondly, an inter-laboratory comparison of calibrations may be undertaken between different institutes operating at a similar level. This paper describes the results of such exercises for free-field calibration of hydrophones in the range from $1 \mathrm{kHz}$ to $500 \mathrm{kHz}$. Firstly, two independent calibration methods are compared: the three-transducer reciprocity method and a method based on optical interferometry. The differences observed in the results are typically less than $0.5 \mathrm{~dB}$, which is of the same order as the overall uncertainties of each of the methods. Secondly, the results are shown of a recent international comparison of hydrophone calibrations involving institutes from Canada, China, Germany, Russia, South Africa, UK, and USA. Here, the agreement was generally within quoted uncertainties, the results generally lying within a $\pm 0.5 \mathrm{~dB}$ band for frequencies up to $300 \mathrm{kHz}$. A discussion is given of the general sources of uncertainties in the calibrations.

2:40

2pUWb3. Phase calibration of hydrophone and its application in the reconstruction of underwater acoustic waveforms. Yuebing Wang (Hangzhou Applied Acoustics Research Institute, 96 Huaxing Road, 310012 Hangzhou, China, wang_yuebing@tom.com), Shui Li (Hangzhou Applied Acoustics Research Institute, 96 Huaxing Road, 310012 Hangzhou, China, lishui9861@163.com), Yongjun Huang (Hangzhou Applied Acoustics Research Institute, 96 Huaxing Road, 310012 Hangzhou, China, pink2701@126.com)

Abstract: In this paper, both the reciprocal method and the optical method for the calibration of plural sensitivities of hydrophones are reviewed firstly. Then an experimental system is introduced in which with a $1.5 \mathrm{MHz}$ focusing transducer acting as a position indicator, phase responses of hydrophones can be calibrated easily and accurately. Measurements are carried out for different types of hydrophones and results are given in the frequency range $20 \mathrm{kHz}$ to $400 \mathrm{kHz}$. Finally, with the plural responses of hydrophones included in the construction for the transfer function of the receiving system, acoustic waveforms produced by a projector are reconstructed in wide band from received signals, and a few conclusions are summarized from the experiment. 


\section{Contributed Papers}

3:00

2pUWb4. The hydrophone free-field calibration in the non-anechoic water tank using continuous radiation mode. Alexander Isaev (VNIIFTRI, Mendeleevo, 141570 Moscow, Russian Federation, isaev@ vniiftri.ru), Anton Matveev (VNIIFTRI, Mendeleevo, 141570 Moscow, Russian Federation, matveev@ vniiftri.ru)

Anechoic chambers are used at free-field measurements in acoustic. Quality of the anechoic chamber estimate by comparing the dependence of sound pressure with distance between a projector and the receiver with the inversely proportional law. Creation of an anechoic water tank is a difficult technical problem. Therefore measurements in a water tank usually use tone burst radiation technique. In a non-anechoic water tank it is possible to provide free-field conditions at a continuous radiation mode. It is achieved by using a signal with the distributed spectrum and mathematical processing of results of measurements. The measurement procedure and results of free field calibration of this method are considered in the paper. Efficiency of measuring procedure was estimated by a check of the inversely proportional law in conditions of a non-anechoic water tank.

3:20

2pUWb5. Calibration of vector sensors. Joseph A. Clark (NSWCCD, Code 7340, 9500 MacArthur Blvd., West Bethesda, MD 20817-5000, USA, joseph.a.clark1@navy.mil)

Vector sensors that combine a hydrophone and a triaxial arrangement of accelerometers in a neutrally buoyant package are currently being used for underwater radiated noise measurements and other applications. Precise amplitude and phase calibration is required to obtain high quality measurements. A basic procedure that has been used successfully to calibrate measured radiated noise spectra in one-third-octave and narrowband formats will be described. Results of validation experiments that compared vector sensor measurements with other reference data will be presented. An iterative calibration scheme for improving the performance of a vector sensor employed as part of an active sonar system will also be presented.

3:40-5:20 Posters

Lecture sessions will recess for the presentation of poster sessions on various topics in acoustics. See poster sessions for topics and abstracts.

\section{Invited Papers}

2pUWb6. Array gain degradation due to nearby bubbles. Richard Lee Culver (ARL Penn State, PO Box 30, State College, PA 16804, USA, rlc5@psu.edu), Mario F. Trujillo (ARL Penn State, PO Box 30, State College, PA 16804, USA, mft10@psu.edu)

Signal-to-noise ratio (SNR) is signal power divided by noise power, usually expressed in $\mathrm{dB}$ (SNR $=20 * \log 10$ (Psig/Pnoise). Array gain $(\mathrm{AG})$ is the increase in SNR at an array output relative to that at a single element. AG assumes spatially-compact signals embedded in spatially-diffuse noise or interference. When the array is steered in the direction of a signal, AG is maximum when the signal is fully coherent across the array (i.e. the signals add in phase for all elements) while the noise or interference is incoherent across the array (i.e. adds with random phase). For an acoustic array operating in the ocean, we would like to understand the degree to which nearby bubbles degrade AG. Scattering by bubbles represents interference at the array. Bubble attenuation can also degrades array performance by attenuating the signal of interest, but that is separate from AG degradation. Previously we applied the single scattering approximation of Ishimaru (1977, Chap. 6) and found that scattering from bubbles very close to the array can generate correlated interference, thereby decreasing AG. Here we validate the theory using in-water measurements of AG as the distance of from the array to the bubbles varies. Work sponsored by Office of Naval Research, Code 321 Undersea Signal Processing.

2pUWb7. A method for calibrating hydrophones immersed in sandy sediment. Gary B. Robb (National Oceanography Centre, University of Southampton Waterfront Campus, European Way, SO14 3ZH Southampton, UK, gbor199@noc.soton.ac.uk), Gary Hayman (National Physical Laboratory, Hampton Road, TW11 OLW Teddington, UK, Gary.Hayman@npl.co.uk), Peter D. Theobald (National Physical Laboratory, Hampton Road, TW11 OLW Teddington, UK, pdt@npl.co.uk), Victor F. Humphrey (Institute of Sound and Vibration, Univ. of Southampton, University Road, Highfield, SO17 1BJ Southampton, UK, vh@isvr.soton.ac.uk), Stephen P. Robinson (National Physical Laboratory, Hampton Road, TW11 OLW Teddington, UK, Stephen.Robinson@npl.co.uk), Timothy G. Leighton (Institute of Sound and Vibration, Univ. of Southampton, University Road, Highfield, SO17 1BJ Southampton, UK, T.G.Leighton@soton.ac.uk), Justin K. Dix (National Oceanography Centre, University of Southampton Waterfront Campus, European Way, SO14 3ZH Southampton, UK, jkd@ noc.soton.ac.uk), Angus I. Best (National Oceanography Centre, University of Southampton Waterfront Campus, European Way, SO14 3ZH Southampton, UK, aib@noc.soton.ac.uk)

Hydrophones are frequently used as receivers for in situ sediment acoustic experiments. At present, processing techniques use receiver sensitivities measured from water-based calibrations. It is, however, accepted that the receive sensitivity will depend on the medium surrounding the hydrophone, particularly at frequencies close to the transducer's resonance frequency. To assess this affect, a series of calibrations were performed over the frequency range of 10 to $200 \mathrm{kHz}$ on two types of hydrophones (with cylindrical and spherical elements) inserted into degassed sandy sediment. Sensitivities were measured using a modified three-transducer reciprocity technique, which uses a co-linear arrangement to allow the sediment attenuation to be omitted from the sensitivity calculation. The insertion of the hydrophones into the sediment reduced the measured receive sensitivities by a maximum value of $3.8 \mathrm{~dB}$ with respect to the equivalent water-based calibrations. The co-linear arrangement adopted allowed the transmission between the outer devices to be recorded with and without the central hydrophone present. Repeat measurements indicated that the sediment disturbance associated with the removal of the central hydrophone caused sensitivity differences of less than $1.2 \mathrm{~dB}$, while the inclusion of the central hydrophone caused a shadowing effect which increased sensitivities by between 1.3 to $4.0 \mathrm{~dB}$. 
2pUWb8. Standard-target method of calibrating active sonars: principles, applications, benefits. Kenneth G. Foote (Woods Hole Oceanographic Institution, Woods Hole, MA 02543, USA, kfoote@whoi.edu)

The standard-target method of calibrating active sonar involves suspending a target at a known position in a sonar beam, insonifying it with a known signal, measuring the scattered pressure field, and relating this to the scattering properties of the target. These properties are known a priori for standard targets, rendering the calibration primary. Repetition of the procedure for different relative sonar-target positions can enable determination of directional and nearfield characteristics. Following a review of standard-target calibration principles, the method is illustrated for both narrowband and broadband scientific echo sounders and multibeam sonars, with operating frequencies in the range 18-3200 kHz. Applications to sidescan sonar and to low-frequency parametric sonars and other mid-frequency sonars in the nominal range 1-10 kHz have not yet been developed, but are evidently feasible given preliminary standard-target designs, which are described. In every case, the standard targets are of a proven manageable size, with immersion weight less than $200 \mathrm{~N}$. Benefits of standard-target calibration, including accuracy, operational convenience, and cost-effectiveness, among others, are emphasized. The method, which is useful for both quantitative and qualitative imaging applications, extends to borehole acoustic sensors and medical ultrasonic devices.

\title{
Contributed Paper
}

6:20

2pUWb9. Calibration of broadband active acoustic systems using a single standard spherical target. Timothy K. Stanton (Woods Hole Oceanographic Institution, Applied Ocean Physics \& Engineering Department, 98 Water Street, MS \#11, Woods Hole, MA 02543, USA, tstanton@whoi.edu), Dezhang Chu (NOAA/NMFS/NWFSC/FRAMD, Montlake Blvd., E. Seattle, WA 98112, USA, dchu@whoi.edu)

When calibrating a broadband active acoustic system with a single standard target such as a sphere, the inherent resonances associated with the scattering by the sphere pose a significant challenge. In this research, a method is developed which completely eliminates the source of resonances through isolating and exploiting the echo from the front interface of a sphere. This echo is relatively insensitive to frequency over a wide range of frequencies, lacking resonances, and relatively insensitive to small changes in material properties and, in the case of spherical shells, shell thickness. The research builds upon the concept of using this echo for calibration in the work of Dragonette et al. (J. Acoust. Soc. Am. 69, 1186-1189 (1981)). This current work generalizes that of Dragonette by 1) incorporating a pulse compression technique to significantly improve the ability to resolve the echo, and 2) rigorously accounting for the scattering physics of the echo so that the technique is applicable over a wide range of frequencies and material properties of the sphere. The utility of the new approach is illustrated through application to data collected at sea with an air-filled aluminum spherical shell and long broadband chirp signals $(30-105 \mathrm{kHz})$.

TUESDAY AFTERNOON, 1 JULY 2008

P3-C, LEVEL 3, 3:40 TO 5:20 P.M.

\section{Session 2pUWd}

\section{Underwater Acoustics and ECUA: Sonar System and Transducer Calibration Methodology II (Poster Session)}

\author{
Kenneth Foote, Cochair \\ Woods Hole Oceanographic Institution \\ Stephen Robinson, Cochair \\ National Physical Laboratory
}

\begin{abstract}
All posters will be on display from 3:40 p.m. to 5:20 p.m. To allow contributors an opportunity to see other posters, contributors of odd-numbered papers will be at their posters from 3:40 p.m. to 4:30 p.m. and contributors of even-numbered papers will be at their posters from 4:30 p.m. to 5:20 p.m.
\end{abstract}

\section{Contributed Paper}

2pUWd1. Low frequency hydrophone calibration with using tensometric pressure sensor. Alexander Isaev (VNIIFTRI, Mendeleevo, 141570 Moscow, Russian Federation, isaev@vniiftri.ru), Alexander Shchelkunov (VNIIFTRI, Mendeleevo, 141570 Moscow, Russian Federation, for_my_reg@mail.ru)

Usually when calibrating a hydrophone in an acoustic coupler the piezoelectric hydrophones use an auxiliary transducer. Widely applicable reciprocity calibration is high-ly accurate, but difficult in realization and laborious. The method of comparison with a reference transducer is more simple and quick, but less accurate. In the paper the method of low frequency hydrophone calibration using the auxiliary transducer with a flat frequency response up to $3 \mathrm{kHz}$ is considered. The auxiliary transducer is calibrated accurately by changing the water column in the acoustic coupler. It finally allows us to calibrate measuring hydrophones more accurately too. The measuring equipment on the basis of tensometric pressure sensor type MPX 5010 is considered. 


\title{
Session 2pUWe
}

\section{Underwater Acoustics and ECUA: Geoacoustic Sediment Modeling I (Poster Session)}

\author{
Nicholas Chotiros, Cochair \\ Applied Research Laboratories, University of Texas \\ Jean-Pierre Sessarego, Cochair \\ Laboratory for Mechanics and Acoustics CNRS
}

\begin{abstract}
All posters will be on display from 3:40 p.m. to 5:20 p.m. To allow contributors an opportunity to see other posters, contributors of odd-numbered papers will be at their posters from 3:40 p.m. to 4:30 p.m. and contributors of even-numbered papers will be at their posters from 4:30 p.m. to 5:20 p.m.
\end{abstract}

\section{Contributed Papers}

2pUWe1. Estimating the frequency dependent behavior of marine sediment sound speeds using low frequency aircraft sound. Alireza Amiri-Simkooei (Acoustic Remote Sensing Group, Delft Institute of Earth Observation and Space Systems, Delft University of Technology, Kluyverweg 1, 2629 HS Delft, Netherlands, a.amirisimkooei@tudelft.nl), Mirjam Snellen (Acoustic Remote Sensing Group, Delft Institute of Earth Observation and Space Systems, Delft University of Technology, Kluyverweg 1, 2629 HS Delft, Netherlands, m.snellen@tudelft.nl), Dick G. Simons (Delft University of Technology, P.O. Box Postbus 5048, 2600 GA Delft, Netherlands, d.g.simons@tudelft.nl), Michael Buckingham (Marine Physical Lab, Scripps Institution of Oceanography, UCSD, 9500 Gilman Dr. M/C 0238, La Jolla, CA 92093-0238, USA, mjb@mpl.ucsd.edu)

For investigating the dispersion behavior of sediment sound speeds, broadband sound speed measurements are required. Sediment sound speed dispersion models predict the largest sound speed variations typically at low frequencies. Aircraft fly-over noise acquired on receivers placed in sediment proved to be a promising approach towards broadband low-frequency measurements. Since the magnitude of the Doppler-shift depends on sound speed at the receiver position, the observed Doppler-shift can be used to derive the sound speed. We propose a new approach using the entire time series instead of employing only the two observed frequencies at approaching and departing. It is based on a model for received signals, accounting for the Doppler-shifted frequency. By maximizing the match between modeled and measured time series as a function of sound speed and frequency in a leastsquares framework, estimates of the frequency-dependent sound speed are obtained. The method is applied to data acquired at receivers placed in air, water and sediment. Statistical tests indicate that the marine sediment sound speed depends indeed on frequency, i.e. sound speed decreases significantly below $300 \mathrm{~Hz}$. A comparison is made with a modified viscous fluid model from which realistic values for the geotechnical parameters of the considered sediment were obtained.

2pUWe2. Imaging of Large-Scale Sediment Transport Dynamics with Multibeam Sonar. Stephen M. Simmons (University of Leeds, School of Earth and Environment, University of Leeds, LS29JT Leeds, UK, s.m.simmons@leeds.ac.uk), Dan R. Parsons (University of Leeds, School of Earth and Environment, University of Leeds, LS29JT Leeds, UK, parsons.daniel@btinternet.com), Jim L. Best (Departments of Geology and Geography and Ven Te Chow Hydrosystems Laboratory, 1301 W. Green St., University of Illinois, Urbana, IL 61801, USA, jimbest@uiuc.edu), Chris Malzone (Reson Inc., Goleta, Santa Barbara, CA 93117, USA, CMalzone @ reson.com), Gareth M. Keevil (University of Leeds, School of Earth and
Environment, University of Leeds, LS29JT Leeds, UK, g.keevil@ see.leeds .ac.uk)

Multibeam Echo-Sounder (MBES) systems have developed rapidly over recent decades and are routinely deployed to provide high-resolution bathymetric imaging. Modern data handling and storage technologies have facilitated the logging of the back-scatter information previously discarded by these systems. This paper develops a novel methodology to exploit this logging capability to quantify the concentration and dynamics of suspended sediment within the water column. This development provides a multipurpose tool for the holistic surveying of sediment transport by imaging suspended sediment concentration, associated flow structures and providing concurrent high-resolution bathymetry. This paper presents the results obtained from the deployment a RESON 7125 MBES in the field, with the aim of examining the dynamics of suspended sediment transport over dune bedforms and in the region of flow mixing between large rivers of significantly different suspended sediment concentration. Results from controlled tank tests are also presented, obtained using different types and mixes of sediment. The results demonstrate the capability of MBES systems to successfully resolve the contrast in suspended sediment concentrations. The large sets of data recorded in the two-dimensional MBES swath enables the real-time monitoring of suspended sediment transport and related flow processes on a scale previously unrealisable with single-beam acoustic systems.

2pUWe3. The influence of topography on sidescan sonar images. Dietmar Bürk (GKSS Research Centre, Max-Planck-Str. 1, Institute for Coastal Research, 21502 Geesthacht, Germany, dietmar.buerk @gkss.de), Ingo Klaucke (IFM-GEOMAR, Leibniz-Institute of Marine Sciences, Wischhofstraße 1-3, 24148 Kiel, Germany, iklaucke@ifm-geomar de)

The backscattering signal on sidescan sonar images is to a large degree dependent on the incident angle of the acoustic beam onto the seafloor. As sidescan sonar systems are often used for mapping seafloor lithologies, it is necessary to reduce or even remove the effect of different backscattering strengths caused by varying incidence angles. This study evaluates the influence of seafloor morphology on the acoustic backscattering signal of the deep-towed IFM-GEOMAR DTS-1 sidescan sonar system. Data used are from the Pacific continental slope offshore Nicaragua in a water depth between 800 and 2400 metres. There authigenic carbonate patches formed by cold fluid venting are imaged with a high backscattering level. The carbonates are often located on top or on the flanks of mound structures with a strong morphology. The specific DTS-1 backscattering function is determined on normal, uniform seafloor sediment, and then applied in a new processing algorithm to the raw data of two test areas. The change in amplitude strength when considering seafloor morphology is calculated. The topographic influence on the backscattering signal can be quite significant when 
imaging mound structures. Nevertheless the high backscatter on mounds is not completely removed, leading to the conclusion that it originates from a different seafloor lithology and roughness.

2pUWe4. Theoretical justification of a coherent forward model for subbottom profiler data inversion. Sandrine Rakotonarivo (Laboratoire E3I2, ENSIETA, 2, rue François Verny, 29806 Brest, France, rakotosa@ensieta.fr), Michel Legris (Laboratoire E3I2, ENSIETA, 2, rue François Verny, 29806 Brest, France, legrismi@ensieta.fr), Rozenn Desmare (Institut de Recherche de l'Ecole Navale, IRENav, BP 600, 29240 Brest Armées, France, desmare@ecole-navale.fr), Frédéric Jean (IXSEA, 46, quai François Mitterrand, 13600 La Ciotat, France, frj@ixsea.com)

Subbottom profiling systems measure and identify sediment layers that exist below the sediment/water interface. Nowadays, very-high resolution chirp profilers provide calibrated wideband signals which may enable quantitative seabed characterisation. In literature, sediment classification is based on attenuation and reflectivity estimation and backscattering models used for the inversion consider plane wave propagation through layered homogeneous attenuated bottom. But this bottom modelling may be in contradiction with the emitted spherical front wave and core samples observations which point out a heterogeneous structure of marine subbottom. Therefore, impacts of profiler features, geometry measurement and subbottom structure (layering, attenuation, rugosity, and volumetric inhomogeneity) on backscattering signal are all reviewed in this paper. This analysis shows that classical model of wave coherent propagation through layered homogeneous attenuated media offers the best compromise between its accuracy and inversion possibility, if bottom heterogeneities are insignificant (Rayleigh scatterers). For bigger heterogeneities (Rayleigh parameter close to unity), coherent backscattering still prevails though wave propagation is affected by scattering. In this case, the same coherent forward model is used with modified reflectivity and transmission coefficients. Then, sensitiviy and inversion possibility of the coherent bottom backscattering model are discussed.

2pUWe5. Use of dual methods to infer methane bubble populations in gassy sediments: Inversion of combination-frequency data. Agni Mantouka (Institute of Sound and Vibration, Univ. of Southampton, University Road, Highfield, SO17 1BJ Southampton, UK, am2@isvr.soton .ac.uk), Timothy G. Leighton (Institute of Sound and Vibration, Univ. of Southampton, University Road, Highfield, SO17 1BJ Southampton, UK, T.G.Leighton@soton.ac.uk), Gary B. Robb (National Oceanography Centre, University of Southampton Waterfront Campus, European Way, SO14 3ZH Southampton, UK, gbor199@noc.soton.ac.uk), Angus I. Best (National Oceanography Centre, University of Southampton Waterfront Campus, European Way, SO14 3ZH Southampton, UK, aib@noc.soton.ac.uk), Justin K. Dix (National Oceanography Centre, University of Southampton Waterfront Campus, European Way, SO14 3ZH
Southampton, UK, jkd@noc.soton.ac.uk),Zygmunt Klusek(Institute of Oceanography, Polish Academy of Science, P.O. Box 148 Sopot, Poland, klusek@iopan.gda.pl), Paul R. White (Institute of Sound and Vibration, Univ. of Southampton, University Road, Highfield, SO17 1BJ Southampton, UK, prw@isvr.soton.ac.uk)

Bubbles can dramatically change the acoustic properties of their host medium even if they are present in very small amounts. This paper describes the combination-frequency component of tank and field measurements taken using a device which measures bubbles in marine sediments using multiple acoustic techniques (allowing the results of the various techniques to be compared). The combination-frequency method uses the nonlinear scattering property of bubbles when insonified by two primary frequencies. For low void fractions, there is a monotonic relationship between the scattered field and the population of bubbles resonant at either of the primary frequencies or combination of these and/or their subharmonics. This principle is used to infer the bubble size distribution. In contrast to the case of gas bubbles in water, in marine sediments the shear properties of the host medium must be incorporated into the model for the bubble dynamics and a new model for this is presented. This model is then inverted to obtain the bubble size distribution. The predictions of this method were compared with the method of inversion of propagation data (detailed in a companion paper), obtained from in situ experiments on the South coast of England.

2pUWe6. Comparisons of Biot and grain-shearing theories: sound speed, attenuation and acoustic impedance. Brian T. Hefner (Applied Physics Laboratory, University of Washington, 1013 NE 40th St, Seattle, WA 98105, USA, hefner@apl.washington.edu), Kevin L. Williams (Applied Physics Laboratory, University of Washington, $1013 \mathrm{NE}$ 40th St, Seattle, WA 98105, USA, williams@apl.washington.edu)

The comparison of sediment sound speed and attenuation measurements to predictions has been the primary method used to test Biot theory and the grain-shearing model. Examples of data/model comparisons will be shown. Subsequent refinements made to these models result in similar predictions for sound speed and attenuation. However, the underlying physics is substantially different suggesting other, more indirect means for discriminating between sediment propagation theories. One technique that has received recent attention is the measurement of forward scattering from the sediment interface. Model predictions of these measurements depend not only the sound speed and attenuation, but also on the acoustic impedance of the medium. Examination of the physics incorporated into Biot Theory shows that the ${ }^{3}$ effective density ${ }^{2}$ seen by the acoustic wave is lower than the bulk density, thus lowering the acoustic impedance. This results in a difference in the predicted flat surface reflection coefficient for Biot-type models as compared to grain-shearing models. The flat surface reflection coefficients derived from experiment will be compared with predictions using the Biot model and the viscosity grain shearing (VGS) model for a sand sediment. The validity of obtaining reflection coefficients using forward scattering from rough surfaces will also be discussed. [Work supported by ONR.] 


\title{
Session 2pUWf
}

\section{Underwater Acoustics, Signal Processing in Acoustics, and ECUA: Acoustic Vector Fields and Sensor Processing I (Poster Session)}

\author{
Kevin Smith, Cochair \\ Naval Postgraduate School/Naval Undersea Warfare Center \\ Jean-Pierre Hermand, Cochair \\ Université libre de Bruxelles (U.L.B.) - Environmental hydroacoustics lab
}

\begin{abstract}
All posters will be on display from 3:40 p.m. to 5:20 p.m. To allow contributors an opportunity to see other posters, contributors of odd-numbered papers will be at their posters from 3:40 p.m. to 4:30 p.m. and contributors of even-numbered papers will be at their posters from 4:30 p.m. to 5:20 p.m.
\end{abstract}

\section{Contributed Papers}

2pUWf1. Vector-phase methods of bottom reflecting properties research in shallow shelf area. Elena Ermolaeva (Moscow Lomonosov State University, Leninskie Gory, Physics Faculty, 119992 Moscow, Russian Federation, ermolaeva@phys.msu.ru), Boris Goncharenko (Moscow Lomonosov State University, Leninskie Gory, Physics Faculty, 119992 Moscow, Russian Federation, goncharenko@phys.msu.ru), Valery Gordienko (Moscow Lomonosov State University, Leninskie Gory, Physics Faculty, 119992 Moscow, Russian Federation, gordienko@phys.msu.ru)

It is known, that parameters of bottom have essential influence on the signal propagation in the shallow water. Variation of the attenuation coefficient can amount to tens $\mathrm{dB}$. Therefore, research of bottom properties and its structure (first of all - thickness and acoustics parameters of alluvial soil) is actual problem, especially at low frequencies. Using of receivers, which record both sound pressure and its gradient make it easier to solve this problem in a number of cases. The following methods are considered: impedance method, which is based on direct measurement of relation between pressure and vertical component of oscillatory velocity in one point on the water/ bottom boundary; method, which is based on measuring phase difference between pressure and horizontal or vertical component of particle velocity; method in which additive combination of sound pressure and vertical component of oscillatory velocity are used. The essence of the last method consists in formation of minima of reception system directional characteristic in the direction at the signals extending from surface to bottom and reflected from bottom. The suggested methods of acoustic characteristics research of a ground repeatedly have been checked in shallow shelf area of the Baltic and the Black seas, and also in conditions of shallow inland water areas.

2pUWf2. Geoacoustic Inversion Based on a Vector Hydrophone Array. Fenghua Li (National Laboratory of Acoustics, Institute of Acoustics, Chinese Academy of Sciences, No 21, Beisihuanxilu, 100080 Beijing, China, lfh@mail.ioa.ac.cn), Renhe Zhang (National Laboratory of Acoustics, Institute of Acoustics, Chinese Academy of Sciences, No 21, Beisihuanxilu, 100080 Beijing, China, zrh@mail.ioa.ac.cn)
Vector sensor has attracted much attention in recent years. However, there are few papers involving in geoacoustic inversion from vector sensor. In this research, a geoacoustic inversion scheme employing a vector hydrophone array has been developed based on the fact that vector hydrophone can provide more acoustic field information than traditional pressure hydrophones. The inversion scheme is the combination of the Matched Field Processing and the difference of transmission losses between pressure and velocity. The advantages of this method are that it can decrease the uncertainty of the inverted sediment sound speed and the inversion of the sediment attenuation is independent on the source level. [Work supported by the National Natural Science Foundation of China under Grand No. 10574136].

2pUWf3. Underwater low frequency sound field simulation with the digital waveguide mesh method. Shengchun Piao (College of Underwater Acoustic Engineering, Harbin Engineering University, 145 Nantong Street, 150001 Harbin, China, shengchunpiao@hrbeu.edu.cn), Lingai Tian (Hangzhou Applied Acoustics Research Institute, 96 Huaxing Road, 310012 Hangzhou, China, tianling_ai@ @rbeu.edu.cn), Jing Yang (College of Underwater Acoustic Engineering, Harbin Engineering University, 145 Nantong Street, 150001 Harbin, China, bambooyang7@163.com)

Digital waveguide mesh method has been widely used to model musical instruments and simulation for room acoustics. In this paper, this method was applied to calculation the acoustic vector fields in the Pekeris waveguide. After introducing a boundary condition treatment method for the ocean bottom in the digital waveguide mesh, the acoustic pressure and particle velocity fields are calculated in spatial and temporal dimensions. Using these calculation results, the waveforms for the received signals and distribution of acoustic intensity in the underwater sound channel can also be obtained. Numerical simulation shows that the digital waveguide mesh method can be used to simulate the low frequency two-dimensional acoustic vector fields in shallow water and this method is easy to applied to threedimensional acoustic fields calculation. 


\title{
Session 2pUWg
}

\section{Underwater Acoustics and ECUA: High Frequency Variability I (Poster Session)}

\author{
Marcia Isakson, Cochair \\ Applied Research Laboratories, University of Texas \\ Thomas Folégot, Cochair \\ NATO Undersea Research Center
}

\begin{abstract}
All posters will be on display from 3:40 p.m. to 5:20 p.m. To allow contributors an opportunity to see other posters, contributors of odd-numbered papers will be at their posters from 3:40 p.m. to 4:30 p.m. and contributors of even-numbered papers will be at their posters from 4:30 p.m. to 5:20 p.m.
\end{abstract}

\section{Contributed Paper}

2pUWg1. Correcting bathymetry measurements for water sound speed effects using inversion theory. Jelle De Plaa (Delft Institute of Earth Observation and Space Systems, Delft University of Technology, Kluyverweg 1, 2629 HS Delft, Netherlands, j.deplaa@tudelft.nl), M. Snellen (Delft Institute of Earth Observation and Space Systems, Delft University of Technology, Kluyverweg 1, 2629 HS Delft, Netherlands, M.Snellen@tudelft.nl), Dick G. Simons (Delft University of Technology, P.O. Box Postbus 5048, 2600 GA Delft, Netherlands, d.g.simons@tudelft.nl)

We present a method to accurately estimate the bathymetry and water sound speed in shallow waters using overlapping swaths obtained from a Multi-Beam Echo Sounder (MBES). The method is designed to correct the errors in the bathymetry manifested mainly in the outer beams of the MBES.
The errors are caused by deviations in sound speed, which occur, for example, in estuaries where fresh river water mixes with seawater. Simulations show that we are able to simultaneously estimate the mean sound speed and the bathymetry. This is accomplished by minimizing the differences between the MBES measurements in the overlap region using a LevenbergMarquardt optimization routine. We also present an application of the method to real data obtained at the fairway to the harbour of Rotterdam, The Netherlands. Analysis shows that the inverted sound speed agrees well with the measured sound speed at the transducer. However, the uncertainties are larger when the track-to-track distance is $\sim 2$ times the depth. Therefore, we also discuss the optimal distance between sailed tracks. The method appears to be a promising tool for the accurate mapping of sea floors in shallowwater areas with a complex sound-speed profile. 